The Use of Synthetic Colloids in

Tracer Transport Experiments in

Saturated Rock Fractures

Paul William Reimus 



\section{DISCLAIMER}

This report was prepared as an account of work sponsored by an agency of the United States Government. Neither the United States Government nor any agency thereof, nor any of their employees, make any warranty, express or implied, or assumes any legal liability or responsibility for the accuracy, completeness, or usefulness of any information, apparatus, product, or process disclosed, or represents that its use would not infringe privately owned rights. Reference herein to any specific commercial product, process, or service by trade name, trademark, manufacturer, or otherwise does not necessarily constitute or imply its endorsement, recommendation, or favoring by the United States Government or any agency thereof. The views and opinions of authors expressed herein do not necessarily state or reflect those of the United States Government or any agency thereof. 


\section{DISCLAIMER}

Portions of this document may be illegible in electronic image products. Images are produced from the best available original document. 


\section{ACKNOWLEDGMENTS}

Many individuals and organizations are gratefully acknowledged for their financial, technical, and emotional support, which enabled me to complete this dissertation. Financial support was provided by the U. S. Department of Energy through the Yucca Mountain Site Characterization Project at Los Alamos National Laboratory. Special thanks are due to Bruce Robinson of Los Alamos, who had direct oversight and ultimate responsibility for the work. He supported the effort from inception to completion, including serving as a key dissertation committee member. I also wish to thank my advisor, Eric Nuttall of the University of New Mexico, for his guidance, gentle pushing, and flexibility in accommodating unconventional circumstances in a dissertation project. The participation and contributions of all my dissertation committee members are greatly appreciated.

Others at Los Alamos whom I would like to thank include: Joe Ladish and John Hererra for making the research possible by establishing a new Los Alamos program for Ph.D. candidates; Phil Thullen, Harold Sullivan, Tom Hirons, Julie Canepa, and Wes Myers for advocating and/or approving my participation in the Ph.D. program; Ed Essington, Darlene Linzey, Everett Springer, and Wayne Hansen for providing laboratory space for the tracer experiments as well as laboratory training, orientation, and advice; Brent Newman for his assistance in obtaining the two fractured core samples from the Nevada Test Site; Robb Habbersett for providing training and assistance in the use of the flow cytometer for fluorescent microsphere analyses; Barbara Carlos for conducting a qualitative mineralogical characterization of the fracture surfaces; Ines Triay for providing technical advice and reviews; Chuck Cotter and Robert Lopez for obtaining the J-13 well water for the tracer experiments, Jose Olivares for conducting the inductively coupled plasma-mass spectrometry analyses of cations in the J-13 water; Jay Thorne for machining the aluminum parts for the fracture flow systems; Dave Mann for offering the use of his rock saws and grinders to prepare the fractures for the experiments; Kelly Tapia and Cindy Sandoval for preparing the graphics for the final manuscript; and Donna Williams, Lyle Wichman, and Steve Bolivar for their assistance with the quality assurance requirements of the Yucca Mountain Site Characterization Project.

Individuals from outside Los Alamos whom I wish to thank include: Bob Glass of Sandia National Laboratories for helping design the fracture flow apparatuses and making the laser profiling of the Bandelier tuff fracture possible; Stephen Brown and Bob Hardy of Sandia for conducting the laser profiling; Mike Nicholl of Sandia for conducting the light transmittance imaging of the RTV silicone cast of the Bandelier tuff fracture void space; and Bill Durham of Lawrence Livermore National Laboratory for providing training and assistance in the use of the PEAK stylus profiler to obtain profiles of all the fracture surfaces. 



\section{CONTENTS}

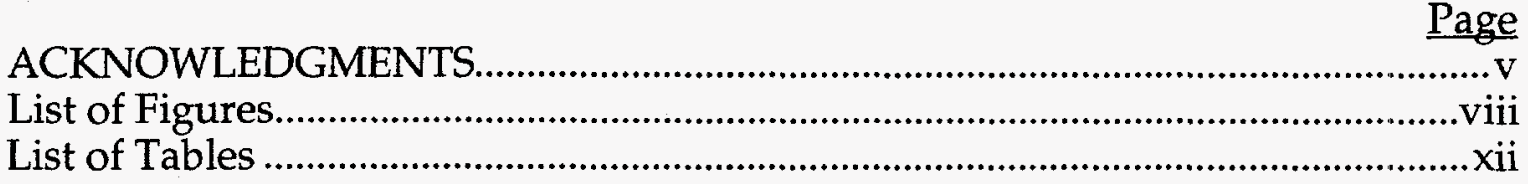

Chapter

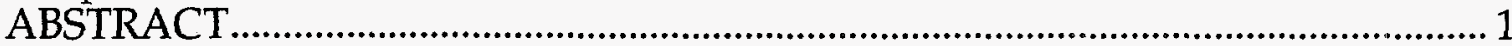

1.0 INTRODUCTION

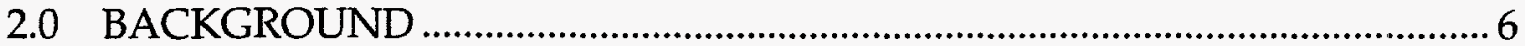

2.1 Colloidal Contaminant Transport............................................................... 6

2.2 Tracer Transport Experiments (with Emphasis on Fractured Media).... 7

2.3 Synthetic Colloids as Tracers ......................................................................... 9

2.4 Existing Models of Colloid Transport in Fractured Media ...................... 10

2.5 Saturated Flow in Natural Fractures ............................................................. 11

2.5.1 The Continuity and Navier-Stokes Equations.................................. 11

2.5.2 Parallel Plates and the Reynolds Equation........................................ 13

2.6 Colloid Transport in Flow Fields ............................................................. 15

2.6.1 Particle Trajectories ............................................................................ 16

2.6.2 Hydrodynamic Corrections Near Walls ............................................. 20

2.6.3 Species Continuity Equation................................................................. 20

2.6.4 Solute Transport vs. Colloid Transport in Fractures......................... 23

2.7 Colloid Interactions with Surfaces......................................................... 23

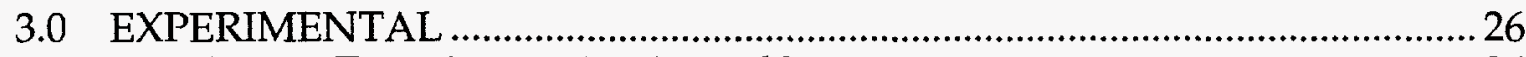

3.1 Tracer Experiments in Natural Fractures ................................................26

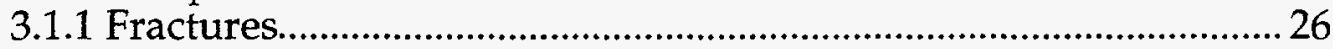

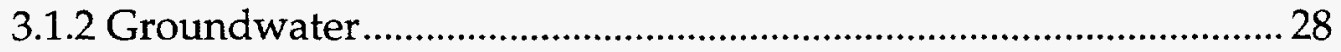

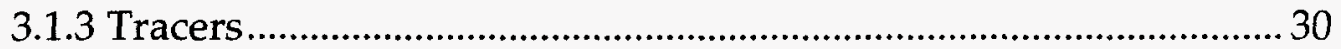

3.1.4 Tracer Analytical Methods.................................................................. 31

3.1.5 Screening of Tracer-Rock-Water Interactions.....................................33

3.1.5.1 J-13 Water Chemistry after Contact with Microspheres... 33

3.1.5.2 Zeta Potential Measurements .................................................34

3.1.5.3 Batch Sorption Measurements of Iodide on Tuffs ............... 36

3.1.6 Tracer Experiments: Apparatus and Procedure .............................. 36

3.1.6.1 Fracture Flow Apparatus ......................................................... 36

3.1.6.2 Experimental Flow System............................................................ 39

3.1.6.3 Experimental Procedure ............................................................ 43

3.1.6.4 Minor Tests to Support Tracer Experiments ........................ 48

3.1.7 Tracer Experiments: Discussion of Results....................................... 49

3.2 Estimation of Aperture Distributions in Fractures......................................5 55

3.2.1 Laser Surface Profiling...................................................................... 55

3.2.2 RTV Silicone Casting and Imaging of the Fracture Void Space ... 56

3.2.3 Mechanical Stylus Profiling of Surfaces ............................................... 58

3.2.4 Aperture Characteristics and Comparison of Measurement

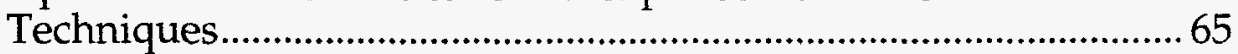

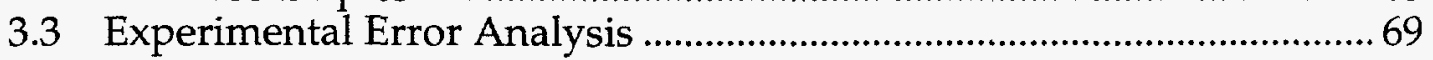

4.0 MODELING AND INTERPRETATION OF EXPERIMENTS...........................73

4.1 Flow Simulations to Estimate Flow Fields in Fractures.............................73 
4.2 Tracer Transport Simulations using Particle-Tracking Methods ...........77

4.2.1 Fluid Residence Time Distributions .....................................................93

4.2.2 Solute Transport without Matrix Diffusion....................................... 94

4.2.3 Solute Transport with Matrix Diffusion..............................................99

4.2.4 Solute Transport with Stagnant Zone along Fracture Walls....... 111

4.2.5 Colloid Transport ............................................................................... 113

4.2.6 Variations in Colloid Particle-Tracking Calculations..................... 116

4.2.7 Verification of Particle-Tracking Models ......................................... 120

4.3 Results of Particle-Tracking Simulations .................................................. 122

4.3.1 Use of Model Predictions to Interpret Experimental

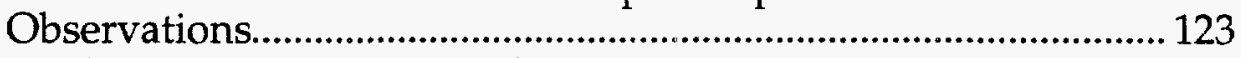

4.3.2 Implications for Larger-Scale Tracer Experiments ........................ 130

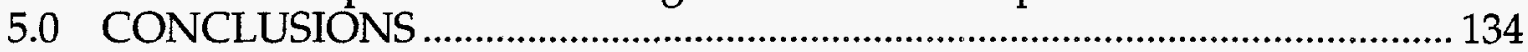

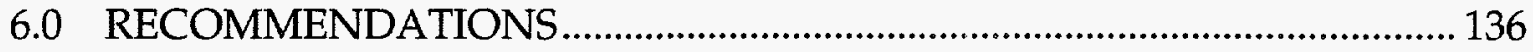

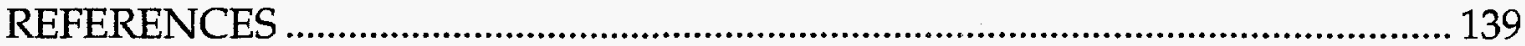

APPENDIX A: SUMMARY OF TRACER EXPERIMENTS ...................................... A-1

APPENDIX B: BREAKTHROUGH CURVES FROM TRACER

EXPERIMENTS..........................................................................................

APPENDIX C: BREAKTHROUGH CURVES AT DIFFERENT FLOW

RATES IN SAME FRACTURE..............................................................

APPENDIX D: COMPARISON OF PARTICLE-TRACKING MODEL

PREDICTIONS AND EXPERIMENTAL DATA ……………………........... D-1

\section{LIST OF FIGURES}

1.1. Depiction of a colloid moving through a saturated natural fracture............ 4

2.1. Hypothetical inlet and outlet concentrations for a pulse input and a step input in a tracer experiment.

2.2. Hydrodynamic correction functions vs. dimensionless surface separation $(H=h / R)$ for a spherical particle near a planar surface

2.3. Depiction of Taylor dispersion and non-Taylor dispersion trajectories and the breakthrough curves resulting from these two transport regimes.

3.1. Top view of aluminum confining plate for fracture flow apparatus...........38

3.2. Side view of fracture flow apparatus showing method of achieving leak-free seal on side of fracture block (for no-flow boundaries..

3.3. Flow apparatus configuration over end of fracture block showing channel cut in gasket material to serve as a flow manifold underneath aluminum bar stock

3.4. Schematic of overall flow system for tracer experiments.............................. 40

3.5. Photograph of flow system just prior to a tracer experiment ....................... 41 
3.6. Simplified schematic of experimental flow system showing only flow into and out of fracture flow apparatus

3.7. Breakthrough curves of 1- $\mu \mathrm{m}$ CML microspheres in the different fractures as a function of void volumes eluted before the damage to the Bullfrog tuff fracture surfaces 54

3.8. Breakthrough curves of 1- $\mu \mathrm{m}$ CML microspheres in the different fractures as a function of void volumes eluted after the damage to the Bullfrog tuff fracture surfaces

3.9. Photograph of PEAK surface profiling instrument showing the Bandelier tuff fracture mounted on the table

3.10. Frequency distributions (histograms) of aperture data sets associated with different fractures. The distributions are normalized so that all histograms have the same area underneath the curve.

3.11. Frequency distributions (histograms) of Bandelier tuff fracture aperture data sets deduced from different aperture measurement techniques. The distributions are normalized so that all histograms have the same area underneath the curve.

3.12. Depiction of a PEAK stylus tip hitting a rough fracture surface.

4.1. Bandelier tuff fracture aperture data set deduced from laser profiling technique and flow field calculated using the Reynolds equation with this aperture data set.

4.2. Bandelier tuff fracture aperture data set deduced from RTV cast/imaging technique and flow field calculated using the Reynolds equation with this aperture data set

4.3. Bandelier tuff fracture aperture data set deduced from first PEAK profile of this fracture and flow field calculated using the Reynolds equation with this aperture data set.

4.4. Bandelier tuff fracture aperture data set deduced from second PEAK profile of this fracture and flow field calculated using the Reynolds equation with this aperture data set.

4.5. Tram tuff fracture aperture data set deduced from PEAK profiling technique and flow field calculated using the Reynolds equation with this aperture data set.

4.6. Bullfrog tuff fracture aperture data set deduced from PEAK profiling technique and flow field calculated using the Reynolds equation with this aperture data set. 
4.7. Rotated Bullfrog tuff fracture aperture data set deduced from PEAK profiling technique and flow field calculated using the Reynolds equation with this aperture data set.

4.8. Predicted fluid residence times in the different fractures as a function of void volumes eluted (based on 2000 particles in the particle-tracking simulations)

4.9. Predicted fluid residence times in the Bandelier tuff fracture using different aperture data sets (based on 2000 particles in the particletracking simulations)

4.10. Illustration of particle trajectory in particle-tracking model when crossing a node boundary

4.11. Depiction of particle movement in a parallel-plate channel according to the particle-tracking model.

4.12. Illustration of the assumed transition from fracture void space to matrix in the particle-tracking model(s) that include matrix diffusion.... 101

4.13. Residence time distributions for particles moving away from a porous wall assuming different transition layer thicknesses.

4.14. Predicted iodide breakthrough curves (based on 1000 particles) in Bandelier tuff fracture assuming (a) nonporous walls and the following transition layer thicknesses: (b) $0.002 \mathrm{~mm}$, (c) $0.025 \mathrm{~mm}$, (d) $0.2 \mathrm{~mm}$, (e) $0.5 \mathrm{~mm}$, (f) $1.5 \mathrm{~mm}$, (g) $5.0 \mathrm{~mm}$, and (h) $\infty$ thickness.

4.15. Predicted iodide breakthrough curves (based on 1000 particles) in Bandelier tuff fracture assuming probabilities of (a) 0.089 and (b) 0.5 of encountering a pore when a particle "hits" a wall

4.16. Illustration of the two-layer model describing the transition from fracture void space to matrix

4.17. Residence time distributions for particles moving away from a wall using different assumptions in the two-layer model shown in Fig. 4.16

4.18. Predicted iodide breakthrough curves (based on 1000 particles) in Bandelier tuff fracture assuming the following overlying layer thicknesses in the two-layer model of Fig. 4.16:

(a) $0.0 \mathrm{~mm}$, (b) $0.01 \mathrm{~mm}$, (c) $0.02 \mathrm{~m}$

4.19. Predicted iodide breakthrough curves (based on 1000 particles) in Bandelier tuff fracture using the two-layer model with a transition layer thickness of $0.01 \mathrm{~mm}$ and an overlying layer thickness of $0.02 \mathrm{~mm}$ 
4.20. Predicted iodide breakthrough curves (based on 1000 particles) in Bandelier tuff fracture using the following models:

(a) particle-tracking with a transition layer thickness of $0.01 \mathrm{~mm}$,

(b) particle-tracking with a transition layer thickness of $0.5 \mathrm{~mm}$, and $(\mathrm{c})$ the Starr method

4.21. Depiction of stagnant layers in solute particle-tracking model

4.22. Predicted iodide breakthrough curves (based on 1000 particles) in Bandelier tuff fracture assuming the following stagnant layer thicknesses: (a) $0.0 \mathrm{~mm}$, (b) $0.005 \mathrm{~mm}$, and (c) $0.01 \mathrm{~mm}$.

4.23. Predicted colloid breakthrough curves (based on 1000 particles) in a parallel-plate channel assuming (a) no hydrodynamic corrections and no gravitational settling, (b) hydrodynamic corrections but no gravitational settling, and (c) hydrodynamic corrections and gravitational settling

4.24. Illustration of possible particle trajectory when a narrow aperture region widens into a large aperture region.....

4.25. Depiction of a colloid moving near a fracture wall showing regions that colloids may be excluded from.

4.26. Depiction of exclusion layers in colloid particle-tracking model

4.27. Predicted 1- $\mu \mathrm{m} C \mathrm{CML}$ microsphere breakthrough curves in Bandelier: tuff fracture using different particle-tracking models/assumptions: (a) standard model described in Section 4.2.5, (b) model without hydrodynamic corrections or gravitational settling, (c) model that assumes "mixing" after crossing each node boundary, (d) model that assumes colloids only enter adjacent nodes (with flow into them) of largest aperture, (e) model with $0.005-\mathrm{mm}$ exclusion layer along walls, (f) model with $0.01-\mathrm{mm}$ exclusion layer along walls, and (g) model with $0.005-\mathrm{mm}$ exclusion layer but with particles maintaining same relative distance from actual walls instead of effective walls when crossing node boundaries

4.28. Comparison of predicted breakthrough curves in a parallel-plate channel using finite-difference and particle-tracking models

4.29. Predicted iodide breakthrough curves (based on 1000 particles) in the Bandelier tuff fracture using variable-aperture and parallel-plate models.

4.30. Predicted and experimental flow-rate dependence of iodide breakthrough curves in Bandelier tuff fracture 
4.31. Predicted and experimental size dependence of CML microsphere breakthrough curves in Bandelier tuff fracture

4.32. Predicted and experimental flow-rate dependence of $0.3-\mu \mathrm{m} \mathrm{CML}$ microsphere breakthrough curves in Bandelier tuff fracture

4.33. Comparison of (a) fluid residence time distribution in Bandelier tuff fracture predicted using aperture data and calculated flow field from laser profiling technique, (b) predicted 1- $\mu \mathrm{m}$ microsphere breakthroucurve (assuming a 0.005 -mm exclusion layer along the fracture walls) using the aperture data and calculated flow field from the first PEAK profile of the Bandelier tuff fracture, and (data) the experimental 1- $\mu \mathrm{m}$ CML microsphere breakthrough curve.

4.34. Comparison of (a) predicted iodide breakthrough curve in the Bandelier tuff fracture assuming a 0.005-m.m stagnant layer along fracture walls and using the aperture data and calculated flow field from the RTV cast/imaging technique, (b) predicted iodide breakthrough curve assuming no stagnant layers and using the aperture data and calculated flow field from the first PEAK profile of the Bandelier tuff fracture, and (data) the experimental iodide breakthrough curve

4.35. Predicted breakthrough curves (based on 1000 particles) of nondepositing 1- $\mu \mathrm{m}$ colloids and a nonsorbing solute over different length scales in a parallel-plate channel.

4.36. Predicted breakthrough curves of a nonsorbing solute over different length scales in a parallel-plate channel using the particle-tracking and Starr methods

\section{LIST OF TABLES}

3.1 Results of Tuff Matrix Porosity Measurements ………...................................28

3.2 J-13 Well Water Chemistry Reported by Ogard and Kerrisk.......................... 29

3.3 Partial Analysis of J-13 Water over a Seven-Month Period............................. 30

3.4 Properties of Polystyrene Microspheres used as Tracers ................................31

3.5 Partial Analysis of J-13 Water after Contact with Microspheres....................34

3.6 Results of Mobility / Zeta Potential Measurements ............................................... 35

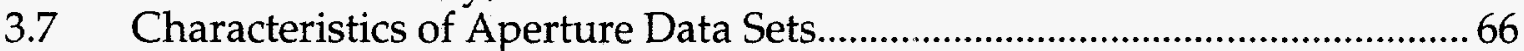

3.8 Relative Standard Deviations of Flow Rates and Normalized Concentrations in the Tracer Experiments ..................................................72

$4.1 \quad$ Measures of Convergence of Flow Simulations............................................... 76

4.2 Calculated and Experimental Hydraulic Apertures ........................................ 76 


\title{
THE USE OF SYNTHETIC COLLOIDS IN TRACER TRANSPORT EXPERIMENTS IN SATURATED ROCK FRACTURES
}

by

\author{
Paul William Reimus
}

\begin{abstract}
Laboratory-scale tracer transport experiments involving $1.0-$ and $0.3-\mu \mathrm{m}$ diameter microspheres and a nonsorbing solute (iodide) were conducted in three different well-characterized natural fractures in tuff. The objectives of the experiments were to

(1) identify a synthetic colloid that could serve as a nondepositing colloid tracer in field-scale tracer experiments in saturated, fractured media;

(2) investigate the potential for using nondepositing colloids to determine how much of the observed dispersion of a nonsorbing solute in fractured media can be attributed to diffusion into the pores of the rock matrix; and

(3) obtain a better understanding of colloid transport in saturated fractures under conditions of minimal deposition, thereby establishing a basis for conducting more thorough studies of the transport of depositing colloids in such systems.

It was determined that carboxylate-modified polystyrene latex (CML) microspheres exhibited less attenuation in the fractures than either polystyrene microspheres with carboxyl surface groups (unmodified latex) or silica microspheres. Thus, the CML microspheres were identified as the best candidate colloid tracer for field-scale experiments, and they were used in all subsequent laboratory experiments in the fractures.

In the tracer experiments, the CML microspheres always arrived earlier in the effluent than the iodide, which can be attributed to (1) Taylor dispersion phenomena and/or (2) the fact that the colloids experienced a smaller effective volume in the fractures because they diffused too slowly to enter low-velocity regions (stagnant zones) along the rough fracture walls. The iodide also approached the inlet concentration in the effluent more slowly than the microspheres, with the concentration at a given elution volume being greater at higher flow rates. By contrast, the rate of approach of the microsphere concentration to the inlet concentration did not vary with flow rate. This behavior is attributed to matrix diffusion of the iodide, with the microspheres being too large/nondiffusive to experience this
\end{abstract}


phenomenon. Dispersion of all tracers was greatest in the fracture of widest average aperture and least in the fracture of narrowest aperture, which is consistent with Taylor dispersion theory.

The tracer experiments were modeled/interpreted using a three-step approach that involved (1) estimating the aperture distribution in each fracture using various experimental measurement techniques, (2) predicting the flow field in the fractures using the deduced aperture distributions, and (3) predicting tracer transport in the fractures using particle-tracking techniques. It was concluded that the best conceptual model for the microspheres involved excluding these tracers from a thin layer of fluid along the fracture walls, and the best conceptual model for the iodide involved adding a stagnant layer of fluid along the fracture walls (to increase the effective volume accessible to the iodide). Iodide was also allowed to diffuse into and out of the porous rock matrix, whereas the microspheres were excluded from the matrix.

The principal implications of the experiments and the modeling exercises were (1) the use of nondepositing colloids in large- or field-scale tracer experiments should provide estimates of mobile fluid residence times in fractured systems, thereby allowing the effects of matrix diffusion of solutes and/or the attenuation of depositing colloids to be studied by comparing the breakthrough curves of these tracers to the breakthrough curves of the nondepositing colloids, and (2) the transport behavior of nonsorbing solutes over large scales in fractured media (with porous matrices) is expected to be dominated by the attenuating effects of matrix diffusion. 


\subsection{INTRODUCTION}

Studies of groundwater flow and contaminant transport in saturated, fractured geologic media are of great interest to researchers studying the potential long-term storage of hazardous wastes in or near such media. A popular technique for conducting such studies is to introduce tracers having different chemical and physical properties into a system and then observe the tracers at one or more downstream locations, inferring flow and transport mechanisms from the breakthrough characteristics of the different tracers. Many tracer studies have been conducted in saturated, fractured media to help develop and/or refine models capable of predicting contaminant transport over large scales in such media. ${ }^{1-10}$

Although it is often assumed that contaminants are mobile in groundwater systems only as solutes, there is considerable evidence that contaminants that exist as colloids (either as true colloids or as solutes adsorbed to natural or anthropogenic colloidal material) can contribute significantly to and even dominate the overall contaminant mass flux in certain systems. ${ }^{11-24}$ Colloids are a special concern in saturated, fractured hydrogeologic systems because they may be excluded from an important mechanism of retardation that applies to solutes: diffusion into the stagnant pores of the rock matrix (or "matrix diffusion"). ${ }^{25-28}$ While most of the groundwater flow in fractured systems occurs in the fractures, most of the groundwater volume is likely to be contained in the matrix and/or in other stagnant regions in the system. ${ }^{29}$ The hydrodynamic dispersion resulting from diffusion of solutes into the matrix and/or these other stagnant regions can cause significant retardation over large length scales25-28 (to be distinguished from chemical retardation in which solutes adsorb to rock surfaces).

Colloids, being large and non-diffusive relative to solutes, are unlikely to experience matrix diffusion or diffusion into other stagnant regions in fractured systems because of size exclusion from the pores as well as a tendency to remain in fluid streamlines rather than collide with the fracture walls. ${ }^{30,31}$ The tendency to remain in fluid streamlines can result in a significant fraction of the total colloid mass moving through a system even more rapidly than the average water velocity in the fractures. ${ }^{31}$ Of course, such enhanced transport can occur over large scales only if the colloids do not tend to deposit onto the rock surfaces. 30,31 A depiction of a colloid moving through a saturated fracture is shown in Fig. 1.1 (some of the forces causing colloid motion are also depicted). The rock matrix is shown with large macropores on its surfaces that may be considered surface roughness features, and it also has a fine pore structure that permeates the entire matrix. Although solutes have access to the entire pore system, colloids may be partially excluded from the macropores (stagnant regions along the fracture walls), and they should be completely excluded from the fine pores.

While the possibility of rapid colloid transport poses significant challenges for performance assessments of potential geologic waste storage/disposal sites in saturated, fractured media, it also suggests an opportunity to conduct more thorough tracer transport studies to characterize such systems. Colloids that 


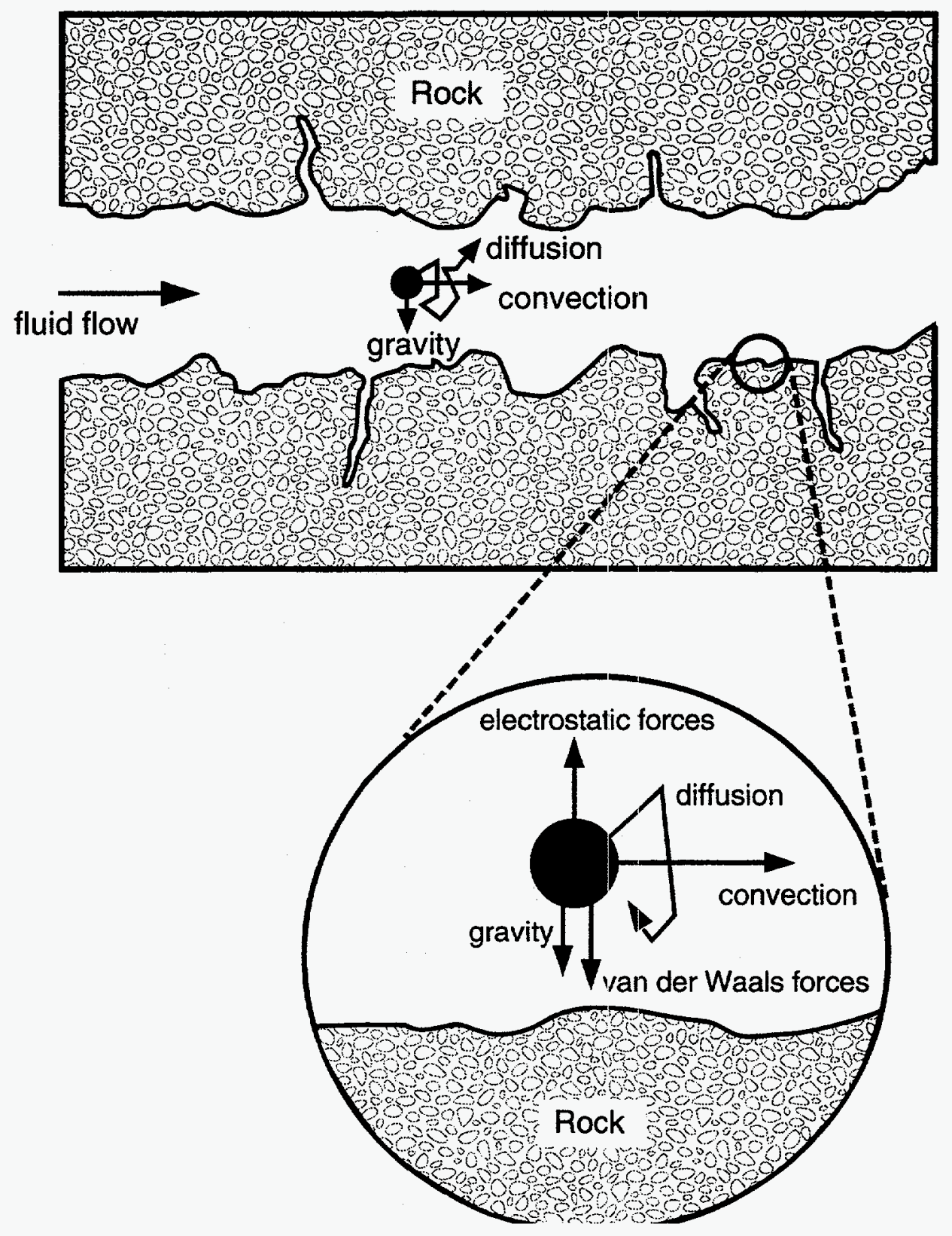

Figure 1.1. Depiction of a colloid moving through a saturated natural fracture. Some of the forces that influence colloid motion are shown. Van der Waals and electrostatic forces (as well as other forces discussed in Section 2.7) are important only when colloids are very close to fracture surfaces (separation distance of much less than one radius).

have unfavorable deposition interactions with the matrix and that can be readily distinguished from natural colloids could, in principle, be used as tracers in conjunction with solutes to provide a means of estimating how much of the observed solute dispersion is due to matrix diffusion and/or diffusion into other stagnant regions in the fracture system. That is, the difference between the 
transport behavior of a nondepositing colloid and a nonsorbing solute could be attributed, at least in part, to solute matrix diffusion or diffusion into stagnant regions. Nondepositing colloids could also be used to determine the amount of pure hydrodynamic dispersion of colloids in a system under conditions of minimal deposition. This would allow the influence of deposition, gravitational settling, coagulation, and perhaps other phenomena associated with real colloids to be studied (again, by observing the difference between the breakthrough behavior of the nondepositing colloid and some other colloid of interest).

Thus, the information derived from tracer studies involving nondepositing colloids could potentially be used in performance assessments to provide a defensible basis for taking credit for (1) solute matrix diffusion and/or diffusion into other stagnant regions in fractured systems, and (2) attenuation/ retardation of colloidal contaminants in fractured systems. These two possibilities inspired the work described in this dissertation. The specific objectives of the research were:

(1) To conduct laboratory-scale tracer experiments in single saturated tuff fractures using various synthetic colloids and a nonsorbing solute to (a) identify a synthetic colloid that is suitable to use as a nondepositing colloid tracer in larger-scale experiments in fractured media, (b) demonstrate the use of nondepositing colloids as tracers in fractured systems to study the effects of solute matrix diffusion or diffusion into other stagnant regions, and (c) provide insights into colloid transport in saturated fractured media; and

(2) To attempt to model/interpret the tracer experiments in the fractures using a three-step approach that involves (a) estimating the aperture distribution in each fracture using various experimental techniques, (b) predicting the flow fields in the fractures (given the aperture distributions) using a localized parallel-plate approximation to solve the Navier-Stokes and continuity equations, and (c) predicting tracer transport in the fractures (given the flow fields) using particle-tracking techniques. 


\subsection{BACKGROUND}

A "colloid" is a substance in the form of finely divided particles that fall roughly within a size range of $1 \mathrm{~nm}$ to just over $1 \mu \mathrm{m}$ (characteristic length scale, typically a diameter or radius). Within the colloid size range, the behavior of particles is strongly influenced by surface properties (e.g., surface electrical charge density, surface chemical groups). However, colloids are small enough to also be influenced by forces generally associated with molecules (e.g., van der Waals forces, Brownian motion/diffusion, migration in an electrical field), and large enough to be influenced by body forces (e.g., gravity, fluid drag). Below 1 $\mathrm{nm}$, the physical and chemical behavior of colloids begin to approach that of large molecular species, and above 5-10 $\mu \mathrm{m}$, colloids are large enough that body forces begin to dominate their behavior.

\subsection{Colloidal Contaminant Transport}

When discussing colloidal contaminant transport, it is useful to distinguish between "true colloids", which consist of a polymerized or crystallized chemical form of the contaminant, ard "psuedocolloids", which consist of natural or anthropogenic colloids that have contaminants adsorbed/attached to their surfaces. ${ }^{32,33}$ True colloids form when chemical conditions are conducive to polymerization or crystallization of compounds containing the contaminant. Natural colloids are ubiquitous in essentially all natural groundwaters, and they can include both organic and inorganic species. Anthropogenic colloids are produced by physical, chemical, or biological processes acting on human-introduced materials in a system (e.g., colloids derived from waste package materials). Natural organic colloids include living entities, such as viruses and bacteria (although the latter are generally at the upper end of the colloid size range) as well as large organic molecules, such as humic or fulvic acids or other complex organic species. Natural inorganic colloids are composed of chemical species present in geologic formations, and include clays and other amorphous or crystalline mineral phases. The mechanism of formation of natural inorganic colloids is unclear (weathering/diagenesis, erosion, precipitation of supersaturated phases are all possibilities), but the fact that they are present in many systems is very evident. ${ }^{32-33}$ The concentration of natural colloids in groundwaters typically ranges from $10^{8}$ to $10^{17}$ particles per liter. ${ }^{32}$ Depending on their concentration, natural or anthropogenic colloids can provide a great deal of surface area for sorption of molecular contaminants. Assuming that such sorption is irreversible (or nearly irreversible), these colloids can facilitate contaminant transport by acting as contaminant carriers. $30-36$

Evidence that colloids can be an important physical/chemical form in which contaminants may be transported through groundwater systems is quite convincing. Means et al. ${ }^{11}$ associated radioactive cobalt and uranium with high molecular weight organic material in groundwater near a disposal trench at Oak Ridge National Laboratory. Killey et al. ${ }^{12}$ associated cobalt- 60 with high molecular weight anionic organic ligands in a contaminant plume at the Chalk River Nuclear Laboratory in Canada. Nyhan et al. ${ }^{13}$ noted colloidal plutonium and americium migration beneath a liquid waste disposal site at Los Alamos National Laboratory. Transition element (manganese, cobalt) and lanthanide (cerium, europium) radionuclides were associated with inorganic colloids in groundwater at the Nevada Test Site by Buddemeier and Hunt. ${ }^{14}$ Penrose et al. ${ }^{15}$ 
have reported that plutonium and americium associated with colloids have migrated distances several orders of magnitude farther (up to $2 \mathrm{~km}$ ) than would be predicted by assuming equilibrium sorption of molecular species in a shallow aquifer at Los Alamos National Laboratory. Payne et al. ${ }^{16}$ reported small but measurable amounts of naturally-occurring radionuclides associated with colloids in the hydrogeologic system of the Koongarra uranium deposit in Australia. Kim et al., in a series of studies, ${ }^{17-20}$ established that actinides associated with colloids (mainly organic) can be readily transported through Gorleben (Germany) aquifer systems.

In the laboratory, Champ et al. ${ }^{21}$ demonstrated rapid groundwater transport of plutonium in core column studies. Saltelli et al.22 observed colloidal americium movement through saturated glauconitic sand columns. Thompson 23 noted colloidal americium eluting through crushed tuff columns more rapidly than tritiated water (though the americium colloid was generally a small fraction of the total americium inventory). Magee et al. ${ }^{24}$ observed that the presence of dissolved organic matter significantly increased the transport rate of phenanthrene through a sand column.

Discussion of many of these studies are provided in review articles by Kim, ${ }^{32}$ McCarthy and Zachara, 34 and Moulin and Ouzounian. ${ }^{35}$ Triay et al.33 have reviewed colloid-facilitated radionuclide transport at the potential highlevel nuclear waste repository at Yucca Mountain, Neveda. Choppin ${ }^{36}$ has reviewed the potential role of humic materials in enhancing radionuclide migration.

\subsection{Tracer Transport Experiments (with Emphasis on Fractured Media)}

Tracer transport studies are usually conducted by introducing either a step or pulse input of tracer(s) into a system and then observing the appearance of the tracer(s) at one or more downstream locations. A plot of tracer concentration vs. time at an observation point is called a breakthrough curve. Hypothetical examples of breakthrough curves for step and pulse inputs are shown in Fig. 2.1. These two breakthrough curves are related in that the response to an ideal impulse function (dirac-delta function) should be the derivative of the response to a step function. 37 Of course, it is not possible to achieve an ideal impulse function experimentally, so a pulse of finite duration is generally used. Laboratory-scale tracer experiments can be conducted with either pulses or steps, but it is usually not practical to conduct large- or fieldscale experiments with steps because of the prohibitive amount of tracer that is needed.

When tracer experiments are carried out using tracers that have different transport characteristics, the differences between breakthrough curves can offer insights into various transport mechanisms in the system. ${ }^{38,39}$ It is customary in tracer experiments to use at least one nonreactive tracer that is assumed to provide a measure of the true hydrodynamic dispersion in a system. In most natural systems, which tend to have negatively-charged surfaces, small anions such as halides or preferably tritiated or deuterated water can serve this purpose. The influence of sorption (as an example) can be deduced by comparing the breakthrough curve of a sorbing tracer with the curve of a nonreactive tracer. In making this comparison, it is assumed that the pure hydrodynamic dispersion of the two tracers is approximately the same, which should be true if the tracers are similar in size and diffusion coefficient (diffusivity). ${ }^{38}$ 

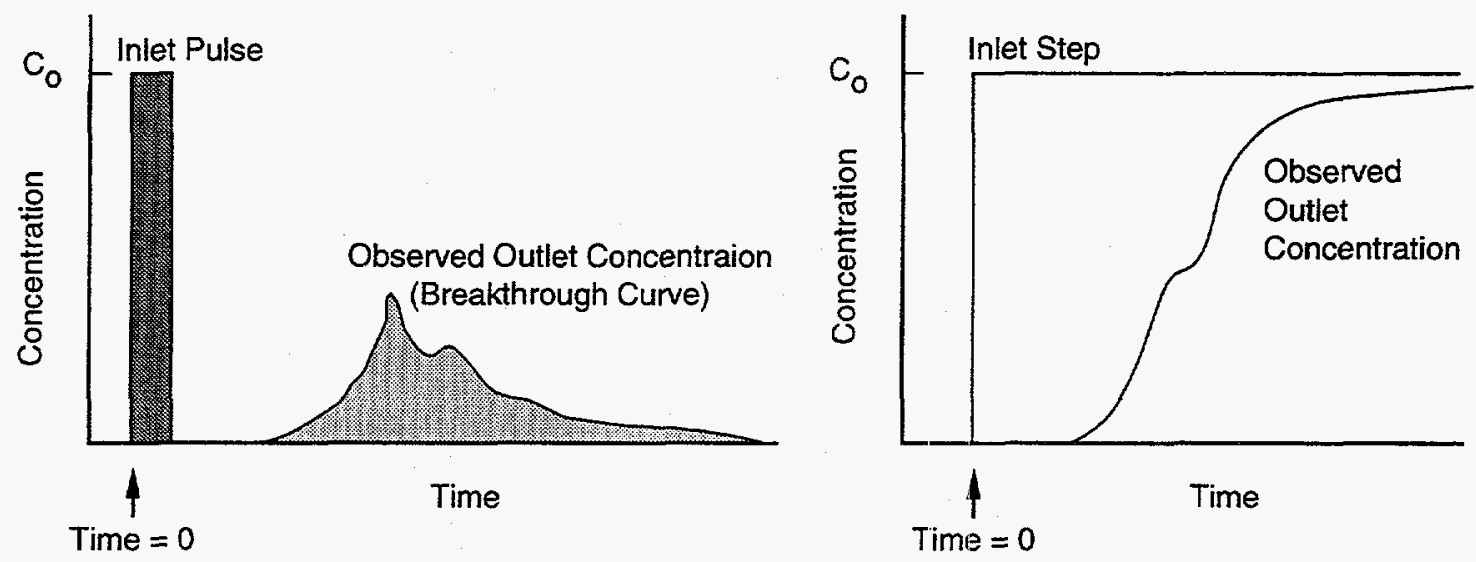

Figure 2.1. Hypothetical inlet and outlet concentrations for a pulse input (left) and a step input (right) in a tracer experiment:.

Although it has long been recognized that diffusion into the rock matrix or into other stagnant regions can significantly retard solute transport in fractured systems, $25-28$ it has been difficult to quantify how much of the observed dispersion of a nonreactive tracer is the result of such processes and how much is the result of other dispersive mechanisms or perhaps incomplete recovery of the tracer. This distinction could be important if credit is to be taken for diffusion into the matrix and/or other stagnant regions in fractured media when performance assessments of potential waste disposal sites are conducted.

One of the problems associated with building a case for matrix diffusion as a mechanism of solute retardation is that there have been a limited number of tracer studies conducted in fractured media, especially in the laboratory. This sparsity of experimental data is at least partially attributable to the fact that it is inherently more difficult to conduct and interpret tracer studies in fractured media than in porous media. Porous media is more easily characterized, more amenable to tracer studies in the field, and it lends itself more readily to packed columns and other idealized geometries in the laboratory than does fractured media.

Neretnieks et al. were among the first to conduct tracer experiments in a single natural fracture in the laboratory. 40 Rundberg and coworkers conducted tracer experiments in both single fractures and small fracture networks in tuff.41-44 Bales et al. used viruses as tracers in experiments in fractured tuff core samples. ${ }^{45}$ Laboratory tracer experiments in natural fractures have also been conducted by Piggott and Elsworth,46 Holtta et al.,47 and Cliffe et al.48 Because of the great current interest in studying transport in fractured systems, many other investigators are currently conducting tracer experiments in laboratory-scale natural fracture systems, but the results of these experiments were not yet documented or published at the time this dissertation was written."

\footnotetext{
* Personal communications in 1993 and 1994 with staff at Los Alamos National Laboratory, Sandia National Laboratory, Lawrence Berkeley Laboratory, U. S. Bureau of Mines, the Canadian Underground Research Laboratory, and researchers in Great Britain.
} 
Field-scale tracer experiments in fractured rock have been conducted by several investigators, including Robinson and Tester ${ }^{49}$ Novakowski et al., ${ }^{1}$ Raven et al., ${ }^{2}$ Shapiro and Nicholas, ${ }^{3}$ Cacas et al., ${ }^{4}$ Abelin et al. (and references therein),, 5 Davison et al., 7 Cady et al., 8 Birgersson et al., ${ }^{9}$ and Novakowski and Lepcevic. ${ }^{10}$ Many of the modeling approaches used to interpret these experiments (including advection-dispersion models, channel models, and channel network models) are reviewed by Moreno and Neretnieks. 50

Maloszewski and Zuber have discussed the concept of using nonreactive solute tracers with different diffusivities to study matrix diffusion in fractured systems. ${ }^{37}$ They have noted that smaller, more diffusive tracers show greater dispersion than larger tracers in fractured media, which they attribute to a greater amount of matrix diffusion of the smaller tracer. It is logical to extend this approach to the extreme of using a tracer that is so large and slowly diffusing that it cannot participate in matrix diffusion at all. The use of a nonreactive colloid tracer (i.e., a colloid with little or no tendency to deposit on rock surfaces) would seem to be an ideal choice. McKay et al.51,52 have, in fact, conducted such a study in a field experiment in "fractured" clay media. They noted that bacteriophages transported through their system many times faster than a nonsorbing solute (the first arrival of bacteriophage was as much as 100 times faster), which they attributed to diffusion of the solute into the pores of the clay matrix. Their work suggests that the use of a nonreactive colloid tracer in conjunction with a nonsorbing solute in tracer studies may allow researchers to estimate how much of the observed dispersion of a solute can be attributed to matrix diffusion.

A secondary benefit of conducting tracer experiments with nonreactive colloid tracers is that the colloid breakthrough curves should help establish the pure hydrodynamic dispersion of colloids in the flow system. This information could then be used to study the influence of colloid-matrix interactions or other physicochemical phenomena (e.g., gravitational settling, coagulation) on the transport of "real" colloids through a system (by comparing the breakthrough curves of the "real" colloids to those of nondepositing colloids). This concept is analogous to studying the transport behavior of reactive solutes by comparing their breakthrough curves to those of nonreactive solutes.

\subsection{Synthetic Colloids as Tracers}

The idea of using colloids as tracers in either laboratory or field experiments is not new. Harvey et al. ${ }^{53,54}$ used both fluorescent-dyed carboxylated polystyrene "microspheres" and fluorescently-tagged bacteria as tracers in field and column studies of bacterial transport through aquifer sediments. O'Melia and coworkers, $, 5,56$ Toran and Palumbo, ${ }^{57}$ Elimelech,, 8 and Wan and Wilson ${ }^{59}$ are among the many researchers that have used polystyrene microspheres in laboratory studies of colloid transport through porous media in packed columns. Thompson ${ }^{23}$ used synthetic plutonium colloid as a tracer in crushed rock column experiments. Rundberg et al. 43 investigated the transport of 3 different-sized fluorescent-dyed carboxylated polystyrene microspheres through a saturated fracture network. Matijevic and coworkers have for many years synthesized inorganic colloids and then used them in column experiments to study deposition under various conditions. ${ }^{60-64}$ Their synthetic colloids have also been used by others in colloid transport studies. ${ }^{65,66}$ Bales and coworkers $45,67-69$ have studied virus and bacteria transport through sandy soil 
columns, columns packed with silica or quartz beads, fractured tuff core samples, and a 30-m-wide wash full of natural se:diments (see also Refs. 51 and 52). Rosta et al..$^{70}$ studied the transport of laponite sols (a synthetic clay material) through columns packed with glass beads, quartz, alumina, and glaciofluvial sediments. Recently, synthetic spherical silica colloids have been used in tracer experiments in both the laboratory and the field, but the results of these studies were not yet published at the time this dissertation was written.*

Certain properties are desirable in a colloid that is to be used as a tracer to study matrix diffusion of solutes and to establish the pure hydrodynamic dispersion of nondepositing colloids in fractured systems. These properties are summarized below:

- The colloid should be approximately spherical, as this greatly simplifies the modeling of the hydrodynamic motion of the colloid in a laminar flow field as well as the modeling of hydrodynamic interactions with surfaces, including both fracture walls and other colloids.

- The colloid should be relatively monodisperse, meaning that the diameters of the particles should have a small standard deviation (5\% or less of the mean). This property allows the transport behavior of all colloids to be treated the same, thus avoiding the complexity of accounting for a population of different-sized particles.

- The colloid should be capable of being detected at very low concentrations because tracers are often highly attenuated in field tests (there may be as much as a factor of $10^{5}$ decrease between the inlet and outlet concentrations of a pulse).

- The colloid should be easily distinguishable from natural colloids that may be present in the system.

- The colloid should be stable against flocculation and against significant attachment to rock surfaces, as both of these phenomena can cause retardation and incomplete recovery of the tracer.

- The colloid should be chemically stable in the suspending solution, meaning that it should not dissolve or grow larger or lose its analytical signal prior to being analyzed.

- The colloid should have near neutral buoyancy to minimize gravitational settling.

Obviously, it may be difficult to satisfy all of these criteria with a single colloid, but to the extent that the criteria can be met, the chances of success in a tracer experiment intended to study matrix diffusion and/or the transport of nondepositing colloids should be maximized.

\subsection{Existing Models of Colloid Transport in Fractured Media}

Few models exist for predicting colloid transport in fractured media, although there is currently much interest in this topic. In keeping with performance assessment objectives, most models have focused on the transport of soluble contaminants in conjunction with mobile colloids to which the solutes are assumed to sorb. $30,31,71$ These models typically assume linear, reversible sorption of solutes to the colloids and to immobile surfaces as well as linear "sorption" of colloids to immobile surfaces. However, Smith and Degueldre ${ }^{30}$

\footnotetext{
* Personal communications in 1993 and 1994 with staff at the University of New Mexico, Los Alamos National Laboratory, the Canadian Underground Research Laboratory, and researchers in Great Britain.
} 
have also considered irreversible sorption of solutes to colloids. Extension of these models to kinetically-controlled sorption of solutes to colloids and/or: colloids to immobile surfaces is, in principle, not difficult; and, in fact, has been done by Corapcioglu and Jiang for porous media. ${ }^{72}$ Castaing ${ }^{73}$ has considered the simultaneous transport of a solute and a colloidal precipitate of the solute in fractures; but aside from different equations describing interactions between the solute and colloids, the resulting model differs very little from the models mentioned above. All of these models are based on the one-dimensional advection-dispersion equation with reaction or equilibrium terms to describe mass transfer between the colloid, solution, and stationary phases (see Eq. 35 in Section 2.6.3, but replace the diffusivity with a "dispersion coefficient" and ignore the torque term). Consequently, these models are mathematically very similar to models that describe simultaneous colloid and solute transport through porous media. ${ }^{72,74}$ In order to realistically simulate differences between colloid and solute transport behavior, all of these models require that different velocities and dispersion coefficients be specified for colloids and solutes. Grindrod ${ }^{31}$ used a colloid velocity equal to 1.3 times the solute velocity and a colloid dispersion coefficient equal to about one-fourth the solute dispersion coefficient based on an asymptotic spectral analysis of dispersive flow in parallel-plate channels. He also used a flux term to account for diffusion of solutes into the rock matrix. ${ }^{31}$

Some investigators have taken a microscopic approach to modeling colloid transport in parallel-plate fractures. ${ }^{75-77}$ This approach allows explicit prediction of differences in colloid and solute dispersion resulting from diffusion across the velocity profile in the fracture (i.e., Taylor dispersion 78,79 -- see Section 2.6.4). Specific colloid-wall interactions and matrix diffusion of solutes can also be included in these models. However, while it offers interesting mechanistic insights, the microscopic approach is of limited use when considering transport over large scales because it does not account for dispersion due to flow channeling in variable-aperture fractures or in fracture networks, which is likely to be more important than Taylor dispersion over long length scales.

Both the advection-dispersion and the microscopic modeling approaches are expected to have shortcomings when trying to model/interpret colloid transport experiments in single natural fractures. The former is limited in its ability to offer mechanistic insights into tracer transport behavior (parameter fitting is generally employed), and the latter cannot account for dispersion due to flow channeling. In this dissertation, an attempt will be made to model transport in single saturated natural fractures using an approach that relies on detailed characterization of the system geometry to predict flow field dispersion and on microscopic modeling to simulate differences between colloid and solute transport over small scales within the fractures.

\subsection{Saturated Flow in Natural Fractures}

\subsubsection{The Continuity and Navier-Stokes Equations}

As with all flow problems, the problem of predicting the flow field in a saturated natural fracture involves the solution of the continuity and NavierStokes (momentum) equations. In saturated groundwater systems, the flow can be assumed incompressible, isothermal, and at steady-state, so these equations become: 80

Continuity:

Momentum:

$$
\begin{gathered}
\nabla \bullet \mathbf{v}=0 \\
\rho \mathbf{v} \bullet \nabla \mathbf{v}=-\nabla p+\mu \nabla^{2} \mathbf{v}+\rho \mathbf{g}
\end{gathered}
$$


where, $\quad \nabla=$ del operator (see Ref. 80, Appendix A)

$\nabla^{2}=$ Laplacian operator 80

$\mathbf{v}=$ fluid velocity vector, $\mathrm{cm} / \mathrm{sec}$

$\rho=$ fluid density, $\mathrm{g} / \mathrm{cm}^{3}$

$\mu=$ fluid viscosity, $\mathrm{g} / \mathrm{cm}$-sec

$\mathrm{p}=$ pressure, $\mathrm{g} / \mathrm{cm}-\mathrm{sec}^{2}$ or dyne $/ \mathrm{cm}^{2}$

$\mathrm{g}=$ gravity vector, $980 \mathrm{~cm} / \mathrm{sec}^{2}$.

The boundary conditions that most often apply to an experimental system involving a single fracture are:

$$
\begin{aligned}
& \mathbf{v}=\mathbf{0} \text { at fracture walls } \\
& \mathrm{p}=\text { constant at fracture entrance and exit, and } \\
& \nabla \mathrm{p}=0 \text { along sides of fracture (no flow boundaries). }
\end{aligned}
$$

In porous media, Darcy's equation, or modifications thereof, is frequently used in place of the momentum equation(s) to describe the macroscopic velocity in a flow system. Darcy's equation is: 81

$$
\mathbf{v}=-\left(\frac{\mathrm{k \rho g}}{\mu}\right) \nabla \mathrm{H}
$$

where, $\mathrm{k}=$ intrinsic permeability, $\mathrm{cm}^{2}$

$\mathrm{g}=$ gravitational constant, $980 \mathrm{~cm} / \mathrm{sec}^{2}$

$\mathrm{H}=(\mathrm{p} / \rho \mathrm{g}+\mathrm{h})=$ hydraulic head, $\mathrm{cm}$

$\mathrm{h}=$ vertical elevation, $\mathrm{cm}$.

This empirically-derived equation makes it relatively easy to predict macroscopic flow fields in porous media. For a large fracture network it is often assumed that the system can be treated as an equivalent porous medium with (frequently) a nonisotropic hydraulic conductivity tensor. 82

For a single fracture, one cannot make such simplifications. However, it is often possible to ignore the inertial terms in the Navier-Stokes equations (the second term on the right-hand side of Eq. 2). This simplification is justified by noting that the Reynolds number in groundwater systems, defined as

$$
\mathrm{N}_{\mathrm{Re}}=\frac{\mathrm{bV} \rho}{\mu}
$$

where, $\quad b=$ characteristic length, e.g., the fracture aperture, $\mathrm{cm}$

$\mathrm{V}=$ characteristic groundwater velocity, $\mathrm{cm} / \mathrm{sec}$

is almost always much less than 1 . The Reynolds number is multiplied by the inertial terms in the dimensionless form of the Navier-Stokes equations, ${ }^{83}$ so these terms become very small compared to the viscous terms, which have a magnitude of order 1. Even for a relatively high groundwater velocity of $1 \times 10^{-4}$ $\mathrm{cm} / \mathrm{sec}^{84}$ and a large fracture aperture of $1 \mathrm{~cm}$, the Reynolds number is only 0.01 . Thus, one is usually justified in dropping the inertial terms from the NavierStokes equations to obtain what are often called the "creeping flow" equations.

Even without the inertial terms in the equations, the flow problem is very difficult to solve because of the highly irregular boundaries corresponding to the fracture walls. One approach to solving this complicated, inherently 3-

dimensional problem, might be to solve the problem numerically using a finiteelement discretization, which would allow approximation of the highly irregular boundaries. Another alternative might be to use lattice-gas and/or latticeBoltzmann calculations ${ }^{85}$ which are rapidly gaining popularity in solving a wide 
variety of small-scale flow and transport problems that have difficult boundaries. Depending on how much detail is assumed in fracture surface roughness, the resulting calculations in either case could be very computationally intensive and may require innovative algorithms to efficiently solve the velocity and pressure fields over the entire fracture domain. Also, current technology for measuring fracture surface roughness may not justify or support the use of these methods.

\subsubsection{Parallel Plates and the Reynolds Equation}

An approximation frequently employed in fracture flow problems is to assume that fractures are parallel-plate channels. In this case, a simple analytical solution for the flow field exists if laminar flow is assumed:

$$
\mathrm{v}_{\mathrm{x}}(\mathrm{z})=1.5 \mathrm{~V}_{\max }\left(1-\frac{\mathrm{z}^{2}}{\mathrm{~b}^{2}}\right)
$$

where, $\quad v_{x}(z)=$ velocity in $x$-direction as a function of $z, \mathrm{~cm} / \mathrm{sec}$

$x=$ coordinate in flow direction

$z=$ coordinate across channel aperture (from $-b$ to $b$ )

$\mathrm{b}=$ channel half-width, $\mathrm{cm}$

$\mathrm{V}_{\max }=-\left[\frac{\mathrm{dp}}{\mathrm{dx}}+\rho \mathrm{g}\left(\frac{\mathrm{dh}}{\mathrm{dx}}\right)\right]\left(\frac{\mathrm{b}^{2}}{2 \mu}\right)=$ maximum velocity (at channel

centerline), $\mathrm{cm} / \mathrm{sec}$.

When integrated over the cross-section of the channel, Eq. (5) gives an expression for the volumetric flow rate in the channel as a function of pressure drop:

$$
\mathrm{Q}=-\mathrm{L}_{z}\left(\frac{\mathrm{a}^{3}}{12 \mu}\right) \nabla \mathrm{P}
$$

where, $Q=$ volumetric flow rate, $\mathrm{cm}^{3} / \mathrm{sec}$

$\mathrm{L}_{\mathrm{z}}=$ width of channel in $\mathrm{z}$-direction (normal to no-flow boundaries), $\mathrm{cm}$

$\mathrm{a}=$ channel aperture $(2 \mathrm{~b}), \mathrm{cm}$

$P=p+\rho g h$

Equation (6) is often called the cubic law. It has the same functional form as Darcy's equation. For a rough-walled fracture, a representative value of the aperture "a" can be obtained by measuring the dependence of the volumetric flow rate on the pressure drop across the fracture, substituting this dependence into Eq. (6), and solving the equation for "a." The value of "a" thus obtained is called the "hydraulic aperture."

Although simple and easy to apply, the parallel-plate approximation cannot predict the existence of flow channeling in the fracture that arises from the spatial variability of the aperture(s). The only velocity variations in a parallel-plate fracture will be due to the parabolic velocity profile across the fracture aperture, and the only dispersion will be that arising from diffusion across this profile (normal to the flow direction); i.e., Taylor dispersion. ${ }^{78,79}$ If attempting to explain tracer breakthrough curves using such an approximation, it may be necessary to introduce an adjustable dispersion coefficient to account for dispersion due to flow channeling within the variable-aperture fracture.

A more refined approach to approximating the flow field in a fracture is to assume that the cubic law applies locally in the fracture and that the continuity equation applies over the entire fracture domain (i.e., $\nabla \bullet Q=0$ ). This approach leads to the following equation: 


$$
\frac{\partial}{\partial x}\left[\frac{L_{z} a^{3}(x, z)}{12 \mu} \frac{\partial p(x, z)}{\partial x}\right]+\frac{\partial}{\partial z}\left[\frac{L_{x} a^{3}(x, z)}{12 \mu} \frac{\partial p(x, z)}{\partial z}\right]=0(7)
$$

where, $a(x, z)=$ fracture aperture as a function of $x$ and $z$ coordinates, $c m$ $p(x, z)=$ pressure as a function of $x$ and $z$, dyne $/ \mathrm{cm}^{2}$.

Equation (7) is known as the Reynolds equation because it is a variation of the equation(s) originally derived in the late 1800's by Reynolds to describe lubrication processes. Actually, Reynolds started his derivation directly from the Navier-Stokes equations, but the result he obtained is the same as that obtained by applying the cubic law locally and imposing conservation of mass. ${ }^{86}$

The Reynolds equation is a 2-dimensional elliptic partial differential equation for the pressure field in a fracture that can be readily solved by numerical methods. The numerical procedure estimates the pressures at the midpoints of the discretized cells or nodes. Once the midpoint pressures are known, the volumetric flow rates across each node boundary can be readily calculated from

$$
Q_{i j}=\left(\frac{w}{6 \mu}\right)\left(\frac{a_{i}^{3} a_{j}^{3}}{a_{i}^{3}+a_{j}^{3}}\right) \frac{\left(p_{i}-p_{j}\right)}{h}
$$

where, $Q_{i j}=$ volumetric flow rate from node $i$ to node $j, \mathrm{~cm}^{3} / \mathrm{sec}$

$p_{i}, p_{j}=$ pressures at midpoints of nodes $i$ and $j, d y n e / \mathrm{cm}^{2}$

$w=$ width of boundary between nodes $i$ and $j, \mathrm{~cm}$

$\mathrm{h}=$ distance between midpoints of nodes $\mathrm{i}$ and $\mathrm{j}, \mathrm{cm}$.

The quantity $\left(\frac{2 a_{i}^{3} a_{j}^{3}}{a_{i}^{3}+a_{j}^{3}}\right)$ is the effective hydraulic aperture between the two node midpoints. It is obtained by solving for the pressure at the node boundary using the cubic law expression for one of the nodes and then substituting the result into the cubic law expression for the other node. Local and global conservation of mass provide a check of the accuracy of the overall solution (the volumetric flow rates across each boundary of a given node should sum to zero, and the overall flow rate into the fracture domain should equal the flow rate out of the domain).

The Reynolds equation has been used by many investigators to solve the flow field in a variable-aperture fracture $84,86-91$. Typically, finite difference methods have been used to discretize the system, and the resulting system of algebraic equations is solved using either a direct or iterative solver. The inherent advantage of using a flow-field solution obtained from the Reynolds equation over a single parallel-plate approximation when doing tracer transport calculations is that dispersion arises naturally from the meandering flow pathways predicted by the Reynolds equation. In principle, if the flow-field solution provides an accurate enough representation of the real flow field in a fracture, it should not be necessary to use an adjustable parameter such as the dispersion coefficient to describe tracer dispersion. Of course, the ability to obtain an accurate flow-field representation will depend on the ability to obtain accurate aperture estimates as a function of position in the fracture (with appropriate spatial resolution), as well as on the validity of the local parallelplate approximation that is inherent in the Reynolds equation. 


\subsection{Colloid Transport in Flow Fields}

It is useful to begin a discussion of colloid transport by considering the motion of an individual spherical colloid in response to forces acting on it. Newton's second law dictates that

$$
F=m a=m\left(\frac{d u}{d t}\right)
$$

where, $\quad \mathbf{F}=$ force acting on colloid, $\mathrm{g}-\mathrm{cm} / \mathrm{sec}^{2}$

$\mathrm{m}=$ colloid mass, $\mathrm{g}$

$\mathrm{a}=$ acceleration, $\mathrm{cm} / \mathrm{sec}^{2}$

$\mathbf{u}=$ colloid velocity, $\mathrm{cm} / \mathrm{sec}$

$\mathrm{t}=$ time, sec.

The net hydrodynamic drag force acting on a spherical particle in a uniform flow field is given by:

$$
\begin{array}{ll}
\qquad \mathbf{F}=\mathrm{f}(\mathbf{v}-\mathbf{u}) \\
\text { where, } \mathrm{f}=\text { friction coefficient, } \mathrm{g} / \mathrm{sec} \\
\mathbf{v}=\text { fluid velocity, } \mathrm{cm} / \mathrm{sec} .
\end{array}
$$

For a spherical particle far away from surfaces, the friction coefficient is given by Stokes Law:80,83,92

$$
\mathrm{f}=6 \pi \mu \mathrm{R}
$$

where, $\mu=$ fluid viscosity, $\mathrm{g} / \mathrm{cm}-\mathrm{sec}$

$$
\mathrm{R}=\text { particle radius, } \mathrm{cm}
$$

Setting Eqs. (9) and (10) equal to each other and integrating (in one direction only), we get

$$
\mathrm{u}=\left(\mathrm{u}_{\mathrm{o}}-\mathrm{v}\right) \exp \left(\frac{-\mathrm{ft}}{\mathrm{m}}\right)+\mathrm{v}
$$

where, $u_{\mathrm{o}}=$ initial velocity, $\mathrm{cm} / \mathrm{sec}$

The ratio $\mathrm{m} / \mathrm{f}$ in Eq. (12) is the characteristic relaxation time for the particle to attain a steady-state velocity given that a constant force acts on it at time zero. For a $1-\mu \mathrm{m}$ diameter spherical particle with a density of $1 \mathrm{~g} / \mathrm{cm}^{3}$ in water at $25^{\circ} \mathrm{C}$, this relaxation time is approximately $6 \times 10^{-8} \mathrm{sec}$. If one considers the velocity due to thermal (Brownian) motion, given by 80,93

$$
u_{t}=\sqrt{\frac{8 \mathrm{kT}}{\pi \mathrm{m}}}
$$

where, $\quad u_{\mathrm{t}}=$ velocity due to thermal motion, $\mathrm{cm} / \mathrm{sec}$

$\mathrm{k}=$ Boltzmann's constant, $1.38 \times 10^{-16} \mathrm{ergs} / \mathrm{K}$

$\mathrm{T}=$ temperature, $\mathrm{K}$

then the distance over which such a particle will move in Brownian motion before attaining a steady velocity will be about $\mathrm{u}_{\mathrm{t}} \mathrm{m} / \mathrm{f}=3 \times 10^{-8} \mathrm{~cm}$, or $0.3 \mathrm{~nm} . .^{93}$ Thus, for a particle to experience accelerations over time or length scales comparable to diffusional accelerations, an external force would have to change significantly over a time scale of $6 \times 10^{-8} \mathrm{sec}$ or over a length scale of $0.3 \mathrm{~nm}$. If forces do not change significantly over these scales, particle inertia can be assumed negligible (i.e., the local velocity will always be F/f). For colloidal particles in water, deterministic forces generally change over scales much larger than this. Thus, particle inertia can usually be ignored (however, there may be exceptions in the vicinity of a surface, where forces can change rapidly over 
small distances). An alternative, though less general, approach to determine the importance of particle inertia is to calculate the particle Reynolds number, 92

$$
\mathrm{N}_{\mathrm{Re}}=\frac{\mathrm{du} \rho}{\mu}
$$

where, $d=$ particle diameter, $\mathrm{cm}$

$$
\rho=\text { particle density }, \mathrm{g} / \mathrm{cm}^{3} \text {. }
$$

If $\mathrm{N}_{\mathrm{Re}} \ll<1$, particle inertia can be ignored. In the example above, $\mathrm{N}_{\mathrm{Re}}=4.5 \times 10^{-3}$.

This analysis of relaxation times is important because if particle inertia is not negligible, the only way one can accurately describe a system of particles is to calculate both the particle concentration and velocity distributions in time using the general Fokker-Planck equation. ${ }^{94}$ The Fokker-Planck equation may be required in aerosol systems, as the pertinent characteristic time and length scales are much larger than in liquids due to the lower viscosity of the medium. The Fokker-Planck equation may also be required in very concentrated particle suspensions, as the time and length scales of interparticle interactions can become comparable to the time and length scales for diffusion. 95 The FokkerPlanck equation should not be needed to describe colloid transport in aqueous solutions provided that their concentration is low enough that particle interactions can be ignored (less than $1 \%$ volume fraction). ${ }^{92}$

\subsubsection{Particle Trajectories}

Given that particle inertia is negligible in liquids, and assuming for the moment that diffusion is negligible compared to deterministic motion, a general force balance on an arbitrarily-shaped particle can be written as: 83,92

$$
\begin{aligned}
& \mathbf{F}-(\mathbf{f} \bullet \mathbf{u}+\mathbf{c} \bullet \boldsymbol{\omega})=\mathbf{0} \\
& \mathbf{T}-\left(\mathbf{c}^{\top} \bullet \mathbf{u}+\mathbf{f}_{\mathbf{r}} \bullet \boldsymbol{w}\right)=0
\end{aligned}
$$

where, $\quad \mathbf{F}=$ force vector acting on particle

$\mathrm{T}=$ torque vector acting on particle

$\mathbf{u}=$ translational velocity vector

$\omega=$ rotational velocity vector

$\mathrm{f}=$ translational resistance tensor

$\mathbf{f}_{\mathrm{r}}=$ rotational resistance tensor

$\mathrm{c}=$ coupling tensor (coupling between translation and rotation)

$\mathrm{c}^{\mathrm{T}}=$ transpose of coupling tensor.

$F$ and $T$ are the forces acting on the particle that cause it to move, and the terms in parentheses in Eqs. (15) are the forces that resist particle movement. The coupling tensor accounts for the fact that, with an arbitrarily-shaped particle, translational motion will induce rotation and vice-versa. For a spherical particle far away from surfaces, all terms of the coupling tensor are zero, and the translational resistance tensor is diagonal with all diagonal terms equal to $6 \pi \mu \mathrm{R}$ (Stokes resistance). ${ }^{83,92}$ Solving Eqs. (15) for u yields

where, $\mathbf{r}=$ position vector

$$
\mathbf{u}=\frac{\mathrm{d} \mathbf{r}}{\mathrm{dt}}=\mathbf{M} \cdot \mathbf{F}-\mathbf{M}^{\prime} \cdot \mathbf{T}
$$

$$
\begin{aligned}
& \mathbf{M}=\left(\mathbf{f}-\mathbf{c} \bullet \mathbf{f}_{\mathrm{r}}^{-1} \bullet \mathbf{c}^{\mathrm{T}}\right)^{-1}=\text { translational mobility tensor } \\
& \mathbf{M}^{\prime}=\mathbf{M} \bullet \mathbf{c} \bullet \mathbf{f}_{\mathrm{r}}^{-1}=\text { rotational mobility tensor. }
\end{aligned}
$$

For an arbitrarily-shaped particle, a diffusion tensor is defined as: 83,92

$$
\mathbf{D}=\mathrm{kTM}
$$


For a spherical particle far away from surfaces $\mathbf{M}=\operatorname{diag}[1 / 6 \pi \mu \mathrm{R}]$, and Eq. (17) reduces to the familiar Stokes-Einstein equation:

$$
\mathrm{D}=\frac{\mathrm{kT}}{6 \pi \mu \mathrm{R}}
$$

where, $\mathrm{D}=$ diffusivity, $\mathrm{cm}^{2} / \mathrm{sec}$

From this equation, the diffusivity is seen to be inversely proportional to the radius of a spherical colloid. Given that the radii of molecular species are typically on the order of $10^{-8}$ to $10^{-7} \mathrm{~cm}$, it is apparent that the diffusivities of colloids are generally two to four orders of magnitude smaller than those for molecular species (at a given temperature in a given fluid). Thus, colloids are very slowly diffusing species compared to molecules.

Assuming that diffusion is negligible, if the forces and torques on a particle as a function of time and position are known, then the set of coupled ordinary differential equations corresponding to Eq. (16) can be solved to obtain the particle position as a function of time given some initial position and a force field. This approach, called the "particle-trajectory" or "particle-tracking" approach, has been used by many investigators to determine colloid trajectories in the vicinity of surfaces. ${ }^{96-104}$ The approach has been used frequently in filtration studies to determine trajectories that lead to colloid deposition on collectors. An overview of the method is provided by Van de Ven. .2

It is informative to examine some of the deterministic velocity expressions that apply to spherical particles being acted upon by various forces. For a spherical particle far away from surfaces $\left(\mathbf{M}=\operatorname{diag}[1 / 6 \pi \mu R], \mathbf{M}^{\prime}=\mathbf{0}\right)$, Eq. (16) reduces to

$$
\mathbf{u}=\mathbf{M} \cdot \mathbf{F}=\frac{\mathbf{F}}{6 \pi \mu R}
$$

If the only force acting on the particle is fluid drag, given by $F=f v$ (where $v=$ fluid velocity, $\mathrm{cm} / \mathrm{sec}$ ), then we get the result

$$
\mathbf{u}_{\text {drag }}=\mathbf{v}
$$

This result is also obtained from Eq. (11) when $t>>\mathrm{m} / \mathrm{f}$. Equation (20) is the mathematical statement that, in the absence of other forces, colloids will tend to follow fluid streamlines. The gravity force acting on a particle is given by

$$
\mathbf{F}=\left(\mathrm{m}_{\mathrm{p}}-\mathrm{m}_{\mathrm{f}}\right) \mathbf{g}=\left(\frac{4}{3}\right) \pi \mathrm{R}^{3}\left(\rho_{\mathrm{p}}-\rho_{\mathrm{f}}\right) \mathbf{g}
$$

where, $m_{p}=$ mass of particle, $g$

$\mathrm{m}_{\mathrm{f}}=$ mass of fluid displaced by particle, $\mathrm{g}$

$\rho_{\mathrm{p}}=$ density of particle, $\mathrm{g} / \mathrm{cm}^{3}$

$p_{\mathrm{f}}=$ density of fluid, $\mathrm{g} / \mathrm{cm}^{3}$

$\mathrm{g}=$ acceleration due to gravity, $\mathrm{cm} / \mathrm{sec}^{2}$.

The particle velocity due to gravity is obtained by substituting Eq. (21) into Eq. (19):

$$
\mathbf{u}_{\text {grav }}=\left(\frac{2}{9 \mu}\right) R^{2}\left(\rho_{\mathrm{p}}-\rho_{\mathrm{f}}\right) \mathbf{g}
$$


In general, a force can be considered the negative gradient of a potential energy, $\nabla \phi$. Thus, the particle velocity due to a potential energy gradient is given by

$$
\mathbf{u}_{\phi}=-\left(\frac{1}{6 \pi \mu R}\right) \nabla \phi
$$

Using Eq. (18), Eq. (23) is equivalent to

$$
\mathbf{u}_{\phi}=-\left(\frac{\mathrm{D}}{\mathrm{kT}}\right) \nabla \phi
$$

In the case of a charged particle in an electric field, Eq. (24) becomes

$$
\mathbf{u}_{\phi_{\text {elec }}}=-\left(\frac{\mathrm{zeD}}{\mathrm{kT}}\right) \nabla \phi_{\text {elec }}
$$

where, $\quad z=$ number of electronic charges on particle (+ or -$)$

$\mathrm{e}=$ electronic charge.

Equations (16) and /or (19) are strictly valid only if diffusion is negligible relative to other forces acting on the particle. To account for diffusion, one must modify the particle-trajectory equation to allow for a random diffusion force. Formally, this requires the solution of a stochastic differential equation called a Langevin equation, 92,94 which is given by

where, $\quad \mathbf{F}_{\text {det }}=$ deterministic force vector,

$$
m a=-f u+F_{\text {det }}+B(t)
$$

$\mathbf{B}(\mathrm{t})=$ random diffusion force.

If particle inertia is negligible ( $\mathrm{ma}=\mathbf{0})$, Eq. (26) becomes

$$
\begin{aligned}
\mathbf{u} & =\frac{\left(\mathbf{F}_{\mathrm{det}}+\mathbf{B}(\mathrm{t})\right)}{\mathrm{f}} \\
\text { or } \mathbf{u} & =\mathbf{u}_{\mathrm{det}}+\frac{\mathbf{B}(\mathrm{t})}{\mathrm{f}}
\end{aligned}
$$

where, $\quad \mathbf{u}_{\text {det }}=\mathbf{u}_{\text {drag }}+\mathbf{u}_{\text {grav }}+\mathbf{u}_{\phi}+\mathbf{u}_{\phi_{\text {elec }}}$

Upon integrating Eq. (27) with respect to time, it can be shown that ${ }^{92}$

$$
x(t+\Delta t)=x(t)+u_{\operatorname{det}} \Delta t+R n[0,2 D \Delta t]
$$

where, $\quad x(t)=$ particle position coordinates at time $t$, and

that

$\operatorname{Rn}[0,2 \mathrm{D} \Delta \mathrm{t}]$ represents a vector of random length and direction has component vectors that are independent and
distributed with a mean of zero and a variance of $2 \mathrm{D} \Delta \mathrm{t}$.

Assuming that the deterministic fluid velocity field and any other potential energy fields are known, Eq. (28) can, in principle, be used to generate stochastic particle trajectories that include the effects of diffusion. This has been done by Van de Ven ${ }^{105}$ and Foister and Van de Ven ${ }^{106}$ for colloids in laminar flow fields. When using this approach, one has to be careful to take time steps small enough that $\mathbf{u}_{\mathrm{det}}$ does not change significantly between the particle positions at the start and end of each time step. Alternatively, if the fluid drag force is the only important deterministic force (given by fv) and wall effects are ignored, Eq. (27) can be solved directly using techniques for stochastic differential equations. 92,106

Recently, particle-tracking techniques have been used to predict solute transport through complex flow fields such as in heterogeneous porous media. 107-111 In these applications, a large number of solute "particles" (assumed to be completely independent) are allowed to move individually through a flow system according to Eq. (28). For macroscopic problems, a dispersion coefficient 
is typically used in place of the diffusivity (D) in Eq. (28). The steady-state flow field is typically solved prior to doing the particle-tracking calculations. MonteCarlo techniques are used to determine the initial position of each particle at the entrance to the flow system as well as the stochastic motion of the particles due to diffusion or dispersion during each time step. For reactive solutes, additional terms can be added to Eq. (28) to account for chemical interactions with the surfaces (e.g., sorption). ${ }^{109-111}$ Tompson and Gelhar ${ }^{108}$ point out that, in systems where diffusivities, dispersion coefficients and/or porosities vary spatially, additional deterministic velocity terms must be added to Eq. (28) to account for the gradients in these variables. If fluid drag is the only deterministic force, these terms are

$$
\begin{gathered}
\mathbf{u}_{\mathrm{D}} \Delta \mathrm{t}=(\nabla \cdot \mathbf{D}) \Delta \mathrm{t} \\
\mathbf{u}_{\varepsilon} \Delta \mathrm{t}=\mathbf{D}(\nabla \cdot \ln \varepsilon) \Delta \mathrm{t}
\end{gathered}
$$

where, $\quad \mathbf{u}_{\mathrm{D}}=$ deterministic velocity due to diffusivity or dispersion coefficient gradient, $\mathrm{cm} / \mathrm{sec}$ $\mathbf{u}_{\varepsilon}=$ deterministic velocity due to porosity gradient, $\mathrm{cm} / \mathrm{sec}$ $\mathbf{D}=$ diffusivity or dispersion coefficient, $\mathrm{cm}^{2} / \mathrm{sec}$ $\varepsilon=$ porosity $\left(\mathrm{cm}^{3}\right.$ fluid $\left./ \mathrm{cm}^{3}\right)$.

The deterministic velocity due to a porosity gradient (Eq. 30 ) becomes more complicated when there are other deterministic forces to consider besides fluid drag. However, for colloids confined to the void space of a fracture there is no porosity gradient, and these complications need not be considered. For solutes that diffuse out of the void space and into the matrix, a porosity gradient must be considered, but all deterministic forces (including fluid drag) become negligible in the matrix, so the complications again need not be considered. The terms represented by Eqs. (29) and (30) arise naturally in the species continuity equation (see Section 2.6.3). If they are not included in Eq. (28), particles will falsely accumulate in stagnant and/or low porosity zones within the flow system. 108

In particle-tracking calculations, concentration profiles or breakthrough curves can be derived from the distribution of the individual particles in space and time. A sufficient number of particles must be simulated in order to obtain a statistically valid representation of the behavior of the system. This approach offers certain advantages over the more traditional approach of solving the species continuity equation in space and time by finite-difference or finiteelement approximations. For instance, heterogeneities in the flow field and in the properties of the geologic media can be explicitly accounted for with minor additional computational burden when compared to traditional finite-difference or finite-element approaches. Complex phenomena such as nonlinear or nonequilibrium sorption and diffusion of particles into the rock matrix can also be simulated with minimal additional computational burden by stochastically sampling residence time distributions that are representative of the delay times associated with the phenomena. ${ }^{110}$ Tompson and Gelhar point out that particletracking calculations become more advantageous relative to finite-difference or finite-element approaches as systems require finer spatial discretization for an acceptable solution. 108 Also, particle-tracking calculations, being independent for each particle, are inherently parallelizable on multiprocessor computers. 
Parallelization of finite-difference or finite-element calculations is more difficult because communication between adjacent nodes is required.

\subsubsection{Hydrodynamic Corrections Near Walls}

A series of mathematical studies in the 1960's and early 1970's established that translation and rotation of spherical particles in the immediate vicinity of planar surfaces differs from that in an unbounded fluid because fluid motion near surfaces is constrained by the no-slip boundary condition. ${ }^{112-118}$ This constraint gives rise to two principal effects: (1) a coupling is established between particle translation and rotation, and (2) movement toward or away from a planar surface is hindered by film thinning between the two surfaces. These effects can be incorporated directly into the mobility tensors $\mathbf{M}$ and $\mathbf{M}^{\prime}$, and Eq. (16) can be solved rigorously to obtain particle trajectories in arbitrary flow fields and geometries. However, because one is typically interested only in the translational motion of a particle, the results are commonly expressed in terms of dimensionless functions that correct the mobility and velocity that the particle would experience in the absence of walls. Thus, the expressions for spherical particle mobilities and velocities near a wall become

$$
\begin{aligned}
& \mathrm{M}_{\perp}=\mathrm{F}_{1}(\mathrm{H}) \mathrm{M}_{\infty} \\
& \mathrm{M}_{\| 1}=\mathrm{F}_{4}(\mathrm{H}) \mathrm{M}_{\infty} \\
& \mathrm{u}_{\| 1}=\mathrm{F}_{3}(\mathrm{H}) \mathrm{v}_{\|} \\
& \mathrm{u}_{\perp}=\mathrm{F}_{1}(\mathrm{H}) \mathrm{F}_{2}(\mathrm{H}) \mathrm{v}_{\perp}
\end{aligned}
$$

where, $\quad \mathrm{M}_{1}=$ mobility perpendicular to wall $\mathrm{cm}^{2} / \mathrm{sec}$

$\mathrm{M}_{\|}=$mobility parallel to wall, $\mathrm{cm}^{2} / \mathrm{sec}$

$\mathrm{M}_{\infty}=$ mobility far from wall, $\mathrm{cm}^{2} / \mathrm{sec}$

$\mathrm{u}_{\perp}=$ particle velocity perpendicular to wall, $\mathrm{cm} / \mathrm{sec}$

$\mathrm{u}_{\|}=$particle velocity parallel to wall, $\mathrm{cm} / \mathrm{sec}$

$\mathrm{v}_{\perp}=$ fluid velocity perpendicular to $\mathrm{wall}, \mathrm{cm} / \mathrm{sec}$

$\mathrm{v}_{\mathrm{II}}=$ fluid velocity parallel to wall, $\mathrm{cm} / \mathrm{sec}$

$\mathrm{F}_{1}(\mathrm{H})=$ correction function given by Brenner ${ }^{112}$ and Bart ${ }^{113}$

$\mathrm{F}_{4}(\mathrm{H})=$ correction function given by Goldman et al.114

$\mathrm{F}_{3}(\mathrm{H})=$ correction function given by Goldman et al.115 and O'Neill and Stewartson ${ }^{116}$

$\mathrm{F}_{2}(\mathrm{H})=$ correction function given by Goren ${ }^{117}$ and Goren and

O'Neill118

$\mathrm{H}=$ dimensionless distance of particle from wall, $\mathrm{h} / \mathrm{R}$

$\mathrm{h}=$ absolute separation between particle and wall, $\mathrm{cm}$.

The functions $\mathrm{F}_{1}(\mathrm{H}), \mathrm{F}_{2}(\mathrm{H}), \mathrm{F}_{3}(\mathrm{H})$, and $\mathrm{F}_{4}(\mathrm{H})$ all have complicated analytical expressions. These functions are depicted graphically in Fig. 2.2 (from Ref. 92). When calculating particle trajectories, these functions can be approximated by simple analytical expressions (see, for example, Ref. 119).

\subsubsection{Species Continuity Equation}

An alternative to using the particle-trajectory approach to calculate the movement of individual particles is to obtain the distribution of particles in space and time by solving the appropriate partial differential equation(s) describing continuum particle behavior. When particle inertia is negligible, the general 


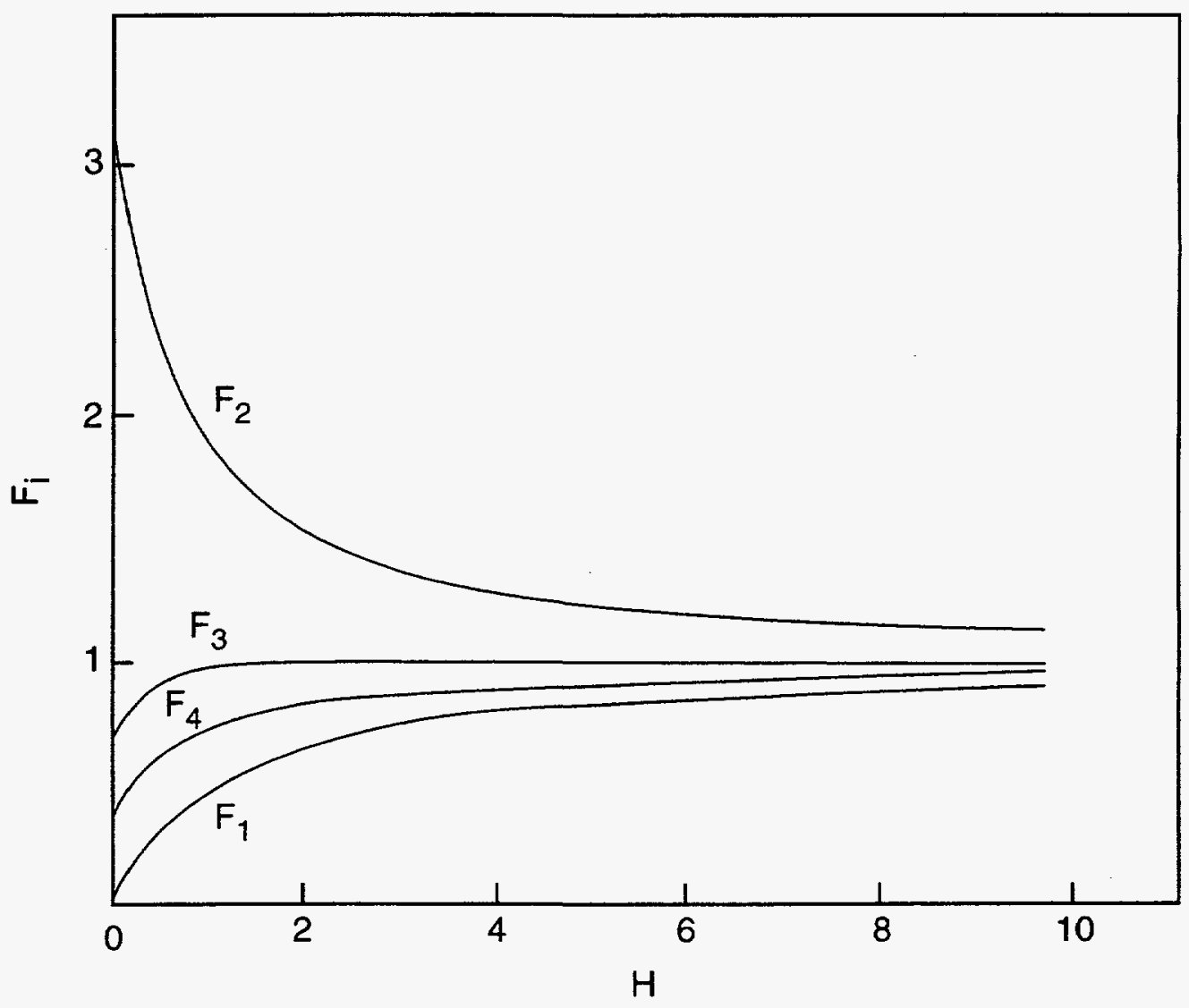

Figure 2.2. Hydrodynamic correction functions vs. dimensionless separation distance $(H=h / R)$ for a spherical particle near a planar surface. (From Ref. 92)

Fokker-Planck equation reduces to the familiar species continuity equation (also known as the convective-diffusion equation): 92

$$
\varepsilon \frac{\partial c_{i}}{\partial t}=\nabla \cdot \varepsilon\left(D \nabla c_{i}-c_{i} M \cdot F+c_{i} M^{\prime} \cdot T\right)+\varepsilon R_{i}
$$

where, $c_{i}=$ concentration of species $i$, number $/ \mathrm{cm}^{3}$

$R_{i}=$ rate of generation of species $i$ by chemical reaction.

For colloids that do not grow or dissolve, the reaction term, $R_{i}$, will be zero (except at surfaces where attachment and detachment occur). If $\mathrm{fv}$ is used for the fluid drag force, Eq. (21) for the gravity force, and $-\nabla \phi$ for a general force, Eq. (35) becomes (assuming that wall effects are negligible):

$$
\varepsilon \frac{\partial c_{i}}{\partial t}=\nabla \cdot \varepsilon\left[D \nabla c_{i}-c_{i} \mathbf{v}+c_{i}\left(\frac{D}{k T}\right) \nabla \phi-c_{i}\left(\frac{2}{9 \mu}\right) R^{2}\left(\rho_{p}-\rho_{f}\right) g\right]
$$

An electrical force is not explicitly included in Eq. (36) because there are generally no electrical fields imposed in tracer studies. The general force given by $-\nabla \phi$ in Eq. (36) is typically important only very close to surfaces.

As an example of how to incorporate hydrodynamic corrections into Eq. (36), it is assumed that there is a single planar surface with which the particles 
can interact, and a rectangular Cartesian coordinate system is used with the $z$ coordinate normal to the planar surface ( $z=0$ at surface). It is also assumed that the general force $-\nabla \phi$ acts only in the $z$-direction (normal to the surface). The component form of Eq. (36) then becomes

$$
\begin{aligned}
\varepsilon \frac{\partial c_{i}}{\partial t} & =\frac{\partial}{\partial x}\left[\varepsilon F_{4}(H) D_{\infty}\left(\frac{\partial c_{i}}{\partial x}\right)\right]-\frac{\partial}{\partial x}\left\{\varepsilon F_{3}(H)\left[v_{x}+\left(\frac{2}{9 \mu}\right) R^{2}\left(\rho_{p}-\rho_{f}\right) g_{x}\right] c_{i}\right\} \\
+ & \frac{\partial}{\partial y}\left[\varepsilon F_{4}(H) D_{\infty}\left(\frac{\partial c_{i}}{\partial y}\right)\right]-\frac{\partial}{\partial y}\left\{\varepsilon F_{3}(H)\left[v_{y}+\left(\frac{2}{9 \mu}\right) R^{2}\left(\rho_{p}-\rho_{f}\right) g_{y}\right] c_{i}\right\} \\
& +\frac{\partial}{\partial z}\left[\varepsilon F_{1}(H) D_{\infty}\left(\frac{\partial c_{i}}{\partial z}\right)\right]-\frac{\partial}{\partial z}\left[\varepsilon F_{1}(H) F_{2}(H) v_{z} c_{i}\right] \\
& -\frac{\partial}{\partial z}\left[\varepsilon F_{1}(H)\left(\frac{2}{9 \mu}\right) R^{2}\left(\rho_{p}-\rho_{f}\right) g_{z} c_{i}\right]+\frac{\partial}{\partial z}\left[\varepsilon F_{1}(H)\left(\frac{D_{\infty}}{k T}\right) c_{i}\left(\frac{\partial \phi}{\partial z}\right)\right]
\end{aligned}
$$

where, $v_{i}=$ component of velocity in i direction

$\mathrm{g}_{\mathrm{i}}=$ component of gravity in i direction

$\mathrm{D}_{\infty}=$ diffusivity far away from wall, $\mathrm{cm}^{2} / \mathrm{sec}$

$\mathrm{H}=$ dimensionless distance between particle and planar surface, $(z-R) / R$

Note: $\quad(x, y, z)$ are coordinates of particle center.

Equation (37) is the most general form of the continuity equation in Cartesian coordinates when there is a single planar surface present. It reduces to the usual continuity equation when wall effects are neglected (i.e., $F_{i}(H)=1, i=1$ to 4 ).

Appropriate boundary conditions must be imposed if a solution to Eq. (36) or (37) is desired. Boundary conditions at the planar surface ( $z=0$, or more properly, $\mathrm{H}=0$ ) can be of various types, including (but not necessarily limited to):

$$
\begin{aligned}
& c_{i}=0 \text { at } z=0 \\
& \frac{\partial c_{i}}{\partial z}=0 \text { at } z=0 \\
& \frac{\partial c_{i}}{\partial z}=-k_{1} c_{i}+k_{2} c_{s} \text { at } z=0
\end{aligned}
$$

where, $k_{1}=$ immobilization rate constant (or expression), $1 / \mathrm{sec}$

$\mathrm{k}_{2}=$ remobilization rate constant (or expression), $1 / \mathrm{cm}$-sec

$\mathrm{c}_{\mathrm{s}}=$ surface concentration, number $/ \mathrm{cm}^{2}$.

Boundary condition (38a) implies that particles that come in contact with the planar surface disappear from the system (i.e., a perfect sink). Boundary condition ( $38 \mathrm{~b}$ ) prevents penetration of the surface and allows particles to arrive at and leave the surface subject to the forces acting on them. Using this boundary condition, immobilization of particles at the surface can occur only if the forces acting on the particles are perfectly balanced (i.e., a net force of zero). Boundary condition (38c) assumes that particles become immobilized and remobilized at the surface according to first order rate expressions (where the rate "constants" may have some functional dependence on other variables such 
as time or surface concentration). When using boundary condition (38c) an additional differential equation is needed to calculate the time dependence of the surface concentration, $\mathrm{c}_{\mathrm{s}}$.

\subsubsection{Solute Transport vs. Colloid Transport in Fractures}

The particle-trajectory equation(s) discussed in Section 2.6.1 and the species continuity equation(s) discussed in Section 2.6 .3 apply to both colloids and solutes. However, solutes are so small relative to colloids that body forces such as gravitational settling are negligible. Also, hydrodynamic corrections for solutes can be ignored because they are important only over infinitesimally small distances near walls. Therefore, the particle-trajectory and species continuity equations are less complex for solutes than for colloids. However, as discussed in Section 2.2, solutes experience matrix diffusion in fractured systems while colloids should not, so the pertinent transport equations for solutes must be extended to the matrix while those for colloids can, as a first approximation, be limited to the fracture void space.

Another important distinction between solutes and colloids is the large difference in the diffusivities of the two species. A solute typically has a diffusivity in water of $10^{-5}$ to $10^{-6} \mathrm{~cm}^{2} / \mathrm{sec}$ while a $1-\mu \mathrm{m}$ diameter colloid has a diffusivity of $\sim 4.4 \times 10^{-9} \mathrm{~cm}^{2} / \mathrm{sec}$ (according to Eq. 18). In fracture flow, this difference has important implications for the amount of dispersion that each species experiences due to the velocity profile across the fracture aperture. Dispersion arising from diffusion across flow streamlines in a long, narrow channel is known as Taylor dispersion. ${ }^{78,79}$ Two extremes of dispersion resulting from diffusion across a parabolic velocity profile are depicted in Fig. 2.3. A species that diffuses rapidly across the width of the profile (e.g., a solute) will sample all of the flow streamlines many times during its residence time in the flow channel, thus experiencing an average velocity very similar to the average fluid velocity. This is classic Taylor dispersion. Alternatively, a slowly diffusing species (a colloid) will stay in approximately the same streamline during its entire time in the channel. The breakthrough curves associated with these two cases are shown at the bottom of Fig. 2.3. In pure streamline flow, the earliest arrival of tracer will be $2 / 3$ rds the average fluid residence time ( $L / V_{\text {ave }}$ ) because the velocity of the streamline at the channel centerline is 1.5 times the average fluid velocity. However, there will be a very long tail associated with streamline flow due to the low velocity streamlines near the channel walls. For Taylor dispersion, the tracer arrival time follows essentially a tight Gaussian distribution centered about the average fluid residence time.

It is important to recognize that the difference in dispersion between the rapidly and slowly diffusing species in the parallel-plate system of Fig. 2.3 is dependent on the time and length scales of the system. As the channel gets narrower or longer or the average flow rate decreases, even species with small diffusivities will eventually tend to follow Taylor dispersion because they will have more opportunity to diffuse across the flow streamlines of the channel. Therefore, differences in tracer dispersion resulting from Taylor dispersion may be far more noticeable at the laboratory scale than at the field scale.

\subsection{Colloid Interactions with Surfaces}

Because the majority of the colloid tracers used in this study were shown to have minimal interactions with fracture surfaces, a detailed discussion of 


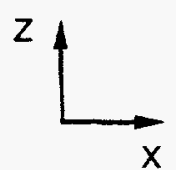

Parallel Plate Flow Channel

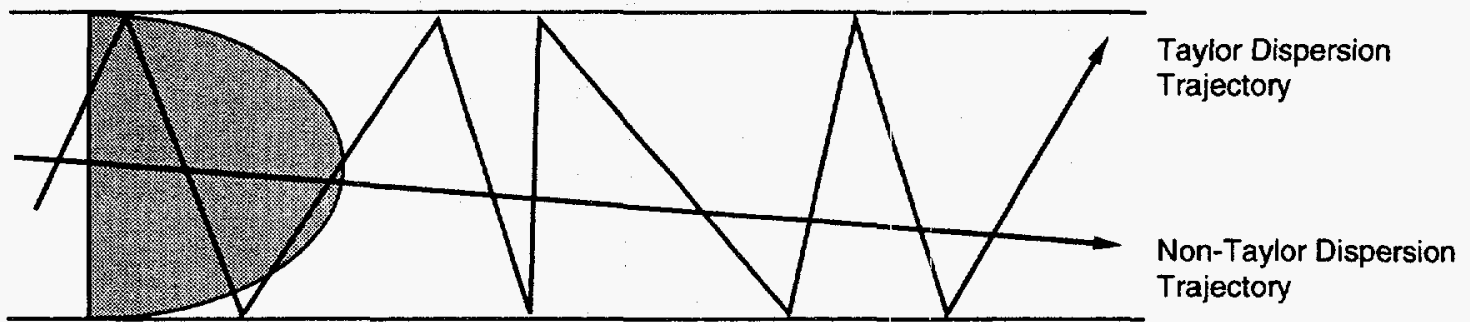

Parabolic Velocity Profile

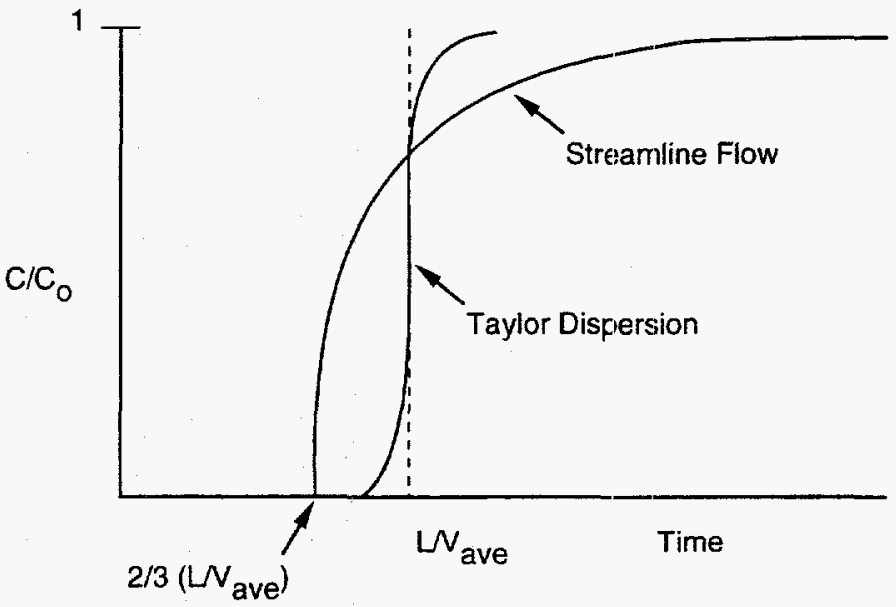

Figure 2.3. Depiction of Taylor dispersion and non-Taylor dispersion trajectories (top) and the breakthrough curves that result from these two transport regimes (bottom). The width of the flow channel (top) has been greatly exaggerated relative to its length $(\mathrm{L})$.

colloid-surface interactions is not included in this dissertation. However, a thorough literature review of this subject is provided in a Los Alamos

manuscript report by the author. ${ }^{120}$ The following discussion is a brief summary of the pertinent material in that report.

Colloid interactions with surfaces are the result of short-range forces that become important when the distance of separation between a colloid and a surface is much less than the colloid radius (e.g., 1/10th of colloid radius). These forces include

- Van der Waals dispersion forces, 121,122

- electrostatic forces, 121,122

- electronic (Born) repulsion forces, 121,123

- steric forces, 121,124

- chemical bonding forces, 125

- structural or hydration forces, ${ }^{121}$ 
- hydrodynamic detachment forces, 126 and

- dynamic forces. ${ }^{92,127-130}$

Each of these forces can be represented as a potential energy that varies as a function of surface separation, and the energies associated with the different forces can be summed linearly to obtain a composite interaction energy "profile" describing the interaction between the surfaces. ${ }^{121,122}$ The composite profile may be entirely attractive or repulsive, or it may have one or more energy maxima (repulsive) or minima (attractive) at various surface separations. In principle, the potential energy gradient associated with the surface interactions can be incorporated directly into either particle-trajectory equations or the species continuity equation as the $\nabla \phi$ term. This has been done by many researchers attempting to model the deposition of colloids onto idealized collector surfaces. ${ }^{119,131-138}$

When macroscopic problems are considered, it is much more practical to approximate colloid-surface interactions using boundary conditions such as Eqs. (38a-c). A series of papers in the 1970's established a theoretical link between certain types of interaction energy profiles and the kinetic boundary condition of Eq. (38c). $93,123,139-145$ The theories lead to considerably more complicated sets of equations than Eq. (38c) if it is assumed that colloids can accumulate in multiple energy minima and be either mobile or immobile while in these minima. 123,144,145 In the simple cases of a deep energy minimum or a large energy maximum, it is probably most appropriate to use boundary conditions (38a) or (38b), respectively.

Direct experimental observations of colloid deposition onto and detachment from surfaces has suggested that deposition/detachment phenomena is considerably more complex than might be inferred from expressions such as Eq. (38c). ${ }^{125,146-154}$ Blocking of portions of the surface by previously-deposited colloids can give rise to a deposition rate constant that is dependent on the number of colloids already deposited. 155,156 Attachment times are often not exponentially distributed (as is implied with a rate "constant"), so real rate "constants" may actually depend on the time a colloid has already spent attached to a surface. ${ }^{155,156}$ Surface roughness, surface chemical heterogeneities, and even the tendency for some colloids to "flatten" near surfaces can all complicate the simplistic view of colloid-surface interactions suggested by interaction energy profiles or the kinetic boundary conditions derived from them. . $^{15-162}$ Nevertheless, these complex phenomena might still be adequately described by an empirical boundary condition that effectively "lumps" their effects.

In this dissertation, boundary condition (38b) will, in effect, be used at the fracture walls in all transport calculations, and it will be assumed that there is no gradient in the interaction energy profile causing movement toward or away from the surfaces. In other words, the surface boundary condition will be purely reflective, and short-range surface interactions will be neglected (or assumed to be purely repulsive over very short distances next to the surfaces). 


\subsection{EXPERIMENTAL}

\subsection{Tracer Experiments in Natural Fractures}

To compare the transport of synthetic colloids and a nonsorbing solute in saturated natural fractures, a total of eighteen tracer experiments were conducted in three different isolated fractures in tuff. These experiments are described in detail in this chapter.

\subsubsection{Fractures}

The three fractures used in the tracer experiments were

(1) A fracture extracted from an outcropping of the Tshirege member of Bandelier Tuff in the Jemez mountains approximately 4 miles west of Los Alamos, NM. The outcropping was unsaturated. This fracture will be referred to as the "Bandelier tuff" fracture.

(2) A fracture from a core sample taken from borehole UE25 C-3 at the Nevada Test Site. The fracture ran through the core at about a 10- to 15-degree angle from the axis of the core (i.e., from vertical). The core sample was collected at a depth of 858 meters below the surface in a geologic formation called the Tram Tuff of the Crater Flat Group. The sample came from below the water table. This fracture will be referred to as the "Tram tuff" fracture.

(3) A fracture from a core sample taken from borehole UE25 C-2 at the Nevada Test Site. The fracture ran through the core almost directly along the axis of the core (essentially vertical). The core sample was collected at a depth of 755 meters below the surface in a geologic formation called the Bullfrog Tuff of the Crater Flat Group. The sample came from below the water table.

This fracture will be referred to as the "Bullfrog tuff" fracture.

Boreholes UE25 C-2 and UE25 C-3 are within 30 meters of each other at the Nevada Test Site, and, along with borehole UE25 C-1, they will ultimately be used to conduct interwell tracer experiments in the fractured tuff below the water table at this location. A Los Alamos National Laboratory mineralogist examined the three fractures and ascertained that they were, indeed, natural fractures and not fractures caused by breakage during the coring or core removal processes.* However, the Bullfrog tuff fracture clearly had some missing material that resulted in very large aperture regions in this fracture (probably the result of multiple fractures in the core, which led to loose pieces breaking free).

Using a diamond-tipped rock saw, the rock surrounding each of the fractures was cut into blocks that were roughly rectangular in shape with the fractures approximately bisecting the blocks. All cuts perpendicular to the fractures were done with the fracture surfaces wedged firmly together (using wood chocks) so that a clean, nonoffsetting cut could be made without damaging the surfaces. The blocks were all approximately $4-$ to $5-\mathrm{cm}$ thick in the direction perpendicular to the plane of the fracture. The surface areas of the fractures were Bandelier: $11.3-\mathrm{x} 10.1-\mathrm{cm}$ (roughly rectangular),

Tram: $9.7-\mathrm{cm}$ long $\times 8.9-\mathrm{cm}$ wide at one end $\times 7.9-\mathrm{cm}$ wide on the other end (a slightly trapezoidal shape because of the angle at which the fractured bisected the core),

Bullfrog: 8.5- $x$ 8.3-cm (roughly rectangular).

Although quantitative mineralogical characterization of the fractures was not conducted, all three fractures were rich in quartz and/or feldspar (see

\footnotetext{
* Informal examination by Barbara Carlos of Los Alamos National Laboratory (1994).
} 
below). Mineralogical characterization of the fracture surfaces was pending at the time this dissertation was written. In a cursory look at the fracture surfaces under an optical microscope, a Los Alamos mineralogist made the following observations: ${ }^{*}$

Bandelier tuff: This fracture had minerals on its surfaces that were not present in the matrix in significant quantities. The matrix was predominantly quartz and/or feldspar. The fracture surfaces had orangered iron oxide specks (probably hematite) scattered all over them. There was also some sort of manganese mineral (black) and possibly some pyroxene (a silicate) on the fracture surfaces.

Tram tuff: Like the Bandelier tuff fracture, this fracture had a lot of coating minerals on its surfaces that were not present in the matrix in significant quantities. The matrix was predominantly quartz and/or feldspar with a significant amount of iron oxide (probably hematite) giving the rock a distinct reddish color. The fracture coating minerals included the iron oxides hematite and magnetite, hollandite (a manganese mineral), quartz, and possibly calcite and clinoptilolite.

Bullfrog tuff: This fracture had very little, if any, coating minerals on its surfaces. There appeared to be some biotite and some iron and/or manganese oxides embedded in a matrix consisting primarily of quartz and /or feldspar.

Information on the bulk mineralogy of the Tram and Bullfrog tuffs is available in a Yucca Mountain Site Characterization Project report. ${ }^{163}$ The mineralogist's observations stated above are in qualitative agreement with this report. There is little specific information available on the mineralogy of the Bandelier tuff sample. Bandelier tuff is known to have a great deal of variability mineralogically, chemically, and lithologically. " The mineralogist's observations stated above are consistent with the current knowledge of most geologic units of Bandelier tuff.*

The incomplete mineralogical characterization of the fractures was not a great concern as long as it could be demonstrated that the tracers did not significantly adsorb to or chemically interact with the surfaces. Investigations of possible interactions between tracers and fracture minerals are discussed in Section 3.1.4.

Because of the potential for matrix diffusion of solutes in the tracer experiments, the porosity of the matrices associated with each fracture was measured experimentally. The measurements were made using some of the larger remnants generated when the fracture blocks were cut with the rock saw. Additional cuts were made on the remnants to get them into convenient shapes for the measurements. Three samples of each type of tuff were prepared so that the measurements could be made in triplicate. The volume of the samples ranged from $\sim 28$ to $\sim 55 \mathrm{~cm}^{3}$. The measurements were made as follows:

(1) The volume of each porosity sample was measured by lowering it slowly into a tilted beaker filled with water. When the sample was lowered into the water, the displaced water overflowed the beaker and was collected in a tared container. After the water stopped overflowing, the tared container was weighed, and the volume of the rock sample was determined from the

\footnotetext{
* Personal communication with Dave Broxton of Los Alamos National Laboratory (1994).
} 
amount of water collected. This measurement was repeated several times for each sample to ensure that a statistically valid mean volume was obtained (standard deviations were less than $1 \%$ of the mean for the Bandelier and Tram tuff samples and 2.0 to $2.5 \%$ for the Bullfrog tuff samples).

(2) After measuring the volume of each sample, the samples were dried for several weeks in an oven at $80^{\circ} \mathrm{C}$. They were weighed periodically (after cooling in a dessicator containing silica dessicant) on an analytical balance until successive weights agreed to within less than $0.03 \mathrm{~g}$ or less than about $0.03 \%$ of the total sample weight. These weights were taken to be the dry weights of the samples.

(3) The samples were then immersed in groundwater (see Section 3.1.2) containing $\sim 0.02 \%$ sodium azide to inhibit biological growth. The immersion was carried out in a plastic dessicator that was hooked up to house vacuum ( 16-20 in. $\mathrm{Hg}$ vacuum) so that the pore space in the samples would saturate more quickly. The samples were periodically removed from the dessicator, patted dry on the surface with a paper towel, and then weighed using an analytical balance. This procedure was repeated until successive weight measurements agreed to within $0.05 \%$, which was about the reproducibility of measurements on the same day due to inconsistencies in patting the surfaces dry and also the continuous drying-out of the samples as the measurements were made. It took about 10 weeks to reach what was believed to be saturation. Occasionally, the dessicator was hooked up to a high-vacuum pump ( $29 \mathrm{in}$. $\mathrm{Hg}$ vacuum) for a 1/2-hour or so to speed up the saturation process.

The results of the porosity measurements are shown in Table 3.1. The relative standard deviations (standard deviation divided by the mean, expressed as a percentage) reported in this table were the greater of (1) the relative standard deviations of the final calculated porosities or (2) the relative standard deviations of just the volume measurements (for the Bullfrog tuff samples, the calculated porosities were in excellent agreement, but the volume measurements had a relative standard deviation of about $2.5 \%$ ). The relative standard deviations in Table 3.1 indicate that the technique had good precision.

Table 3.1. Results of Tuff Matrix Porosity Measurements.

\begin{tabular}{|c|c|c|}
\hline Tuff & Mean Porosity & Rel. Standard Deviation \\
\hline Bandelier & 0.089 & $0.89 \%$ \\
\hline Tram & 0.192 & $3.2 \%$ \\
\hline Bullfrog & 0.208 & $2.5 \%$ \\
\hline
\end{tabular}

\subsubsection{Groundwater}

The water used in all but one of the tracer experiments was collected from Well J-13 at the Nevada Test Site on 7/8/93 (the water used in the first experiment was also from Well J-13, but it was collected on $7 / 27 / 91$ ). This water should have been reasonably representative of the groundwater at the UE25 C-holes location, as Well J-13 is only about 2 miles from the C-holes. The water was collected in two polyethylene-lined 55-gallon drums and then shipped to 
Los Alamos where the tracer experiments were carried out. Prior to filling the drums, approximately 10-15 gallons of well water were "sloshed" around in each drum by shaking, rolling and finally emptying them. This procedure was intended to rid the drums of any residual surface contamination they might contain. When the drums were filled, they were allowed to overflow for several minutes before capping them to purge them of any dust or other foreign material that might have settled on the air-water interface during filling.

As reported by Ogard and Kerrisk, $164 \mathrm{~J}-13$ well water is essentially a sodium/calcium bicarbonate water that is saturated with silica (silicic acid). The composition reported by Ogard and Kerrisk is given in Table 3.2. The ionic strength of this water is approximately $0.003 \underline{\mathrm{M}}$. The composition of the J-13 water collected for the tracer experiments was not analyzed. However, samples were analyzed for several cations immediately after collection and again after all tracer experiments (about seven months apart) by inductively coupled plasmamass spectrometry, or ICP-MS ( $\mathrm{pH}$ was also measured). The results of these analyses are given in Table 3.3. The curious aspect of these results is that the concentration of calcium in the J-13 water appears to double with time; and magnesium, though never a major constituent, essentially disappears with time. An explanation for this behavior cannot be provided. Either there was some phenomenon occurring in the 55-gallon drums that resulted in these changes with time, or the samples were somehow compromised during handling or analysis. All samples were filtered with a $0.2-\mu \mathrm{m}$ syringe filter (Gelman Sterile Acrodisc, Gelman Sciences) and then submitted in 30-ml polypropylene bottles to the inductively coupled plasma-mass spectrometry lab at Los Alamos.

Treatment and handling of the samples once they reached the lab was unknown.

Table 3.2. J-13 Well Water Chemistry Reported by Ogard and Kerrisk. ${ }^{164}$

\begin{tabular}{|c|c|}
\hline Constituent & Concentration, $\mathrm{mg} / \mathrm{L}^{\mathrm{a}}$ \\
\hline $\mathrm{Ca}$ & 11.5 \\
\hline $\mathrm{Mg}$ & 1.76 \\
\hline $\mathrm{Na}$ & 45 \\
\hline $\mathrm{K}$ & 5.3 \\
\hline $\mathrm{Li}$ & 0.06 \\
\hline $\mathrm{Fe}$ & 0.04 \\
\hline $\mathrm{Mn}$ & 0.001 \\
\hline $\mathrm{Al}$ & 0.03 \\
\hline $\mathrm{Si}$ & 30.0 \\
\hline Fluoride & 2.1 \\
\hline Chloride & 6.4 \\
\hline Sulfate & 18.1 \\
\hline Bicarbonate & 124 \\
\hline Nitrate & 10.1 \\
\hline $\mathrm{O}_{2}$ & 5.7 \\
\hline $\mathrm{pH}$ & 6.9 \\
\hline
\end{tabular}

a All cation concentrations are based on the element, not ionic/molecular species. bFrom Fuentes et al. ${ }^{165}$ (not reported by Ogard and Kerrisk ${ }^{164}$ ). 
Table 3.3. Partial Analysis of J-13 Water over a Seven-Month Period.

\begin{tabular}{|c|c|c|}
\hline Constituent & ppm, Aug. 1993a, & ppm, March 1994a \\
\hline $\mathrm{Li}$ & below det. limit & 0.12 \\
\hline $\mathrm{Na}$ & 40.7 & 39.1 \\
\hline $\mathrm{Mg}$ & 1.57 & $<0.02$ \\
\hline $\mathrm{Al}$ & 0.053 & 0.055 \\
\hline $\mathrm{Ca}$ & 17.1 & 34.3 \\
\hline $\mathrm{Mn}$ & below det. limit & 0.012 \\
\hline $\mathrm{Fe}$ & 0.072 & 0.072 \\
\hline $\mathrm{Cs}$ & below det. limit & $<0.04$ \\
\hline $\mathrm{pH}$ (range) & $6.7-6.9$ & $6.7-6.9$ \\
\hline
\end{tabular}

a Values are means; relative standard deviations ranged from 5 to $10 \%$. bJ-13 water collected on 7/27/91 had essentially this composition.

Unfortunately, all that can be said about the calcium concentration in the $\mathrm{J}-13$ water during the tracer experiments is that it should have been somewhere between $17 \mathrm{ppm}$ and $34 \mathrm{ppm}$ over the course of the experiments. The cation concentrations after contact with the colloid tracers were also measured (see Section 3.1.5.1). Despite the apparent changes in the cation concentrations with time, there was no evidence that there was any change in the behavior of the tracers over time. Thus, it was concluded that the changes in cation concentrations did not affect the tracer behavior in the experiments. It should be noted that the J-13 water used in all tracer experiments was filtered with a $1-\mu \mathrm{m}$ cartridge prefilter followed by a $0.2-\mu \mathrm{m}$ Nylon cartridge finishing filter (Whatman Polycap 75 AS, Arbor Technologies, Inc.). The filtering was done to sterilize the water and to reduce the amount of background colloidal material in the water.

\subsubsection{Tracers}

The tracers used in the tracer experiments were

(1) $1-\mu \mathrm{m}$ diameter carboxylate-modified polystyrene latex (CML) microspheres tagged with a fluorescent blue dye (Interfacial Dynamics Corp., Portland, OR).

(2) $0.3-\mu \mathrm{m}$ diameter CML microspheres tagged with a fluorescent yellow dye (Interfacial Dynamics Corp., Portland, OR).

(3) $1-\mu \mathrm{m}$ diameter polystyrene microspheres having carboxyl surface groups tagged with a fluorescent yellow dye (Interfacial Dynamics Corp., Portland, $\mathrm{OR})$.

(4) $0.3-\mu \mathrm{m}$ diameter silica microspheres (Nissan Chemical, Ltd., Japan).

(5) Lithium iodide (Fluka Chemical Co., Germany), with the iodide being used as a nonsorbing solute (lithium was not analyzed).

Additional details on the properties of the polystyrene microspheres are given in Table 3.4. The first two microspheres in Table 3.4 (CML) were very similar in every respect except size. The third microsphere (the polystyrene with carboxyl surface groups, or non-CML polystyrene) was similar to the first two in that it had carboxyl groups as the surface functional group. However, as reported by Wan and Wilson, ${ }^{99}$ the CML microspheres have a hydrophilic surface (as determined from macroscopic contact angle measurements), while the carboxylated polystyrene has a hydrophobic surface. Wan and Wilson ${ }^{59}$ noted 
distinct differences in the transport behavior of these two types of microspheres in tracer experiments in saturated and unsaturated packed column experiments, with the hydrophobic microspheres being retained to a much greater degree in the columns. The silica microspheres should have hydrophilic surfaces like the CML microspheres, but they also have a density of $2.65 \mathrm{~g} / \mathrm{cm}^{3}$ (compared to $1.055 \mathrm{~g} / \mathrm{cm}^{3}$ for the polystyrene microspheres). This greater density gives the silica microspheres a significantly greater gravitational settling velocity than the polystyrene microspheres (approximately 32 times greater than $0.3-\mu \mathrm{m}$ polystyrene and 3 times greater than $1-\mu \mathrm{m}$ polystyrene -- see Eq. 22).

Table 3.4. Properties of Polystyrene Microspheres used as Tracers.a

\begin{tabular}{|c|c|c|c|}
\hline Property & $1-\mu \mathrm{m}$ CML & $0.3-\mu \mathrm{m} \mathrm{CML}$ & $1-\mu \mathrm{m}$ non-CML \\
\hline $\begin{array}{l}\text { Surface Functional } \\
\text { Group }\end{array}$ & Carboxyl & Carboxyl & Carboxyl \\
\hline $\begin{array}{l}\text { Mean Diameter }(\mu \mathrm{m}) \pm \\
\text { Rel. Standard Deviation }\end{array}$ & $0.977 \pm 2.6 \%$ & $0.282 \pm 2.6 \%$ & $0.984 \pm 5.1 \%$ \\
\hline Density, $\mathrm{g} / \mathrm{cm}^{3}$ & 1.055 & 1.055 & 1.055 \\
\hline $\begin{array}{l}\text { Surface Charge } \\
\text { Density, }, \mu \mathrm{C} / \mathrm{cm}^{2}\end{array}$ & 6.52 & 4.83 & 2.88 \\
\hline
\end{tabular}

aAll properties taken from certificates of analyses provided by Interfacial Dynamics Corp.

bFrom transmission electron microscopy of 500 randomly-selected particles. cDetermined by conductometric titration.

The CML, carboxylated polystyrene, and silica microspheres were used as tracers in initial experiments in the Bandelier tuff fracture to determine which type of microsphere would best serve as a nondepositing colloid in subsequent tracer experiments. As discussed in Section 3.1.7, it was quickly determined that the carboxylated polystyrene and the silica microspheres exhibited unsatisfactory deposition or attenuation behavior (also the silica could not be analyzed with as much sensitivity or precision as the polystyrene microspheres), so these microspheres were not used in subsequent experiments.

\subsubsection{Tracer Analytical Methods}

Iodide was analyzed using an ion-selective electrode (Orion Model 94-53 iodide electrode in conjunction with an Orion Model 90-02 double junction reference electrode). This technique was chosen because of its simplicity, speed, and excellent detection limit, which was about 5 parts per billion in the J-13 water. Based on the manufacturer's instruction manual, the expected precision of the technique was about $4 \%$ (unclear whether this was a standard deviation or other measure of error). However, repeat measurements indicated a relative standard deviation of about 2\% (see Section 3.3, Error Analysis). The exact procedure used to measure iodide concentrations was documented in a detailed technical procedure prepared for the Yucca Mountain Site Characterization Project by the author. ${ }^{166}$ The procedure involved recalibrating the electrodes with a series of standards at least once an hour to minimize errors caused by electrode drift or temperature changes in the laboratory. When the readings associated with the calibration standards changed, a linear change with time was 
assumed for the purpose of calculating the concentrations of all unknowns measured between the two calibrations. Dilutions of the inlet tracer solution for a given tracer experiment were always used as the calibration standards. In this way, the measured iodide concentration was automatically normalized to the inlet concentration.

The $0.3-\mu \mathrm{m}$ diameter silica microspheres were analyzed by single-angle light scattering (or turbidity) using a Coulter N4IMD laser photon correlation spectroscopy instrument at the University of Ner $N$ Mexico. The effective detection limit of this technique was somewhere between $10^{8}$ and $10^{9}$ spheres/liter (which was also confirmed by flow cytometry -- see next paragraph). The chief limitation of this technique was that it was very difficult to reduce residual colloid concentrations in water samples to less than about $10^{8}$ particles/liter. Thus, to effectively conduct a tracer experiment, the system had to be "swamped" with the colloid tracer to ensure its detection in the presence of the background. Another limitation was that the relative standard deviation of repeat measurements was typically about $5 \%$, which is significantly less precision than other methods, such as flow cytometry, offer.

The fluorescent polystyrene microspheres were analyzed by flow cytometry. ${ }^{167}$ This technique takes advantage of the fluorescent signature of the dyed microspheres to discriminate them from natural or background colloidal material. In flow cytometry, a liquid sample is passed through a series of orifices and a fluid sheath, collectively known as a "hydrodynamic focusing chamber," which produces a very narrow stream of the fluid in which all particles of appreciable size are lined up in "single file." This stream is passed through one or more laser beams, which excite the fluorescing molecules in the tagged microspheres. Fluorescence signals are detected by photomultiplier tubes at a 90-degree angle from the incident laser beam(s). These signals, or events, can be counted using digital electronic equipment, and the number of events per unit volume of fluid sample can be determined by weighing the sample before and after the measurement. Microspheres tagged with different dyes can be analyzed simultaneously by using multiple laser beams and multiple photomultipliers (in combination with light filters) to excite and detect fluorescent dyes over various wavelength ranges in the electromagnetic spectrum. Discrimination of tagged microspheres from background colloidal material is excellent provided the natural material does not fluoresce significantly over the wavelength range of interest (concentrations as much as 3 orders of magnitude below background levels can be quantitatively analyzed).

The "Big Geek" flow cytometer at Los Alamos National Laboratory was used for all the fluorescent microsphere analyses reported in this dissertation. The analytical procedure was documented in a detailed technical procedure prepared for the Yucca Mountain Site Characterization Project by the author. ${ }^{168}$ The detection limit of the technique was approximately $10^{5}$ spheres/liter (dependent on the amount of background present and on the intensity of the fluorescence signals associated with the microspheres). The precision of the technique was excellent, with relative standard deviations of approximately $1 \%$ for repeat measurements (see Section 3.3, Error A.nalysis).

\footnotetext{
* From repeat measurements on the Coulter N4MD and also personal communications with Rahul Kale of the University of New Mexico (1993).
} 


\subsubsection{Screening of Tracer-Rock-Water Interactions}

The intent of the tracer experiments was to study differences between colloid and solute transport under conditions where neither tracer interacted (attached or adsorbed) appreciably with the fracture surfaces. A number of separate experiments were carried out to investigate possible interactions between the tracers, the groundwater, and the rock surfaces to determine if any such interactions might occur. These experiments included (1) analyzing the cation concentrations in the J-13 water after contact with the microspheres to determine if the microspheres in any way changed the water chemistry, (2) measuring the zeta potentials of the colloids and the tuff surfaces in the J-13 water to determine if electrostatic attraction between the surfaces was possible, and (3) conducting batch sorption experiments to investigate the sorption of iodide onto the tuff surfaces in J-13 water. These experiments are discussed in the following three sections.

\subsubsection{J-13 Water Chemistry after Contact with Microspheres}

At three different times (August 1993, February 1994, and March 1994), some of the microspheres used as tracers were immersed for $\sim 24$ hours at ambient temperature in J-13 water that had been filtered with a $0.2-\mu \mathrm{m}$ filter (Whatman Polycap 75 AS, Arbor Technologies, Inc.). A 24-hr contact period was chosen because this exceeded the expected contact time between the microspheres and the J-13 water prior to and during a tracer experiment. The dispersions were then filtered so that the filtrate could be analyzed in the same way as described in Section 3.1.2 for the J-13 water (ICP-MS and pH). Filtered J13 water that had not been in contact with the microspheres was analyzed at the same time as the water that had been in contact with the microspheres. However, the uncontacted J-13 water sample from the February 1994 analyses was compromised by the analytical laboratory, so no results are reported for that time period.

Table 3.5 shows the results of ICP-MS analyses of the water samples after contact with microspheres at the three different times (refer to Table 3.3 for corresponding results for the J-13 water that was not in contact with the microspheres). In all cases, the concentration of microspheres in contact with the water samples was greater than or equal to that in any of the tracer experiments. The non-CML polystyrene microspheres were not used in any of the contact experiments because they were eliminated from consideration as tracers prior to these experiments.

The differences between the J-13 water cation concentrations before and after contact with the microspheres are inconsistent at the various times. It must be remembered that the measured calcium concentration in the J-13 water doubled (from $\sim 17 \mathrm{ppm}$ to $\sim 34 \mathrm{ppm}$ ) and the magnesium concentration essentially went from 2 ppm to zero between August 1993 and March 1994. The following conclusions can be drawn from the data in Tables 3.3 and 3.5:

(1) The CML microspheres sometimes had no noticeable effect on the J-13 water chemistry (August 1993), and at other times they seemed to significantly increase the calcium concentration (by $50 \%$ ) in the water (March 1994).

(2) The silica microspheres seemed to increase both the sodium (by $\sim 40 \%$ ) and the calcium (by $\sim 50 \%$ ) concentrations in the J-13 water. 
Table 3.5. Partial Analysis of J-13 Water after Contact with Microspheres (ppm). ${ }^{\text {a }}$

\begin{tabular}{|c|c|c|c|c|c|}
\hline Constituent & $\frac{\text { CML }_{x}}{\text { Aug. } 1993}$ & $\frac{\text { CML }}{\text { Feb. } 1994}$ & $\begin{array}{c}\text { Silica, } \\
\text { Feb. } 1994 \\
\end{array}$ & $\frac{\text { CML }_{6}}{\text { March } 1994}$ & 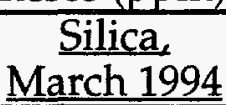 \\
\hline $\mathrm{Li}$ & below det. & 0.05 & 0.04 & 0.18 & 0.16 \\
\hline $\mathrm{Na}$ & 41.2 & 51.2 & 62.4 & 38.1 & 54.9 \\
\hline $\mathrm{Mg}$ & 1.40 & 2.66 & 2.71 & $<0.2$ & $<0.03$ \\
\hline $\mathrm{Al}$ & 0.038 & 0.12 & 0.28 & 0.07 & 0.08 \\
\hline $\mathrm{Ca}$ & 15.3 & $\overline{33.4}$ & 30.7 & $\overline{52.4}$ & 48.8 \\
\hline$\overline{\mathrm{Mn}}$ & $<0.003$ & 0.09 & $<0.03$ & $<0.02$ & 0.02 \\
\hline $\mathrm{Fe}$ & 0.089 & 1.68 & 1.45 & 0.11 & 0.12 \\
\hline Cs & below det. & 0.09 & 0.28 & $<0.03$ & $<0.03$ \\
\hline$\overline{\mathrm{pH}}$ & 6.8 & 6.7 & 6.7 & not done & not done \\
\hline
\end{tabular}

a Values are means; relative standard deviations ranged from 5 to $15 \%$.

(3) Neither microsphere affected the $\mathrm{pH}$ of the J-13 water.

(4) Concentrations of minor constituents seemed to vary more as a function of time than as a function of contact with the microspheres.

It is suspected that some of these inconsistencies may have been the result of poor sample handling and analyses techniques in the Los Alamos ICP-MS laboratory. Other researchers at Los Alamos had similar problems with analytical consistency from this laboratory during the time period that the analyses were done." Because of these problems, it was difficult to put much confidence in the results of the contact experiments. However, as noted in Section 3.1.2, there was no evidence of any changes in the transport behavior of the microspheres or iodide over time, so it was concluded that changes in water chemistry, if real, did not affect the behavior of the tracers in the experiments.

An interesting observation made during the March 1994 contact experiment was that the water that had been in contact with the CML microspheres appeared to fluoresce under ultraviolet light even after the microspheres had been removed by filtration. The fluorescence persisted after several filtrations with a $0.2-\mu \mathrm{m}$ filter, indicating that it was probably not coming from microspheres that had passed through the filter. This observation suggests that some of the fluorescent dyes used to tag the microspheres may have come off/out of the microspheres during the 24 -hr contact time. This finding may be significant if microspheres are ultimately used in field experiments because there is often a significant lag time between sample collection and sample analyses in field experiments, and it is conceivable that the microspheres could lose their fluorescent signal during this time.

\subsubsection{Zeta Potential Measurements}

To investigate the possibility of electrostatic attraction between the colloid and fracture surfaces, the zeta potentials associated with these surfaces were measured in J-13 water and in J-13 water spiked with $0.001 \underline{\mathrm{M}}$ LiI (the LiI concentration in all tracer experiments) using a Coulter Delsa 440 (Doppler electrophoretic light-scattering analyzer) at the University of New Mexico. The zeta potential is the electrostatic potential at the surface of hydrodynamic shear

\footnotetext{
* Personal communications with Ines Triay and Chuck Cotter of Los Alamos National Laboratory (1994).
} 
of a moving particle (relatively close to the actual particle surface). ${ }^{121}$ The Coulter Delsa 440 actually measures particle mobilities, and the mobilities are converted to zeta potentials using the Helmholtz-Smoluchowski equation:121

where, $\zeta=$ zeta potential, $\mathrm{V}$

$$
\zeta=\frac{u \mu}{\varepsilon \varepsilon_{\mathrm{o}}}
$$

$\mathrm{u}=$ mobility, $\mathrm{m}^{2} / \mathrm{V}$-sec

$\mu=$ viscosity, $\mathrm{kg} / \mathrm{m}$-sec

$\varepsilon=$ dielectric constant of medium (dimensionless)

$\varepsilon_{\mathrm{o}}=$ permittivitiy of vacuum, $\mathrm{C}^{2} / \mathrm{J}-\mathrm{m}$.

The results of the mobility / zeta potential measurements are given in Table 3.6. The tuff samples in the J-13 water (and spiked J-13 water) were prepared by grinding two rock surfaces together (not the actual fracture surfaces) to generate fines and then suspending the fines in the water. The microspheres were simply dispersed in the water.

Table 3.6. Results of Mobility/Zeta Potential Measurements. ${ }^{\mathrm{a}, \mathrm{b}}$

\begin{tabular}{|c|c|c|}
\hline Microsphere/Tuff/Water & Mobility $\mu \mathrm{m}-\mathrm{cm} / \mathrm{v}-\mathrm{s}$ & Zeta Pot., $\mathrm{mV}$ \\
\hline $1-\mu \mathrm{m}$ CML in J-13 water & -2.58 & -33.0 \\
\hline $1-\mu \mathrm{m}$ CML in J-13 with 0.001 M LiI & -2.64 & -33.9 \\
\hline $0.3-\mu \mathrm{m}$ CML in J-13 water & -2.41 & -31.0 \\
\hline $0.3-\mu \mathrm{m}$ CML in J-13 with 0.001 M LiI & -2.25 & -28.9 \\
\hline $1-\mu \mathrm{m}$ non-CML in J-13 water & -2.71 & -34.8 \\
\hline $1-\mu \mathrm{m}$ non-CML in J-13 with 0.001 M & -2.39 & -30.7 \\
LiI & & \\
\hline $0.3-\mu \mathrm{m}$ Silica in J-13 water & -3.03 & -38.9 \\
\hline $0.3-\mu \mathrm{m}$ Silica in J-13 with 0.001 M LiI & -3.25 & -41.7 \\
\hline Bandelier Tuff in J-13 water & -2.05 & -26.3 \\
\hline Bandelier Tuff in J-13 with 0.001 M LiI & -1.64 & -21.1 \\
\hline Tram Tuff in J-13 water & -1.65 & -21.2 \\
\hline Tram Tuff in J-13 with 0.001 M LiI & -1.61 & -20.7 \\
\hline Bullfrog Tuff in J-13 water & -1.63 & -20.9 \\
\hline Bullfrog Tuff in J-13 with 0.001 M LiI & -1.71 & -21.9 \\
\hline
\end{tabular}

aSix measurements on one sample gave a relative standard deviation of $1.3 \%$. bAll measurements were conducted at $25^{\circ} \mathrm{C}$.

The results in Table 3.6 indicate that all of the surfaces had significant negative electrostatic potentials in the J-13 water and that the presence of $0.001 \mathrm{M}$ LiI had little or no effect on these potentials. These results indicate that electrostatic interactions between the microspheres and the rock surfaces should have been primarily repulsive rather than attractive, which is a necessary (but not sufficient) condition for the colloid tracers to not attach to the rock surfaces. 


\subsubsection{Batch Sorption Measurements of Iodide on Tuffs}

To investigate the potential for adsorption of iodide to the fracture surfaces, 24-hour batch sorption experiments were conducted using each of the three tuffs. The 24-hour duration was deemed sufficiently long because the contact time between the iodide solution and the tuffs in the tracer experiments never exceeded 1 hour. The procedure was as follows:

(1) Tuff chunks were crushed and pulverized (using an ore crusher and a pulverizer), and the crushed rock was sieved into various size fractions.

(2) 20.00 grams of tuff in the size range $45-$ to $250-\mu \mathrm{m}$ (weighed out using a top-loading balance) was poured into a plastic centrifuge container (with screw cap).

(3) $25.0 \mathrm{ml}$ of $0.2-\mu \mathrm{m}$ filtered $\mathrm{J}-13$ water was added to the container and shaken with the tuff for $\sim 60$ hours to condition the tuff prior to the introduction of iodide.

(4) $25.0 \mathrm{ml}$ of $0.002 \mathrm{M} \mathrm{LiI}$ in $0.2-\mu \mathrm{m}$ filtered J-13 water was added to the container to bring the total amount of water to $50.0 \mathrm{ml}$ and the total concentration of iodide in the water to $0.001 \underline{\mathrm{M}}$ (the same as in the tracer experiments).

(5) The container was again shaken for $\sim 24$ hours, and then the water was analyzed for iodide using the ion-selective electrode technique discussed in Section 3.1.4.

(6) Control samples containing no tuff were subjected to the same steps as above to check for iodide interactions with the containers.

All tests were run in triplicate ( 3 containers for each tuff and 3 containers with no tuff for a total of 12 containers).

The electrode measurements indicated no sorption of iodide onto any of the tuffs. In fact, the solutions that had been in contact with the tuffs tended to have slightly higher concentration readings than the control samples, although the difference was within the precision of the analytical technique. It was concluded that there was no measurable sorption of iodide onto the tuffs in the 24-hour time period of the experiments. However, it must be remembered that the experiments were carried out using minerals from the tuff matrices and not from the fracture surfaces. Thus, it is not possible to conclude unequivocally that sorption could not occur in the tracer experiments based on the results of these batch tests.

3.1.6 Tracer Experiments: Apparatus and Procedure

Eighteen tracer experiments were conducted in the three tuff fractures over a six-month period. This section describes (1) the fracture flow apparatus(es), (2) the experimental flow system, and (3) the procedure followed to conduct the experiments. The details of the conditions under which each experiment was run are provided in Appendix A. The results of the experiments are discussed in Section 3.1.7.

\subsubsection{Fracture Flow Apparatus}

A flow apparatus was built around each of the tuff fracture blocks with the intent of sealing two opposite sides of each fracture to serve as no-flow boundaries and allowing the remaining two sides (ends) to be constant-pressure boundaries. For each fracture, a pair of $1 / 2$-inch-thick aluminum plates were cut so that they were approximately 3 inches longer and 3 inches wider than the fracture block itself (in the plane of the fracture). Holes were drilled through 
these plates at corresponding locations in each corner. On one plate the holes were large enough to allow a 1/4-28 NPT bolt to pass through them, and on the other plate the holes were tapped so that a 1/4-28 NPT bolt could be screwed directly into the plate. The fracture block was placed between the plates, and then the plates were bolted together to force the fracture surfaces together under a small normal load. A separate set of such plates, called "confining plates," were machined for each fracture.

Four 3/16-inch-wide and 3/16-inch-deep rectangular grooves were machined into one side of each confining plate. The grooves ran parallel to the edges of the confining plates and down the entire length of the plates. In general, the grooves were very close to the edges of the plates (within 1/4-inch). Fig. 3.1 shows the grooves and bolt holes in a typical confining plate. The grooves on each plate faced inward toward each other when the confining plates were bolted together to confine a fracture.

Fig. 3.2 is a cutaway view looking at the side of a confined fracture block. This figure shows the key features of a fracture flow apparatus. For clarity, the bolts holding the confining plates together are not shown. The grooves in the confining plates were designed to allow $1 / 8$-inch-thick aluminum plates to be slid into them parallel to the fracture block surfaces. These thin plates were about 1 -inch wide and as long as necessary to fit comfortably into the grooves on both the top and bottom confining plate. The plates had a pem nut mounted in them to allow a 1/4-28 NPT bolt to be screwed through them as shown in Fig. 3.2. Three such plates were slid into the grooves on each side of the fracture, and four were slid into the grooves on the ends of the fracture.

To provide a no-flow boundary along the side of the fracture, a piece of 3/16-inch thick silicone gasket material (Groendyk, 30 durometer shore hardness) was cut to the same length as the fracture block and about 3/4-inch wide. This piece of gasket material was placed over the fracture trace along the edge of the block, and then a piece of 3/4-inch-wide, 1/4-inch-thick aluminum bar stock (cut to the same length as the gasket material) was placed over the gasket material. The grooves in the confining plates kept the thin plates in position so that the bolts threaded through the pem nuts could be tightened down onto the aluminum bar stock to compress the gasket material over the side of the fracture and thus seal the fracture. In some cases, dimples were machined into the bar stock to provide a "seat" for the bolts, but it was found that these were often not used because the bolt positions were changed frequently to try to minimize leaks.

Although not explicitly shown in Fig. 3.2, the edges of the fracture block just above and below the fracture were coated with a thin layer of silicone sealant. It was found that leakage could be significantly reduced when the silicone gasket material was pressed down onto the silicone sealant rather than the bare rock surface. Thicker beads of silicone were used to coat the corners, as leaks at the corners were a persistent problem.

The ends of the fractures were sealed in a manner similar to the sides, with the following exceptions:

(1) The gasket material and aluminum bar stock were both 1-inch wide, and they were about 1 inch longer than the fracture block (rather than the same length as the fracture block). Also, the gasket material was 40 or 50 durometer shore hardness to give it enough deformation resistance to keep 


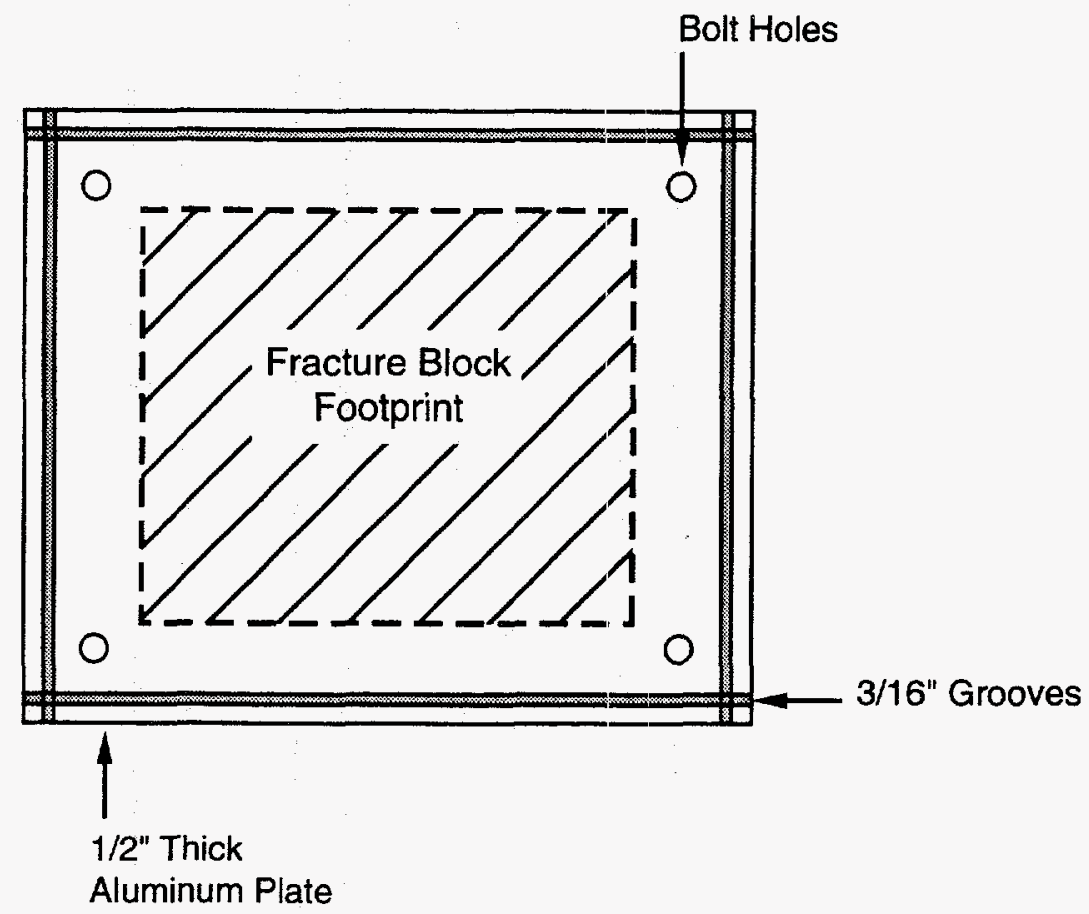

Figure 3.1. Top view of aluminum confining plate for fracture flow apparatus.

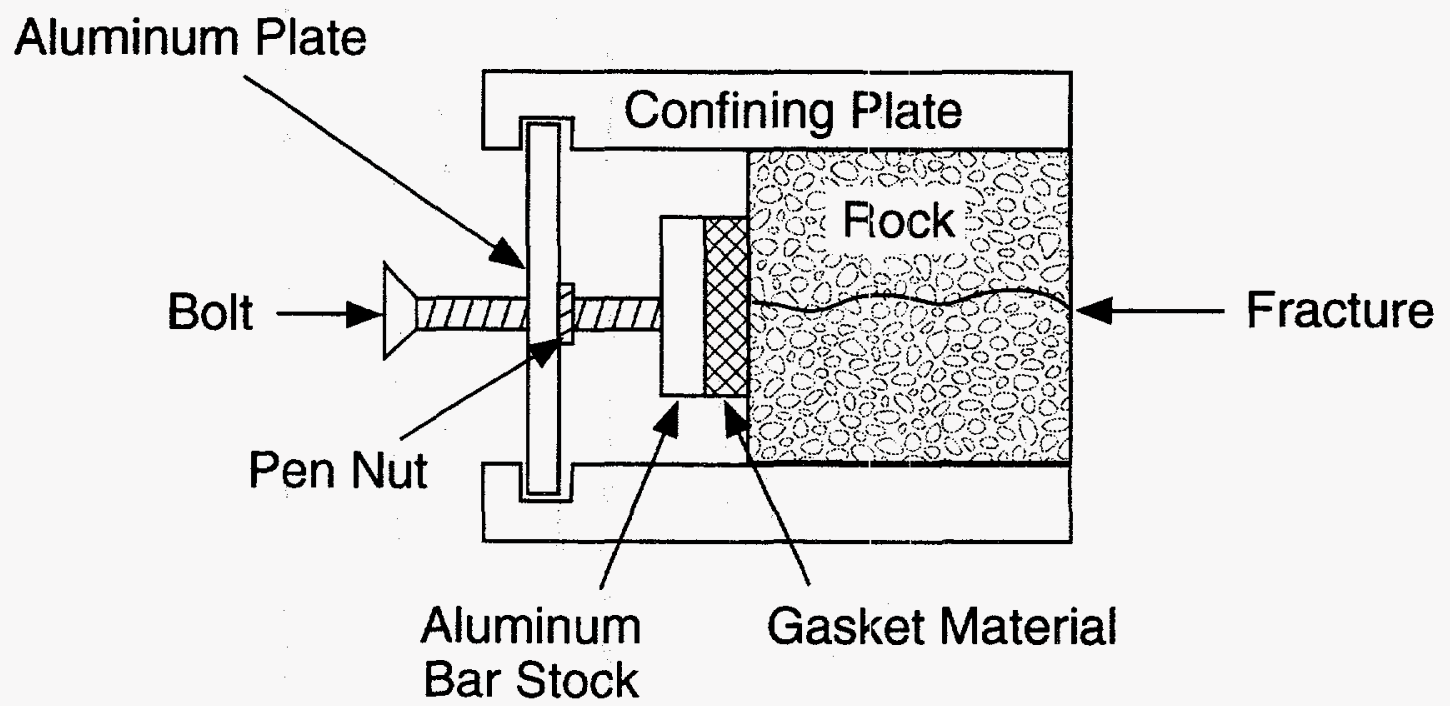

Figure 3.2. Side view of fracture flow apparatus showing method of achieving leak-free seal on side of fracture block (for no-flow boundaries). 
the flow manifold (described below) from collapsing under load from the bolts.(2) A 1/4-inch-wide "channel" was cut out of the center of the gasket material such that it would fit exactly over the fracture when the gasket material was placed over the end of the fracture block. This channel served as a constant-pressure flow manifold in the tracer experiments.

(3) Two holes were drilled and tapped (for 1/4-28 NPT fittings) in the aluminum bar stock at positions corresponding to just inside the corners of the fracture block. These holes served as flow ports on either end of the channel in the gasket material when the bar stock was properly positioned over the gasket material.

Fig. 3.3 shows the configuration over the end of a fracture block, with everything under the aluminum bar stock being shown in dashed lines. The gasket material and bar stock on the ends overlap those on the sides of the fracture block in order to achieve a good seal at the corners of the block. The four bolts used to press the gasket material down onto the ends of the block were positioned such that two were just outside the port holes (right at the corners of the block) and two were inside the port holes. Polypropylene fittings that allowed connections to $1 / 8-$ inch ID Tygon tubing were screwed into the port holes using Teflon tape to achieve a good seal.

\subsubsection{Experimental Flow System}

The fracture flow apparatus described in the previous section was a subset of the overall flow system used to conduct tracer experiments. Fig. 3.4 is a schematic of the entire system used to conduct the experiments. The valves in Fig. 3.4 are numbered for reference in Section 3.1.6.3, which provides a detailed description of the procedure(s) for conducting tracer experiments. Although not shown in Fig. 3.4, the fracture flow apparatus was always completely immersed in a water bath to help minimize outward leakage from the system (and also to minimize the effect of temperature fluctuations in the room during the experiments). The fracture was oriented horizontally in all of the tracer experiments. Two reservoirs (2-liter aspirator bottles) containing J-13 water with tracers and tracer-free J-13 water, respectively, were used as feeds to the fracture flow apparatus (see upper-right of Fig. 3.4). All tubing shown in Fig. 3.4 was

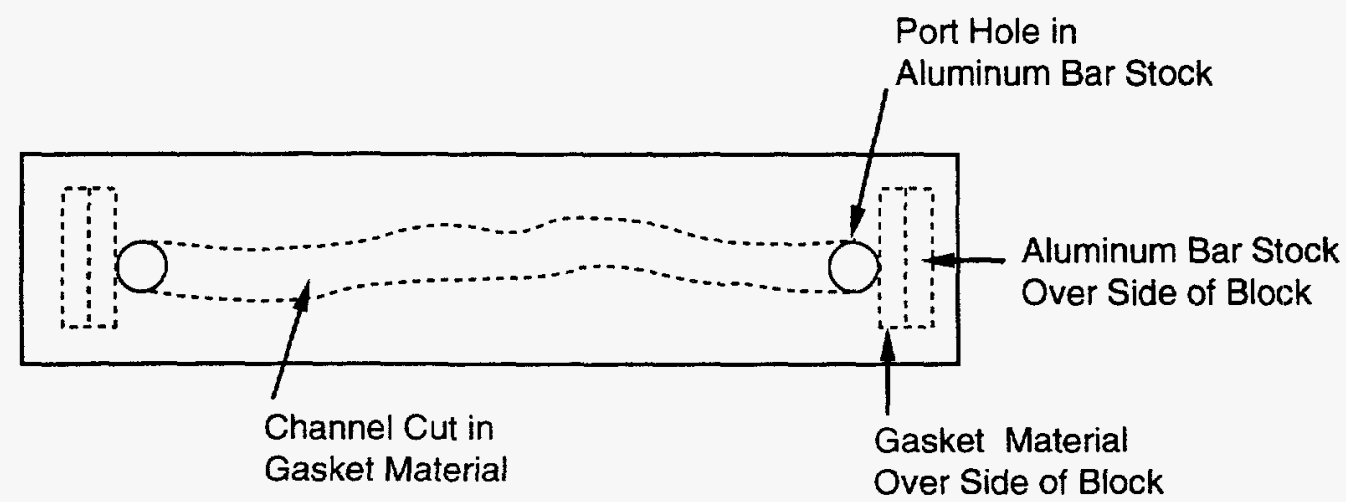

Figure 3.3. Flow apparatus configuration over end of fracture block showing channel cut in gasket material to serve as a flow manifold underneath aluminum bar stock. The channel is cut to fit exactly over the fracture. 


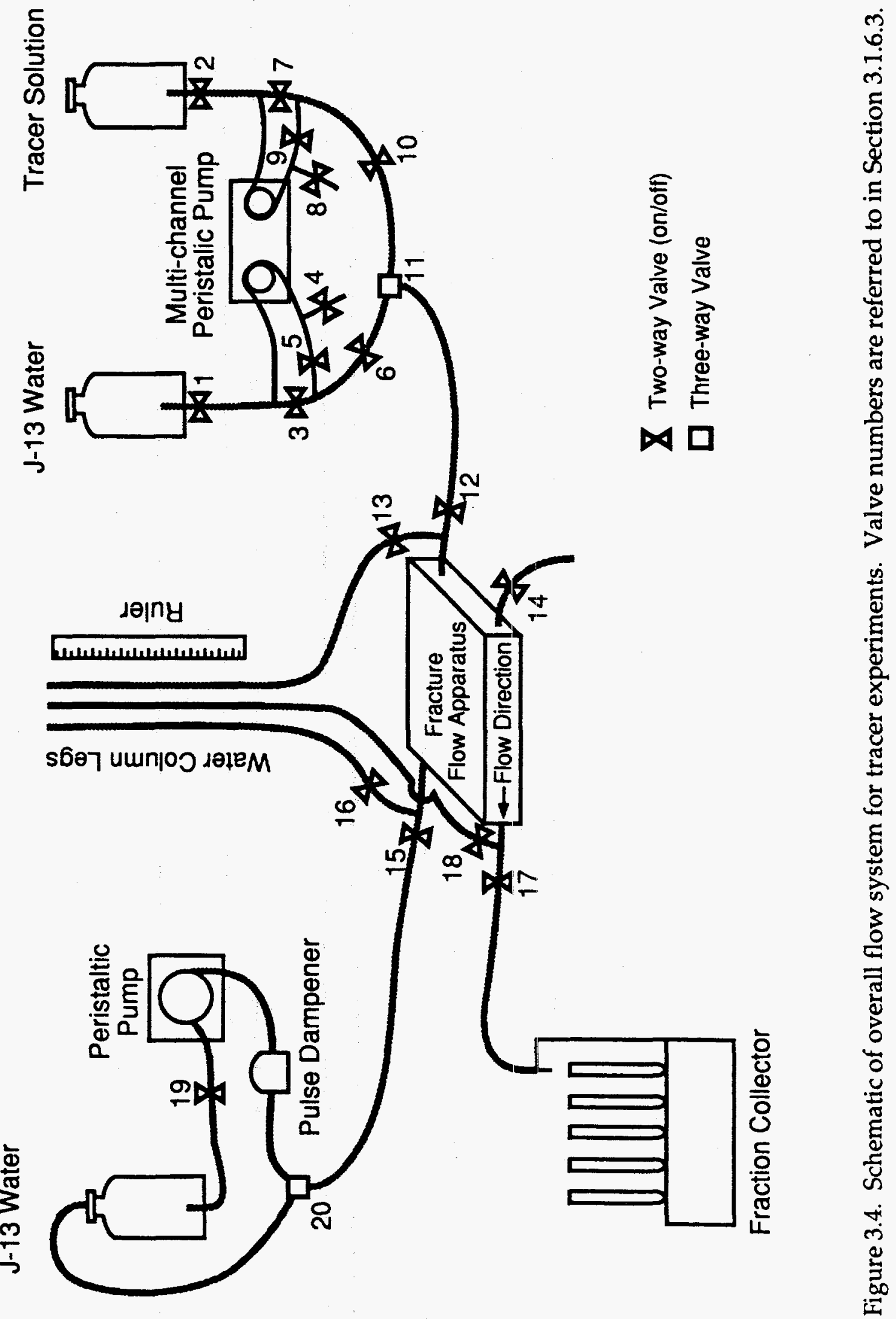


flexible Tygon R-3603 plastic tubing (with the exception of the tubing used in the high-flow-rate peristaltic pump head, which was Tygon R-1000 plastic tubing). All valves and fittings were made of polypropylene (from Cole-Parmer, Inc. or Value Plastics, Inc.). The multichannel peristaltic pump used to control flow rates through the fracture was a Buchler/Labconco Multistaltic Pump, Cat. No. 426-2000). This pump had four channels, each with eight rollers to minimize pulsations; and it had good rotation speed control. Only two channels were ever used at the same time in the tracer experiments. The channels accepted either 1/32-inch ID or 1/16-inch ID Tygon tubing, depending on the desired flow rate(s). Other tubing in the system was $1 / 8$-inch ID to minimize tubing pressure drop. The tubing labeled "water column legs" in Fig. 3.4 was used to determine pressure drops (by water column height difference) across either the fracture or the outlet manifold (see Section 3.1.6.3).

The peristaltic pump on the outlet side of the fracture was a standard Cole-Parmer Masterflex variable-speed drive equipped with a size 7016 Masterflex head, which accepted 1/4-inch OD, 1/8-inch ID flexible tubing. This pump was fed by a reservoir (2-liter aspirator bottle) filled with tracer-free J-13 water, which was used to flush the outlet manifold of the fracture flow apparatus (discussed in Section 3.1.6.3). A pulsation dampener (Cole-Parmer, Inc.) was used to eliminate pressure pulses in the outlet manifold that would have been caused by the pump. The samples from the tracer experiments were collected in 18- $x$ 150-mm test tubes using a Spectra/Chrom CF-1 automated fraction collector. A photograph of the experimental flow system is shown in Fig. 3.5.

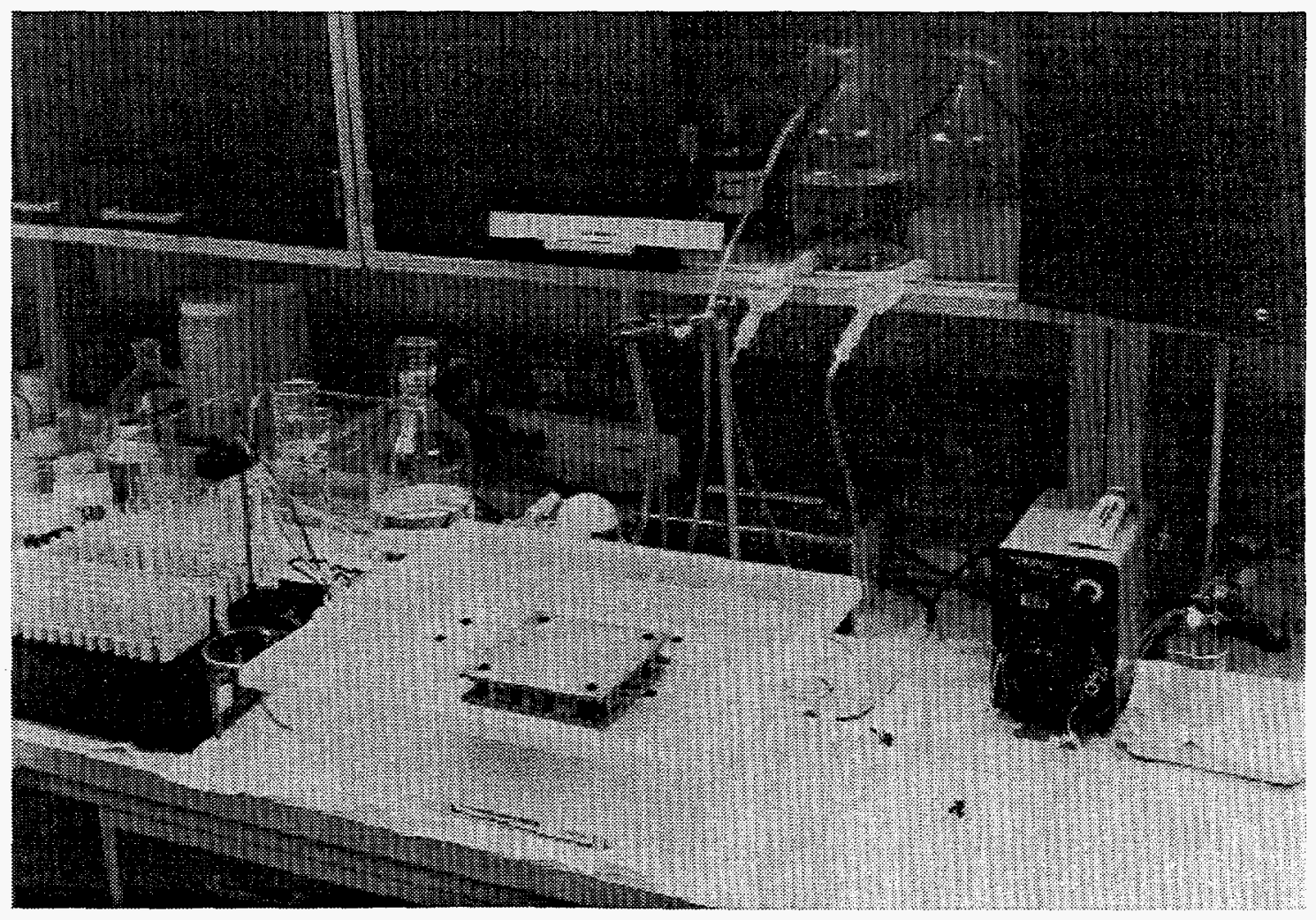

Figure 3.5. Photograph of flow system just prior to a tracer experiment. Fracture flow apparatus is immersed in a pan of water to minimize leakage. 



\subsubsection{Experimental Procedure}

The tracer experiments were conducted by introducing a uniform step of tracer solution into the inlet manifold of the fracture flow apparatus, forcing a constant flow rate through the fracture, and collecting samples from the outlet manifold. In most experiments, the tracers were subsequently flushed out of the fracture by introducing a uniform step of tracer-free J-13 water and again collecting samples at the outlet to monitor the decay of the tracer concentrations in the system. Although not explicitly mentioned in the following discussion, all $\mathrm{J}-13$ water used in the tracer experiments was filtered with a $0.2-\mu \mathrm{m}$ Whatman Polycap 75 AS Nylon cartridge filter (Arbor Technologies, Inc.). The procedure for conducting an experiment was as follows:

First, prior to an experiment, the following steps were taken:

(1) The fracture blocks were saturated over several months by placing them in $\mathrm{J}-13$ water in a dessicator and drawing a vacuum $(\sim 16-20$ in. $\mathrm{Hg}$ house vacuum) on the solution to speed up the saturation process. The water was spiked with 0.02 to $0.05 \%$ sodium azide to prevent biological growth on the rock surfaces. The rocks were not weighed periodically to check for saturation, but air bubbles stopped evolving on the rock surfaces after a few weeks.

(2) The day before an experiment, the fracture flow apparatus was assembled completely under water in a large sink to avoid any inadvertent introduction of air bubbles to the system. Deionized (DI) water was used to fill the sink, as there was not enough J-13 water available to fill the sink more than a few times.

(3) Immediately after assembly, the fracture flow apparatus was placed into a large pan full of DI water and then hooked up to the rest of the experimental flow system (shown in Fig. 3.4).

(4) J-13 water was allowed to flow by gravity through the fracture for several minutes to displace the DI water. As a measure to avoid the introduction of air bubbles into the flow system, the J-13 water was degassed by drawing house vacuum on it prior to using it. The three water column legs were allowed to fill with the J-13 water by opening Valves 13,16 and 18 in sequence. The level in each leg was adjusted so that it was approximately $40 \mathrm{~cm}$ above the fracture.

(5) $40 \mathrm{~cm}$ of head was applied to the fracture flow apparatus by closing Valves $12,14,15$, and 17 to isolate the fracture from the rest of the flow system. This head was kept on the flow apparatus for an extended time, and the level in the legs was monitored to check for leaks in the system. The goal was to achieve a leak rate of less than $1-2 \%$ of the minimum flow rate planned for the subsequent tracer experiment(s). Unacceptable leaks could generally be fixed by simply tightening the bolts pressing down on the gasket material along the sides and ends of the fracture. Leaks were most common at the corners of the fracture blocks.

(6) After achieving an acceptable leak rate, the fracture was usually allowed to sit overnight (waiting for a tracer experiment to be conducted the next morning).

(7) 1-liter of tracer solution was prepared by adding enough Lil to J-13 water to make a $0.001 \mathrm{M}$ solution (about $0.134 \mathrm{~g}$ of Lil per liter). A $0.001 \mathrm{M}$ solution was used because this concentration would not appreciably affect the ionic 
strength of the J-13 water $(0.003 \underline{\mathrm{M}})$. There was a concern that the colloid tracers might be more susceptible to flocculation if the ionic strength was increased significantly by the addition of the solute tracer (because of thinning of the electrical double layers that help provide stability). The solution was transferred to a 2-liter aspirator bottle and placed under vacuum to degas it (generally overnight) prior to the tracer experiments. Two other 2-liter aspirator bottles full of tracer-free J-13 water were also degassed at the same time.

(8) Colloid tracers were generally added to the tracer solution immediately before a tracer experiment. Approximately $0.5 \mathrm{ml}$ of $2 \%$ (by volume) stock solution of $1-\mu \mathrm{m}$ microspheres and 2 or 3 drops of $0.3-\mu \mathrm{m}$ CML microsphere stock solution were added to the tracer solution. These additions resulted in concentrations of $1.5-4 \times 10^{10} /$ liter for the $1-\mu \mathrm{m}$ microspheres and 1-3 $\mathrm{x}$ $10^{11} /$ liter for the $0.3-\mu \mathrm{m}$ microspheres. Several $\mathrm{ml}$ of the $0.3-\mu \mathrm{m}$ silica microspheres were added to the tracer solution to produce a concentration estimated to be about $10^{13} /$ liter. The much higher silica concentration was necessary because of the analytical limitations of the turbidity measurement technique discussed in Section 3.1.4. The tracer concentrations (including iodide) were not intended to be reproducible or even known exactly because the breakthrough curves were all normalized to the inlet concentrations.

After these setup procedures, a tracer experiment was conducted as follows:

(9) The peristaltic pumps were started well in advance of an experiment to allow them to stabilize.

(10) Tracer-free J-13 water was allowed to flow through the fracture by gravity with the following valves open: $1,3,6,11,12,13,17$, and 18 . Valves 13 and 18 were opened to allow the pressure drop (head difference) across the fracture to be measured. The flow rate through the fracture was determined by weighing the amount of water collected in a tared beaker at the outlet in a given amount of time. The hydraulic aperture of the fracture could then be calculated using a rearrangement of Eq. (6):

$$
a=\left(\frac{12 Q \mu L}{W \Delta P}\right)^{1 / 3}
$$

where, $\mathrm{a}=$ hydraulic aperture, $\mathrm{cm}$

$\mathrm{L}=$ length of fracture, $\mathrm{cm}$

$\mathrm{W}=$ average width of fracture, $\mathrm{cm}$

$\Delta \mathrm{P}=$ pressure drop across fracture, dyne $/ \mathrm{cm}^{2}$.

(11) After measuring the hydraulic aperture, the flow rates of the tracer solution and the tracer-free J-13 water were adjusted and measured using the collectweigh-time technique mentioned in Step 10. To do this, Valves 3, 5, 7, and 9 were closed, and Valves 4 and 8 were opened. The flow rates of the two solutions were intended to be identical, but there was almost always some difference between them, presumably due to slightly different tubing diameters in the pump heads or perhaps due to stretching or wear of the tubing.

(12) The flow rate of the tracer-free solution being pumped by the peristaltic pump on the outlet side of the fracture flow apparatus was adjusted and measured using the collect-weigh-time technique mentioned in Steps 10 and 11. After adjustment to the desired flow rate, flow was briefly diverted into 
the outlet manifold by changing the position of Valve 20, opening Valves $15-18$, and closing Valves 12-14 (to prevent flow through the fracture). This was done to determine if there was excessive pressure drop across the outlet manifold. A pressure drop of no more than $2-4 \mathrm{~cm}$ of water (depending on flow rate) was desired to ensure that their was no blockage in the flow manifold due to incorrect positioning of the aluminum bar stock over the gasket material forming the manifold. If the pressure drop exceeded $2-4 \mathrm{~cm}$ of water, the fracture flow apparatus was disassembled and reassembled until an acceptable pressure drop was obtained.

(13) At this point, Valves 13 and 15-18 were closed to prevent flow through the fracture or into the water column legs. Reference is now made to Fig. 3.6, which is a simplified schematic showing only a top view of the fracture flow apparatus (note that the flow direction is reversed from that in Fig. 3.4 to be consistent with the convention for presenting aperture distributions and flow fields in Chapter 4). The valving arrangement shown in Fig. 3.6 is greatly simplified over that in Fig. 3.4 to make it easier to describe the procedure for conducting a tracer experiment. From this point on, when reference is made to opening or closing Valves 1 through 4 , the reference is being made to Fig. 3.6, not Fig. 3.4. It is also understood that other valves in the overall system must to be manipulated in order to accomplish the described actions (to allow the pumps to run continuously and still have somewhere to pump liquid without pressurizing lines), but these manipulations will not be described. The closing of Valves 13 and 15-18 mentioned in the first sentence of this paragraph now becomes simply the into and out of fracture flow apparatus. The fracture block is not rectangular to emphasize that nonrectangular fractures can be accommodated in the flow system (e.g., the Tram tuff fracture).

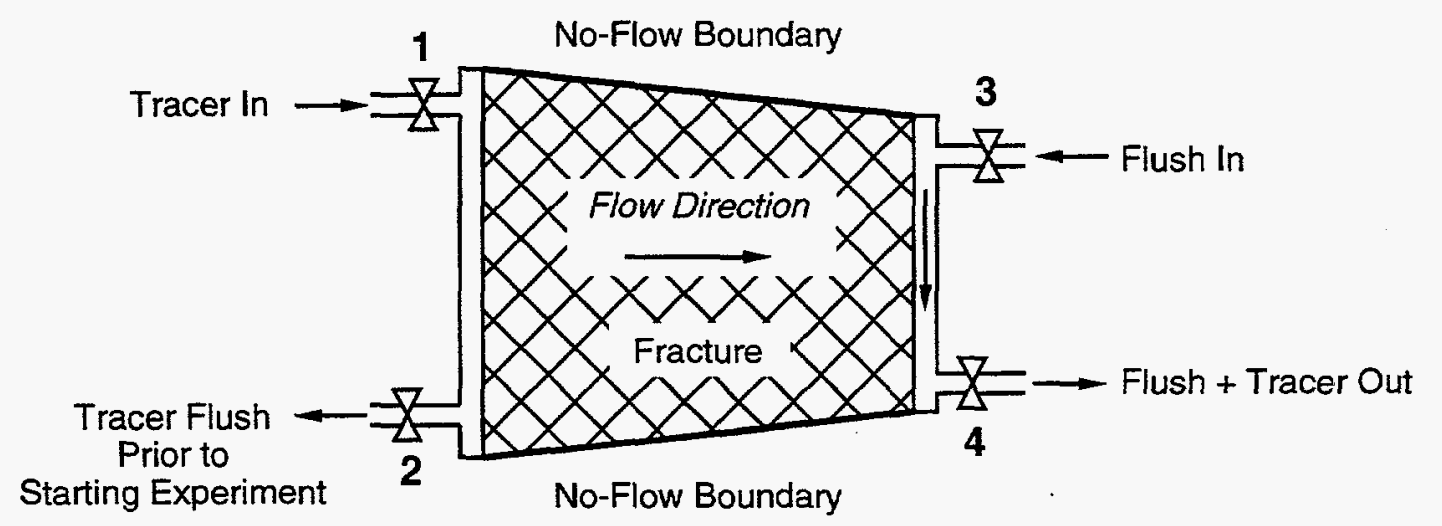

Figure 3.6. Simplified schematic of experimental flow system showing only flow closing of Valves 3 and 4 . The valves allowing access to the water column legs (Valves 13, 16, and 18 in Fig. 3.4) were kept closed for the remainder of the experiment. 
(14) After closing Valves 3 and 4, Valves 1 and 2 were opened and tracer solution was allowed to flow rapidly through the inlet manifold by gravity. These actions allowed a uniform step of tracer solution to be introduced essentially simultaneously throughout the entire inlet manifold prior to allowing flow of tracer solution into the fracture. After enough time had elapsed to adequately flush the manifold (several seconds), a sample of the tracer solution was collected downstream of Valve 2. This sample was ultimately used to determine the tracer inlet concentrations (to which all outlet concentrations were normalized).

(15) Valves 1 and 2 were closed, and Valve 4 was opened. The following steps were then conducted in very rapid succession (within 1-2 seconds of each other):

- Valve 3 was opened to allow the outlet manifold to be flushed with tracer-free J-13 water from the reservoir at the left of Fig. 3.4,

- Valve 1 was opened to allow tracer solution to be pumped into the fracture by the multichannel peristaltic pump, and

- the fraction collector was started (programmed to collect samples every 20 or 30 seconds, depending on the flushing flow rate). The flushing of the outlet manifold was necessary to reduce the tracer residence time in the manifold and in the tubing downstream of the manifold to only a small fraction of the residence time in the fracture. Without such a flush, the tracers would have spent more time in the outlet manifold and tubing than in the fractures (the volume of the manifold and tubing was generally much greater than that of the fractures), making it impossible to distinguish between transport phenomena occurring in the fracture and that occurring in the manifold and tubing. The flush rate varied from 50 to 600 times the tracer flow rate (in different experiments). This ratio had to be known accurately in order to account for dilution when normalizing the tracer concentrations in the effluent samples to the inlet concentrations.

(16) The step phase of the tracer experiment was terminated by simultaneously closing Valves 1 and 3 (and redirecting flow upstream of these valves to avoid overpressurizing lines). If only a step phase was to be conducted, Step 17 was completed next. If a flush-out (or flush) phase was to be conducted, Steps 14 and 15 were repeated, but this time with tracer-free J-13 water displacing the tracer solution already in the fracture and manifolds. A sample of the tracer-free solution exiting the inlet manifold (after flushing the manifold) was collected and later analyzed to check the degree of completeness of flushing the tracer solution out of the manifold. The elapsed time between completion of the step phase and initiation of the flush phase was typically 2 to 3 minutes.

(17) After the completion of the tracer experiment, all flow rates from the peristaltic pumps were again checked using the collect-weigh-time method to determine if (or how much) the flow rates had changed during the course of the experiment. The fracture was flushed by gravity with tracer-free J-13 water, and the hydraulic aperture was again measured as described in Step 10 to check for changes.

(18) The test tubes containing the effluent samples were capped with wax "parafilm" (which had previously been determined to not contaminate the 
samples) and placed in a refrigerator $\left(4^{\circ} \mathrm{C}\right)$ until they could be analyzed. The sample(s) of the inlet tracer solution were immediately diluted by 100:1, 1000:1, and sometimes 10000:1 using serial dilution with J-13 water. These diluted samples were later used for inlet tracer concentration measurements and/or as standards for analyzing the effluent samples.

(19) The fracture flow apparatus was taken apart, and the two fracture surfaces were examined under an intense ultraviolet (UV) light (UVP, Inc., Model B$100 \mathrm{AP}$ ) in a dark room to see if there was any evidence of microspheres on the surfaces. It was known from previous scoping experiments that this was a fairly sensitive technique to determine if microspheres were present in significant quantities on surfaces. Although only a qualitative measurement, knowledge of the presence and spatial locations of any microspheres on the surfaces was useful in interpreting the experiments. After examining the surfaces, the fractures were gently scrubbed with a clean brush to remove residual microspheres and then returned to the dessicator containing J-13 water (with 0.02 to $0.05 \%$ sodium azide) to keep them saturated.

(20) The effluent samples from the tracer experiment were analyzed for microspheres and iodide generally within a week of the experiment. This quick turnaround time on analyses helped avoid any problems that might be associated with aging of the samples (there was some evidence in early scoping tests on the flow cytometer that the fluorescent signals associated with the microspheres degraded with time).

On four occasions, three experiments were conducted in rapid succession on the same day in the same fracture without disassembling the fracture flow apparatus. When this was done, the fracture surfaces were examined under UV light only after the third tracer experiment. The flow rates in the three experiments were varied in approximately a 4:2:1 ratio to help elucidate mechanisms affecting tracer transport behavior in the fractures (see Section 3.1.7). The experiments were run in order from highest to lowest flow rate because it was felt that this would minimize cross-contamination between the experiments (it was assumed that the fractures would flush better at higher flow rates). The average linear flow velocities in the experiments ranged from about 0.1 to $0.8 \mathrm{~mm} / \mathrm{sec}$, which is much higher than natural gradient conditions but spans the expected range of flow rates in most forced gradient field-scale tracer experiments. All experiments were run at ambient temperature, which ranged from 21 to $26^{\circ} \mathrm{C}$.

Tracer experiments were run in two different flow orientations in the Bullfrog tuff fracture. The fracture block was rotated 90 degrees in the fracture flow apparatus to achieve the second orientation (relative to the first). That is, the no-flow boundaries in the first orientation became constant-pressure boundaries in the second and vice-versa. The Bullfrog tuff fracture was the only fracture for which this was done because it was nearly square and hence the machined pieces of aluminum bar stock could be used interchangeably on either set of sides. The other fractures would have required additional machining of aluminum parts to change the flow orientation. 


\subsubsection{Minor Tests to Support Tracer Experiments}

A number of minor tests were conducted to provide information that would help interpret the tracer experiments or to check assumptions made in the experiments. These are discussed briefly in this section:

(1) It was assumed in all tracer experiments that there was no significant tracer dispersion due to stagnant regions in the outlet manifolds (as they were being flushed). This assumption was checked by clamping the gasket material and aluminum bar stock that comprised a flow manifold over a small plexiglass plate and filling the manifold with a dyed solution. The manifold was then flushed at approximately the flow rate that it was flushed during a tracer experiment, and the dye displacement was observed through the plexiglass. Although the displacement was not always uniform over the cross-section of the manifold, in all cases the dye was completely flushed out in a very short time ( $a$ few seconds) compared to the residence time of the tracers in the fractures.

(2) It was assumed that there was negligible pressure drop across the outlet manifolds as they were flushed during a tracer experiment (the outlet manifolds were assumed to be constant pressure boundaries when modeling flow through the fractures -- see Section 4.1). It was known that the pressure drop measured in Step 12 of Section 3.1.6.2 was not the true pressure drop across the outlet manifold because there were fittings and sharp bends in the flow pathway between the two water column legs. To estimate the pressure drop across only the rnanifold, the pressure drop across various lengths of 1/8-inch ID Tygon tubing was measured using water column legs. It was assumed that the pressure drop across the Tygon tubing would provide an upper bound estimate of the pressure drop across the manifolds because the cross-section of the manifolds was approximately 1/4-inch by 1/8-inch (assuming some compression of the 3/16-inch gasket material under the aluminum bar stock). By measuring the pressure drop across various lengths of the tubing, it was determined that the pressure drop at a flow rate of $1.17 \mathrm{ml} / \mathrm{sec}$ was about $0.065 \mathrm{~cm}$ water $/ \mathrm{cm}$ of tubing, and the pressure drop at a flow rate of $0.70 \mathrm{ml} / \mathrm{sec}$ was about $0.04 \mathrm{~cm}$ water $/ \mathrm{cm}$ of tubing. These two flow rates bounded the flush flow rates in all of the tracer experiments. These results indicate that, in many experiments, the pressure drop across the outlet manifold could have been nearly as great as that across the fracture (particularly in the lower flow rate experiments in the Bullfrog tuff fracture where the two pressure drops were calculated to be about equal). The implication is that flow may have been forced to exit the fracture at the downstream end of the outlet manifold, with very little flow exiting out of the upstream end because the pressure there would have been about the same as the pressure in the inlet manifold. Whether or not this actually occurred was impossible to determine without directly observing the flow through the fractures. However, the microsphere breakthrough curves at different flow rates through the fractures and at different flow rates through. the outlet manifolds were in almost exact agreement with each other (for a given fracture) when plotted on a volume eluted basis (see Section 3.1.7), which suggests that the actual pressure drops across the outlet manifolds may have been significantly lower than the pressure drops calculated based on the Tygon tubing 
measurements. The estimated pressure drops across the fracture and across the outlet manifold (based on the Tygon tubing measurements) are listed in Appendix A for each tracer experiment.

(3) The volume of the tubing downstream of the outlet manifold in each tracer experiment was measured by filling the tubing with water, and then draining the water into a tared beaker so that the mass of the water could be measured. This measurement was made so that the tracer delay time in the tubing between the outlet manifold and the fraction collector could be estimated and then subtracted from the actual collection times of the effluent samples. The dead volumes ranged from 3.40 to $4.25 \mathrm{ml}$, with corresponding delay times of approximately 3 to 5 seconds (depending on the flush flow rate).

(4) Because the effluent samples were not always capped immediately after collection, a quick study of evaporation rates at room temperature was conducted by weighing water drops over a 1-hour time period. The evaporation rate was estimated to be $\sim 6-7 \times 10^{-6} \mathrm{ml} / \mathrm{sec}$. It was concluded that losses due to evaporation from the samples (which were $20-25 \mathrm{ml}$ ) were negligible.

\subsubsection{Tracer Experiments: Discussion of Results}

The details of the conditions under which each of the eighteen tracer experiments were run as well as some notes pertaining to the experiments are provided in Appendix A. Appendices B and C contain plots of all the experimental data, with Appendix B showing comparisons between the breakthrough curves of different tracers in the same experiment and Appendix $C$ showing comparisons of the breakthrough curves of the same tracer in the same fracture at different flow rates (i.e., different experiments). These appendices will be referred to repeatedly in the following discussion. As a space-saving measure, the raw data from the experiments are not tabulated in this dissertation, but they can be obtained from the author on request.

The first two experiments were run with the intent of determining which type of colloid tracer exhibited the least amount of attenuation in the fractures. It is clear from Figs. B.I through B.3 that the CML microspheres were less attenuated than both the non-CML polystyrene and the silica microspheres in the Bandelier tuff fracture. This fracture was used for the two scoping experiments because it had the narrowest average aperture, which should have resulted in more collisions between the microspheres and the fracture walls and hence the greatest possibility of observing unfavorable colloid-wall interactions. The differences between the transport behavior of the microspheres are perhaps better illustrated in Figs. C.1 and C.2, which compare the breakthrough curves of microspheres of the same diameter in the Bandelier tuff fracture at different flow rates.

The fractional recovery of the non-CML microspheres could not be calculated because the experiment involving these microspheres did not have a flush phase. However, the recovery can be estimated at between 0.70 and 0.80 based on the final concentration reached in the step phase. The fraction of silica microspheres recovered was approximately 0.89 , although the noise in the data suggests a standard deviation of at least $5 \%$. The silica data was somewhat questionable because an undesirably long time ( $\sim 2$ weeks) elapsed between the completion of the tracer experiment and the analyses of the samples. Also, the 
analyses of the inlet solution samples (used to normalize the effluent sample concentrations) were conducted $\sim 2$ weeks after the effluent sample analyses. For unknown reasons, the measured silica concentrations were found to decrease slightly with time, so the actual recovery of silica may have been lower than that suggested by normalizing the effluent concentrations to the delayed inlet concentration measurement.

In contrast to the non-CML and silica microspheres, the fractional recovery of CML microspheres was always greater than 0.95 , and it was statistically indistinguishable from 1.0 in the Bandelier tuff and Tram tuff fractures. The greater dispersion in the Bullfrog tuff fracture and the finite duration of the experiments were believed to be the reasons for the lower recovery in this fracture.

The reasons for the greater attenuation of the non-CML polystyrene and silica microspheres relative to the CML microspheres in the Bandelier tuff fracture can only be speculated. Wan and Wilson ${ }^{59}$ noted similar differences between non-CML polystyrene and CML microspheres in porous-media column experiments. They attributed the greater attenuation of the non-CML microspheres to the greater hydrophobicity of the non-CML surface (they measured hydrophobicity by macroscopic contact angles on surfaces coated with the microspheres). ${ }^{59}$ They speculated that the hydrophobicity results in an attractive structural or hydration force ${ }^{122}$ that can overcome electrostatic repulsion between the colloids and media surfaces. The zeta potentials of these two types of microspheres in the J-13 water were nearly the same (see Table 3.6), and both would be expected to be electrostatically repelled from the fracture surfaces. In addition to the hydrophilic/hydrophobic effects, the CML microspheres may have also experienced a greater amount of steric repulsion from the rock surfaces than the non-CML microspheres, as the CML surfaces are known to be "fuzzier" than most other types of polystyrene surfaces (according to Interfacial Dynamics Corp.).

The attenuation of the non-CML microspheres was confirmed by the examination of the fracture surfaces under UV light after the first experiment; both surfaces glowed with the color of the yellow dye used to tag these microspheres. The microspheres were distributed uniformly over the top and bottom surfaces, indicating no spatial preference for attachment. With the exception of the Bullfrog tuff fracture, the CML microspheres were never observed on the fracture surfaces after an experiment. In the case of the Bullfrog tuff fracture, CML microspheres were observed only on the lower surface and only in areas corresponding to regions of very large aperture where there was missing matrix material (i.e., the red-colored areas in the aperture plots of Figs. 4.6 and 4.7). This result suggests that the CML microspheres in the Bullfrog tuff fracture were probably attenuated by dispersive mechanisms in the large aperture regions (as opposed to actually attaching to the fracture surfaces). When flow was stopped, the microspheres apparently settled to the lower surface before being observed under the UV light.

The differences in the transport behavior of the CML and silica microspheres cannot be attributed to differences in the hydrophilic/hydrophobic surface characteristics of these microspheres. Both of these microspheres were expected to have hydrophilic surfaces, and the silica had a zeta potential in J-13 water even more negative than that of the CML. It is speculated that the 
differences in the transport behavior of these microspheres may have been due to the much greater gravitational settling velocity of the silica relative to the CML $(\sim 32$ times greater). The more rapid settling may have made it easier for the silica to get "hung up" on asperities on the lower surface or to settle into crevices in the lower surface where they could no longer be advected with the fluid. Greater steric repulsion of the CML microspheres from the fracture surfaces is another possible explanation for the observed differences.

After the first two experiments, only the 1- and $0.3-\mu \mathrm{m}$ CML microspheres and iodide were used as tracers. The two microspheres were used to determine if there was any effect of size on colloid transport through the fractures. The iodide was used as a nonsorbing solute that would presumably be subject to diffusion into the rock matrix or into stagnant zones along the fracture walls.

The results of the tracer experiments in all three fractures had many common themes:

(1) The two microspheres always eluted essentially simultaneously, with their breakthrough curves nearly falling on top of each other (see figures in Appendix B). However, on close examination of the data, the $1-\mu \mathrm{m}$ microspheres usually arrived slightly earlier than the $0.3-\mu \mathrm{m}$ microspheres (the ratio of $1-\mu \mathrm{m}$ to $0.3-\mu \mathrm{m}$ microspheres was greater during the early stages of the breakthrough curves than in the inlet solution). This behavior might be attributable to the tendency of the smaller, more diffusive microspheres to more closely follow Taylor dispersion than the larger microspheres.

(2) The microspheres always arrived earlier in the effluent than the iodide, and they approached the inlet concentration $\left(C / C_{0}=1\right)$ more quickly than the iodide (see figures in Appendix B). The earlier arrival of microspheres was probably the result of the iodide more closely following Taylor dispersion than the microspheres (similar to the behavior shown in Fig. 2.3). However, it could also be the result of the iodide experiencing a larger volume in the fractures because it had diffusive access to stagnant regions along the fracture walls that the microspheres were excluded from. The relative difference between the first arrival times of the colloids and the iodide decreased as the average fracture aperture became wider. This result is consistent with both of the above explanations: a wider aperture would make it harder for iodide to diffuse across all the flow streamlines in the fracture and experience Taylor dispersion, and it would also make the stagnant zones along the fracture walls a smaller fraction of the total void volume in the fracture. The slower approach of the iodide concentration to the inlet concentration (i.e., the long tails in the breakthrough curves) is consistent with the hypothesis that the iodide is diffusing into the matrix and/or into stagnant regions along the fracture walls. The colloids should be too large/too slowly diffusing to experience this phenomenon.

(3) In experiments featuring both step and flush phases, the breakthrough curves of all species during the step phase were nearly identical to the reflection of the breakthrough curves (plots of $1-C / C_{0}$ vs. volume eluted) during the flush phase (see figures in Appendix B). The exception to this behavior was when $C / C_{o}$ during the step phase never got close to 1 . In these cases, the starting value of $\left(1-C / C_{0}\right)$ for the flush phase was greater than 0 , and often $\left(1-C / C_{o}\right)_{\text {flush }}$ remained greater than $\left(C / C_{o}\right)_{\text {step }}$ over the 
entire range of the breakthrough curves. The close agreement between the step and flush breakthrough curves implies that hydrodynamic dispersion and diffusion phenomena (as opposed to interactions with the fracture walls) were principally responsible for the observed transport behavior of all tracers. For the microspheres, this conclusion is supported by the empirical observation that no fluorescence was visible under UV light on the fracture surfaces after the tracer experiments (and only on the lower surface of the Bullfrog tuff fracture), suggesting that attachment of microspheres to the fracture surfaces was minimal. For iodide, chemical interactions such as sorption cannot be unequivocally ruled out, but the batch sorption experiments showed no evidence of this. Also, identical breakthrough curves during the step and flush phases would be expected only if sorption were linear and reversible, and this sort of behavior would not, in general, result in the long tails of the solute breakthrough curves or the observed flow rate dependence of the breakthrough curves discussed in item 4 below.

(4) The colloid breakthrough curves at different flow rates in the same fracture were essentially identical when plotted as a function of volume eluted through the fracture (see figures in Appendix $\mathrm{C}$ ). The iodide breakthrough curves, on the other hand, showed a flow rate dependence when plotted in this manner, with the tails of the curves approaching the inlet concentration more slowly at lower flow rates. These results are consistent with the hypothesis that the iodide is diffusing into the rock matrix and/or into stagnant regions along the fracture walls, while the microspheres do not experience these phenomena. Diffusion into the matrix/stagnant regions will cause longer tailing at lower flow rates because solutes will have more opportunity to diffuse into the matrix/stagnant regions when they have a greater residence time in the system. The agreement between microsphere breakthrough curves at different flow rates is also consistent with the hypothesis that the transport behavior of these tracers is primarily the result of hydrodynamic dispersion in the fractures and not interactions with the fracture walls.

(5) In many of the experiments that had a flush phase, there was a significant "spike" in the concentration of all tracers in the first sample collected after the flush was initiated (see figures in Appendix B). This spike was believed to be the result of a pressure pulse that forced some additional tracer solution through the fracture as the inlet manifold was being flushed to displace the tracer solution with tracer-free J-13 water. The pressure pulse apparently forced only tracer solution into the fracture because the arrival time of the tracer-free solution in the effluent was always essentially the same as the arrival time of the tracer solution during the step phase, regardless of the magnitude of the spike. In one instance (in the Bullfrog tuff fracture), one of the valves on the outlet manifold was inadvertently left open for a few seconds after initiating the inlet manifold flush. This action allowed considerable flow through the fracture during the flush. However, even though the "spike" reached a concentration of nearly 8 times $C / C_{0}$, the tracer-free solution still arrived in the effluent at the same time that the tracer solution had arrived during the step phase. Therefore, the valve on the outlet manifold must have been closed before the tracer solution was 
flushed out of the inlet manifold. It was concluded that the pressure pulses causing the concentration spikes did not compromise the breakthrough curves (except for the first sample after the flush phase was initiated). After the experiments were completed, it was suggested that the pressure pulse might be avoided by using mild suction to flush the inlet manifold rather than forcing the tracer-free J-13 water through by gravity.*

An exception to the observation that the microsphere breakthrough curves were identical at all flow rates in each fracture (item 4 above) is apparent in Figs. C.7, C.8, C.10 and C.11, which show breakthrough curves in the Bullfrog tuff fracture in the two different flow orientations on different days. The differences between the breakthrough curves on the different days might be explained by the fact that a few small pieces of matrix broke off the surfaces of this fracture sometime between the first set of experiments and the second set of experiments. These pieces were too small to significantly change the void volume of the fracture, but they could have (and apparently did) change the dominant flow pathways in the fracture, resulting in different tracer dispersion behavior. Of course, it is possible that the differences in the observed dispersion on the different days could have been the result of some anomaly such as an inadvertent bypass in the fracture flow apparatus or an air bubble somewhere in the system on one of the days, but these possibilities are considered unlikely because they should have affected the apparent volume of the system, and there was no evidence of this. Also, this discrepancy never occurred with the other two fractures, and there was good agreement between tracer tests conducted in the Bullfrog tuff fracture on two different days after the surface damage had occurred.

The relative amount of hydrodynamic dispersion in the different fractures can be compared by examining the microsphere breakthrough curves in each fracture plotted as a function of the number of void volumes eluted through the fracture. It is not advisable to use the iodide breakthrough curves to make this comparison because they are complicated by the effects of matrix diffusion and/or diffusion into stagnant zones, which is different for fractures of different average aperture and matrix porosity. Void volumes in each fracture were estimated from the volume corresponding to the "center of mass" of the stepphase portion of the microsphere breakthrough curves -- that is, the eluted volume at which the integrated area under the breakthrough curve to the left (mass already eluted) was equal to the integrated area over the curve to the right (mass remaining to be eluted). This was a subjective determination because of scatter in the data and because the tails of the breakthrough curves (which can account for a great deal of area) were truncated in most of the experiments. The following void volumes were estimated:

Bandelier tuff fracture: $0.6 \mathrm{~cm}^{3}$ (average aperture of $\sim 55 \mu \mathrm{m}$ )

Tram tuff fracture: $1.2 \mathrm{~cm}^{3}$ (average aperture of $\sim 155 \mu \mathrm{m}$ )

Bullfrog tuff fracture: $3.7 \mathrm{~cm}^{3}$ (average aperture of $\sim 560 \mu \mathrm{m}$ )

Plots of the $1-\mu \mathrm{m}$ microsphere breakthrough curves on a void volume basis in each fracture are shown in Figs. 3.7 and 3.8. Fig. 3.7 shows the comparison before the damage to the Bullfrog tuff surfaces, and Fig. 3.8 shows the comparison after the damage. In both cases, it is apparent that the greatest

\footnotetext{
* Personal communication with Mike Nicholl of Sandia National Laboratories, who had noticed the pressure pulse problem when working with dyes in transparent model fractures (1994).
} 


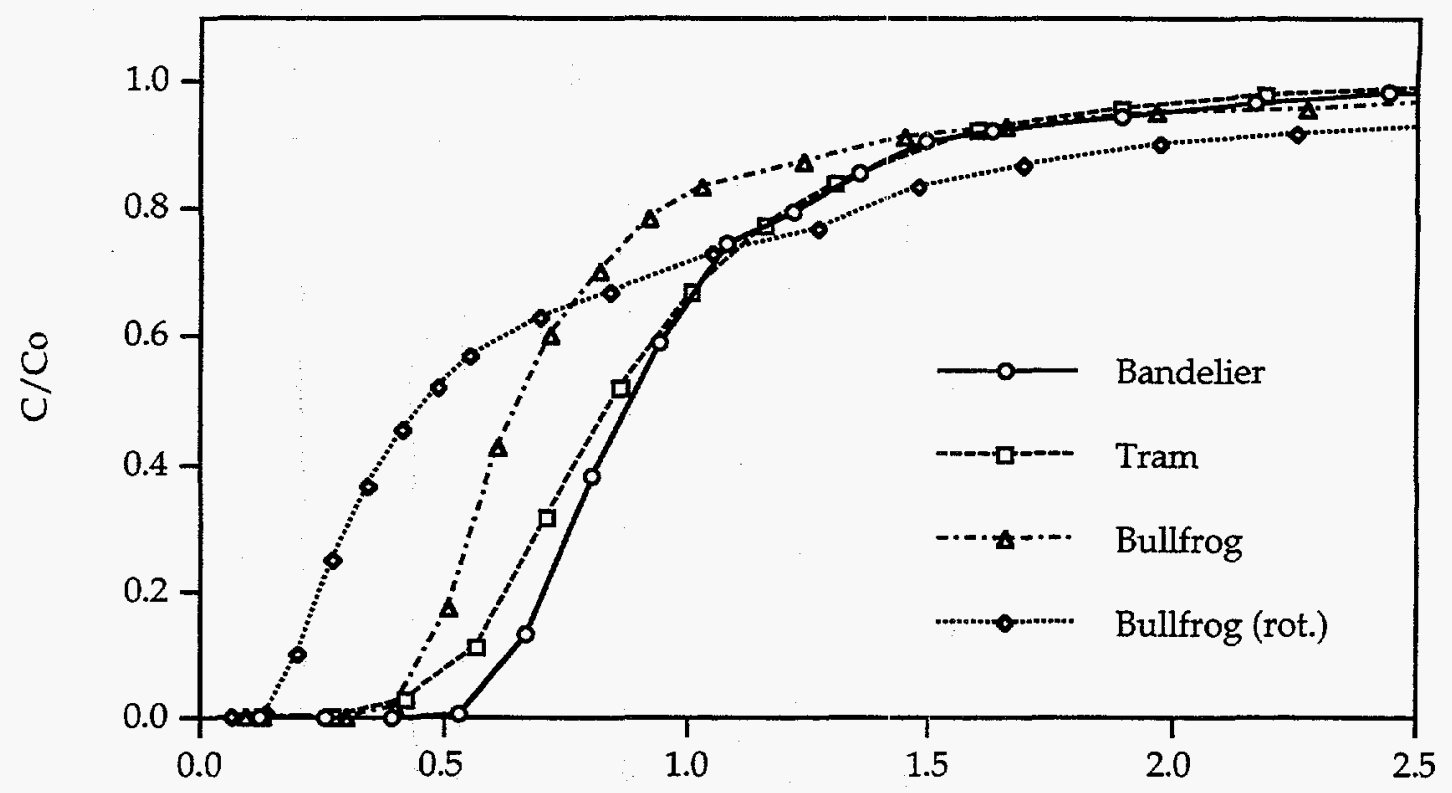

Void Volumes

Figure 3.7. Breakthrough curves of 1- $\mu \mathrm{m}$ CML microspheres in the different fractures as a function of void volumes eluted before the damage to the Bullfrog tuff fracture surfaces.

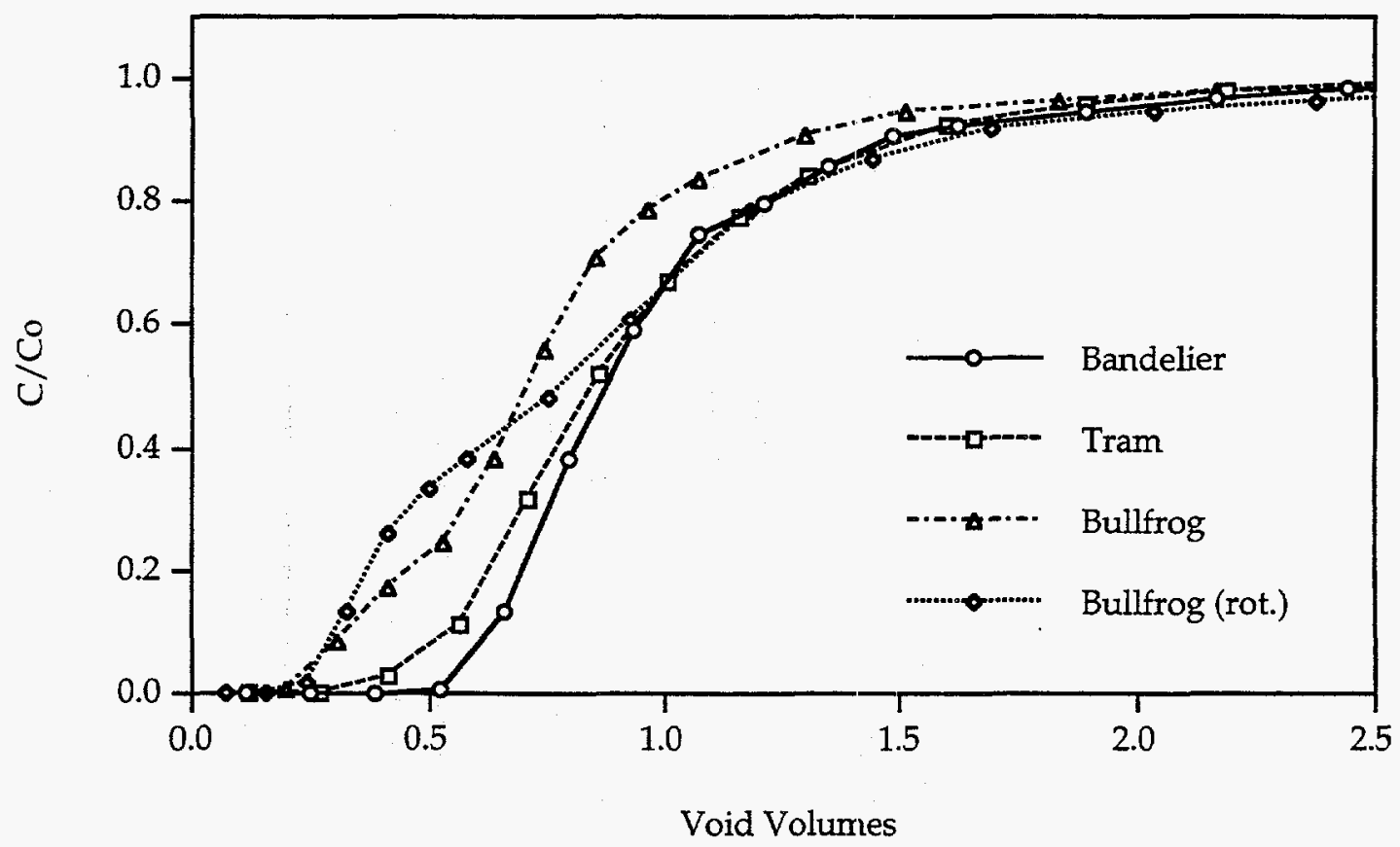

Figure 3.8. Breakthrough curves of 1- $\mu \mathrm{m}$ CML microspheres in the different fractures as a function of void volumes eluted after the damage to the Bullfrog tuff fracture surfaces. 
amount of dispersion is in the rotated Bullfrog tuff fracture, with the unrotated Bullfrog tuff fracture being second in relative dispersion, the Tram tuff fracture third, and the Bandelier tuff fracture last. This result is not surprising if one considers the average apertures in each fracture and uses Taylor dispersion. arguments. If the residence times are approximately the same in each fracture (as they are), one would expect greater dispersion in the widest aperture fracture because the microspheres should sample a smaller fraction of the flow streamlines in the velocity profile than in the narrower aperture fractures, resulting in a breakthrough curve that more closely approximates streamline flow (i.e., has greater dispersion). However, this argument cannot explain the differences in the breakthrough curves associated with the two different flow orientations in the Bullfrog tuff fracture, as the average aperture in both cases is the same and the residence time was actually a bit longer in the case that exhibited more dispersion (rotated), which is contrary to what Taylor dispersion theory would predict for parallel-plate channels. Clearly, this behavior can only be explained by the existence of flow channels in the variable-aperture fracture that result in different flow pathways and hence different dispersion in the two flow orientations. This possibility is examined in more detail in Chapter 4, where dispersion is predicted from models that use actual estimates of the aperture distributions in each fracture.

\subsection{Estimation of Aperture Distributions in Fractures}

In an attempt to model tracer transport through the three fractures without relying on a parallel-plate approximation or using an adjustable dispersion coefficient to "fit" the data, the aperture distributions within the fractures were estimated using three different techniques. The strategy was to use the estimated aperture distributions to predict flow fields in the fractures (by solving the Reynolds equation, as discussed in Section 2.5.2) and then use the predicted flow fields to simulate tracer transport through the fractures using particle-tracking techniques (discussed in Chapter 4).

The three techniques used to estimate aperture distributions in the fractures are discussed in the following three sections. The first two techniques (laser surface profiling and RTV silicone casting/imaging) were used only with the Bandelier tuff fracture, as there was not enough time or availability of resources to use them with the other two fractures. The third technique (stylus profiling) was used with all three fractures and was repeated once with the Bandelier tuff fracture to get a qualitative measure of the reproducibility of the technique. Thus, it was possible to compare three different techniques with one fracture, and to compare the aperture distributions in all three fractures using one technique.

\subsubsection{Laser Surface Profiling}

With the laser surface profiling technique, the two surfaces of the fracture were profiled separately using a noncontact laser system to provide a threedimensional mapping of the surfaces. The surface data were then matched and further manipulated to estimate apertures as a function of position within the fracture. A profiling instrument at Sandia National Laboratories was used for data acquisition.* This instrument was very similar to one described by Huang et al. ${ }^{169}$ It operated on optical principles, using a $\sim 25-\mu \mathrm{m}$ diameter (slightly

\footnotetext{
* Steven Brown and Robert Hardy of Sandia National Laboratories did the profiling in 1992.
} 
ellipsoidal cross-section) laser beam to illuminate the surface at a single location and a lens/photodiode array system to detect changes in the elevation of the illuminated point. The laser and lens/photodiode systems were mounted on a head that was driven by high-precision stepper motors to allow precise control over the spatial location(s) of the surface elevation measurements. The system was automated by interface to a computer that controlled the movement of the head and performed data acquisition/reduction. The vertical precision of the system was estimated from repeat measurements on a flat opaque surface to be $\pm 8 \mu \mathrm{m}$ (standard deviation). ${ }^{* *}$ Precision can be degraded by translucent crystals on the surfaces because the laser beam can penetrate these and cause the system to think the surface is actually lower than it is.

Both surfaces of the Bandelier tuff fracture were profiled at a 0.5- $\times 0.5-\mathrm{mm}$ resolution in the horizontal plane (2-dimensional grid). Manipulation of the raw surface data to obtain apertures as a function of position was described by Reimus et al. ${ }^{170}$ The deduced apertures were adjusted by adding or subtracting a constant to get an average aperture equal to the mean aperture estimated from the microsphere breakthrough curves (see Section 3.1.7 for how this was done). Interpenetrating surfaces (i.e., negative apertures) resulting from this procedure were assumed to be contact points, and the apertures were set equal to zero at these points.

The characteristics of the aperture data set deduced from the laser profiling technique are discussed in Section 3.2.4. A depiction of the deduced aperture distribution is provided in Fig. 4.1, where the apertures are shown along with the flow field predicted using the apertures in the Reynolds equation. The laser profiling was conducted prior to the tracer experiments. This was possible because the technique did not involve physical contact with the surfaces, which might have compromised the surfaces.

3.2.2 RTV Silicone Casting and Imaging of the Fracture Void Space

The second technique employed to estimate fracture aperture

distributions was that of making a cast of the fracture void space using RTV moldmaking silicone and then measuring light transmittance through the cast as a function of position to deduce the apertures. This technique has been described by Cox et al. ${ }^{171}$

A cast of the Bandelier tuff fracture void space was made using a two-part addition cure RTV silicone rubber (Silpak R-2350A and R-2350B, Pomona, CA). The components were mixed together in the specified ratio, and then a very small amount of compatible blue dye was added to the mixture to give the normally translucent silicone a tint that would partially block light transmission through the cured cast. The mixture was degassed under a high vacuum (less than 1 inch $\mathrm{Hg}$ absolute pressure) to eliminate air: bubbles that could degrade the quality of the cast and cause errors in the light transmission measurements. The fracture surfaces were prepared by coating them with a thin layer of a mold release mixture consisting of petroleum jelly dissolved in a volatile hydrocarbon (Silpak, proprietary mixture). This treatment helped minimize tearing of the silicone cast when the fracture surfaces were separated after the silicone cured.

To make the cast, the degassed silicone mixture was poured over one end of one surface of the fracture, and then the surface was inclined to allow the

\footnotetext{
** Personal communication with Steven Brown of Sandia National Laboratories (1992).
} 
material to flow slowly over it. The slow flow helped minimize the entrapment of air bubbles. The second fracture surface was then slowly "folded" onto the first surface (in a motion similar to closing a book) while the first surface was held at a steep incline. Once the space between the surfaces was completely filled with silicone, the surfaces were squeezed together until they were nearly touching. The silicone was then allowed to cure for $\sim 24$ hours at room temperature with a small amount of pressure holding the surfaces together and preventing relative motion between them.

The unused portion of the RTV silicone mixture was then poured into two wedge-shaped depressions that had been precision-machined out of $1 / 2$-inchthick aluminum plate. Both wedges were approximately 4 inches long by 1 inch wide. One was $0.375 \pm 0.001$ inch deep at one end (no depth at the other end), and the other was $0.124 \pm 0.001$ inch deep at one end (also no depth at the other end). A plexiglass plate was forced down over the top of the wedges to form a mold for the RTV silicone. Openings in the ends of the wedges allowed excess silicone to be squeezed out of the molds as the plexiglass plate was bolted down onto the aluminum plate.

The cured wedges were used to obtain a calibration curve of cast thickness vs. light transmittance, which was then used to determine the thickness of the fracture cast as a function of position based on light transmittance. The wedges and fracture cast were always made from the same batch of RTV silicone because the transmittance properties of the casts were so sensitive to the amount of dye added to the mixture that it was essentially impossible to achieve batch-to-batch reproducibility.

Light transmittance through the wedges and casts were measured at Sandia National Laboratories using a light table and a charge-coupled-device (CCD) camera to take digitizable images. With this system, the light intensity associated with each "pixel" of an image was assigned a value from 0 to 255 . When taking an image, the fracture cast and wedges were immersed together in degassed DI water in a custom-made transparent, flat-bottomed plexiglass dish that was placed on the light table. The casts were immersed in water to achieve better matching of their index of refraction with the surrounding medium, which helped sharpen the images. When measurements were made in air, the images were considerably fuzzier than in water (the index of refraction of RTV silicone is $\sim 1.43$, water is 1.33, and air is 1.00). An image was also taken of the water bath without the cast or wedges in it to measure the incident light intensity as a function of position. The incident intensity was not necessarily spatially uniform because of defects and imperfections in the light diffuser. follows:*

Once the images were taken, the method of data reduction was as

(1) The ratio of the incident light intensity (image without cast and wedges) to transmitted light intensity (image with cast and wedges) was calculated for each pixel in the two corresponding images and stored in a file.

(2) Average values of the intensity ratio at various thicknesses of the RTV silicone cast were obtained by averaging all the pixels at specified distances from the ends of the wedges (where the thicknesses were constant and known). This information was used to generate a calibration curve of cast

\footnotetext{
* Based on discussions with Mike Nicholl of Sandia National Laboratories (1994), who ran the light table/CCD camera system and did the data reduction.
} 
thickness vs. logarithm of intensity ratio. This relationship should have been linear according to the Beer-Lambert law of light transmittance through an absorbing medium: 172

$$
\log \left(\frac{I_{\mathrm{o}}}{\mathrm{I}}\right)=\mathrm{kx}
$$

where, $I=$ transmitted light intensity,

$\mathrm{I}_{0}=$ incident light intensity,

$\mathrm{k}=$ absorbtivity of medium, $\mathrm{cm}^{-1}$

$\mathrm{x}=$ thickness of medium, $\mathrm{cm}$.

However, when a calibration curve was fitted to the data, a second-order polynomial (in the logarithm of intensity ratio) was used to account for any curvature due to nonidealities.

(3) The aperture associated with each pixel in the fracture cast was calculated from the intensity ratio using the calibration curve fitted to the wedge data.

(4) The deduced aperture data set was detrended by fitting a plane to the data (multiple linear regression) and then subtracting the plane from the data. This procedure eliminated trends in the data due to uneven thinning of the RTV silicone during casting. When detrending, the largest apertures were filtered out and not fitted because they tended to bias the detrending in such a way that there were still trends in the small aperture data (something that was deemed unrealistic because it was felt that the contact points should have been evenly distributed over the fracture void space rather than clustered in one region of the fracture).

The apertures deduced in this manner had a spatial resolution of approximately $0.175-\mathrm{mm}$ (that is, the pixels were $0.175-\times 0.175-\mathrm{mm}$ in the plane of the fracture). As with the laser profiling technique, the deduced apertures had to be adjusted by a constant to get an average aperture corresponding to the mean aperture estimated from the microsphere breakthrough curves (negative apertures were set equal to zero and assumed to be contact points). The precision of this technique was not assessed -- this would have required repeat measurements, which were not done.

The characteristics of the Bandelier tuff fracture aperture data set deduced from the RTV casting/imaging technique are discussed in Section 3.2.4. A depiction of the deduced aperture distribution is provided in Fig. 4.2, where the apertures are shown along with the flow field predicted using the apertures in the Reynolds equation. Although the Bandelier tuff fracture was the only fracture that was fully characterized by the RTV casting/imaging technique, casts of the other two fractures were prepared and could be imaged at any time. These casts were not imaged because the light table/CCD camera system and key Sandia staff were not available after the casts were prepared.

\subsubsection{Mechanical Stylus Profiling of Surfaces}

The third technique used to estimate aperture distributions in the fractures was surface profiling using a mechanical stylus. This technique was used to obtain aperture data sets for each of the three fractures, and it was repeated once for the Bandelier tuff fracture (a total of 4 profiles). As with the laser surface profiling technique, the data from two fractures surfaces had to be matched and then further manipulated to deduce aperture distributions. The profiler, dubbed the PEAK profilometer, was located at Lawrence Livermore National Laboratory, and all profiling was done there. Durham and Bonner ${ }^{173}$ 
have described PEAK in considerable detail, so only a summary description is provided here.

The PEAK instrument can be equipped with up to four styli that simultaneously profile a surface, although only three styli were ever used to profile the fractures described in this dissertation. Surfaces to be profiled are mounted on a table that is moved in two orthogonal horizontal directions ( $x$ - and $\mathrm{y}$-directions) by high-precision stepper motors while a head holding the styli remains stationary above the table (except to move the styli up and down to take elevation measurements). The styli are arranged in approximately a straight line perpendicular to the primary direction of table movement. A photograph of the PEAK instrument is shown in Fig. 3.9.

The styli used to profile the fracture surfaces had either tungsten carbide or sintered diamond tips, which were initially ground to a sharp point $(<25 \mu \mathrm{m}$ in diameter) at a 30-degree angle from the axes of the styli. The tungsten carbide tips typically flattened to $\sim 60-80 \mu \mathrm{m}$ after completing a profile and had to be resharpened before the next profile. Flattening or chipping of the diamond tips was negligible, and over the course of the profiling it became clear that these tips were far superior to the tungsten carbide ones.

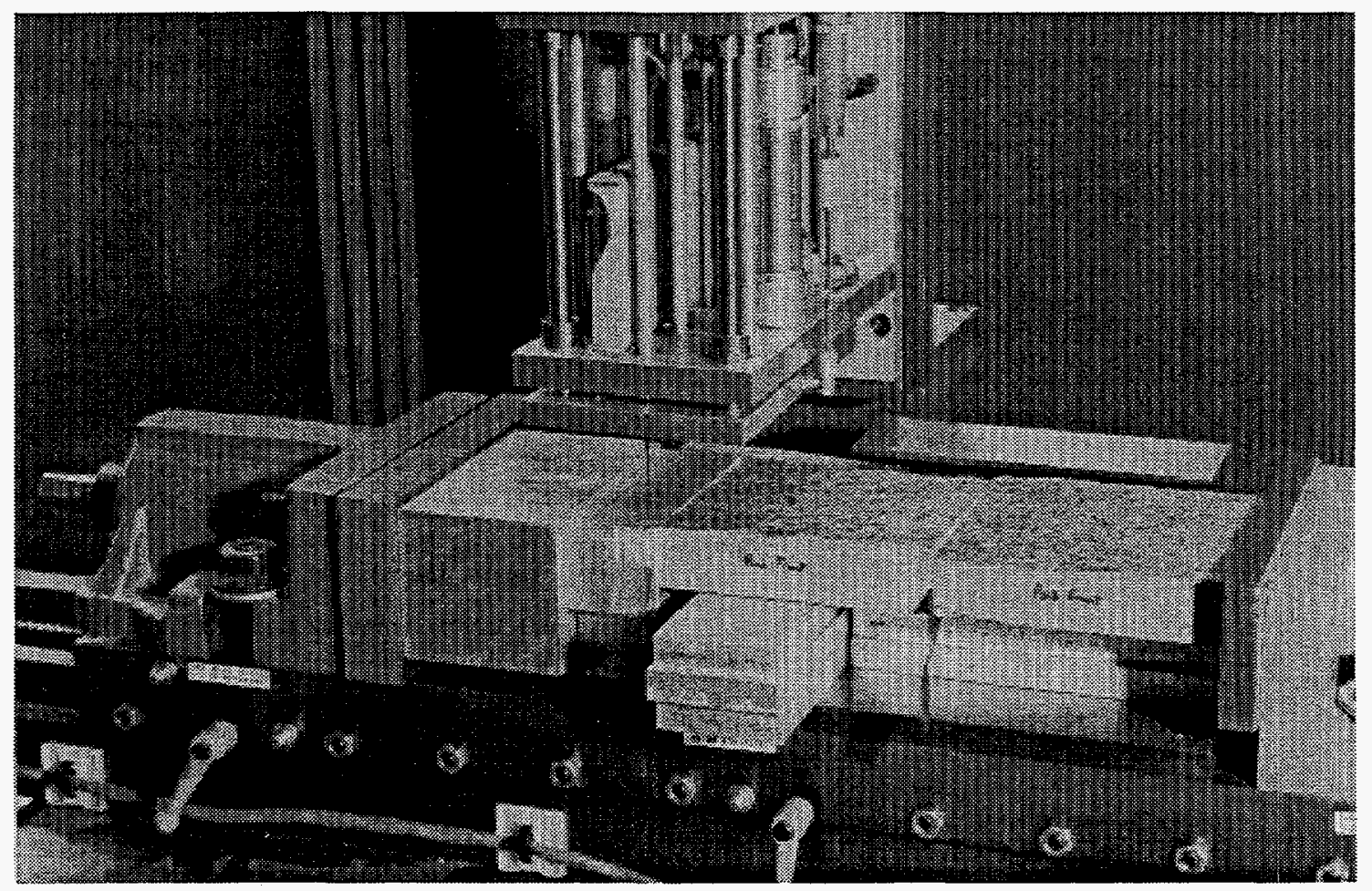

Figure 3.9. Photograph of PEAK surface profiling instrument showing the Bandelier tuff fracture mounted on the table. The front stylus can be seen pointing down toward the table. The flat surface mounted on the table behind the fracture was also profiled to allow the raw data to be corrected for systematic errors due to temperature fluctuations in the lab (see text). 

The following procedure was used to obtain surface elevation data for a pair of fracture surfaces:

(1) The fracture blocks were prepared for profiling by grinding their tops and bottoms (surfaces parallel to the fracture) flat and parallel to each other using a surface grinder. The edges corresponding to the entrance and exit ends during the tracer experiments were also ground flat and parallel, and one of the sides of the block (no flow boundary) was ground flat and exactly perpendicular to the entrance and exit edges.

(2) The two halves of the fracture block were mounted on the PEAK table endto-end (either both entrance edges or both exit edges touching) with the side edge that had been ground flat facing the front of the instrument and aligned such that there was no offset between the two halves of the block. After clamping the rocks into place, the table was moved manually back and forth in the direction parallel to the front edge, and a micrometer equipped with a ball tip was used to determine if the front edges were indeed flush and exactly parallel to the direction of table movement (the $x$ direction). It was sometimes necessary to use thin plastic shim stock at the corners of the block to get the edges parallel to the $x$-direction. This procedure ensured that the styli would "hit" points on both surfaces that corresponded to exactly the same distance from the front edge, thereby minimizing mismatch of the surface data in the direction perpendicular to the front edge (the $y$-direction).

(3) Short ( $2-\mathrm{mm})$ linear profiles were done at a fine resolution ( $50-\mu \mathrm{m}$ spacing) over the touching edges of the two halves of the fracture block to determine the exact position of the edges relative to the "home" $x$-position for each of the three styli. The position where the edges met was used as the "center" position for the profiles (i.e., the same number of data points were taken on either side of the center position). The styli were typically not aligned exactly perpendicular to the direction of table movement (they might lead or trail each other by as much as $0.1 \mathrm{~mm}$ in the $x$-direction), so the determination of a center position was dependent on which stylus was chosen (if two styli were aligned with each other, these were generally used to define the center position).

(4) Once the center $x$-position was determined, a profile was initiated with the styli starting at a position very close to the left edge of the left fracture surface and proceeding across the surfaces to the right edge of the right fracture surface. The starting location was chosen so that the styli would hit exactly where the two surfaces met (i.e., the center position), and therefore all points to the left of the center position would correspond exactly to points to the right of the center position. This procedure minimized the $x$ direction mismatch between the surface data. Of course, this minimization could not be accomplished for all three styli unless they were all aligned exactly perpendicular to the direction of table movement, which was never the case.

(5) All profiles were done using a $0.25-\mathrm{mm}$ spacing in both the $\mathrm{x}$ - and $\mathrm{y}$ directions. The table was moved in the $x$-direction until the end of a linear trace was reached, and then the table was stepped $0.25 \mathrm{~mm}$ in the $y-$ direction and another trace was initiated. The distance between styli was approximately $38 \mathrm{~mm}$, so 156 traces $(38.75-\mathrm{mm}$ movement in the y- 
direction) were done to ensure that the styli would overlap each other on the surfaces. The "home" $x$-position was checked every 4 traces to ensure that the positioning system was not "drifting." The y-position could not be checked in this way, but the y-distance between the front edge of the table and a fixed location was checked using a telescoping micrometer before and after each profile to check for drift (the table was supposed to return its starting position after each profile was completed).

(6) For the last two profiles (see below), a fourth stylus having an unsharpened tip was used to "profile" a flat surface mounted on the table. Measuring the elevation of the flat surface allowed corrections to be made for errors caused by expansion and contraction of the stylus head resulting from diurnal temperature fluctuations in the laboratory. After analyzing the data from the first profile, it was apparent that these temperature fluctuations (which had unknown magnitude because the temperature in the lab was not recorded) were causing periodic fluctuations of $\sim 10-\mu \mathrm{m}$ in the surface data. These periodic errors actually caused errors of $\sim 20-\mu \mathrm{m}$ in the deduced apertures because the high points were matched to high points and the low points were matched to low points when the surface data were combined to deduce apertures (an entire linear trace on both fracture surfaces was either high or low).

Once the surface data were obtained in this manner, an aperture data set was deduced using the following procedure:

(7) First, the surface data were corrected for errors resulting from the diurnal temperature fluctuations by subtracting a correction factor from each $x-$ direction trace of the data set. The correction factors associated with each trace were determined from the flat surface data provided by the fourth stylus. The flat surface generally wasn't perfectly horizontal, so a plane was fit to the data (multiple linear regression) or a line was fit to individual $y$ direction traces of the data (linear regression). The correction factors were determined from the difference between the data points and the fitted line/plane at those points. All of the differences associated with a given $x-$ direction trace were averaged to obtain the value of the correction factor for that trace.

Note: This procedure was used only for those fractures in which a flat surface was profiled (see discussion of the unique aspects of each profile [later in this section] for how corrections were made when a flat surface was not profiled).

(8) The corrected surface data from the three styli were "patched" together to generate a complete surface data set. The first step in the "patching" process was to find the best trace-to-trace matches between adjacent styli. Matching was accomplished by a sum-of-squares minimization procedure in which individual traces were first detrended by fitting a line to them (linear regression) and then subtracting the line from the data. Detrended traces from two adjacent styli were compared by summing the squares of their differences on a point-by-point basis. Points near the edges were not included in this procedure because the differences were often unrealistically large when one stylus went off a surface and the other stayed on (because the styli were not aligned perfectly in the $x$-direction). The sum-of-squares procedure was used to compare the last $\mathrm{z}$ traces of a lower numbered stylus 
to the first $z$ traces of a higher numbered stylus, with the resulting sum of squares being divided by $z$ to normalize the result for different values of $z$. The $z$ value giving the lowest value of the weighted sum of squares was used as the best trace-to-trace match. For example, if $z=4$ gave the lowest weighted sum of squares between styli 1 and 2, then trace 153 of stylus 1 was matched to trace 1 of stylus 2, trace 154 of stylus 1 was matched to trace 2 of stylus 2 , and so on. In cases of overlap, the data from the higher numbered stylus was generally used, and the last few traces from the lower numbered stylus were discarded. Once the best matches between detrended traces were found, the average elevations of corresponding undetrended traces from different styli were calculated and the difference between these averages was taken to be the difference between the elevations of the two styli. The differences between styli elevations were used to adjust all surface data to the same height basis.

(9) The matching of data from the two fracture surfaces was accomplished by first multiplying all of the data from one surface by -1 , and then reversing the order of the data on this surface in the $x$-direction so that points near the beginning of each trace would be matched with points near the end of the traces on the other surface. The point-to-point matching of the surface data was accomplished by both visual examination of detrended traces and by a sum-of-squares minimization of the differences between traces from the two surfaces. The matching was done separately for each stylus, as the mismatches were often different for different styli. The visual examinations and sum-of-squares minimizations were generally in agreement on what the best match should be for each stylus. The visual examinations were important because large aperture regions could sometimes cause erroneous conclusions to be drawn from the sum-of-squares minimizations. Care also had to be taken to avoid including points near edges or traces that ran off the back edge of the fracture surfaces. The locations of the edges were determined by visual examination of the surface data prior to doing the surface-to-surface matching.

(10) Once the surface data were matched, each surface was detrended by fitting a plane to the matched data (multiple linear regression). The point-to-point differences between the two detrended surfaces yielded the unadjusted aperture data set (having a mean aperture of zero). This data set was examined using a two-dimensional computer graphics software package in which color could be used to represent apertures in the third dimension (Spyglass Transform, Spyglass, Inc.). Often there were unrealistic trends in the data set due to large aperture regions that had biased the surface detrending procedure. To correct for this, the aperture data set was detrended by fitting a plane (multiple linear regression) to only a narrow range of the data that was near the lower end of the total range (i.e., the apertures that should have been in contact or nearly in contact). This detrending procedure generally produced a data set that looked much more realistic than the original data set when plotted.

(11) Even after Step 4, there were sometimes peculiar trends in the data that did not look real. For instance, after the first profile of the Bandelier tuff fracture, the aperture data set had "stripes" of high and low aperture in the $x$-direction. These were eventually deduced to be the result of the diurnal 
temperature fluctuations in the laboratory. However, the stripes also had a second-degree (parabolic) curvature to them, which suggested that the surfaces may have moved during profiling. In other instances, there was an obvious discontinuity in the data at the point where two styli overlapped. In the second profile of the Bandelier tuff fracture surfaces, the apertures associated with traces in the middle of the fracture were clearly larger than apertures near the front and back edges (i.e., a convex appearance) despite the fact that there were no other trends or discontinuities in the data. All of these inconsistencies in the aperture data sets were handled by detrending individual traces and then reconstructing the aperture data using the detrended traces. The detrending was sometimes linear and sometimes parabolic (multiple regression), depending on whether curvature was apparent in the data (e.g., the first Bandelier: tuff fracture profile). Also, the detrending procedure generally included only a narrow range of apertures so that the regressions would not be biased by large apertures. The results were always an improved aperture data set (based on visual examination with the plotting software). Of course, there was always the risk that real trends were being removed from the data, but this was impossible to assess.

(12) As a final step, the detrended aperture data sets were adjusted by a constant so that the average aperture matched the mean aperture estimated from the microsphere breakthrough curves. Any negative apertures were set equal to zero and assumed to be contact points.

The unique aspects of each of the four PEAK profiles (and the data reduction methods associated with them) are described below in chronological sequence.

(1) Bandelier tuff fracture, first profile: The first and third styli (from the front of the instrument) had sintered diamond tips, and the second stylus had a tungsten carbide tip. This was the only case in which the edges of the fracture block were not ground flat and perpendicular using a surface grinder, although they appeared to be reasonably flat and perpendicular. A flat surface was not profiled with this fracture (a fourth stylus was not used). The problems with diurnal temperature fluctuations became apparent when "stripes" in the x-direction appeared in the aperture data set. The stripes were removed from the aperture data by fitting a second-order polynomial to each trace of data (some curvature in the $x$-direction was also noted). Very high and very low apertures were filtered out of the fitting procedure to avoid bias due to large apertures. The resulting detrended data set looked realistic when plotted.

(2) Tram tuff fracture: The styli tips were the same as for the first profile of the Bandelier tuff fracture (above). A flat surface was not profiled with this fracture, but it was still possible to make corrections for diurnal temperature fluctuations by using the last few y-direction traces for each stylus, which "profiled" the flat vice-clamp surface at the right edge of the PEAK table. The aperture data set deduced from the detrended surface data had no apparent trends or discontinuities, so detrending of the apertures was not necessary. However, there were a few locations on the surfaces where the stylus tips did not hit the true surface because the surface was so steeply inclined (i.e., a "cliff") that a portion of the stylus hit the edge of the incline and did not allow the tip to go any lower. In places where this occurred, the aperture data set had unrealistically small apertures that resulted in flow 
blockages or "baffles" when the aperture data were used to calculate flow fields. To remedy this problem, all unrealistically small apertures that were known to be associated with "cliffs" were set equal to the average aperture. The unrealistically small apertures were easy to determine by examining plots of the data.

(3) Bullfrog tuff fracture: This fracture was profiled using three diamondtipped styli, and a fourth stylus was used to profile a flat surface for calculating temperature corrections. Because of a rather large area of extremely large apertures (see Figs. 4.6 and 4.7 ), the resulting aperture data set had to be detrended twice (with "filtering" of high and low apertures) to obtain a reasonable data set. There was also a discontinuity in the data from styli 2 and 3, with the apertures deduced from stylus 3 having a significant linear trend relative to the apertures from stylus 2 . Therefore, the apertures from stylus 3 were detrended (linear regression) on a trace-by-trace basis (filtering out very large and small apertures), and the detrended data were substituted for the original data. The resulting data set looked more realistic than the original data set.

(4) Bandelier tuff fracture, second profile: This profile was done because of the problems associated with the first Bandelier tuff fracture profile (the stripes and parabolic trends in the aperture data). This time, three diamond-tipped styli were used, the edges of the fracture block were ground flat and perpendicular, and a flat bar was profiled by a fourth stylus to allow calculation of temperature corrections. Unfortunately, as mentioned in Step (11) above, the deduced aperture data set had a convex appearance, with larger apertures in the center of the fracture and smaller apertures near the front and back edges. To remedy this problem, each $\mathrm{x}$-direction trace in the aperture data set was linearly detrended after filtering out very high and low apertures. After detrending, the aperture data looked reasonable.

Depictions of the aperture data sets deduced from the PEAK surface profile data are provided in Figs. 4.3 through 4.7, where the apertures are shown along with the flow fields predicted using the apertures in the Reynolds equation. The characteristics of the PEAK fracture aperture data sets and a discussion of the advantages and disadvantages of the PEAK profiling technique relative to the laser profiling and RTV cast/imaging techniques is provided in the next section.

\subsubsection{Aperture Characteristics and Comparison of Measurement Techniques}

As mentioned in the previous sections, depictions of the aperture data sets deduced in each fracture by each measurement technique are provided in Figs. 4.1 through 4.7. The data sets are projected onto rectangular regions because the computer model used to calculate flow fields required a rectangular domain (see Section 4.1). In cases where a fracture was not rectangular-shaped (e.g., the Tram tuff fracture), large regions of zero aperture were used to fill in spaces that were not actually part of the fracture.

The size of the aperture data sets, the mean apertures, and the fraction of the surfaces in contact in each data set are given in Table 3.7. The mean apertures associated with different data sets for the same fracture are in good agreement because the data were adjusted to approximately match the mean aperture estimated from the microsphere breakthrough curves. Standard 
Table 3.7. Characteristics of Aperture Data Sets.

\begin{tabular}{|c|c|c|c|}
\hline $\begin{array}{c}\text { Data Set } \\
\text { (Fracture - Technique) }\end{array}$ & $\begin{array}{c}\text { No. of Data Points } \\
\text { (Spacing betw. Pts.) }\end{array}$ & $\begin{array}{c}\text { Mean } \\
\text { Aperture, } \mu \mathrm{m}\end{array}$ & $\begin{array}{c}\text { Fractional } \\
\text { Contact Area }\end{array}$ \\
\hline Bandelier - Laser & $194 \times 221(0.5 \mathrm{~mm})$ & 56.9 & 0.080 \\
\hline Bandelier - RTV & $282 \times 310(0.35 \mathrm{~mm})^{\mathrm{b}}$ & 55.2 & 0.019 \\
\hline Bandelier - PEAK 1 & $399 \times 444(0.25 \mathrm{~mm})$ & 55.1 & 0.0075 \\
\hline Bandelier - PEAK 2 & $394 \times 438(0.25 \mathrm{~mm})$ & 55.5 & 0.016 \\
\hline Tram - PEAK & $337 \times 367(0.25 \mathrm{~mm})$ & 155.3 & 0.051 \\
\hline Bullfrog - PEAK & $330 \times 324(0.25 \mathrm{~mm})$ & 555.8 & 0.041 \\
\hline Rot. Bullfrog - PEAK & $324 \times 324(0.25 \mathrm{~mm})$ & $563.7 \mathrm{c}$ & $0.031^{\mathrm{c}}$ \\
\hline
\end{tabular}

aSecond number corresponds to flow direction.

bRTV data set was "shrunk" from $565 \times 620$ to $282 \times 310$ by averaging every 4 adjacent data points. The Reynolds equation solver (discussed in Section 4.1) could not handle the larger data set.

c"Rotated Bullfrog" data differs from "Bullfrog" data because the latter data set includes several zero aperture points along one edge that were not included in the "rotated" data set.

deviations of the data sets were not calculated because they were influenced by the fraction of the surface area in contact. A qualitative measure of the "spread" in the data is provided in Fig. 3.10, which shows histograms of the aperture distributions in each of the three fractures, as determined from the PEAK surface profiles. It is clear from this figure that the data sets for the fractures with larger mean apertures have greater variance, and that the means of the Tram and Bullfrog tuff fracture data sets are significantly greater than the modes. These properties indicate that the Tram and Bullfrog tuff data sets have long tails that extend out to very large apertures (the tails must be long because the total area under all histograms is the same). The Bullfrog tuff fracture data set had many apertures greater than $6 \mathrm{~mm}$ (all from a few locations where matrix material was missing). Spatial correlations in the aperture data sets were not calculated, although it is apparent from examination of Figs. 4.1 through 4.7 that the correlations appear to be greater in the fractures with larger mean apertures.

Fig. 3.11 shows a comparison of the histograms of the aperture data deduced for the Bandelier tuff fracture by the three different measurement techniques. In this figure, it is clear that the PEAK data sets had the smallest variance, while the laser data set had the largest variance. It is also clear that the histograms associated with the two PEAK data sets are not in very good agreement, although they both have smaller variances than the other two data sets. The reason for the smaller variance of the PEAK data sets might be the exclusion of the styli tips from fine surface features that the other methods can more easily "probe." This hypothesized situation is illustrated in Fig. 3.12, which shows that the finite size of a stylus tip excludes it from crevices that the RTV might be able to flow into or the laser beam could illuminate. However, because the comparison of techniques was done using only a single fracture, this explanation should be considered speculative.

The differences between the aperture data. sets obtained by the different techniques suggest that there is considerable error associated with the aperture 


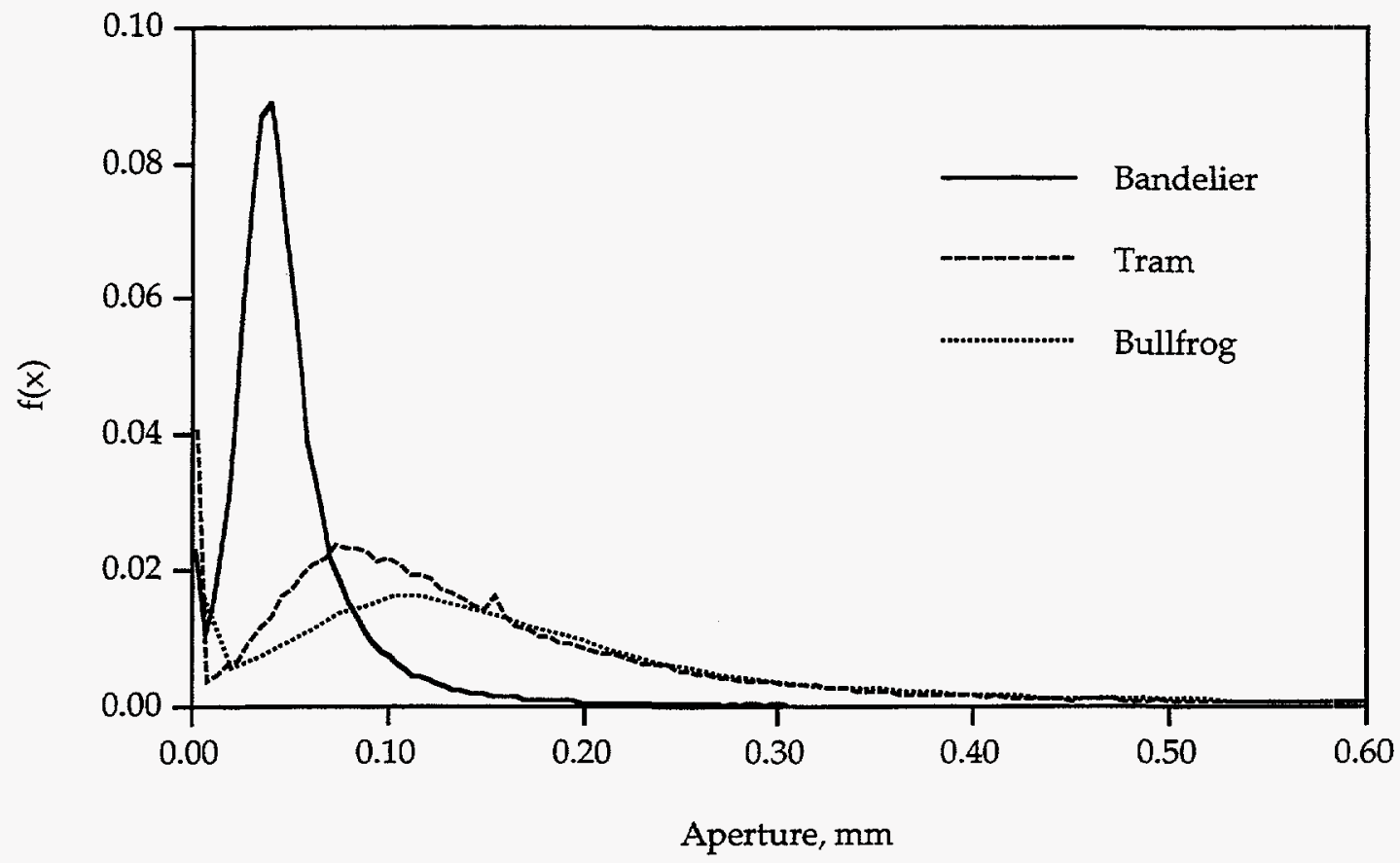

Figure 3.10. Frequency distributions (histograms) of aperture data sets associated with different fractures. The distributions are normalized so that all histograms have the same area underneath the curve. The data set for the Tram tuff fracture has a "spike" in the histogram at about $155 \mu \mathrm{m}$ (the mean aperture) because unrealistically low apertures resulting from "cliffs" on the fracture surfaces were set equal to the mean aperture (see text).

measurements. As mentioned in Section 3.2.1, the precision of the laser profiling technique was about $\pm 8 \mu \mathrm{m}$ (standard deviation of repeat measurements). The precision of the RTV cast/imaging technique was not determined. Repeat measurements on a flat surface with the PEAK profilometer (after temperature corrections) indicated a standard deviation of about $\pm 1.7 \mu \mathrm{m}$ in the vertical direction. However, the errors associated with each technique are not entirely attributable to measurement precision. The PEAK profilometer is probably somewhat limited by the finite size of the stylus tips (see above), and by the fact that the three styli cannot all be aligned in a straight line. The laser profilometer is prone to errors if there are translucent crystals on the surfaces. Both of these surface profiling techniques are also subject to errors associated with matching of the surfaces and limitations in being able to measure elevations at exactly corresponding points on the two surfaces. The RTV cast/imaging technique is subject to errors due to light refraction during imaging, air bubbles or tears in the casts, inability of the silicone to penetrate small crevices in the surfaces, and errors in the imaging system due to differences in sensitivity over different ranges of light intensity.

The apparent advantages and disadvantages of the three techniques (relative to each other) are listed below: 


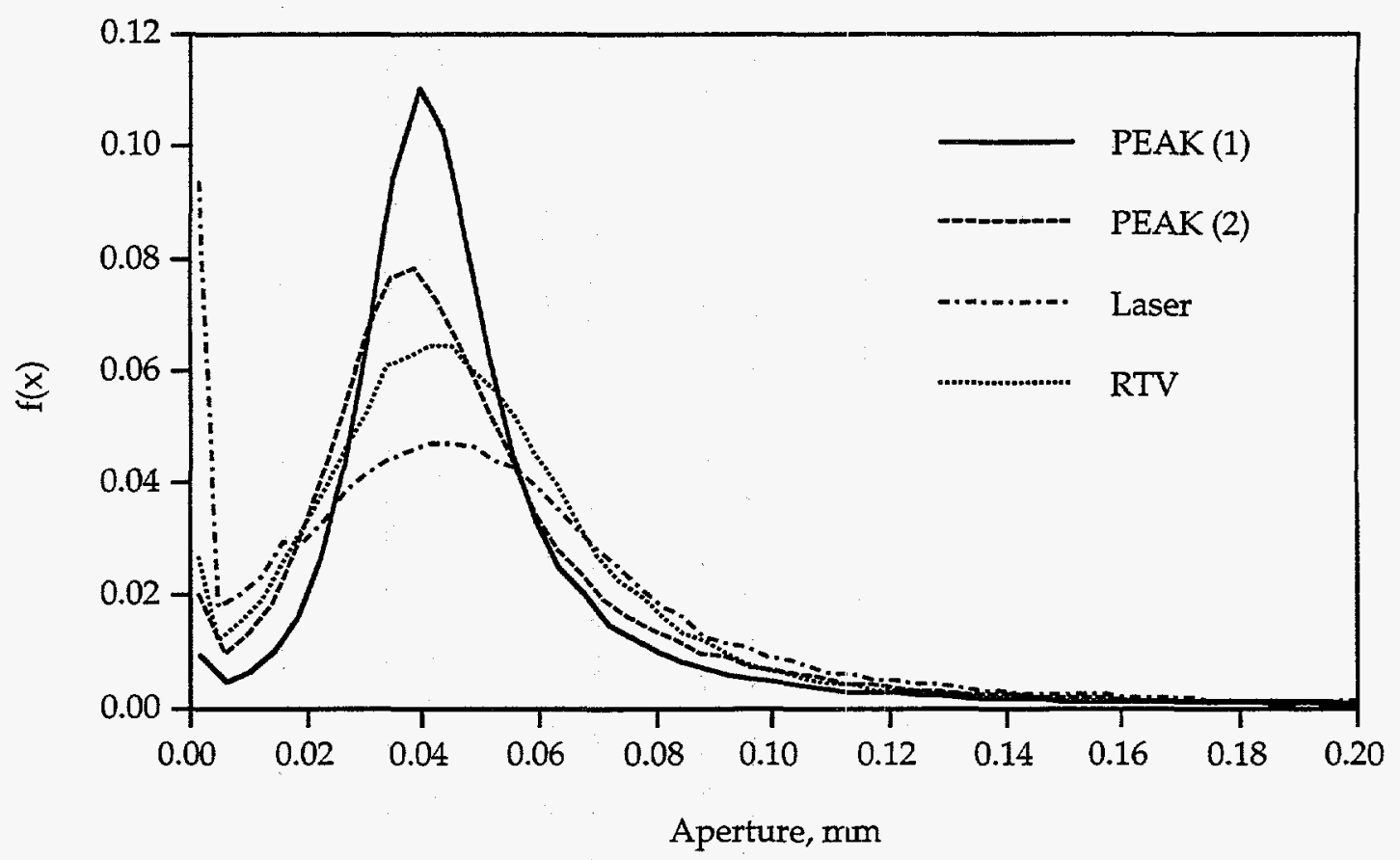

Figure 3.11. Frequency distributions (histograms) of Bandelier tuff fracture aperture data sets deduced from different aperture measurement techniques. The distributions are normalized so that all histograms have the same area underneath the curve. PEAK (1) and PEAK(2) refers to the first and second PEAK profiles of the fracture surfaces.

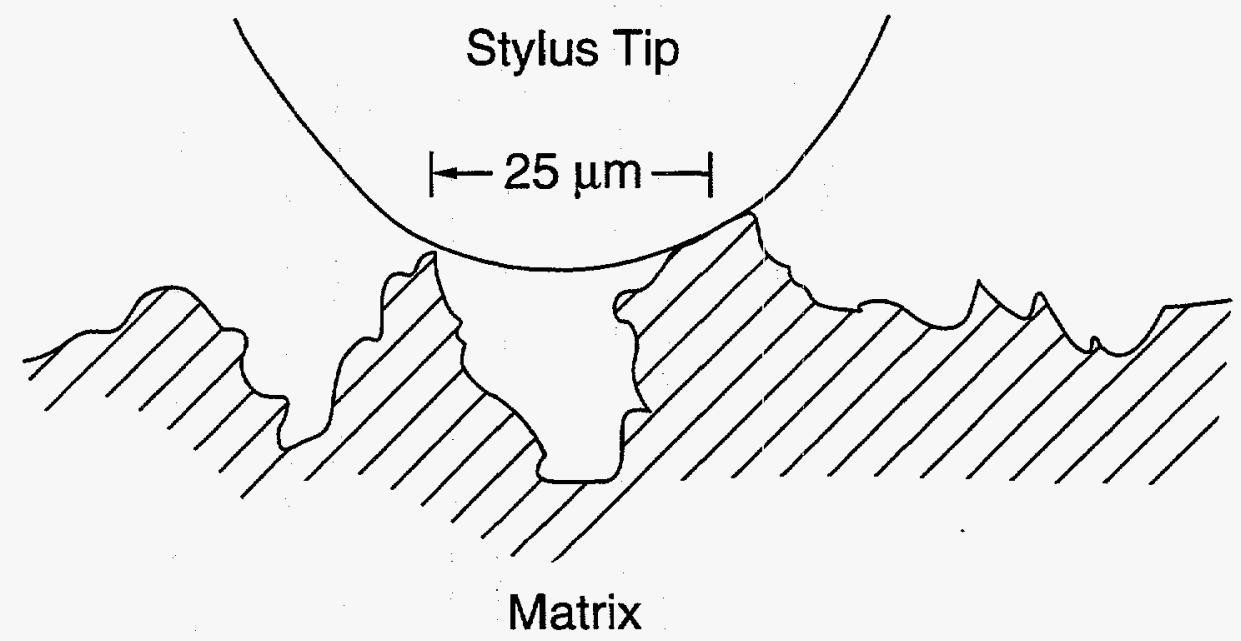

Figure 3.12. Depiction of a PEAK stylus tip hitting a rough fracture surface. The tip is too large to "see" fine surface detail that the other aperture measurement techniques may be able to "recognize." 
Laser Profiling, Advantages:

(1) There is no physical contact with the surfaces, so there is minimal contamination or physical alteration of the surfaces.

(2) The measurements are completely automated.

Laser Profiling, Disadvantages:

(1) There is a considerable amount of work and potential introduction of error associated with deducing apertures from the raw surface data. Great care must be taken during measurement and data reduction to ensure that the surface data are properly matched. Note: The measures taken to ensure matching of surface data from the PEAK profiles (i.e., the grinding of flat surfaces, aligning of surfaces on the table, and simultaneous profiling of the two surfaces) were much better than those taken for the laser profile(s).

(2) There is no easy way to correct for errors due to temperature fluctuations (as there is with the PEAK profilometer).

(3) The technique cannot be used on surfaces with translucent crystals.

RTV Cast/Imaging, Advantages:

(1) Aperture measurements are obtained directly -- there is no time consuming matching of surfaces or manipulation of surface data.

(2) A physical record of the fracture void space geometry is generated. RTV Cast/Imaging, Disadvantages:

(1) The casting process is difficult, with great care required to eliminate air bubbles and to avoid tearing of the cast after cure. Also, the difficulty of casting varies from fracture to fracture, with more porous matrices increasing the difficulty in eliminating air bubbles.

(2) The casting process irreversibly alters the surface chemistry of the fracture surfaces (e.g., the surfaces are obviously more hydrophobic after casting).

(3) The casting process can result in physical surface alterations when "chunks" of the surface stick to the cast. Because of this problem and item (2), RTV casting should be attempted only after all flow and tracer experiments have been completed.

Stylus Profiling, Advantages:

(1) This technique probably offers more precise positioning of the measurements in the plane of the fracture ( $x$ - and $y$-directions) than laser profiling, although there is no reason why the positioning associated with laser profiling could not be improved to the same level.

(2) The technique does not chemically alter the surfaces.

(3) The measurements are automated.

Stylus Profiling, Disadvantages:

(1) There is a considerable amount of work and potential introduction of error associated with deducing apertures from the raw surface data. Because of the necessity to "patch" the surface data from multiple styli, the amount of work is even greater than that for the laser profiling technique.

(2) There is a possibility of physically altering fracture surfaces because of the contact of the styli with the surfaces. Therefore, it is advisable to profile fracture surfaces after all flow and tracer experiments have been completed.

\subsection{Experimental Error Analysis}

The methods of estimating errors associated with the tracer experiments and the aperture measurements are discussed in this section. These methods address only measurement errors or measurement uncertainty. Systematic 
errors and errors of method (such as invalid assumptions, bias, or poor technique) are not formally addressed, although they are discussed qualitatively in appropriate sections of this dissertation. Errors associated with the tuff porosity measurements are discussed in Section 3.1.1.

Experimental errors associated with quantities that were dependent on more than one measured variable were estimated by propagation of error methods. For a general functional relationship, $F=f(x, y, z, \ldots)$, the expression for the variance of $F$ (assuming that the errors in the independent variables are random and uncorrelated) is ${ }^{174}$

$$
\sigma^{2}(F)=\overline{\left(\frac{\partial F}{\partial x}\right.}^{2} \sigma^{2}(x)+\overline{\left(\frac{\partial F}{\partial y}\right.}^{2} \sigma^{2}(y)+\overline{\left(\frac{\partial F}{\partial z}\right.}^{2} \sigma^{2}(z)+\ldots
$$

where, $\quad \sigma^{2}(x)=$ variance in variable $x$, and

$$
\begin{aligned}
& \left(\frac{\partial \mathrm{F}}{\partial \mathrm{x}}\right) \\
& \text { independent variables. }
\end{aligned}
$$

Equation (42) can be applied to both the calculation of apertures from surface profile data and the calculation of normalized concentrations $\left(\mathrm{C} / \mathrm{C}_{\mathrm{o}}\right)$. The aperture calculation involves subtraction of one surface elevation from another:

where, $\mathrm{a}=$ aperture, $\mathrm{mm}$

$$
a=b-c
$$

$\mathrm{b}=$ top surface elevation, $\mathrm{mm}$

$\mathrm{c}=$ bottom surface elevation, $\mathrm{mm}$.

If Eq. (43) is substituted into Eq. (42), the result is

$$
\begin{aligned}
& \sigma^{2}(a)=\sigma^{2}(b)+\sigma^{2}(c), \text { or } \\
& \sigma(a)=\sqrt{\sigma^{2}(b)+\sigma^{2}(c)}
\end{aligned}
$$

where, $\quad \sigma(\mathrm{a})=$ standard deviation of $\mathrm{a}$.

This is a well known result for the variance/standard deviation of a sum or difference of uncorrelated random variables. Using Eq. (44b) and the standard deviations of the surface profiling techniques reported in Section $3.2 .4(8 \mu \mathrm{m}$ for laser profiling and $1.7 \mu \mathrm{m}$ for stylus profiling), it can be concluded that the standard deviations associated with the aperture measurements should have been $11.3 \mu \mathrm{m}$ for the laser profiling technique and $2.4 \mu \mathrm{m}$ for the stylus profiling technique. However, these standard deviations (particularly for the stylus technique) do not necessarily reflect the true errors associated with the techniques, as there was often clear evidence of trends and discontinuities in the aperture data sets that could not be the result of random errors. A standard deviation calculation for the RTV cast/imaging technique was not possible because there were no repeat measurements using this technique.

The calculation of normalized concentrations $\left(C / C_{o}\right)$ was accomplished using the following formula:

$$
\frac{C}{C_{o}}=F=\left(\frac{x+y}{y}\right) \frac{c}{c_{o}}=\left(\frac{x}{y}+1\right) \frac{c}{c_{o}}
$$

where, $x=$ flow rate through outlet manifold, $\mathrm{ml} / \mathrm{sec}$

$\mathrm{y}=$ flow rate through fracture, $\mathrm{ml} / \mathrm{sec}$

$c=$ measured concentration of tracer in effluent sample, 


$$
\mathrm{c}_{\mathrm{o}}=\text { measured inlet concentration. }
$$

Because the ratio of $x$ to $y$ always exceeded 50, Eq. (45) was simplified to

$$
F=\left(\frac{x}{y}\right) \frac{c}{c_{0}}
$$

Substitution of Eq. (46) into Eq. (42) (recognizing that the errors in the independent variables are uncorrelated) and dividing by $\overline{\mathrm{F}}^{2}$ yields

$$
\begin{aligned}
& \frac{\sigma^{2}(\mathrm{~F})}{\overline{\mathrm{F}}^{2}}=\frac{\sigma^{2}(\mathrm{x})}{\overline{\mathrm{x}}^{2}}+\frac{\sigma^{2}(\mathrm{y})}{\overline{\mathrm{y}}^{2}}+\frac{\sigma^{2}(\mathrm{c})}{\mathrm{c}^{2}}+\frac{\sigma^{2}\left(\mathrm{c}_{\mathrm{o}}\right)}{\bar{c}_{\mathrm{o}}^{2}} \\
& \frac{\sigma(\mathrm{F})}{\overline{\mathrm{F}}}=\sqrt{\frac{\sigma^{2}(\mathrm{x})}{\overline{\mathrm{x}}^{2}}+\frac{\sigma^{2}(\mathrm{y})}{\overline{\mathrm{y}}^{2}}+\frac{\sigma^{2}(\mathrm{c})}{\bar{c}^{2}}+\frac{\sigma^{2}\left(\mathrm{c}_{\mathrm{o}}\right)}{\bar{c}_{\mathrm{o}}^{2}}}
\end{aligned}
$$

where, $\quad \bar{x}=$ mean of general variable $x$.

Equation (47b) was used to calculate the relative standard deviations associated with the normalized concentrations. The "error bars" shown in the breakthrough curve plots in Appendices B and D correspond to \pm 2 times these relative standard deviations. Assuming that the errors were normally distributed, these error bars correspond to $95.5 \%$ confidence limits. That is, the probability that the true value of the normalized concentration falls between the error bars is 0.955 .

The values used for the relative standard deviations of the independent variables in Eq. (47b) were based on repeat measurements of the variables. For the concentration measurements, the relative standard deviations were calculated from the weighted average of the standard deviations of repeat measurements taken over the course of all eighteen tracer experiments (except for the silica measurements, which were all done on the same day). The relative standard deviations of measured concentrations of the different tracers were:

1- $\mu \mathrm{m}$ fluorescent microspheres: 0.0098

$0.3-\mu \mathrm{m}$ fluorescent microspheres: 0.0127

$0.3-\mu \mathrm{m}$ silica microspheres: 0.048

Iodide: 0.0217

However, the scatter in the iodide data was generally greater than the standard deviation of the repeat measurements suggested, so a relative standard deviation of 0.04 was used for iodide based on the measurement precision stated in the iodide electrode instruction manual.

The relative standard deviations of the flow rates were calculated separately for each tracer experiment based on measurements made before and after the experiments. These relative standard deviations actually had a systematic error component in them because the flow rates generally tended to increase or decrease during the course of an experiment. Thus, even though the actual random measurement errors should have been about the same in all experiments, some experiments had considerably larger relative standard deviations in flow rates than others because there was a greater increase or decrease in the flow rates during the test (presumably due to wear or stretching of the tubing in the peristaltic pumps). Also, the relative standard deviations in flow rates were sometimes different in the step and flush phases of a test because the flow rates of the tracer solution and the tracer-free J-13 water changed by different amounts. In cases where the flow rate was measured only once before and after a test, it was impossible to calculate a formal standard deviation. In 
these cases, the standard deviation was assumed to be the absolute value of half the difference between the two measurements.

The values of the relative standard deviations of the flow rates in each tracer experiment and the relative standard deviations of the normalized concentrations calculated from Eq. (47b) are provided in Table 3.8. The error bars in the breakthrough curves of Appendices $B$ and D are \pm 2 times the values in the last 3 columns of Table 3.8 .

To be complete, it should be mentioned that the data points in the breakthrough curves of the appendices should also have error bars in the $x$-axis direction as well as in the y-axis direction. Errors associated with the time scales should have never exceeded \pm 2 seconds (the time error associated with manually changing valve positions and starting the fraction collector). However, when times were converted to eluted volumes, the conversion involved multiplying the time by the flow rate through the fracture, which had considerable error associated with it in some cases (see Table 3.8). Error bars in the $\mathrm{x}$-axis direction were not plotted because they caused the plots to look excessively cluttered. Also, the $x$-direction error bars were thought to be less important than the $y$-direction error bars.

Table 3.8. Relative Standard Deviations of Flow Rates and Normalized Concentrations in the Tracer Experiments.

\begin{tabular}{|c|c|c|c|c|c|c|}
\hline $\begin{array}{l}\text { Experi- } \\
\text { ment }^{\mathrm{a}}\end{array}$ & $\begin{array}{l}\frac{\text { Tracer }}{\text { Flow }} \\
\text { lon }\end{array}$ & $\frac{\text { I-13 }}{\text { Flow }}$ & $\frac{\text { Manifold }}{\text { Flow }}$ & $\frac{C / \bar{C}_{0}}{1-\mu m^{b}}$ & $\frac{C / C_{g}}{0.3-\mu m^{b}}$ & $\frac{\mathrm{C} / \mathrm{C}_{\underline{Q}}}{\text { lodide }^{b}}$ \\
\hline 1 & $0.025^{c}$ & $\mathrm{~N} / \mathrm{A}$ & 0.0061 & 0.029 & $\mathrm{~N} / \mathrm{A}$ & 0.048 \\
\hline 2 & 0.0182 & 0.0181 & 0.0034 & $\bar{N} / \bar{A}$ & $0.102 / 0.102$ & $0.044 / 0.044$ \\
\hline 3 & 036 & 0.0340 & 0.0109 & $0.018 / 0.038$ & $0.021 / 0.040$ & $0.042 / 0.054$ \\
\hline 4 & 0.0790 & 0.0087 & 0.0032 & $0.080 / 0.017$ & $0.081 / 0.020$ & $0.089 / 0.041$ \\
\hline 5 & 0.0298 & 0.0038 & 0.0057 & $0.033 / 0.015$ & $0.035 / 0.019$ & $0.050 / 0.041$ \\
\hline 6 & 0.0047 & 0.0022 & 0.0034 & $0.015 / 0.014$ & $0.019 / 0.018$ & $0.040 / 0.040$ \\
\hline 7 & 0.0117 & 0.0048 & 0.0039 & $0.019 / 0.015$ & $0.022 / 0.019$ & $0.042 / 0.040$ \\
\hline$\overline{8}$ & 0.0136 & 0.0105 & 0.0039 & $0.020 / 0.018$ & $0.023 / 0.021$ & $0.042 / 0.042$ \\
\hline 9 & 0.0113 & 0.0133 & 0.0044 & $0.018 / 0.020$ & $0.022 / 0.023$ & $0.042 / 0.042$ \\
\hline 10 & 0.0308 & 0.0243 & 0.0035 & $0.034 / 0.028$ & $0.036 / 0.030$ & $0.051 / 0.047$ \\
\hline$\overline{11}$ & 0.0151 & $-0-$ & 0.0005 & $0.021 / 0.014$ & $0.023 / 0.018$ & $0.043 / 0.040$ \\
\hline$\overline{12}$ & 0.0076 & 0.0036 & 0.0101 & $0.019 / 0.018$ & $0.022 / 0.021$ & $0.042 / 0.041$ \\
\hline 13 & 0.0136 & 0.0165 & 0.0013 & $0.019 / 0.022$ & $0.023 / 0.024$ & $0.042 / 0.043$ \\
\hline 14 & 0.0067 & 0.0074 & 0.0020 & $0.016 / 0.016$ & $0.019 / 0.020$ & $0.041 / 0.041$ \\
\hline 15 & 0.0022 & $\mathrm{~N} / \mathrm{A}$ & 0.0043 & 0.015 & 0.019 & 0.040 \\
\hline 16 & 0.0059 & N/A & 0.0026 & 0.015 & 0.019 & 0.041 \\
\hline 17 & 0.0097 & N/A & 0.0026 & 0.017 & 0.021 & 0.041 \\
\hline 18 & 0.0050 & $\mathrm{~N} / \mathrm{A}$ & 0.0124 & 0.019 & 0.022 & 0.042 \\
\hline
\end{tabular}

aSee Appendix $\mathrm{A}$ for description of tracer experiments.

bWhere there are two values, the first refers to the step phase, and the second refers to the flush phase. Only one value appears when there was no flush phase.

cAssumed-- there was only one flow rate measurement. 


\subsection{MODELING AND INTERPRETATION OF EXPERIMENTS}

A two-step approach was used to model fluid flow and tracer transport through the fractures: (1) steady-state flow fields in the fractures were estimated by solving the Reynolds equation (Eq. 7) using the aperture distributions deduced from the techniques discussed in Section 3.2, and (2) tracer transport in the fractures was predicted using particle-tracking techniques (Section 2.6.1) with the deterministic motion of the particles being dictated by the steady-state flow fields. The objective of these modeling exercises was to provide insights into the conceptual modeling of colloid and solute transport through variable-aperture fractures. Although model parameters were sometimes varied to investigate or illustrate their influence on model predictions, the intent was not to obtain the best parametric fit to the experimental data. Instead, emphasis was placed on identifying (as opposed to quantifying) important mechanisms of colloid and solute transport in fractures. Thus, the insights gained from the modeling exercises were qualitative in nature.

In the interest of saving space, the computer models used to conduct the modeling exercises are described only conceptually in the following sections. Computer source code listings are not included in this dissertation because they total several thousand lines and would significantly increase the length of the dissertation. Source code listings can be obtained by contacting the author.

\subsection{Flow Simulations to Estimate Flow Fields}

A computer model was developed to solve the Reynolds equation in a rectangular region with no-flow boundaries on the sides and constant pressure boundaries on the ends. The model solved a finite-difference discretization of Eq. (7) using aperture data corresponding to the data sets discussed in Section 3.2. Each node was assumed to be a set of parallel plates. The discretization at a given node was a simple forward-backward differencing scheme involving only the four immediately adjacent nodes in each direction in the fracture plane (i.e.,

$\frac{\partial^{2} P}{\partial x^{2}}=\frac{P_{i-1, j}-2 P_{i, j}+P_{i+1, j}}{(\Delta x)^{2}}$, and $\left.\frac{\partial^{2} P}{\partial y^{2}}=\frac{P_{i, j-1}-2 P_{i, j}+P_{i, j+1}}{(\Delta y)^{2}}\right)$. The effective aperture between any two nodes ( $i$ and $j$ ) was assumed to be $\left(\frac{2 a_{i}^{3} a_{j}^{3}}{a_{i}^{3}+a_{j}^{3}}\right)$ (see Section 2.5.2). Once the pressures were obtained from the solution of Eq. (7), flow rates across node boundaries were calculated using Eq. (8). This approach has been used by others to obtain flow fields in variable-aperture fractures, $84,86-91$ but the aperture distributions used by others have typically been generated mathematically rather than deduced from actual measurements of void space geometry.

The Reynolds equation can be expected to offer a valid approximation of the pressure distribution in a fracture only if the Reynolds number in the fracture (Eq. 4) remains relatively low. At higher Reynolds numbers, the local parabolic velocity profile assumed in the Reynolds equation will break down in a roughwalled fracture because the inertial terms in the Navier-Stokes equation will become too large. Unfortunately, the criteria for when the Reynolds equation breaks down is problem dependent. In a channel with very smooth walls (e.g., parallel plates or slowly converging/diverging plates), the equation is likely to 
be valid for flow rates approaching the transition to turbulent flow (a Reynolds number of about 2000). 80 However, in rough-walled fractures, the Reynolds equation can be expected to break down at much lower Reynolds numbers, and a good rule-of-thumb is probably that the Reynolds number should not exceed 1 (i.e., "creeping" flow). The maximum Reynolds number in any of the tracer experiments was about 0.24 (averaged over entire fracture), so the flow in the fractures should have been essentially "creeping." Thus, the experimental flow rates were low enough that the use of the Reynolds equation should have been valid.

Because of (1) the wide range of apertures in each aperture data set (from zero to 2 or more $\mathrm{mm}$ ) and (2) the cubic dependence of permeability on aperture (Eq. 6), the range of local permeabilities in the fractures was typically 8 to 10 orders-of-magnitude. This wide range resulted in a very "stiff" problem that was difficult to solve numerically. To reduce the effort necessary to obtain a solution, apertures less than $0.5 \mu \mathrm{m}$ in the Bandelier tuff data sets were set equal to $0.5 \mu \mathrm{m}$, and apertures less than $1.0 \mu \mathrm{m}$ in the Tram and Bullfrog tuff data sets were set equal to $1.0 \mu \mathrm{m}$. This scheme greatly simplified the computer coding and significantly reduced computation times. The modified apertures were small enough that negligible flow was calculated in the corresponding nodes, and the mean aperture of the data sets were essentially unaffected.

Ideally, the best way to solve the linear system of equations resulting from the discretization of the Reynolds equation would be to use direct matrix inversion. Direct inversion can be most efficiently accomplished by a lowerupper decomposition of the coefficient matrix followed by forward and back substitutions to obtain the solution vector. ${ }^{175}$ This method provides a completely converged solution (within machine roundoff) with no dependence of computation time on the "stiffness" of the coefficient matrix. Unfortunately, the method is very memory intensive, and the practical upper limit in problem size that can be accommodated on a computer with 128 megabytes of memory is about 16,000 nodes (determined on a Sun Sparcstation 690MP). The problem sizes associated with the fracture aperture data sets ranged from $\sim 43,000$ to $\sim 177,000$ nodes, so an iterative method of solution was required.

The iterative method selected was the implicit alternating-direction (IAD) method. ${ }^{176,177}$ This method made two "passes" through the linear system of equations for each iteration, with each pass solving the equations resulting from discretization in only one direction of the domain. The advantage of this technique is that the calculations for each pass involve a tridiagonal system of equations, which can be solved very efficiently by direct methods. ${ }^{177}$ In the IAD method, the diagonal terms of the two tridiagonal coefficient matrices are perturbed by constants to accelerate convergence. An optimum sequence of perturbation parameters for a given number of iterations was calculated using an algorithm developed by Saylor and Sebastian, ${ }^{178}$ which required estimates of the upper and lower eigenvalues of the coefficient matrices. These estimates were obtained using subroutines from the Common Los Alamos Mathematical Software (CLAMS) FORTRAN library. ${ }^{179}$ It was determined that convergence could be accelerated the most by repeating short sequences of perturbation parameters rather than using one long sequence. Also, the truncation of the sequences calculated by the Saylor and Sebastian algorithm (at some lower cutoff value) sometimes helped speed convergence. These empirical modifications 
were often necessary to obtain a converged solution. The computer code used to obtain the solutions was verified by comparing the computed solution for a small problem (e.g., 10,000 nodes) with a solution obtained for the same problem using direct matrix inversion.

Convergence of the iterative method could also be accelerated by providing a good initial guess of the pressure distribution in the fracture. Good initial guesses were obtained as follows:

(1) The fracture domain was divided into "blocks" consisting of a small number of nodes (typically $5 \times 5$ or $6 \times 6$ nodes). The volumetric flow rate for a given pressure drop across each "block" was calculated in both the $\mathrm{x}$ - and $\mathrm{y}$ directions using direct matrix inversion. These results were then used to calculate hydraulic apertures in each direction of each "block" using Eq. (40).

(2) The direction-dependent hydraulic apertures of the "blocks" were used as apertures in a coarsened representation of the overall fracture domain. The pressure distribution in the coarsened domain was calculated using direct methods. For example, if the entire domain was $200 \times 300$ nodes, and Step (1) involved "blocks" of 5- x 5-nodes, then the coarsened domain would be $40 \times 60$ nodes, which was a small enough problem that it could be solved by direct matrix inversion.

(3) The pressures at the boundaries of the coarsened nodes were calculated and then used as boundary conditions to directly solve the pressure distribution in each of the small "blocks" from Step (1). The resulting pressures at each node were mapped into the overall fracture domain as the initial guesses for the iterative method.

These initial guesses resulted in more than a two-fold decrease in computation time compared to when initial guesses were provided by simply assuming a linear pressure drop across the fracture. The computation time to provide the guesses was typically only about $1 / 50$ th of the time required to obtain a converged solution, so the extra effort was well justified. Total computation times ranged from about 2 to 8 hours on a Sun Sparcstation 690MP for the various aperture data sets (given appropriate modifications to the perturbation parameter sequence).

Convergence of the iterative solution was checked by various methods. The first method was a check of the mass flow balance at each node in the problem domain. The convergence criteria was that the volumetric flow rate out of each node and the volumetric flow rate into each node agree to within a specified percentage. This method worked well for the Bandelier tuff fracture flow simulations, but the criteria was too stringent for the Tram and Bullfrog tuff fracture simulations, which were inherently stiffer problems. For these simulations, iterations were continued until the value of the "error norm" dropped below a specified value. The error norm is given by

$$
\langle e\rangle=\left[\sum(\mathrm{b}-\overline{\overline{\mathbf{A}} \mathbf{x}})^{2}\right]^{1 / 2}
$$

where, $\langle\mathrm{e}\rangle=$ error norm,

$\mathbf{b}=$ right-hand-side vector in the problem $\overline{\overline{\mathbf{A x}}}=\mathbf{b}$,

$\overline{\overline{\mathbf{A}}}=$ coefficient matrix , and

$\mathbf{x}=$ solution vector at current iteration. 
Values of the error norm giving acceptable solutions were somewhat problem dependent, so the criteria ultimately used to test convergence (if the node-bynode mass balance method failed) was that the total volumetric flow rate into the fracture domain agree with the total flow rate out of the domain to within less than $1 \%$. This criteria did not guarantee that all nodes would have a reasonable mass balance; and, in fact, there was typically a small percentage of nodes that had no flow into or out of them (i.e., source or sink nodes). However, it was confirmed that these nodes always had very low flow rates and hence did not contribute much to the overall flow field. Convergence was typically checked only every 20 or so iterations because the checks were computationally very costly compared to the iterations themselves (one check took as much time as about 5 or 6 iterations).

Measures of the degree of convergence achieved for the flow simulations associated with each aperture data set are provided in Table 4.1. These measures are (1) the percent error between the total volumetric flow into and out of the fracture domain, (2) the percentage of nodes that have no mass balance (source or sink nodes), and (3) the percentage of nodes that have $<1 \%$ mass balance error.

Table 4.2 shows the hydraulic apertures calculated from the flow simulations (using Eq. 40) as well as the corresponding range of experimental hydraulic apertures. The hydraulic apertures calculated for the Bandelier tuff

Table 4.1. Measures of Convergence of Flow Simulations.

\begin{tabular}{|c|c|c|c|}
\hline $\begin{array}{c}\text { Flow Simulation } \\
\text { (Fracture - Data Set) }\end{array}$ & $\begin{array}{c}\frac{\text { Overall Flow }}{\text { Rate Error (\%) }} \\
\text { Bandelier - Laser }\end{array}$ & $\begin{array}{c}\% \text { of Nodes with } \\
\text { No Mass Bal. }\end{array}$ & $\begin{array}{c}\text { \% of Nodes with } \\
\leq 1 \% \text { M. Bal. Error }\end{array}$ \\
\hline Bandelier - RTV & $4 \times 10^{-5}$ & $-0-$ & $\sim 100$ \\
\hline Bandelier - PEAK 1 & $8 \times 10^{-6}$ & $-0-$ & $\sim 100$ \\
\hline Bandelier - PEAK & $4 \times 10^{-5}$ & $-0-$ & $\sim 100$ \\
\hline Tram - PEAK & 0.025 & $-0-$ & 99.77 \\
\hline Bullfrog - PEAK & 0.084 & 0.40 & 95.13 \\
\hline rot. Bullfrog - PEAK & 0.019 & 1.33 & 94.76 \\
\hline
\end{tabular}

Table 4.2. Calculated and Experimental Hydraulic Apertures.

\begin{tabular}{|c|c|c|}
\hline $\begin{array}{l}\text { Flow Simulation } \\
\text { (Fracture - Data Set) }\end{array}$ & $\frac{\text { Calc. Hydraulic }}{\text { Aperture, } \mu \mathrm{m}}$ & $\begin{array}{l}\text { Range of Exp. } \\
\text { Hyd. Apers. } \mu \mathrm{m}\end{array}$ \\
\hline Bandelier - Laser & 39.8 & $27.4-37.6^{\mathrm{a}}$ \\
\hline Bandelier - RTV & 40.4 & $27.4-37.6^{a}$ \\
\hline Bandelier - PEAK 1 & 41.2 & $27.4-37.6^{a}$ \\
\hline Bandelier - PEAK 2 & 38.9 & $27.4-37.6^{a}$ \\
\hline Tram - PEAK & 112.8 & $88.9-92.5$ \\
\hline Bullfrog - PEAK & 167.8 & $108.4-114.7$ \\
\hline rotated Bullfrog-PEAK & 162.5 & $111.0-114.0$ \\
\hline
\end{tabular}

aThe hydraulic aperture of $37.6 \mu \mathrm{m}$ was measured after inadvertently pressurizing the fracture; all other hydraulic aperture measurements in the Bandelier tuff fracture were $29.0 \mu \mathrm{m}$ or less. 
fracture using different aperture data sets are all in reasonably good agreernent, indicating that the predicted flow resistance in this fracture is relatively insensitive to the aperture measurement technique (at least when the apertures are adjusted to approximately the same mean value). However, the calculated hydraulic apertures for all data sets are consistently greater than the experimentally observed hydraulic apertures, indicating that the adjustment of the aperture data to match the mean apertures deduced from microsphere breakthrough curves results in overprediction of the hydraulic apertures. This discrepancy is difficult to explain without further study. Perhaps the aperture measurement techniques are incapable of picking up fine-scale flow obstructions that profoundly affect flow resistance in the fractures.

Depictions of the aperture data sets and the corresponding predicted flow fields are provided in Figs. 4.1 through 4.7. When modeling tracer transport, the calculated flow rates across each node boundary were scaled by the ratio of overall experimental flow rate to overall calculated flow rate to obtain a flow field with appropriate flow magnitudes to match a given experiment (this allowed one flow simulation to be used for multiple experimental flow rates in a given fracture).

Evidence of flow channeling is apparent in each of the flow fields depicted in Figs. 4.1 through 4.7. However, the channeling appears to be more dominant in the fractures of larger average aperture (which also appear to have greater aperture correlation lengths). It is apparent that despite the good agreement of the calculated hydraulic apertures from each data set for the Bandelier tuff fracture (Table 4.2), there are discrepancies in the predicted flow pathways through this fracture. A more stringent test of the agreement between the data sets is provided by comparing the fluid residence time distributions predicted using the models described in Section 4.2.

\subsection{Tracer Transport Simulations using Particle-Tracking Methods}

Particle-tracking methods were used in conjunction with the flow field solutions presented in Section 4.1 to model tracer transport through the fractures. The intent was to model tracer transport using actual estimates of the fracture flow fields to account for hydrodynamic dispersion rather than using the more traditional approach of assuming a linear flow velocity and then "fitting" the experimental data with an adjustable dispersion coefficient. The latter approach does not provide the mechanistic insights into the problem that were desired in this study. The particle-tracking models were sometimes modified to investigate the influence of different modeling assumptions. The model predictions were always compared to the predictions of models in which it was assumed that the fractures were single parallel plates. These comparisons provided perspective on the advantages of using variable-aperture flow fields as the basis for transport calculations. It is important to note that the models were greatly simplified by assuming that the tracers did not adsorb (iodide) or attach (microspheres) to the fracture surfaces. The experimental data supported these simplifying assumptions (see Section 3.1.7). The various particle-tracking models are described in the following sections. The model predictions are discussed in Section 4.3. 



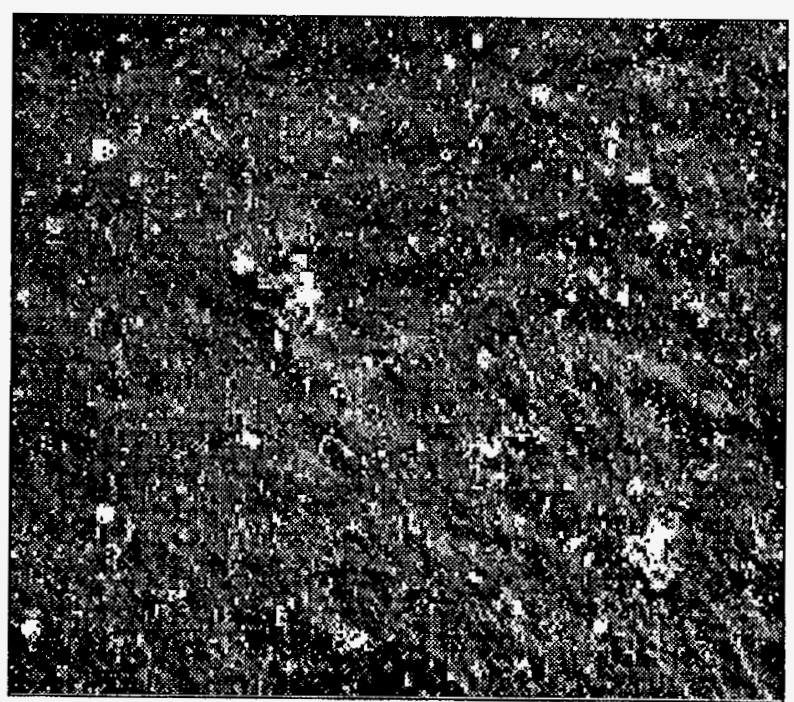

ฟु
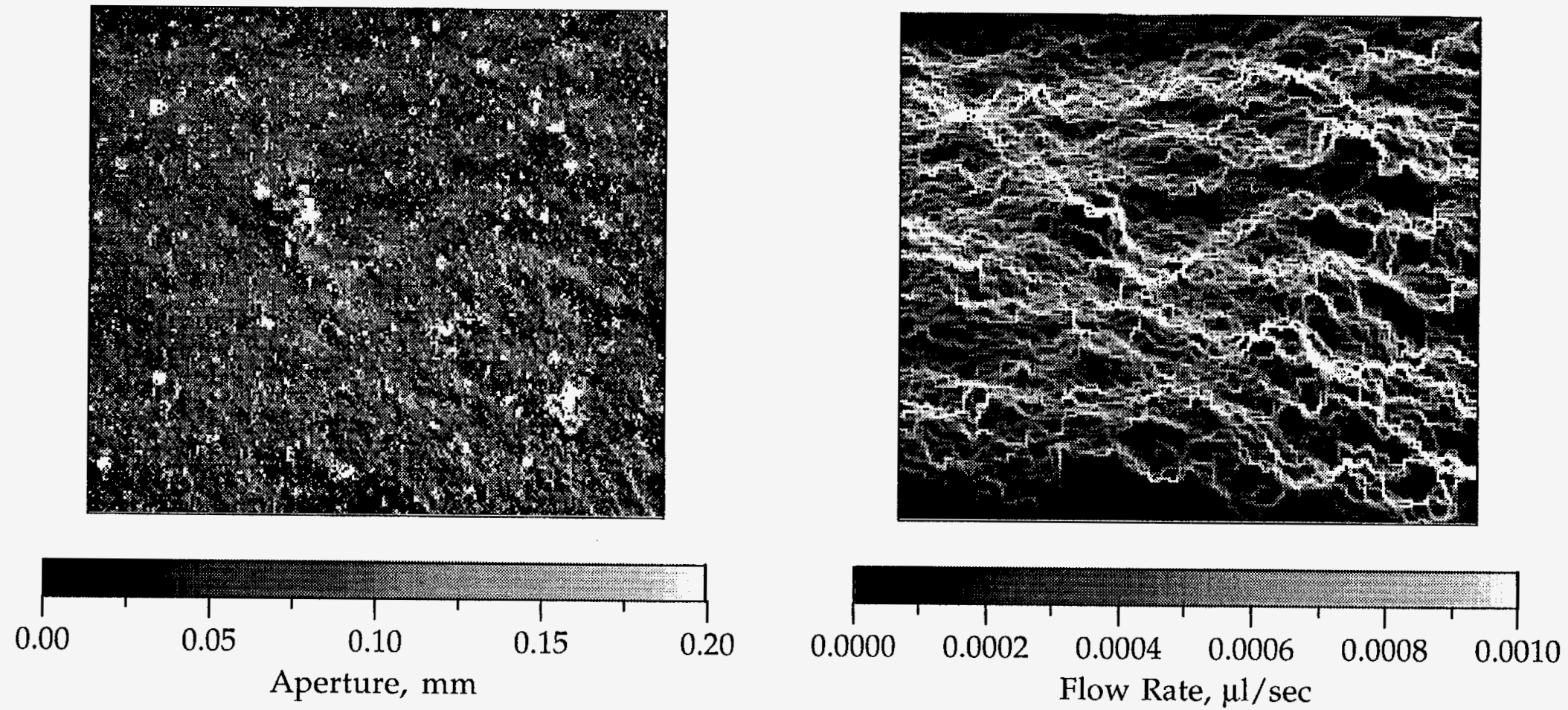

Figure 4.1. Bandelier tuff fracture aperture data set deduced from laser profiling technique (left) and the flow field calculated using this aperture data set in the Reynolds equation (right). The flow field was calculated assuming constant pressure boundaries on the left and right edges of the plots and no-flow boundaries on the top and bottom edges, with flow being from left to right to match the flow direction in the tracer experiments. Each pixel in both plots represents a $0.5-x 0.5-\mathrm{mm}$ area. All flow rates in the right-hand plot must be multiplied by the ratio of experimental to calculated flow rate to match the overall flow rate in a given tracer experiment. 



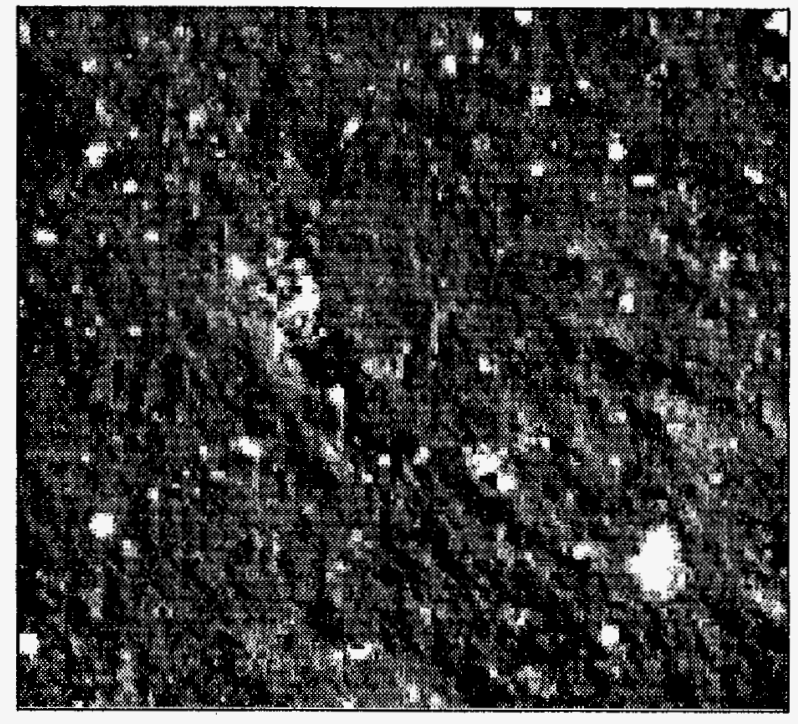

$\stackrel{\infty}{\infty}$

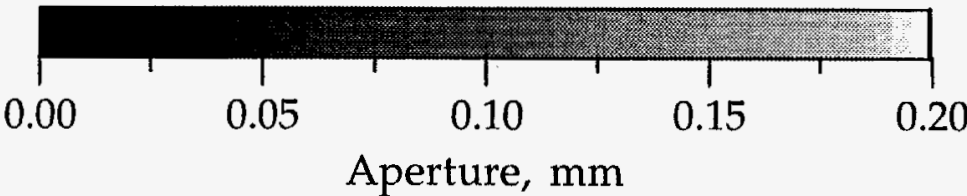

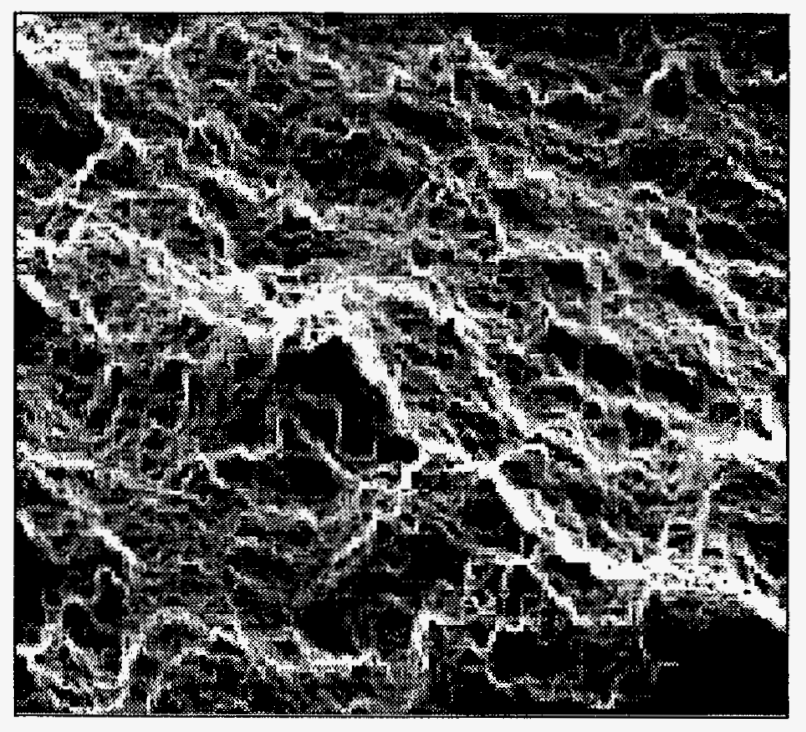

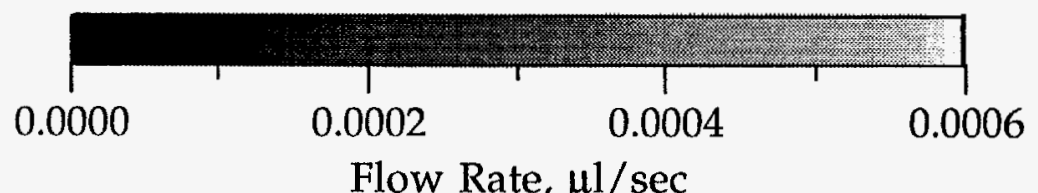

Figure 4.2. Bandelier tuff fracture aperture data set deduced from RTV cast/imaging technique (left) and the flow field calculated using this aperture data set in the Reynolds equation (right). The flow field was calculated assuming constant pressure boundaries on the left and right edges of the plots and no-flow boundaries on the top and bottom edges, with flow being from left to right to match the flow direction in the tracer experiments. Each pixel in both plots represents a $0.35-\times 0.35-\mathrm{mm}$ area. All flow rates in the right-hand plot must be multiplied by the ratio of experimental to calculated flow rate to match the overall flow rate in a given tracer experiment. 


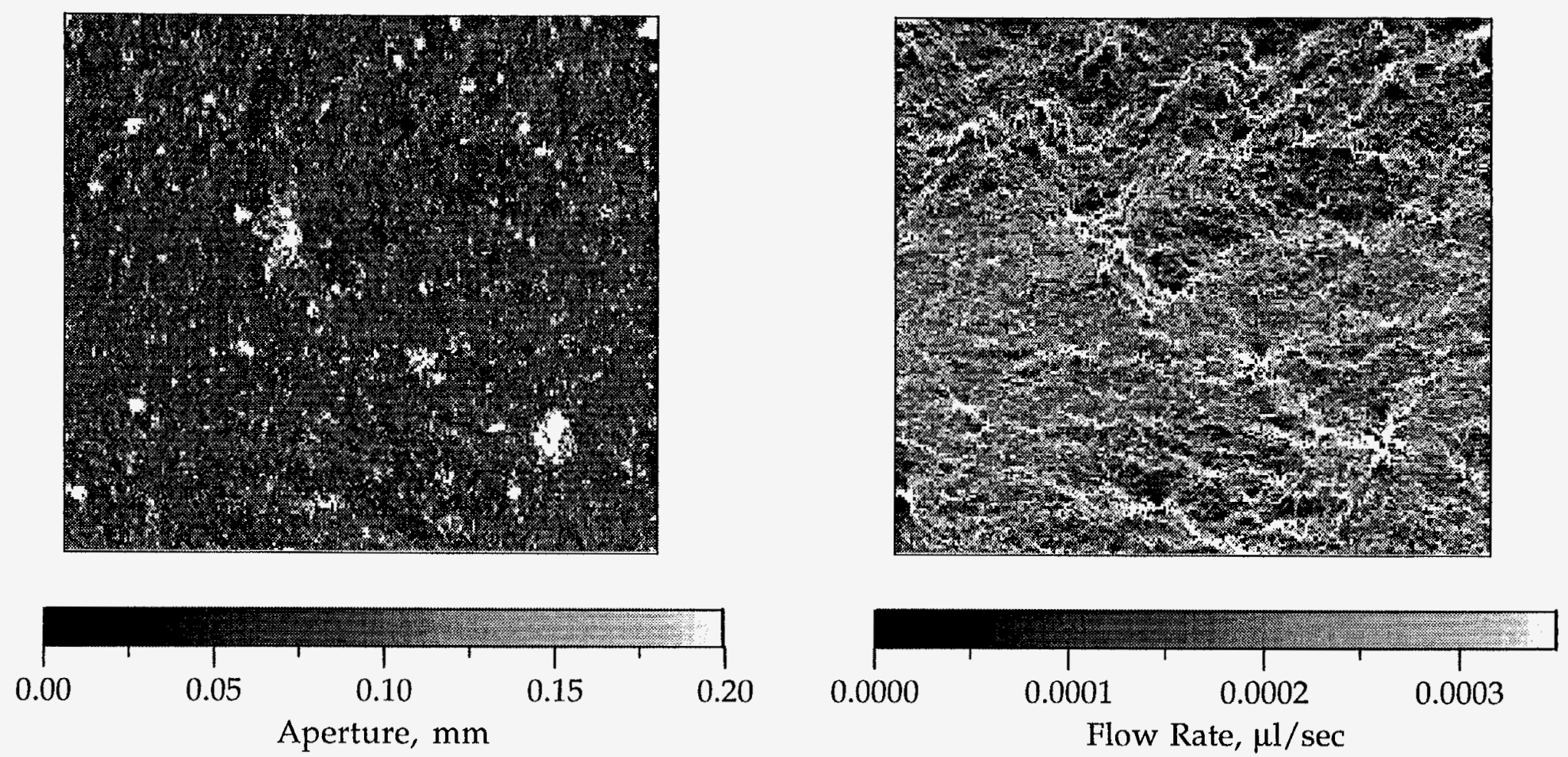

Figure 4.3. Bandelier tuff fracture aperture data set deduced from first PEAK profile of this fracture (left) and the flow field calculated using this aperture data set in the Reynolds equation (right). The flow field was calculated assuming constant pressure boundaries on the left and right edges of the plots and no-flow boundaries on the top and bottom edges, with flow being from left to right to match the flow direction in the tracer experiments. Each pixel in both plots represents a $0.25-\times 0.25-\mathrm{mm}$ area. All flow rates in the right-hand plot must be multiplied by the ratio of experimental to calculated flow rate to match the overall flow rate in a given tracer experiment. 


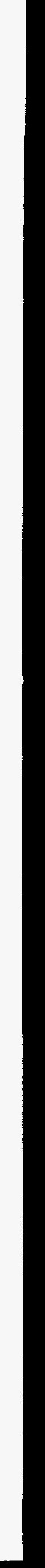



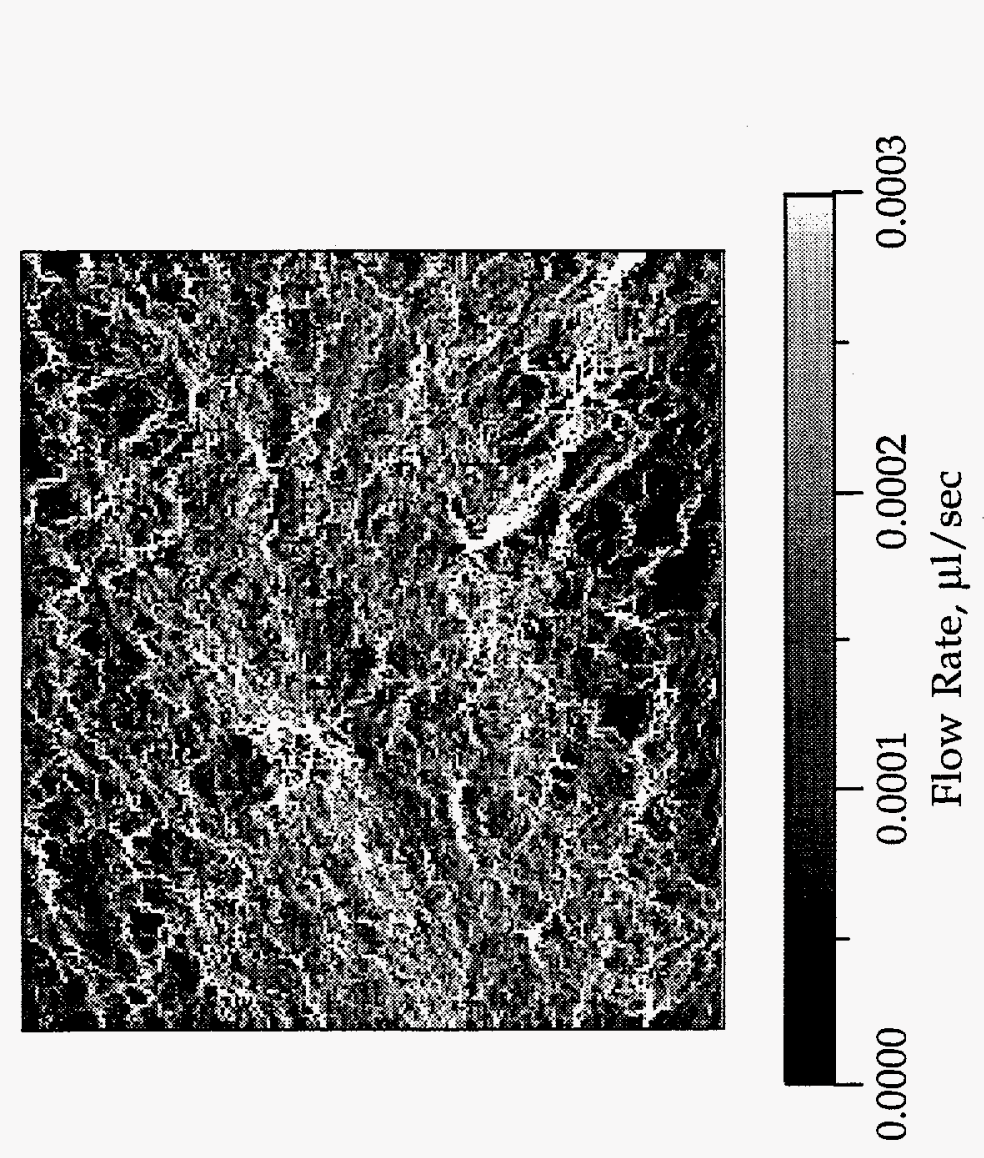

3.

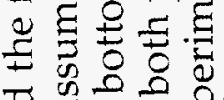

몰

๘

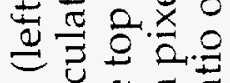

过艺苛

苞芯藏焉

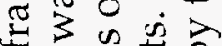

可记

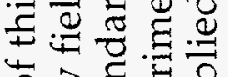

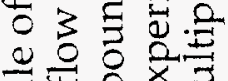

월을 일

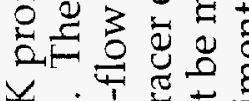

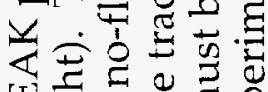

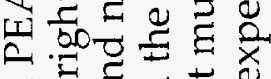

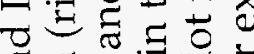

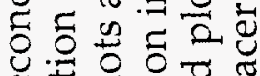

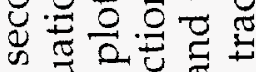

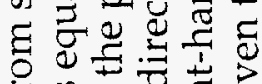

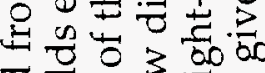

प्रि की

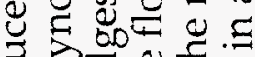

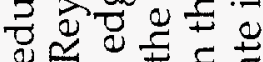

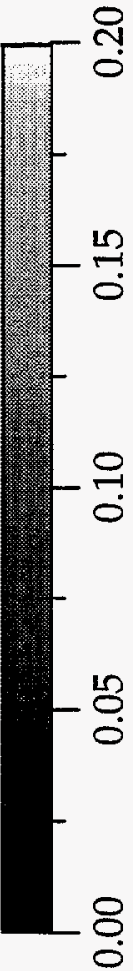

뫂

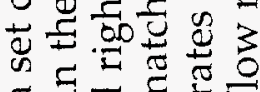

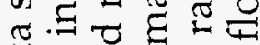

元记

屯艺芹氖

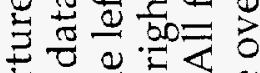

E

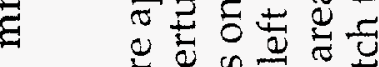

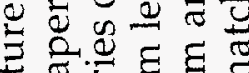

艺产导导

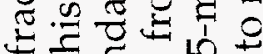

$\pm 00000$ I.

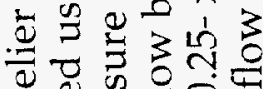

웡

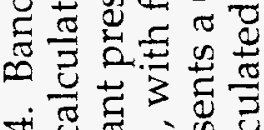

+ن 吾 के

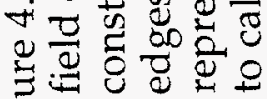
क्ष 


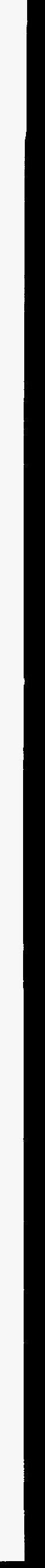




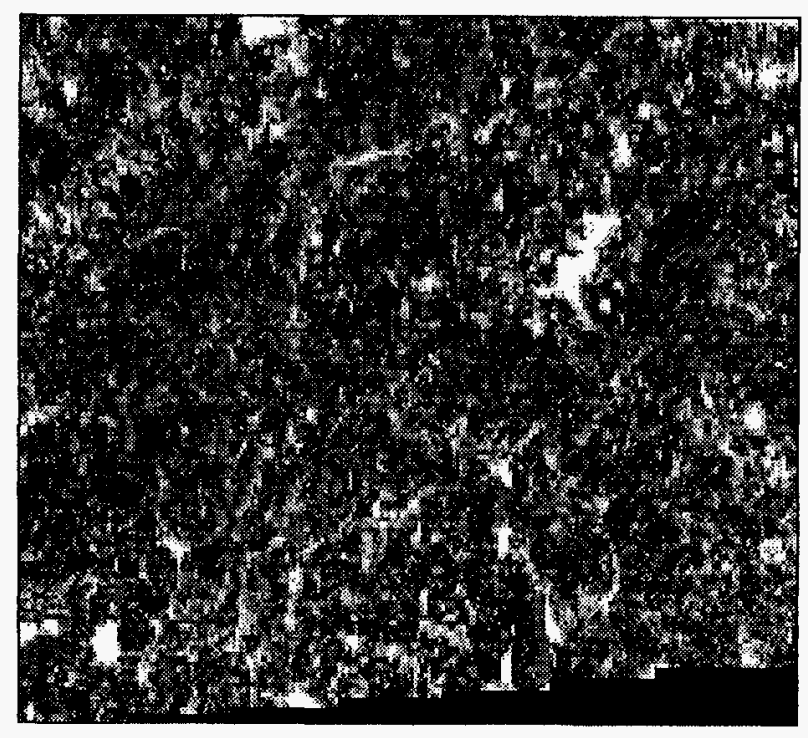

$\stackrel{\infty}{y}$

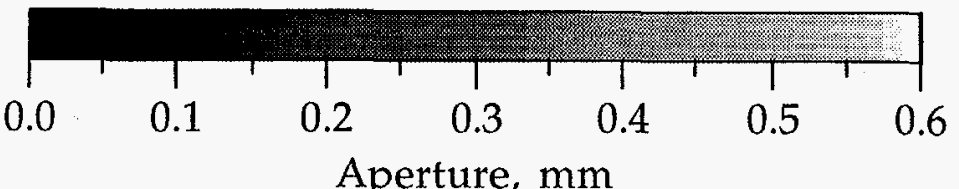

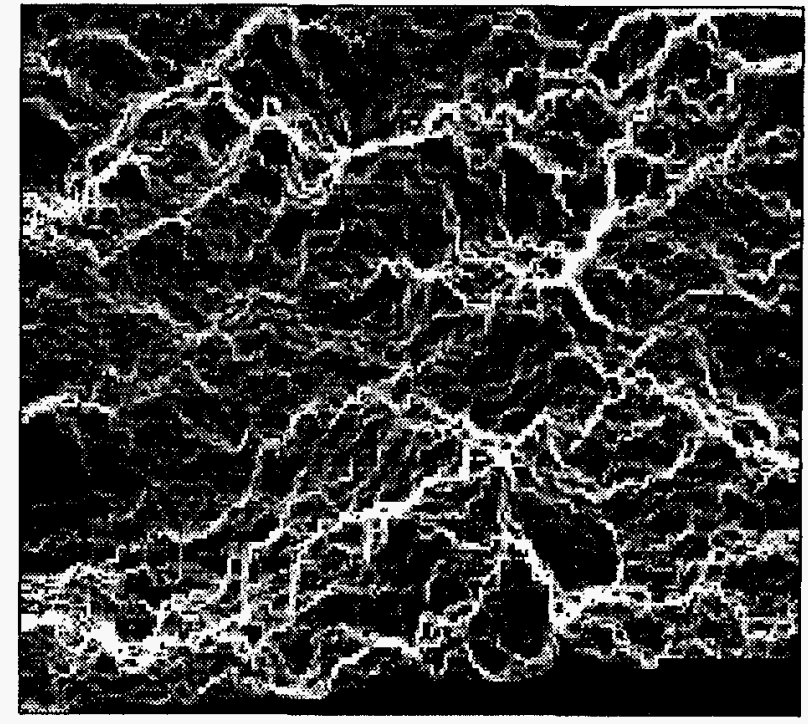

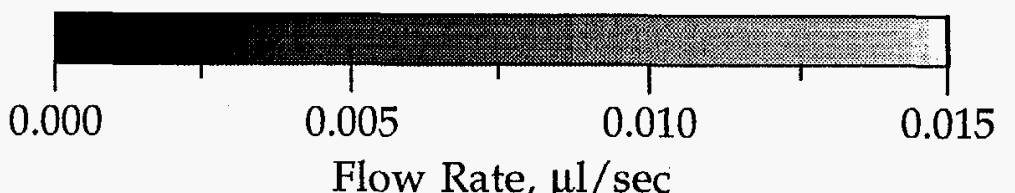

Figure 4.5. Tram tuff fracture aperture data set deduced from PEAK profiling technique (left) and the flow field calculated using this aperture data set in the Reynolds equation (right). The flow field was calculated assuming constant pressure boundaries on the left and right edges of the plots and no-flow boundaries on the top and bottom edges, with flow being from left to right to match the flow direction in the tracer experiments. Each pixel in both plots represents a $0.25-\times 0.25-\mathrm{mm}$ area. All flow rates in the right-hand plot must be multiplied by the ratio of experimental to calculated flow rate to match the overall flow rate in a given tracer experiment. 


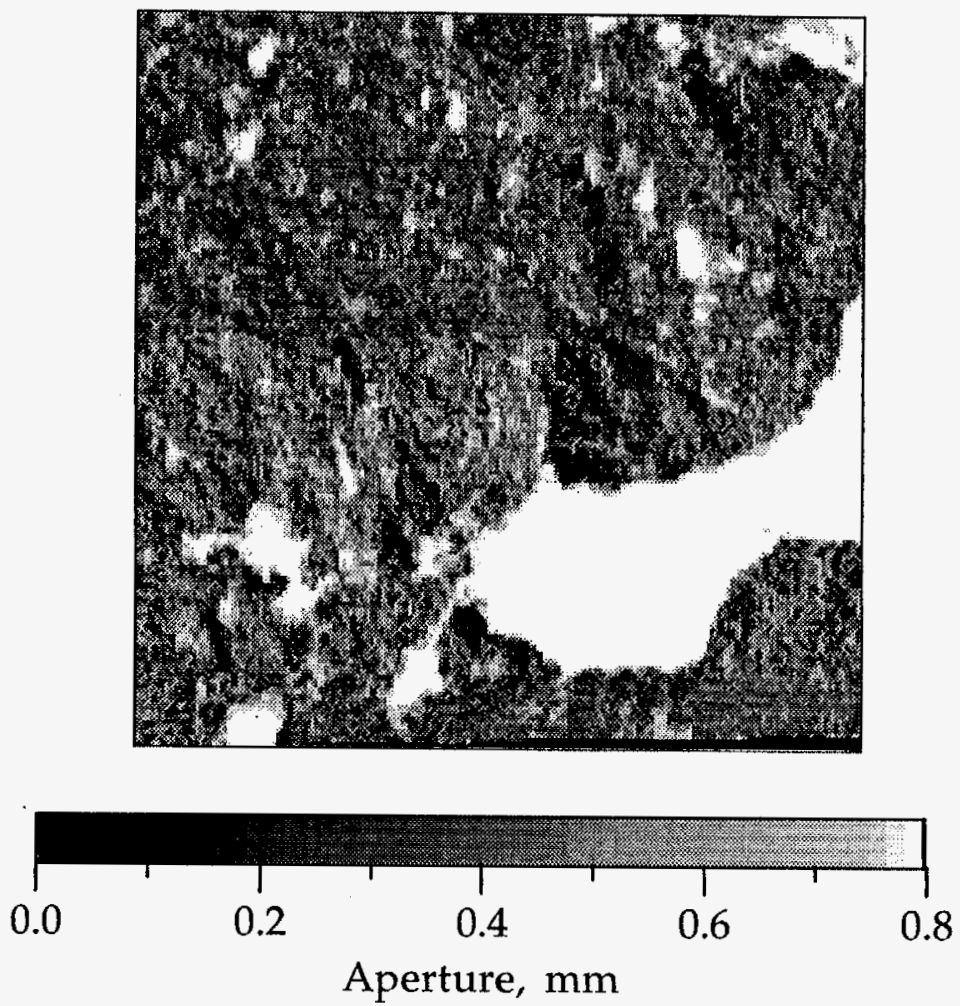
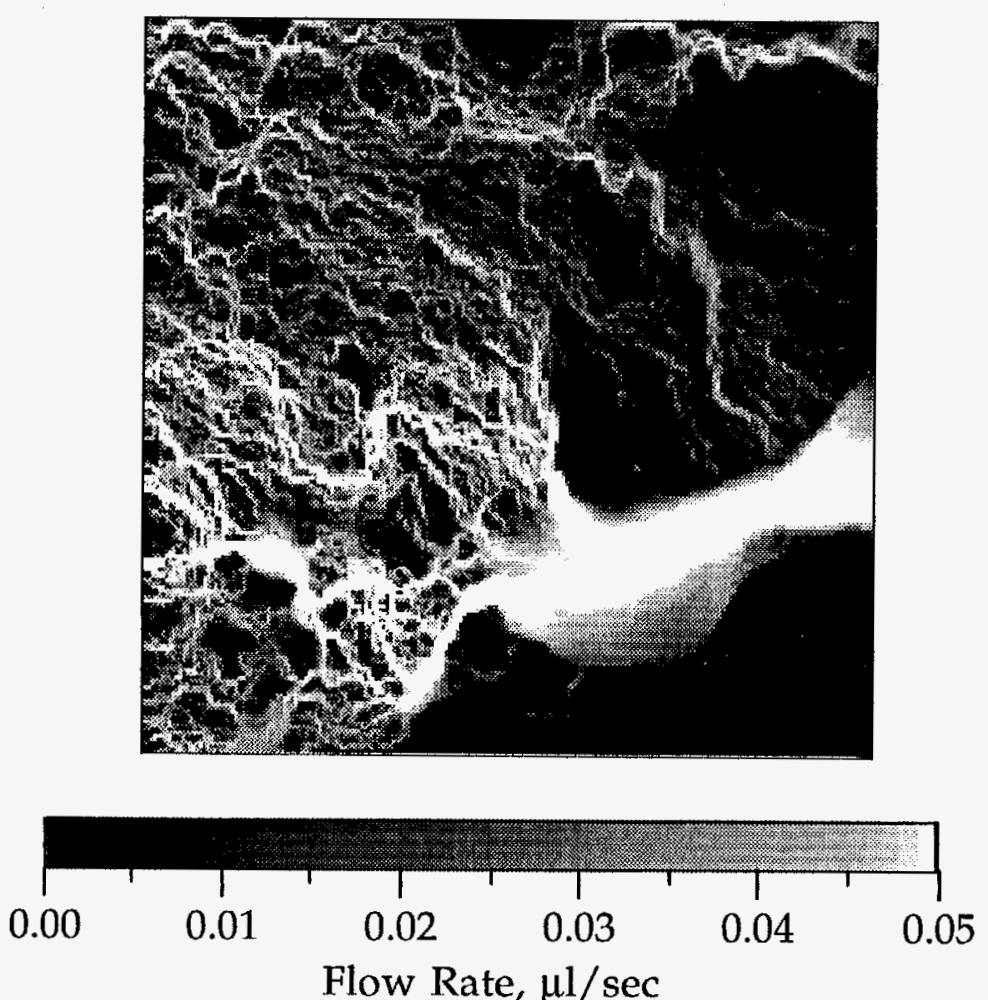

Figure 4.6. Bullfrog tuff fracture aperture data set deduced from PEAK profiling technique (left) and the flow field calculated using this aperture data set in the Reynolds equation (right). The flow field was calculated assuming constant pressure boundaries on the left and right edges of the plots and no-flow boundaries on the top and bottom edges, with flow being from left to right to match the flow direction in the tracer experiments. Each pixel in both plots represents a $0.25-\times 0.25-\mathrm{mm}$ area. All flow rates in the right-hand plot must be multiplied by the ratio of experimental to calculated flow rate to match the overall flow rate in a given tracer experiment. 


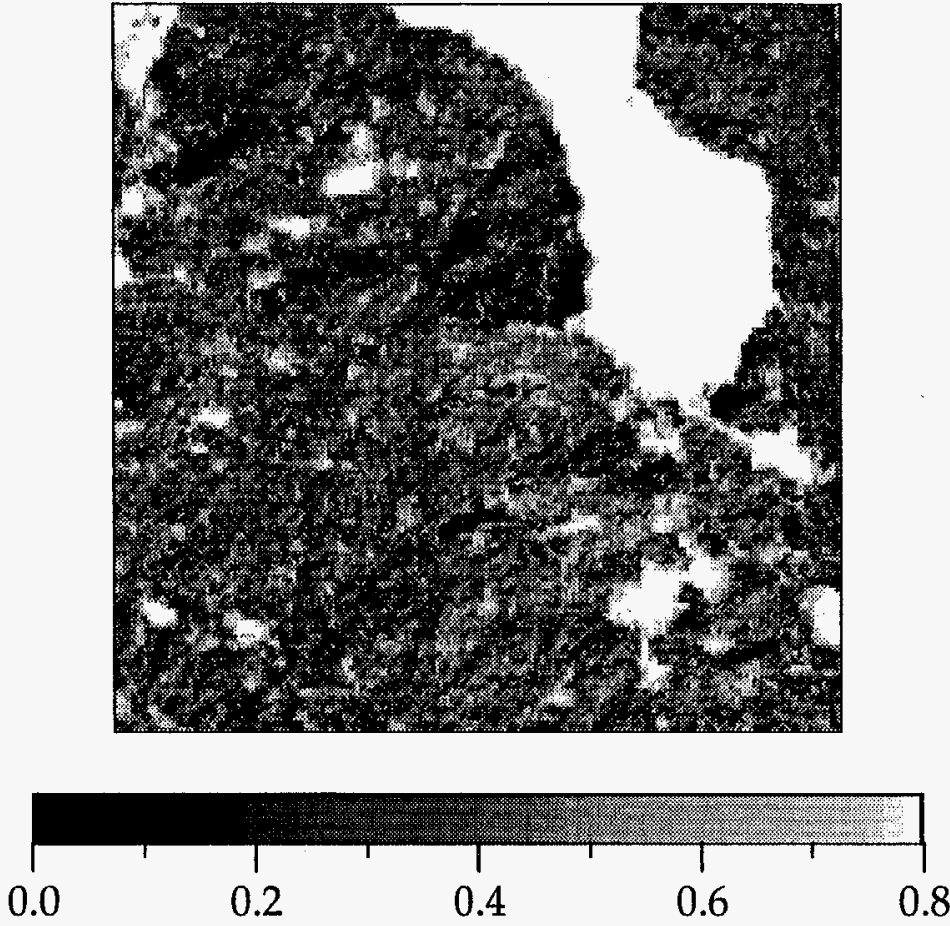

Aperture, $\mathrm{mm}$
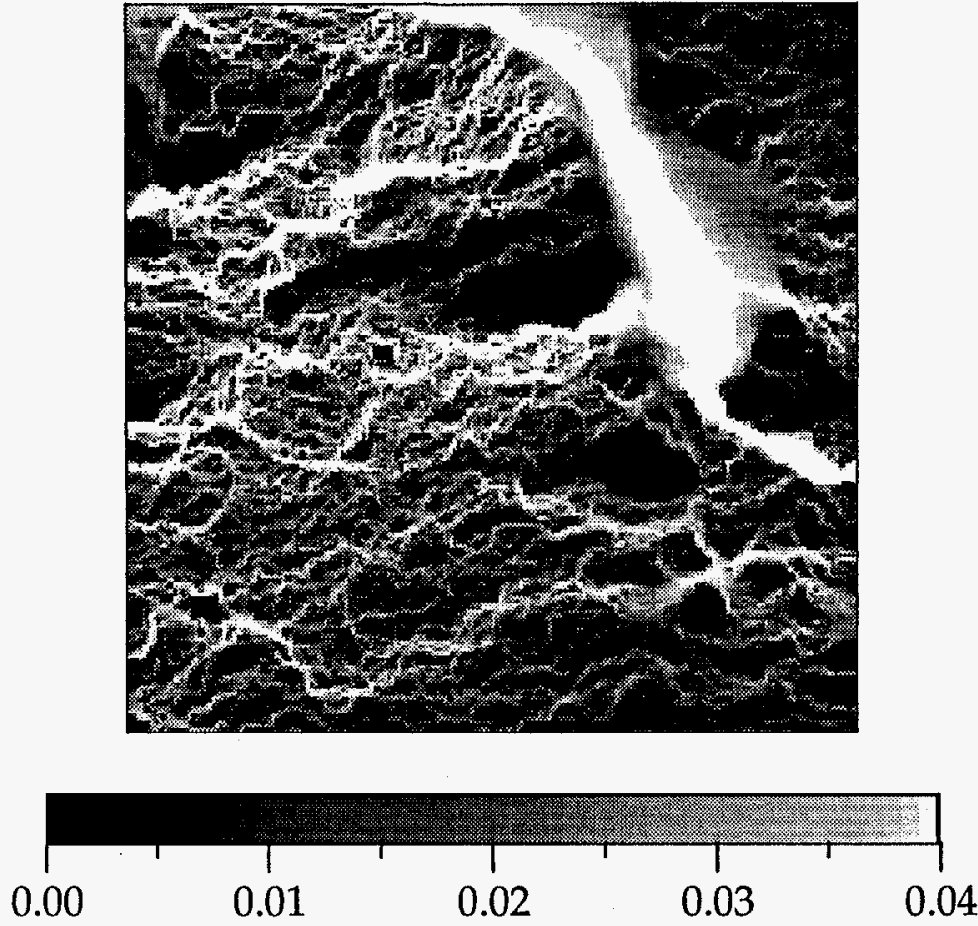

Flow Rate, $\mu \mathrm{l} / \mathrm{sec}$

Figure 4.7. Rotated Bullfrog tuff fracture aperture data set deduced from PEAK profiling technique (left) and the flow field calculated using this aperture data set in the Reynolds equation (right). The flow field was calculated assuming constant pressure boundaries on the left and right edges of the plots and no-flow boundaries on the top and bottom edges, with flow being from left to right to match the flow direction in the tracer experiments. Each pixel in both plots represents a $0.25-\times 0.25-\mathrm{mm}$ area. All flow rates in the right-hand plot must be multiplied by the ratio of experimental to calculated flow rate to match the overall flow rate in a given tracer experiment. 



\subsubsection{Fluid Residence Time Distribution}

The fluid residence time distribution in each fracture was calculated to offer comparisons with the predicted microsphere and iodide breakthrough curves and to allow comparisons of the relative amounts of predicted dispersion in each fracture. The fluid residence time distributions provided estimates of the pure flow-field dispersion in each fracture without the complicating effects of Taylor dispersion/streamline flow or matrix diffusion. The method of calculating the residence time distributions was essentially identical to that used by others who have done particle-tracking calculations in variable-aperture fractures. $88,89,180$ This method is based on the premise that the breakthrough curve for a step function input to a flow system can be constructed from the cumulative residence time distribution of individual "particles" allowed to independently move through the system subject to the flow field. The method was implemented for fractures as follows:

(1) Particles were introduced to the inlet side of the fracture flow domain, with the probability of entering a given node assumed to be proportional to the volumetric flow rate into that node. A discrete cumulative probability density function was constructed by sequentially adding the probabilities of entering each inlet boundary node. As a simple example, if there were three nodes at the inlet boundary with flow rates 1,2 , and 3 (arbitrary units), the probability of entering each node was $1 / 6,1 / 3$, and $1 / 2$, respectively; and the cumulative probability density function (the probability of entering a node of equal or lower number) was $1 / 6,1 / 2$, and 1 for the three nodes. A uniform random number between 0 and 1 was then generated using an appropriate algorithm. 181 The node that the particle entered was determined by where the random number fell in the cumulative distribution. For instance, if the random number was 0.336 in the above example, the particle entered the second node because 0.336 is greater than $1 / 6$ but less than $1 / 2$.

(2) Once a particle entered a node, the time it spent in that node was assumed to be equal to the volume of the node divided by the average volumetric flow rate in the node:

$$
\Delta t_{i, j}=\left(\frac{a_{i, j} \Delta x \Delta y}{Q_{i, j}}\right)
$$

where, $\Delta t_{i, j}=$ time particle spends in node $(i, j)$, sec

$a_{i, j}=$ aperture of node $(i, j), m m$

$Q_{i, j}=$ volumetric flow rate in node $(i, j), \mu l / s e c$

$\Delta x, \Delta y=$ dimensions of node $(i, j)$ in $x$ - and $y$-directions, $\mathrm{mm}$.

The next node that the particle entered was selected in the same way as in Step (1) except that now only adjacent nodes receiving flow from the current node were considered in the Monte-Carlo process. The total particle residence time in the fracture was incremented by Eq. (49) each time a particle entered a new node.

(3) If a particle entered a node that had no outlet flow (because of an unconverged flow solution), then the particle residence time was incremented by Eq. (49), and the particle was "jumped" to the next downstream node (the particle could not escape the node otherwise). 
Special logic prevented the particle from going back into such a node from a downstream node. This feature was in place in all of the particle-tracking models, but it was never actually exercised because the entry of a such a sink node was apparently an extremely rare event.

(4) When a particle crossed over the outlet boundary, it was assumed to disappear from the system. The total residence time of the particle in the system was stored to allow calculation of the cumulative residence time distribution of all particles when the simulation was completed. Another particle was then started at the inlet boundary.

(5) Many particles (typically 1000 or more) were allowed to move through the fracture domain according to Steps (1-4), and the cumulative distribution of their residence times (i.e., a plot of the probability of having a residence time less than some value vs. that value) was taken to be the fluid residence time distribution in the fracture.

Fig. 4.8 shows the fluid residence time distributions calculated for the four different fractures (the rotated Bullfrog tuff fracture is considered to be a separate fracture) on a void volume eluted basis. By comparing this figure to Fig. 3.8 , it is apparent that the predicted fluid dispersion in the fractures is in good qualitative agreement with the observed colloid dispersion in the fractures. This result suggests that the aperture data sets can be used to provide a qualitative measure of the dispersion in the fractures. However, Fig. 4.9 shows that there is considerable discrepancy in the predicted fluid dispersion using the different aperture data sets for the Bandelier tuff fracture. The 1- $\mu \mathrm{m}$ CML microsphere and iodide data from the tracer experiment with a flow rate of $4.11 \mu \mathrm{l} / \mathrm{sec}$ in this fracture are shown in this figure for comparison.

\subsubsection{Solute Transport without Matrix Diffusion}

For solute transport, it is recognized that particles will not experience the average fluid residence time in individual nodes unless the flow rate is sufficiently low that they can diffuse across all the streamlines in the velocity profile during their time in the node; that is, unless the particles experience Taylor dispersion (see Section 2.6.4). A quick check of the flow-field solutions indicated that the Taylor dispersion assumptions would not be satisfied for every node in any of the fractures at any of the flow rates (although the assumptions would, in general, be valid for the fractures as a whole). Therefore, a particletracking method was developed to allow particles to move through individual nodes while also diffusing across the streamlines in the parabolic velocity profile in the nodes. The method was implemented as follows:

(1) When a particle was introduced to the inlet side of the fracture domain, the entry node was selected in the same way as for the fluid particles in Step (1) of Section 4.2.1. However, it was also necessary to select the starting position of the particle in the direction perpendicular to the fracture walls (the $z$-direction). The probability of a given starting position was assumed to be proportional to the flow rate at that position. The probability of having a starting position less than $z$ (between $+b$ and $-b$, where $b$ is half the aperture) is given by 


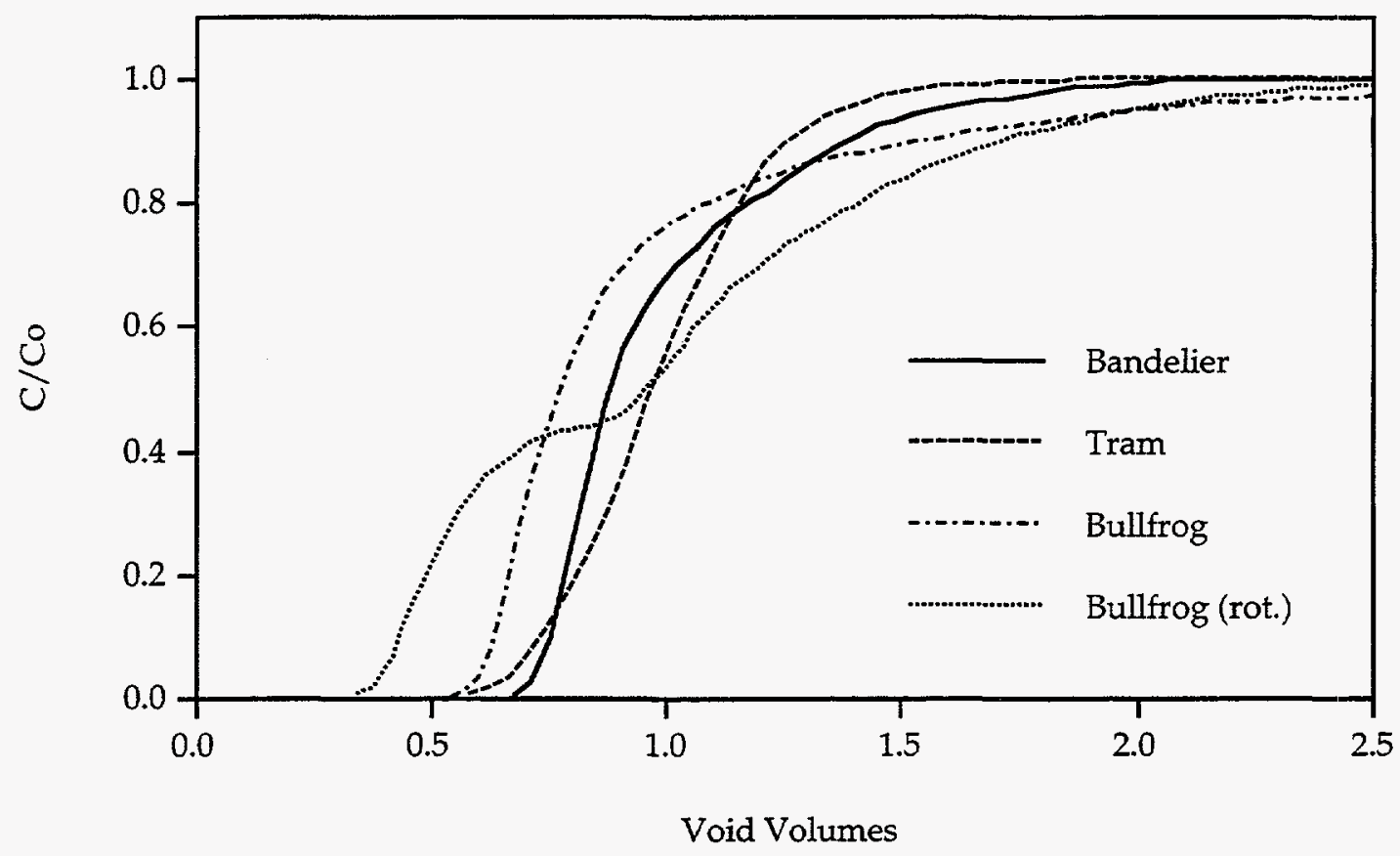

Figure 4.8. Predicted fluid residence times in the different fractures as a function of void volumes eluted (based on 2000 particles in the particle-tracking simulations). The aperture data set and calculated flow field from the second PEAK profile were used for the Bandelier tuff fracture predictions.

$$
P(z)=\frac{\int_{-b}^{z} v_{x}(z) d z}{\int_{-b}^{b} v_{x}(z) d z}
$$

If Eq. (5) is substituted for $v_{x}(z)$ in Eq. (50), the result is

$$
\mathrm{P}(\mathrm{z})=\frac{1.5 \mathrm{~V}_{\mathrm{ave}}\left(\mathrm{z}-\frac{\mathrm{z}^{3}}{3 \mathrm{~b}^{2}}+\frac{2 \mathrm{~b}}{3}\right)}{1.5 \mathrm{~V}_{\mathrm{ave}}\left(\frac{4 \mathrm{~b}}{3}\right)}=\left(\frac{3}{4 \mathrm{~b}}\right) z-\left(\frac{1}{4 \mathrm{~b}^{3}}\right) \mathrm{z}^{3}+\frac{1}{2}
$$

A uniform random number between 0 and 1 was substituted for $P(z)$ into Eq. (51), and the root of the resulting polynomial in $\mathrm{z}$ was solved (by Newton's method) to determine a starting position that was appropriately weighted by flow rate.

(2) Once a starting $z$-position was determined, the particle was stepped across the aperture of the node in $\mathrm{z}$-direction distance increments, and the resulting distance traveled in the $x$-direction (the direction of flow) was calculated using Eq. (28) after determining the time to move the $z$-direction distance increment. This approach differed from most particle-tracking methods discussed in the literature, which advance particles in time increments rather than distance increments. However, when matrix diffusion is considered, there is a need to know exactly when and where a particle "hits" 


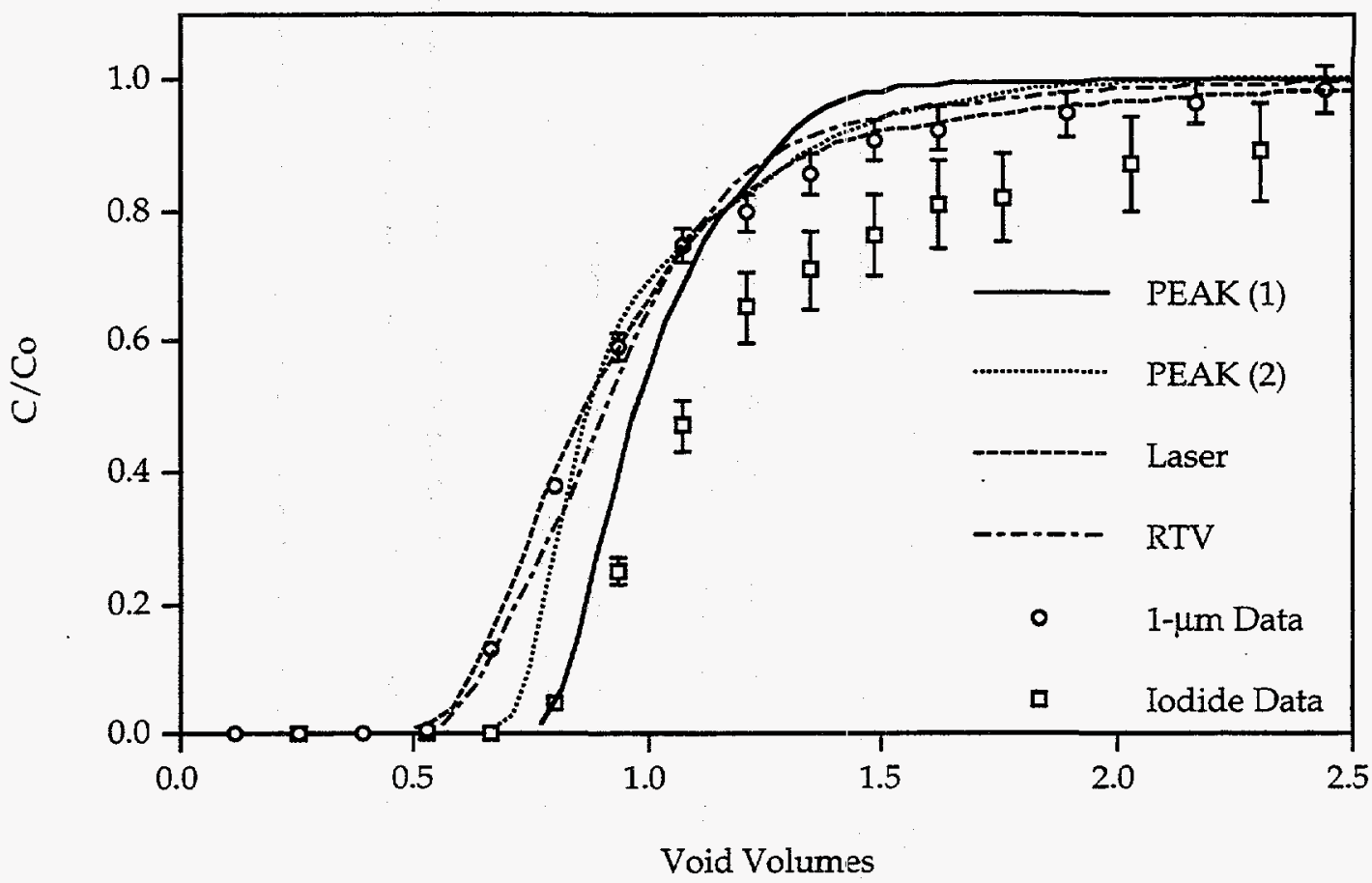

Figure 4.9. Predicted fluid residence times in the Bandelier tuff fracture using different aperture data sets (based on 2000 particles in the particle-tracking simulations). The 1- $\mu \mathrm{m} C \mathrm{CML}$ microsphere and iodide data from a tracer experiment $(4.11 \mu \mathrm{l} / \mathrm{sec}$ flow rate) are shown for comparison.

a wall. This is not possible when using time steps unless the steps are reduced to extremely small values when particles are near walls. Because small time steps are computationally very costly, an alternative was devised in which particles were allowed to diffuse across the parallel-plate channel in increments of $1 / 20$ th of the channel aperture until they got close to walls. Increments of this size were used because it was shown in finite-difference calculations (see Section 4.2.7) that a discretization of 21 nodes across a parallel-plate channel was sufficient to obtain a realistic residence time distribution in the channel. When a particle got to within less than $1 / 20$ th of the aperture from a wall, it was simply moved an increment equal to the distance to the wall. Once a particle "hit" a wall, it was moved a specified distance away from the wall (generally $0.5 \mu \mathrm{m}$ ), and the process was started again. Although the distance increments were always specified before a particle was moved, the direction that the particle moved was determined by generating a random normal variate (a random number with a mean of zero and a standard deviation of 1) and then moving the particle in the $-\mathrm{z}$ direction if the variate was negative or in the $+z$ direction if the variate was positive (an equal probability of either direction).

(3) The time required to move a specified distance increment in the $z$-direction was determined using the following formula:

$$
\Delta t=\exp \left[\ln \left(\frac{z^{2}}{2 D}\right)-0.2+(0.79) \operatorname{Rn}(0,1)\right]
$$


where, $\exp =$ exponential

$\ln =$ natural logarithm

$\mathrm{z}=$ distance increment, $\mathrm{cm}$

$\mathrm{D}=$ particle diffusivity, $\mathrm{cm}^{2} / \mathrm{sec}$

$\operatorname{Rn}(0,1)=$ random normal variate

This formula was deduced by fitting the time distributions resulting from a large number of random-walk simulations in which particles were moved a specified distance in very small time increments. The time increments in the simulations were small enough that it would take several of them to move the specified distance even if the normal variates were all +1 or -1 . In accordance with Eq. (28) with $\mathbf{u}_{\text {det }}=0$, the distance moved during each time increment was "Rn $(0,1) \sqrt{2 \mathrm{D} \Delta \mathrm{t}}$." Equation (52) implies that the times required to move a distance $z$ under random-walk conditions are lognormally distributed with a mean of $\ln$ (time) equal to $\ln \left(\frac{z^{2}}{2 D}\right)-0.2$ and a standard deviation of $\ln$ (time) equal to 0.79 . Residence time distributions calculated using the random-walk method and those calculated directly from Eq. (52) were found to be in excellent agreement regardless of the distance or the time increment used (as long as the time increment was small enough that the distance steps were a small fraction of the total distance moved). In retrospect, there is probably some way of mathematically deriving or proving Eq. (52) for random-walk processes, but no attempt was made to find such a proof.

(4) After the time associated with a $z$-direction distance increment was obtained from Eq. (52), the distance moved in the x-direction during the time increment was determined using a one-dimensional version of Eq. (28). The fluid velocity at the most recent $z$-position of the particle (at the beginning of the time step) was used as the deterministic particle velocity during the time step. However, if a particle started at a wall, the particle velocity was assumed to be the flow velocity at a distance from the wall equal to 0.4 times the distance moved during the time step (determined by trial and error to give more reasonable results than assuming zero velocity). Particle movement in the $y$-direction (parallel to the walls but perpendicular to the flow direction) was ignored. A particle was required to move forward in the $x$-direction a distance equal to the length of the side of a node before it could enter another node. When a particle crossed a boundary into another node during a time step, the time increment to reach the boundary was calculated from

$$
\Delta \mathrm{t}_{\mathrm{b}}=\left(\frac{\Delta \mathrm{x}_{\mathrm{b}}}{\Delta \mathrm{x}}\right) \Delta \mathrm{t}
$$

where, $\Delta t_{b}=$ time to reach boundary,

$\Delta t=$ time calculated from Eq. (52),

$\Delta \mathrm{x}_{\mathrm{b}}=$ distance to boundary, and

$\Delta \mathrm{x}=$ distance particle would have moved in absence of boundary.

The $z$-direction distance moved during the time to reach the boundary was calculated from 


$$
\Delta \mathrm{z}_{\mathrm{b}}=\left(\sqrt{\frac{\Delta \mathrm{t}_{\mathrm{b}}}{\Delta \mathrm{t}}}\right) \Delta \mathrm{z}
$$

where, $\Delta \mathrm{z}_{\mathrm{b}}=\mathrm{z}$-direction distance increment during $\Delta \mathrm{t}_{\mathrm{b}}$, and

$\Delta \mathrm{z}=\mathrm{z}$-direction distance increment during $\Delta \mathrm{t}$.

Equation (54) is a mathematical statement that diffusion distances are proportional to the square root of time.

(5) The selection of the next node for the particle to enter was done in exactly the same way as for particles in the fluid residence time calculations (Steps 1 and 2 of Section 4.2.1).

(6) The relative distance between a particle and a wall (in the $\mathrm{z}$-direction) upon entering a new node was assumed to be the same as it was upon exiting the previous node. This situation is illustrated in Fig. 4.10. Happel and Brenner ${ }^{83}$ have shown that this assumption is valid under creeping flow conditions in slowly converging or diverging channels.

(7) Special logic was necessary to avoid excessive or unnecessary computations in the following situations:

(a) If a node was encountered in which the fluid residence time was greater than the time required for a particle to diffuse the length of the node, a diffusion time was calculated and used for the residence time in the node. The relative $z$-position of the particle upon exiting the node was determined randomly in these situations.

\section{Fracture Wall}

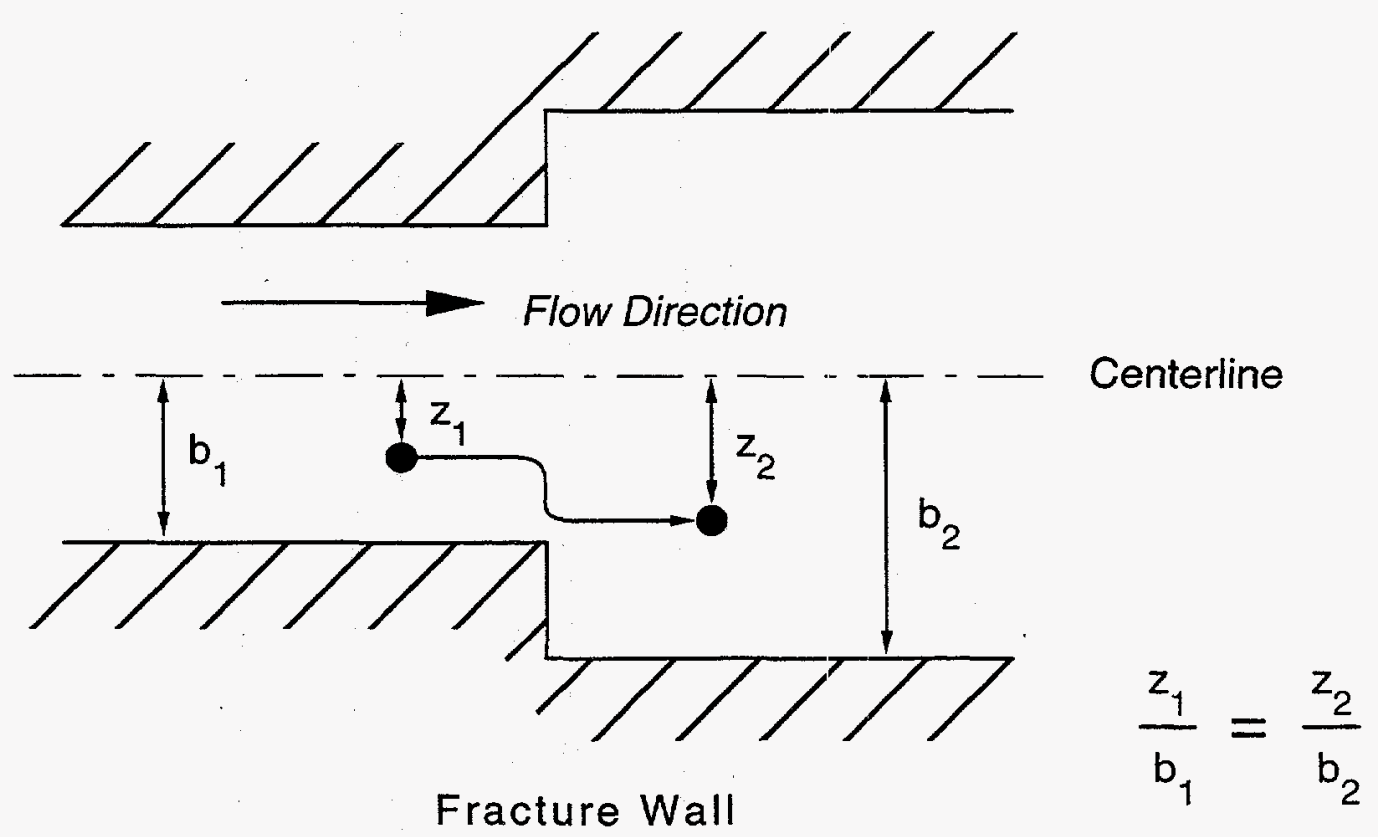

Figure 4.10. Illustration of particle trajectory in particle-tracking model when crossing a node boundary. 
(b) If condition (a) was not met, but the quantity $\frac{\sqrt{D \Delta t_{i, j}}}{a_{i, j}}$ was greater than 10 , the particle residence time in the node was assumed to be equal to the fluid residence time plus a correction for the distance that a particle would have diffused in the direction of flow during this time. The rationale for this assumption was that the particles should experience approximately the average fluid residence time in a node if they can diffuse across the flow channel several times during their residence time in the node. In this case, the z-position of the particle upon exiting the node was determined as in Step (1).

(8) Steps (1-7) were repeated until the particle crossed the outlet boundary of the flow domain. Another particle was then started at the inlet boundary.

It is interesting to note that particle trajectories obtained by following Steps (1-7) do not look at all like the illustration in Fig. 2.3. A depiction of a typical particle trajectory in a parallel-plate channel is shown in Fig. 4.11. It is apparent in this figure that both the time steps and the distance increments in the direction of flow become smaller as particles move closer to walls. It is also clear that once a particle "hits" a wall, it has a much greater probability of hitting the wall again than of moving back into the middle of the channel. However, given enough time, particles will eventually move back and forth across the channel as a random walk would dictate.

\subsubsection{Solute Transport with Matrix Diffusion}

To simulate particle diffusion into pores in the fracture walls, it was necessary to modify the particle-tracking model described in Section 4.2.2. At a superficial level, the two models differed only in the time distributions assumed for particles when they moved away from walls and back into the parallel-plate

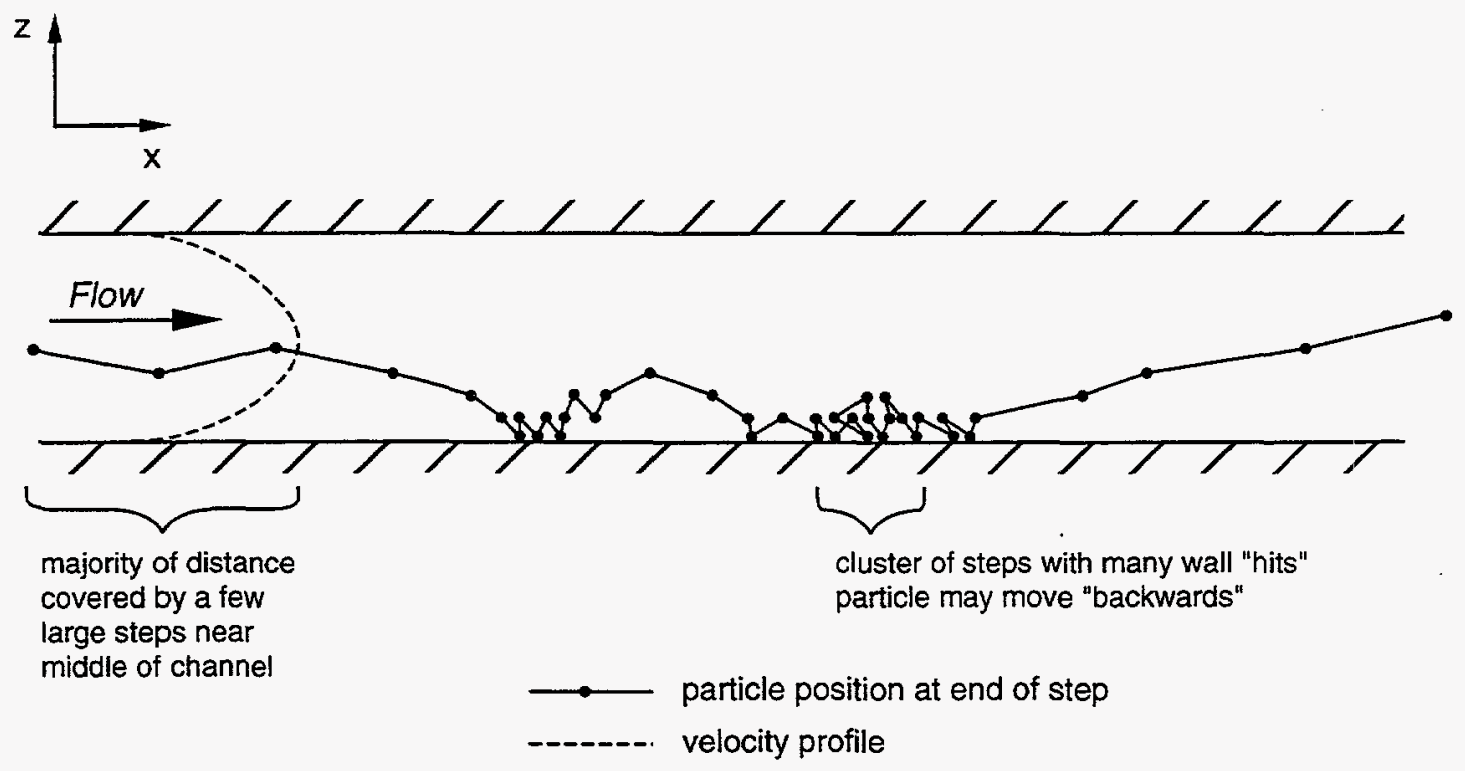

Figure 4.11. Depiction of particle movement in a parallel-plate channel according to the particle-tracking model. The particle moves a fixed distance in the $\mathrm{z}$ direction during each step. The distance moved in the $x$-direction is determined using a one-dimensional version of Eq. (28). 
channel. Thus, the steps required to implement the porous-wall model were exactly the same as in Section 4.2.2, with the exception that Eq. (52) could not be used to determine the time for a particle to move away from a wall when there was a pore present at that location in the wall. It was a non-trivial matter to obtain the time distribution(s) for this situation.

In the porous-wall model, the probability of a particle encountering a pore when it "hit" a wall was assumed to be equal to the porosity of the matrix. A random number between 0 and 1 (uniformly distributed) was generated every time a particle hit a wall, and if this number was less than the porosity, a pore encounter was simulated. There was no attempt to try determine if a particle moved far enough between wall hits to encounter the same position along a wall; it was simply assumed that all wall hits had an equal probability of being pore encounters. When a pore was not encountered, Eq. (52) was used to determine the time to diffuse a specified distance back into the parallel-plate channel. A distance of $0.5 \mu \mathrm{m}$ was used as this "specified distance" in all simulations because this was a small fraction of the aperture of most nodes, but it was a large enough distance that computation times were not excessively long (computation time increased significantly as this distance decreased).

When a pore was encountered, it was necessary to sample a time distribution generated from an independent random-walk simulation in which a particle was initially placed right at the porous wall and allowed to move either into the wall or away from the wall. The random-walk movement of an individual particle was terminated when the particle moved the specified distance back into the flow channel $(0.5 \mu \mathrm{m})$. The particle times could be very short if the particles moved quickly into the flow channel, but they could also be excessively long if particles moved into the pores and spent a long time in the matrix before coming back out. The resulting time distributions could not be fit with any reasonable function, so an interpolation scheme was devised in which a uniformly distributed random number from 0 to 1 was generated, and the time associated with that number was linearly interpolated from a table of $\ln$ (time) vs. cumulative probability. This scheme was checked by repeated random sampling to confirm that the resulting times were distributed in the same way as the time distribution generated by the random-walk simulation.

It was quickly realized that assuming an instantaneous change in the matrix porosity and particle diffusivity at the fracture wall (from free water values to matrix values) resulted in time distributions that were biased toward times that were too long. As discussed in Section 2.6.1, when there are gradients in porosity and diffusivity, it becomes necessary to add Eqs. (29) and (30) to Eq. (28) or there will be false accumulation of particles in stagnant regions (in this case, the porous matrix). When using Eqs. (29) and (30), gradients of porosity and diffusivity must be assumed in the matrix. The situation is illustrated in Fig. 4.12, which shows the pertinent particle-tracking equations for different regions in the problem domain. In this figure, the porosity and diffusivity are assumed to vary linearly across a transition layer from free values to matrix values. The particle residence time distribution should be sensitive to the transition layer thickness because this thickness will influence the magnitude of the diffusivity and porosity gradients that tend to "push" particles out of the matrix.

Fig. 4.13 shows calculated time distributions from random-walk simulations (of 10,000 particles) in which the thickness of the transition layer was 
$\uparrow$

Open Space in Fracture, $\varepsilon=1.0$

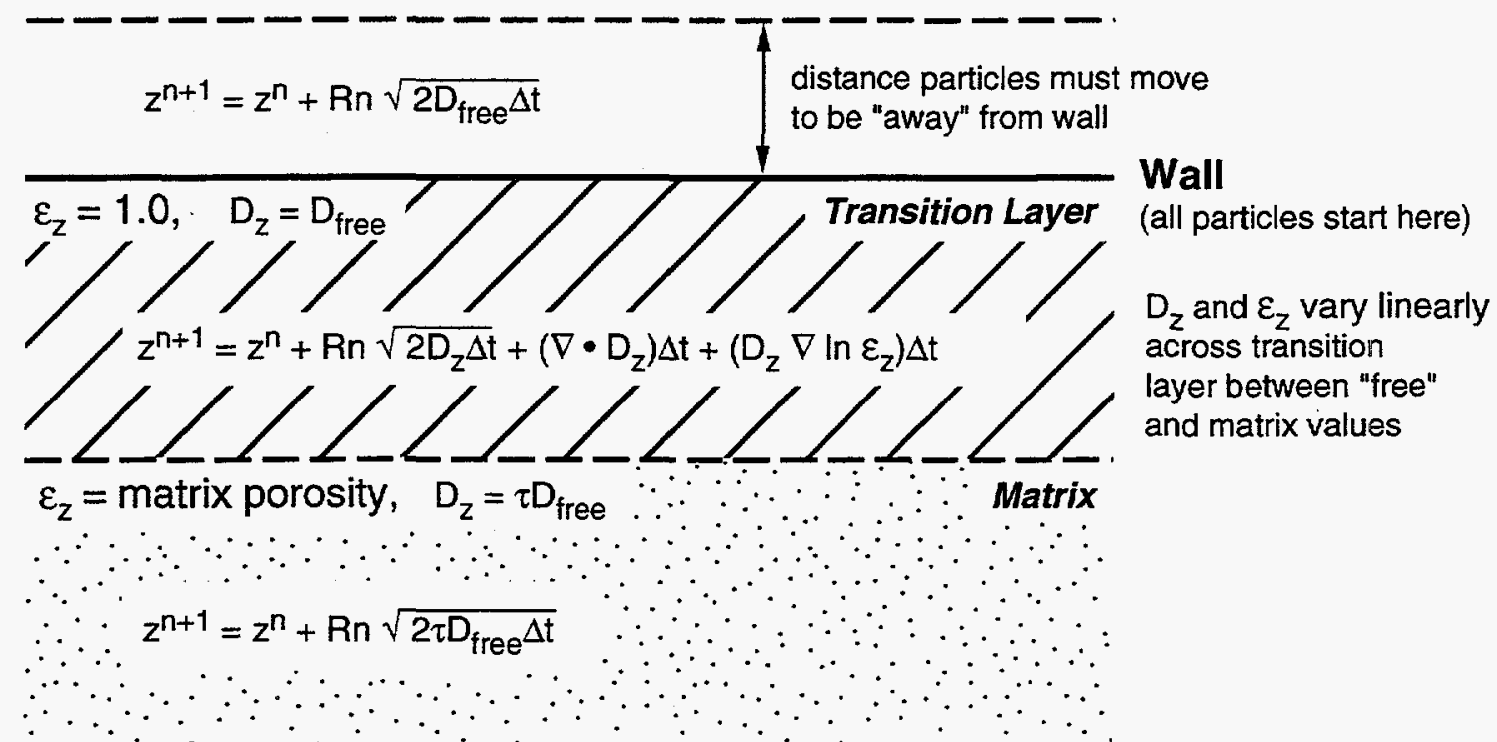

Figure 4.12. Illustration of the assumed transition from fracture void space to matrix in the particle-tracking model(s) that include matrix diffusion.

varied. The time distributions associated with a nonporous wall (i.e., the distribution fit by Eq. 52) and for a case with no porosity or diffusivity gradient terms in Eq. (28) are shown for comparative purposes. In all of these simulations, the specified distance for particles to move into the flow channel before ending their random walk was $0.5 \mu \mathrm{m}$. The bulk matrix porosity was assumed to be 0.089 (corresponding to the Bandelier tuff), the particle diffusivity in free-water was assumed to be $0.0015 \mathrm{~mm}^{2} / \mathrm{sec}$, and the particle diffusivity in the matrix was assumed to be $0.00015 \mathrm{~mm}^{2} / \mathrm{sec}$, or 0.1 times the free-water diffusivity. This factor of 0.1 , called the tortuosity factor $(\tau),{ }^{38}$ corrects for reductions in diffusivity due to the tortuous pathways that particles must negotiate within the pores of the matrix (compared to the unhindered situation in free water). A tortuosity factor of 0.1 was assumed in all three tuff matrices based on measurements of matrix diffusivities made by Triay et al. 182 A freewater diffusivity of $0.0015 \mathrm{~mm}^{2} / \mathrm{sec}$ was used because it was representative of the iodide diffusivity reported by others. $38,183,184$

It is apparent from Fig. 4.13 that the calculated time distributions were quite sensitive to the assumed thickness of the transition layer, and that the inclusion of porosity and diffusivity gradient terms in Eq. (28) noticeably decreased the calculated residence times of particles in the matrix. The lower plot in Fig. 4.13 shows that the differences in the time distributions were most significant in the upper tails of the distributions (especially considering that the $\mathrm{x}$-axis is a logarithmic scale). Fig. 4.14 shows the profound effect of using these different time distributions in particle-tracking simulations of iodide transport through the Bandelier tuff fracture (using the flow field solution from the first PEAK aperture data set -- see Fig. 4.3). It is clear from Fig. 4.14 that the assumed 

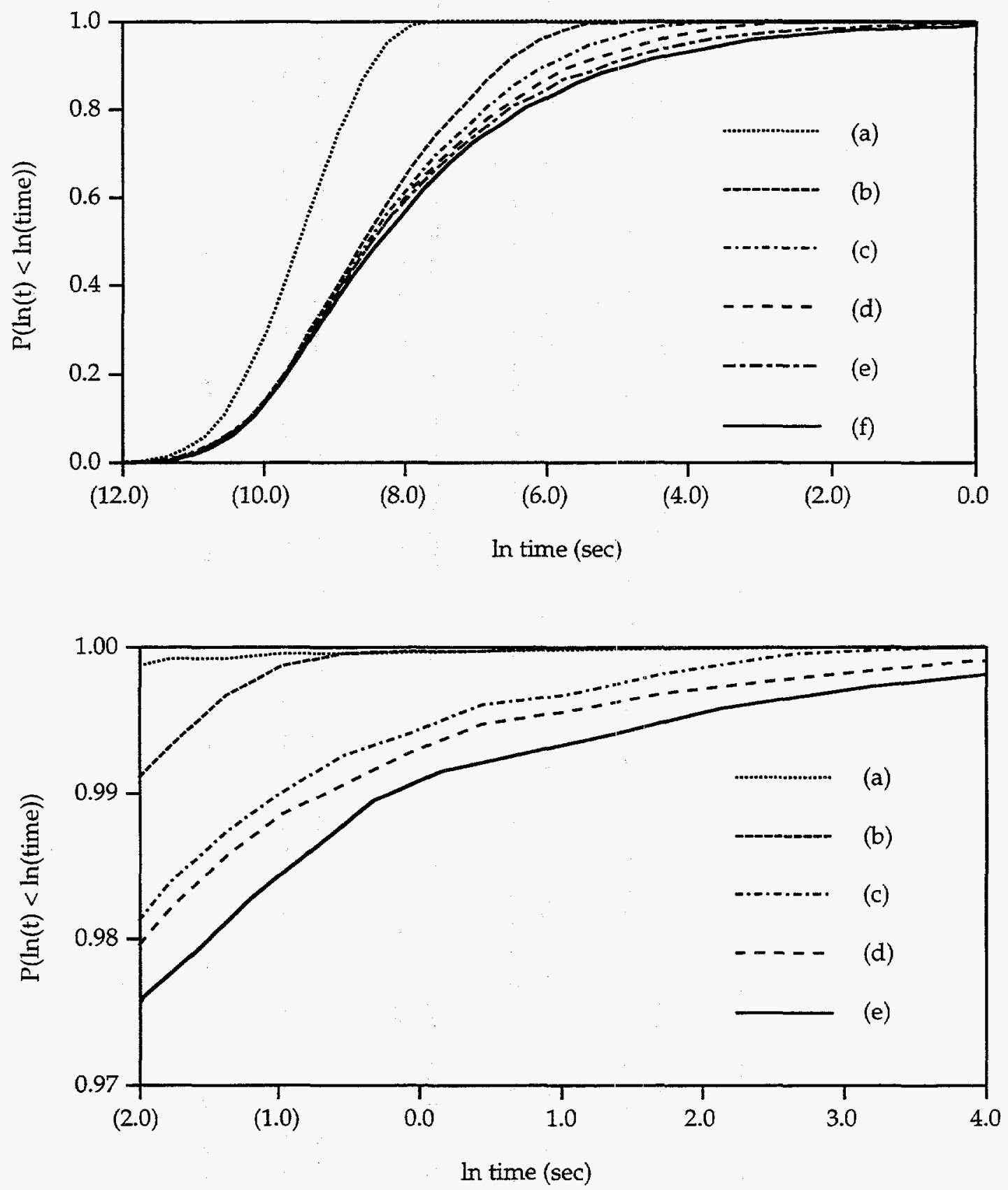

Figure 4.13. Residence time distributions for particles moving away from a porous wall assuming different transition layer thicknesses. The wall porosity was assumed to be 0.089 (corresponding to the Bandelier tuff fracture). The upper plot shows residence times for (a) a nonporous wall, and for transition layer thicknesses of (b) $0.002 \mathrm{~mm}$, (c) $0.005 \mathrm{~mm}$, (d) $0.01 \mathrm{~mm}$, (e) $0.2 \mathrm{~mm}$, and (f) $\infty$ (i.e., no transition layer). The lower plot shows the tails of the residence time distributions for transition layer thicknesses of (a) 0.01 $\mathrm{mm}$, (b) $0.025 \mathrm{~mm}$, (c) $0.2 \mathrm{~mm}$, (d) $5.0 \mathrm{~mm}$, and (e) $\infty$. Parentheses indicate negative numbers. See text for other model assumptions. 


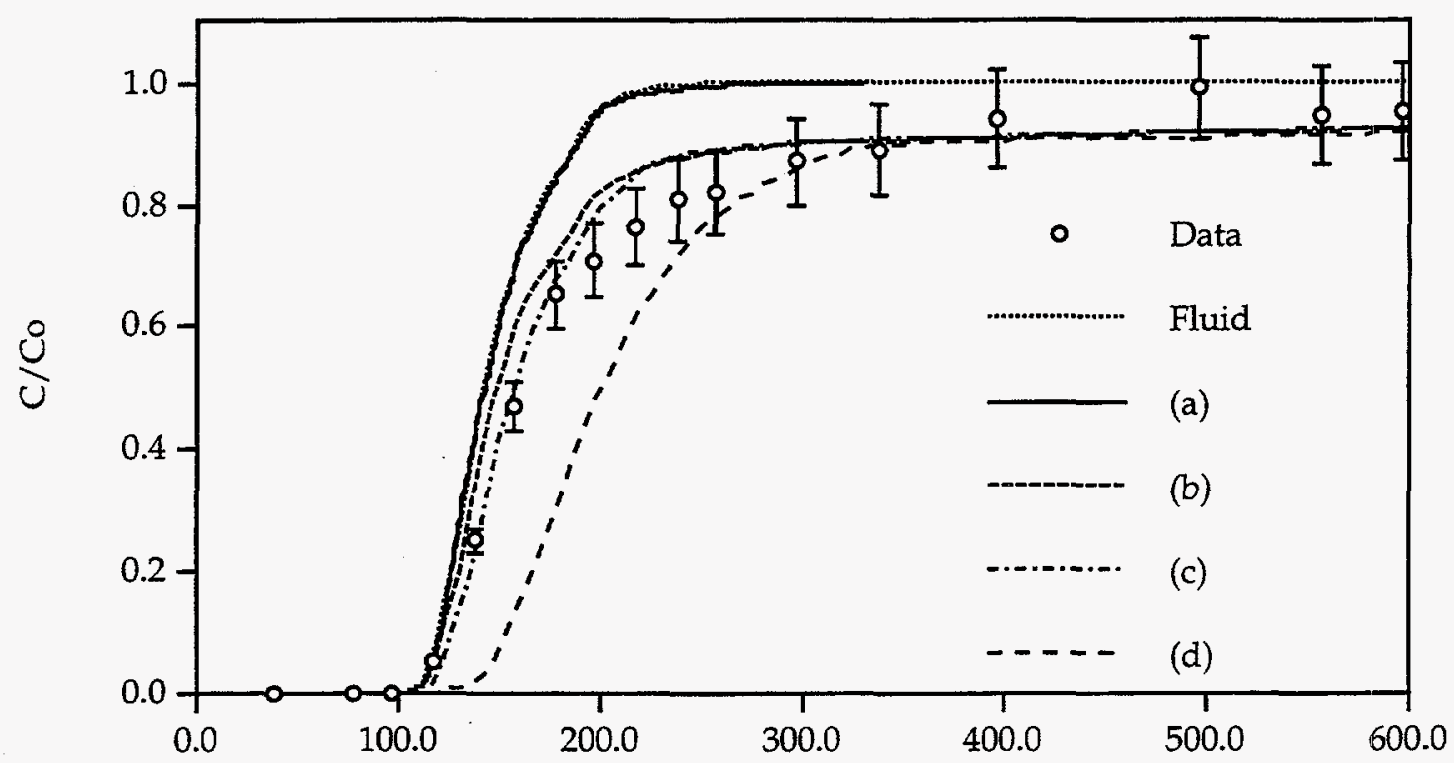

Time, sec

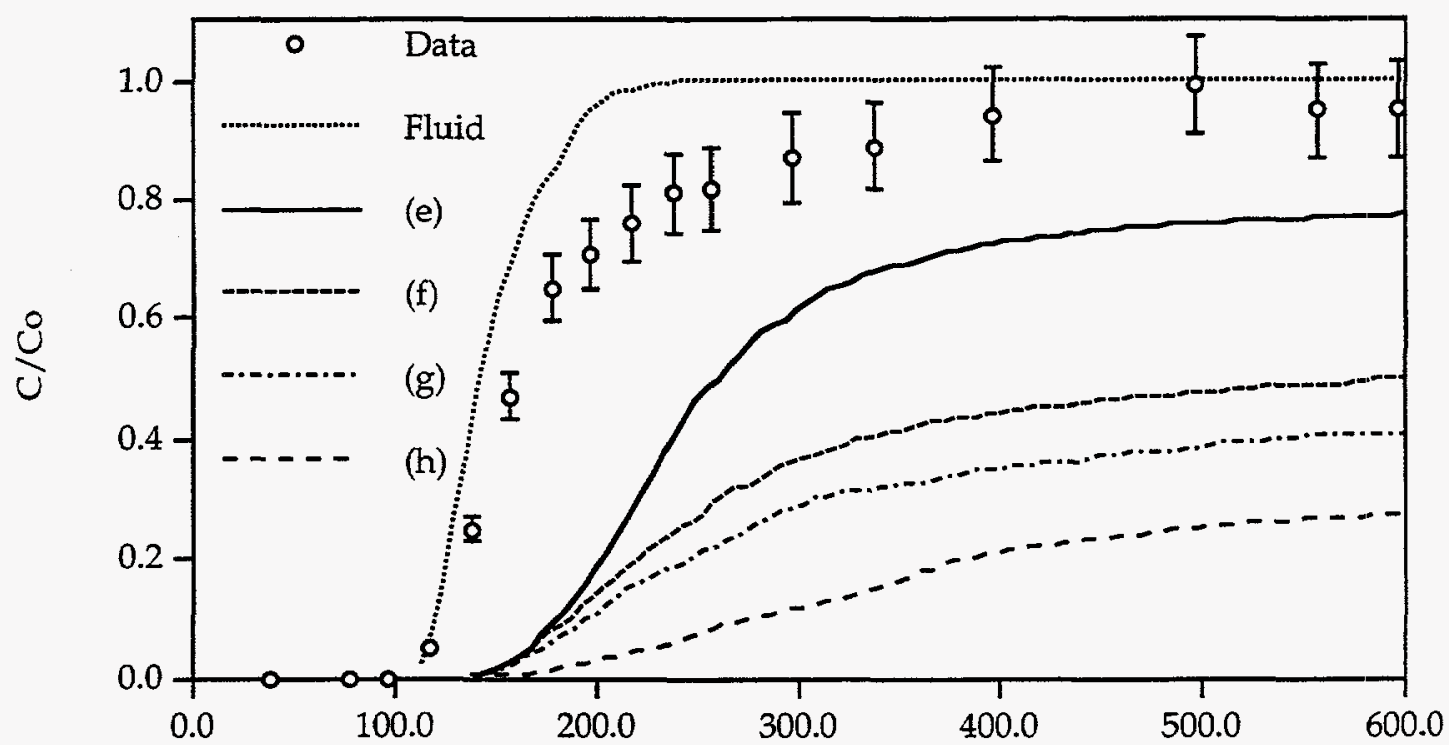

Time, sec

Figure 4.14. Predicted iodide breakthrough curves (based on 1000 particles) in Bandelier tuff fracture assuming (a) nonporous walls and the following transition layer thicknesses: (b) $0.002 \mathrm{~mm}$, (c) $0.025 \mathrm{~mm}$, (d) $0.2 \mathrm{~mm}$, (e) 0.5 $\mathrm{mm}$, (f) $1.5 \mathrm{~mm}$, (g) $5.0 \mathrm{~mm}$, and (h) $\infty$ thickness. The experimental data and predicted fluid residence time are shown in both plots for comparison. The flow rate was $4.11 \mu \mathrm{l} / \mathrm{sec}$. The predictions were made using the aperture data and calculated flow field from the first PEAK profile of this fracture. 


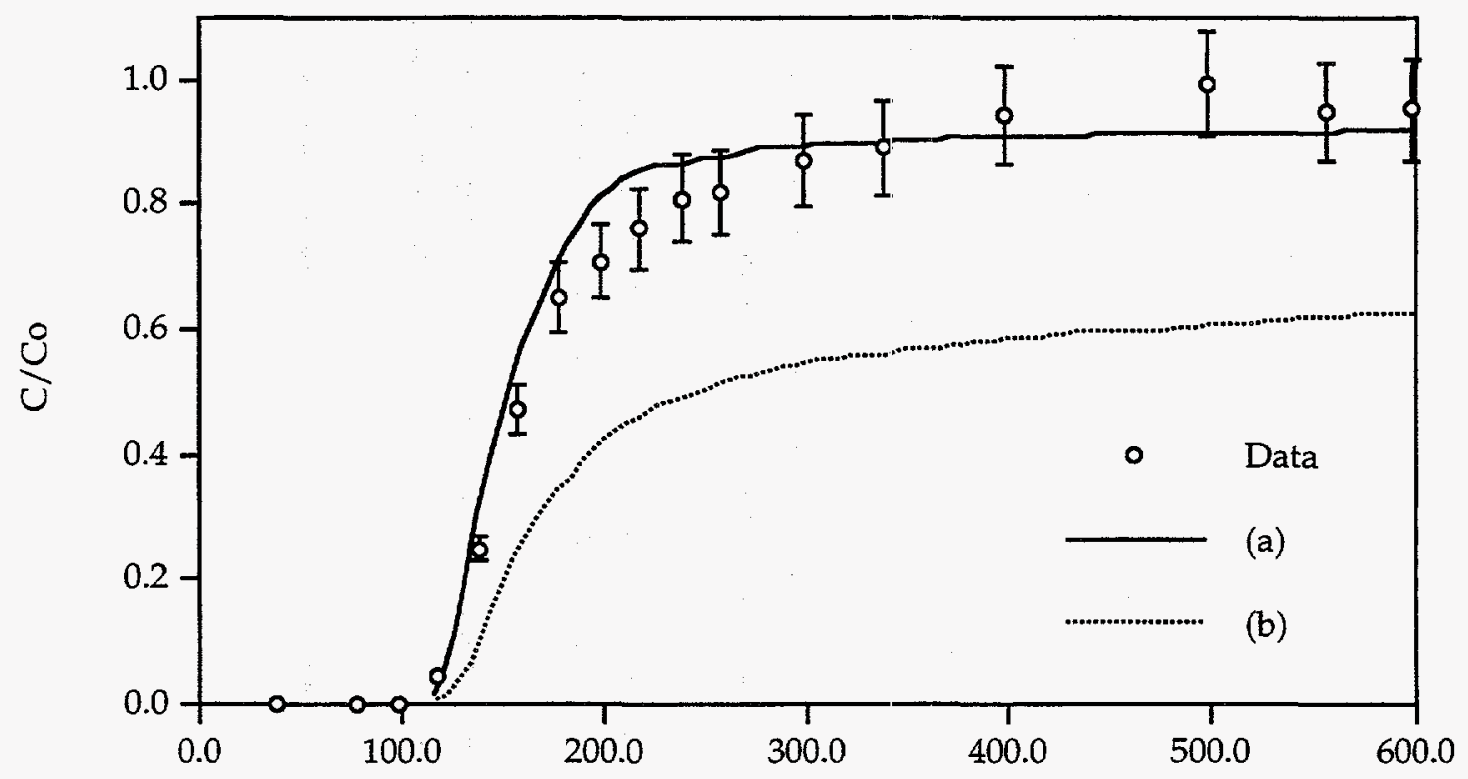

Time, sec

Figure 4.15. Predicted iodide breakthrough curves (based on 1000 particles) in Bandelier tuff fracture assuming probabilities of (a) 0.089 and (b) 0.5 of encountering a pore when a particle "hits" a wall. The transition layer thickness in both cases was $0.005 \mathrm{~mm}$. The flow rate was $4.11 \mu \mathrm{l} / \mathrm{sec}$. The predictions were made using the aperture data and calculated flow field from the first PEAK profile of this fracture. The experimental data are shown for comparison.

transition layer thickness can affect both the first arrival time and the height of the tail in the predicted breakthrough curves, making the former increase and the latter decrease as the transition layer thickness increases. Thus, the transition layer thickness could serve as an adjustable parameter to "fit" experimental breakthrough curves having different characteristics, although this was not the intent here.

There is an inconsistency in assuming that the probability of a particle encountering a pore when it hits a wall is equal to the matrix porosity even though the porosity at the outer boundary of the transition layer is assumed equal to 1 . This contradiction can be justified by arguing that when a particle initially enters a pore it still "sees" an effective porosity of 1 and its diffusivity is not altered from that in free water. Fig. 4.15 shows the effect of using a number greater than the matrix porosity as the probability of a particle encountering a pore when it hits a wall in the Bandelier tuff fracture (in this case, a probability of 0.5 was used). It is clear that the greater probability of encountering a pore resulted in a significant decrease in the predicted breakthrough concentrations of the iodide, and that this parameter, like the transition layer thickness, could be adjusted to "fit" different experimental data sets.

An alternative conceptual model of the void-space-to-matrix transition is shown in Fig. 4.16. In this model, there is still a transition layer, but it is assumed to be underneath a layer where the effective porosity and particle diffusivity are 


\section{Open Space in Fracture}

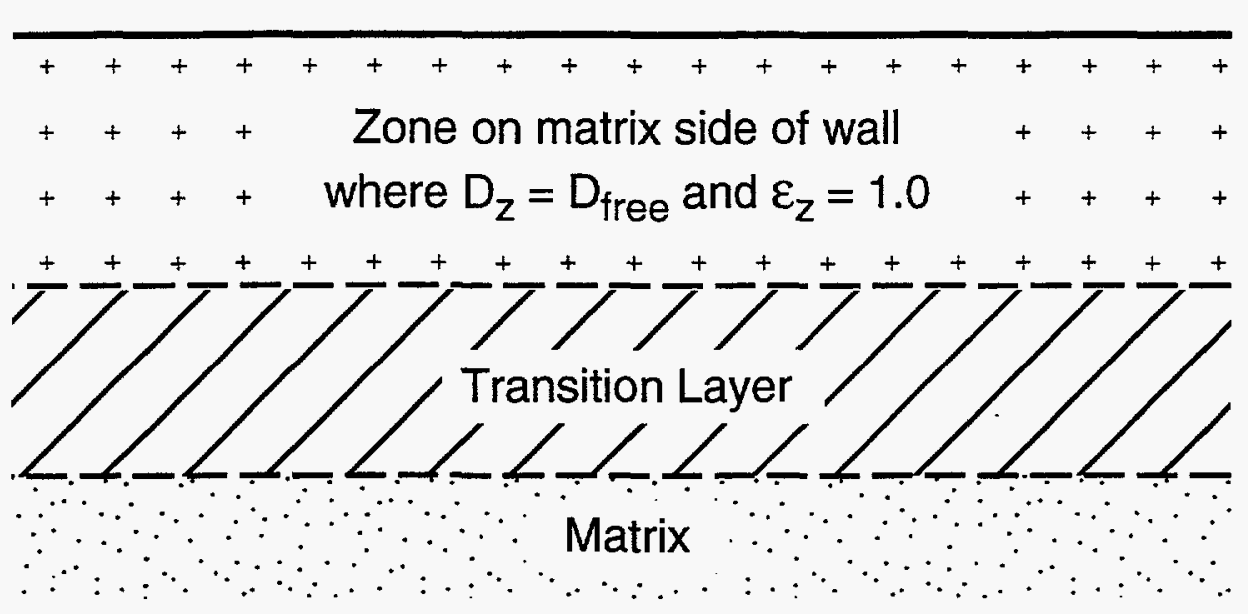

\section{Wall}

Figure 4.16. Illustration of the two-layer model describing the transition from fracture void space to matrix.

equal to the free water values. The rationale behind such a model is that there may be a pore "entrance region" where the particles do not behave any differently than in the void space, but once they move a certain distance into the pore, they begin to feel the effects of the pore walls and experience an effective decrease in diffusivity (and porosity). Fig. 4.17 shows calculated residence time distributions for 10,000 particles moving away from a wall assuming various thicknesses of the overlying layer and a constant transition layer thickness of 10 $\mu \mathrm{m}$. Not surprisingly, the inclusion of an overlying layer having the properties of the fracture void space increased the residence times of the particles, with the increase being most noticeable in the upper tails of the distributions. Fig. 4.18 shows the effect of assuming various thicknesses of the overlying layer on the predicted iodide breakthrough curves in the Bandelier tuff fracture. Although not shown in this figure, further increases in the thickness of the overlying layer (beyond $20 \mu \mathrm{m}$ ) had an effect similar to increasing the transition layer thickness in the absence of an overlying layer (i.e., Fig. 4.14). Because the predicted breakthrough curves using the single transition layer model could effectively be matched to any breakthrough curves predicted by the double-layer model, it was concluded that the use of the more complicated double-layer model was not justified. However, the double-layer model is discussed because it may actually provide a more accurate physical description of the transition between the void space and the matrix. Also, the double-layer model can easily be reduced to the single-layer model by simply specifying an overlying layer of zero thickness.

It is worthwhile to point out that the concentrations in the tails of the predicted breakthrough curves in the Bandelier tuff fracture are very sensitive to subtle differences in the tails of the residence time distributions from the random-walk simulations. This point is important because the tails in the random-walk time distributions were strongly influenced by the behavior of a very small number of particles in the simulations. To avoid excessively long computation times in the random-walk simulations, it was necessary to put an 

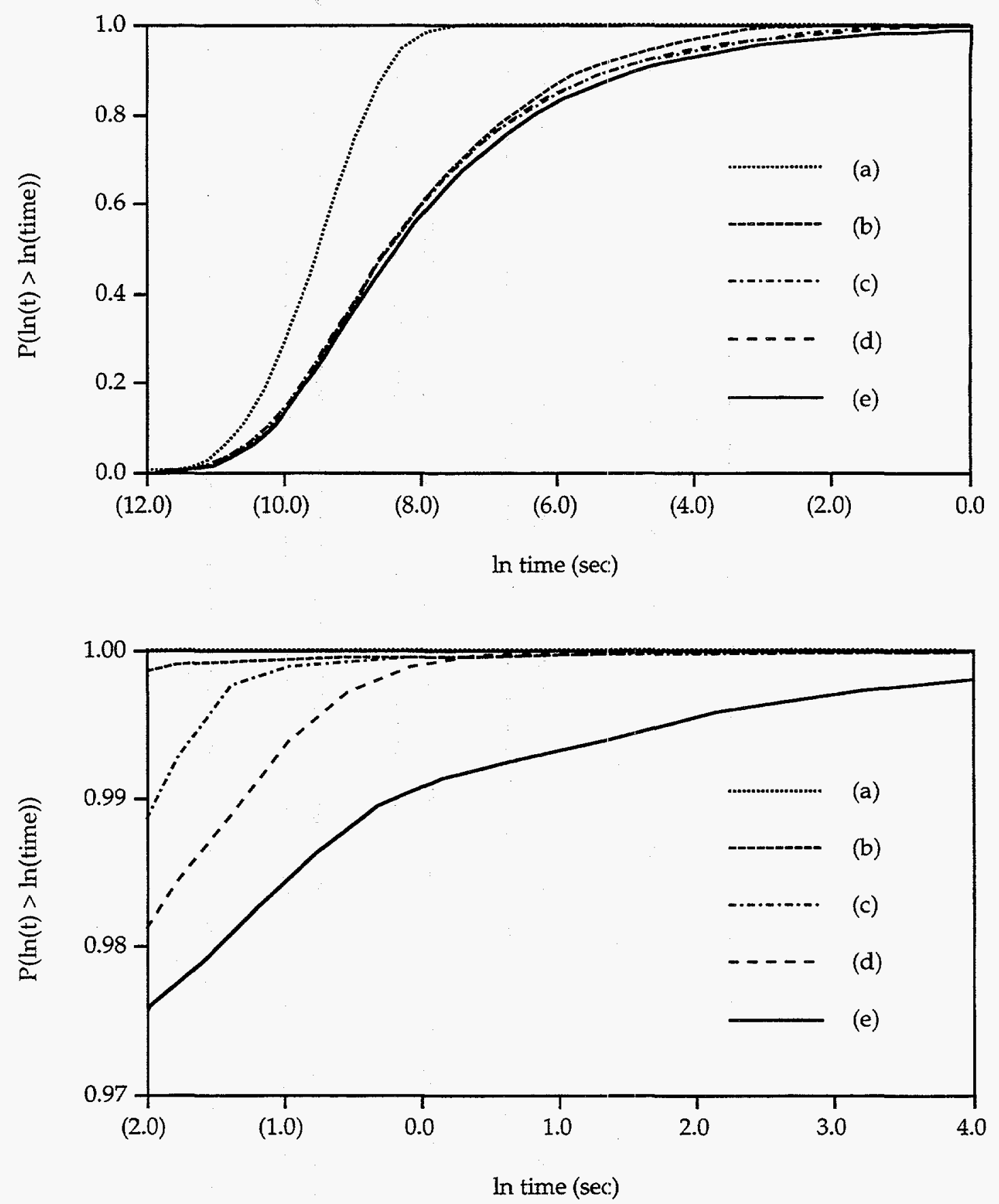

Figure 4.17. Residence time distributions for particles moving away from a wall using different assumptions in the two-layer model shown in Fig. 4.16. The wall porosity was assumed to be 0.089 (corresponding to the Bandelier tuff fracture), and the transition layer thickness was assumed to be $0.01 \mathrm{~mm}$ in all cases. The upper plot shows residence times for (a) a nonporous wall, and for overlying layer thicknesses of (b) $0.0 \mathrm{~mm}$, (c) $0.01 \mathrm{~mm}$, (d) $0.02 \mathrm{~mm}$, and (e) $\infty$. The lower plot shows the tails of curves (a) through (e). Parentheses indicate negative numbers. 


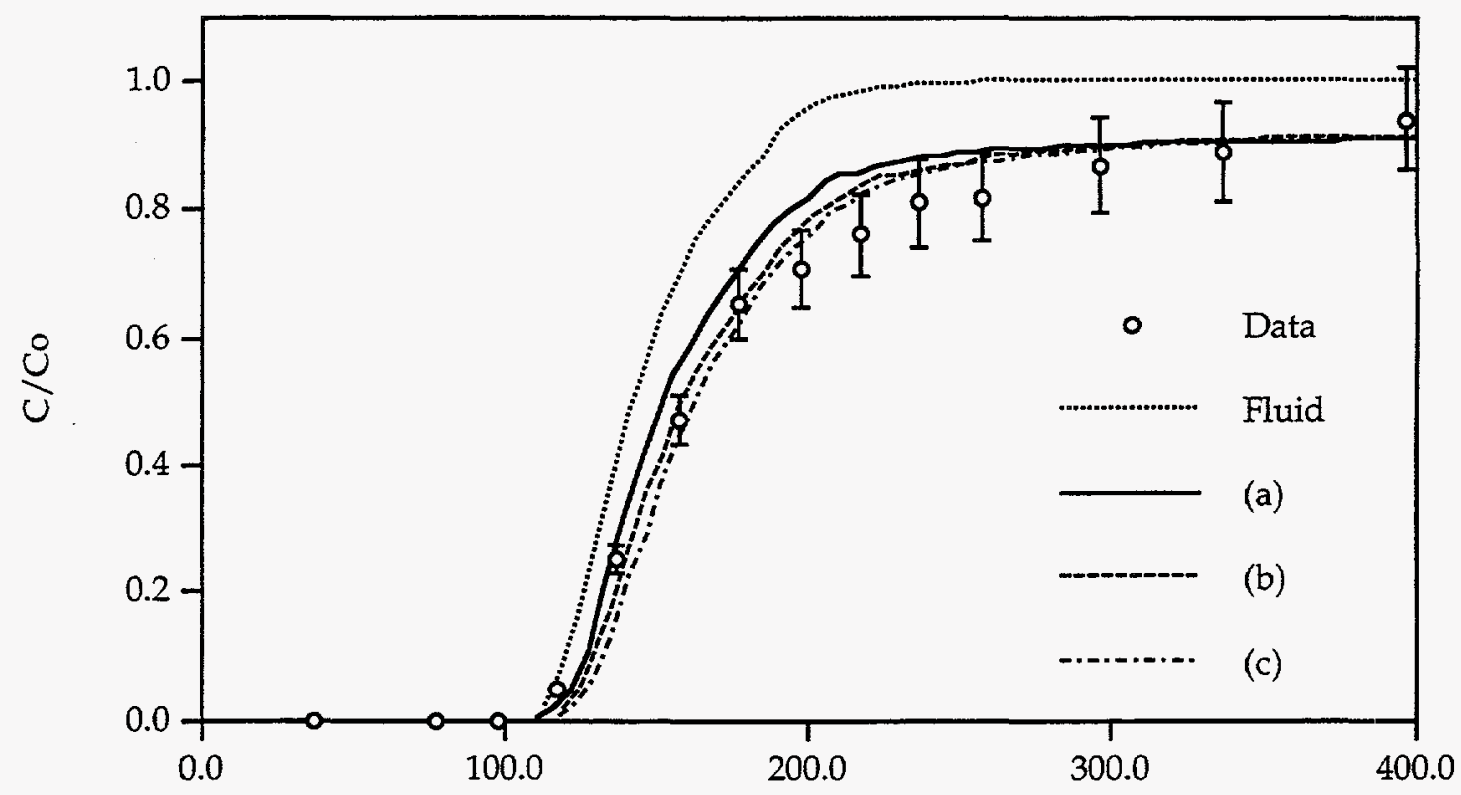

Time, sec

Figure 4.18. Predicted iodide breakthrough curves (based on 1000 particles) in Bandelier tuff fracture assuming the following overlying layer thicknesses in the two-layer model of Fig. 4.16: (a) $0.0 \mathrm{~mm}$, (b) $0.01 \mathrm{~mm}$, (c) $0.02 \mathrm{~mm}$. The transition layer thickness was assumed to be $0.01 \mathrm{~mm}$ in all cases. The flow rate was $4.11 \mu \mathrm{l} / \mathrm{sec}$. The predictions were made using the aperture data and calculated flow field from the first PEAK profile of this fracture. The experimental data and predicted fluid residence time are also plotted.

upper time limit on the particle residence times (that is, once a particle time exceeded some specified cutoff time, the calculations for that particle were terminated and the cutoff time was accepted as the residence time for that particle). Because of this "truncation" procedure, the residence time distributions had to be arbitrarily extrapolated beyond the cutoff time to some finite time at which it was assumed that all particles would have diffused out of the matrix (actually, an extremely small fraction of particles would be expected to have infinite residence times). This arbitrary extrapolation and the small number of simulated particles having long residence times resulted in an undesirably large uncertainty associated with the tails of the residence time distributions, which contributed to an undesirably large uncertainty in the predicted breakthrough curves. The following discussion illustrates this point. Typically, only one particle out of 10,000 had a residence time greater than 50 seconds (the cutoff time) in any of the random-walk simulations with a transition layer thickness of $200 \mu \mathrm{m}$ or less. Fig. 4.19 shows the effect on the overall predicted breakthrough curves in the Bandelier tuff fracture of having two particles instead of one with residence times exceeding 50 seconds in the random-walk simulations (in every other respect the two random-walk time distributions were identical). The difference between the two breakthrough curves can only be attributed to the fact that an additional $0.0009 \%$ of the particle-wall collisions ( 1 in 10,000 of the 


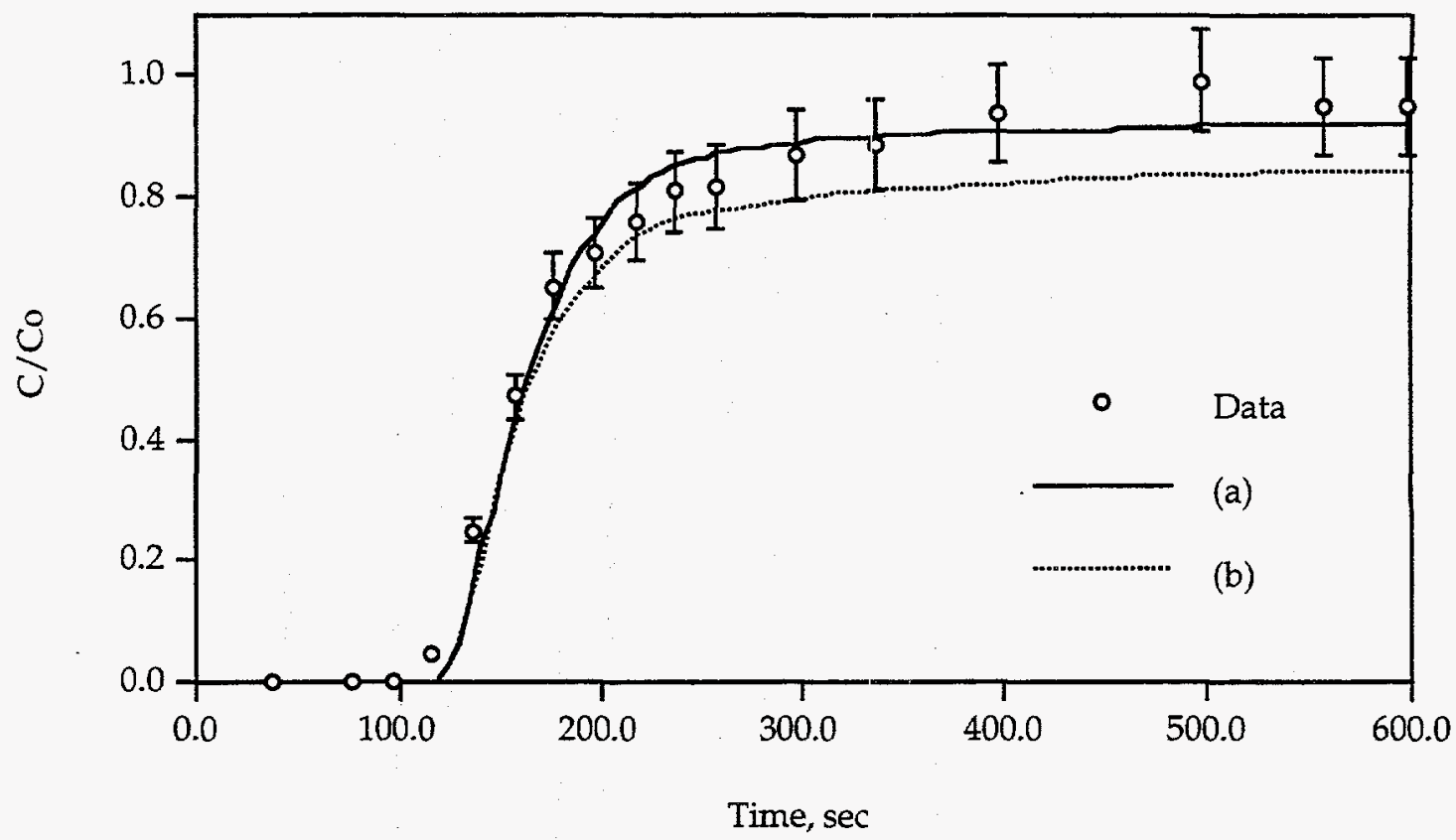

Figure 4.19. Predicted iodide breakthrough curves (based on 1000 particles) in Bandelier tuff fracture using the two-layer model with a transition layer thickness of $0.01 \mathrm{~mm}$ and an overlying layer thickness of $0.02 \mathrm{~mm}$. In (a) only one particle has a residence time greater than 50 seconds in the random-walk simulation of matrix diffusion, and in (b) two particles have residence times greater than 50 seconds. The two residence time distributions are identical in every other respect. The flow rate was $4.11 \mu . / \mathrm{sec}$. The predictions were made using the aperture data and calculated flow field from the first PEAK profile of this fracture. The experimental data are shown for comparison.

$\sim 9 \%$ of the collisions that occur where there is a pore) resulted in sampling the extrapolated tail of the random-walk time distribution. The levels of the plateaus in the tails of the predicted breakthrough curves of Fig. 4.14 can be shown to be inversely related to the number of particles that exceeded the time cutoffs in the random-walk simulations. Obviously, there is a large enough number of particle-wall collisions in the breakthrough curve simulations that the extreme values in the tails of the random-walk time distributions are occasionally sampled. Although not shown here, the predicted breakthrough curves were also sensitive to the upper time limit assumed in the extrapolations of the random-walk time distributions. The fact that such rare events and subtle assumptions can have such a profound impact on predicted breakthrough curves suggests that a better method of determining the exact characteristics of the tails of the random-walk time distributions is needed to improve confidence in the particle-tracking calculations. Also, the above observations suggest that the breakthrough curves could be sensitive to subtle biases or periodicity in the random number generation algorithms. This possibility was not investigated.

When the special situations mentioned in Step (7) of Section 4.2.2 were encountered in the porous-wall particle-tracking model, the residence time 
distributions in the nodes were calculated using a rearrangement of an analytical expression attributable to Starr et al: 185

$$
\Delta t=\Delta t_{0}+\frac{\Delta t_{0}{ }^{2} \varepsilon^{2} \tau D}{a^{2}\{\text { inverfc }[U(0,1)]\}^{2}}
$$

where, $\Delta t=$ residence time in node,

$\Delta \mathrm{t}_{\mathrm{o}}=$ residence time in node in absence of matrix diffusion,

$\varepsilon=$ matrix porosity,

$\tau \mathrm{D}=$ effective diffusivity in matrix,

$a=$ aperture of node,

inverfc $[U(0,1)]=$ the argument of the complementary error function when

the function is equal to $U(0,1)$,

$\mathrm{U}(0,1)=\mathrm{a}$ uniform random number between 0 and 1 .

This analytical expression assumes plug flow (no velocity gradient) in the void space of the fracture with no dispersion in the direction of flow and no

concentration gradients across the fracture aperture. These assumptions roughly

correspond to Taylor dispersion in a very narrow channel where the particles are constantly hitting the walls. In cases of very low fracture flow rates, when Taylor dispersion occurs on local scales throughout the fracture, it might be possible to obtain realistic breakthrough curve simulations using Eq. (55) in each node of the fracture domain. This method has been used in fracture network models by Moreno and Neretnieks. ${ }^{50}$ The advantage of such a method is that the calculation of individual particle trajectories through nodes is not required; the particle residence times in each node are obtained by simply generating one random number and evaluating Eq. (55).

It is interesting to compare the breakthrough curves predicted by the porous-wall particle-tracking model to those obtained by using Eq. (55) in each node of the fracture domain. Fig. 4.20 shows such a comparison for the Bandelier tuff fracture. It is apparent that the method of Eq. (55) (hereafter called the Starr method) predicts lower concentrations than the particle-tracking calculations when the transition layer thickness is assumed to be $10 \mu \mathrm{m}$ (curve (a)). This result can be attributed to the fact that the Starr method implicitly assumes more particle-wall collisions than the particle-tracking method. The earlier arrival time predicted by the particle-tracking calculations can not be attributed to nonTaylor dispersion effects because Fig. 4.14 shows that the breakthrough curves of the fluid and the particles (in the absence of matrix diffusion) are essentially identical. If some particles were staying in streamlines near the center of the channel, they would arrive significantly before the fluid, and these two curves (in Fig. 4.14) would not agree. Instead, the later arrival time predicted by the Starr method (in Fig. 4.20) must be attributed to the fact that every particle in this model spends a significant time in the matrix, whereas with the particle-tracking model, some particles spend negligible time in the matrix. However, Fig. 4.14 suggests that by adjusting the assumed transition layer thickness in the particletracking model, the two models could be made to nearly agree with each other. Curve (b) in Fig. 4.20 is an example of a particle-tracking model breakthrough curve that predicts lower concentrations than the Starr method (a transition layer thickness of $0.5 \mathrm{~mm}$ was assumed). Thus, the comparison between the models is made somewhat ambiguous by the fact that the particle-tracking model can be 


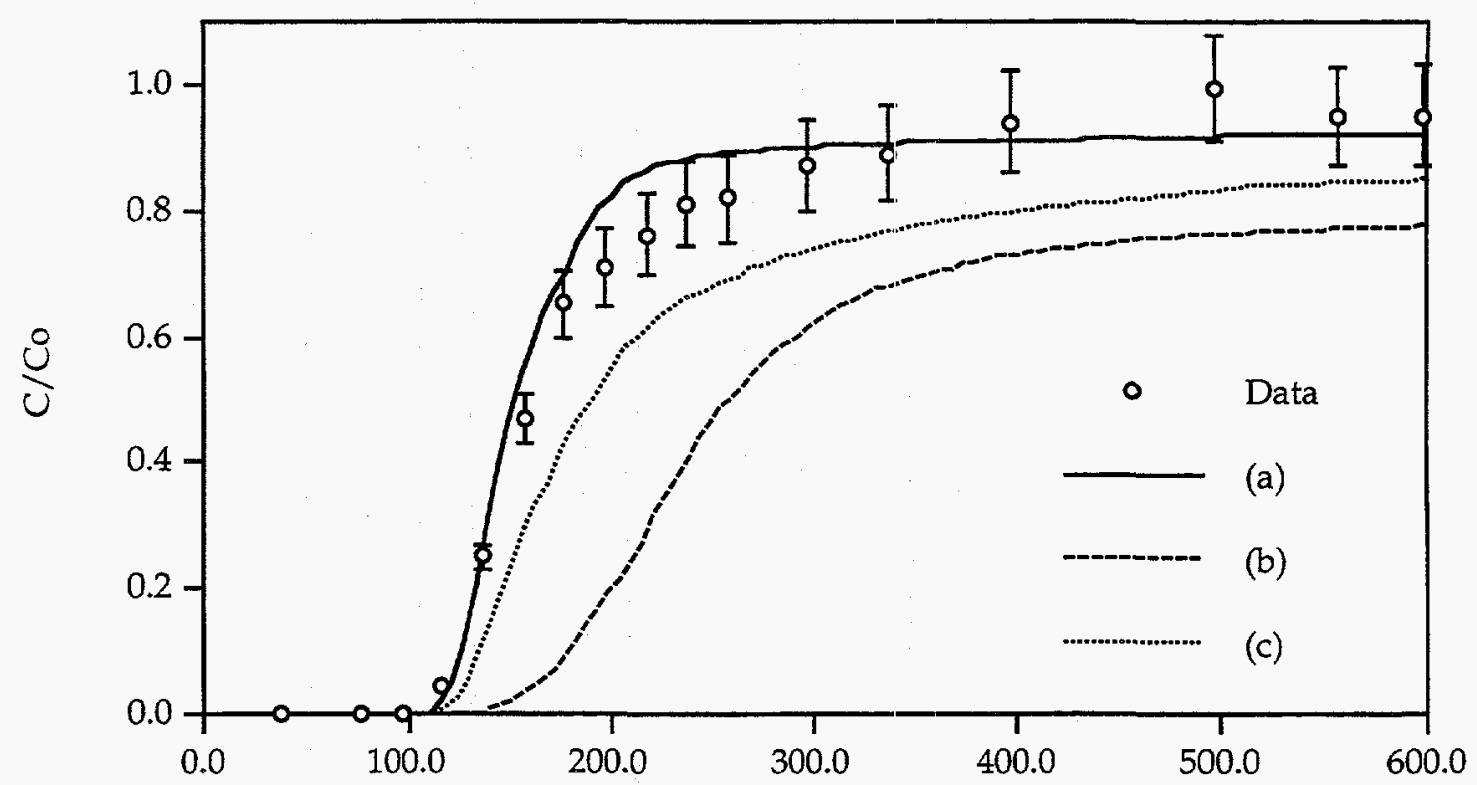

Time, sec

Figure 4.20. Predicted iodide breakthrough curves (based on 1000 particles) in Bandelier tuff fracture using the following models: (a) particle-tracking with a transition layer thickness of $0.01 \mathrm{~mm}$, (b) particle-tracking with a transition layer thickness of $0.5 \mathrm{~mm}$, and (c) the Starr method. The predictions of the Starr method are bracketed by the particle-tracking model using the different transition layer thicknesses. The flow rate was $4.11 \mu \mathrm{l} / \mathrm{sec}$. The predictions were made using the aperture data and calculated flow field from the first PEAK profile of this fracture. The experimental data are shown for comparison.

adjusted to fit just about any shape of breakthrough curve as long as there is freedom to specify the transition layer thickness.

It is appropriate to wrap up this discussion of solute particle-tracking models that account for matrix diffusion with the following observations:

(1) Calculated particle residence time distributions for diffusion into the matrix are quite sensitive to assumptions about the porosity and diffusivity gradients between the fracture void space and the matrix. Subtle differences in the upper tails of these time distributions can have a profound influence on the breakthrough curves predicted by the particletracking models. Consequently, a wide range of experimental breakthrough curve characteristics can be "fit" by simply changing the assumptions in the random-walk simulations used to calculate particle residence time distributions in the matrix.

(2) The random-walk simulations were incapable of providing detailed information about the all-important tails of the particle residence time distributions in the matrix. More work is needed to better define the characteristics of these tails and to ensure that the rare events associated with the tails are sampled in an accurate and unbiased manner. 
(3) Without some knowledge of the microscale characteristics of the pore structure at the fracture surfaces (and their spatial variability), there is no physical basis for determining which assumptions about porosity and diffusivity gradients are most appropriate. It is not even clear how one would use such knowledge to make these determinations. However, based on visual observations of the fracture surfaces under an optical microscope, it seems reasonable that the transition layer thicknesses should have been no greater than about $100 \mu \mathrm{m}$. Fig. 4.14 indicates that the concentration plateaus in the tails of predicted breakthrough curves were approximately the same for transition layer thicknesses up to at least $200 \mu \mathrm{m}$ (probably because all random-walk simulations for these cases had only one particle exceed the cutoff time), and the only difference between the breakthrough curves was a slight lag in arrival time for thicker transition layers. It is also apparent from these figures that transition layer thicknesses less than 200 $\mu \mathrm{m}$ resulted in reasonably good fits to the tail of the experimental breakthrough curve. Therefore, a transition layer thickness of $10 \mu \mathrm{m}$, which offered a reasonable fit to the early portion of the experimental breakthrough data as well as to the tail of the data, was chosen for all subsequent random-walk simulations used to generate residence time distributions for particle diffusion into the matrix.

\subsubsection{Solute Transport with Stagnant Zone along Fracture Walls}

A minor variation of the solute particle-tracking models was developed to allow for the possibility that there may be more void volume in the fractures than suggested by the deduced aperture data sets. This possibility is suggested by the situation shown in Fig. 3.12, where the stylus profiler tip is incapable of detecting small crevices and pits in the fracture surfaces. This same sort of inability to detect fine-scale surface features may have also been a characteristic of the other aperture measurement techniques. This limitation would tend to result in underestimation of the volume of the fracture void space, with the overlooked volume being concentrated along the fracture walls. The flow velocity in such regions would be expected to be very low.

To determine the potential impact of overlooking such stagnant regions along the fracture walls, a modification of the solute particle-tracking model was developed in which a layer of stagnant fluid of uniform thickness could be specified along the fracture walls. This layer was assumed to penetrate into the fracture walls so that the effective local aperture was increased by two times the specified thickness of the layer. However, the parabolic velocity profile was assumed to remain confined between the original channel walls, and the new volume along the walls was assumed stagnant. This situation is illustrated in Fig. 4.21. The stagnant layers differed from the transition layers described in Section 4.2 .3 in that they had a porosity of 1 throughout. Thus, the probability of a particle entering a stagnant layer when it approached from the original portion of the flow channel was 1 (rather than equal to the matrix porosity). The boundary between a stagnant layer and the matrix was treated in exactly the same way as the channel-matrix boundary in Section 4.2.3. 
Solute Stagnant Layer(s)

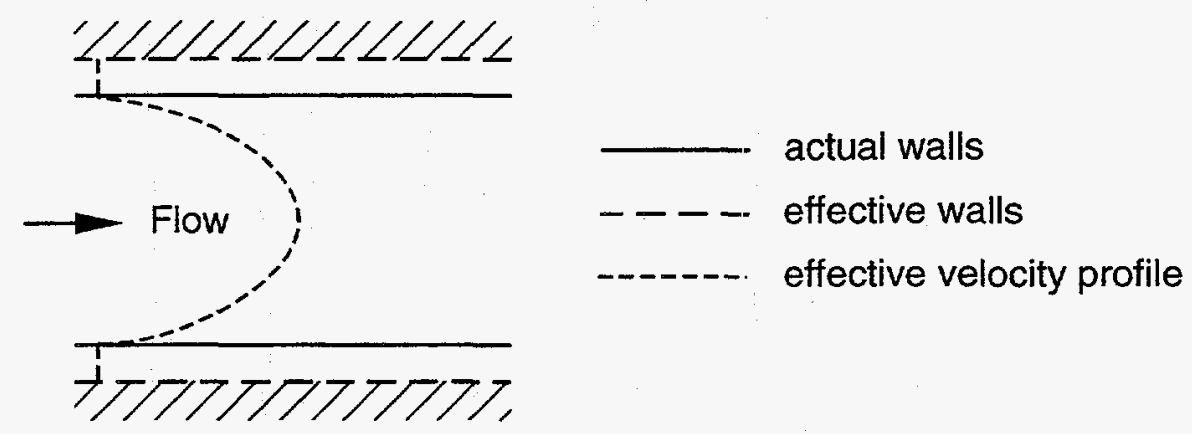

Figure 4.21. Depiction of stagnant layers in solute particle-tracking model.

Fig. 4.22 shows breakthrough curves predicted by particle-tracking models in which stagnant layers of various thicknesses were included in the calculations. Not surprisingly, the addition of the stagnant layers delayed the arrival times of the solute by an amount that was roughly proportional to the volume added to the system by the layers. The layers, in general, did not affect the heights of the plateaus in the tails of the predicted breakthrough curves.

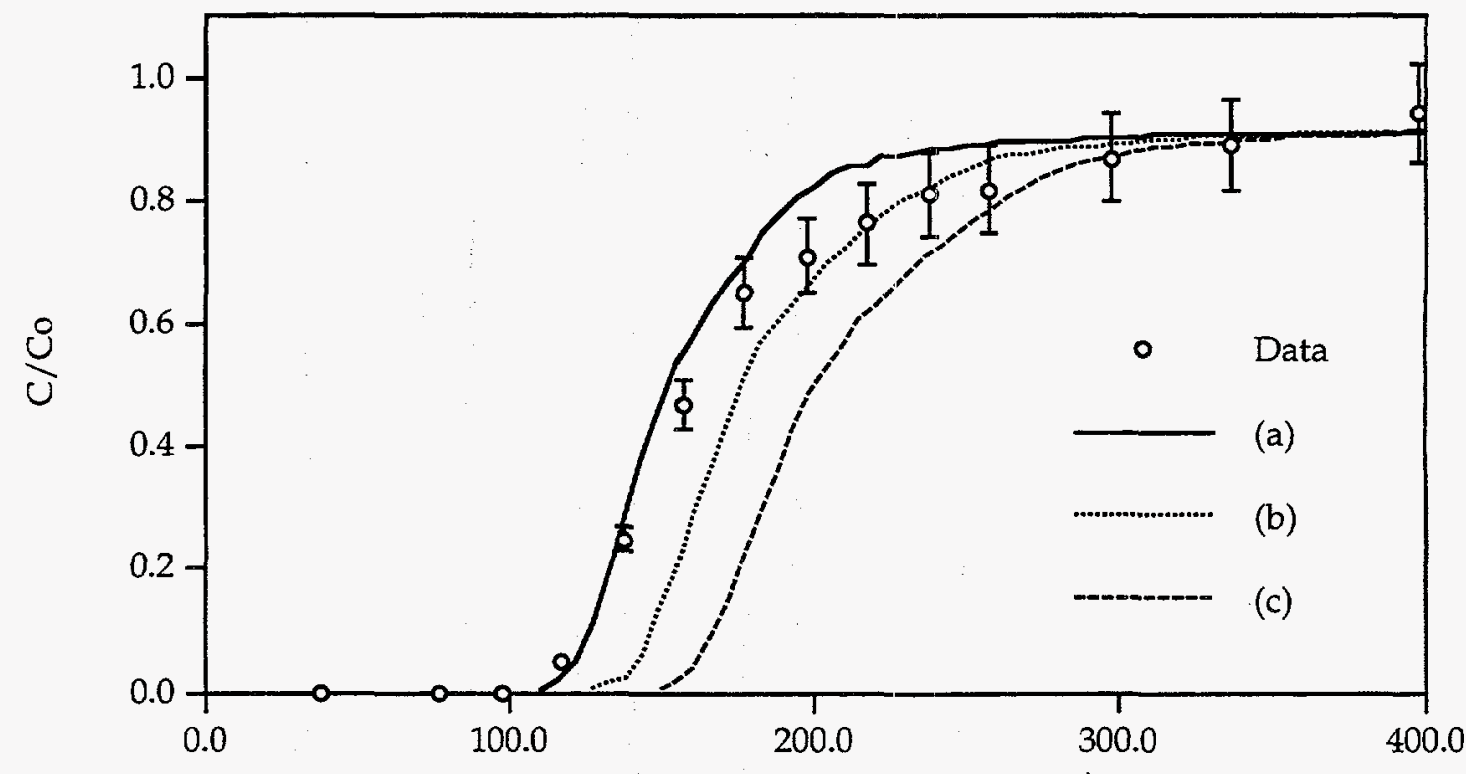

Time, sec

Figure 4.22. Predicted iodide breakthrough curves (based on 1000 particles) in Bandelier tuff fracture assuming the following stagnant layer thicknesses: (a) $0.0 \mathrm{~mm}$, (b) $0.005 \mathrm{~mm}$, and (c) $0.01 \mathrm{~mm}$. The transition layer thickness in all cases was assumed to be $0.01 \mathrm{~mm}$ (no overlying layer). The flow rate was $4.11 \mu \mathrm{l} / \mathrm{sec}$. The predictions were made using the aperture data and calculated flow field from the first PEAK profile of this fracture. The experimental data are shown for comparison. 


\subsubsection{Colloid Transport}

The particle-tracking method described in Section 4.2.2 for modeling solute transport through fractures was also used to model colloid transport through fractures. However, there were the following important differences:

(1) Colloids were subject to hydrodynamic corrections near walls, (2)

Colloids were subject to gravitational settling, and

(3) Colloids were not allowed to diffuse into the matrix.

The gravitational settling of colloids was incorporated into the colloid particle-tracking calculations as follows: First, the gravitational settling velocity was calculated from Eq. (22). The distance settled during a time step was determined by multiplying the settling velocity by the time increment. Because the fractures were always oriented horizontally in the tracer experiments, gravitational settling was always assumed to be in the negative $z$-direction. That is, the gravitational settling distance was always subtracted from the z-direction distance increments in the particle-tracking calculations.

Incorporation of hydrodynamic corrections near walls into the colloid particle-tracking calculations was accomplished as follows:

(1) First, the location of a colloid particle in the model was always assumed to be the location of the center of the colloid. Because colloids had a finite radius $(R)$, they were assumed to "hit" a wall when they reached a distance $R$ from the wall. A dimensionless variable, $H=h / R$ (where $h=$ separation distance between colloid surface and nearest wall), was calculated after each time step and used in Step 2 to estimate the hydrodynamic corrections at the current particle position.

(2) The following analytical expressions were used to approximate the hydrodynamic correction functions $F_{1}(H), F_{3}(H)$, and $F_{4}(H)$, which were discussed in Section 2.6.2:

$$
\begin{gathered}
F_{1}(H)=\frac{H}{H+1} \\
F_{3}(H)=\frac{(H+1)^{6}}{(H+1)^{6}+0.75} \\
F_{4}(H)=1-\frac{9}{16}\left(\frac{1}{H+1}\right)+\frac{1}{8}\left(\frac{1}{H+1}\right)^{3}-0.01758\left(\frac{1}{H+1}\right)^{4}-\frac{1}{16}\left(\frac{1}{H+1}\right)^{5}
\end{gathered}
$$

Equations (56) and (58) were taken from Goldman et al.115 and Chari and Rajagopalan, ${ }^{119}$ respectively. Equation (57) was deduced by trial-and-error calculations. These expressions should have no more than a $10 \%$ error at any value of $\mathrm{H} . \mathrm{F}_{2}(\mathrm{H})$ was not needed in the particle-tracking calculations because this correction function is applicable only when there is a fluid velocity component directed toward a wall, and flow was always assumed to be parallel to the walls.

(3) The hydrodynamic correction functions were used in the following ways to correct the movement of the colloid particles:

(a) The particle diffusivity given by Eq. (18) (the diffusivity in the absence of walls) was multiplied by $F_{1}(H)$ to determine the effective diffusivity in the $\mathrm{z}$-direction. This effective diffusivity, $\mathrm{F}_{1}(\mathrm{H}) \mathrm{D}$, was used in Eq. (52) in place of $D$ to calculate the time required to move a distance increment in the $z$-direction. The settling velocity was also multiplied 
by $F_{1}(H)$ to correct for wall effects. Thus, settling velocities and diffusivities both decreased as a particle neared a wall.

(b) The particle diffusivity given by Eq. (18) was multiplied by $\mathrm{F}_{4}(\mathrm{H})$ to determine the effective diffusivity in the $x$-direction. This effective diffusivity, $F_{4}(H) D$, was used in Eq. (28) in place of $D$ to determine the $x$-direction distance increment due to diffusion during each time step.

(c) The fluid velocity at a given z-position was multiplied by $\mathrm{F}_{3}(\mathrm{H})$ to obtain the deterministic particle velocity at that position. This velocity was used as $\mathbf{u}_{\text {det }}$ in Eq. (28) to determine the $x$-direction distance increment due to advection during each time step.

(4) Because the hydrodynamic correction function $F_{1}(H)$ introduced a diffusivity gradient in the $\mathrm{z}$-direction, an expression corresponding to Eq. (29) had to be incorporated into the particle-tracking calculations to account for the deterministic velocity caused by this gradient. The following expression was used in place of Eq. (29):

$$
u_{\mathrm{D}} \Delta \mathrm{t}=\frac{\partial \mathrm{F}_{1}(\mathrm{H})}{\partial \mathrm{z}} \mathrm{D} \Delta \mathrm{t}= \pm\left[\frac{1}{\mathrm{H}+1}-\frac{\mathrm{H}}{(\mathrm{H}+1)^{2}}\right] \mathrm{R}^{-1} \mathrm{D} \Delta \mathrm{t}
$$

The \pm sign indicates that the deterministic velocity $u_{D}$ could be directed in either the + or $-z$ direction, with the appropriate direction always being away from the nearest wall. Because there was a diffusivity gradient only in the $z$-direction, $\mathbf{u}_{\mathrm{D}}$, like the gravitational settling velocity, acted only to modify the $\mathrm{z}$-direction distance increments.

As with the solute particle-tracking calculations, special logic was required to handle the situations described in Step (7) of Section 4.2.2. Special situation (a) in this section was treated the same for colloids as for solutes. Special situation (b) was modified so that the criteria for invoking the special logic was that $\frac{\sqrt{\mathrm{D} \Delta t_{i, j}}}{a_{i, j}}$ be greater than 2 rather than greater than 10 , (also, $F_{1}(H) D$, evaluated at the center of the channel, was substituted for $D$ in this expression). The difference between the node residence times for $\frac{\sqrt{D \Delta t_{i, j}}}{a_{i, j}}$ values of 2 and 10 was not significant if the walls were assumed impermeable to particles (as they were for colloids). Also, the fluid velocity used to estimate particle residence times was multiplied by $\mathrm{F}_{3}(\mathrm{H})$ (evaluated at the center of the channel) to correct the particle velocity for wall effects.

Another special situation arising for colloids was when the particle diameter actually exceeded the aperture of a node, making it impossible for the particle to enter the node. Exclusion of particles from nodes was not formally implemented, but colloid particles were forced to move through such nodes at a velocity equal to 0.57 times the average fluid velocity in the node because the value of the hydrodynamic correction function $\mathrm{F}_{3}(\mathrm{H})$ is 0.57 when $\mathrm{H}=0$ (that is, when the particle surface is in contact with a wall).

The effect of hydrodynamic corrections and gravitational settling on predicted breakthrough curves could be investigated by comparing breakthrough curves with these effects included in the calculations to 
breakthrough curves obtained using the solute particle-tracking model discussed in Section 4.2.2. Fig. 4.23 shows the results of such a comparison for a parallelplate channel having a length of $100 \mathrm{~cm}$, an aperture of $0.01 \mathrm{~cm}$, and an average flow velocity of $1.667 \mathrm{~cm} / \mathrm{sec}$ (average fluid residence time of 60 seconds). The particle diffusivity in this case was assumed to be $2 \times 10^{-7} \mathrm{~mm}^{2} / \mathrm{sec}$, which corresponds to a particle of radius $1.09 \mu \mathrm{m}$; and the particle density was assumed to be $1.055 \mathrm{~g} / \mathrm{cm}^{3}$, which is the density of the polystyrene microspheres (a large particle size was chosen so that the effect of settling could be clearly seen). Fig. 4.23 shows that the effect of including hydrodynamic corrections in the calculations is to lower the tail of the breakthrough curve by a slight amount, and the incremental effect of including gravitational settling is to lower the tail further still.

It is easy to understand how gravitational settling could lower the breakthrough curve tail: settling particles will tend to accumulate along the bottom wall and stay in the low velocity streamlines next to the wall. The effect of hydrodynamic corrections is not so obvious. The deterministic velocity due to the diffusivity gradient in the z-direction (Eq. 59) should always tend to push particles away from walls into higher velocity streamlines. However, the decrease in diffusivity as particles approach walls also tends to make it harder for particles to diffuse away from walls. It can be shown that, as a particle approaches a wall, the magnitude of the rate of change in diffusivity is actually greater than the magnitude of the rate of change in diffusivity gradient (at least

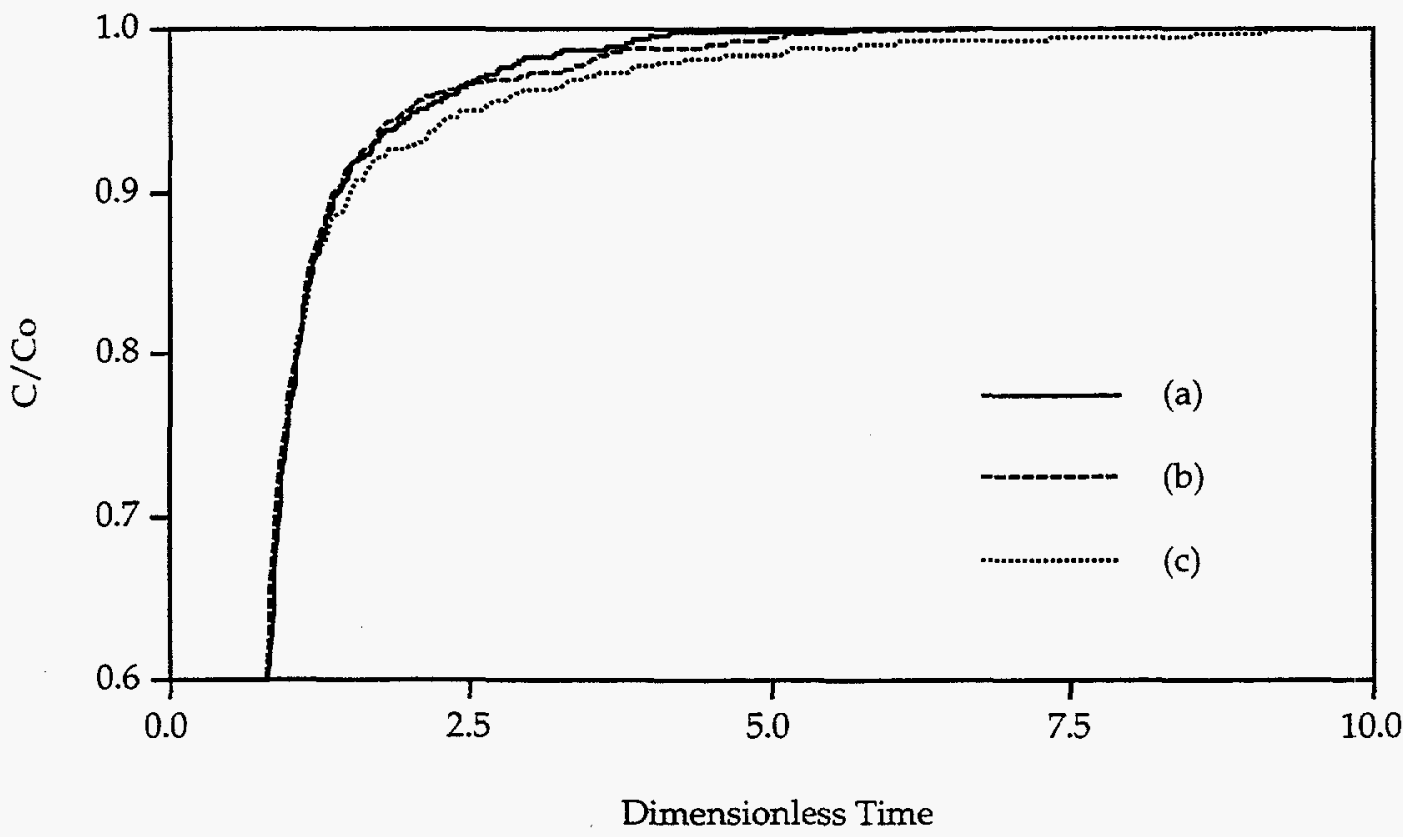

Figure 4.23. Predicted colloid breakthrough curves (based on 1000 particles) in a parallel-plate channel assuming (a) no hydrodynamic corrections and no gravitational settling, (b) hydrodynamic corrections but no gravitational settling, and (c) hydrodynamic corrections and gravitational settling. See text for assumptions. Note that the $y$-axis starts at $C / C_{0}=0.6$. Dimensionless time is $\left(\mathrm{V}_{\text {ave }}\right)$ (time)/(length of channel). 
until the particle gets very close to a wall), so the net effect of hydrodynamic corrections is actually to "hold" a particle near a wall rather than "push" it away. The amount of imbalance between "holding" and "pushing" is biased toward "holding" if larger time steps are taken, so time steps should be kept small to avoid numerical errors.

From a practical standpoint, the differences between the breakthrough curves with and without hydrodynamic corrections in Fig. 4.23 are so small that it would be very difficult to obtain experimental data to verify the differences (the experimental errors and uncertainties would be greater than the differences between the predicted curves). Also, the differences between the curves would be expected to be less for the smaller microspheres used in the tracer experiments $(0.15-$ to $0.5-\mu \mathrm{m}$ radius vs. the $1.09-\mu \mathrm{m}$ radius assumed in the calculations to obtain the curves in Fig. 4.23). Thus, it was concluded that hydrodynamic corrections were relatively unimportant in the colloid particle-tracking calculations. However, because the trouble had already been taken to incorporate them, these corrections were included in all colloid breakthrough curve calculations.

\subsubsection{Variations in Colloid Particle-Tracking Calculations}

Several variations of the colloid particle-tracking method were developed to investigate the effects of various modeling assumptions on the colloid transport predictions. These variations are described below:

(1) In the first variation, it was assumed that the colloid particles were distributed across the velocity profile in the same way as described in Step (1) of Section 4.2.2 after each crossing of a node boundary. Thus, instead of particles remaining at the same relative distance from the walls when crossing node boundaries, a semi-random mixing (weighted by the flow rate across the channel) was assumed for particles as they crossed node boundaries. The rationale for this variation was that there may be some cross-streamline mixing due to the rough fracture walls and perhaps some bias toward particles redistributing into the center of the flow channel, especially when regions of narrow aperture widen into large apertures. The latter situation is illustrated in Fig. 4.24.

(2) In the second variation, particles were allowed to move only into adjacent nodes (with flow into them) that had the largest aperture. The exception was at the inlet boundary, where the node selection was the same as in Step (1) of Section 4.2.1. The rationale for this variation was that colloids may be excluded from smaller apertures because of size considerations and hydrodynamic corrections.

(3) In the third variation, it was assumed that there was a layer of fluid along the fracture walls from which colloids were excluded. The rationale for this variation is shown in Fig. 4.25. In this figure, the rough surfaces give rise to regions along the fracture walls that the colloids cannot access because there is no flow into them and because the colloid diffusivities are too small to allow the colloids to diffuse into them. The exclusion layers were implemented in the particle-tracking models by simply not allowing 

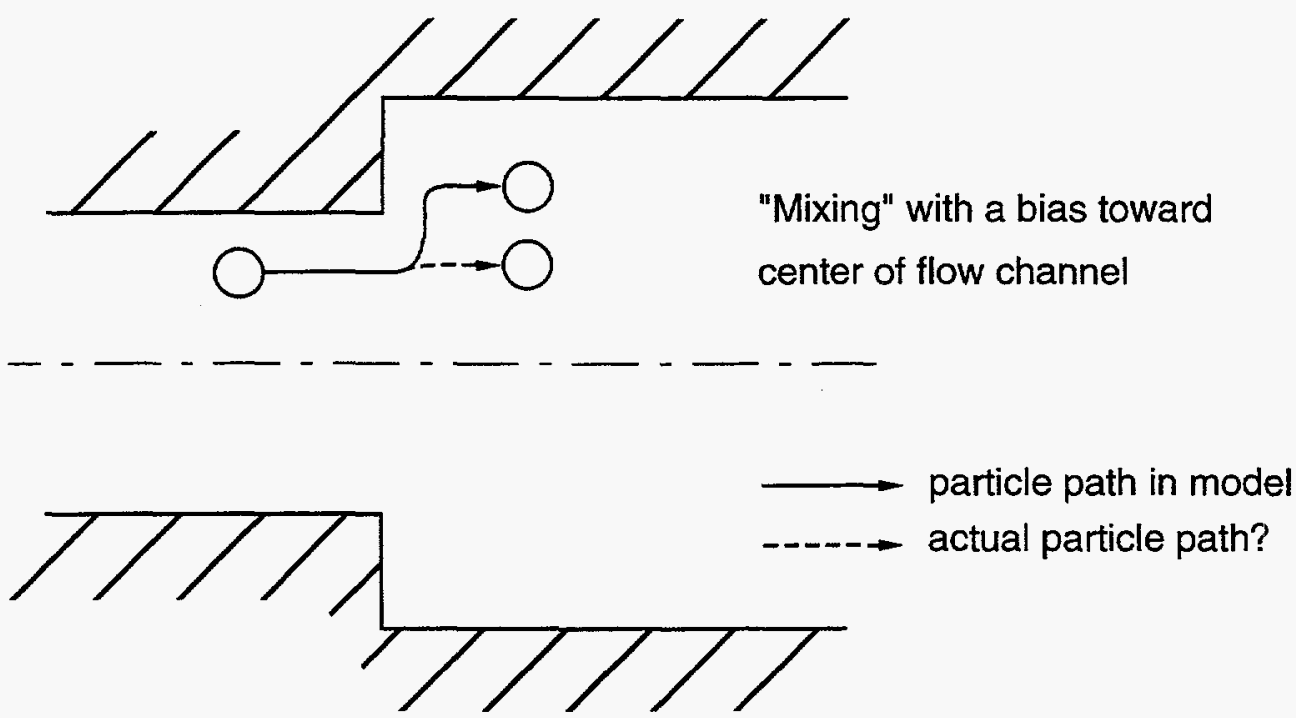

Figure 4.24. Illustration of possible particle trajectory when a narrow aperture region widens into a large aperture region (dashed line). The solid line shows the trajectory assumed in the particle-tracking models.

colloids to move within a specified distance of the walls. When colloid particles moved across node boundaries, they maintained their position relative to the "effective wall" rather than to the actual wall (i.e., the ratio of "the distance from particle to channel centerline" to "the distance from. effective wall to centerline" was kept constant). The velocity profile across the flow channel was not altered to make the velocity at the effective wall equal to zero. Instead the original velocity profile was preserved, and the colloid particles were simply excluded from the low velocity regions along the actual walls. This situation is illustrated in Fig. 4.26. In nodes where the aperture was less than twice the exclusion layer thickness, the exclusion layers were ignored and the particles were assumed to have access to the entire width of the flow channel.

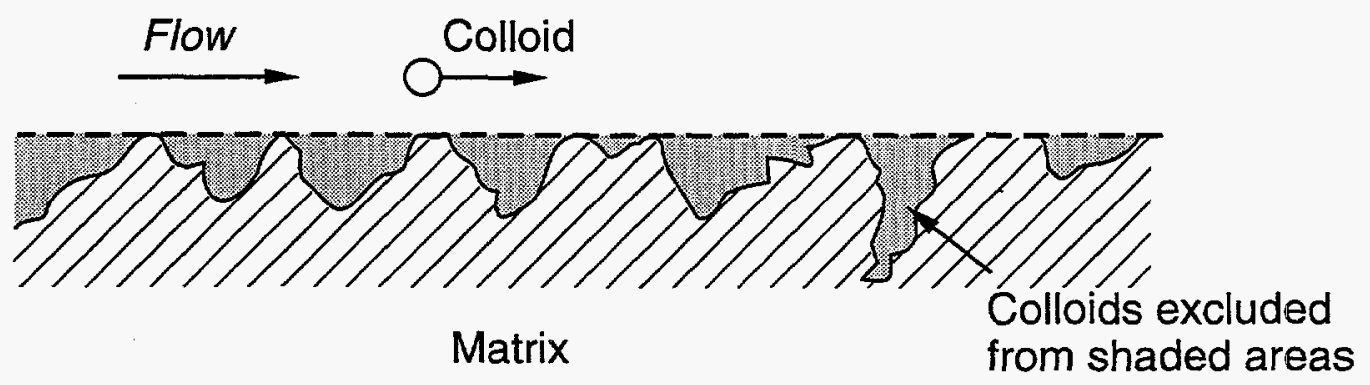

Figure 4.25. Depiction of a colloid moving near a fracture wall showing regions that colloids may be excluded from. 
Colloid Exclusion Layer(s)
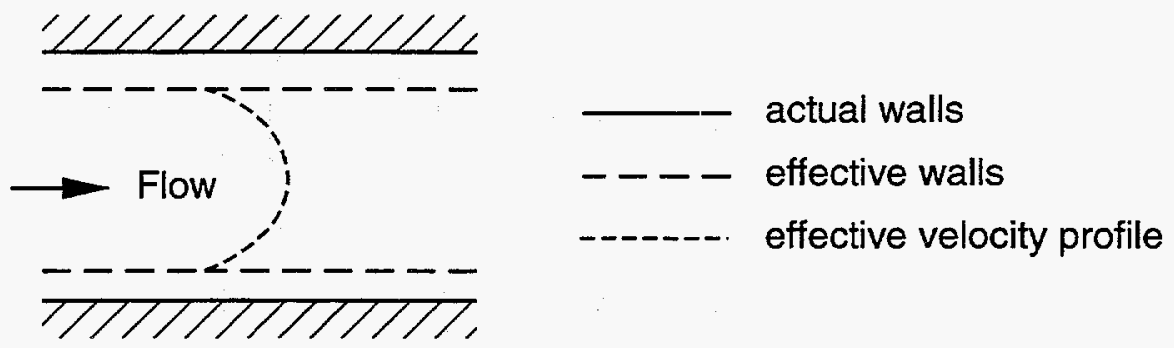

Figure 4.26. Depiction of exclusion layers in colloid particle-tracking model.

(4) A final variation was implemented because it was discovered that variation (3) could actually result in slowing down the colloid particles rather than speeding them up when there was a significant trend of flow from small apertures into large apertures in a fracture. This trend was especially apparent in the Bullfrog tuff fracture simulations because of the large aperture region near the outlet end of this fracture. When flowing from small to large apertures, the logic for maintaining particle positions at the same relative distance from the effective walls when crossing node boundaries resulted in the particles actually moving closer (in relative distance) to the actual walls. This movement resulted in the particles experiencing a slower velocity as they moved into larger aperture nodes. Of course, the opposite was true when particles moved from larger aperture nodes to smaller aperture nodes (i.e., a faster velocity was experienced). To deal with this problem, the logic was changed back to the original logic of maintaining particles at the same relative distance from the actual walls instead of from the effective walls when crossing node boundaries. However, the particles were still excluded from a layer of specified thickness along the walls (except when node apertures were less than twice the exclusion layer thickness). This variation generally resulted in shorter particle residence times than variation (3) in all the fractures, although the effect was most pronounced in the Bullfrog tuff fracture.

Fig. 4.27 shows calculated breakthrough curves for the $1-\mu \mathrm{m}$ microspheres in the Bandelier tuff fracture (based on 1000 particles) using the particle-tracking variations discussed in this section as well as the approach discussed in Section 4.2 .5 and the solute particle-tracking model, which ignores hydrodynamic corrections and gravitational settling. The aperture data set and flow-field solution used to obtain the breakthrough curves in Fig. 4.27 were from the first PEAK profile of this fracture. The calculated fluid residence time distribution and the experimental data are shown in both plots for comparison. The following conclusions can be drawn from the breakthrough curves in Fig 4.27:

(1) The breakthrough curve obtained using the method of Section 4.2.5 correctly predicts the first arrival time of the colloids, but it underpredicts the rest of the experimental breakthrough curve. The curve obtained by using the solute particle-tracking model, which ignores hydrodynamic 


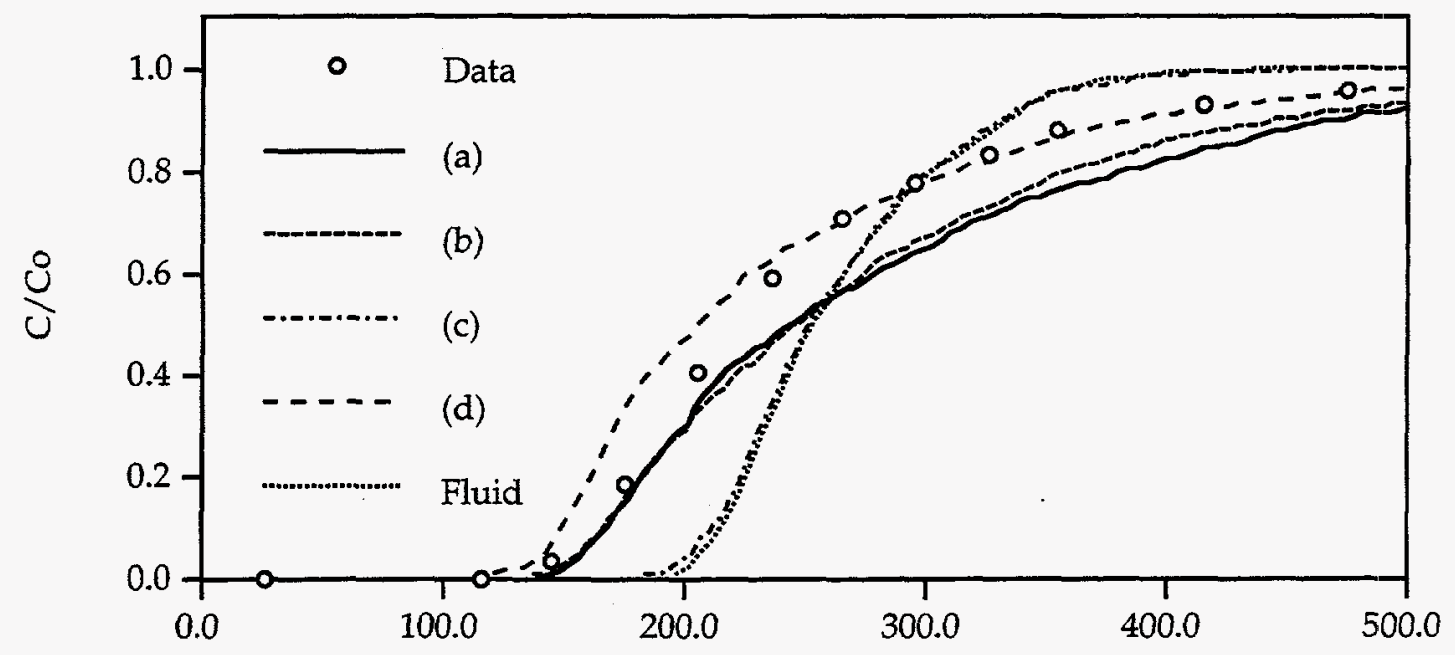

Time, sec

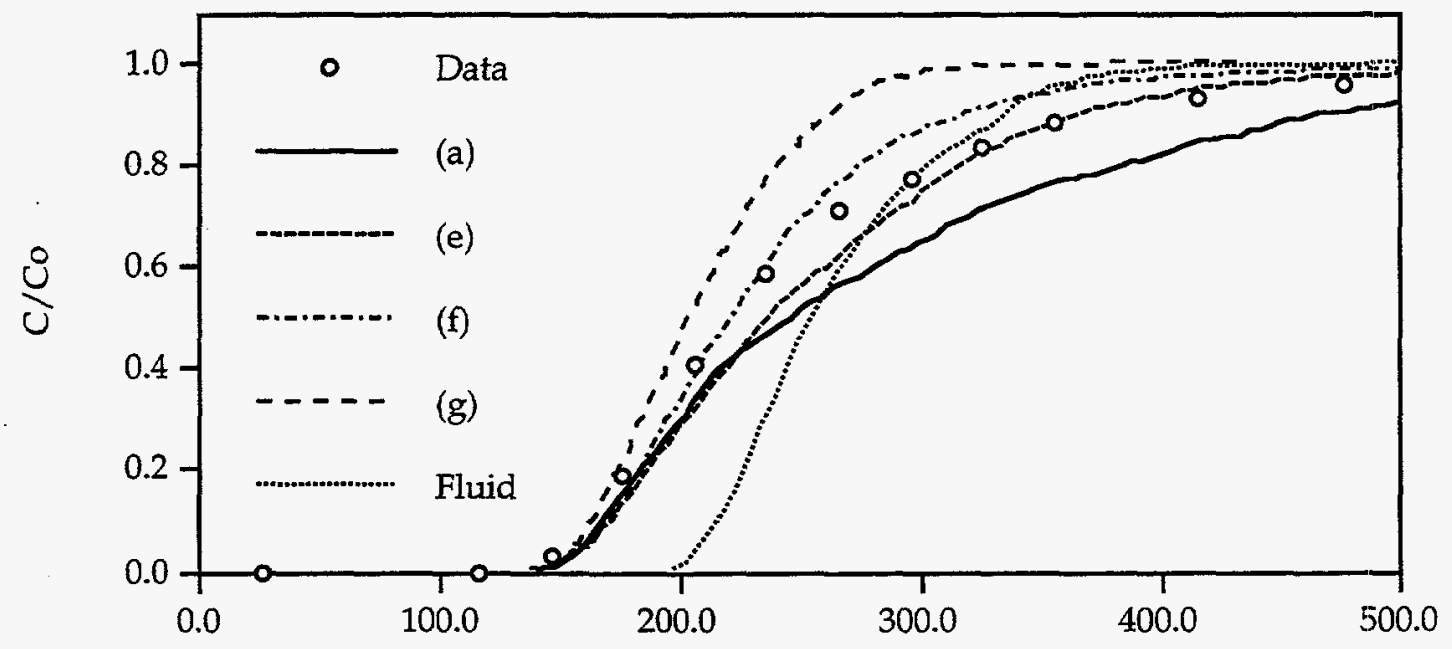

Time, sec

Figure 4.27. Predicted 1- $\mu \mathrm{m}$ CML microsphere breakthrough curves in Bandelier tuff fracture using different particle-tracking models/assumptions: (a) standard model described in Section 4.2.5, (b) model without hydrodynamic corrections or gravitational settling, (c) model that assumes "mixing" after crossing each node boundary, (d) model that assumes colloids only enter adjacent nodes (with flow into them) of largest aperture, (e) model with 0.005$\mathrm{mm}$ exclusion layer along walls, (f) model with $0.01-\mathrm{mm}$ exclusion layer along walls, and $(\mathrm{g})$ model with $0.005-\mathrm{mm}$ exclusion layer but with particles maintaining same relative distance from actual walls instead of effective walls when crossing node boundaries. The flow rate was $2.30 \mu \mathrm{l} / \mathrm{sec}$. The predictions were made using the aperture data and calculated flow field from the first PEAK profile of this fracture. The experimental data (no error bars) and the predicted fluid residence time are shown for comparison. 
corrections and gravitational settling, is only slightly different from the curve obtained using the method of Section 4.2 .5 , with the differences being consistent with the differences noted in Fig. 4.23.

(2) The assumption of semi-random mixing of particles after each crossing of a node boundary results in a breakthrough curve that is almost identical to the fluid residence time curve. Therefore, this assumption is equivalent to saying that colloids provide a good estimate of the mobile fluid residence time in the fractures. However, the resulting predicted breakthrough curve is not in good agreement with the experimental data.

(3) The assumption that particles move only into adjacent nodes of largest aperture results in earlier colloid arrival times than any of the other models and the experimental data suggest.

(4) The assumption of an exclusion layer along the fracture walls shifts the predicted breakthrough curves "upward" so that they are in better agreement with the experimental data. There are significant differences between the curves generated when assuming that particles maintain the same relative distance from the "effective" walls (when crossing node boundaries) and curves generated assuming that particles maintain the same relative distance from the actual walls. In either case, the experimental data could be "fit" by varying the exclusion layer thickness, but the "best fit" thickness would likely be different in the two cases. Additional discussion of the results of the colloid particle-tracking models is provided in Section 4.3 .

\subsubsection{Verification of Particle-Tracking Models}

The particle-tracking models were verified by comparing their predictions to the results of a 2-dimensional finite-difference model. The finite-difference model was limited in its ability to represent a variable-aperture fracture, so the comparisons were made for a single parallel-plate channel (this limitation was the reason for developing particle-tracking models in the first place). The finitedifference model solved the time-dependent species continuity equation (Eq. 36) in a parallel-plate channel having the parabolic velocity gradient given by Eq. (5). Only the diffusion and advection terms were included in the continuity equation (no gravitational settling or arbitrary forces). A simple backward differencing scheme was used to approximate the advection term, and a forward-backward differencing scheme identical to that used for pressure in the Reynolds equation (see Section 4.1) was used to approximate the diffusion term. The resulting set of ordinary differential equations in time were solved using a fourth-order RungeKutta method. 175 It was found that a discretization of at least 20 nodes across the fracture aperture ( 21 were actually used) was necessary in order to avoid a "stair step" appearance in the tails of the breakthrough curves when streamline flow was approximated. The "stair steps" occurred because each node across the fracture aperture had a different flow velocity, and the difference in the velocities of adjacent nodes was greater with a smaller number of nodes, leading to discrete "jumps" in the residence times associated with different node positions.

A comparison of the breakthrough curves predicted by the particletracking method and the finite-difference model is shown in Fig. 4.28. The calculations were done for a 100-cm-long parallel-plate channel with an aperture of $0.01 \mathrm{~cm}$, and an average flow velocity of $1.667 \mathrm{~cm} / \mathrm{sec}$. Simulations were done for two different values of diffusivities: $2 \times 10^{-7} \mathrm{~mm}^{2} / \mathrm{sec}$ and $2 \times 10^{-4} \mathrm{~mm}^{2} / \mathrm{sec}$. 


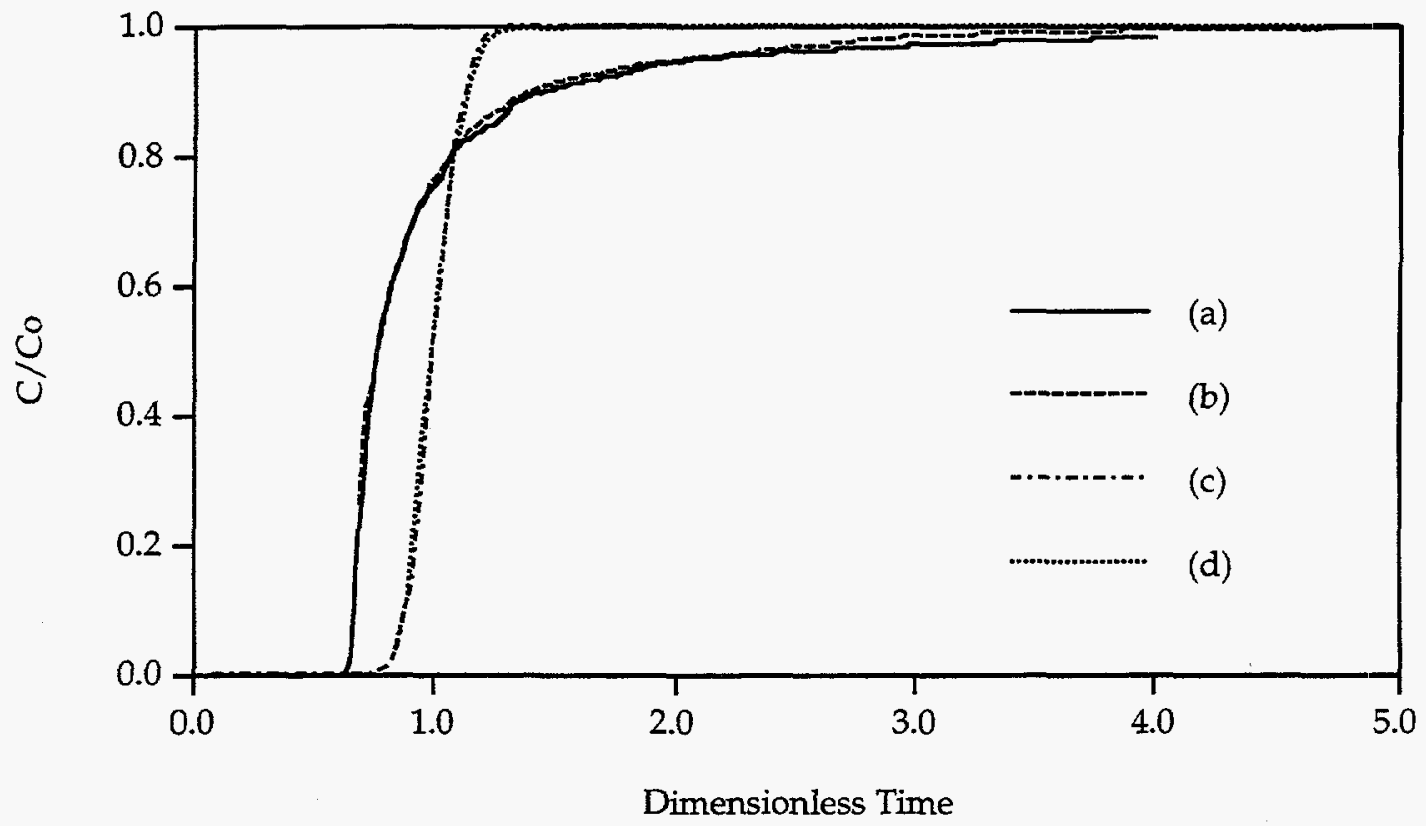

Figure 4.28. Comparison of predicted breakthrough curves in a parallel-plate channel using finite-difference and particle-tracking models. The models and particle diffusivities corresponding to each curve are (a) finite difference with diffusivity $=2 \times 10^{-7} \mathrm{~mm}^{2} / \mathrm{sec}$, (b) particle tracking with diffusivity $=2 \times 10^{-7}$ $\mathrm{mm}^{2} / \mathrm{sec}$, (c) finite difference with diffusivity $=2 \times 10^{-4} \mathrm{~mm}^{2} / \mathrm{sec}$, and (d) particle tracking with diffusivity $=2 \times 10^{-4} \mathrm{~mm}^{2} / \mathrm{sec}$. See text for other assumptions and model parameters.

These two diffusivities were chosen because they roughly correspond to streamline flow and Taylor dispersion (or to colloids and solutes), respectively. The values of the quantity $\frac{\sqrt{\mathrm{D} \Delta t}}{\mathrm{a}}$ associated with these diffusivities were 0.0346 and 1.095, respectively. To be consistent with the finite-difference model, hydrodynamic corrections and gravitational settling were not included in the particle-tracking calculations.

Fig. 4.28 shows that the two models were in very good agreement for both diffusivities. The only perceptible difference between the models was that the finite-difference model predicted slightly lower concentrations in the tail of the breakthrough curve in the low diffusivity case. However, this result might be attributable to the fact that the nodes at the channel walls in the finite-difference model were assumed to have zero flow velocity, while in the particle-tracking model, particles that moved away from walls were assumed to have a velocity equal to the flow velocity at a distance from the wall equal to 0.4 times the distance moved during the time step (see Step 4 of Section 4.2.2). It was determined from simulations with larger diffusivities that the logic in the particle-tracking model was actually more accurate (the mean residence time in the channel was closer to the volume divided by the volumetric flow rate when this logic was used). 
Based on the results shown in Fig. 4.28, it was concluded that the particletracking model was working properly at least for a parallel-plate channel. It was not possible to check the logic for hydrodynamic corrections, gravitational settling, or node-to-node movement in a variable-aperture fracture because the finite-difference model was not capable of simulating these phenomena.

Therefore, verification of these features had to be done on a semi-empirical basis by exercising subroutines and/or checking the reasonableness of results (as in Fig. 4.23 for hydrodynamic corrections and gravitational settling).

\subsection{Results of Particle-Tracking Simulations}

Many particle-tracking simulations were conducted using the predicted flow fields from each fracture aperture data set and adjusting the flow rates so that they corresponded to the flow rates in the tracer experiments. The only experiment that was not simulated was the one in the Bandelier tuff fracture in which the $1-\mu \mathrm{m} C M L$ and the $1-\mu \mathrm{m}$ non-CML polystyrene microspheres were used as tracers (the first experiment). This experiment was not simulated because (1) it was clear that to properly model the transport of the non-CML microspheres, interactions with the fracture walls would have to be included in the models, and (2) the 1- $\mu \mathrm{m} \mathrm{CML}$ and iodide breakthrough curves in this experiment were essentially identical to the breakthrough curves of these tracers in another Bandelier tuff fracture experiment that had a similar flow rate (the fifth experiment).

Only the step portions of the experimental breakthrough curves were modeled because the cumulative residence time distributions generated by the particle-tracking models corresponded to step tracer inputs of infinite duration. The flush phases were not modeled because they would have required more sophisticated logic to account for material that was already in the fractures when the flushes began. However, the fact that the step and flush breakthrough curves were essentially mirror images of each other (see Appendix B) suggests that the flush phase could have been modeled by subtracting one step phase curve from another, with a time offset between the two curves corresponding to the length of the step phase.

The predictions of the particle-tracking simulations are presented in Appendix D. A separate plot is presented for each tracer in each experiment (except for the first experiment), with each plot showing predicted breakthrough curves obtained using different modeling assumptions (the experimental data are also shown for comparison). For the microspheres, breakthrough curves obtained using the following variations in the particle-tracking models are plotted in each of the figures:

(1) the unaltered (or baseline) colloid calculations described in Section 4.2.5,

(2) the variation described in Section 4.2.6 in which colloids were excluded from a layer of fluid along the fracture walls, and

(3) a parallel-plate model in which the aperture was assumed to be equal to the average aperture estimated from the experimental breakthrough curves of the 1- $\mu \mathrm{m}$ CML microspheres (see Section 3.1.7).

For iodide, the following breakthrough curves are plotted in Appendix D:

(1) a curve generated using the solute particle-tracking method described in Section 4.2.3, which included the effects of matrix diffusion, 
(2) a curve generated using the solute particle-tracking method described in Section 4.2.4, in which a stagnant layer of fluid along the fracture walls is included (as well as the effects of matrix diffusion),

(3) a curve generated using a parallel-plate particle-tracking model (which included the effects of matrix diffusion) and

(4) a curve generated using the Starr method described in Section 4.2.3. The predicted fluid residence time distributions, calculated as described in Section 4.2.1, are also included in each plot of Appendix D. These curves show the amount of pure flow-field dispersion in each fracture (for each aperture data set). The differences between the fluid residence time distributions and the predicted microsphere or iodide breakthrough curves can be attributed to either (1) streamline flow of the tracers, (2) matrix diffusion (iodide only), or (3) the effects of variations in the tracer particle-tracking models (e.g., stagnant layers for iodide, exclusion layers for microspheres). The fluid residence time distributions also provide a basis for comparing model predictions associated with different tracers in the same fracture (which are shown on different plots in Appendix D).

In the solute models, the porosities of the tuff matrices were assumed to be equal to the experimentally determined porosities in Table 3.1, and the transition layer thickness (discussed in Section 4.2.3) was assumed to be $10 \mu \mathrm{m}$ in all fractures. The iodide diffusivity was assumed to be $0.0015 \mathrm{~mm}^{2} / \mathrm{sec}$, and the tortuosity factor in the matrix was assumed to be 0.1 . The microsphere diffusivities (calculated from Eq. 18) were $4.36 \times 10^{-7} \mathrm{~mm}^{2} / \mathrm{sec}$ and $1.45 \times 10^{-6}$ $\mathrm{mm}^{2} / \mathrm{sec}$ for the $1-\mu \mathrm{m}$ and $0.3-\mu \mathrm{m}$ microspheres, respectively.

The results of the parallel-plate models allowed an assessment of the advantages of using actual aperture data instead of a simple parallel-plate representation of the fracture to predict tracer transport behavior. If the parallelplate models offered predictions that were just as good as the variable-aperture models, it would suggest that the aperture data sets provided no better representation of the fractures than parallel-plate approximations. The results of the Starr method offered a comparison with an alternative approach to solute particle tracking in which Taylor dispersion was assumed to be valid at the scale of individual nodes within the fractures.

\subsubsection{Use of Model Predictions to Interpret Experimental Observations}

The following conclusions can be drawn from the plots in Appendix D:

(1) The colloid particle-tracking model with exclusion layers along the fracture walls consistently offered better quantitative predictions of the experimental microsphere breakthrough curves than the particle-tracking model without exclusion layers. The model without exclusion layers generally underpredicted the experimental breakthrough curves. The effect of including exclusion layers became less important when the ratio of average aperture to exclusion layer thickness became larger. Thus, including exclusion layers in the Tram and Bullfrog tuff fractures had less effect on the predicted breakthrough curves than it did in the Bandelier tuff fracture. The parallel-plate version of the colloid particle-tracking model was in relatively good agreement with the experimental breakthrough curves, suggesting that this much simpler modeling approach may provide reasonable estimates of colloid transport in some situations. However, the parallel-plate model was incapable of predicting the differences in 
microsphere dispersion in the two different flow orientations in the Bullfrog tuff fracture.

(2) The colloid particle-tracking models did not predict the attenuation of the silica microspheres that was experimentally observed. If this attenuation was due to gravitational settling, the silica microspheres would have likely settled into pits or crevices along the bottom fracture wall where they could no longer be advected by the flow field. The particle-tracking models were unable to account for this phenomenon because it was assumed that the fracture walls were parallel plates.

(3) The solute particle-tracking model with stagnant layers along the fracture walls generally provided better predictions of the experimental iodide breakthrough curves than the other solute models. The Starr method consistently underpredicted the experimental breakthrough curves, presumably because it overpredicted diffusion into the matrix. The parallel-plate solute model was in relatively poor agreement with the experimental breakthrough curves, as it consistently underpredicted the amount of dispersion experienced by the iodide. An example corresponding to the Bandelier tuff fracture is shown in Fig. 4.29. Thus, for iodide it was clear that the use of the flow fields calculated from the variable-aperture representations of the fractures offered better predictions than were offered by parallel-plate representations. The reason for the greater discrepancy of the parallel-plate and variable-aperture predictions for the iodide relative to the microspheres is probably that the iodide more closely followed Taylor dispersion in the parallel-plate model and hence it had much lower predicted dispersion than the microspheres. In retrospect, it is probably somewhat coincidental that the microsphere dispersion in the parallel-plate model (which was entirely due to non-Taylor dispersion effects) was in relatively good agreement with the dispersion in the variable-aperture model(s). Over larger length or time scales, one would expect the two models to diverge because the relative colloid dispersion in the parallel-plate model should tend to decrease as the microspheres sample all of the streamlines across the flow channel while the dispersion in the variable-aperture model should increase in accordance with well-known theories of increasing flow field dispersion with increasing scale. 186

(4) The flow rate dependence of the experimental iodide breakthrough curves (i.e., the lower tails at lower flow rates when plotted on a volume eluted basis) was qualitatively predicted by the variable-aperture particle-tracking models. However, the tendency for the tails of the experimental breakthrough curves at the two lower flow rates to be much closer together than the breakthrough curves at the two higher flow rates was generally the opposite of what was predicted. An example corresponding to the Bandelier tuff fracture is shown in Fig. 4.30. It is not clear why this discrepancy between model and experiment occurred, but two speculations are offered: (1) the tails of the predicted breakthrough curves are somehow compromised by the uncertainty associated with sampling the extreme upper end of the tail of the residence time distributions for particle diffusion into the matrix (see Section 4.2.3), and/or (2) the iodide may experience a certain amount of anion exclusion from the negatively charged rock 


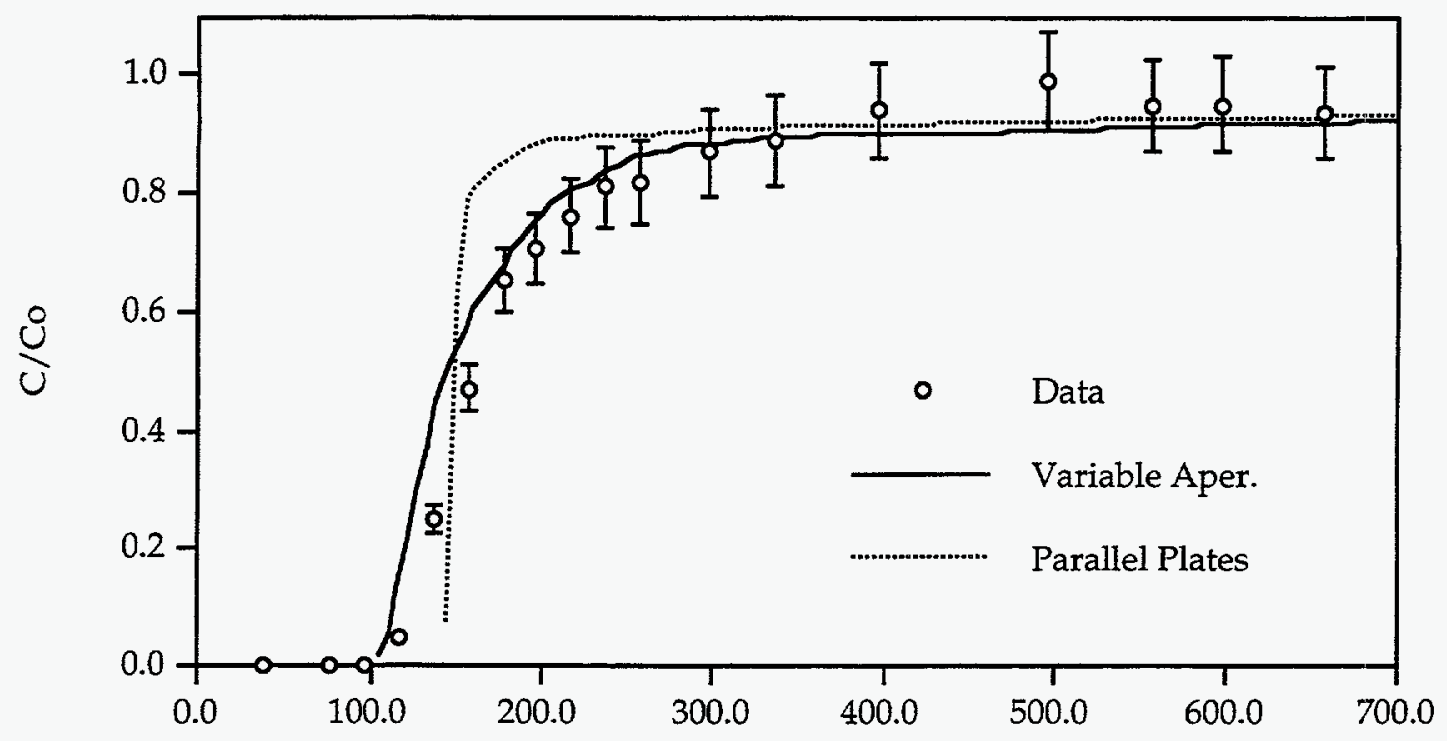

Time, sec

Figure 4.29. Predicted iodide breakthrough curves (based on 1000 particles) in the Bandelier tuff fracture using variable-aperture and parallel-plate models. The flow rate was $4.11 \mu \mathrm{l} / \mathrm{sec}$. The aperture data and calculated flow field from the second PEAK profile of this fracture were used to obtain the variable-aperture predictions. A transition layer thickness of $0.01 \mathrm{~mm}$ was assumed in the models (no stagnant layer). The experimental data are shown for comparison.

surfaces, 187,188 resulting in less matrix diffusion than predicted by the models, particularly at lower flow rates.

(5) The predicted breakthrough curves in the Bullfrog tuff fracture are in much better agreement with the experimental data after the damage to the fracture surfaces occurred than with the data before the damage occurred. This is understandable because the PEAK aperture data sets were obtained after the damage had occurred.

(6) The colloid particle-tracking method with exclusion layers along the fracture walls predicted the flow rate and size dependence of the microsphere breakthrough curves better than the method without exclusion layers. The differences between the model predictions and the experimental data for the $1-\mu \mathrm{m}$ and $0.3-\mu \mathrm{m}$ microspheres at a flow rate of $1.17 \mu \mathrm{l} / \mathrm{sec}$ are shown in Fig. 4.31. In the upper plot of Fig. 4.31, it is apparent that the predicted dispersion of the $1-\mu \mathrm{m}$ microspheres is significantly greater than that of the $0.3-\mu \mathrm{m}$ microspheres even though the data suggest little or no difference. The greater predicted dispersion of the larger microspheres is a direct result of their smaller diffusivity, which prevents them from sampling as many streamlines across the width of the fracture as the smaller microspheres. The lower plot of Fig. 4.31 shows that when exclusion layers are included in the particle-tracking models, the predicted dispersion of the two microspheres is almost the same, which is consistent with the 


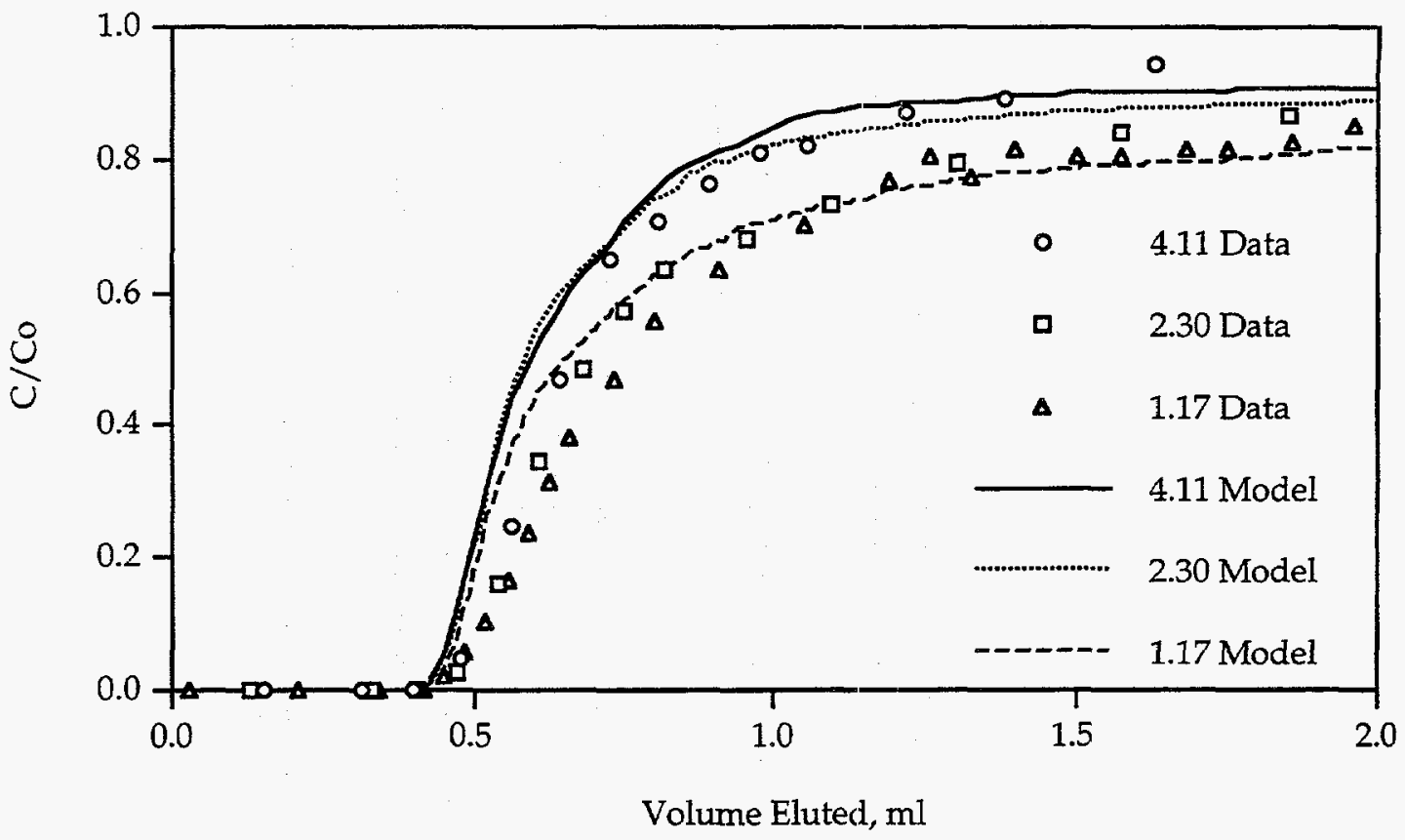

Figure 4.30. Predicted and experimental flow-rate dependence of iodide breakthrough curves in Bandelier tuff fracture. The predictions were made using the aperture data and calculated flow field from the second PEAK profile of this fracture. A transition layer thickness of $0.01 \mathrm{~mm}$ was assumed in the models (no stagnant layer). Numbers in legend refer to flow rates in $\mu \mathrm{l} / \mathrm{sec}$. Predictions were based on 1000 particles in the simulations.

experimental data. The predictions of the exclusion layer model are also quantitatively much better that those of the model without exclusion layers. Fig. 4.32 shows the differences between the model predictions and the experimental data for the $0.3-\mu \mathrm{m}$ microspheres at three different flow rates in the Bandelier tuff fracture. Here again, it is apparent that the particle-tracking model with exclusion layers predicts the trends in the experimental data (or the lack thereof) better than the model without exclusion layers. The exclusion layers obviously suppress some of the flow rate (and size) dependence of dispersion that is predicted when the particles have access to all of the streamlines in the velocity profile within the fracture. The Bandelier tuff fracture was used to make these comparisons because the differences in predicted dispersion were greatest for this fracture (a consequence of having a narrower aperture than the other fractures). (7) By examining the predicted breakthrough curves associated with the different aperture data sets for the Bandelier tuff fracture, two separate conclusions can be drawn about the appropriate conceptual model(s) to use for microsphere and iodide transport:

(a) By looking at the breakthrough curves associated with the laser profile and RTV cast data sets, it could be concluded that (1) the microspheres provide a measure of the mobile fluid residence time distribution in the fracture, and (2) the iodide is best modeled using a stagnant fluid layer 


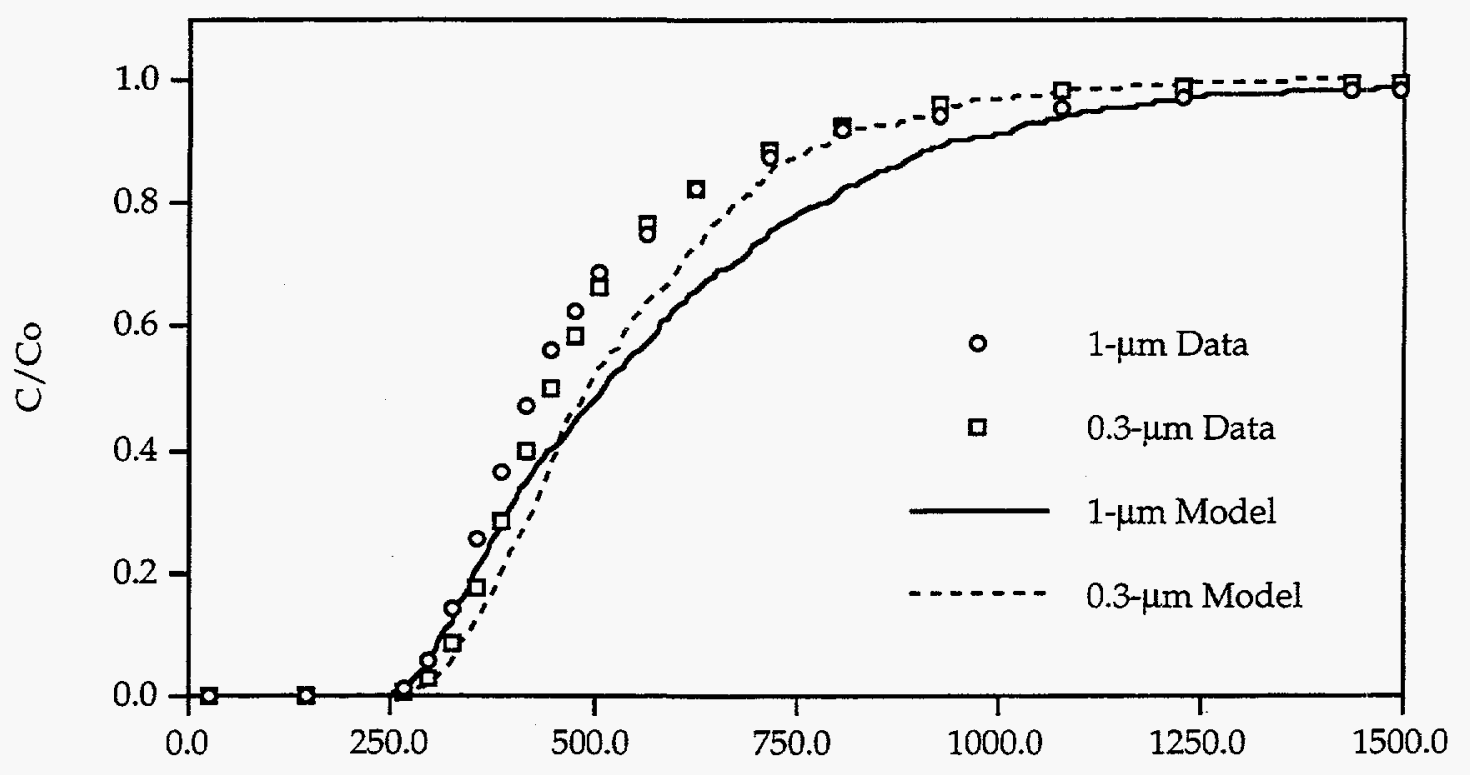

Time, sec

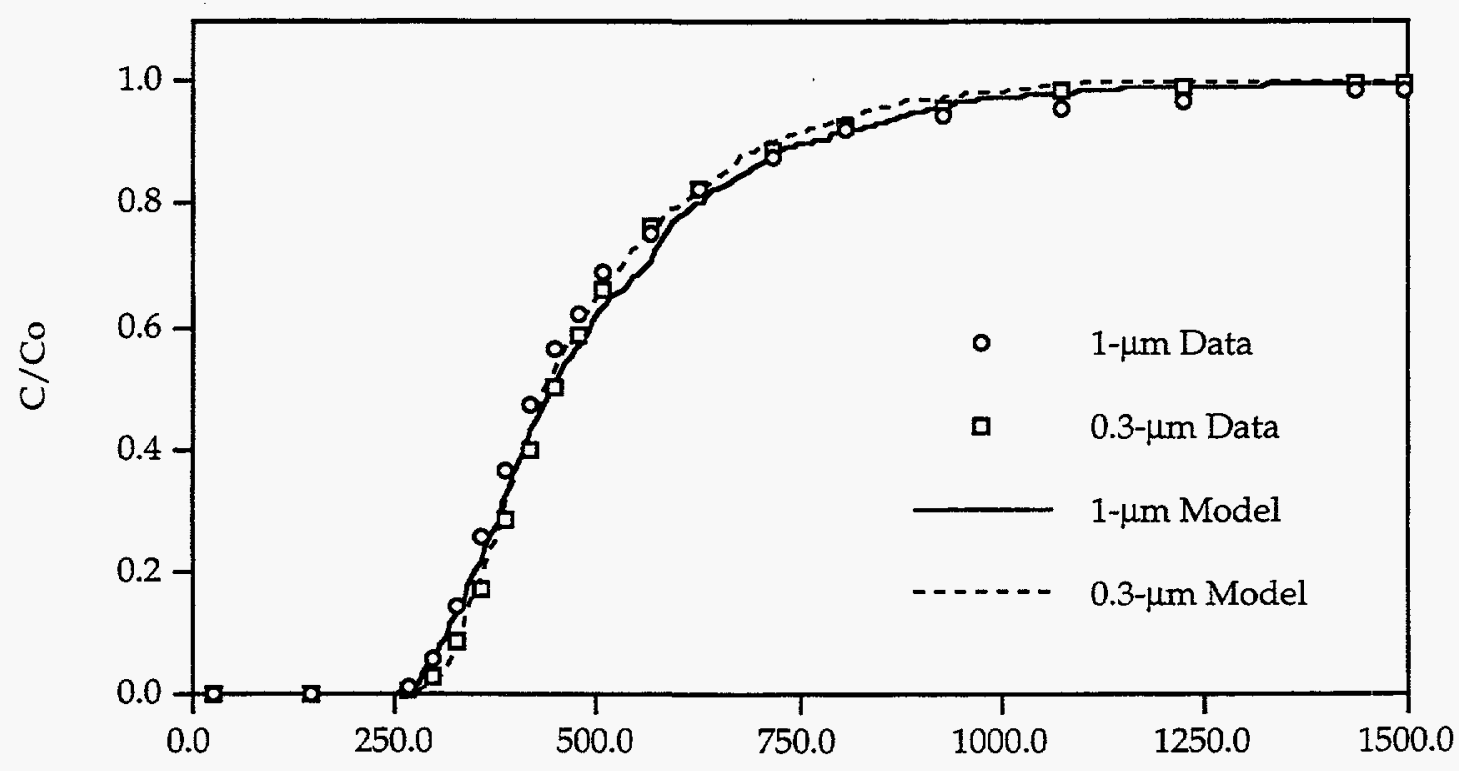

Time, sec

Figure 4.31. Predicted and experimental size dependence of CML microsphere breakthrough curves in Bandelier tuff fracture. Upper plot shows predictions of particle-tracking model without exclusion layers, and lower plot shows predictions of model with 0.005 - $\mathrm{mm}$ exclusion layers. The flow rate was 1.17 $\mu \mathrm{l} / \mathrm{sec}$. The predictions were made using the aperture data and calculated flow field from the second PEAK profile of this fracture. 


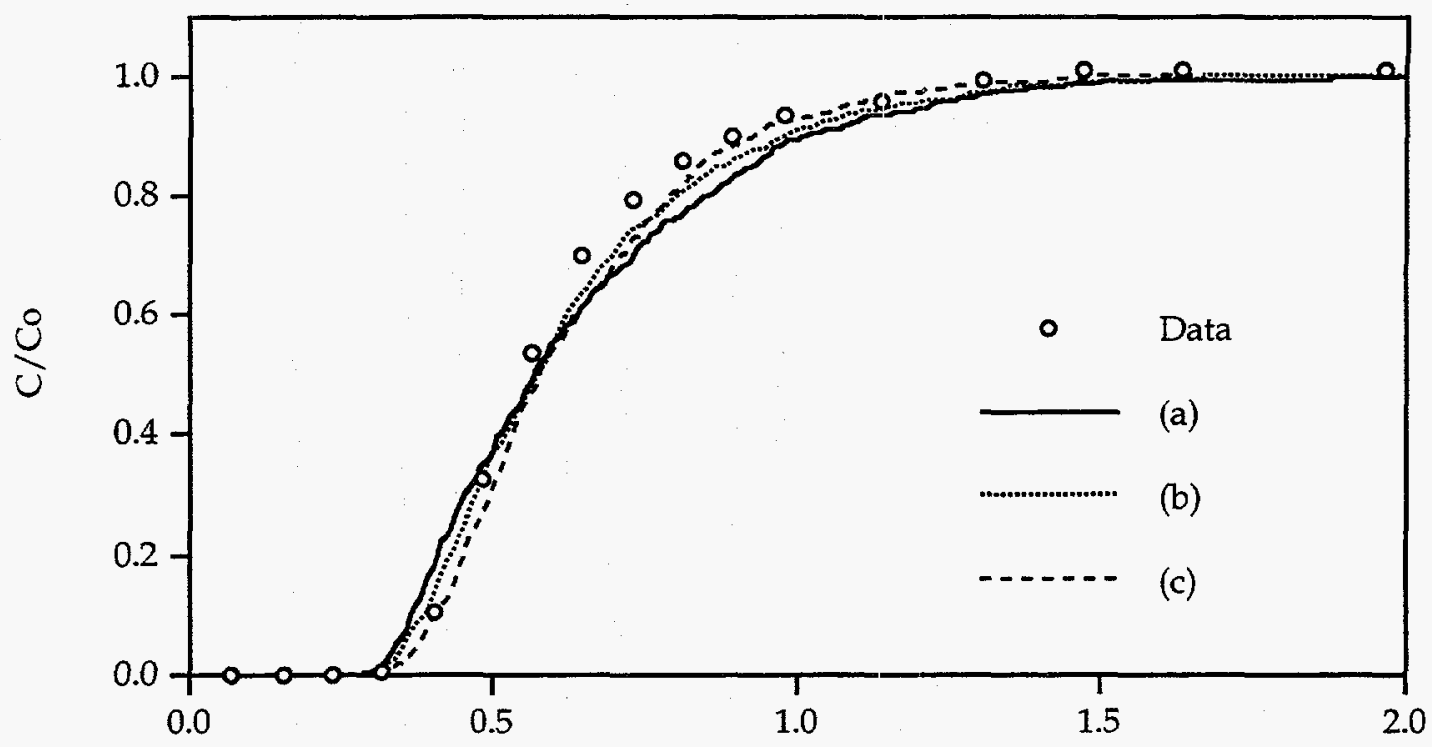

Volume Eluted, $\mathrm{ml}$

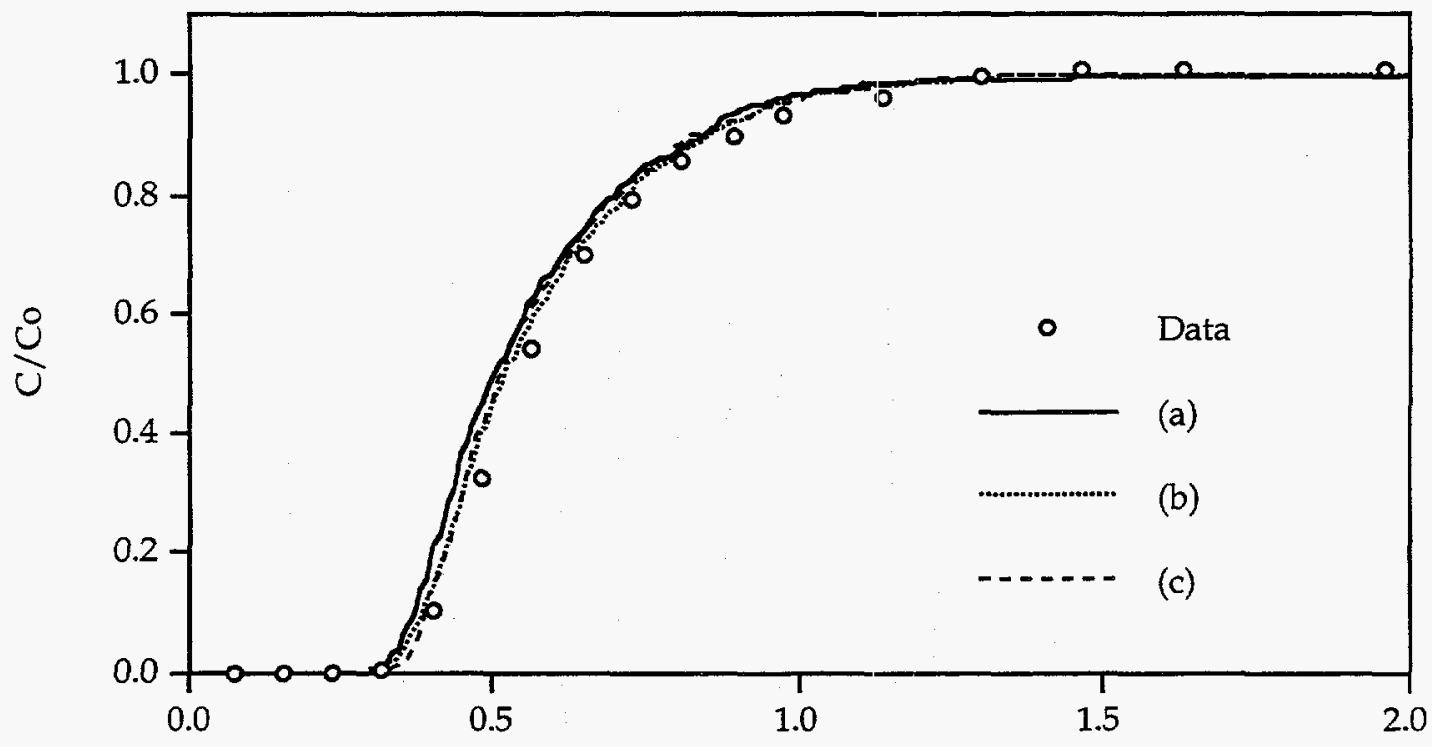

Volume Eluted, $\mathrm{ml}$

Figure 4.32. Predicted and experimental flow-rate dependence of $0.3-\mu \mathrm{m} C M L$ microsphere breakthrough curves in Bandelier tuff fracture. Upper plot shows predictions of particle-tracking model without exclusion layers, and lower plot shows predictions of model with $0.005-\mathrm{mm}$ exclusion layers. The flow rates were (a) $4.11 \mu \mathrm{l} / \mathrm{sec}$, (b) $2.30 \mu \mathrm{l} / \mathrm{sec}$, and (c) $1.17 \mu \mathrm{l} / \mathrm{sec}$. The predictions were made using the aperture data and calculated flow field from the second PEAK profile of this fracture. The experimental data at $4.11 \mu \mathrm{l} / \mathrm{sec}$ are shown for comparison (data at other flow rates were essentially the same). 
along the fracture walls and assuming matrix diffusion. In this case, the stagnant layers provide the explanation for the difference in the first arrival times of the microspheres and iodide, and matrix diffusion is the explanation for the lower tails of the iodide breakthrough curves.(b)

By looking at the breakthrough curves associated with the PEAK aperture data sets, it is apparent that the experimental microsphere breakthrough curves do not agree with the calculated fluid residence times as they did for the laser profile and RTV cast aperture data sets. In this case, it could be concluded that the differences in microsphere and iodide arrival times are primarily the result of differences in the dispersion of the two tracers resulting from diffusion across streamlines in the fracture (i.e., the microspheres tend to stay in fluid streamlines and the iodide tend to more closely follow Taylor dispersion). The differences in the tails of the breakthrough curves are still attributable to matrix diffusion of the iodide and perhaps some diffusion into stagnant zones along the fracture walls. Also, the microspheres are probably partially excluded from some of the fluid along the fracture walls (this assumption results in better "fits" to the experimental data). Figures 4.33 and 4.34 illustrate how the different modeling assumptions result in equally good agreement with the experimental data when different aperture data sets are used for the Bandelier tuff fracture.

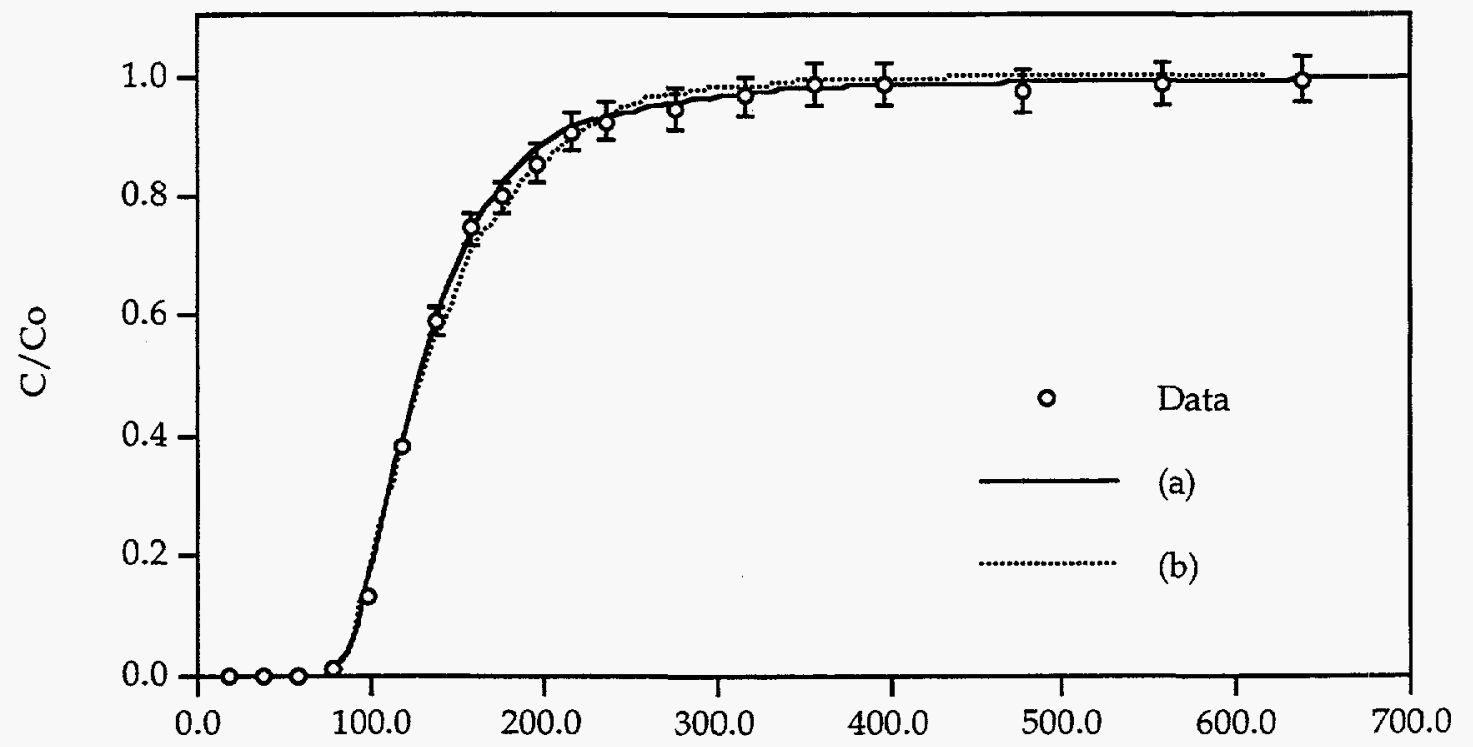

Time, sec

Figure 4.33. Comparison of (a) fluid residence time distribution in Bandelier tuff fracture predicted using aperture data and calculated flow field from laser profiling technique, (b) predicted 1- $\mu \mathrm{m}$ microsphere breakthrough curve (assuming a $0.005-\mathrm{mm}$ exclusion layer along the fracture walls) using the aperture data and calculated flow field from the first PEAK profile of the Bandelier tuff fracture, and (data) the experimental 1- $\mu \mathrm{m}$ CML microsphere breakthrough curve. The flow rate was $4.11 \mu \mathrm{l} / \mathrm{sec}$. 


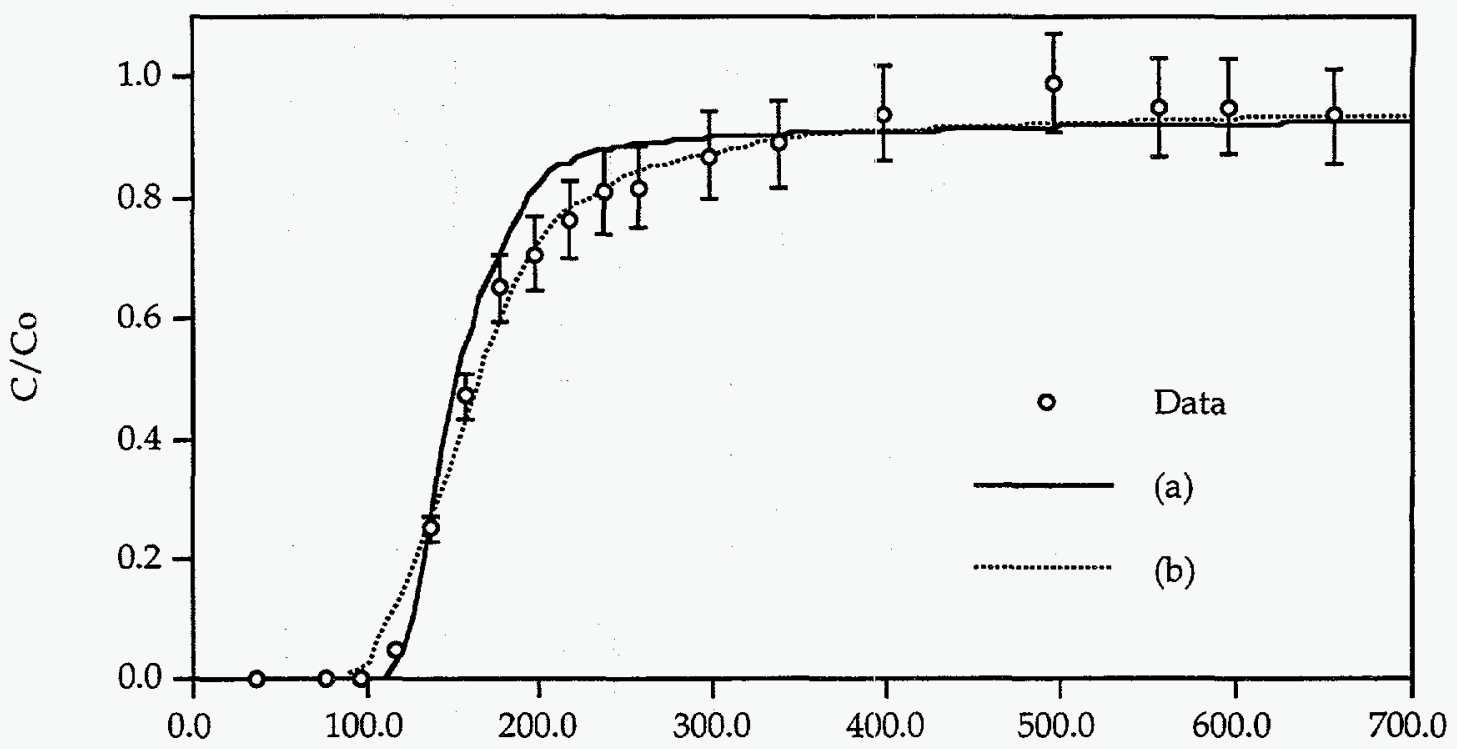

Time, sec

Figure 4.34. Comparison of (a) predicted iodide breakthrough curve assuming no stagnant layers and using the aperture data and calculated flow field from the first PEAK profile of the Bandelier tuff fracture, (b) predicted iodide breakthrough curve in the Bandelier tuff fracture assuming a $0.005-\mathrm{mm}$ stagnant layer along fracture walls and using the aperture data and calculated flow field from the RTV cast/imaging technique, and (data) the experimental iodide breakthrough curve. The flow rate was $4.11 \mu \mathrm{l} / \mathrm{sec}$.

Unfortunately, the experimental data from the Tram and Bullfrog tuff fractures do not allow the competing conceptual models to be sorted out. The smaller difference in the first arrival times of the microspheres and the iodide in these fractures compared to the Bandelier tuff fracture could be attributed to either (a) the fact that the stagnant regions along the fracture walls are a smaller fraction of the total volume in these larger aperture fractures, resulting in less difference in the volumes experienced by the two tracers, or (b) the inability of some of the iodide to diffuse across all the streamlines in the larger aperture fractures, resulting in an earlier iodide arrival time due to partial streamline flow. It is likely that the differences can be attributed to a combination of both phenomena. Perhaps the appropriate conceptual model(s) could be better sorted out if laser profile and/or RTV cast aperture data sets were available for the Tram and Bullfrog tuff fractures. It is speculated that Tram and Bullfrog tuff aperture data sets measured using different techniques would be in better agreement with each other than the Bandelier tuff aperture data sets because the ratio of measurement error to average aperture would be less in these fractures than in the Bandelier tuff fracture.

\subsubsection{Implications for Larger-Scale Tracer Experiments}

A key question that remains to be answered is: "What are the implications of the experimental results and modeling conclusions of this study when 
considering the use of nondepositing microspheres in large- or field-scale experiments to study the effects of matrix diffusion and/or colloid transport in fractured media?" To help answer this question, particle-tracking simulations were conducted in (1) a parallel-plate representation of the Bandelier tuff fracture and (2) a parallel-plate representation of a fracture that had the same average aperture but was 10 times longer than the Bandelier tuff fracture. The flow rates were assumed to be the same in the two cases so that the residence time was 10 times longer in the larger fracture. These simulations cannot be expected to show the effects of increasing dispersion over longer length scales (due to flow channeling in a variable-aperture fracture), but they should qualitatively predict the effects of matrix diffusion and diffusion across streamlines over different length scales.

The results of the simulations are shown in Fig. 4.35. The breakthrough curves in this figure are plotted as a function of void volumes eluted so that the curves associated with different length scales can be compared on an equivalent basis. It is apparent that matrix diffusion can result in both a delay in the first arrival time and a decrease in the height of the tail of a nonsorbing solute breakthrough curve as length scales increase. Nondepositing colloids, on the

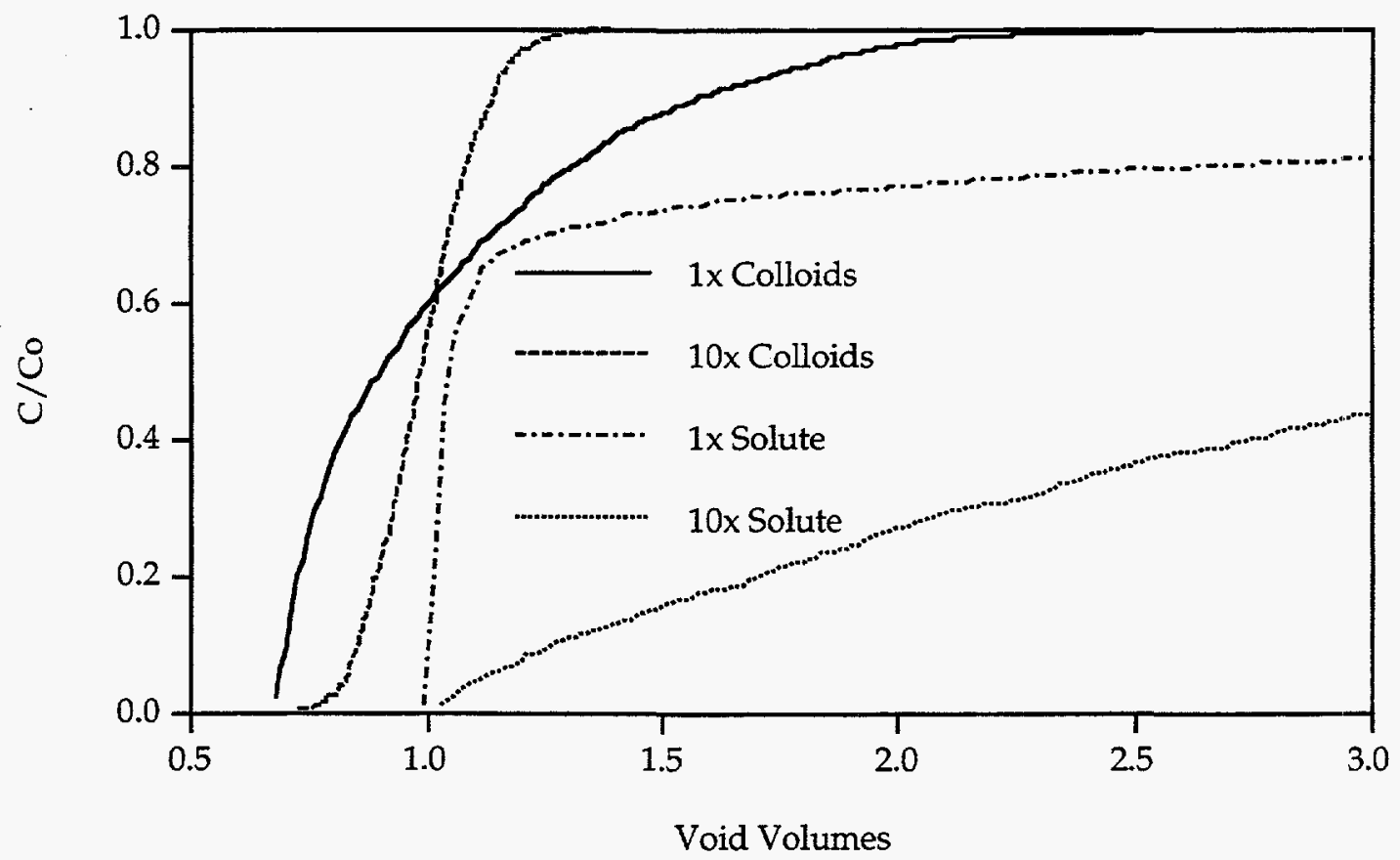

Figure 4.35. Predicted breakthrough curves (based on 1000 particles) of nondepositing $1-\mu \mathrm{m}$ colloids and a nonsorbing solute over different length scales in a parallel-plate channel. Predictions were made using particletracking models without stagnant layers or exclusion layers. Channel aperture was $\sim 55-\mu \mathrm{m}$ (corresponding to the mean aperture in the Bandelier tuff fracture), and the volumetric flow rate was $1.17 \mu \mathrm{l} / \mathrm{sec}$. "1x" refers to a channel with the same length and width as the Bandelier tuff fracture, and "10x" refers to a channel with the same width but 10 times the length of the Bandelier tuff fracture. 
other hand, simply experience a decrease in dispersion over longer length and time scales because they have more time to diffuse across the streamlines in the fracture. Although it is unlikely that dispersion would actually decrease with length scale in a variable-aperture fracture, the significant implication of Fig. 4.35 is that, over longer length scales, colloids should tend to more closely follow the mobile fluid residence time distribution in a fracture. Thus, longer length scales should actually increase the validity of assuming that nondepositing colloids provide a measure of the mobile fluid residence time in a fracture. Also, it is expected that the breakthrough behavior of a nonsorbing solute will be dominated by matrix diffusion and/or diffusion into stagnant zones along the fracture walls as length scales increase. These conclusions should hold for fracture networks as well as single fractures. Of course, it is critical that both the colloid and solute tracers do not significantly deposit onto or adsorb to the fracture surfaces, or the interpretation of the experiments will be significantly more complicated. The results of the laboratory-scale tracer experiments conducted in this study suggest that the CML microspheres are good candidates for nondepositing colloid tracers because they were essentially completely recovered in the experimental systems.

Another issue of potential importance for larger-scale experiments is the applicability of using the Starr method to predict: solute breakthrough curves over large length scales. This method offers significant computational advantages over the more sophisticated particle-tracking approach. It was hoped that it could be demonstrated that predictions from the Starr and particletracking methods tend to approach each other as length scales increase because solutes should more closely follow Taylor dispersion over longer scales and there should also be a larger number of particle-wall collisions over longer scales. Both of these trends should result in the solute more closely satisfying the assumptions of the Starr method. To investigate the differences between the two modeling approaches over different scales, simulations were conducted for the same two parallel-plate fractures (and same flow conditions) as described above for the comparison of colloid and solute breakthrough curves over different length scales.

Fig. 4.36 shows the results of the nonsorbing solute simulations using the Starr and particle-tracking methods at the two different length scales. Unfortunately, although both methods predict the same trends with scale, the Starr method predicts significantly lower concentrations at both length scales, and there is no evidence of a "convergence" of the two approaches. The discrepancies between the two methods should be studied further if serious consideration is given to using the Starr method to predict large-scale transport behavior in fractures. The discrepancies could be partly due to the uncertainties associated with sampling the extreme upper ends of the tails of the residence time distributions for particle diffusion into the matrix in the particle-tracking calculations (discussed in Section 4.2.3). However, it is also likely that the assumptions implicit in the Starr method (no velocity or concentration gradients in the fracture void space) result in more particle-wall collisions per unit length of fracture than in the particle-tracking method. This difference would tend to produce the trends observed in Fig. 4.36. Of course, as mentioned in Section 4.2.3, the particle-tracking method can be made to agree with the Starr method 
by simply adjusting the assumed thickness of the transition layer in the matrix or by changing other somewhat arbitrary assumptions in the model(s).

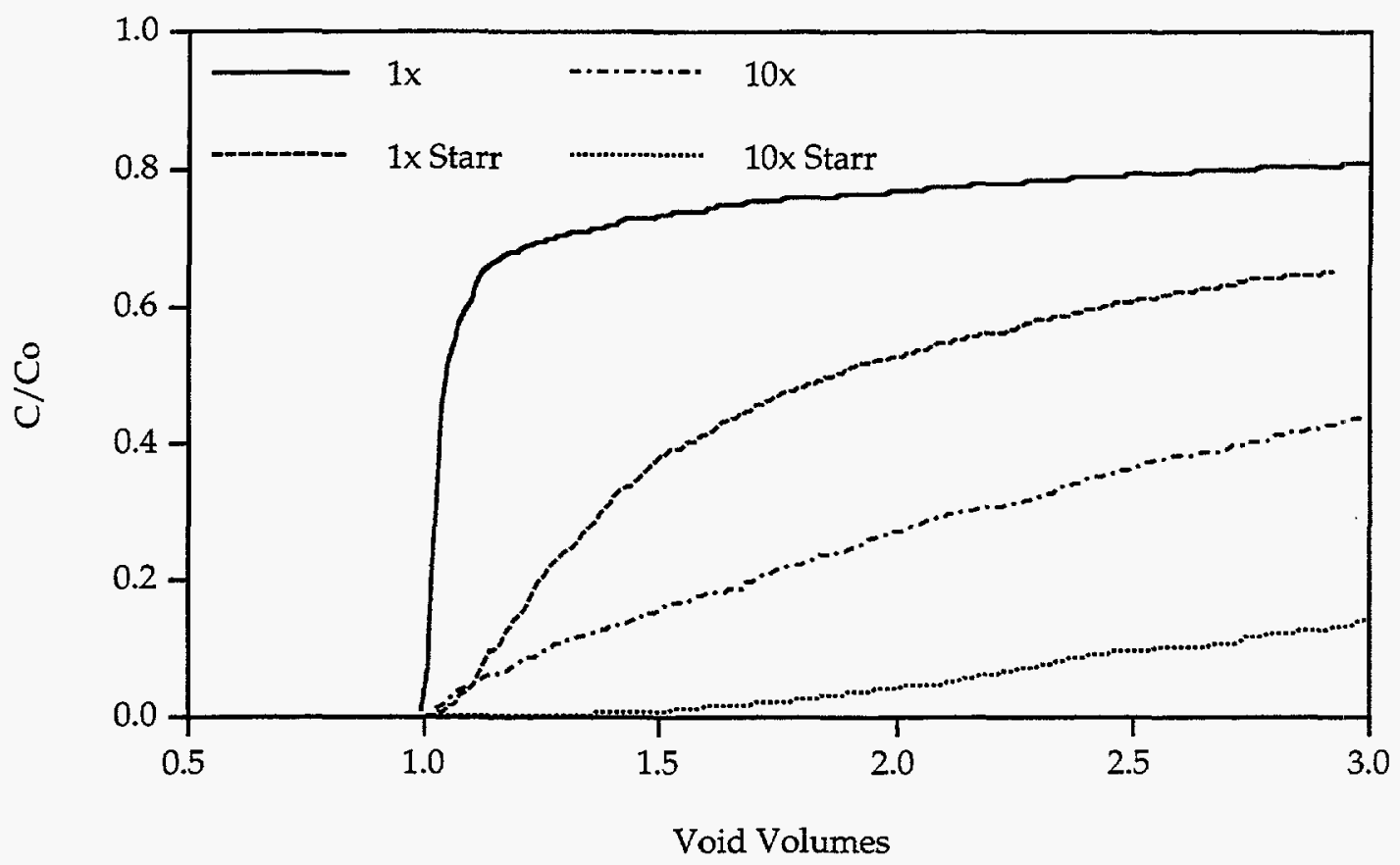

Figure 4.36. Predicted breakthrough curves of a nonsorbing solute over different length scales in a parallel-plate channel using the particle-tracking and Starr methods. The channel aperture and flow rate are the same as in Fig. 4.35. " $1 x "$ refers to a channel with the same length and width as the Bandelier tuff fracture, and "10x" refers to a channel with the same width but 10 times the length of the Bandelier tuff fracture. "Starr" refers to predictions of the Starr model. 1000 particles were used in the particle-tracking simulations, and 2000 particles were used in the Starr model simulations. 


\subsection{CONCLUSIONS}

The research conducted for this dissertation resulted in the following conclusions:

(1) The CML microspheres were identified as the best candidates for nondepositing colloid tracers in large- or field-scale tracer experiments because they were essentially completely recovered in small-scale fracture experiments. Non-CML polystyrene microspheres with carboxyl surfaces and silica microspheres were both significantly attenuated in the small-scale experiments. However, the silica attenuation was less certain than that of the non-CML microspheres because of delays in analyzing the samples and also because of the rather large uncertainty associated with the analytical technique (turbidity).

(2) Flow cytometry worked superbly as a method of detecting and quantitatively measuring concentrations of fluorescently-tagged microspheres in solutions. This method would be ideal for field-scale tracer experiments because it would allow quantitative analyses of low concentrations of tracer colloids in the presence of a large background of natural colloidal material.

(3) The results of the tracer experiments in the single fractures and the subsequent flow-field and particle-tracking simulations to predict/interpret the experiments led to the following conclusions:

(a) There were significant discrepancies between the predicted breakthrough curves using the aperture data sets obtained by different measurement techniques for the same fracture, which indicated that there was a considerable amount of uncertainty in the measurements. However, the breakthrough curve predictions using the aperture data sets were in better qualitative agreement with the experimental results than predictions based on simple parallel-plate models. Also, the predicted trends of tracer dispersion in the different fractures were in qualitative agreement with the observed experimental trends. There was better quantitative agreement between the model predictions and the experimental data when (1) iodide was assumed to have access to a stagnant layer of fluid along the fracture walls and (2) the microspheres were assumed to be excluded from a thin layer of fluid along the fracture walls. These assumptions tended to increase the predicted dispersion and average residence time of the iodide and to decrease the predicted dispersion and average residence time of the microspheres.

(b) The first arrival of microspheres in the tracer experiments was consistently earlier than that of the iodide because of one or a combination of the following: (1) the low-diffusivity microspheres tended to remain in high-velocity fluid streamlines near the centerline of the flow channel longer than the iodide (that is, iodide followed Taylor dispersion more closely than the microspheres), and (2) the iodide experienced a greater effective volume and longer residence time in the fractures than the microspheres because it had access to more stagnant or low-velocity regions along the fracture walls (as a result of being smaller and more diffusive than the microspheres).

(c) The iodide breakthrough curves had longer tails than the microsphere breakthrough curves because the iodide diffused into the pores of the 
rock matrix, whereas the colloids were too large and / or too slowly diffusing to experience this phenomenon.

(d) The dispersion of all tracers tended to be greater in fractures having larger average apertures and greater aperture correlation lengths (estimated by visual examination of the aperture data sets). These aperture characteristics resulted in predicted flow fields that had a smaller number of high-velocity flow channels through the fractures. Other investigators have also predicted greater dispersion in such flow fields. ${ }^{88-90,189}$ However, dispersion due to diffusion across streamlines (or lack thereof) in the assumed parabolic velocity profiles is also expected to be greater in larger aperture fractures, so this phenomenon may offer a partial explanation for the dependence of dispersion on average aperture.

(4) By using particle-tracking models to extrapolate the experimental results to larger scales, it was concluded that, as scales increase,

(a) nondepositing microspheres should provide an increasingly better estimate of the mobile fluid residence time distribution in a fractured system, and

(b) matrix diffusion will dominate the behavior of nonsorbing solutes in such systems (assuming a large enough matrix porosity and/or a long enough time or length scale).

These conclusions suggest that it should be possible to study the effects of solute matrix diffusion in saturated, fractured systems by comparing the breakthrough curves of nondepositing colloids and nonsorbing solutes.

(5) Nondepositing colloid tracers should prove useful in investigations of the effects of deposition and perhaps gravitational settling, coagulation, and other attenuating phenomena on the transport of "real" colloids in saturated, fractured media. The nondepositing colloid breakthrough curves should provide a measure of colloid dispersion in the system in the absence of the attenuating phenomena, which would allow the effects of the phenomena on the "real" colloids to be studied in a less ambiguous manner than current approaches offer. 


\subsection{RECOMMENDATIONS}

Based on the results and conclusions presented in this dissertation, numerous recommendations can be made for further study:

(1) It would be informative to conduct additional tracer experiments in single fractures with silica microspheres to try to determine their mechanism of attenuation (or even if they are significantly attenuated). There are at least two ways this could be done. First, separate experiments could be conducted in the same fracture in both horizontal and vertical orientations so that any differences in the breakthrough curves could be attributed to gravitational settling. Second, the silica microspheres could be tagged with fluorescent dyes $190, *$ before being used in tracer experiments. This would allow the microspheres to be analyzed precisely by flow cytometry, and the fracture surfaces could be easily and quickly examined under UV light (post-test) for attenuated microspheres.

(2) Because of the advantages offered by flow cytometry in determining colloid concentrations in solution samples, it would be worthwhile to develop ways of tagging colloids other than those used in this study with fluorescent dyes so that they could be used in future tracer experiments in either the laboratory or the field. If tagged colloids that have characteristics of natural colloids could be developed, the effects of various attenuation mechanisms on the colloids could be studied by comparing their breakthrough curves with those of nondepositing colloids.

(3) A possible experimental improvement would be the use of pulsation-free pumps (e.g., large piston pumps manufactured by ISCO, Inc. of Lincoln, NB) to force solutions through the fractures. Pulsations in the tracer experiments should have been minimal, but: completely pulsation-free flow is desirable to better simulate natural conditions and/or conditions in forced-gradient field-scale tracer experiments. Also, pulsations may influence the amount of attachment of microspheres to surfaces (possibly decreasing the amount of attachment that would occur in the absence of pulsations).

(4) It would be informative to estimate the aperture distributions in the Tram and Bullfrog tuff fractures using the laser profiling and/or RTV cast/imaging techniques to see if the differences between these techniques and the PEAK profiling technique follow the same trends for these fractures as they did for the Bandelier tuff fracture. RTV silicone casts of the Tram and Bullfrog tuff fractures have already been prepared.

(5) Better characterization of the aperture data sets (e.g., calculation of statistical parameters such as correlation lengths in various directions ${ }^{170}$ ) should be conducted so that the observed tracer dispersion in the experiments might be more quantitatively related to the aperture characteristics.

(6) Quantitative mineralogical characterization of the three fractures used in the tracer experiments should be completed (with emphasis on how the mineralogy of the fracture surfaces differs from that of the matrices).

(7) To better understand and quantify colloid-sirface interactions, an experimental technique similar to that used by Sjollema and Busscher ${ }^{151}$

- Personal communication with $\mathrm{H}$. Eric Nuttall of the University of New Mexico, where techniques of synthesizing fluorescently-tagged silica microspheres are being developed (1994). 
and Meinders et al., 154 in which colloids are observed in real time in a parallel-plate flow channel, should be considered. Such an experimental flow system could conceivably be set up with one transparent plate and one "plate" consisting of a rock surface. Epifluorescence microscopy could be used to detect the movement of colloids near the rock surface and study the colloid-rock interactions under different flow conditions. Visualization experiments such as these would provide valuable insights into colloid-rock interactions.

(8) Tracer transport experiments in epoxy replicas of fractures, prepared as described by Persoff et al. 191 , should be considered. If carefully made, these replicas should have approximately the same void space geometry as the real fractures from which they are cast, but they will have a nonporous matrix. Thus, matrix diffusion should be eliminated as a mechanism affecting solute transport behavior in the replicas, and the resulting breakthrough curves could be compared with breakthrough curves from the real fractures to deduce the effects of solute matrix diffusion. Also, experiments in such replicas might yield additional insights into the appropriate conceptual model(s) to use for colloid transport in fractures.

(9) It would be desirable to develop a capability to specify a linear pressure drop across the inlet and/or outlet boundaries of the fracture domain in the flow-field calculations so that the effects of a pressure drop across the flow manifolds could be investigated. Although it is believed that the pressure drops across the outlet manifolds did not affect the experimental results (because the microsphere breakthrough curves showed no dependence on either the fracture or manifold flow rates), it would be interesting to examine the predicted sensitivity of the breakthrough curves to this phenomenon. This type of sensitivity analysis might help in the design of future tracer experiments.

(10) It would be desirable to explore other computational approaches to simulating the flow field in a variable-aperture fracture, particularly approaches that relax some of the assumptions in the Reynolds equation approximation. One possibility is the use of lattice-gas and/or latticeBoltzmann calculations, ${ }^{85}$ which are gaining popularity in solving a wide variety of small-scale flow and transport problems that have difficult boundaries. The deduced apertures could be linearly interpolated in 2dimensions to generate a variable-aperture flow region that does not have local parallel plates. However, the ability of this or any other method to provide better approximations of fracture flow fields is likely to be limited by the errors/uncertainties in the aperture data.

(11) The problems associated with sampling the extreme upper ends of the tails of the residence time distributions for particle diffusion into the matrix (see Section 4.2.3) require further attention if particle-tracking methods are to be used to quantitatively predict solute transport through saturated fractures. Specifically, the characteristics of the tails must be more precisely determined, and the method of sampling the tails must be shown to be unbiased.

(12) The differences between the solute transport predictions of the Starr ${ }^{185}$ and particle-tracking methods should be further investigated so that the 
limitations of each model and the conditions under which each model is applicable are better understood. 


\section{REFERENCES}

1. Novakowski, K., Evans, G. V., Lever, D. A., and Raven, K. G. "A Field Example of Measuring Hydrodynamic Dispersion in a Single Fracture," Water Resour. Res. 21(8), 1165-1174 (1985).

2. Raven, K. G., Novakowski, K. S., and Lepcevic, P. A. "Interpretation of Field Tracer Tests of a Single Fracture Using a Transient Solute Storage Model," Water Resour. Res. 24(12), 2019-2032 (1988).

3. Shapiro, A. M. and Nicholas, J. R. "Assessing the Validity of the Channel Model of Fracture Aperture under Field Conditions," Water Resour. Res. 25(5), 817-828 (1989).

4. Cacas, M. C., Ledoux, E., DeMarsily, G., Tillie, B., Barbreau, A., Durand, E., Feuga, B., and Peaudecerf, P. "Modeling Fracture Flow with a Stochastic Discrete Fracture Network: Calibration and Validation 1. The Flow Model," Water Resour. Res. 26(3), 479-489 (1990).

5. Abelin, H., Birgersson, L., Gidlund, J., and Neretnieks, I. "A Large-Scale Flow and Tracer Experiment in Granite 1. Experimental Design and Flow Distribution," Water Resour. Res. 27(12), 3107-3117 (1991).

6. Abelin, H., Birgersson, L., Widen, H., Agren, T., Moreno, L., and Neretnieks, I. "Channeling Experiments in Crystalline Fractured Rocks," J. Contam. Hydrol. 15 129-138 (1994).

7. Davison, C. C., Frost, L. H., Kozak, E. T., Scheier, N. W., and Martin, C. D. "Groundwater Transport Characteristics of Fracture Zones in a Granite Batholith," in High-Level Radioactive Waste Management, Proceedings of the 4th Annual International Conference, American Nuclear Society, LaGrange Park, Illinois, pp. 418-424 (1993).

8. Cady, C. C., Silliman, S. E., and Shaffern, E. "Variation in Aperture Estimate Ratios from Hydraulic and Tracer Tests in a Single Fracture," Water Resour. Res. 29(9), 2975-2982 (1993).

9. Birgersson, L., Moreno, L., and Neretnieks, I. "A Tracer Migration Experiment in a Small Fracture Zone in Granite," Water Resour. Res. 29(12), 3867-3878 (1993).

10. Novakowski, K. S. and Lepcevic, P. A. "Field Measurement of Radial Solute Transport in Fractured Rock," Water Resour. Res. 30(1), 37-44 (1994).

11. Means, J. L., Crerar, D. A., and Duguid, J. O. "Migration of Radioactive Wastes: Radionuclide Mobilization by Complexing Agents," Science 200, 1477-1481 (1978). 
12. Killey, R. W. D., McHugh, J. O., Champ, D. R., Cooper, E. L., and Young, J. L. "Subsurface Cobalt-60 Migration from a Low-Level Waste Disposal Site," Environ. Sci. Technol., 18(3), 148-157 (1984).

13. Nyhan, J. W., Drennon, B. J., Abeele, W. V., Wheeler, M. L., Purtymun, W. D., Trujillo, G., Herrara, W. J., and Booth, J. W. "Distribution of Plutonium and Americium Beneath a 33-yr-old Liquid Waste Disposal Site," J. Environ. Qual., 14(4), 501-508 (1985).

14. Buddemeier, R. W. and Hunt, J. R. "Transport of Colloidal Contaminants in Groundwater: Radionuclide Migration at the Nevada Test Site," Appl. Geochem., 3, 535-548 (1988).

15. Penrose, W. R., Polzer, W. L., Essington, E. H., Nelson, D. M., and Orlandini, K. A. "Mobility of Plutonium and Americium through a Shallow Aquifer in a Semiarid Region," Environ. Sci. Technol., 24(2), 228-234 (1990).

16. Payne, T. E., Edis, R., and Seo, T. "Radionuclide Transport by Groundwater Colloids at the Koongarra Uranium Deposit," Mat. Res. Soc. Symp. Proc., 257, 481-488 (1992).

17. Kim, J. I., Buckau, G., Baumgartner, F., Moon, H. C., and Lux, D. "Colloid Generation and the Actinide Migration in Gorleben Groundwaters," Mat. Res. Soc. Symp. Proc., 26, 31-40 (1984).

18. Kim, J. I., Buckau, G., and Klenze, R. "Natural Colloids and Generation of Actinide Pseudocolloids in Groundwater," in Natural Analogues in Radioactive Waste Disposal, B. Come and N. Chapman, eds., pp. 289-299, Graham + Trotman, London (1987).

19. Kim, J. I., Buckau, G., Rommel, H. and Sohnius, B. "The Migration Behaviour of Transuranium Elements in Gorleben Aquifer Systems: Colloid Generation and Retention Process," Mat. Res. Soc. Symp. Proc., 127, 849-854 (1989).

20. Dearlove, J. P. L., Longworth, G., Ivanovich, M., Kim, J. I., Delakowitz, B., and Zeh, P. "A Study of Groundwater-Colloids and their Geochemical Interactions with Natural Radionuclides in Gorleben Aquifer Systems," Radiochim. Acta, 52/53(P1), 83-89 (1991).

21. Champ, D. R., Merritt, W. F., and Young, J. L. "Potential for the Rapid Transport of Plutonium in Groundwater as Demonstrated by Core Column Studies," Mat. Res. Soc. Symp. Proc. 11, 745-754 (1982).

22. Saltelli, A., Avogadro, A. and Bidoglio, G. "Americium Filtration in Glauconitic Sand Columns," Nuclear Tech., 67, 245-254 (1984).

23. Thompson, J. L. "Actinide Behavior on Crushed Rock Columns," J. Radioanal. and Nucl. Chem. 130(2), 353-364 (1989). 
24. Magee, B. R., Lion, L. W., and Lemley, A. T. "Transport of Dissolved Organic Macromolecules and Their Effect on the Transport of Phenanthrene in Porous Media," Environ. Sci. Technol. 25(2), 323-331 (1991).

25. Neretnieks, I. "Diffusion into the Rock Matrix: An Important Factor in Radionuclide Retardation?," J. Geophys. Res. 85, 4379-4397 (1980).

26. Rasmuson, A. and Neretnieks, I. "Migration of Radionuclides in Fissured Rock: The Influence of Micropore Diffusion and Longitudinal Dispersion," J. Geophys. Res., 86(B5), 3749-3758 (1981).

27. Neretnieks, I. "A Note on Fracture Flow Dispersion Mechanisms in the Ground," Water Resour. Res. 19(2), 364-370 (1983).

28. Maloszewski, P. and Zuber, A. "On the Theory of Tracer Experiments in Fissured Rock with a Porous Matrix," J. Hydrol. 79, 333-358 (1985).

29. Neretnieks, I. "Some Concepts of Transport and Interaction of Dissolved Species in Fractured Rock," in Flow and Contaminant Transport in Fractured Rock, Bear, J., Tsang, C-F. and DeMarsily, G., eds., pp. 45-52, Academic Press, New York (1993).

30. Smith, P. A. and Degueldre, C. "Colloid-Facilitated Transport of Radionuclides through Fractured Media," J. Contam. Hydrol. 13, 143-166 (1993).

31. Grindrod, P. "The Impact of Colloids on the Migration and Dispersal of Radionuclides within Fractured Rock," J. Contam. Hydrol. 13, 167-181 (1993).

32. Kim, J. I. "Actinide Colloid Generation in Groundwater," Radiochim. Acta, 52/53(P1), 71-81 (1991).

33. Triay, I., Simmons, A., Levy, S., Nuttall, H., Robinson, B., Steinkampf, W., and Viani B. "Colloid-Facilitated Radionuclide Transport at Yucca Mountain," LA-12779-MS, Los Alamos National Laboratory, Los Alamos, NM (in press).

34. McCarthy, J. F. and Zachara, J. M. "Subsurface Transport of Contaminants," Environ. Sci. Technol. 23(5), 496-502 (1989).

35. Moulin, V. and Ouzounian, G. "Role of Colloids and Humic Substances in the Transport of Radionuclides through the Geosphere," Appl. Geochem., Suppl. Issue No. 1, 179-186 (1992).

36. Choppin, G. R. "The Role of Natural Organics in Radionuclide Migration in Natural Aquifer Systems," Radiochim. Acta 58/59, 113-120 (1992). 
37. Levenspiel, O. Chemical Reaction Engineering, 2nd ed. John Wiley and Sons, New York (1972).

38. Maloszewski, P. and Zuber, A. "Mathematical Modeling of Tracer Behavior in Short-Term Experiments in Fissured Rocks," Water Resour. Res. 26(7), $1517-1528$ (1990).

39. Maloszewski, P. and Zuber, A. "Tracer Experiments in Fractured Rocks: Matrix Diffusion and the Validity of Models," Water Resour. Res. 29(8), 2723-2735 (1993).

40. Neretnieks, I., T. Eriksen, and P. Tahtinen. "Tracer Movement in a Single Fissure in Granitic Rock: Some Experimental Results and Their Interpretation," Water Resour. Res. 18(4), 849-858 (1982).

41. Rundberg, R. S., Thompson, J. L., and Maestas, S. "Radionuclide Migration: Laboratory Experiments with Isolated Fractures," Scientific Basis for Nuclear Waste Management, Vol. 6, pp. 239-248, North-Holland, New York (1982).

42. Rundberg, R., Ogard, A. E., Mroz, E. J., and Mitchell, A. J. "Fracture Flow under Saturated Conditions," NNWSI Milestone Report M318, Los Alamos National Laboratory, Los Alamos, NM (1986).

43. Rundberg, R. S., D. R. Janecky, M. Ott, and A. Mitchell. "Dynamic Transport of Colloidal Tracers through Fractured Tuff: 0.10 to $9.55 \mu \mathrm{m}$ Diameter Carboxylated Polystyrene Spheres," NNWSI Milestone Report R743, Los Alamos National Laboratory, Los Alamos, NM (1989).

44. Janecky, D. R., Rundberg, R. S., Ott, M., and Mitchell, A. "Update Report on Fracture Flow in Saturated Tuff: Dynamic Transport Task for the Nevada Nuclear Waste Investigations," LA-11957-MIS, Los Alamos National Laboratory, Los Alamos, NM, (1990).

45. Bales, R. C., Gerba, C. P., Grondin, G. H., and Jensen, S. L. "Bacteriophage Transport in Sandy Soil and Fractured Tuff," Appl. Environ. Microbiol. 55(8), 2061-2067 (1989).

46. Piggott, A. R. and Elsworth, D. "Laboratory Studies of Transport within a Single Rock Fracture," in Rock Joints, Barton and Stephansson, eds., Balkema, Rotterdam, pp. 397-404 (1990).

47. Holtta, P., Hautojarvi, A., and Hakanen, M. "Transport and Retardation of Non-Sorbing Radionuclides in Crystalline Rock Fractures," Radiochim. Acta 58/59, 285-290 (1992).

48. Cliffe, K. A., Gilling, D., Jefferies, N. L., and Lineham, T. R. "An Experimental Study of Flow and Transport in Fractured Slate," J. Contam. Hydrol. 13, 73-90 (1993). 
49. Robinson, B. A. and Tester, J. "Dispersed Fluid Flow in Fractured Reservoirs: An Analysis of Tracer Determined Residence Time Distributions," J. Geophys. Res. 89(B12), 10374-10384 (1984).

50. Moreno, L. and Neretnieks, I. "Flow and Nuclide Transport in Fractured Media: The Importance of the Flow-Wetted Surface for Radionuclide Migration," J. Contam. Hydrol. 13, 49-71 (1993).

51. McKay, L. D., Cherry, J. A., Bales, R. C., Yahya, M. T., and Gerba, C. P. "A Field Example of Bacteriophage as Tracers of Fracture Flow," Environ. Sci. Technol. 27, 1075-1079 (1993).

52. McKay, L. D., Gillham, R. W., and Cherry, J. A. "Field Experiments in a Fractured Clay Till 2. Solute and Colloid Transport," Water Resour. Res. 29(12), 3879-3890 (1993).

53. Harvey, R. W., George, L. H., Smith, R. L., and LeBlanc, D. R. "Transport of Microspheres and Indigenous Bacteria through a Sandy Aquifer: Results of Natural- and Forced-Gradient Tracer Experiments," Environ. Sci. Technol. 23(1), 51-56 (1989).

54. Harvey, R. W., Kinner, N. E., MacDonald, D., Metge, D. W., and Bunn, A. "Role of Physical Heterogeneity in the Interpretation of Small-Scale Laboratory and Field Observations of Bacteria, Microbial-Sized Microsphere, and Bromide Transport through Aquifer Sediments," Water Resour. Res. 29(8), 2713-2721 (1993).

55. Tobiason, J. E. and O'Melia, C. R. "Physicochemical Aspects of Particle Removal in Depth Filtration," Journal AWWA 80(Dec.), 54-64 (1988).

56. Elimelech, M. and O'Melia, C. R. "Kinetics of Deposition of Colloidal Particles in Porous Media," Environ. Sci. Technol. 24(10), 1528-1536 (1990).

57. Toran, L. and Palumbo, A. V. "Colloid Transport through Fractured and Unfractured Laboratory Sand Columns," J. Contam. Hydrol. 9, 289-303 (1992).

58. Elimelech, M. "Effect of Particle Size on the Kinetics of Particle Deposition under Attractive Double-Layer Interactions," J. Colloid Interface Sci. 164, 190-199 (1994).

59. Wan, J. and Wilson, J. L. "Colloid Transport in Unsaturated Porous Media," Water Resour. Res. 30(4), 857-864 (1994).

60. Kolakowski, J. E. and Matijevic, E. "Particle Adhesion and Removal in Model Systems. Part 1. - Monodispersed Chromium Hydroxide on Glass," J. C. S. Faraday_I 75, 65-78 (1979). 
61. Kuo, R. J. and Matijevic, E. "Particle Adhesion and Removal in Model Systems. III. Monodispersed Ferric Oxide on Steel," J. Colloid Interface Sci. 78(2), 407-421 (1980).

62. Kallay, N. and Matijevic, E. "Particle Adhesion and Removal in Model Systems. IV. Kinetics of Detachment of Hematite Particles from Steel," J. Colloid Interface Sci., 83(1), 289-300 (1981).

63. Thompson, G., Kallay, N., and Matijevic, E. "Particle Adhesion and Removal in Model Systems - VIII Deposition of Rod-Like Beta-FeOOH Particles on Steel," Chem. Eng. Sci. 38(11), 1901-1908 (1983).

64. Ryde, N., Kihira, H., and Matijevic, E. "Particle Adhesion in Model Systems 15. Effect of Colloid Stability in Multilayer Deposition," J. Colloid Interface Sci., 151(2), 421-432 (1992).

65. Puls, R. W. and Powell, R. M. "Transport of Inorganic Colloids through Natural Aquifer Material: Implications for Contaminant Transport," Environ. Sci. Technol. 26, 614-621 (1992).

66. Ryan, J. N. and Gschwend, P. M. "Effects of Ionic Strength and Flow Rate on Colloid Release: Relating Kinetics to Intersurface Potential Energy," J. Colloid Interface Sci. 164, 21-34 (1994).

67. Bales, R. C., Shimin, L., Maguire, K. M., Moyasar, T. Y., and Gerba, C. P. "MS-2 and Poliovirus Transport in Porous Media: Hydrophobic Effects and Chemical Perturbations," Water Resour. Res. 29(4), 957-963 (1993).

68. Kinoshita, T., Bales, R. C., Maguire, K. M., and Gerba, C. P. "Effect of pH on Bacteriophage Transport through Sandy Soils," J. Contam. Hydrol. 14, 55-70 (1993).

69. McCaulou, D. R., Bales, R. C., and McCarthy, J. F. "Use of Short-Pulse Experiments to Study Bacteria Transport through Porous Media," J. Contam. Hydrol. 15, 1-14 (1994).

70. Rosta, L., Von Gunten, H. R., and Giovanoli, R. "Packed Bed Filtration of Laponite Sols," Colloids and Surfaces 55, 205-221 (1991).

71. Hwang, Y., Chambre, P. L., Lee, W. W.-L., and Pigford, T. H. "Analytic Solution of Colloid Transport in Fractured Porous Media," Mat. Res. Soc. Symp. Proc., 176, 599-605 (1990).

72. Corapcioglu, M. Y. and Jiang, S. "Colloid-Facilitated Groundwater Contaminant Transport," Water Resour. Res. 29(7), 2215-2226 (1993).

73. Castaing, R. "Un Modele Simple pour la Migration de Radionucleides par Transport Colloidal dans un Milieu Fracture," (in French) J. Hydrol. 125, 5592 (1991). 
74. Mills, W. B., Liu, S., and Fong, F. K. "Literature Review and Model (COMET) for Colloid/Metals Transport in Porous Media," Groundwater 29(2), 199-208 (1991).

75. Nuttall, H. E., Jain, R., and Fertelli, Y. "Radiocolloid Transport in Saturated and Unsaturated Fractures," in High-Level Radioactive Waste Management, Proceedings of the 2nd Annual International Conference, American Nuclear Society, LaGrange Park, Illinois, pp. 189-196 (1991).

76. Jain, R. "A Model and Computer Code for Particulate Systems." M. S. Thesis, University of New Mexico (1992).

77. Van der Lee, J., DeMarsily, G., and Ledoux, E. "Are Colloids Important for Transport Rate Assessment of Radionuclides? A Microscopic Modeling Approach," in High-Level Radioactive Waste Management, Proceedings of the 4th Annual International Conference, American Nuclear Society, LaGrange Park, Illinois, pp. 646-652 (1993).

78. Taylor, G. I. "Dispersion of Soluble Matter in Solvent Flowing Slowly through a Tube," Proc. Roy. Soc. A. 219, 186-203 (1953).

79. Aris, R. "On the Dispersion of a Solute in a Fluid Flowing through a Tube," Proc. Roy. Soc. A. 235, 67-77 (1956).

80. Bird, R. B., Stewart, W. E., and Lightfoot, E. N. Transport Phenomena. John Wiley \& Sons, New York (1960).

81. DeMarsily, G. Quantitative Hydrogeology. Academic Press, New York (1986).

82. Long, J. C. S., Remer, J. S., Wilson, C. R., and Witherspoon, P. A. "Porous Media Equivalents for Networks of Discontinuous Fractures," Water Resour. Res. 18(3), 645-658 (1982).

83. Happel, J. and Brenner, H. Low Reynolds Number Hydrodynamics. Prentice-Hall, Englewood Cliffs, NJ (1965).

84. Thompson, M. E. "Numerical Simulation of Solute Transport in Rough Fractures," J. Geophys. Res. 96(B3), 4157-4166 (1991).

85. Grunau, D. W. "Lattice Methods for Modeling Hydrodynamics," Ph.D. Dissertation, Dept. of Math, Colorado State University (1993).

86. Brown, S. R. "Fluid Flow through Rock Joints: The Effect of Surface Roughness," J. Geophys. Res. 92(B2), 1337-1347 (1987).

87. Brown, S. R. "Transport of Fluid and Electrical Current Through a Single Fracture," L. Geophys. Res. 94(B7), 9429-9438 (1989). 
88. Moreno, L., Tsang, Y. W., Tsang, C. F., Hale, F. V., and Neretnieks, I. "Flow and Tracer Transport in a Single Fracture: A Stochastic Model and Its Relation to Some Field Observations," Water Resour. Res. 24(12), 2033-2048 (1988).

89. Moreno, L., Tsang, C. F., Tsang, Y., and Neretnieks, I. "Some Anomalous Features of Flow and Solute Transport Arising from Fracture Aperture Variability," Water Resour. Res. 26(10), 2377'-2391 (1990).

90. Tsang, Y.W. and Tsang, C. F. "Flow Channeling in a Single Fracture as a Two-Dimensional Strongly Heterogeneous Permeable Medium," Water Resour. Res. 25(9), 2076-2080 (1989).

91. Stratford, R. G., Herbert, A. W., and Jackson., C. P. "A Parameter Study of the Influence of Aperture Variation on Fracture Flow and the Consequences in a Fracture Network," in Rock Joints, Barton and Stephansson, eds., Balkema, Rotterdam, pp. 413-422 (1990).

92. Van de Ven, T. G. M. Colloidal Hydrodynamics. Academic Press, New York (1989).

93. Dahneke, B. "Kinetic Theory of the Escape of Particles from Surfaces," J. Colloid Interface Sci. 50(1), 89-107 (1975).

94. Chandrasekhar, S. "Stochastic Problems in Physics and Astronomy. Chapter II. The Theory of Brownian Motion," Rev. Mod. Physics 15, 20-44 (1943).

95. Peters, M. H. "Adsorption of Interacting Brownian Particles onto Surfaces. I. General Formulation of the Macroscopic Diffusion Equation and Results for Nonhydrodynamic Force Interactions," J. Colloid Interface Sci. 121(1), 179-192 (1988).

96. Spielman, L. A. and Goren, S. L. "Capture of Small Particles by London Forces from Low-Speed Liquid Flows," Environ. Sci. Tech. 4(2), 135-140 (1970).

97. Spielman, L. A. and Fitzpatrick, J. A. "Theory for Particle Collection under London and Gravity Forces," J. Colloid Interface Sci. 42(3), 607-623 (1973).

98. Spielman, L. A. and Cukor, P. M. "Deposition of Non-Brownian Particles under Colloidal Forces," J. Colloid Interface Sci. 43(1), 51-65 (1973).

99. Payatakes, A. C., Tien, C., and Turian, R. M. "A New Model for Granular Porous Media: Part 1. Model Formulation," AIChE J. 19(1), 58-67 (1973).

100. Payatakes, A. C., Tien, C., and Turian, R. M. "A New Model for Granular Porous Media: Part 2. Numerical Solution of Steady State Incompressible 
Newtonian Flow Through Periodically Constricted Tubes," AIChE J. 19(1), 67-76 (1973).

101. Payatakes, A. C., Tien, C., and Turian, R. M. "Trajectory Calculation of Particle Deposition in Deep Bed Filtration, Part I. Model Formulation," AIChE J. 20(5), 889-900 (1974).

102. Payatakes, A. C., Tien, C., and Turian, R. M. "Trajectory Calculation of Particle Deposition in Deep Bed Filtration, Part II. Case Study of the Effect of the Dimensionless Groups and Comparison with Experimental Data," AIChE J. 20(5), 900-905 (1974).

103. Rajagopalan, R. and Tien, C. "Trajectory Analysis of Deep-Bed Filtration with the Sphere-in-Cell Porous Media Model," AIChE J. 22(3), 523-533 (1976).

104. Rajagopalan, R. and Tien, C. "Single Collector Analysis of Collection Mechanisms in Water Filtration," Can. J. Chem. Eng. 55, 246-255 (1977).

105. Van de Ven, T. G. M. "Diffusion of Brownian Particles in Shear Flow," J. Colloid Interface Sci. 62(2), 352-355 (1977).

106. Foister, R. T. and Van de Ven, T. G. M. "Diffusion of Brownian Particles in Shear Flows," J. Fluid Mech. 96(1), 105-132 (1980).

107. Kinzelbach, W. "The Random Walk Method in Pollutant Transport Simulation," in Groundwater Flow and Quality Modeling, E. Custodio, A. Gurgui, and J. P. Lobo Ferriera, eds., D. Riedel, Dordrecht, The Netherlands, pp. 227-245 (1987).

108. Tompson, A. F. B. and Gelhar, L. W. "Numerical Simulation of Solute Transport in Three-Dimensional, Randomly Heterogeneous Porous Media," Water Resour. Res. 26(10), 2541-2562 (1990).

109. Fabriol, R., Sauty, J.-P., and Ouzounian, G. "Coupling Geochemistry with a Particle Tracking Transport Model," J. Contam. Hydrol. 13, 117-129 (1993).

110. Valocchi, A. J. and Quinodoz, H. A. M. "Application of the Random Walk Method to Simulate the Transport of Kinetically Adsorbing Solutes," in Groundwater Contamination, L. M. Abriola, ed., LAHS Press, Wallingford, Oxfordshire, pp. 35-42 (1993).

111. Tompson, A. F. B. "Numerical Simulation of Chemical Migration in Physically and Chemically Heterogeneous Porous Media," Water Resour. Res. 29(11), 3709-3726 (1993).

112. Brenner, $\mathrm{H}$. "The Slow Motion of a Sphere through a Viscous Fluid Towards a Plane Surface," Chem. Eng. Sci. 16(3/4), 242-251 (1961). 
113. Bart, E. "The Slow Unsteady Settling of a Fluid Sphere toward a Flat Fluid Interface," Chem. Eng. Sci. 23, 193-210 (1968).

114. Goldman, A. J., Cox, R. G., and Brenner, H. "Slow Viscous Motion of a Sphere Parallel to a Plane Wall - I Motion through a Quiescent Fluid," Chem. Eng. Sci. 22, 637-651 (1967).

115. Goldman, A. J., Cox, R. G., and Brenner, H. "Slow Viscous Motion of a Sphere Parallel to a Plane Wall - II Couette Flow," Chem. Eng. Sci. 22, 653660 (1967).

116. O'Neill, M. E. and Stewartson, K. "On the Slow Motion of a Sphere Parallel to a Nearby Plane Wall," J. Fluid Mech. 27(4), 705-724 (1967).

117. Goren, S. L. "The Normal Force Exerted by Creeping Flow on a Small Sphere Touching a Plane," J. Fluid Mech. 41(3), 619-625 (1970).

118. Goren, S. L. and O'Neill, M. E. "On the Hydrodynamic Resistance to a Particle of a Dilute Suspension when in the Neighbourhood of a Large Obstacle," Chem. Eng. Sci. 26, 325-338 (1971).

119. Chari, K. and Rajagopalan, R. "Deposition of Colloidal Particles in Stagnation-Point Flow," J. C. S. Faraday II 81(1), 1345-1366 (1985).

120. Reimus, P. W. "Transport of Synthetic Colloids through Single Saturated Fractures, A Literature Review," LA-12707-MS, Los Alamos National Laboratory, Los Alamos, NM (in press).

121. Hiemenz, P. C. Principles of Colloid and Surface Chemistry, 2nd ed. Marcel Dekker, Inc., New York (1986).

122. Israelachvili, J. Intermolecular and Surface Forces, 2nd ed. Academic Press, New York (1992).

123. Ruckenstein, E. and Prieve, D. C. "Adsorption and Desorption of Particles and Their Chromatographic Separation," AIChE J. 22(2), 276-283 (1976).

124. Warszynski, P. "The Influence of Polymer Adsorption on Deposition Kinetics of Colloid Particles. 1. Theory," Colloids and Surfaces 39(1-3), 7992 (1989).

125. Van de Ven, T. G. M., Dabros, T. and Czarnecki, J. "Flexible Bonds between Latex Particles and Solid Surfaces," J. Colloid Interface Sci. 93(2), 580-581 (1983).

126. Hubbe, M. A. "Theory of Detachment of Colloidal Particles from Flat Surfaces Exposed to Flow," Colloids and Surfaces 12, 151-178 (1984). 
127. Warszynski, P. and Van de Ven, T. G. M. "Effect of Electroviscous Drag on the Coagulation and Deposition of Electrically Charged Colloidal Particles," Adv. Colloid Interface Sci. 36, 33-63 (1991).

128. Bike, S. G. and Prieve, D. C. "Electrohydrodynamic Lubrication with Thin Double-Layers," J. Colloid Interface Sci. 136(1), 95-112 (1990).

129. Bike, S. G. and Prieve, D. C. "Electrohydrodynamics of Thin Double Layers: A Model for the Streaming Potential Profile," J. Colloid Interface Sci. 154(1), 87-96 (1992).

130. Dukhin, S. S. and Lyklema, J. "Dynamics of Colloid Particle Interaction," Langmuir 3(1), 94-98 (1987).

131. Prieve, D. C. and Ruckenstein, E. "Effect of London Forces upon the Rate of Deposition of Brownian Particles," AIChE J. 20(6), 1178-1187 (1974).

132. Adamczyk, Z., Dabros, T., and Czarnecki, J. "Theory for Particle Deposition onto the Rotating Disc Surface," Acta Physica Pol. A 53(2), 347-356 (1978).

133. Adamczyk, Z. "Long Nonstationary Transitions in Particle Deposition under External Forces," J. Colloid Interface Sci. 79(2), 381-389 (1981).

134. Adamczyk, Z. and Van de Ven, T. G. M. "Deposition of Particles under External Forces in Laminar Flow through Parallel-Plate and Cylindrical Channels," J. Colloid Interface Sci. 80(2), 340-356 (1981).

135. Adamczyk, Z., Dabros, T., Czarnecki, J., and Van de Ven, T. G. M. "Particle Transfer to Solid Surfaces," Adv. Colloid Interface Sci. 19, 183-252 (1983).

136. Adamczyk, Z. and Van de Ven, T. G. M. "Kinetics of Particle Accumulation at Collector Surfaces. I. Approximate Analytical Solutions," J. Colloid Interface Sci. 97(1), 68-90 (1984).

137. Adamczyk, Z., Dabros, T., Czarnecki, J., and Van de Ven, T. G. M. "Kinetics of Particle Accumulation at Collector Surfaces. II. Exact Numerical Solutions," J. Colloid Interface Sci. 97(1), 91-104 (1984).

138. Chari, K. and Rajagopalan, R. "Transport of Colloidal Particles over Energy Barriers," J. Colloid Interface Sci. 107(1), 278-282 (1985).

139. Ruckenstein, E. and Prieve, D. C. "Rate of Deposition of Brownian Particles under the Action of London and Double-layer Forces," J. C. S. Faraday II 69, 1522-1536 (1973).

140. Spielman, L. A. and Friedlander, S. K. "Role of the Electrical Double Layer in Particle Deposition by Convective Diffusion," J. Colloid Interface Sci. 46(1), 22-31 (1974). 
141. Dahneke, B. "Diffusional Deposition of Particles," J. Colloid Interface Sci. 48(3), 520-522 (1974).

142. Dahneke, B. "Resuspension of Particles," J. Colloid Interface Sci. 50(1), 194196 (1975).

143. Prieve, D. C. and Ruckenstein, E. "Rates of Deposition of Brownian Particles Calculated by Lumping Interaction Forces into a Boundary Condition," J. Colloid Interface Sci. 57(3), 547-550 (1976).

144. Ruckenstein, E. and Prieve, D. C. "On Reversible Adsorption of Hydrosols and Repeptization," AIChE J. 22(6), 1145-1147 (1976).

145. Ruckenstein, E. "Reversible Rate of Adsorption or Coagulation of Brownian Particles - Effect of the Shape of the Interaction Potential," J. Colloid Interface Sci. 66(3), 531-543 (1978).

146. Bowen, B. D. and Epstein, N. "Fine Particle Deposition in Smooth ParallelPlate Channels," J. Colloid Interface Sci. 72(1), 81-97 (1979).

147. Dabros, T. and Van de Ven, T. G. M. "A Direct Method for Studying Particle Deposition onto Solid Surfaces," Colloid Polymer Sci. 261(8), 694-707 (1983).

148. Albery, W. J., G. R. Kneebone, and A. W. Foulds. "Kinetics of Colloidal Deposition Studied by a Wall-Jet Cell," J. Colloid Interface Sci. 108(1), 193198 (1985).

149. Dabros, T. and Van de Ven, T. G. M. "Deposition of Latex Particles on Glass Surfaces in an Impinging Jet," Physicochem. Hydrodynamics 8(2), 161-172 (1987).

150. Varennes, S. and Van de Ven, T. G. M. "Deposition and Detachment of Latex Particles at Glass Surfaces Exposed to Flow," Physicochem. Hydrodynamics 9(3/4), 537-559 (1987).

151. Sjollema, J. and Busscher, H. J. "Deposition of Polystyrene Latex Particles Toward Polymethylmethacrylate in a Parallel Plate Flow Cell," J. Colloid Interface Sci. 132(2), 382-394 (1989).

152. Sjollema, J. and Busscher, H. J. "Deposition of Polystyrene Particles in a Parallel Plate Flow Cell. 1. The Influence of Collector Surface Properties on the Experimental Deposition Rate," Colloids and Surfaces 47, 323-336 (1990).

153. Albery, W. J., Fredlein, R. A., Kneebone, G. R., O'Shea, G. J., and Smith, A. L. "The Kinetics of Colloidal Deposition Under Conditions of Controlled Potential," Colloids and Surfaces 44, 337-356 (1990).

154. Meinders, J. M., Noordmans, J. and Busscher, H. J. "Simultaneous Monitoring of the Adsorption and Desorption of Colloidal Particles during 
Deposition in a Parallel Plate Flow Chamber" J. Colloid Interface Sci. 152(1), 265-280 (1992).

155. Dabros, T. and Van de Ven, T. G. M. "Kinetics of Coating by Colloidal Particles," J. Colloid Interface Sci. 89(1), 232-244 (1982).

156. Dabros, T. and Van de Ven, T. G. M. "On the Effects of Blocking and Particle Detachment on Coating Kinetics," J. Colloid Interface Sci. 93(2), 576579 (1983).

157. Prieve, D. C. and Lin, M. M. J. "The Effect of a Distribution of Surface Properties on Colloid Stability," J. Colloid Interface Sci. 86(1), 17-25 (1982).

158. Adamczyk, Z., Czarnecki, J., and Warszynski, P. "The Effect of Fluctuations of the Energy Barrier on Colloid Stability," J. Colloid Interface Sci. 106(2), 299-306 (1985).

159. Czarnecki, J. and Warszynski, P. "The Evaluation of Tangential Forces Due to Surface Inhomogeneities in the Particle Deposition Process," Colloids and Surfaces 22, 207-214 (1987).

160. Adamczyk, Z. and Petlicki, J. "Adsorption and Desorption Kinetics of Molecules and Colloidal Particles," J. Colloid Interface Sci. 118(1), 20-49 (1987).

161. Sharma, M. M., Chamoun, H., Sita Rama Sarma, D. S. H., and Schechter, R. S. "Factors Controlling the Hydrodynamic Detachment of Particles from Surfaces," J. Colloid Interface Sci. 149(1), 121-134 (1992).

162. Das, S. K., Schechter, R. S., and Sharma, M. M. "The Role of Surface Roughness and Contact Deformation on the Hydrodynamic Detachment of Particles from Surfaces," J. Colloid Interface Sci. 164, 63-77 (1994).

163. Geldon, A. L. "Preliminary Hydrogeologic Assessment of Boreholes UE-25c \#1, UE-25c \#2, and UE-25c \#3, Yucca Mountain, Nye County, Nevada," Water-Resources Investigations Report 92-4016, U. S. Geological Survey, Denver, CO (1993).

164. Ogard, A. E., and Kerrisk, J. F. "Groundwater Chemistry along Flow Paths Between a Proposed Repository Site and the Accessible Environment," LA10188-MS, Los Alamos National Laboratory, Los Alamos, NM (1984).

165. Fuentes, H. R., Polzer, W. L., Essington, E. H., and Newman, B. D. "Characterization of Reactive Tracers for C-Wells Field Experiments I: Electrostatic Sorption Mechanism, Lithium," LA-11691-MS, Los Alamos National Laboratory, Los Alamos, NM (1989).

166. Reimus, P. W. "Use of an Ion-Selective Electrode to Determine Ion Concentrations in Solution," LANL-EES-DP-328, Yucca Mountain Site 
Characterization Project Detailed Procedure, Los Alamos National Laboratory, Los Alamos, NM (1993).

167. Steinkamp, J. A., Habbersett, R. C., and Hiebert, R. D. "Improved Multilaser/Multiparameter Flow Cytometer for Analysis and Sorting of Cells and Particles," Rev. Sci. Instrum. 62(11), 2751-2764 (1991).

168. Reimus, P. W. "Use of a Flow Cytometer to Determine Particle Concentrations in Solution," LANL-EES-DP-327, Yucca Mountain Site Characterization Project Detailed Procedure, Los Alamos National Laboratory, Los Alamos, NM (1993).

169. Huang, C., White, I., Thwaite, E. G., and Bendeli, A. "A Noncontact Laser System for Measuring Soil Surface Topography," Soil Sci. Am. J. 52, 350-355 (1988).

170. Reimus, P. W., Robinson, B. A., and Glass, R. J. "Aperture Characteristics, Saturated Fluid Flow, and Tracer Transport Calculations for a Natural Fracture," in High-Level Radioactive Waste Management, Proceedings of the 4th Annual International Conference, American Nuclear Society, LaGrange Park, Illinois, pp. 2009-2016 (1993).

171. Cox, B. L., Pruess, K., and Persoff, P. "A Casting and Imaging Technique for Determining Void Geometry and Relative Permeability Behavior of a Single Fracture Specimen," LBL-28485, Lawrence Berkeley Laboratory, Berkeley, CA (1990).

172. Mann, C. K., Vickers, T. J., and Gulick, W. M. Instrumental Analysis, Harper and Row, New York (1974).

173. Durham, W. B. and Bonner, B. P. "PEAK: A New Kind of Surface Microscope," Int. J. Rock Mech. Min. Sci. \& Geomech. Abstr. 30(7), 699-702 (1993).

174. Shoemaker, D. P., Garland, C. W., and Steinfeld, J. I. Experiments in Physical Chemistry, 3rd ed., McGraw-Hill, New York (1974).

175. Johnson, L. W. and Riess, R. D. Numerical Analysis, 2nd ed., AddisonWesley, Reading, MA (1982).

176. Peaceman, D. W. and Rachford, H. H. "The Numerical Solution of Parabolic and Elliptic Differential Equations," J. Soc. Ind. Appl. Math., 3, 28-41 (1955).

177. Lapidus, L. and Pinder, G. F. Numerical Solution of Partial Differential Equations in Science and Engineering, John Wiley and Sons, New York (1982). 
178. Saylor, P. E. and Sebastian, J. D. "Calculation of Optimum Parameters for Alternating Direction Implicit Procedures," Comm. ACM 16(10), 633-635 (1973).

179. Los Alamos National Laboratory. Common Los Alamos Mathematical Software Compendium, CIC \#148, Los Alamos National Laboratory, Los Alamos, NM (1988).

180. Tsang, C.-F. "Flow and Transport in Single Fractures," in Flow and Contaminant Transport in Fractured Rock, J. Bear, C.-F. Tsang, and C. DeMarsily, eds., Academic Press, New York, pp. 242-250 (1993).

181. Kahaner, D., Moler, C., and Nash, S. Numerical Methods and Software, Ch. 10, Prentice-Hall, Englewood Cliffs, NJ (1989).

182. Triay, I. R., Birdsell, K. H., Mitchell, A. J., and Ott, M. A. "Diffusion of Sorbing and Non-Sorbing Radionuclides," in High-Level Radioactive Waste Management, Proceedings of the 4th Annual International Conference, American Nuclear Society, LaGrange Park, Illinois, pp. 1527-1532 (1993).

183. Newman, J. Electrochemical Systems, Prentice-Hall, Englewood Cliffs, NJ (1973).

184. Weast, R. C. and Astle, M. J. eds. CRC Handbook of Chemistry and Physics, CRC Press, Boca Raton, FL (1981).

185. Starr, R. C., Gillham, R. W., and Sudicky, E. A. "Experimental Investigation of Solute Transport in Stratified Porous Media 2. The Reactive Case," Water Resour. Res. 21(7), 1043-1050 (1985).

186. Molz, F. J., Guven, O., and Melville, J. G. "An Examination of ScaleDependent Dispersion Coefficients," Groundwater 21(6), 715-725 (1983).

187. Treher, E. N. and Raybold, N. A. "The Elution of Radionuclides through Columns of Crushed Rock from the Nevada Test Site," LA-9329-MS, Los Alamos National Laboratory, Los Alamos, NM (1982).

188. Rundberg, R., Janecky, D., and Mitchell, A. "Anion Exclusion in Yucca Mountain Tuff," NNWSI Milestone Report R313, Los Alamos National Laboratory, Los Alamos, NM (1987).

189. Moreno, L. and Tsang, C.-F. "Flow Channeling in Strongly Heterogeneous Porous Media: A Numerical Study," Water Resour. Res. 30(5), 1421-1430 (1994).

190. Nyffenegger, R., Quellet, C., and Ricka, J. "Synthesis of Fluorescent, Monodisperse, Colloidal Silica Particles," J. Colloid Interface Sci. 159, 150157 (1993). 
191. Persoff, P., Pruess, K., and Myer, L. "Two-Phase Flow Visualization and Relative Permeability Measurement in Transparent Replicas of RoughWalled Rock Fractures," in Proceedings of the Sixteenth Workshop on Geothermal Reservoir Engineering, Stanford University, Stanford, CA, pp. 203-210 (1991). 


\section{APPENDIX A: SUMMARY OF TRACER EXPERIMENTS}

The test conditions in each of the 18 tracer experiments are summarized in this appendix. The objectives of each experiment and some miscellaneous notes about the experiments are also recorded. The experiments are listed in the order in which they were conducted.

\section{Experiment 1:}

Date: $7 / 27 / 93$

Fracture: Bandelier Tuff

Tracers and Tracer Inlet Concentrations:

- $1-\mu \mathrm{m}$ Yellow Polystyrene Microspheres with Carboxyl Surface Groups $\left(1.61 \times 10^{10} /\right.$ liter $)$

- $\quad 1-\mu \mathrm{m}$ Blue Carboxylate-Modified Latex (CML) Microspheres (2.12 x $10^{10} /$ liter $)$

- $\quad$ Iodide $(\sim 0.001 \underline{\mathrm{M}})$

Temperature: $\sim 24^{\circ} \mathrm{C}$

Hydraulic Aperture: $27.4 \mu \mathrm{m}$

Tracer Flow Rate: $1.36 \mu \mathrm{l} / \mathrm{sec}$ (only one measurement)

J-13 Water Flow Rate: N/A

Manifold Flush Flow Rate: $0.808 \mathrm{ml} / \mathrm{sec}$ (range: 0.801 to $0.816 \mathrm{ml} / \mathrm{sec}$ )

Leak Rate: $\sim 0.025 \mu \mathrm{l} / \mathrm{sec}$ under $\sim 40 \mathrm{~cm} \mathrm{H}_{2} \mathrm{O}$ head

Estimated Pressure Drop across Fracture: $9.2 \mathrm{~cm} \mathrm{H}_{2} \mathrm{O}$

Estimated Pressure Drop across Outlet Manifold:* $0.46 \mathrm{~cm} \mathrm{H}_{2} \mathrm{O}$

Objective(s): Investigate the transport differences between the two types of polystyrene microspheres to determine which is more suitable for field-scale tracer experiments.

Notes: This experiment was conducted as a single tracer step in the inlet solution lasting 1230 seconds with no samples collected during flush out. Samples were collected every 30 seconds. Extensive flushing of the fracture was done by gravity after the last sample was collected. This test was the only one in which neoprene was used instead of silicone as the gasket material to seal the sides and ends of the fracture. The neoprene was found to contribute background levels of both iodide and blue colloids, which had to be subtracted out from the analytical measurements to obtain the breakthrough curve data. It was clear from the breakthrough curves that the CML microspheres transported much more readily through the fracture than the unmodified polystyrene microspheres. There was a lot of yellow color observed on the fracture surfaces under UV light after the test, indicating significant attachment of the non-CML microspheres to the fracture surfaces. These results, combined with earlier observations (on the flow cytometer) that the nonCML microspheres tended to be much more aggregated than the CML microspheres, led to a decision to eliminate the non-CML microspheres from further consideration as tracers. This was the only test in which the non-CML polystyrene microspheres were used.

\footnotetext{
* Based on pressure drop measurements in 1/8-inch ID Tygon tubing.
} 
Experiment 2:

Date: $9 / 28 / 93$

Fracture: Bandelier Tuff

Tracers and Tracer Inlet Concentrations:

- $\quad 0.3-\mu \mathrm{m}$ Silica Microspheres ( 1013/liter)

- Iodide $(\sim 0.001 \underline{\mathrm{M}})$

Temperature: $\sim 21^{\circ} \mathrm{C}$

Hydraulic Aperture: $37.6 \mu \mathrm{m}$ (after inadvertently pressurizing the fracture)

Tracer Flow Rate: $1.65 \mu \mathrm{l} / \mathrm{sec}$ (range: 1.68 to $1.62 \mu \mathrm{l} / \mathrm{sec}$ - decreasing)

J-13 Water Flow Rate: $1.66 \mu \mathrm{l} / \mathrm{sec}$ (range: 1.69 to $1.63 \mu \mathrm{l} / \mathrm{sec}$ - decreasing)

Manifold Flush Flow Rate: $0.750 \mathrm{ml} / \mathrm{sec}$ (range: 0.746 to $0.752 \mathrm{ml} / \mathrm{sec}$ )

Leak Rate: $\sim 0.03 \mu \mathrm{l} / \mathrm{sec}$ under $\sim 40 \mathrm{~cm} \mathrm{H}_{2} \mathrm{O}$ head

Estimated Pressure Drop across Fracture: $4.3 \mathrm{~cm} \mathrm{H}_{2} \mathrm{O}$

Estimated Pressure Drop across Outlet Manifold: $0.43 \mathrm{~cm} \mathrm{H}_{2} \mathrm{O}$

Objective(s): Screen the transport behavior of silica colloids through the Bandelier tuff fracture.

Notes: This test was conducted as a tracer step lasting 1440 seconds followed by a flush phase lasting 1440 seconds. Samples in both phases were collected every 30 seconds. The inlet silica concentration was approximately 2 orders of magnitude greater than that of the dyed $0.3-$ $\mu \mathrm{m}$ polystyrene microspheres in other tests so that the silica could be detected against the background concentration of colloids in the samples. The imprecision of the silica analytical technique and a 2-week delay in analyzing the samples made it clifficult to quantitatively interpret this test. An initial estimate of $\sim 80 \%$ recovery of the silica was based on normalization of the effluent sample turbidities to a single questionable turbidity measurement of a diluted inlet solution sample. Repeat measurements of inlet solution samples conducted 2 weeks after the effluent sample measurements indicated a lower turbidity of the inlet solution and hence greater normalized concentrations of the effluent samples (bringing the estimated recovery up to $~ 89 \%$ ). Because of the seemingly incomplete recovery of the silica and the various analytical problems, this was the only test in which silica microspheres were used.

Experiment 3:

Date: $10 / 5 / 93$

Fracture: Bandelier Tuff

Tracers and Tracer Inlet Concentrations:

- 1- $\mu \mathrm{m}$ Blue CML Microspheres (2.99 x 1010/liter)

- $0.3-\mu \mathrm{m}$ Yellow CML Microspheres (2.45 x 1011/liter)

- Iodide $(\sim 0.001 \underline{\mathrm{M}})$

Temperature: $24^{\circ} \mathrm{C}$

Hydraulic Aperture: $27.2 \mu \mathrm{m}$ (before test) $-28.0 \mu \mathrm{m}$ (after test)

Tracer Flow Rate: $4.11 \mu \mathrm{l} / \mathrm{sec}$ (range: 4.11 to $4.14 \mu \mathrm{l} / \mathrm{sec}$ - increasing)

J-13 Water Flow Rate: $3.85 \mu \mathrm{l} / \mathrm{sec}$ (range: 4.11 to $3.84 \mu \mathrm{l} / \mathrm{sec}$ - decreasing)

Flush Flow Rate: $1.162 \mathrm{ml} / \mathrm{sec}$ (range: 1.151 to $1.173 \mathrm{ml} / \mathrm{sec}$ - increasing)

Leak Rate: $0.04 \mu \mathrm{l} / \mathrm{sec}$ under $\sim 40 \mathrm{~cm}$ head

Estimated Pressure Drop across Fracture: $27.2 \mathrm{crn} \mathrm{H}_{2} \mathrm{O}$ 
Estimated Pressure Drop across Outlet Manifold: $0.66 \mathrm{~cm} \mathrm{H}_{2} \mathrm{O}$

Objective(s): Investigate the transport differences between the two sizes of CML microspheres, and compare the transport behavior of the microspheres and iodide at different flow rates in the Bandelier tuff fracture (the other flow rates were run on the same day in Experiments 4 and 5).

Notes: This test was conducted as a tracer step lasting 720 seconds followed by a flush phase lasting 720 seconds. Samples were collected every 20 seconds in both phases. Both microspheres reached the inlet concentration in the effluent during the step portion of the test (within experimental error), and both arrived earlier than the iodide. The $1-\mu \mathrm{m}$ microspheres arrived slightly ahead of the $0.3-\mu \mathrm{m}$ microspheres. The iodide did not reach the inlet concentration during the step, presumably because of matrix diffusion.

Experiment 4:

Date: $10 / 5 / 93$

Fracture: Bandelier Tuff

Tracers and Tracer Inlet Concentrations:

- $1-\mu \mathrm{m}$ Blue CML Microspheres $\left(2.95 \times 10^{10} /\right.$ liter $)$

- $\quad 0.3-\mu \mathrm{m}$ Yellow CML Microspheres $\left(2.43 \times 10^{11} /\right.$ liter $)$

- Iodide $(\sim 0.001 \underline{\mathrm{M}})$

Temperature: $26^{\circ} \mathrm{C}$

Hydraulic Aperture: $28.0 \mu \mathrm{m}$ (before test) - $28.9 \mu \mathrm{m}$ (after test)

Tracer Flow Rate: $2.30 \mu \mathrm{l} / \mathrm{sec}$ (range: 2.39 to $2.04 \mu \mathrm{l} / \mathrm{sec}$ - decreasing)

J-13 Water Flow Rate: $2.29 \mu \mathrm{l} / \mathrm{sec}$ (range: 2.27 to $2.31 \mu \mathrm{l} / \mathrm{sec}$ - increasing)

Flush Flow Rate: $0.790 \mathrm{ml} / \mathrm{sec}$ (range: 0.787 to $0.793 \mathrm{ml} / \mathrm{sec}$ - constant)

Leak Rate: $0.04 \mu \mathrm{l} / \mathrm{sec}$ under $\sim 40 \mathrm{~cm}$ head

Estimated Pressure Drop across Fracture: $13.7 \mathrm{~cm} \mathrm{H} \mathrm{H}_{2} \mathrm{O}$

Estimated Pressure Drop across Outlet Manifold: $0.46 \mathrm{~cm} \mathrm{H}_{2} \mathrm{O}$

Objective(s): Same as Experiment 3.

Notes: This test was conducted as a tracer step lasting 1080 seconds followed by a flush phase lasting 1080 seconds. Samples were collected every 30 seconds in both phases. The results were similar to Experiment 3 except that the iodide did not reach as high a concentration during the step portion of the test, which was further evidence of matrix diffusion.

\section{Experiment 5:}

Date: $10 / 5 / 93$

Fracture: Bandelier Tuff

Tracers and Tracer Inlet Concentrations:

- 1- $\mu \mathrm{m}$ Blue CML Microspheres (2.97 x 1010/liter)

- $\quad 0.3-\mu \mathrm{m}$ Yellow CML Microspheres $\left(2.44 \times 10^{11} /\right.$ liter $)$

- Iodide $(\sim 0.001 \underline{\mathrm{M}})$

Temperature: $24^{\circ} \mathrm{C}$

Hydraulic Aperture: $28.9 \mu \mathrm{m}$ (before test) $-29.0 \mu \mathrm{m}$ (after test)

Tracer Flow Rate: $1.17 \mu \mathrm{l} / \mathrm{sec}$ (range: 1.21 to $1.14 \mu \mathrm{l} / \mathrm{sec}$ - decreasing)

J-13 Water Flow Rate: $1.32 \mu \mathrm{l} / \mathrm{sec}$ (range: 1.33 to $1.32 \mu \mathrm{l} / \mathrm{sec}$ - constant)

Flush Flow Rate: $0.787 \mathrm{ml} / \mathrm{sec}$ (range: 0.793 to $0.783 \mathrm{ml} / \mathrm{sec}$ - decreasing) 
Leak Rate: $0.04 \mu \mathrm{l} / \mathrm{sec}$ under $\sim 40 \mathrm{~cm}$ head

Estimated Pressure Drop across Fracture: $7.0 \mathrm{~cm} \mathrm{H} \mathrm{H}_{2} \mathrm{O}$

Estimated Pressure Drop across Outlet Manifold: $0.45 \mathrm{~cm} \mathrm{H}_{2} \mathrm{O}$

Objective(s): Same as Experiment 3.

Notes: This test was conducted as a tracer step lasting 1440 seconds followed by a flush phase lasting 1440 seconds. Samples were collected every 30 seconds in both phases. The results were similar to Experiment 3 except that the iodide did not reach as high a concentration during the step portion of the test, which was further evidence of matrix diffusion. After this test, the flow system was disassembled and the fracture surfaces were observed under UV light. There were no microspheres apparent on the surfaces.

Experiment 6:

Date: $11 / 5 / 93$

Fracture: Bullfrog Tuff

Tracers and Tracer Inlet Concentrations:

- $1-\mu \mathrm{m}$ Blue CML Microspheres (3.93 x 1010/liter)

- $\quad 0.3-\mu \mathrm{m}$ Yellow CML Microspheres (1.55 x 1011/liter)

- Iodide $(\sim 0.001 \underline{\mathrm{M}})$

Temperature: $21^{\circ} \mathrm{C}$

Hydraulic Aperture: $108.7 \mu \mathrm{m}$ (before test) - $108.7 \mu \mathrm{m}$ (after test)

Tracer Flow Rate: $19.30 \mu \mathrm{l} / \mathrm{sec}$ (range: 19.21 to $19.39 \mu \mathrm{l} / \mathrm{sec}$ - increasing)

J-13 Water Flow Rate: $20.18 \mu \mathrm{l} / \mathrm{sec}$ (range: 20.22 to $20.13 \mu \mathrm{l} / \mathrm{sec}$ - constant)

Flush Flow Rate: $1.161 \mathrm{ml} / \mathrm{sec}$ (range: 1.157 to $1.165 \mathrm{ml} / \mathrm{sec}$ - constant)

Leak Rate: $0.08 \mu \mathrm{l} / \mathrm{sec}$ under $\sim 40 \mathrm{~cm}$ head

Estimated Pressure Drop across Fracture: $1.9 \mathrm{~cm} \mathrm{H} \mathrm{H}_{2} \mathrm{O}$

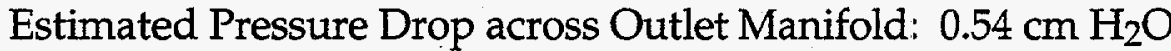

Objective(s): Investigate the transport differences between the two sizes of CML microspheres, and compare the transport behavior of the microspheres and iodide at different flow rates in the Bullfrog tuff fracture (other flow rates were run on the same day in Experiments 7 and 8).

Notes: This test was conducted as a tracer step lasting 720 seconds followed by a flush phase lasting 720 seconds. Samples were collected every 20 seconds in both phases. A large "spike" occurred in the data at 720 seconds because there was some flow through the fracture during the flush out of the inlet manifold. Both microspheres reached the inlet concentration during the step portion of the test (within experimental error), and both arrived slightly earlier than the iodide. The delay in the iodide arrival time (relative to the microspheres) was much less than in the Bandelier tuff fracture. The iodide did not reach the inlet concentration in the outlet, but the difference between the iodide and microsphere concentrations was less in this test than in the Bandelier tuff tests.

Experiment 7:

Date: $11 / 5 / 93$

Fracture: Bullfrog Tuff 
Tracers and Tracer Inlet Concentrations:

- 1- $\mu \mathrm{m}$ Blue CML Microspheres (3.94 x 1010/liter)

- $\quad 0.3-\mu \mathrm{m}$ Yellow CML Microspheres (1.56 x 1011/liter)

- Iodide $(\sim 0.001 \underline{\mathrm{M}})$

Temperature: $21^{\circ} \mathrm{C}$

Hydraulic Aperture: $108.7 \mu \mathrm{m}$ (before test) - $109.1 \mu \mathrm{m}$ (after test)

Tracer Flow Rate: $9.23 \mu \mathrm{l} / \mathrm{sec}$ (range: 9.34 to $9.14 \mu \mathrm{l} / \mathrm{sec}$ - decreasing)

J-13 Water Flow Rate: $9.81 \mu \mathrm{l} / \mathrm{sec}$ (range: 9.86 to $9.77 \mu \mathrm{l} / \mathrm{sec}$ - constant)

Flush Flow Rate: $0.771 \mathrm{ml} / \mathrm{sec}$ (range: 0.768 to $0.774 \mathrm{ml} / \mathrm{sec}$ - constant)

Leak Rate: $0.08 \mu \mathrm{l} / \mathrm{sec}$ under $\sim 40 \mathrm{~cm}$ head

Estimated Pressure Drop across Fracture: $0.89 \mathrm{~cm} \mathrm{H} \mathrm{H}_{2} \mathrm{O}$

Estimated Pressure Drop across Outlet Manifold: $0.37 \mathrm{~cm} \mathrm{H}_{2} \mathrm{O}$

Objective(s): Same as Experiment 6.

Notes: This test was conducted as a tracer step lasting 1080 seconds followed by a flush phase lasting 1080 seconds. Samples were collected every 30 seconds in both phases. The tracers did not reach as high a concentration during the step up portion of the test as they did in Experiment 6 . This can be attributed to the fact that the total volume eluted through the fracture was less than in Experiment 6. Also, the lower iodide concentration can be attributed to more matrix diffusion at the lower flow rate in this test.

Experiment 8:

Date: $11 / 5 / 93$

Fracture: Bullfrog Tuff

Tracers and Tracer Inlet Concentrations:

- 1- $\mu \mathrm{m}$ Blue CML Microspheres (3.94 x 1010/liter)

- $\quad 0.3-\mu \mathrm{m}$ Yellow CML Microspheres (1.56 x 1011/liter)

- Iodide $(\sim 0.001 \underline{\mathrm{M}})$

Temperature: $22^{\circ} \mathrm{C}$

Hydraulic Aperture: $109.1 \mu \mathrm{m}$ (before test) $-109.2 \mu \mathrm{m}$ (after test)

Tracer Flow Rate: $4.88 \mu \mathrm{l} / \mathrm{sec}$ (range: 4.94 to $4.82 \mu \mathrm{l} / \mathrm{sec}$ - decreasing)

J-13 Water Flow Rate: $5.24 \mu \mathrm{l} / \mathrm{sec}$ (range: 5.29 to $5.18 \mu \mathrm{l} / \mathrm{sec}$ - decreasing)

Flush Flow Rate: $0.773 \mathrm{ml} / \mathrm{sec}$ (range: 0.770 to $0.776 \mathrm{ml} / \mathrm{sec}$ - constant)

Leak Rate: $0.08 \mu \mathrm{l} / \mathrm{sec}$ under $\sim 40 \mathrm{~cm}$ head

Estimated Pressure Drop across Fracture: $0.47 \mathrm{~cm} \mathrm{H}_{2} \mathrm{O}$

Estimated Pressure Drop across Outlet Manifold: $0.37 \mathrm{~cm} \mathrm{H}_{2} \mathrm{O}$

Objective(s): Same as Experiment 6.

Notes: This test was conducted as a tracer step lasting 1440 seconds followed by a flush phase lasting 1440 seconds. Samples were collected every 30 seconds in both phases. The tracers did not reach as high a concentration during the step up portion of the test as they did in Experiment 6 . This can be attributed to the fact that the total volume eluted through the fracture was less than in Experiment 6. Also, the lower iodide concentration can be attributed to more matrix diffusion at the lower flow rate in this test. After this test, the flow system was disassembled and the fracture surfaces were observed under UV light. It was apparent that there was a significant amount of microspheres (primarily blue) on the bottom surface and a hint of microspheres on the 
top surface. The distribution of the microspheres on both surfaces was recorded in a drawing in a laboratory notebook. The distribution on the bottom surface corresponded almost exactly to the aperture distribution shown in Fig. 4.6, with microspheres being more concentrated in regions of large aperture.

Experiment 9:

Date: $11 / 29 / 93$

Fracture: Tram Tuff

Tracers and Tracer Inlet Concentrations:

- 1- $\mu \mathrm{m}$ Blue CML Microspheres (4.24 x 1010/liter)

- $0.3-\mu m$ Yellow CML Microspheres (3.06 x 1011/liter)

- Iodide $(\sim 0.001 \underline{\mathrm{M}})$

Temperature: $22^{\circ} \mathrm{C}$

Hydraulic Aperture: $90.7 \mu \mathrm{m}$ (before test) - $90.8 \mu \mathrm{m}$ (after test)

Tracer Flow Rate: $8.85 \mu \mathrm{l} / \mathrm{sec}$ (range: 8.95 to $8.75 \mu \mathrm{l} / \mathrm{sec}$ - decreasing)

J-13 Water Flow Rate: $9.01 \mu \mathrm{l} / \mathrm{sec}$ (range: 9.13 to $8.89 \mu \mathrm{l} / \mathrm{sec}$ - decreasing)

Flush Flow Rate: $1.130 \mathrm{ml} / \mathrm{sec}$ (range: 1.126 to $1.135 \mathrm{ml} / \mathrm{sec}$ - increasing)

Leak Rate: $0.015 \mu \mathrm{l} / \mathrm{sec}$ under $\sim 40 \mathrm{~cm}$ head

Estimated Pressure Drop across Fracture: $1.64 \mathrm{~cm} \mathrm{H} \mathrm{H}_{2} \mathrm{O}$

Estimated Pressure Drop across Outlet Manifold: $0.46 \mathrm{~cm} \mathrm{H}_{2} \mathrm{O}$

Objective(s): Investigate the transport differences between the two sizes of $\mathrm{CML}$ microspheres, and compare the transport behavior of the microspheres and iodide at different flow rates in the Tram tuff fracture (other flow rates were run on the same day in Experiments 10 and 11 ).

Notes: This test was conducted as a tracer step lasting 720 seconds followed by a flush phase lasting 720 seconds. Samples were collected every 20 seconds in both phases. Both microspheres reached the inlet concentration during the step up portion of the test (within experimental error), and both arrived slightly earlier than the iodide. The iodide almost reached the inlet concentration, and its delayed arrival relative to the microspheres was less than in the Bandelier tuff fracture but more than in the Bullfrog tuff fracture.

\section{Experiment 10:}

Date: $11 / 29 / 93$

Fracture: Tram Tuff

Tracers and Tracer Inlet Concentrations:

- $\quad 1-\mu \mathrm{m}$ Blue CML Microspheres (4.25 x 1010/liter)

- $\quad 0.3-\mu \mathrm{m}$ Yellow CML Microspheres (3.07 x 1011/liter)

- Iodide $(\sim 0.001 \underline{\mathrm{M}})$

Temperature: $22^{\circ} \mathrm{C}$

Hydraulic Aperture: $90.8 \mu \mathrm{m}$ (before test) $-91.8 \mu \mathrm{m}$ (after test)

Tracer Flow Rate: $4.57 \mu \mathrm{l} / \mathrm{sec}$ (range: 4.69 to $4.44 \mu \mathrm{l} / \mathrm{sec}$ - decreasing)

J-13 Water Flow Rate: $4.53 \mu \mathrm{l} / \mathrm{sec}$ (range: 4.64 to $4.42 \mu \mathrm{l} / \mathrm{sec}$ - decreasing)

Flush Flow Rate: $0.760 \mathrm{ml} / \mathrm{sec}$ (range: 0.763 to $0.758 \mathrm{ml} / \mathrm{sec}$ - increasing)

Leak Rate: $0.015 \mu \mathrm{l} / \mathrm{sec}$ under $\sim 40 \mathrm{~cm}$ head

Estimated Pressure Drop across Fracture: $0.83 \mathrm{~cm} \mathrm{H}_{2} \mathrm{O}$ 
Estimated Pressure Drop across Outlet Manifold: $0.34 \mathrm{~cm} \mathrm{H}_{2} \mathrm{O}$

Objective(s): Same as Experiment 9.

Notes: This test was conducted as a tracer step lasting 1080 seconds followed by a flush phase lasting 1080 seconds. Samples were collected every 30 seconds in both phases. The results of the test were very similar to Experiment 9, except that iodide clearly did not reach the inlet concentration during the step portion of the test.

Experiment 11:

Date: $11 / 29 / 93$

Fracture: Tram Tuff

Tracers and Tracer Inlet Concentrations:

- 1- $\mu \mathrm{m}$ Blue CML Microspheres (4.23 x 1010/liter)

- $0.3-\mu \mathrm{m}$ Yellow CML Microspheres (3.07 x 1011/liter)

- Iodide $(\sim 0.001 \underline{\mathrm{M}})$

Temperature: $22^{\circ} \mathrm{C}$

Hydraulic Aperture: $91.8 \mu \mathrm{m}$ (before test) - $92.5 \mu \mathrm{m}$ (after test)

Tracer Flow Rate: $2.50 \mu \mathrm{l} / \mathrm{sec}$ (range: 2.53 to $2.46 \mu \mathrm{l} / \mathrm{sec}$ - decreasing)

J-13 Water Flow Rate: $2.51 \mu \mathrm{l} / \mathrm{sec}$ (range: 2.51 to $2.51 \mu \mathrm{l} / \mathrm{sec}-\sim$ constant)

Flush Flow Rate: $0.763 \mathrm{ml} / \mathrm{sec}$ (range: 0.764 to $0.763 \mathrm{ml} / \mathrm{sec}$ - constant)

Leak Rate: $0.015 \mu \mathrm{l} / \mathrm{sec}$ under $\sim 40 \mathrm{~cm}$ head

Estimated Pressure Drop across Fracture: $0.45 \mathrm{~cm} \mathrm{H}_{2} \mathrm{O}$

Estimated Pressure Drop across Outlet Manifold: $0.34 \mathrm{~cm} \mathrm{H}_{2} \mathrm{O}$

Objective(s): Same as Experiment 9.

Notes: This test was conducted as a tracer step lasting 1680 seconds followed by a flush phase lasting 1680 seconds. Samples were collected every 30 seconds in both phases. The results of the test were very similar to Experiment 9, except that iodide clearly did not reach the inlet concentration during the step portion of the test. After this test, the flow system was disassembled and the fracture surfaces were observed under UV light. There were no microspheres apparent on the surfaces.

Experiment 12:

Date: $12 / 15 / 93$

Fracture: Bullfrog Tuff (rotated)

Tracers and Tracer Inlet Concentrations:

- 1- $\mu \mathrm{m}$ Blue CML Microspheres $\left(4.17 \times 10^{10} /\right.$ liter $)$

- 0.3- $\mu \mathrm{m}$ Yellow CML Microspheres (2.50 x 1011/liter)

- Iodide $(\sim 0.001 \underline{\mathrm{M}})$

Temperature: $22^{\circ} \mathrm{C}$

Hydraulic Aperture: $111.2 \mu \mathrm{m}$ (before test) - $111.7 \mu \mathrm{m}$ (after test)

Tracer Flow Rate: $13.10 \mu \mathrm{l} / \mathrm{sec}$ (range: 13.12 to $13.01 \mu \mathrm{l} / \mathrm{sec}$ - constant)

J-13 Water Flow Rate: $13.80 \mu \mathrm{l} / \mathrm{sec}$ (range: 13.71 to $13.84 \mu \mathrm{l} / \mathrm{sec}$ - constant)

Flush Flow Rate: $1.172 \mathrm{ml} / \mathrm{sec}$ (range: 1.161 to $1.183 \mathrm{ml} / \mathrm{sec}$ - increasing)

Leak Rate: $0.042 \mu \mathrm{l} / \mathrm{sec}$ under $\sim 40 \mathrm{~cm}$ head

Estimated Pressure Drop across Fracture: $1.13 \mathrm{~cm} \mathrm{H}_{2} \mathrm{O}$

Estimated Pressure Drop across Outlet Manifold: $0.55 \mathrm{~cm} \mathrm{H}_{2} \mathrm{O}$

Objective(s): Investigate differences in tracer dispersion in two different flow orientations in the Bullfrog tuff fracture by comparing the results of 
this experiment (and Experiments 13 and 14, which were done at different flow rates on the same day) with the results of

Experiments 6 through 8.

Notes: This test was conducted as a tracer step lasting 720 seconds followed by a flush phase lasting 720 seconds. Samples were collected every 20 seconds in both phases. None of the tracers reached their inlet concentration during the step portion of the test, and it was apparent that the dispersion of all tracers was greater than it had been in the three previous tests in the Bullfrog tuff fracture (Experiments 6 through 8). The iodide arrival was delayed relative to the microspheres, and the iodide did not reach as high a concentration as the microspheres. These results are qualitatively consistent with those of the other tests.

\section{Experiment 13:}

Date: $12 / 15 / 93$

Fracture: Bullfrog Tuff (rotated)

Tracers and Tracer Inlet Concentrations:

- 1- $\mu \mathrm{m}$ Blue CML Microspheres (4.19 x 1010/liter)

- $\quad 0.3-\mu \mathrm{m}$ Yellow CML Microspheres $\left(2.52 \times 10^{11} /\right.$ liter $)$

- Iodide $(\sim 0.001 \underline{\mathrm{M}})$

Temperature: $21^{\circ} \mathrm{C}$

Hydraulic Aperture: $111.4 \mu \mathrm{m}$ (before test) - $111.8 \mu \mathrm{m}$ (after test)

Tracer Flow Rate: $6.66 \mu \mathrm{l} / \mathrm{sec}$ (range: 6.75 to $6.58 \mu \mathrm{l} / \mathrm{sec}$ - decreasing)

J-13 Water Flow Rate: $7.18 \mu \mathrm{l} / \mathrm{sec}$ (range: 7.27 to $7.10 \mu \mathrm{l} / \mathrm{sec}$ - decreasing)

Flush Flow Rate: $0.769 \mathrm{ml} / \mathrm{sec}$ (range: 0.770 to $0.768 \mathrm{ml} / \mathrm{sec}$ - constant)

Leak Rate: $0.042 \mu \mathrm{l} / \mathrm{sec}$ under $\sim 40 \mathrm{~cm}$ head

Estimated Pressure Drop across Fracture: $0.57 \mathrm{~cm} \mathrm{H}_{2} \mathrm{O}$

Estimated Pressure Drop across Outlet Manifold: $0.38 \mathrm{~cm} \mathrm{H}_{2} \mathrm{O}$

Objective(s): Same as Experiment 12.

Notes: This test was conducted as a tracer step lasting 1080 seconds followed by a flush phase lasting 1080 seconds. Samples were collected every 30 seconds in both phases. The results of the test were very similar to Experiment 12, except that iodide did not reach as high a concentration during the step portion of the test as in Experiment 12.

Experiment 14:

Date: $12 / 15 / 93$

Fracture: Bullfrog Tuff (rotated)

Tracers and Tracer Inlet Concentrations:

- $1-\mu \mathrm{m}$ Blue CML Microspheres $\left(4.20 \times 10^{10} /\right.$ liter $)$

- $0.3-\mu \mathrm{m}$ Yellow CML Microspheres $\left(2.54 \times 10^{11} /\right.$ liter $)$

- Iodide $(\sim 0.001 \underline{\mathrm{M}})$

Temperature: $21^{\circ} \mathrm{C}$

Hydraulic Aperture: $111.8 \mu \mathrm{m}$ (before test) $-111.8 \mu \mathrm{m}$ (after test)

Tracer Flow Rate: $3.66 \mu \mathrm{l} / \mathrm{sec}$ (range: 3.68 to $3.63 \mu \mathrm{l} / \mathrm{sec}$ - constant)

J-13 Water Flow Rate: $3.92 \mu \mathrm{l} / \mathrm{sec}$ (range: 3.94 to $3.89 \mu \mathrm{l} / \mathrm{sec}-\sim$ constant)

Flush Flow Rate: $0.766 \mathrm{ml} / \mathrm{sec}$ (range: 0.767 to $0.764 \mathrm{ml} / \mathrm{sec}$ - constant)

Leak Rate: $0.042 \mu \mathrm{l} / \mathrm{sec}$ under $\sim 40 \mathrm{~cm}$ head

Estimated Pressure Drop across Fracture: $0.34 \mathrm{~cm} \mathrm{H}_{2} \mathrm{O}$ 
Estimated Pressure Drop across Outlet Manifold: $0.38 \mathrm{~cm} \mathrm{H}_{2} \mathrm{O}$

Objective(s): Same as Experiment 12.

Notes: This test was conducted as a tracer step lasting 1680 seconds followed by a flush phase lasting 1680 seconds. Samples were collected every 30 seconds in both phases. The results of the test were very similar to Experiment 12, except that iodide did not reach as high a concentration during the step portion of the test as in Experiment 12. After the test, the flow system was disassembled and the fracture surfaces were observed under UV light. It was apparent that there was a significant amount of microspheres (primarily blue) on the bottom surface and a hint of microspheres on the top surface. The distribution of the microspheres on both surfaces was recorded in a drawing in a laboratory notebook. The distributions were very similar to those observed after Experiment 8.

\section{Experiment 15:}

Date: $1 / 4 / 94$

Fracture: Bullfrog Tuff

Tracers and Tracer Inlet Concentrations:

- $1-\mu \mathrm{m}$ Blue CML Microspheres (3.89 x 1010/liter)

- $0.3-\mu \mathrm{m}$ Yellow CML Microspheres $\left(1.90 \times 10^{11} /\right.$ liter $)$

- Iodide $(\sim 0.001 \underline{\mathrm{M}})$

Temperature: $21^{\circ} \mathrm{C}$

Hydraulic Aperture: $110.7 \mu \mathrm{m}$ (before test) $-110.4 \mu \mathrm{m}$ (after test)

Tracer Flow Rate: $20.19 \mu \mathrm{l} / \mathrm{sec}$ (range: 20.23 to $20.14 \mu \mathrm{l} / \mathrm{sec}$ - constant)

J-13 Water Flow Rate: N/A

Flush Flow Rate: $1.157 \mathrm{ml} / \mathrm{sec}$ (range: 1.154 to $1.161 \mathrm{ml} / \mathrm{sec}$ - constant)

Leak Rate: $0.14 \mu \mathrm{l} / \mathrm{sec}$ under $\sim 40 \mathrm{~cm}$ head

Estimated Pressure Drop across Fracture: $1.87 \mathrm{~cm} \mathrm{H}_{2} \mathrm{O}$

Estimated Pressure Drop across Outlet Manifold: $0.54 \mathrm{~cm} \mathrm{H}_{2} \mathrm{O}$

Objective(s): This test essentially served as a repeat of Experiment 6 . The intent was to see how reproducible the tracer experiments were on two separate days and for two separate times assembling the flow system. The fracture surfaces had been slightly damaged since the earlier experiments (a few small pieces had broken off), so the effect of this damage could be assessed.

Notes: This test was conducted as a tracer step lasting 880 seconds, with samples collected every 20 seconds. There was no flush phase, although extensive flushing by gravity was conducted after collecting the last sample. The tracer arrival times in this test were earlier and the tails in the breakthrough curves were lower than in the earlier tests in this fracture (Experiments 6 through 8), indicating an increase in dispersion in the fracture that was perhaps due to the surface damage.

Experiment 16:

Date: $1 / 4 / 94$

Fracture: Tram Tuff

Tracers and Tracer Inlet Concentrations: 
- $\quad 1-\mu \mathrm{m}$ Blue CML Microspheres (3.91 x 1010/liter)

- $0.3-\mu m$ Yellow CML Microspheres $\left(1.91 \times 10^{11} /\right.$ liter $)$

- Iodide $(\sim 0.001 \underline{\mathrm{M}})$

Temperature: $22^{\circ} \mathrm{C}$

Hydraulic Aperture: $88.9 \mu \mathrm{m}$ (before test) - $90.8 \mu \mathrm{m}$ (after test)

Tracer Flow Rate: $9.33 \mu \mathrm{l} / \mathrm{sec}$ (range: 9.36 to $9.30 \mu \mathrm{l} / \mathrm{sec}-\sim$ constant)

J-13 Water Flow Rate: N/A

Flush Flow Rate: $1.164 \mathrm{ml} / \mathrm{sec}$ (range: 1.167 to $1.161 \mathrm{ml} / \mathrm{sec}-\sim$ constant)

Leak Rate: $\sim 0 \mu \mathrm{l} / \mathrm{sec}$ (not measurable) under $\sim 40 \mathrm{~cm}$ head

Estimated Pressure Drop across Fracture: $1.78 \mathrm{~cm} \mathrm{H}_{2} \mathrm{O}$

Estimated Pressure Drop across Outlet Manifold: $0.49 \mathrm{~cm} \mathrm{H}$

Objective(s): This test essentially served as a repeat of Experiment 9 . The intent was to see how reproducible the tracer experiments were on two separate days and for two separate times assembling the flow system.

Notes: This test was conducted as a tracer step lasting 640 seconds, with samples collected every 20 seconds. There was no flush phase, although extensive flushing by gravity was conducted after collecting the last sample. The dispersion of all tracers in this test was essentially identical to that observed the earlier tests in this fracture (Experiments 9 through 11), indicating good experimental reproducibility. After the test, the flow system was disassembled and the fracture surfaces were observed under UV light. There were no microspheres apparent on the surfaces.

Experiment 17:

Date: $1 / 4 / 94$

Fracture: Bullfrog Tuff (rotated)

Tracers and Tracer Inlet Concentrations:

- $\quad 1-\mu \mathrm{m}$ Blue CML Microspheres (3.92 x 1010/liter)

- $0.3-\mu \mathrm{m}$ Yellow CML Microspheres (1.92 x 1011/liter)

- Iodide $(\sim 0.001 \underline{\mathrm{M}})$

Temperature: $22^{\circ} \mathrm{C}$

Hydraulic Aperture: $114.0 \mu \mathrm{m}$ (before test) - $113.7 \mu \mathrm{m}$ (after test)

Tracer Flow Rate: $15.80 \mu \mathrm{l} / \mathrm{sec}$ (range: 15.92 to $15.69 \mu \mathrm{l} / \mathrm{sec}$ - decreasing)

J-13 Water Flow Rate: N/A

Flush Flow Rate: $1.169 \mathrm{ml} / \mathrm{sec}$ (range: 1.172 to $1.166 \mathrm{ml} / \mathrm{sec}$ - constant)

Leak Rate: $0.084 \mu \mathrm{l} / \mathrm{sec}$ under $\sim 40 \mathrm{~cm}$ head

Estimated Pressure Drop across Fracture: $1.28 \mathrm{~cm} \mathrm{H}_{2} \mathrm{O}$

Estimated Pressure Drop across Outlet Manifold: $0.55 \mathrm{~cm} \mathrm{H} \mathrm{H}_{2} \mathrm{O}$

Objective(s): This test essentially served as a repeat of Experiment 12 . The intent was to see how reproducible the tracer experiments were on two separate days and for two separate times assembling the flow system. The fracture surfaces had been slightly damaged since the earlier experiments (a few small pieces had broken off), so the effect of this damage could be assessed.

Notes: This test was conducted as a tracer step lasting 1120 seconds, with samples collected every 20 seconds. There was no flush phase, although extensive flushing by gravity was conducted after collecting the last sample. The tracer arrival times in this test were significantly later and 
the tails in the breakthrough curves were higher than in the earlier tests in this fracture (Experiments 12 through 14), indicating a decrease in dispersion in the fracture that was perhaps due to the surface damage. After this test, the flow system was disassembled and the fracture surfaces were observed under UV light. It was apparent that there was a significant amount of microspheres (primarily blue) on the bottom surface and a hint of microspheres on the top surface. The distribution of the microspheres on the surfaces was very similar to that observed after Experiments 8 and 14.

Experiment 18:

Date: $1 / 6 / 94$

Fracture: Bullfrog Tuff

Tracers and Tracer Inlet Concentrations:

- $1-\mu \mathrm{m}$ Blue CML Microspheres $\left(3.95 \times 10^{10} /\right.$ liter $)$

- $0.3-\mu \mathrm{m}$ Yellow CML Microspheres $\left(1.92 \times 10^{11} /\right.$ liter $)$

- Iodide $(\sim 0.001 \underline{\mathrm{M}})$

Temperature: $22^{\circ} \mathrm{C}$

Hydraulic Aperture: $114.2 \mu \mathrm{m}$ (before test) $-114.7 \mu \mathrm{m}$ (after test)

Tracer Flow Rate: $19.95 \mu \mathrm{l} / \mathrm{sec}$ (range: 20.10 to $19.85 \mu \mathrm{l} / \mathrm{sec}$ - decreasing)

J-13 Water Flow Rate: N/A

Flush Flow Rate: $1.183 \mathrm{ml} / \mathrm{sec}$ (range: 1.166 to $1.197 \mathrm{ml} / \mathrm{sec}$ - increasing)

Leak Rate: $0.097 \mu \mathrm{l} / \mathrm{sec}$ under $\sim 40 \mathrm{~cm}$ head

Estimated Pressure Drop across Fracture: $1.66 \mathrm{~cm} \mathrm{H}_{2} \mathrm{O}$

Estimated Pressure Drop across Outlet Manifold: $0.54 \mathrm{~cm} \mathrm{H}_{2} \mathrm{O}$

Objective(s): This test essentially served as a repeat of Experiment 15. The intent was to see how reproducible the tracer experiments were on two separate days and for two separate times assembling the flow system. This time, however, no apparent damage to the surfaces had occurred in the two days since Experiment 15.

Notes: This test was conducted as a tracer step lasting 880 seconds, with samples collected every 20 seconds. There was no flush phase, although extensive flushing by gravity was conducted after collecting the last sample. The results of this test were very similar to the results of Experiment 15, suggesting that there was good reproducibility of tracer experiments as long as no surface damage occurred. After this test, the flow system was disassembled and the fracture surfaces were observed under UV light. It was apparent that there was a significant amount of microspheres (primarily blue) on the bottom surface and a hint of microspheres on the top surface. The distribution of the microspheres on the surfaces was very similar to that observed after Experiments 8,14, and 17. 


\section{APPENDIX B: BREAKTHROUGH CURVES FROM TRACER EXPERIMENTS}

This appendix contains plots of the experimental breakthrough curves associated with each of the 18 tracer experiments summarized in Appendix A. For each experiment, the following plots are provided:

- A plot showing the breakthrough curves of all tracers over the entire duration of the test. For tests in which there was a flush phase, this plot shows the rise and decay of all tracer concentrations as a function of time. For tests in which there was no flush phase, only the rise in tracer concentrations is shown.

- For tests in which there was a flush phase, a plot is included that shows only the rise of all tracer concentrations during the step phase of the test.

- For tests in which there was a flush phase, a plot of $\left(1-C / C_{0}\right)$ vs. volume eluted during the flush phase is superimposed on a plot of $C / C_{o} v s$. volume eluted during the step phase. These plots are provided for each tracer in a given experiment, so there are as many plots as there are tracers. The plots are intended to show the equivalence of the step and flush curves. This equivalence suggests that the primary mechanism affecting tracer transport through the fractures is hydrodynamic dispersion and not adsorption or attachment reactions with the fracture walls (which would likely cause discrepancies between the step and flush curves - see discussion in Section 3.1.7).

All concentrations in the breakthrough curves are normalized to the inlet concentration of the experiment, $C_{0}$. Error bars that correspond to \pm 2 relative standard deviations are shown in the plots comparing the step and flush phases (errors were typically different in the step and flush phases). For experiments in which there was no flush phase, error bars are shown in the plot of the breakthrough curves of all tracers (the only plot provided). The relative standard deviations used to calculate the error bars are given in the last column of Table 3.8. The reader is referred to Appendix A for the experimental conditions associated with each plot. 


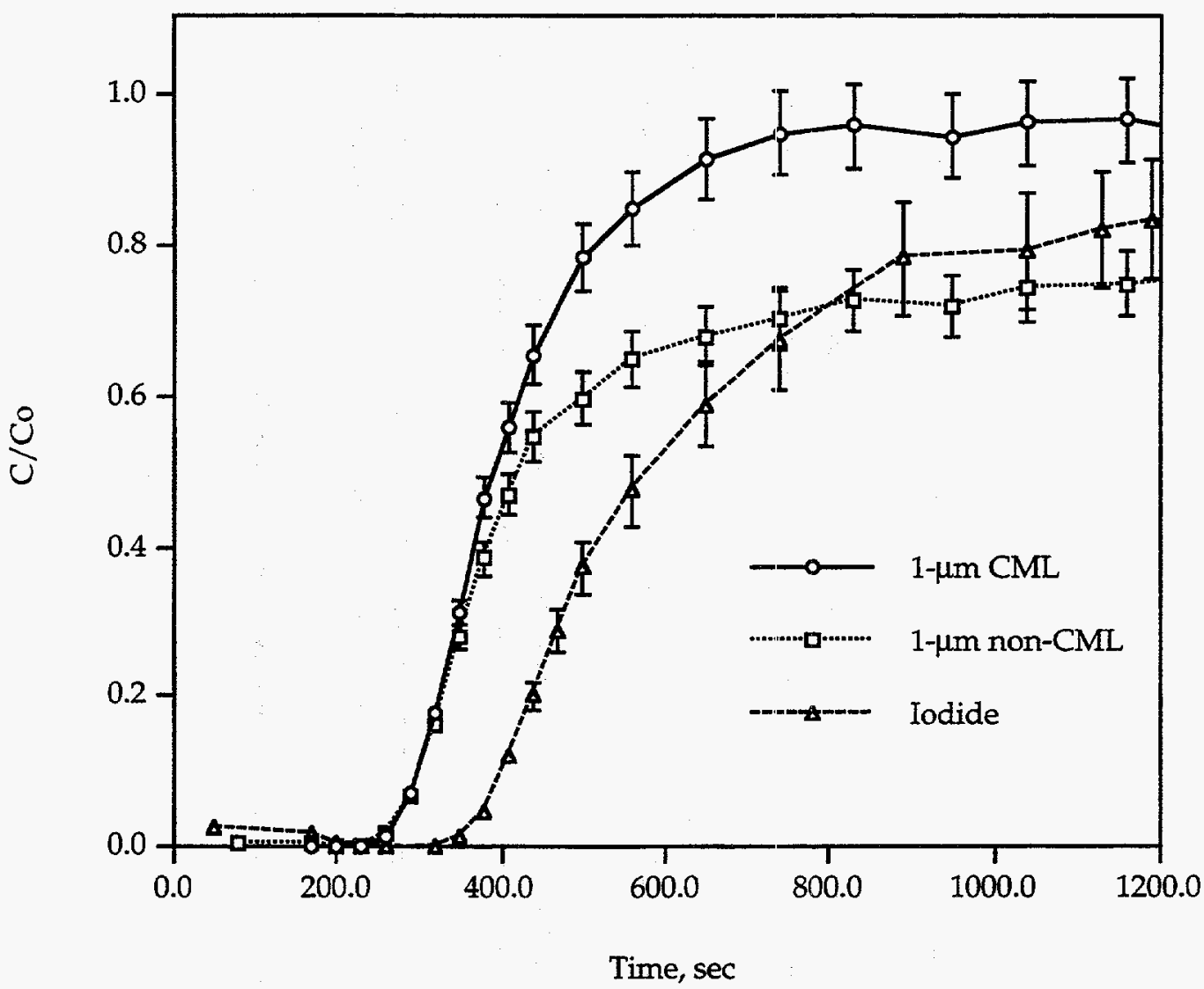

Figure B.1. Breakthrough curves of the 1- $\mu \mathrm{m}$ CML microspheres, the 1- $\mu \mathrm{m}$ nonCML (polystyrene) microspheres, and iodide in Experiment 1 (Bandelier tuff fracture). 


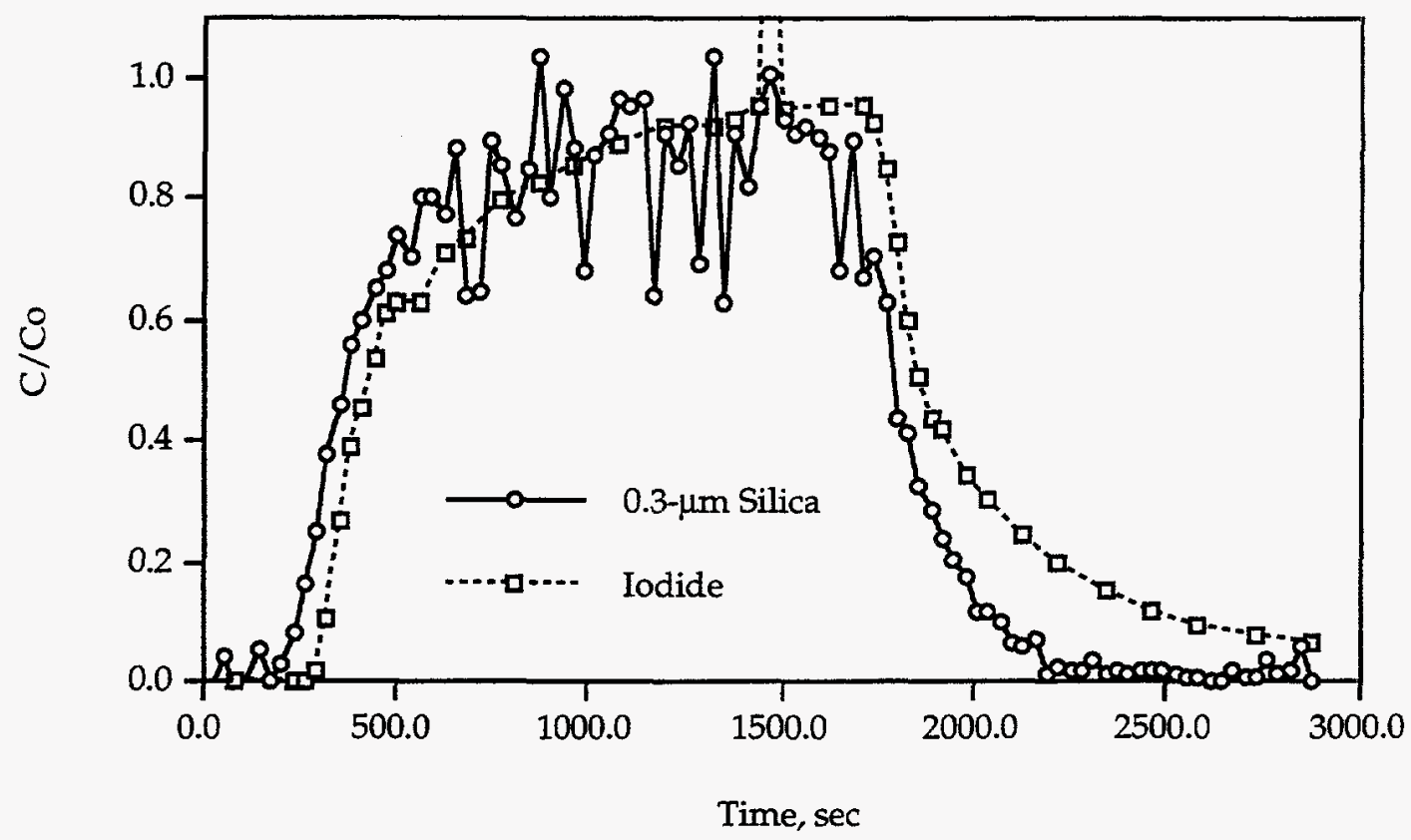

Figure B.2. Breakthrough curves of $0.3-\mu \mathrm{m}$ silica microspheres and iodide in Experiment 2 (Bandelier tuff fracture).

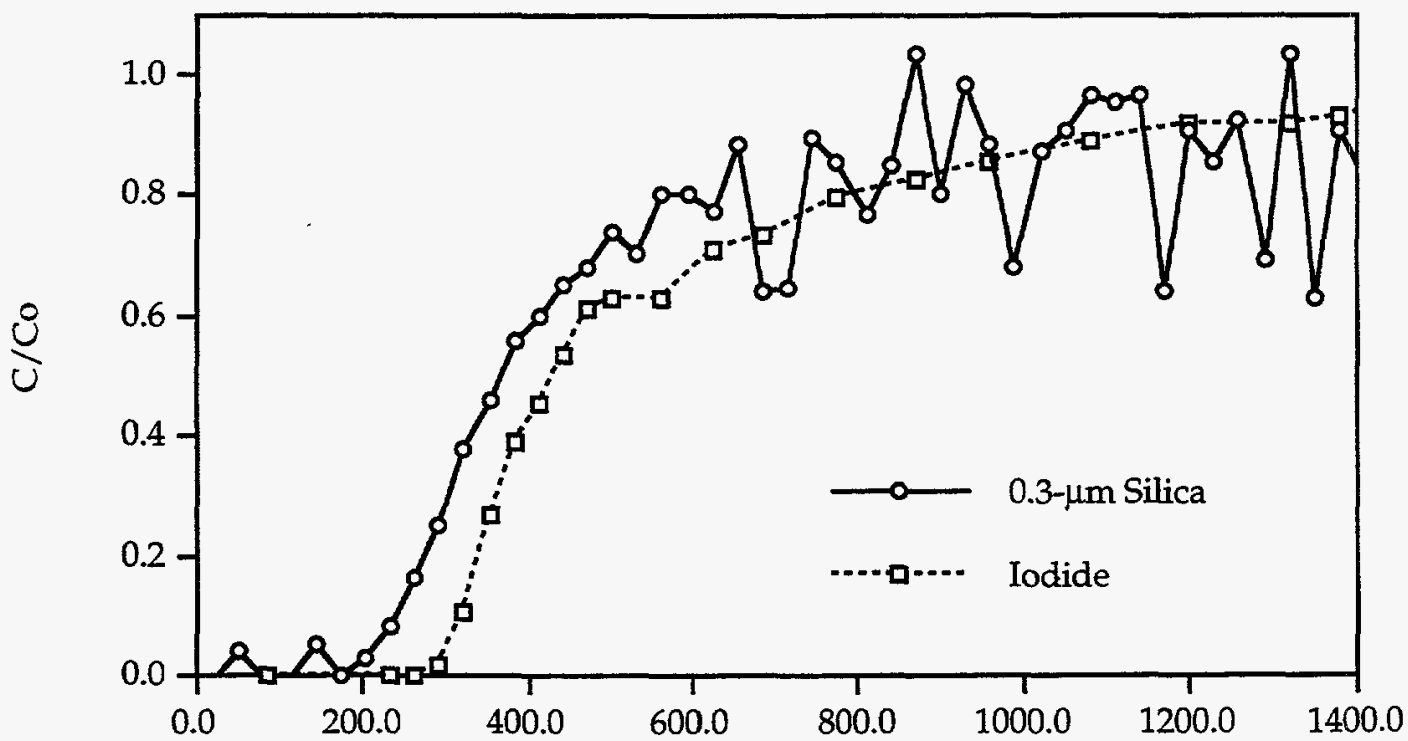

Time, sec

Figure B.3. Step portion of breakthrough curves for $0.3-\mu \mathrm{m}$ silica microspheres and iodide in Experiment 2 (Bandelier tuff fracture). 


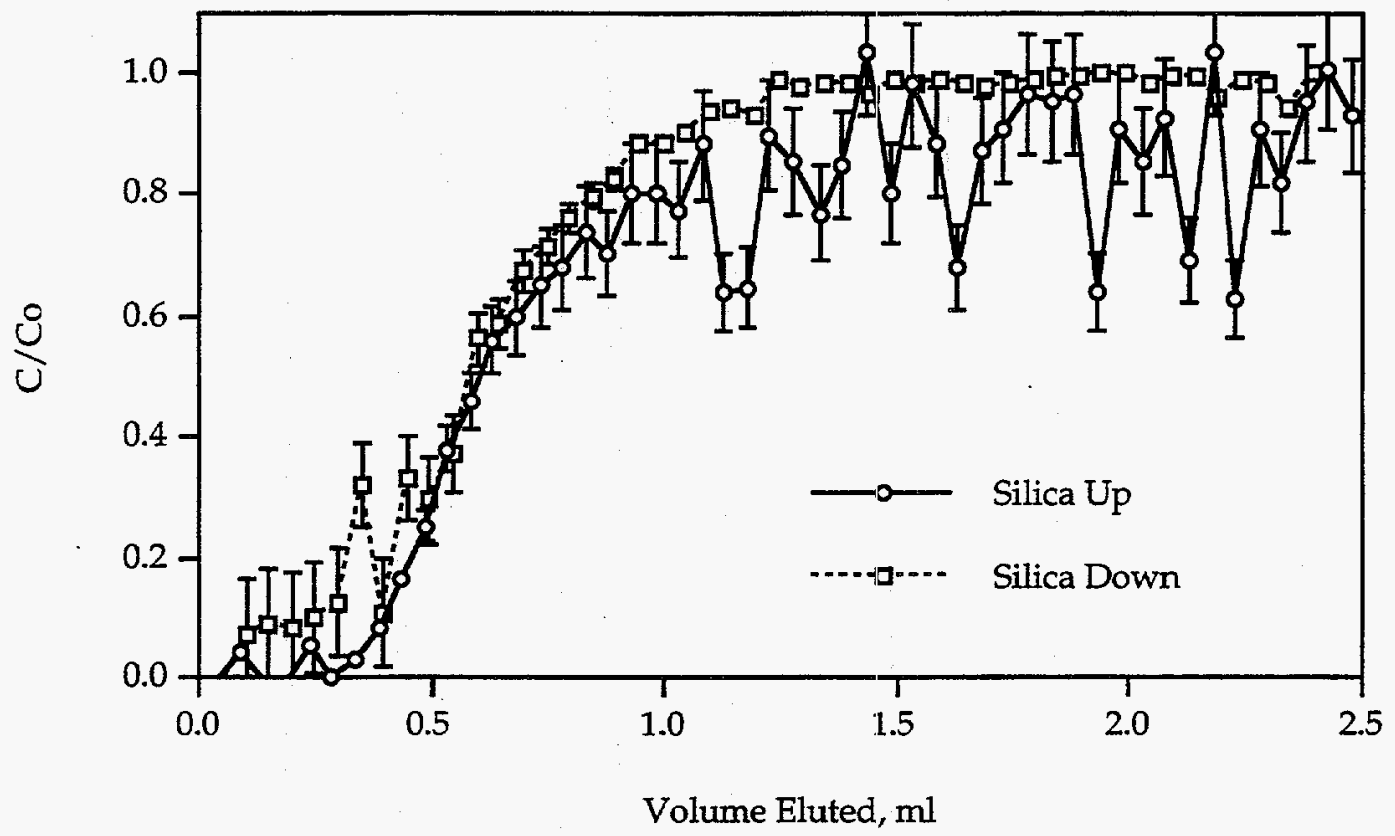

Figure B.4. Comparison of $\left(C / C_{0}\right)_{\text {step }}(u p)$ and $\left(1-C / C_{0}\right)$ flush $($ down $)$ for the 0.3$\mu \mathrm{m}$ silica microspheres in Experiment 2 (Bandelier tuff fracture).

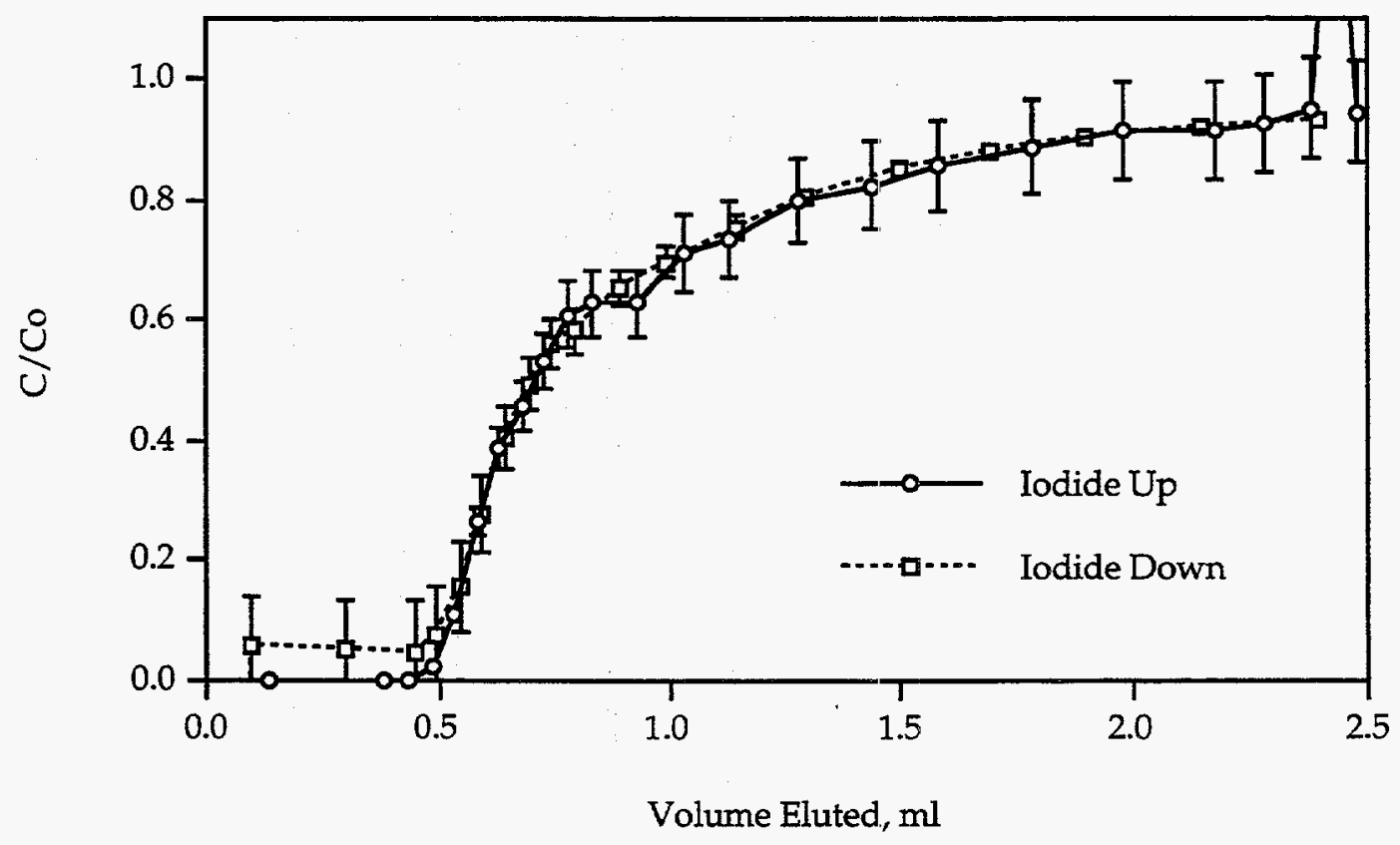

Figure B.5. Comparison of $\left(C / C_{0}\right)_{\text {step }}$ (up) and $\left(1-C / C_{0}\right)_{\text {flush }}($ down $)$ for iodide in Experiment 2 (Bandelier tuff fracture). 


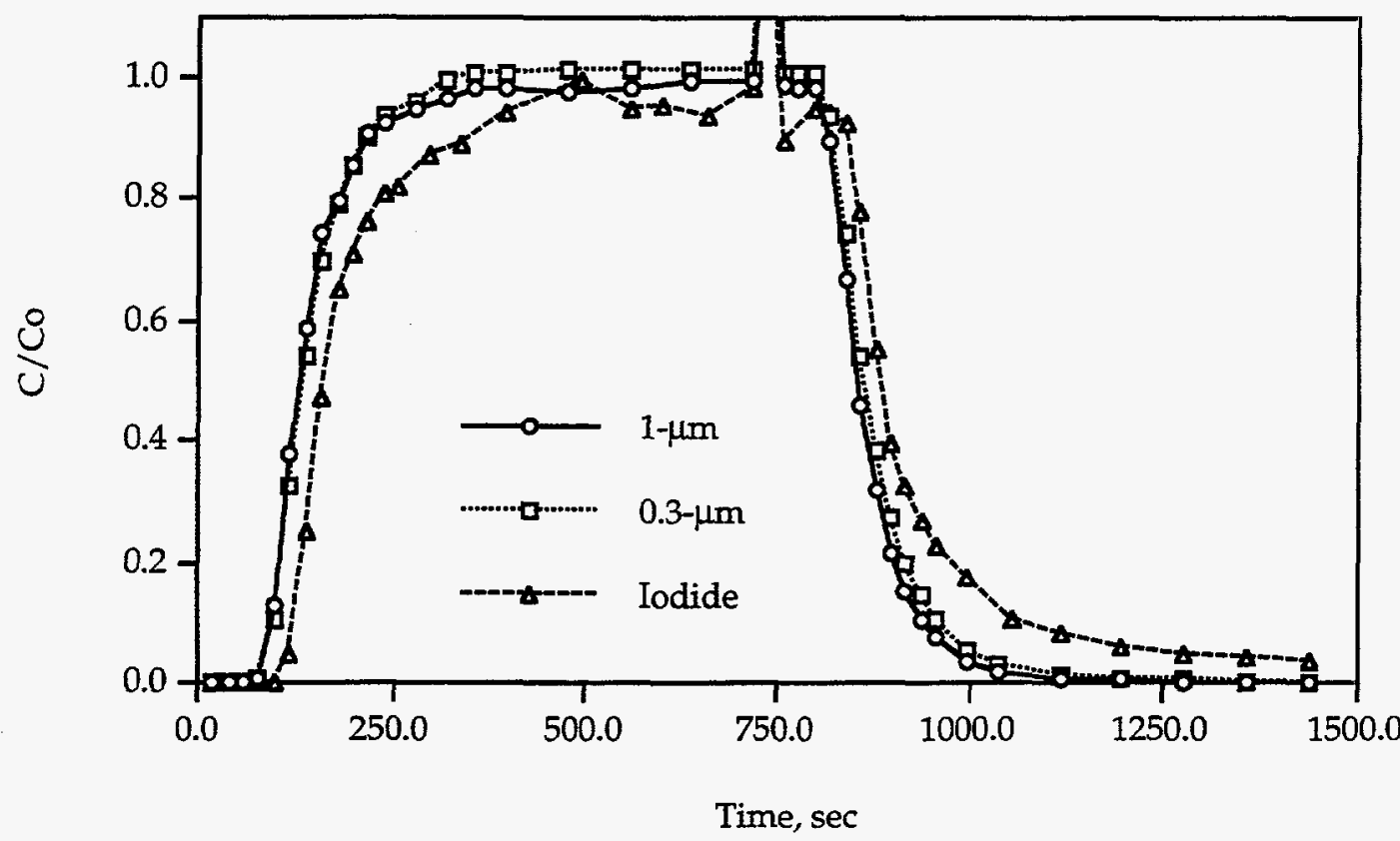

Figure B.6. Breakthrough curves of 1- $\mu \mathrm{m}$ CML microspheres, $0.3-\mu \mathrm{m}$ CML microspheres, and iodide in Experiment 3 (Bandelier tuff fracture).

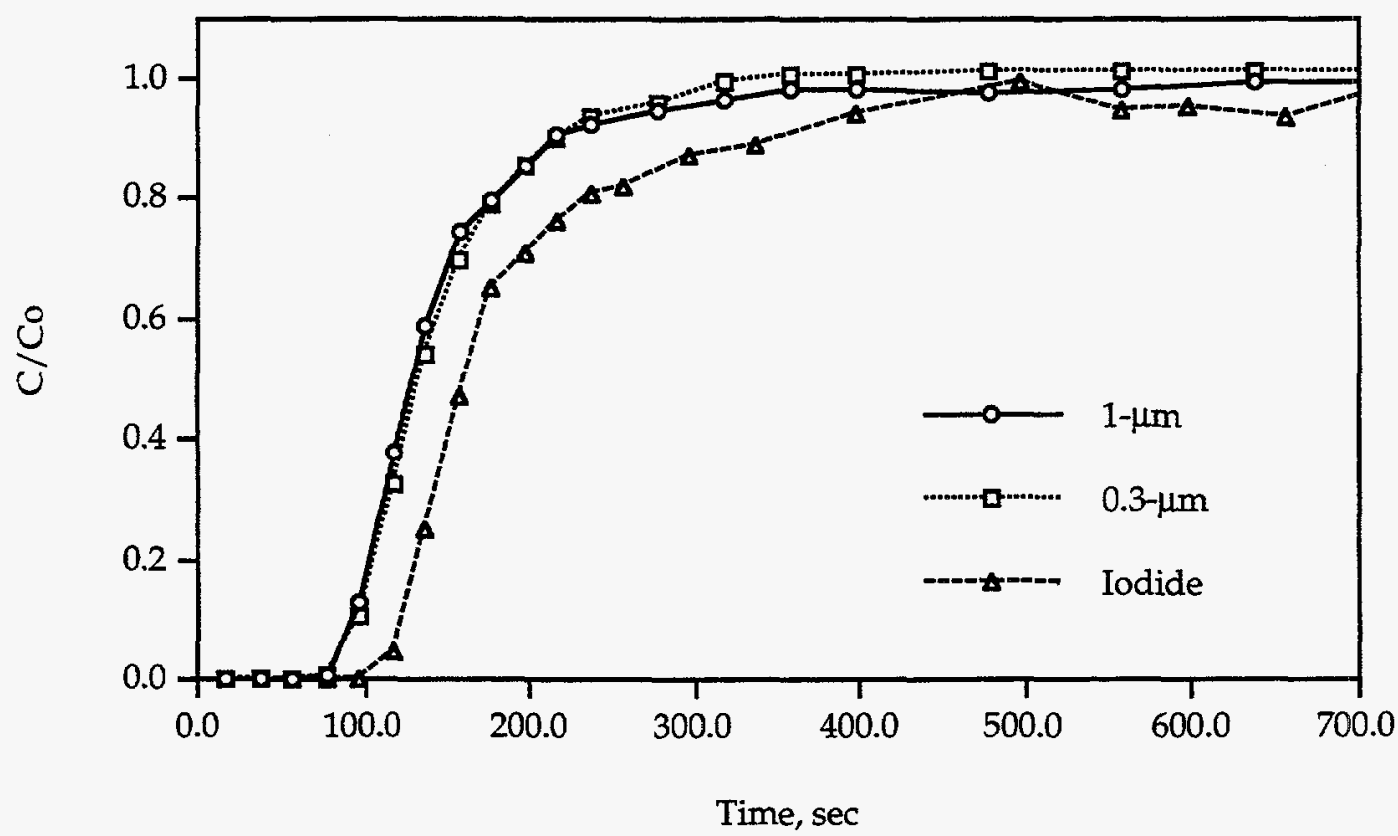

Figure B.7. Step portion of breakthrough curves for 1- $\mu \mathrm{m}$ CML microspheres, $0.3-\mu \mathrm{m}$ CML microspheres, and iodide in Experiment 3 (Bandelier tuff fracture). 


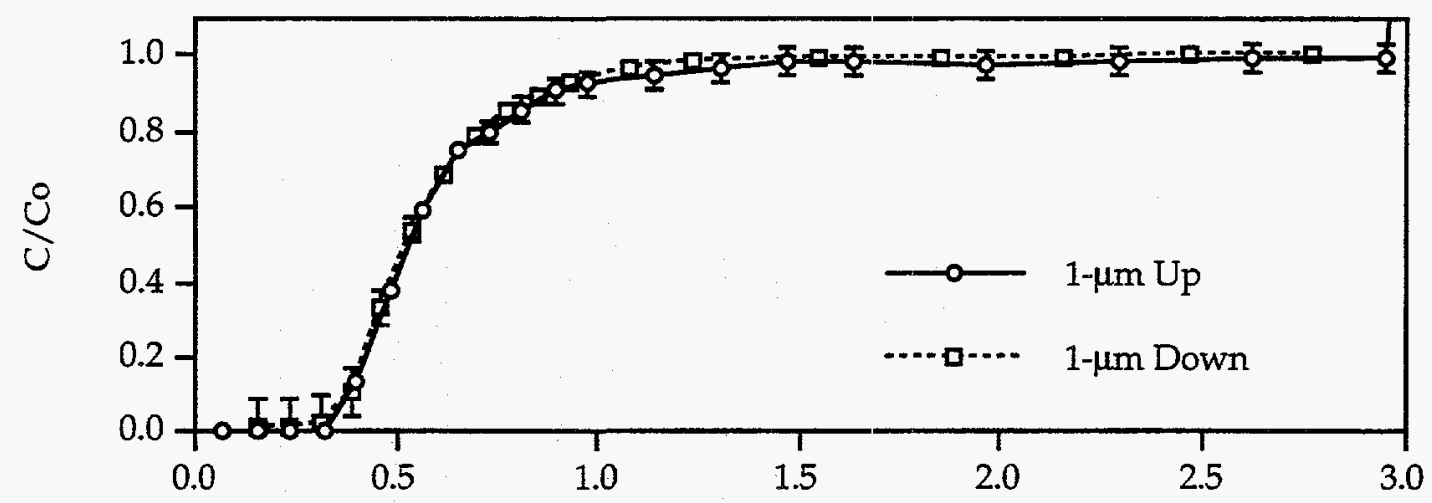

Volume Eluted, $\mathrm{ml}$

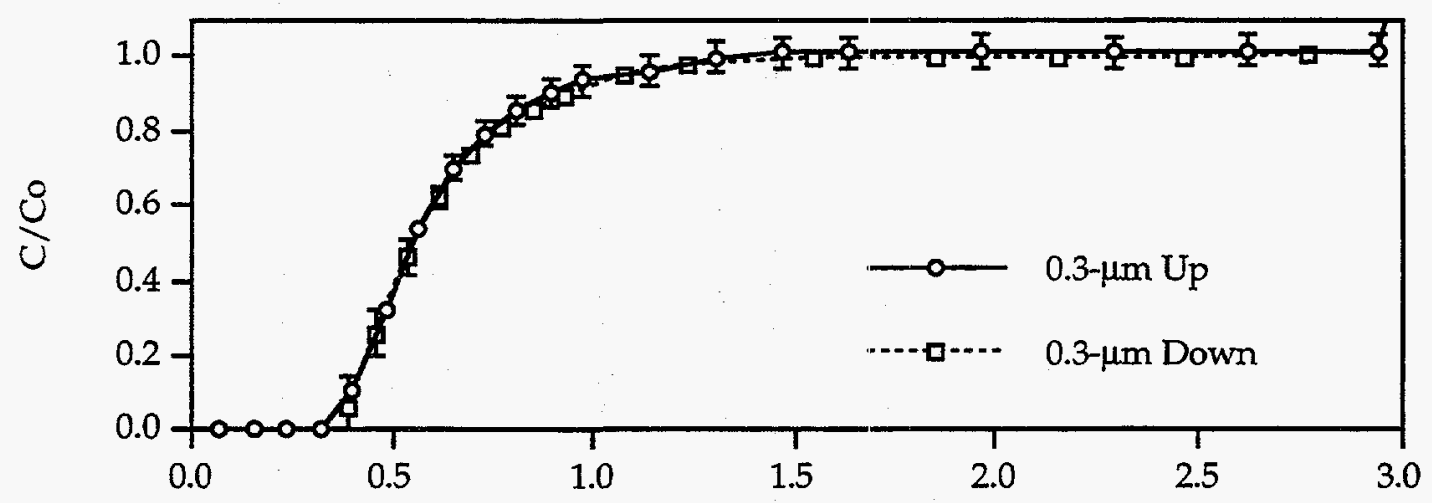

Volume Eluted, $\mathrm{ml}$

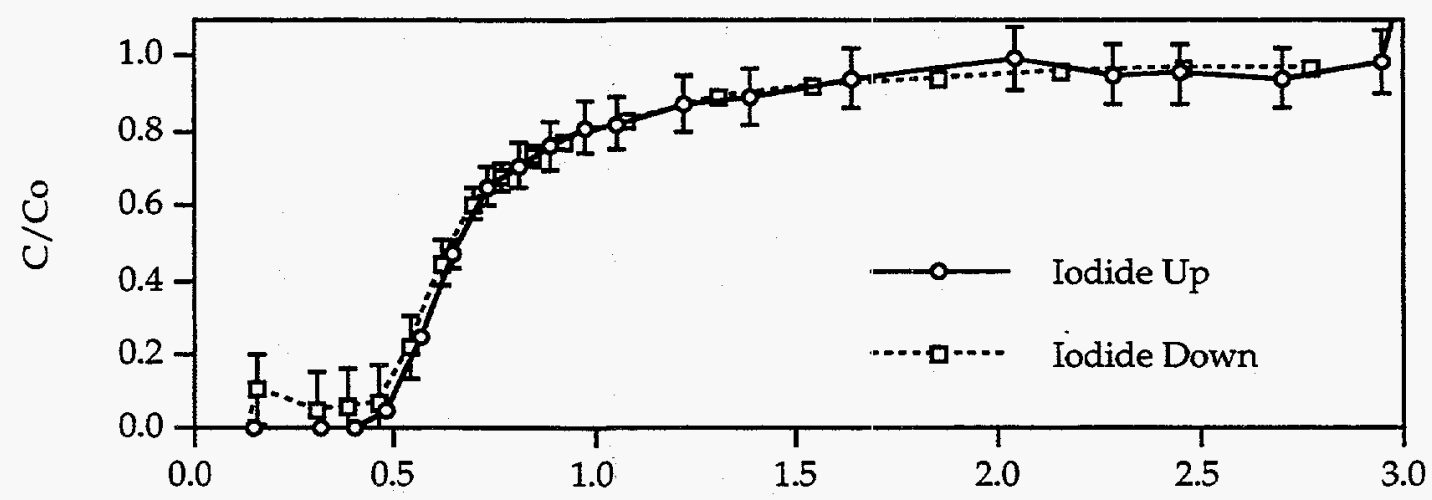

Volume Eluted, ml

Figures B.8, B.9, B.10. Comparison of $\left(\mathrm{C} / \mathrm{C}_{0}\right)_{\text {step }}$ (up) and $\left(1-\mathrm{C} / \mathrm{C}_{0}\right)_{\text {flush }}$ (down) for the 1- $\mu \mathrm{m}$ CML microspheres, $0.3-\mu \mathrm{m}$ CML microspheres, and iodide, respectively, in Experiment 3 (Bandelier tuff fracture). 


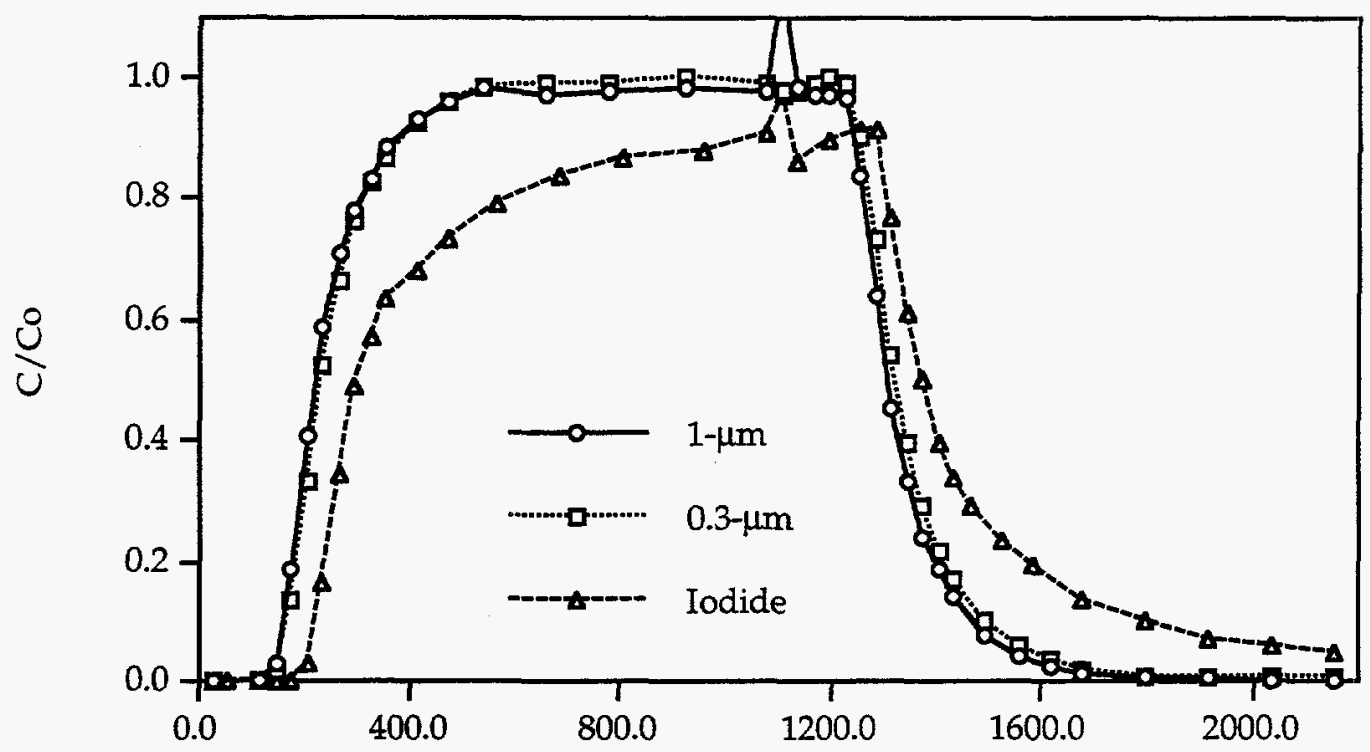

Time, sec

Figure B.11. Breakthrough curves of $1-\mu \mathrm{m}$ CML microspheres, $0.3-\mu \mathrm{m} C M L$ microspheres, and iodide in Experiment 4 (Bandelier tuff fracture).

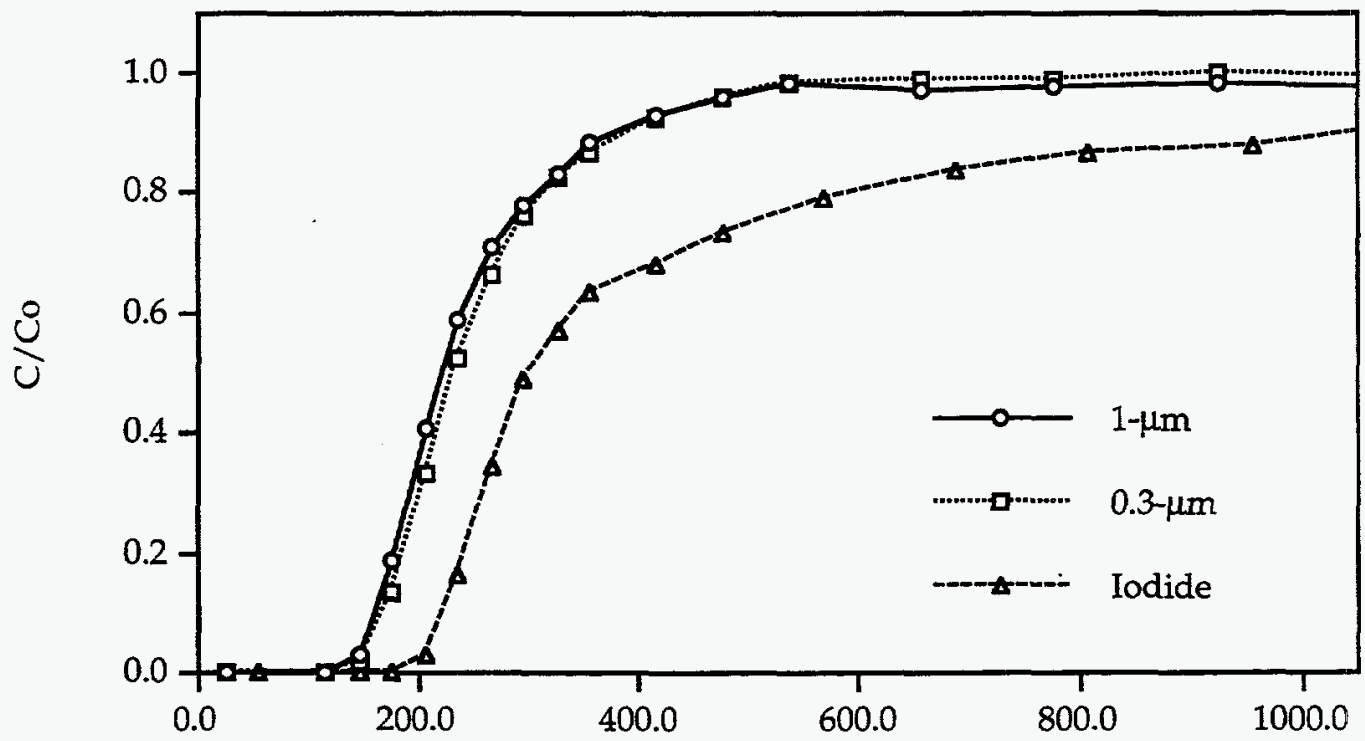

Time, sec

Figure B.12. Step portion of breakthrough curves for 1- $\mu \mathrm{m}$ CML microspheres, $0.3-\mu \mathrm{m}$ CML microspheres, and iodide in Experiment 4 (Bandelier tuff fracture). 


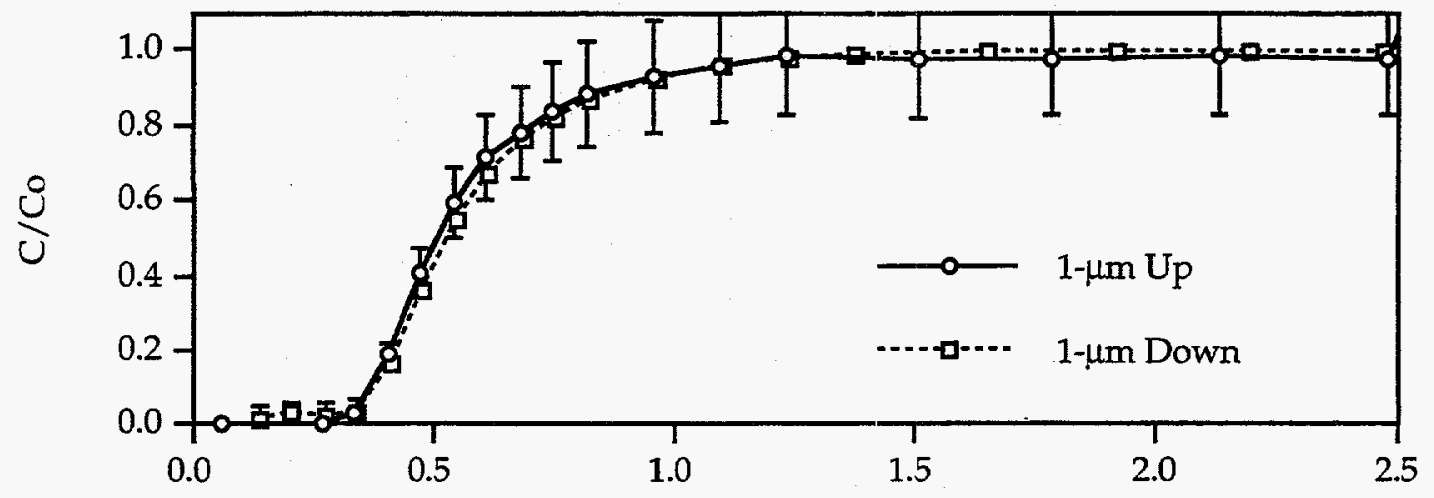

Volume Eluted, ml
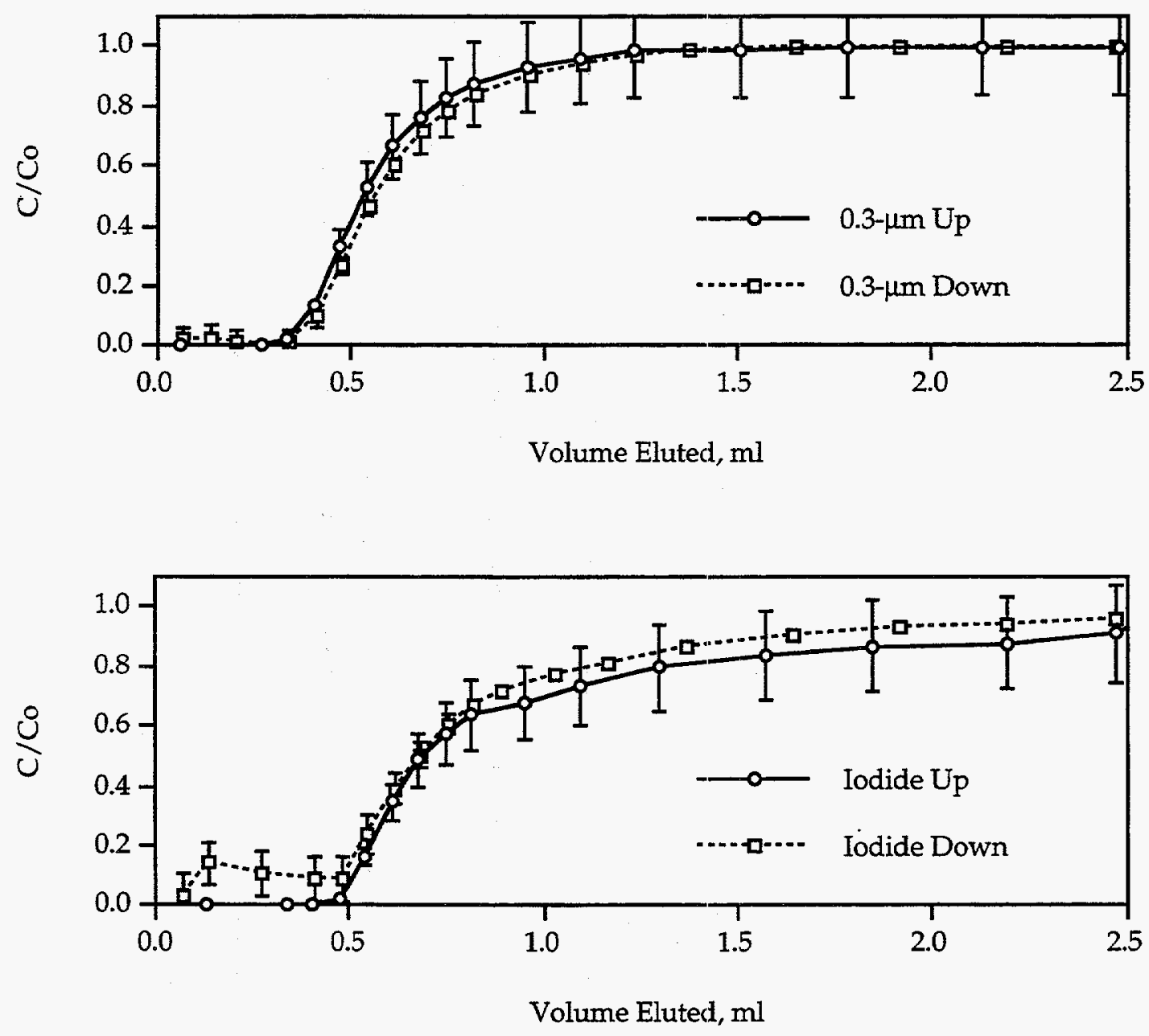

Figures B.13, B.14, B.15. Comparison of $\left(C / C_{0}\right)_{\text {step }}$ (up) and $\left(1-C / C_{0}\right)_{\text {flush }}$ (down) for the 1- $\mu \mathrm{m}$ CML microspheres, $0.3-\mu \mathrm{m}$ CML microspheres, and iodide, respectively, in Experiment 4 (Bandelier tuff fracture). 


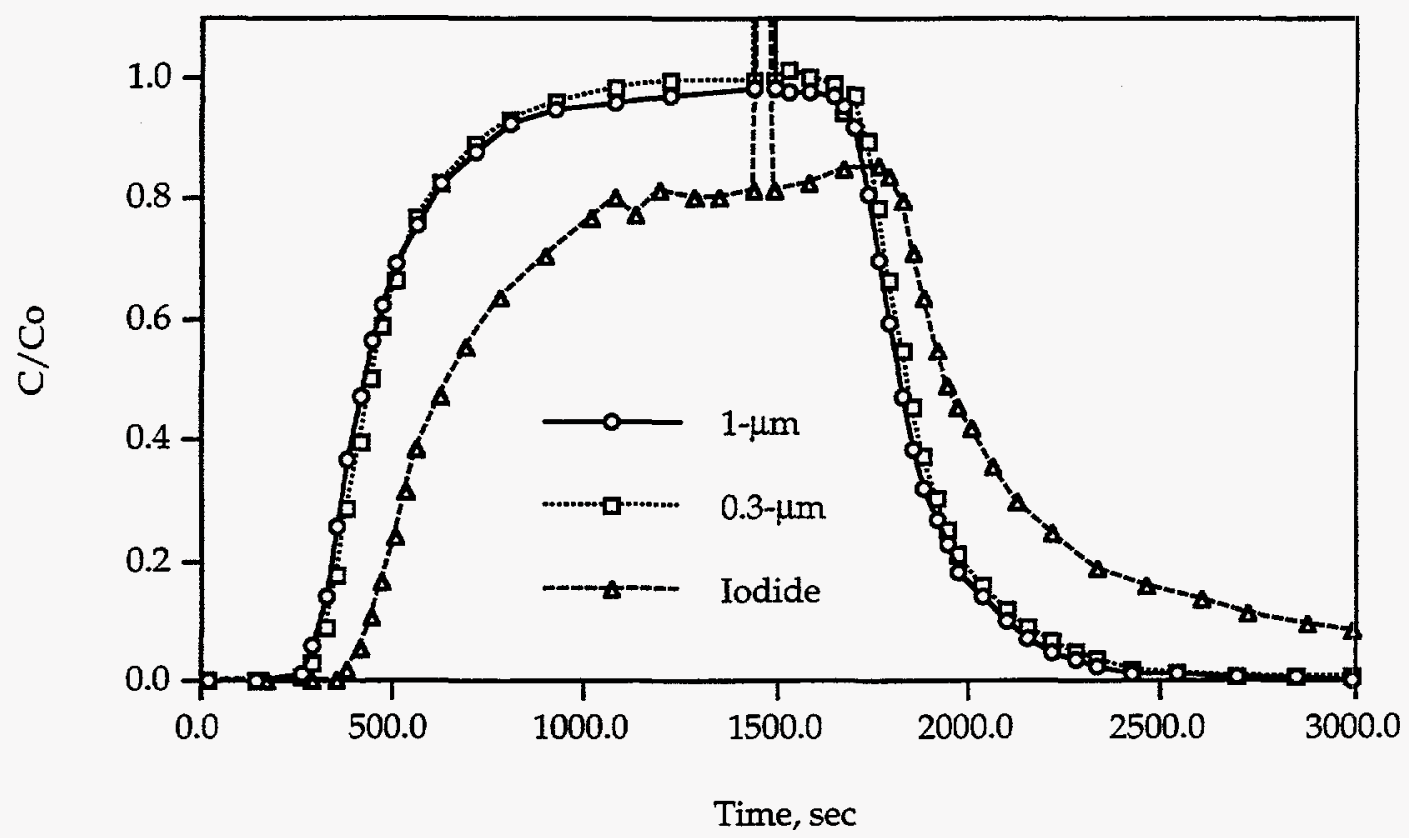

Figure B.16. Breakthrough curves of 1- $\mu \mathrm{m}$ CML microspheres, $0.3-\mu \mathrm{m}$ CML microspheres, and iodide in Experiment 5 (Bandelier tuff fracture).

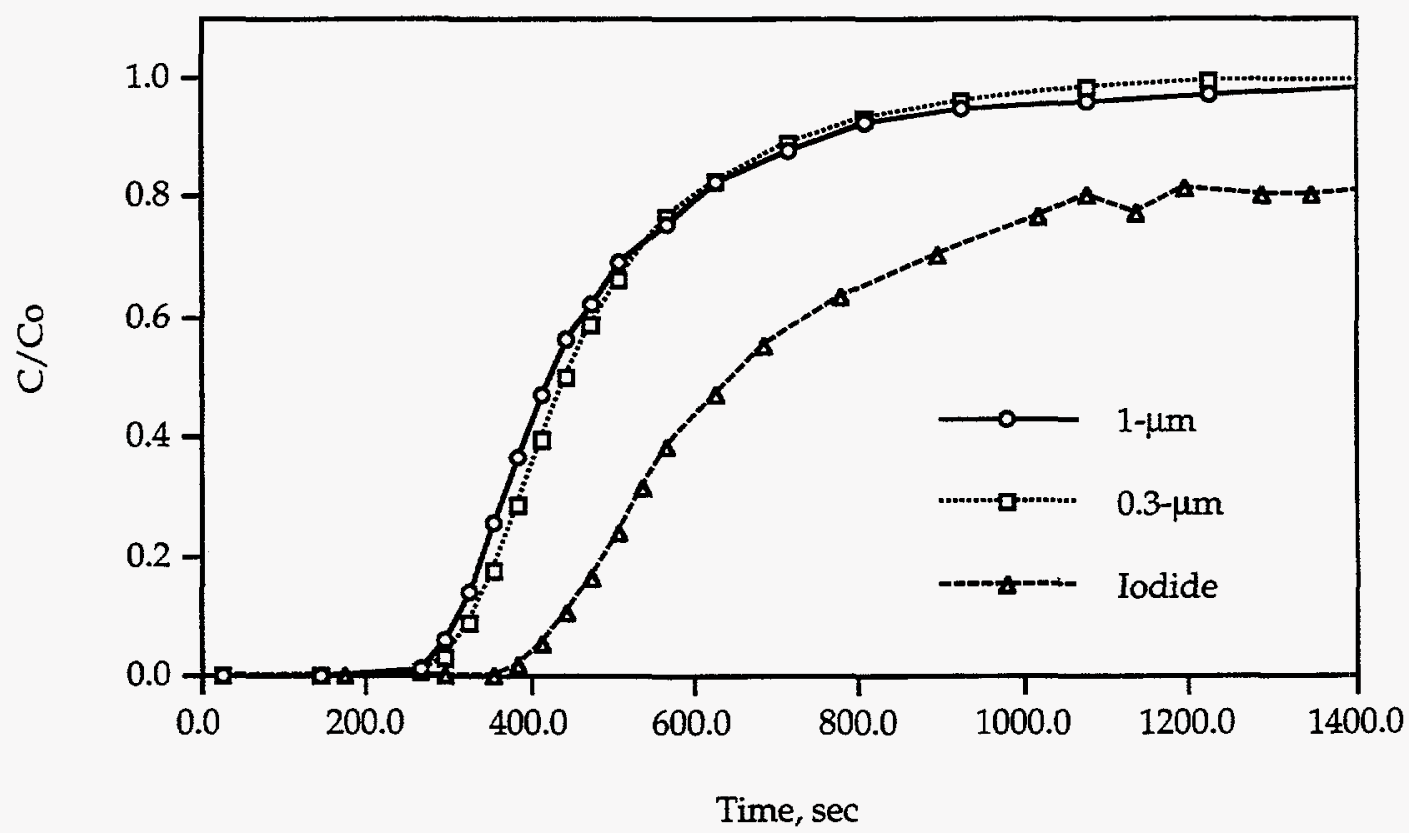

Figure B.17. Step portion of breakthrough curves for 1- $\mu \mathrm{m} C M L$ microspheres, $0.3-\mu \mathrm{m}$ CML microspheres, and iodide in Experiment 5 (Bandelier tuff fracture). 


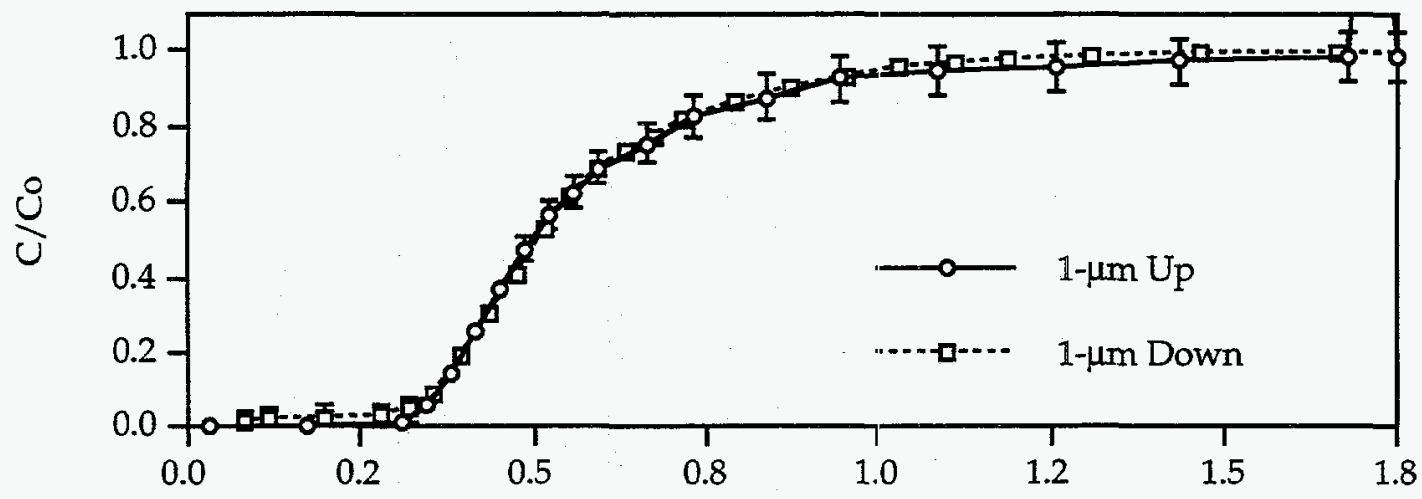

Volume Eluted, $\mathrm{ml}$

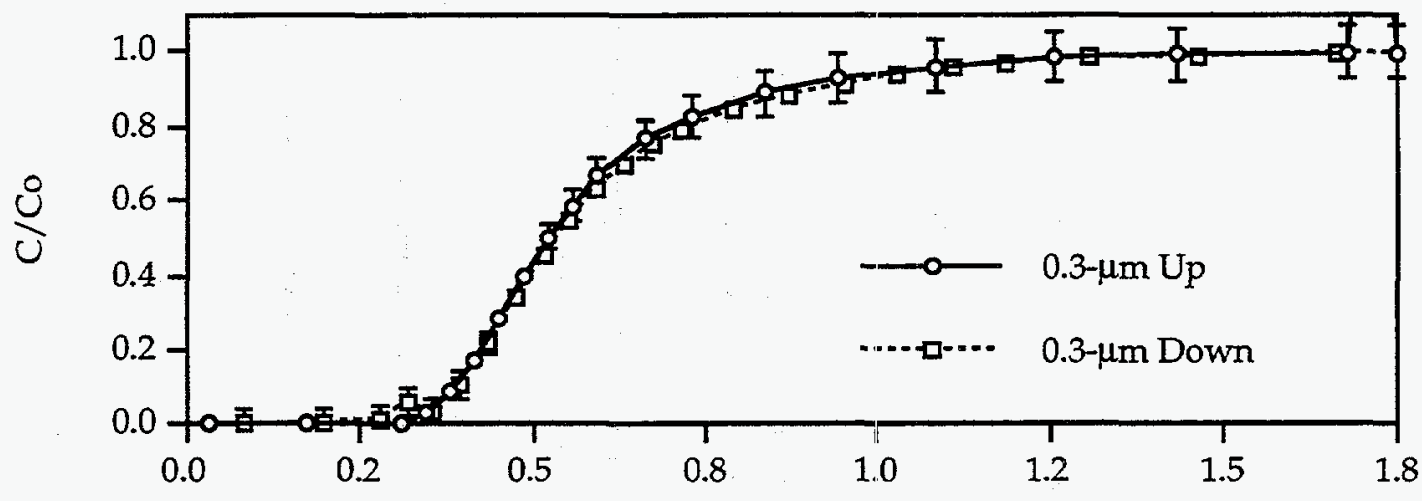

Volume Eluted, ml

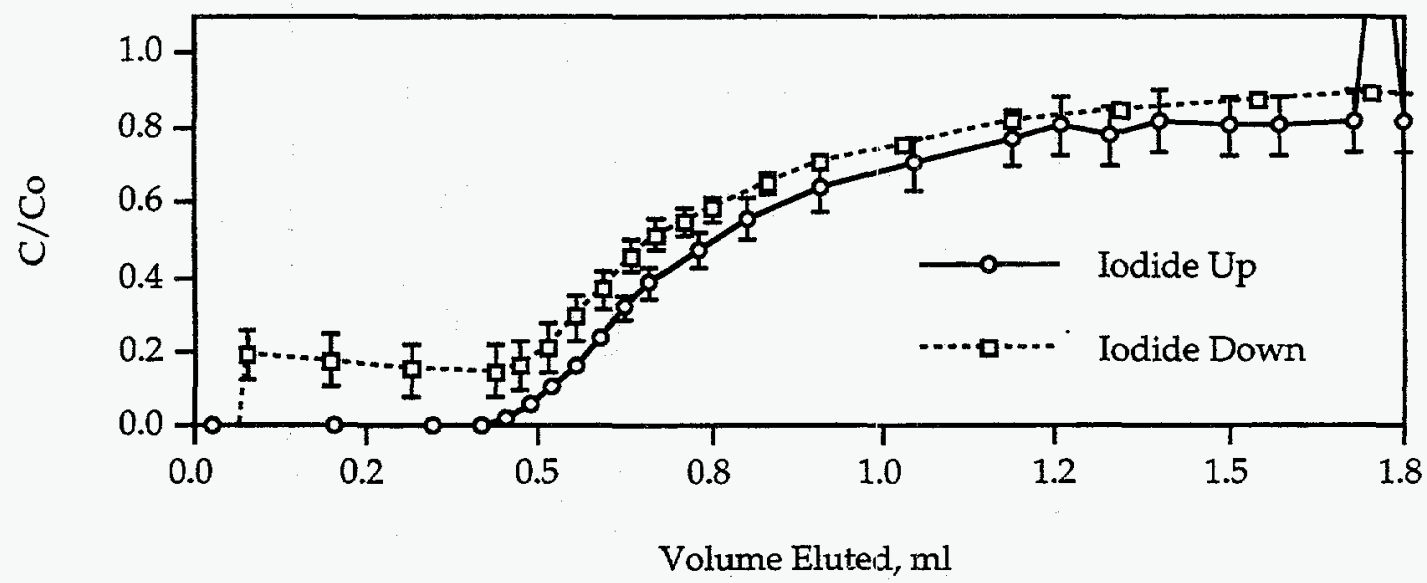

Figures B.18, B.19, B.20. Comparison of $\left(C / C_{0}\right)_{\text {step }}$ (up) and $\left(1-C / C_{0}\right)_{\text {flush }}$ (down) for the 1- $\mu \mathrm{m}$ CML microspheres, $0.3-\mu \mathrm{m}$ CML microspheres, and iodide, respectively, in Experiment 5 (Bandelier tuff fracture). 


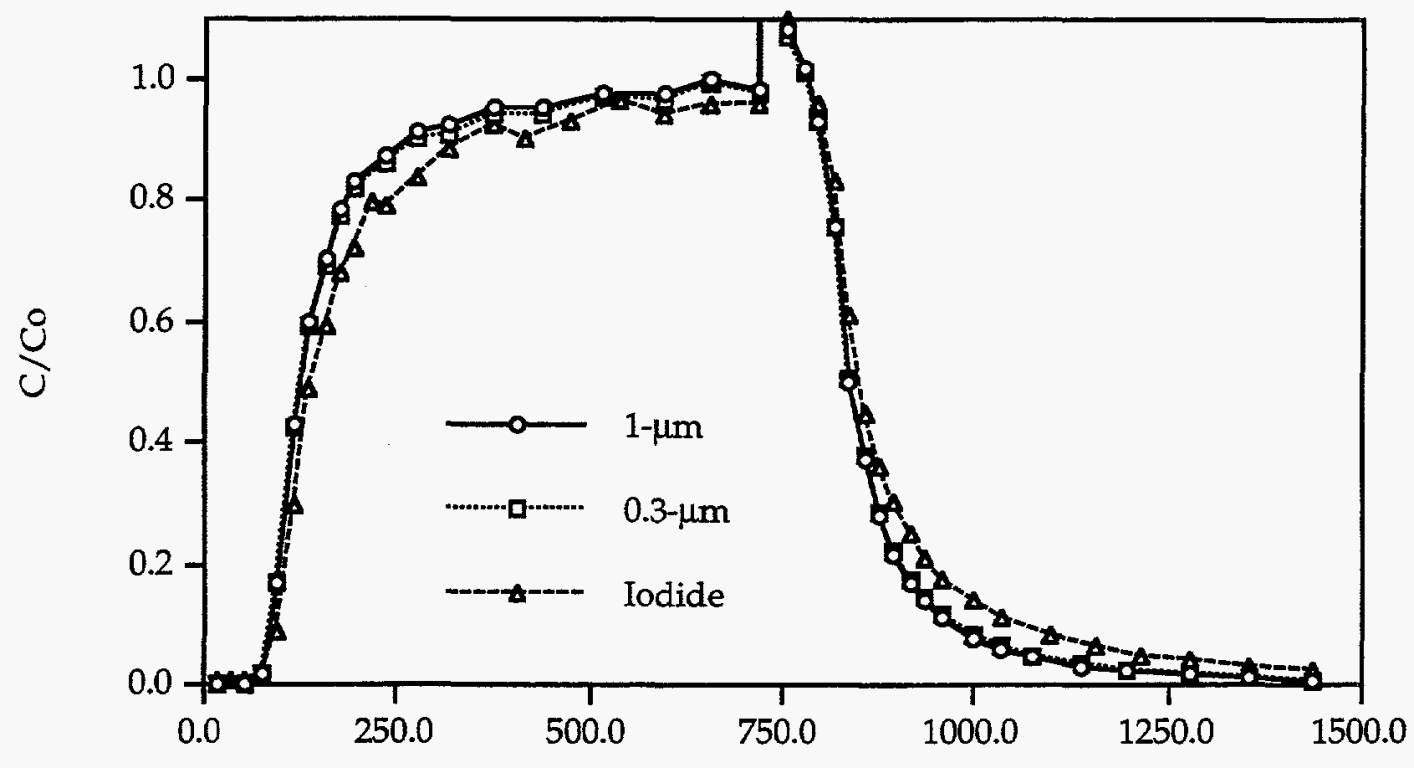

Time, sec

Figure B.21. Breakthrough curves of 1- $\mu \mathrm{m}$ CML microspheres, $0.3-\mu \mathrm{m} C \mathrm{CML}$ microspheres, and iodide in Experiment 6 (Bullfrog tuff fracture).

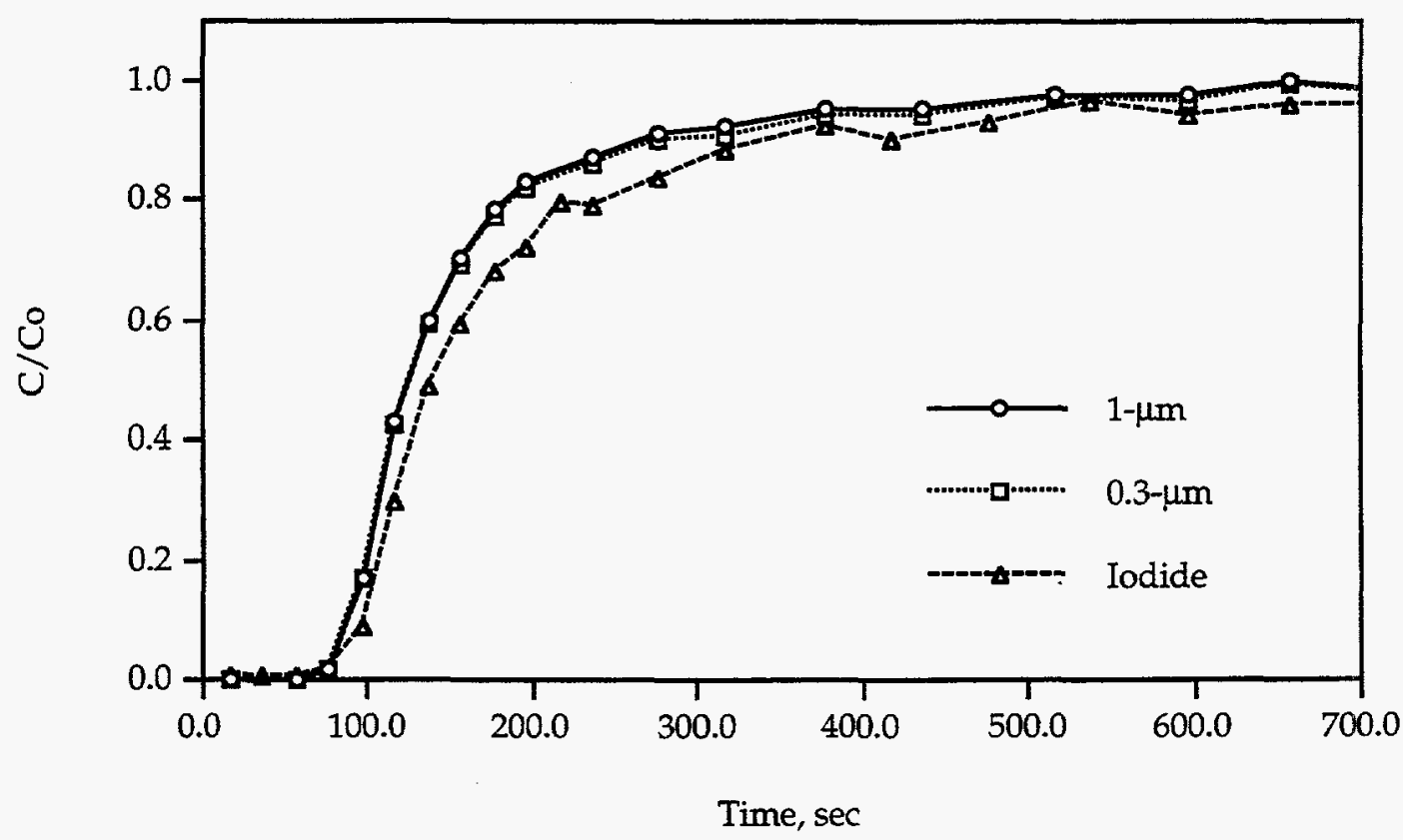

Figure B.22. Step portion of breakthrough curves for $1-\mu \mathrm{m}$ CML microspheres, $0.3-\mu \mathrm{m} C \mathrm{CML}$ microspheres, and iodide in Experiment 6 (Bullfrog tuff fracture). 


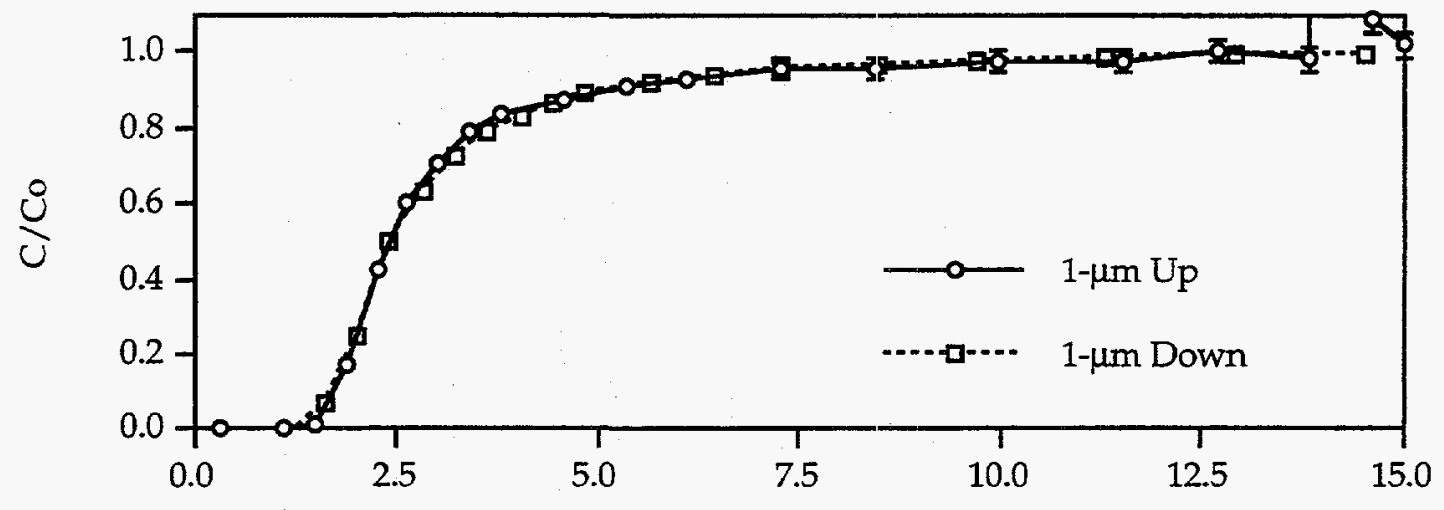

Volume Eluted, $\mathrm{ml}$

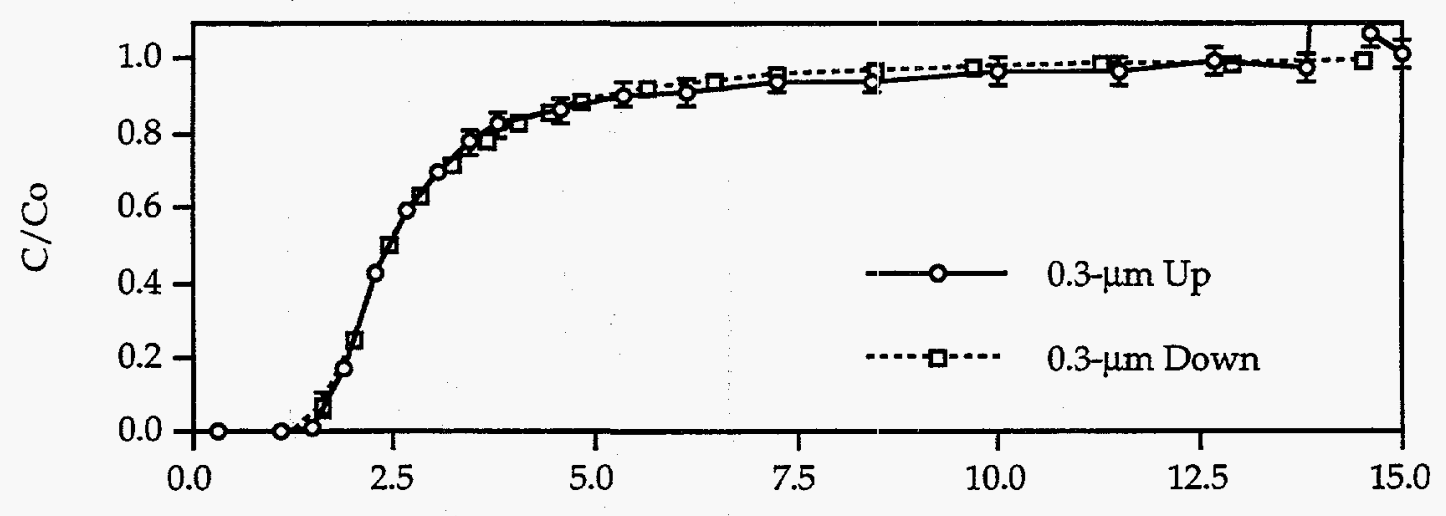

Volume Eluted, $\mathrm{ml}$

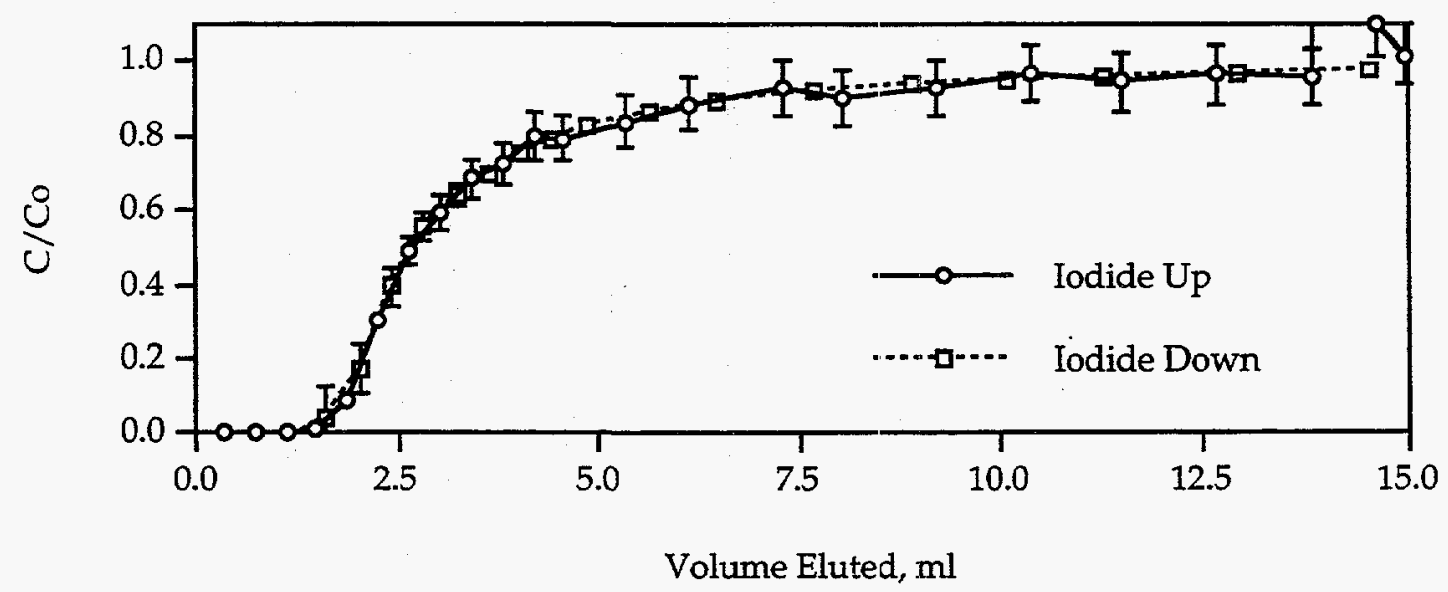

Figures B.23, B.24, B.25. Comparison of $\left(C / C_{0}\right)_{\text {step }}$ (up) and (1-C/ $\left.C_{0}\right)$ flush (down) for the 1- $\mu \mathrm{m} C M L$ microspheres, $0.3-\mu \mathrm{m} C M L$ microspheres, and iodide, respectively, in Experiment 6 (Bullfrog tuff fracture). 


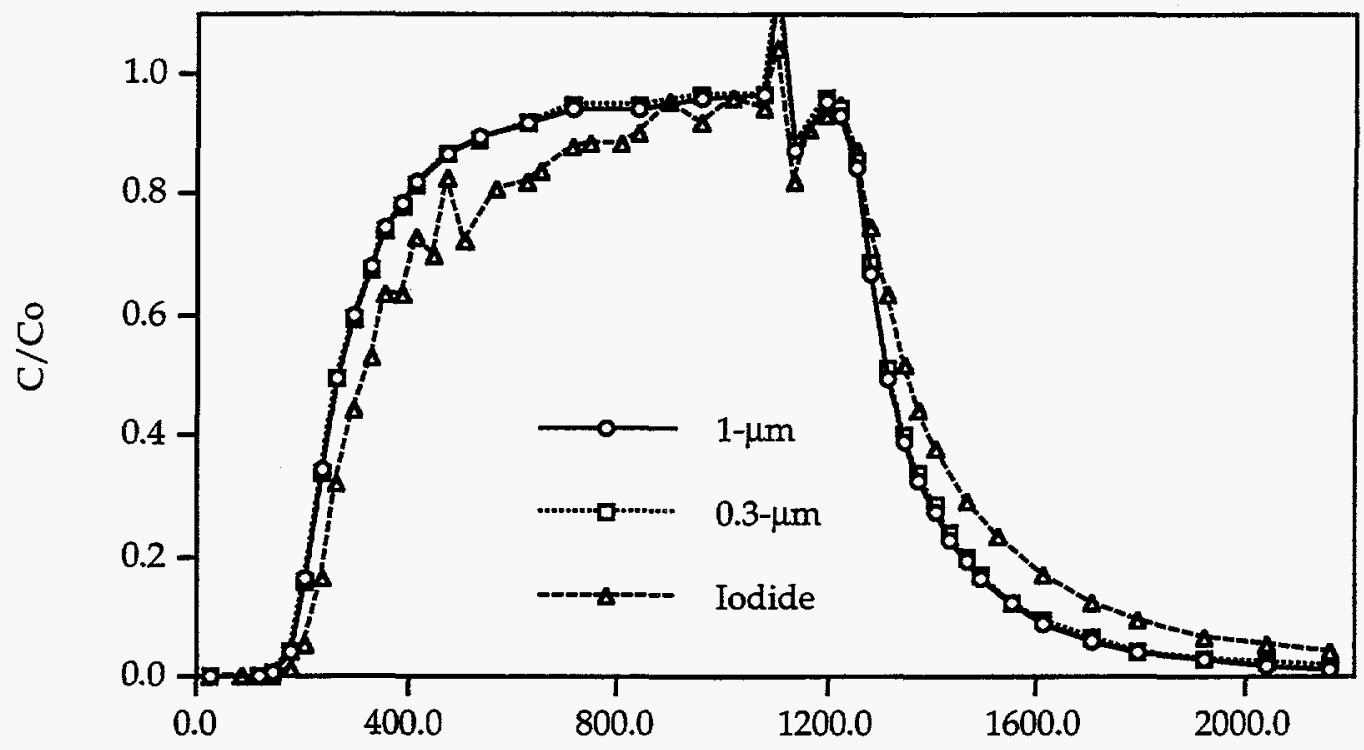

Time, sec

Figure B.26. Breakthrough curves of 1- $\mu \mathrm{m}$ CML microspheres, $0.3-\mu \mathrm{m}$ CML microspheres, and iodide in Experiment 7 (Bullfrog tuff fracture).

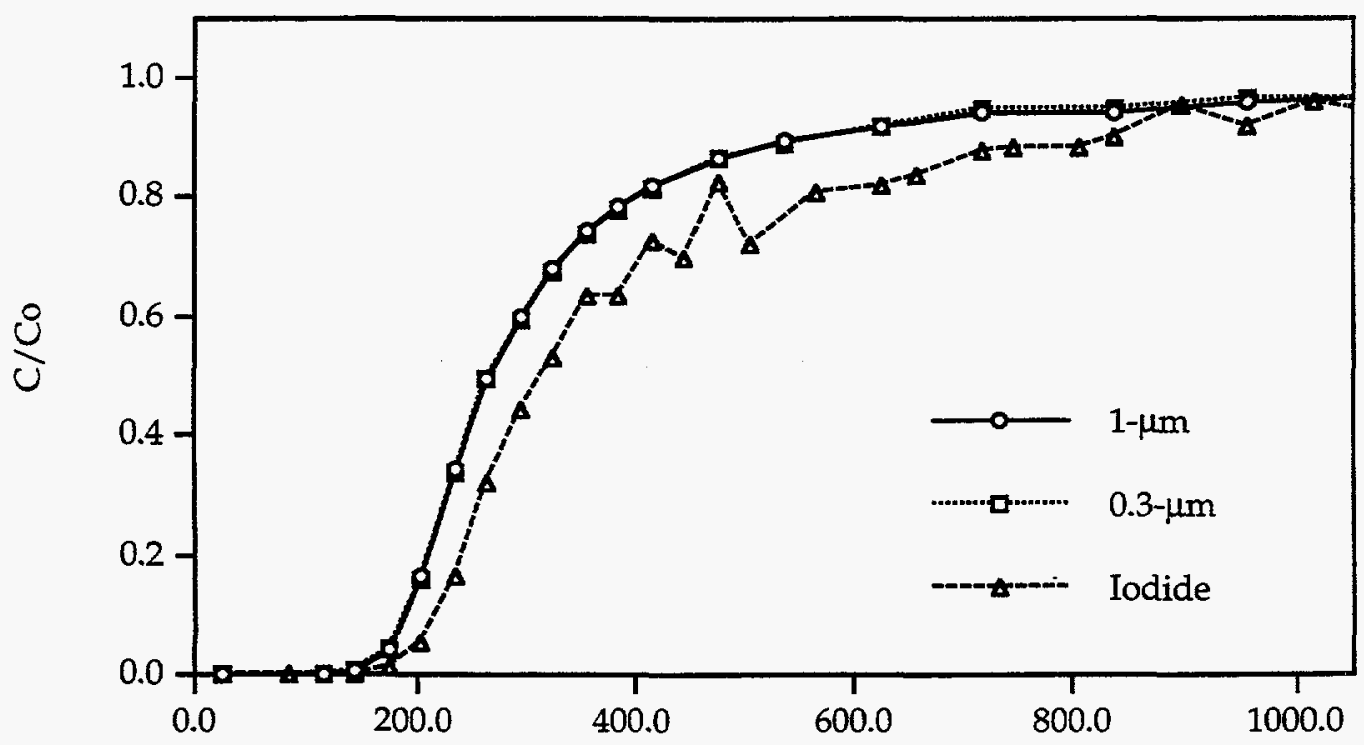

Time, sec

Figure B.27. Step portion of breakthrough curves for 1- $\mu \mathrm{m}$ CML microspheres, $0.3-\mu \mathrm{m}$ CML microspheres, and iodide in Experiment 7 (Bullfrog tuff fracture). 


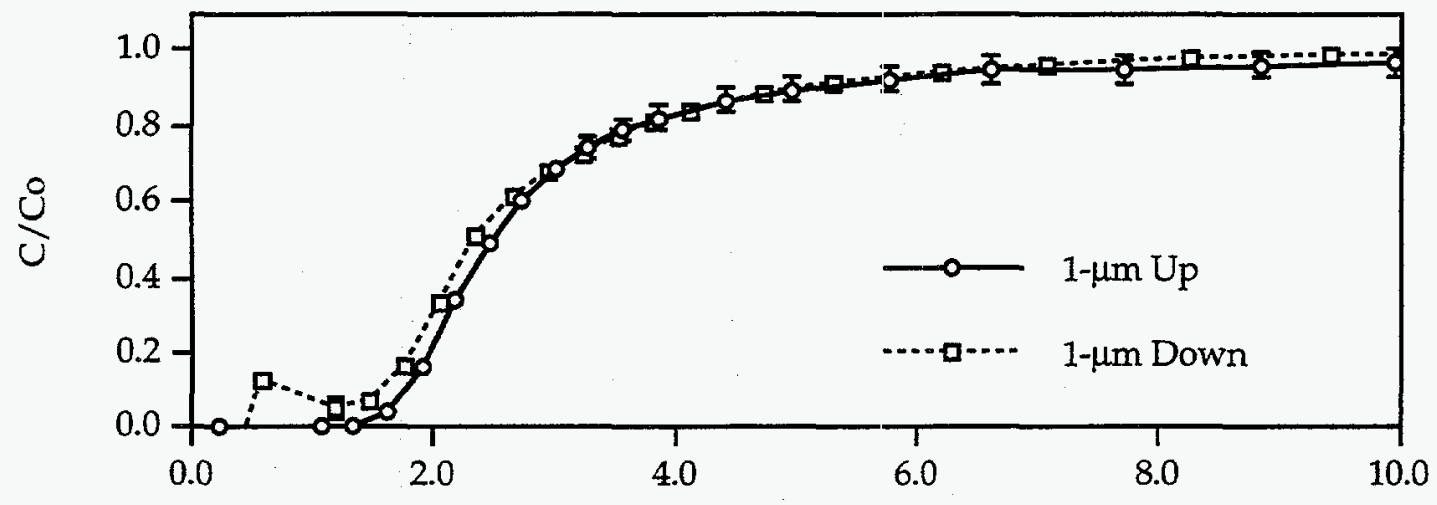

Volume Eluted, $\mathrm{ml}$

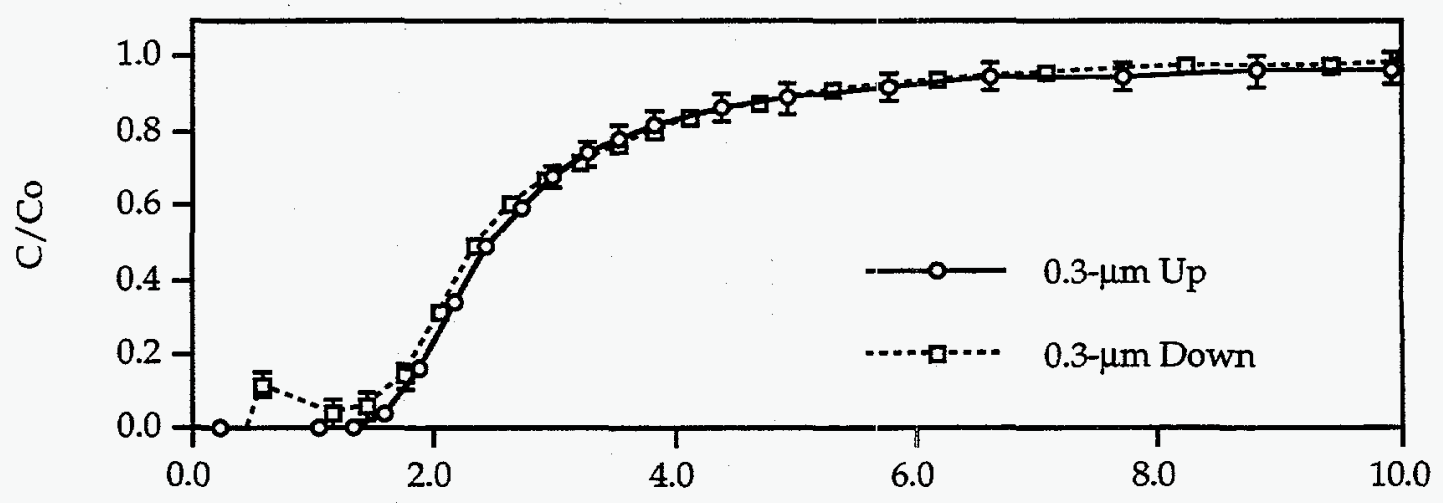

Volume Eluted, $\mathrm{ml}$

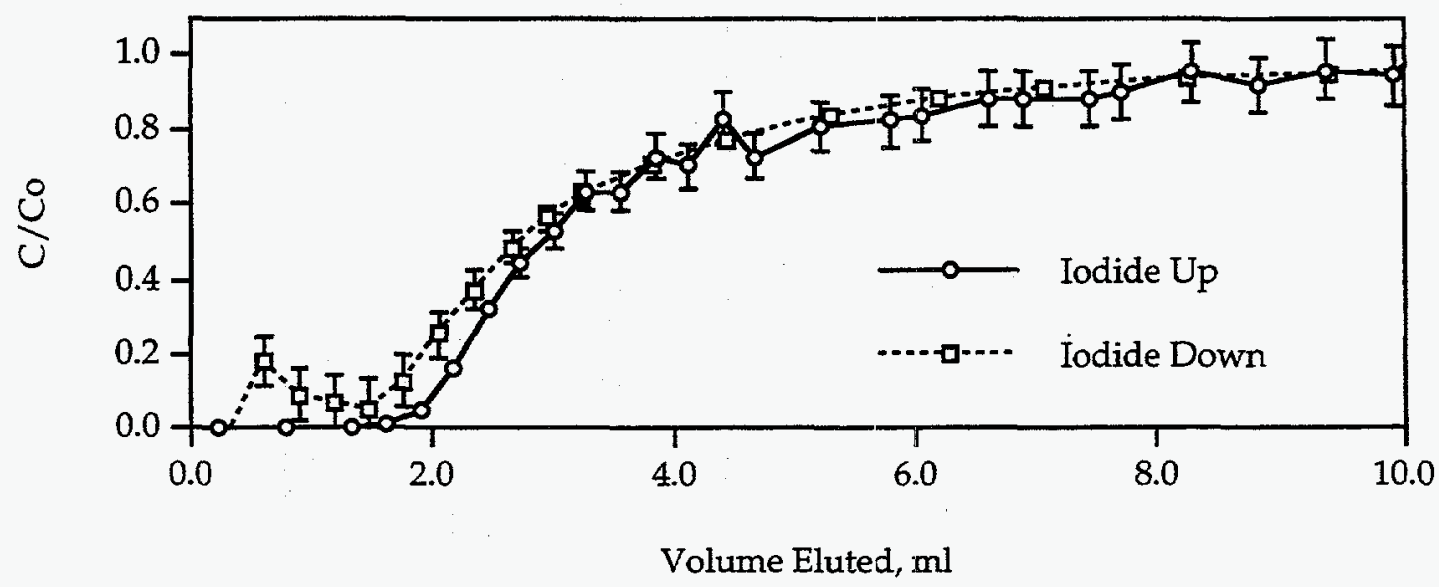

Figures B.28, B.29, B.30. Comparison of $\left(C / C_{0}\right)_{\text {step }}(\mathrm{up})$ and $\left(1-\mathrm{C} / \mathrm{C}_{0}\right)_{\text {flush }}$ (down) for the 1- $\mu \mathrm{m}$ CML microspheres, $0.3-\mu \mathrm{m}$ CML microspheres, and iodide, respectively, in Experiment 7 (Bullfrog tuff fracture). 


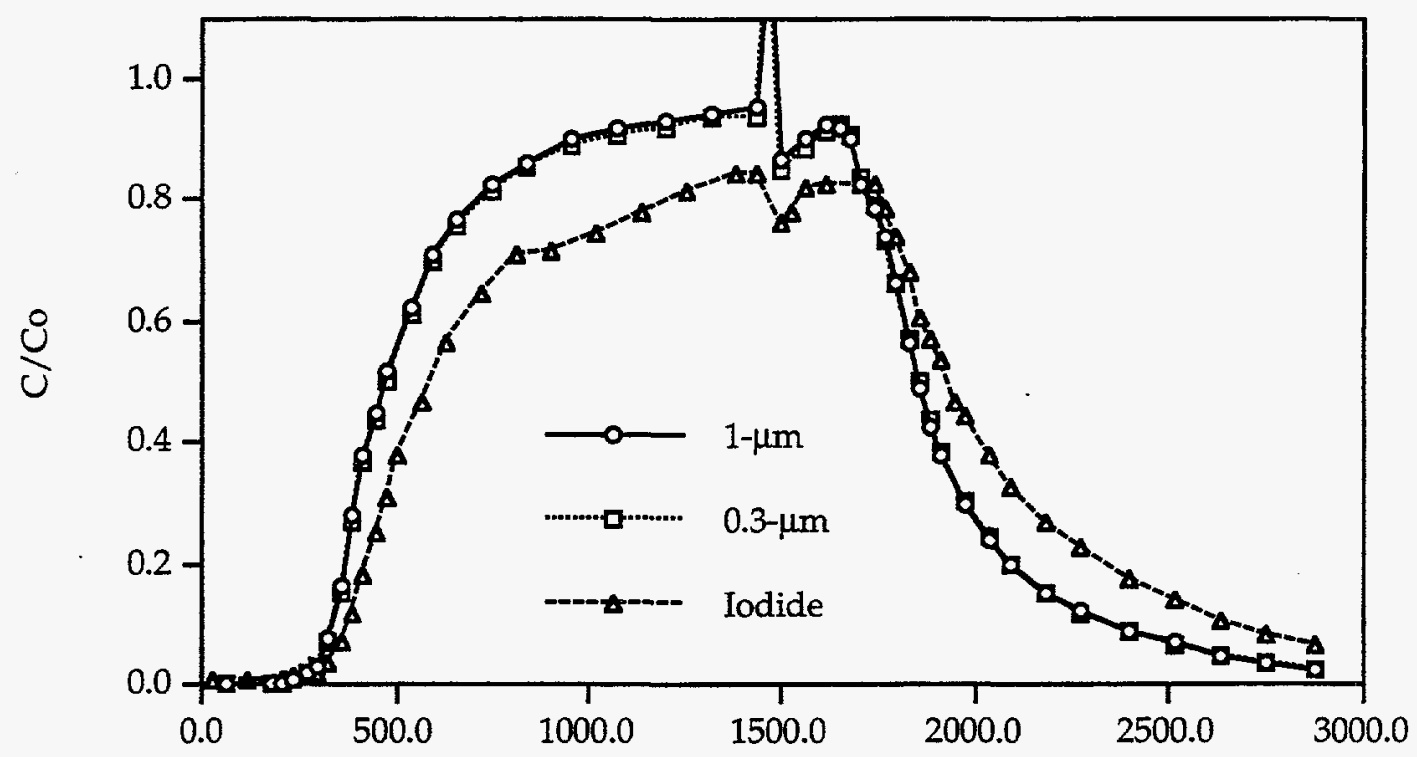

Time, sec

Figure B.31. Breakthrough curves of 1- $\mu \mathrm{m}$ CML microspheres, $0.3-\mu \mathrm{m} C M L$ microspheres, and iodide in Experiment 8 (Bullfrog tuff fracture).

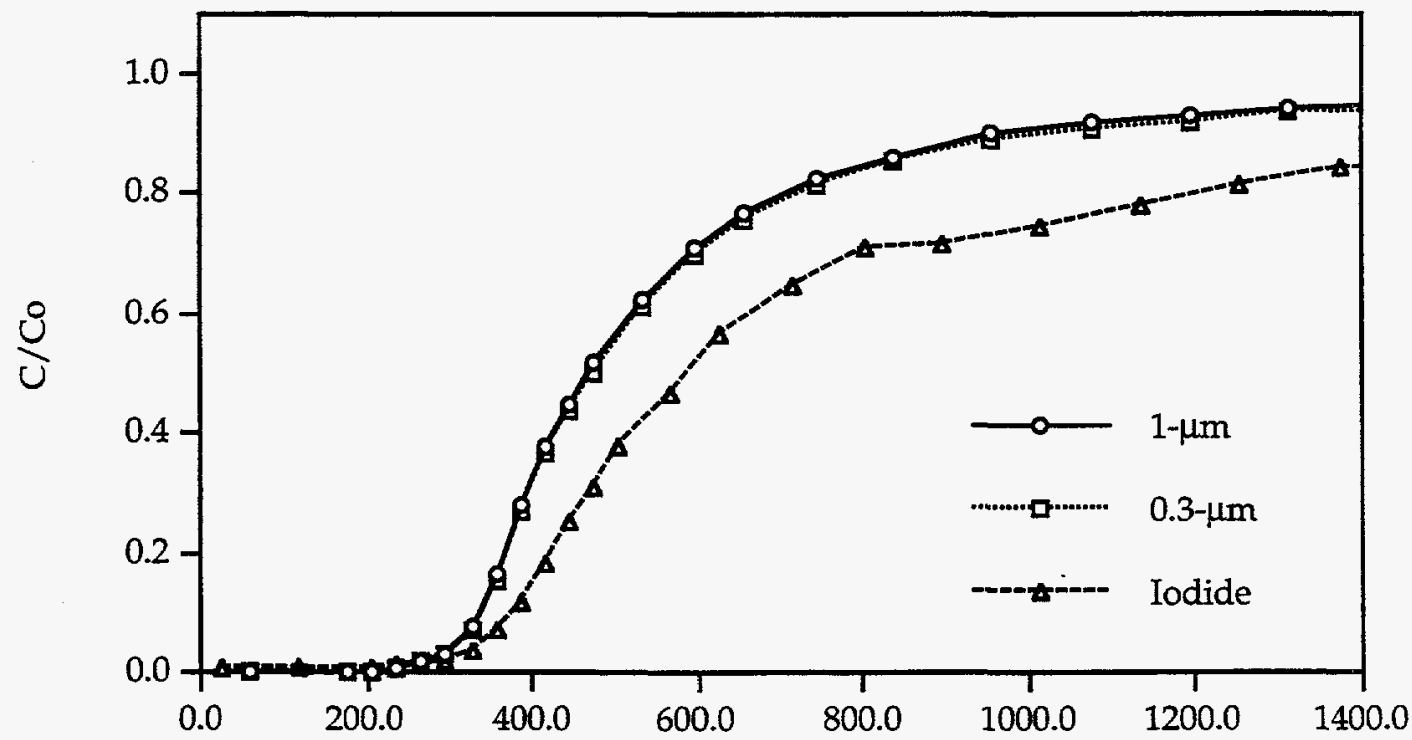

Time, sec

Figure B.32. Step portion of breakthrough curves for 1- $\mu \mathrm{m}$ CML microspheres, $0.3-\mu \mathrm{m}$ CML microspheres, and iodide in Experiment 8 (Bullfrog tuff fracture). 


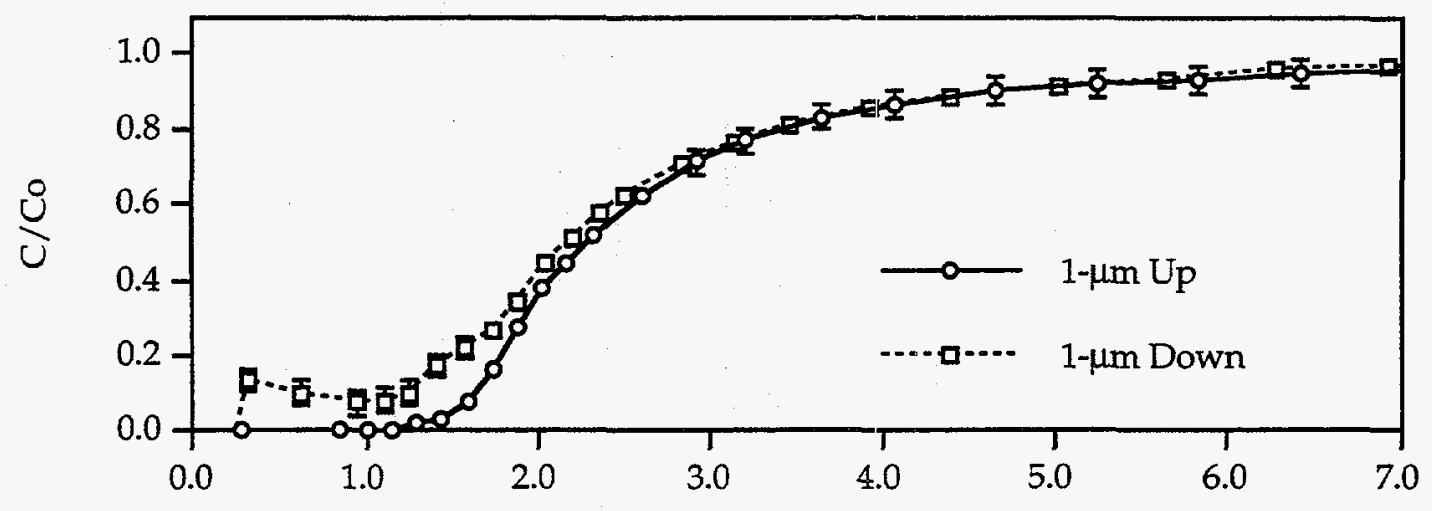

Volume Eluted, $\mathrm{ml}$

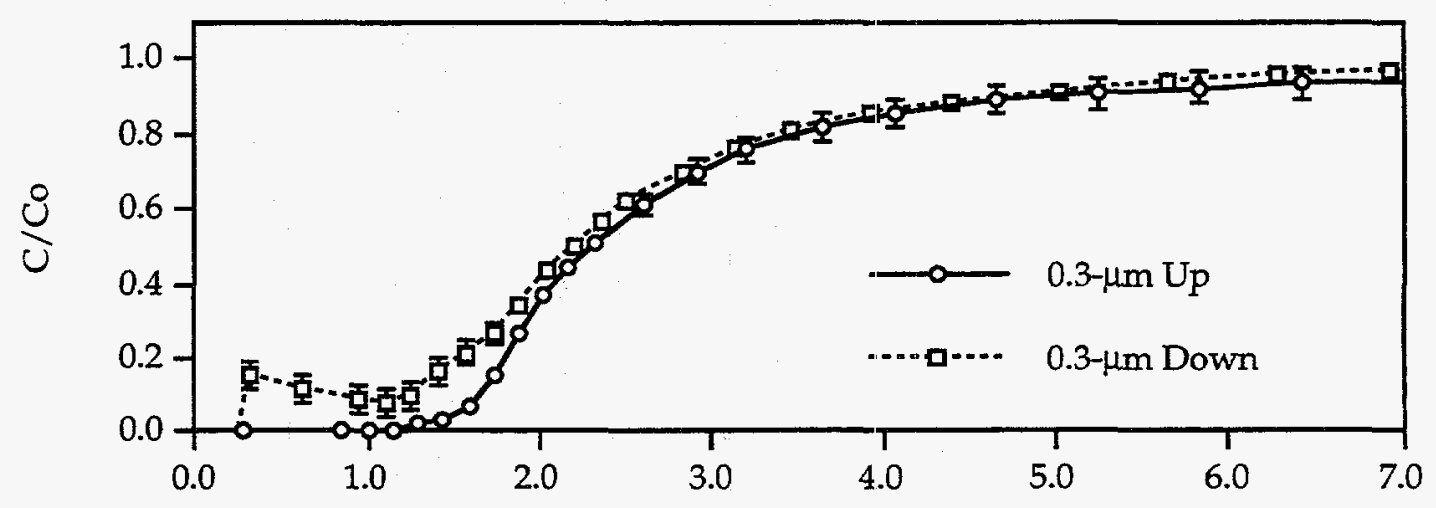

Volume Eluted, $\mathrm{ml}$

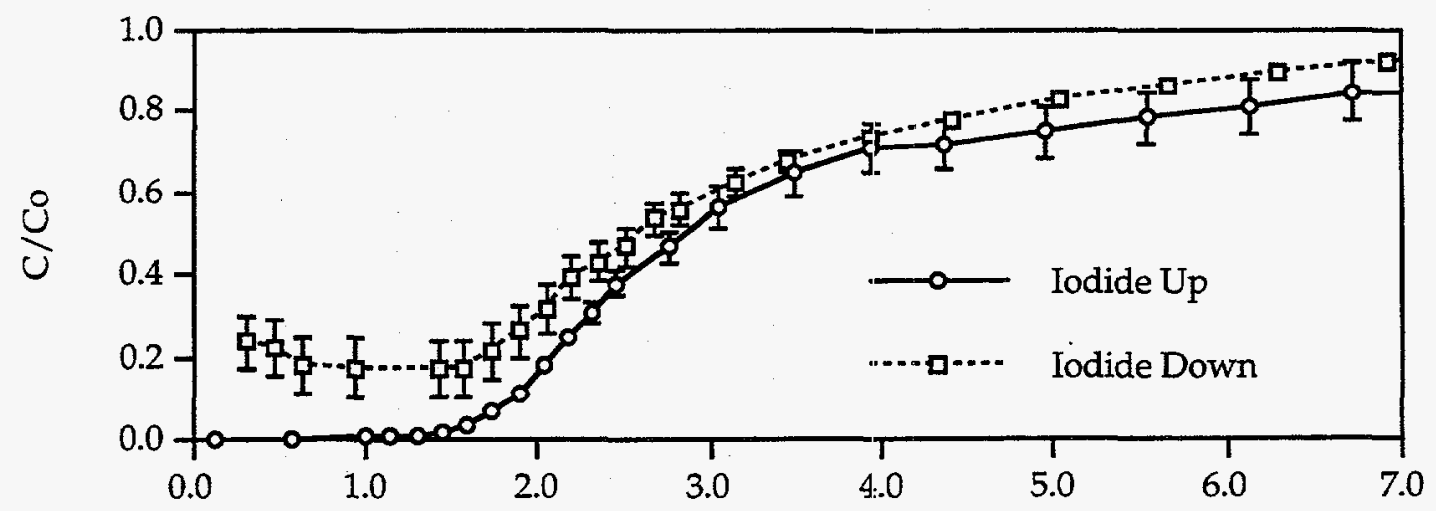

Volume Eluted, ml

Figures B.33, B.34, B.35. Comparison of $\left(C / C_{0}\right)_{\text {step }}$ (up) and $\left(1-C / C_{0}\right)_{\text {flush }}$ (down) for the 1- $\mu \mathrm{m}$ CML microspheres, $0.3-\mu \mathrm{m}$ CML microspheres, and iodide, respectively, in Experiment 8 (Bullfrog tuff fracture). 


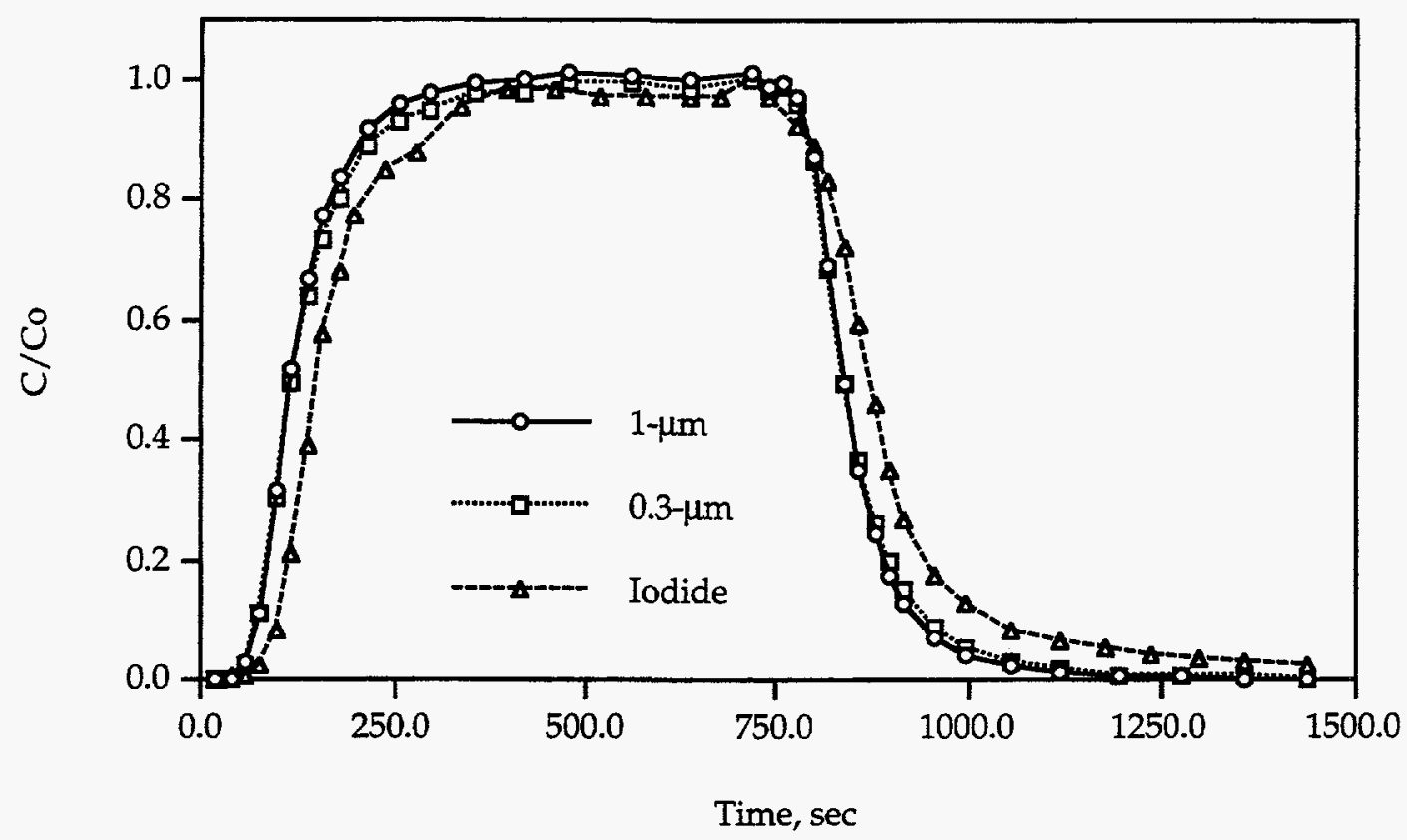

Figure B.36. Breakthrough curves of 1- $\mu \mathrm{m}$ CML microspheres, 0.3- $\mu \mathrm{m}$ CML microspheres, and iodide in Experiment 9 (Tram tuff fracture).

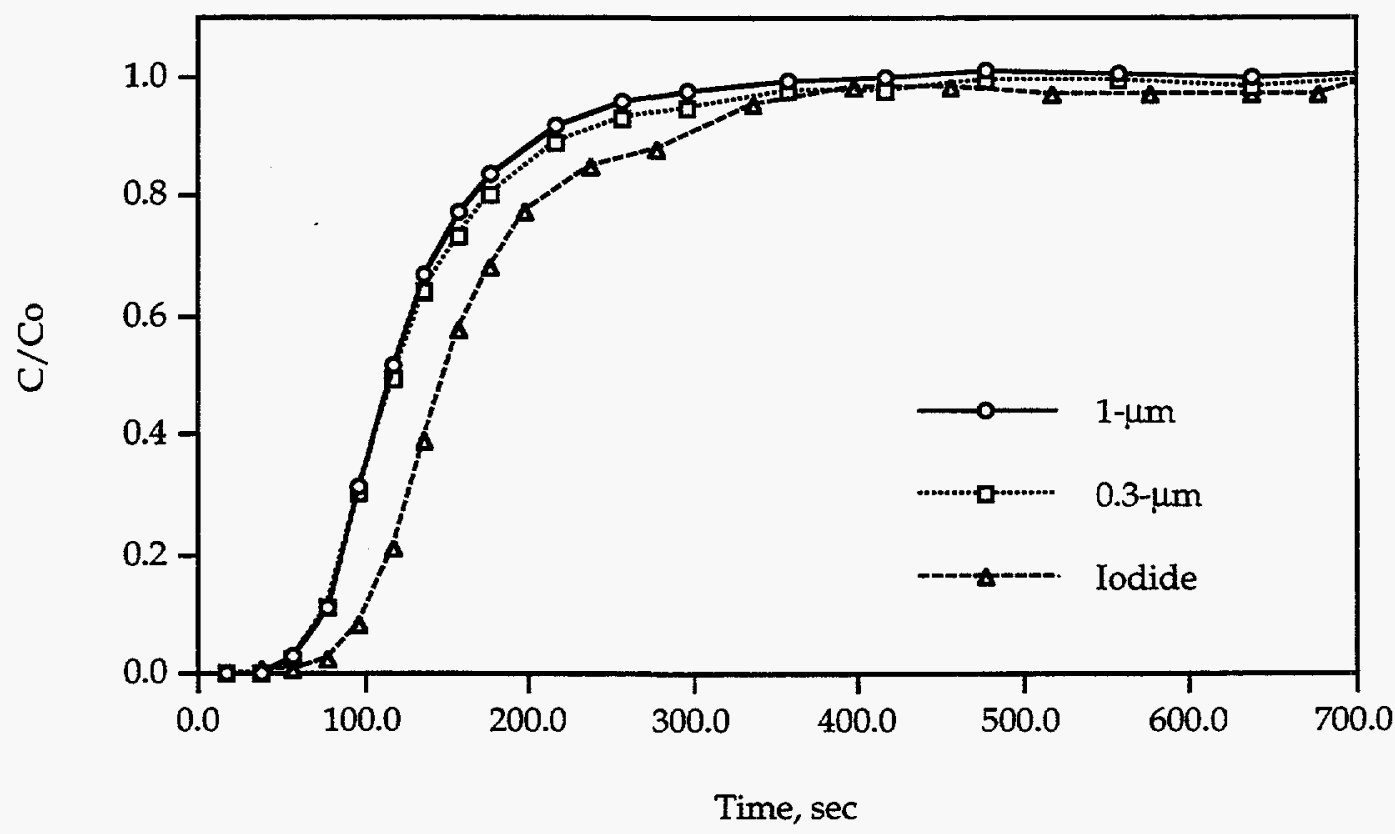

Figure B.37. Step portion of breakthrough curves for 1- $\mu \mathrm{m}$ CML microspheres, $0.3-\mu \mathrm{m}$ CML microspheres, and iodide in Experiment 9 (Tram tuff fracture). 


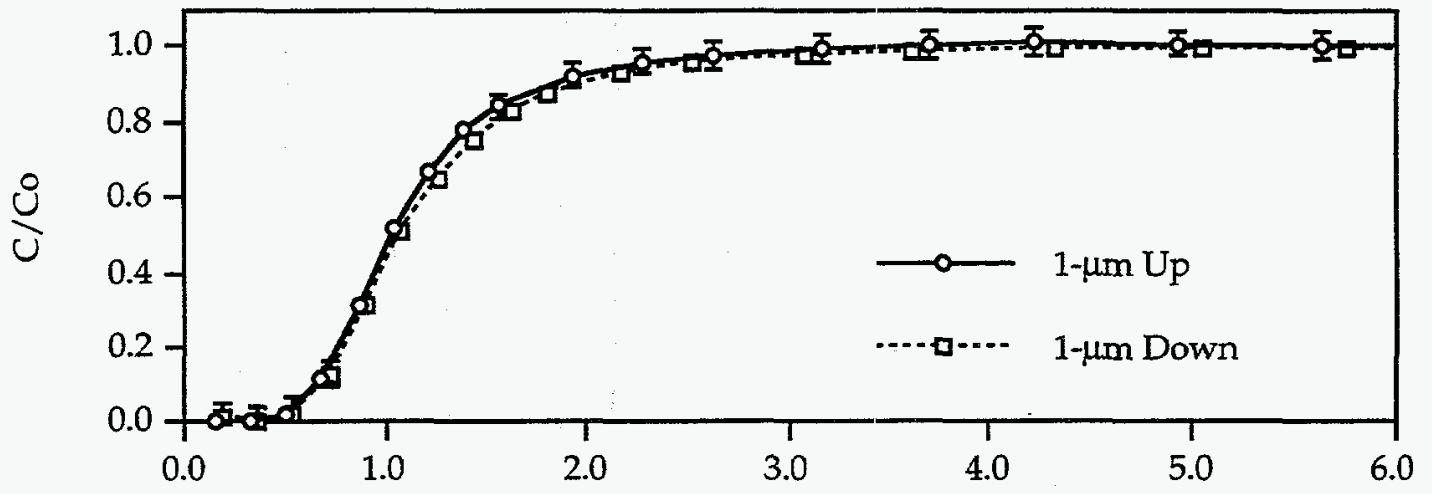

Volume Eluted, $\mathrm{ml}$

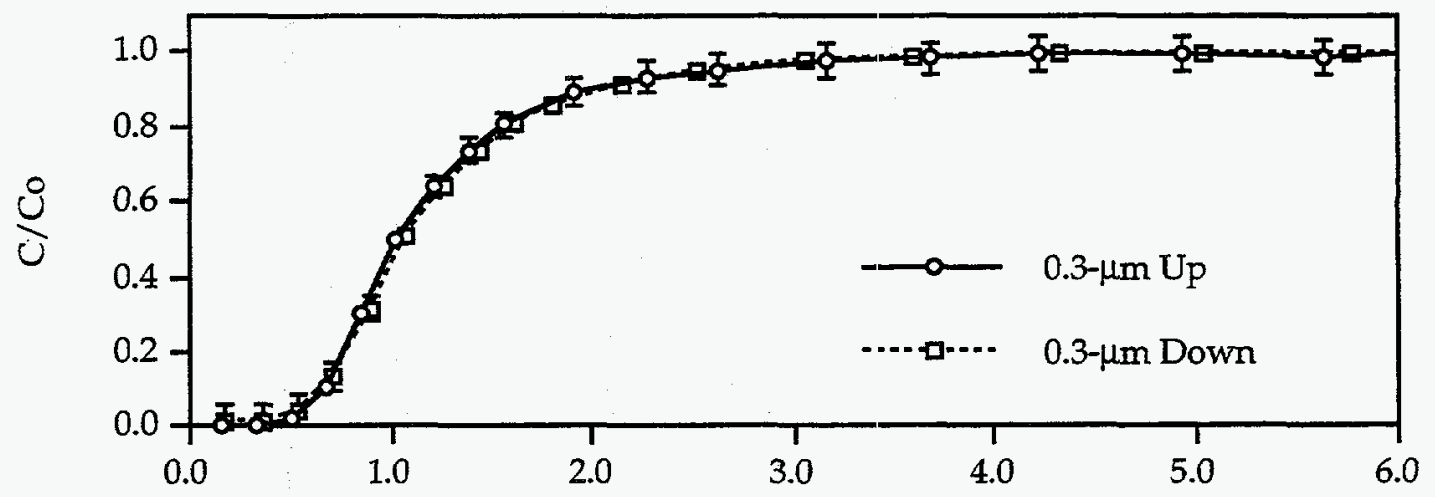

Volume Eluted, ml

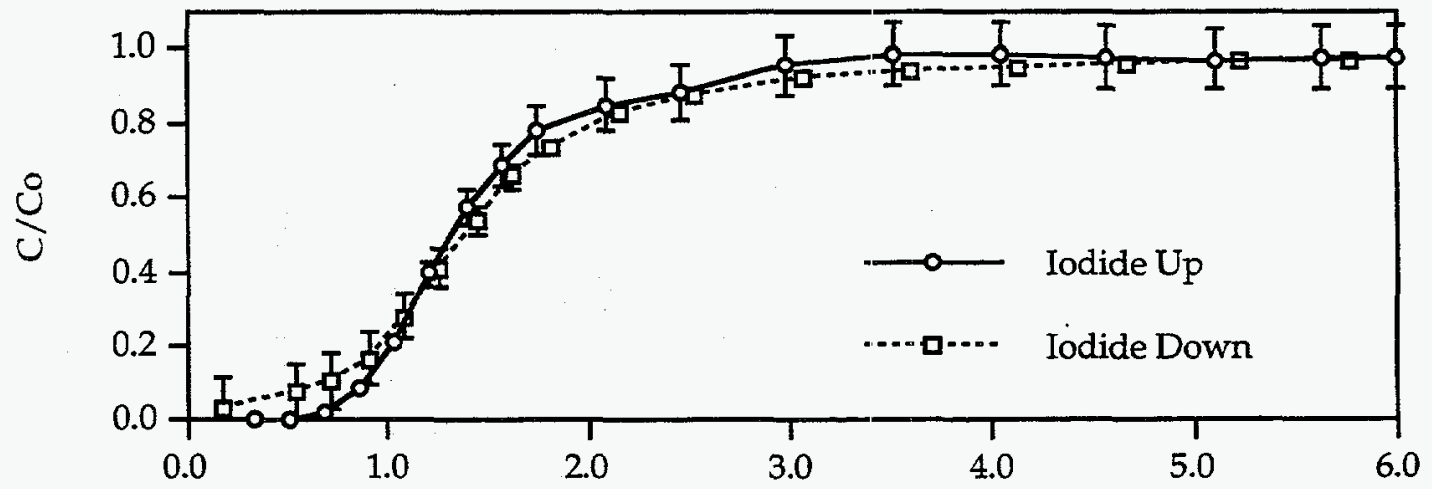

Volume Eluted, $\mathrm{ml}$

Figures B.38, B.39, B.40. Comparison of $\left(\mathrm{C} / \mathrm{C}_{0}\right)_{\text {step }}(\mathrm{up})$ and $\left(1-\mathrm{C} / \mathrm{C}_{0}\right)_{\text {flush }}$ (down) for the 1- $\mu \mathrm{m}$ CML microspheres, $0.3-\mu \mathrm{m}$ CML microspheres, and iodide, respectively, in Experiment 9 (Tram tuff fracture). 


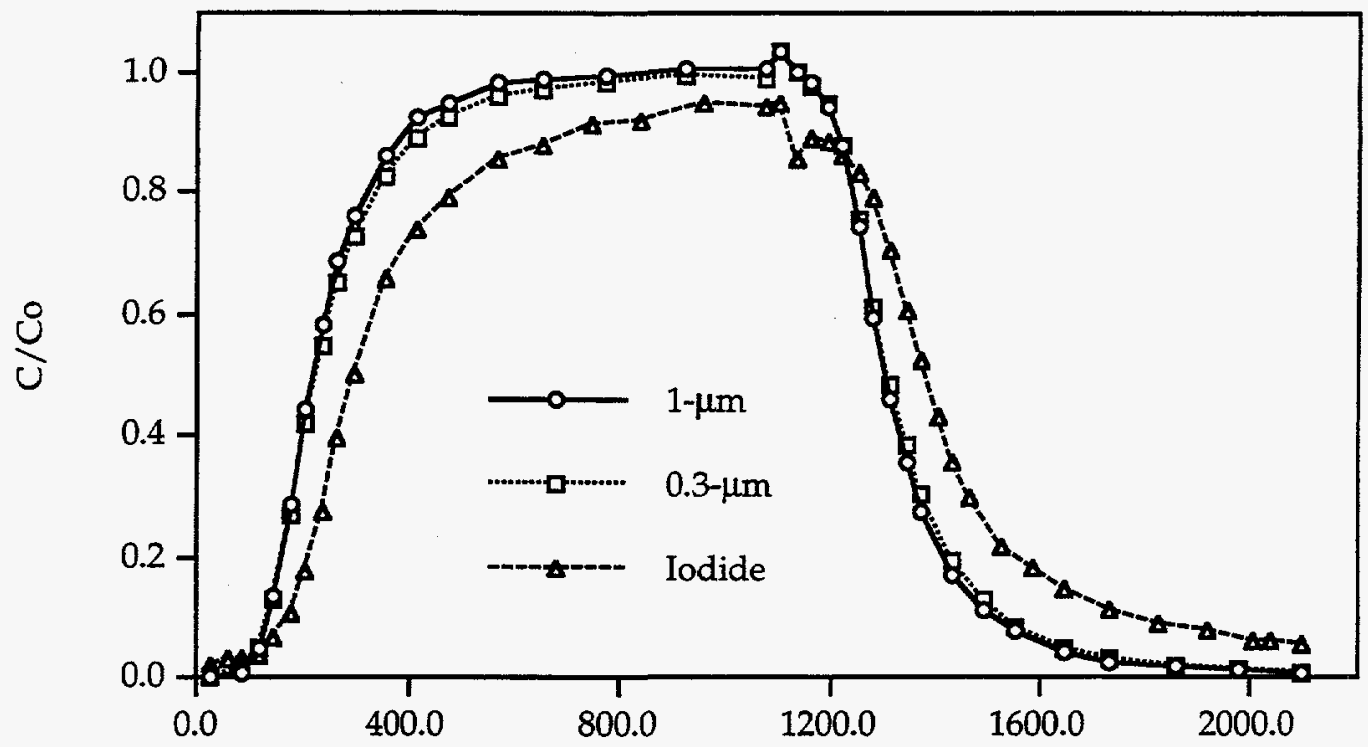

Time, sec

Figure B.41. Breakthrough curves of $1-\mu \mathrm{m}$ CML microspheres, $0.3-\mu \mathrm{m}$ CML microspheres, and iodide in Experiment 10 (Tram tuff fracture).

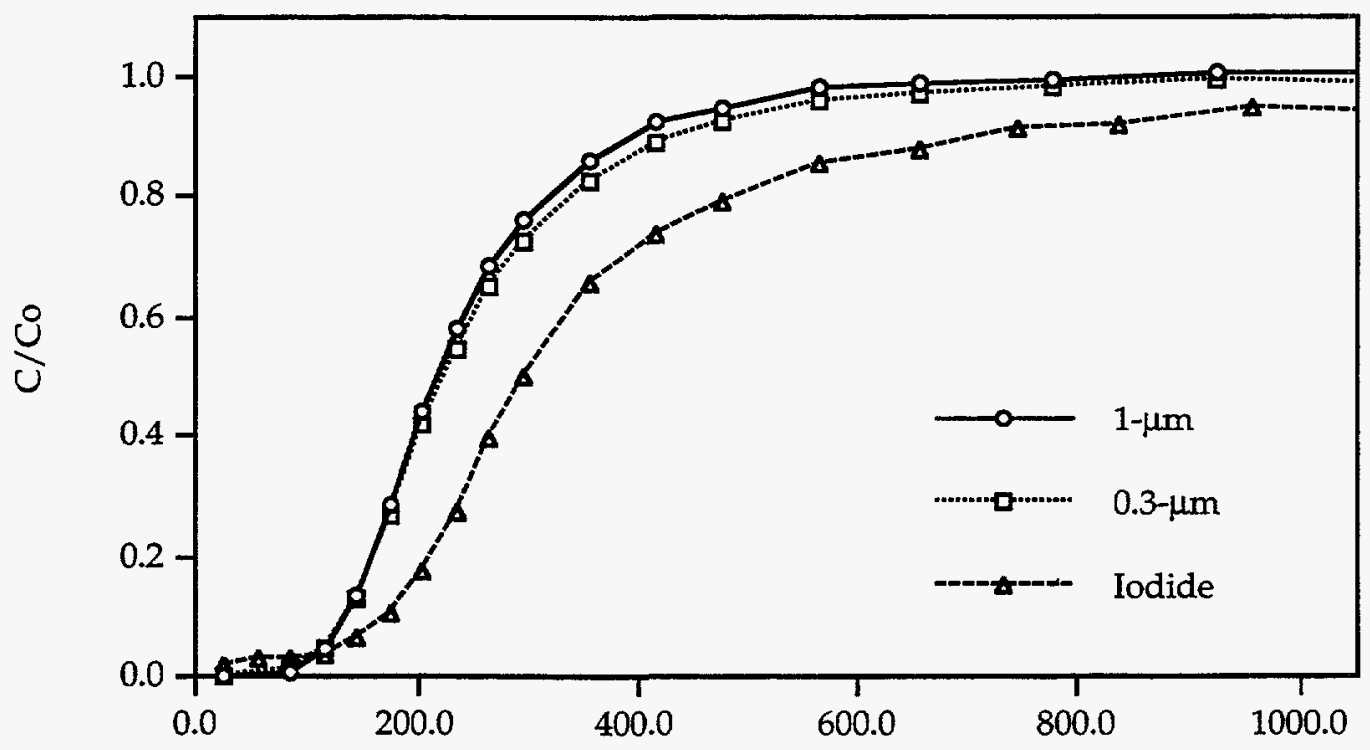

Time, sec

Figure B.42. Step portion of breakthrough curves for $1-\mu \mathrm{m}$ CML microspheres, $0.3-\mu \mathrm{m} C M L$ microspheres, and iodide in Experiment 10 (Tram tuff fracture). 


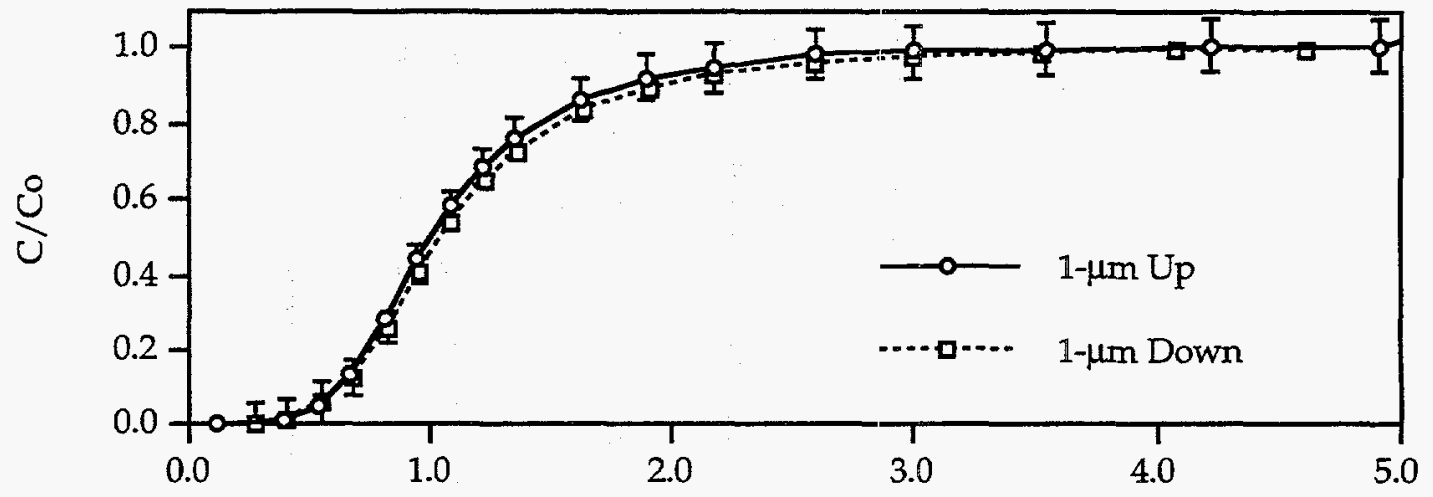

Volume Eluted, $\mathrm{ml}$

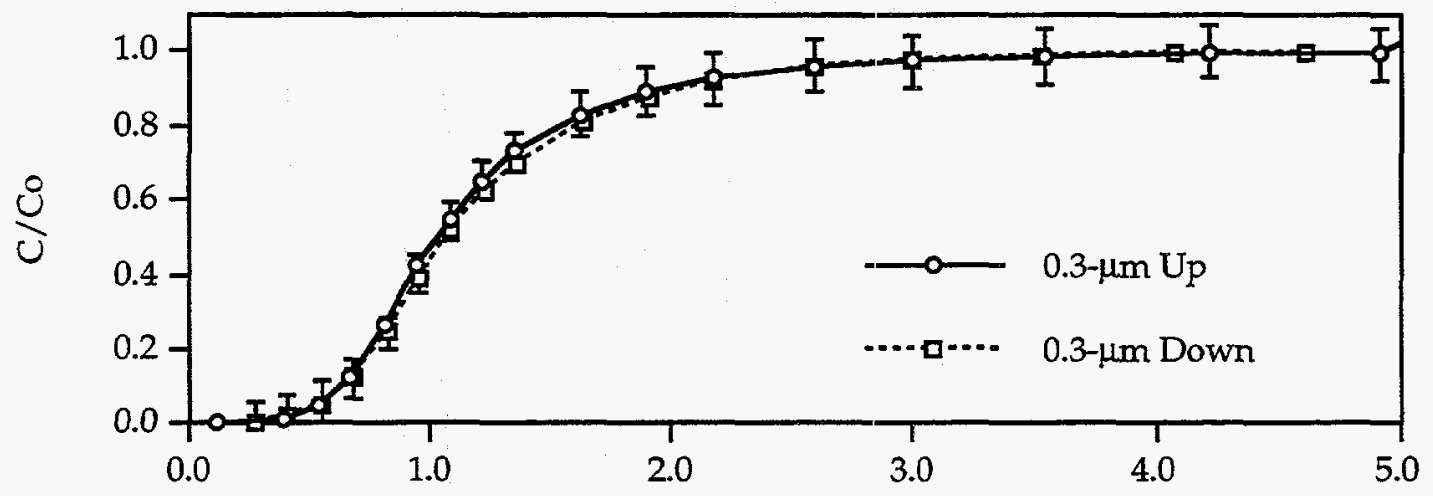

Volume Eluted, $\mathrm{ml}$

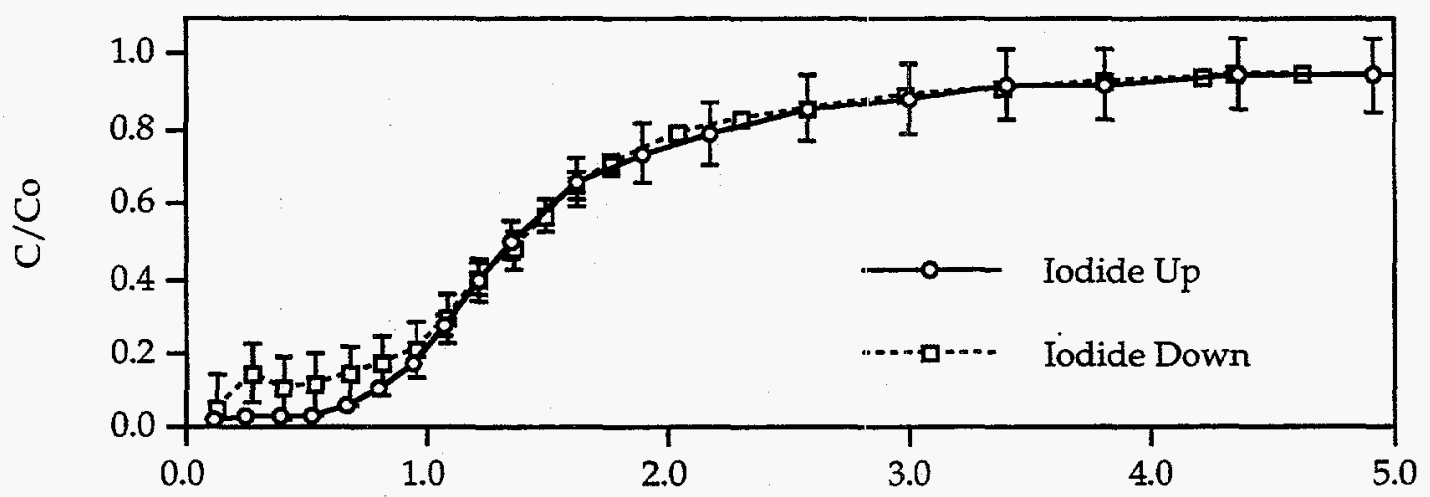

Volume Eluted, $\mathrm{ml}$

Figures B.43, B.44, B.45. Comparison of $\left(\mathrm{C} / \mathrm{C}_{0}\right)_{\text {step }}$ (up) and $\left(1-\mathrm{C} / \mathrm{C}_{0}\right)_{\text {flush }}$ (down) for the 1- $\mu \mathrm{m}$ CML microspheres, $0.3-\mu \mathrm{m}$ CML microspheres, and iodide, respectively, in Experiment 10 (Tram tuff fracture). 


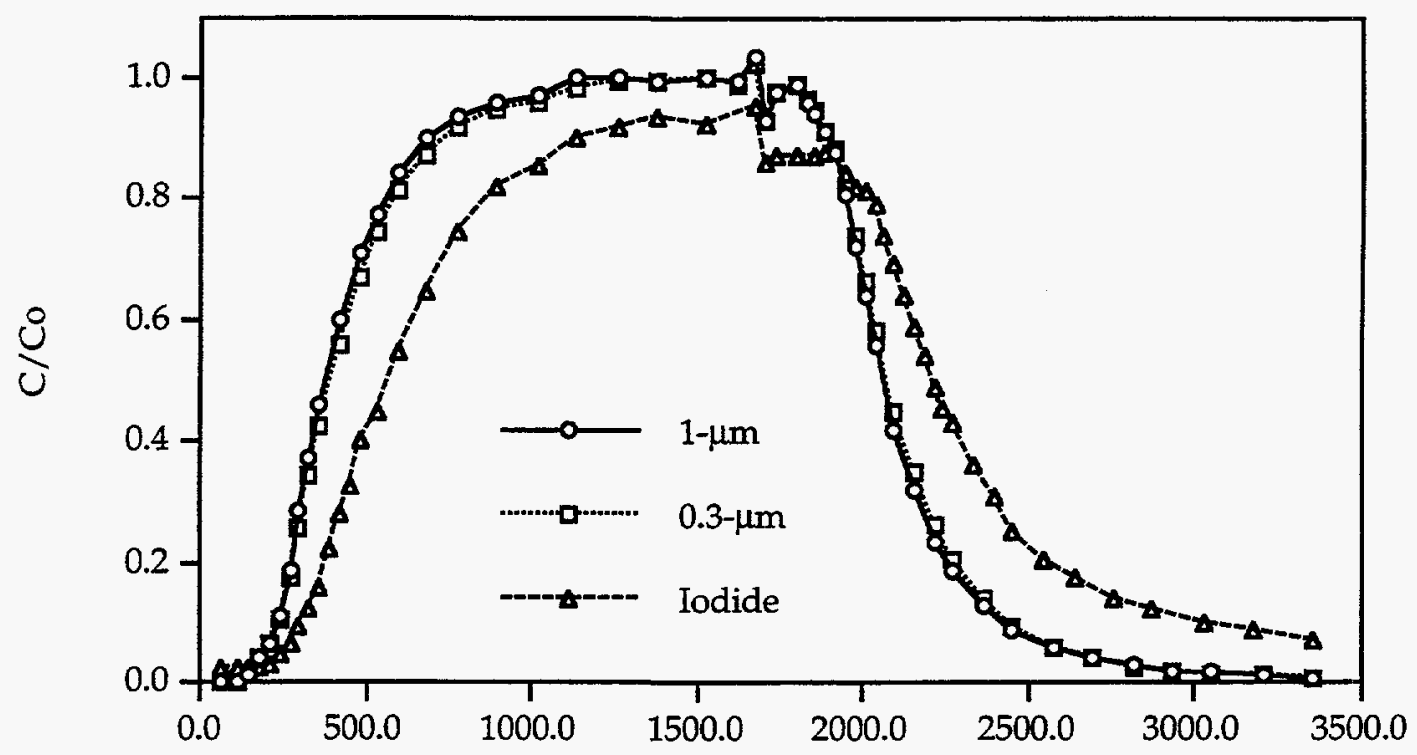

Time, sec

Figure B.46. Breakthrough curves of 1- $\mu \mathrm{m}$ CML microspheres, $0.3-\mu \mathrm{m}$ CML microspheres, and iodide in Experiment 11 (Tram tuff fracture).

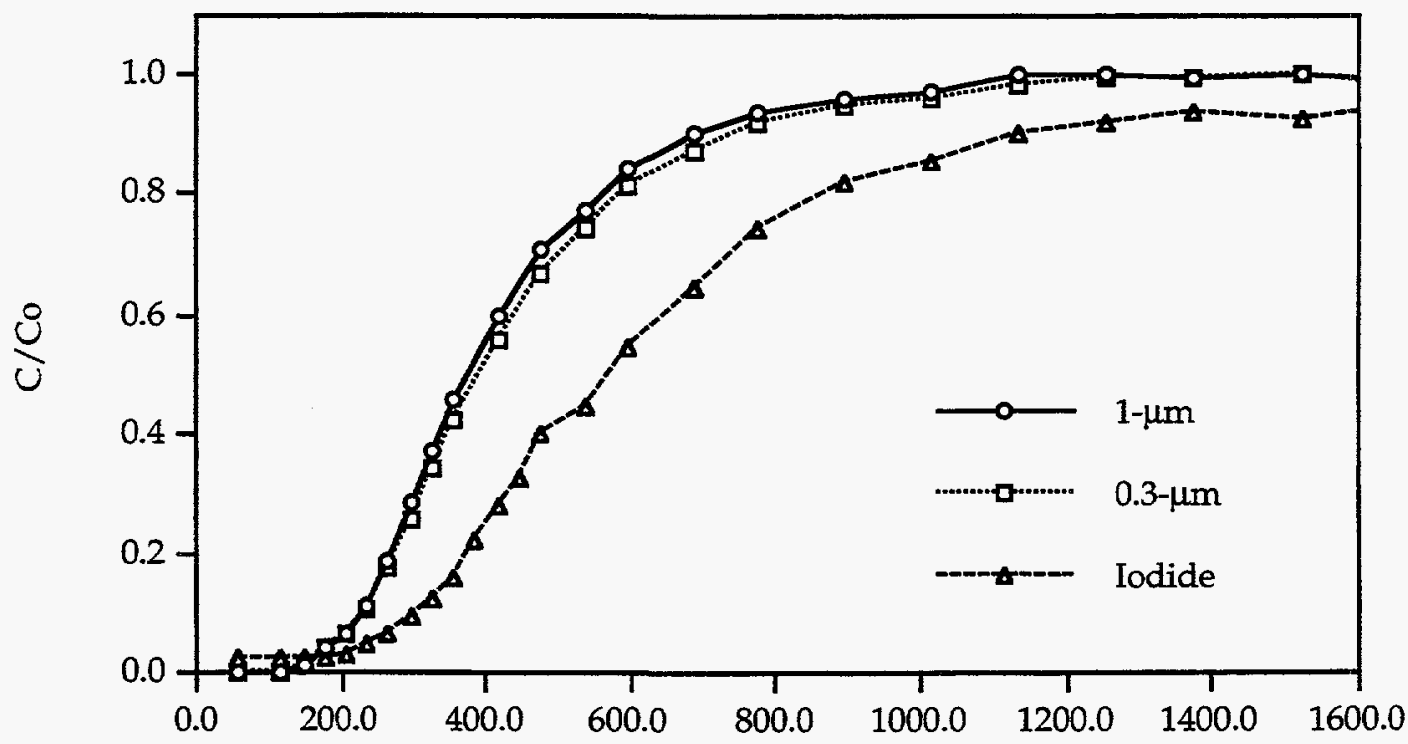

Time, sec

Figure B.47. Step portion of breakthrough curves for 1- $\mu \mathrm{m}$ CML microspheres, $0.3-\mu \mathrm{m}$ CML microspheres, and iodide in Experiment 11 (Tram tuff fracture). 


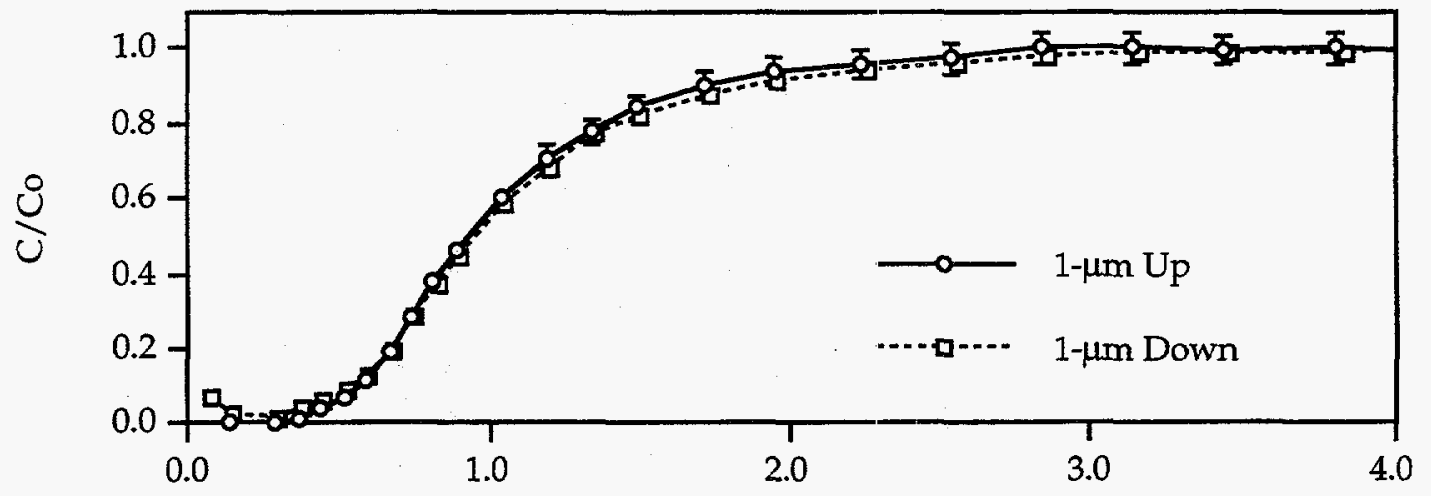

Volume Eluted, $\mathrm{ml}$

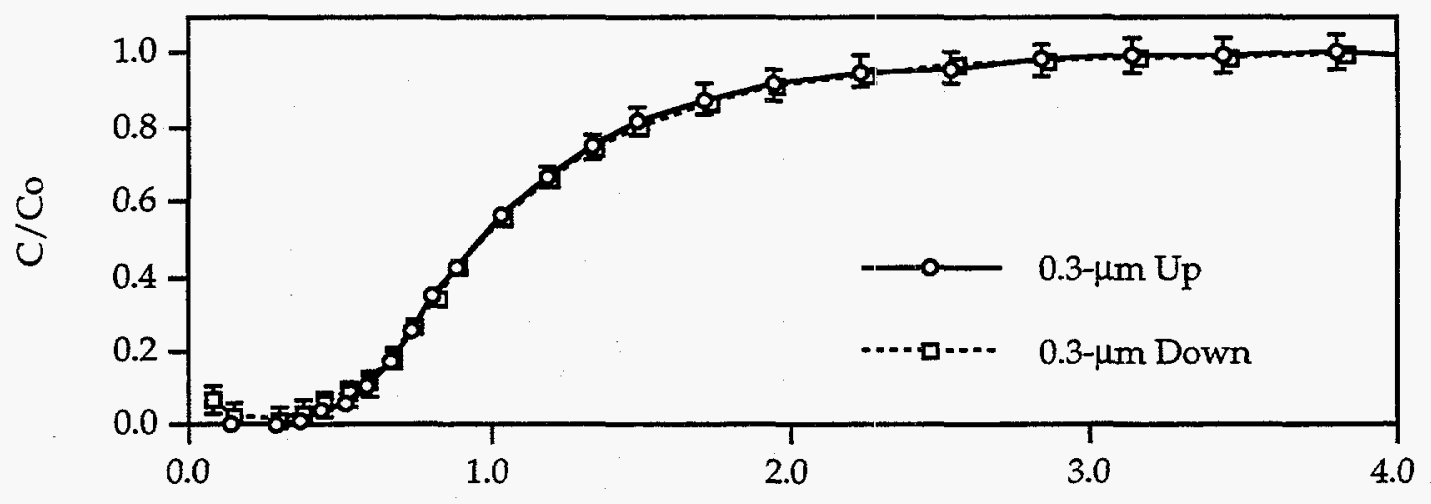

Volume Eluted, $\mathrm{ml}$

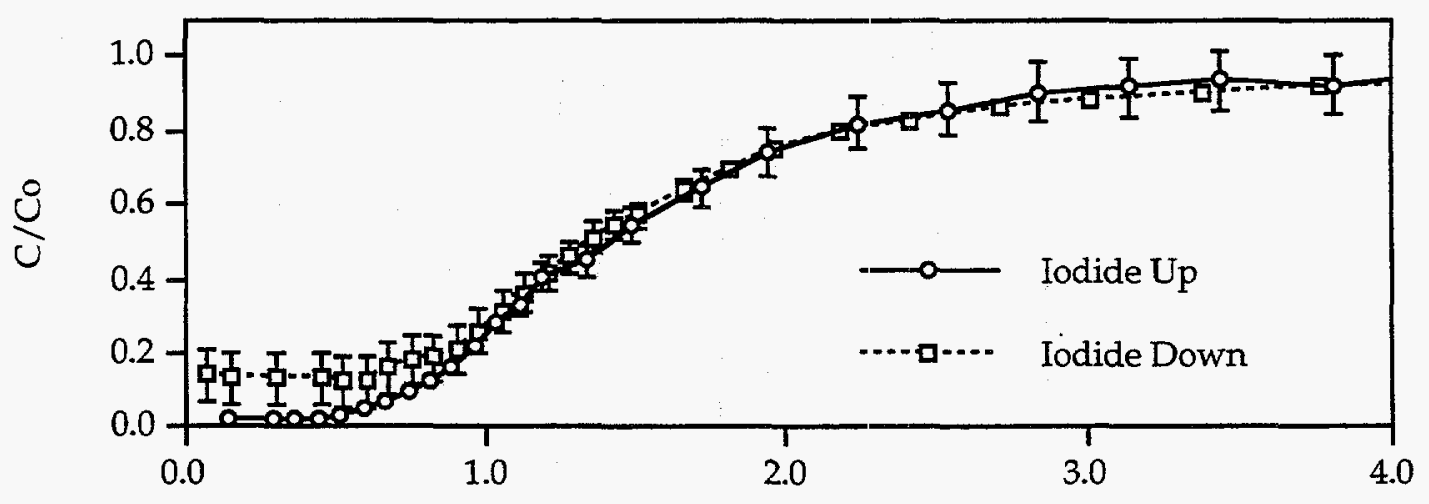

Volume Eluted, $\mathrm{ml}$

Figures B.48, B.49, B.50. Comparison of $\left(C / C_{0}\right)_{\text {step }}$ (up) and $\left(1-C / C_{0}\right)_{\text {flush }}$ (down) for the 1- $\mu \mathrm{m}$ CML microspheres, $0.3-\mu \mathrm{m}$ CML microspheres, and iodide, respectively, in Experiment 11 (Tram tuff fracture). 


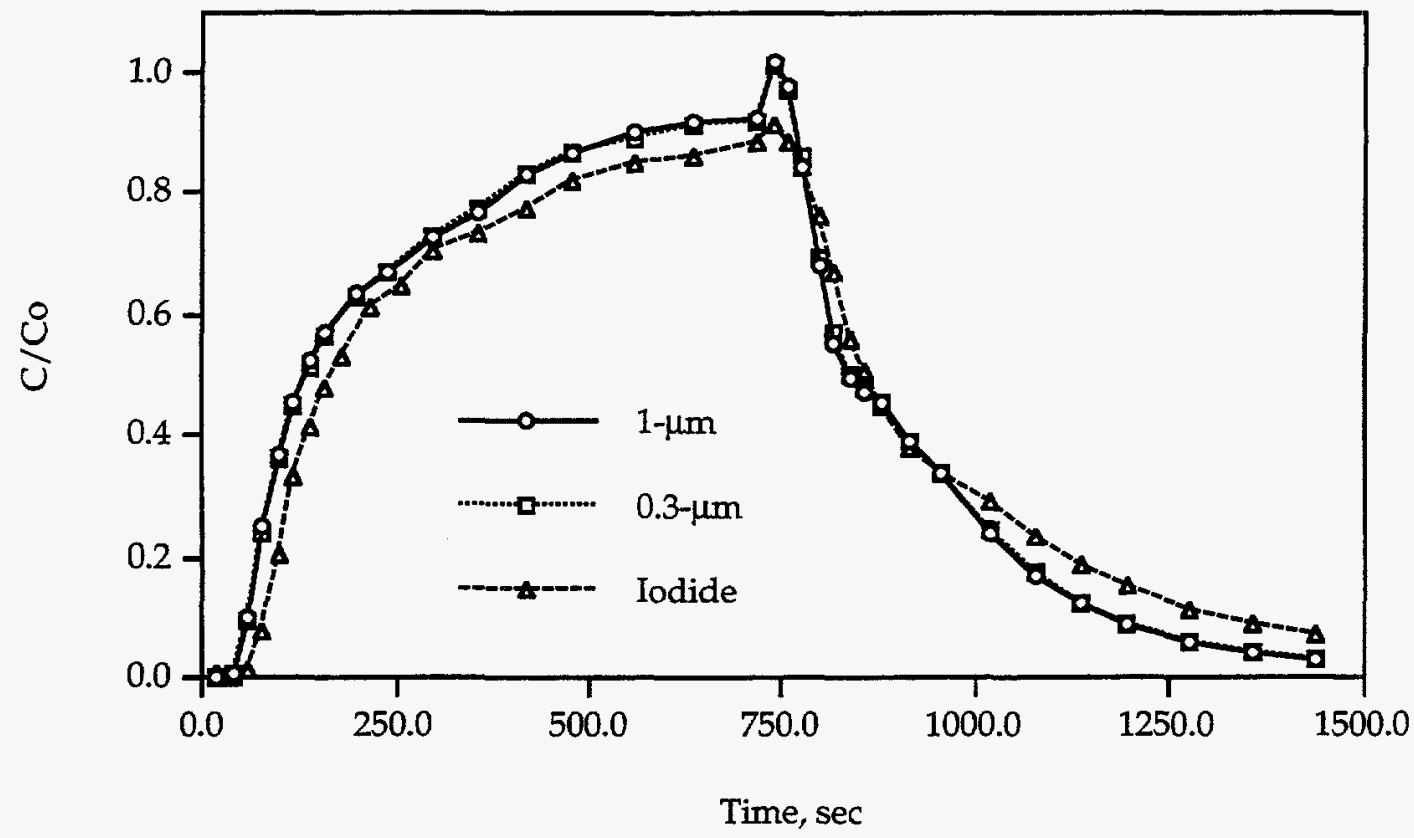

Figure B.51. Breakthrough curves of $1-\mu \mathrm{m}$ CML microspheres, $0.3-\mu \mathrm{m}$ CML microspheres, and iodide in Experiment 12 (rotated Bullfrog tuff fracture).

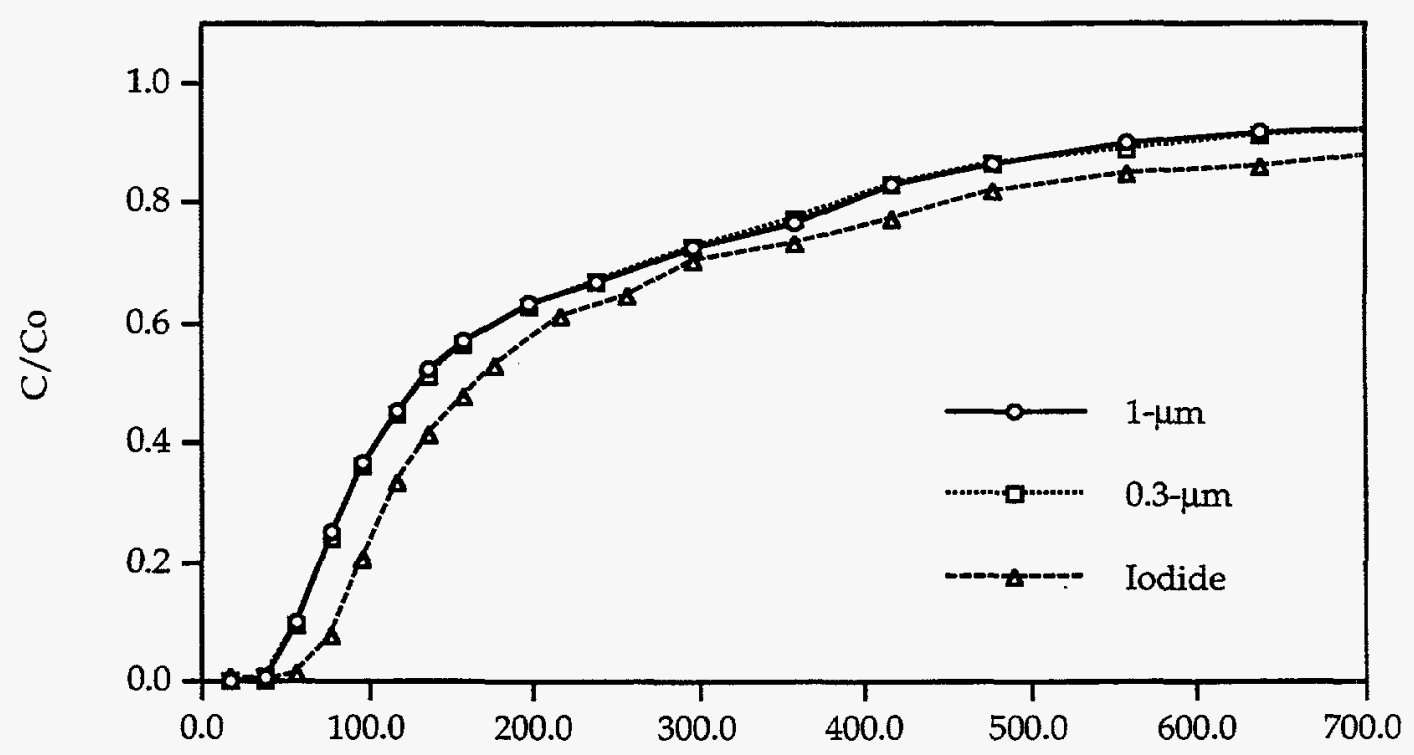

Time, sec

Figure B.52. Step portion of breakthrough curves for 1- $\mu \mathrm{m}$ CML microspheres, $0.3-\mu \mathrm{m}$ CML microspheres, and iodide in Experiment 12 (rotated Bullfrog tuff fracture). 


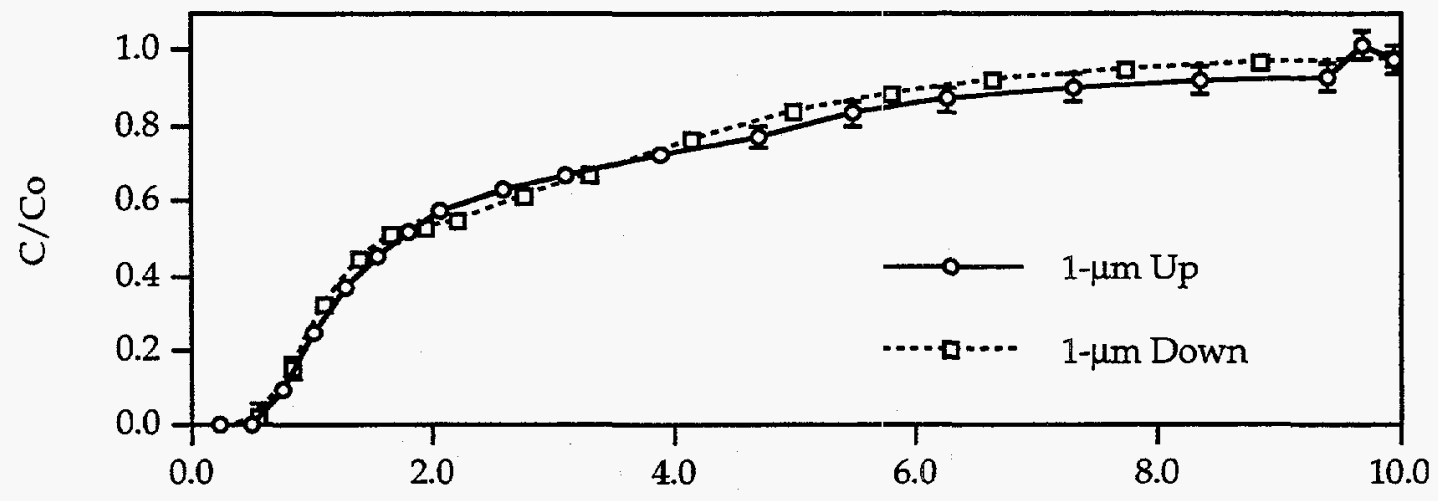

Volume Eluted, $\mathrm{ml}$

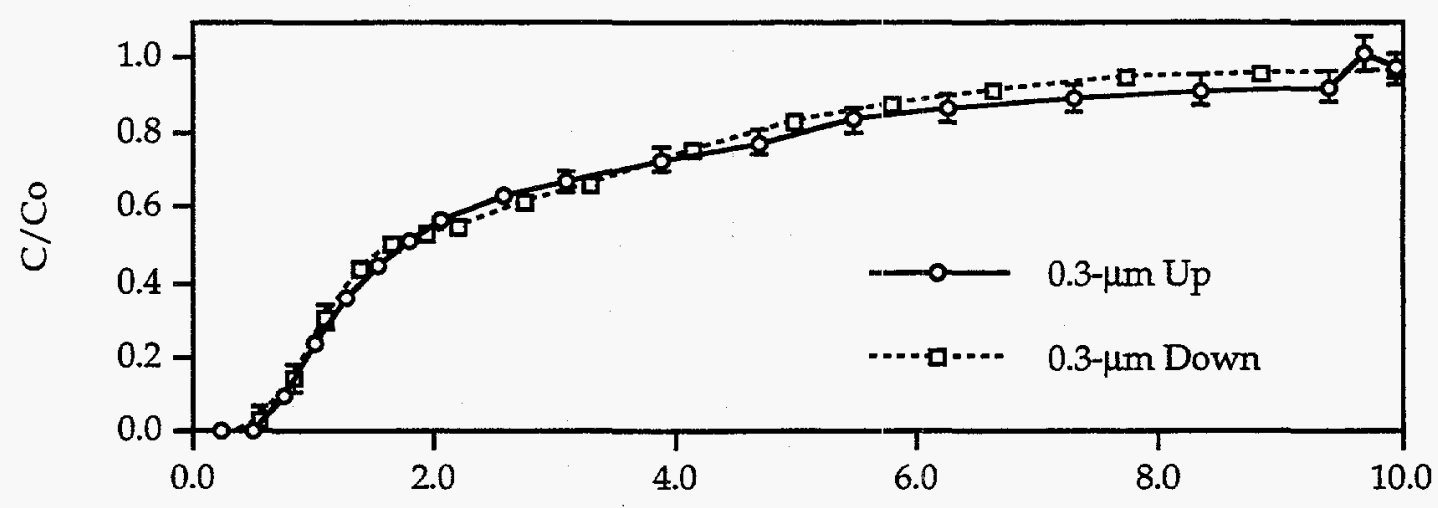

Volume Eluted, $\mathrm{ml}$

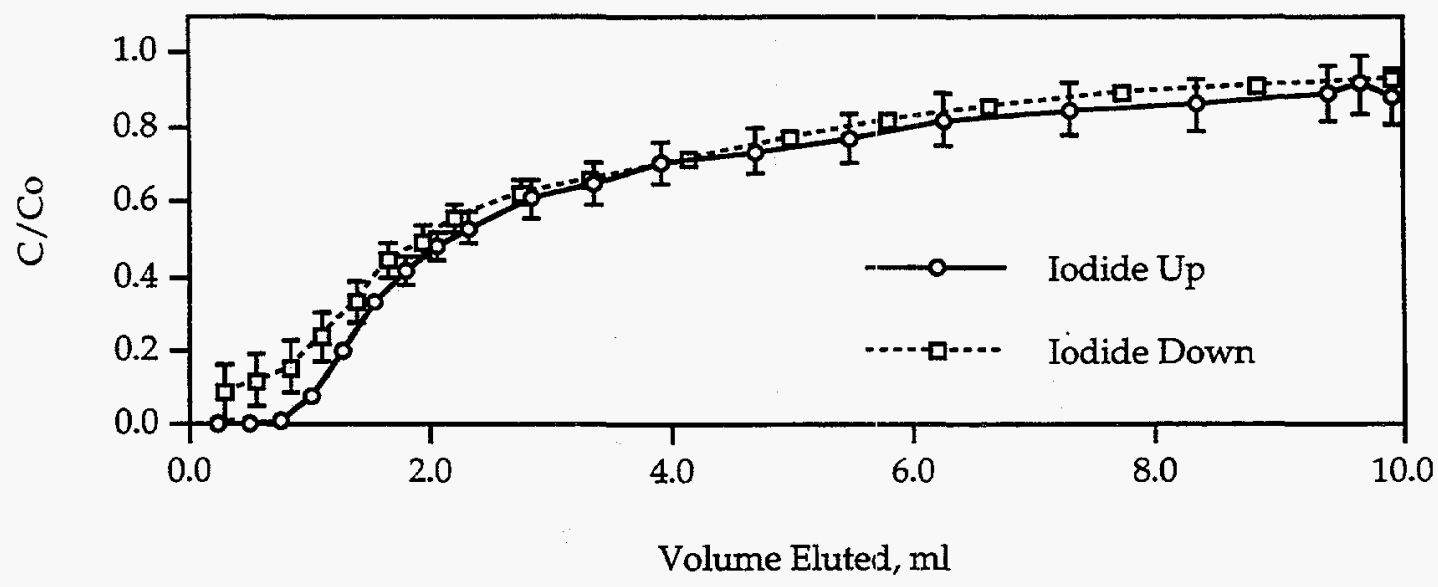

Figures B.53, B.54, B.55. Comparison of $\left(C / C_{0}\right)_{\text {step }}$ (up) and $\left(1-C / C_{0}\right)_{\text {flush }}$ (down) for the 1- $\mu \mathrm{m}$ CML microspheres, $0.3-\mu \mathrm{m}$ C.ML microspheres, and iodide, respectively, in Experiment 12 (rotated Bullfrog tuff fracture). 


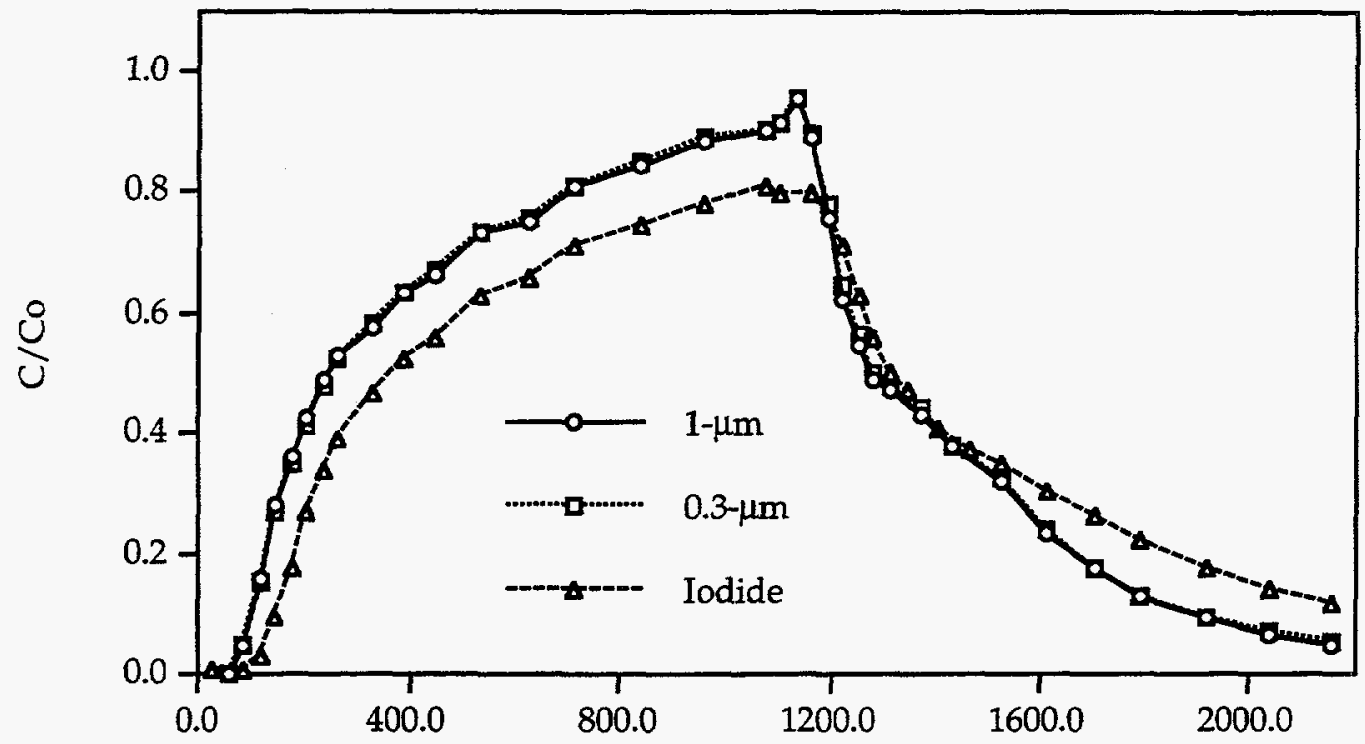

Time, sec

Figure B.56. Breakthrough curves of 1- $\mu \mathrm{m}$ CML microspheres, $0.3-\mu \mathrm{m} \mathrm{CML}$ microspheres, and iodide in Experiment 13 (rotated Bullfrog tuff fracture).

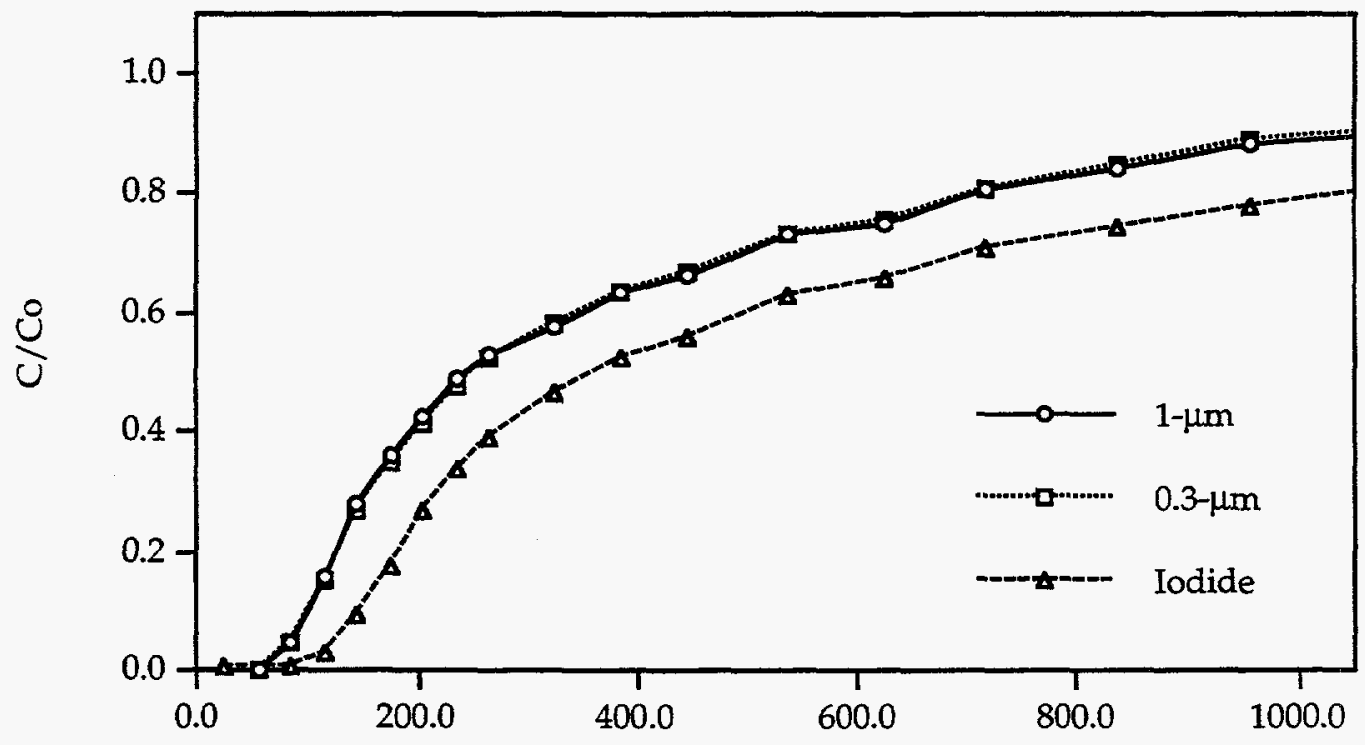

Time, sec

Figure B.57. Step portion of breakthrough curves for 1- $\mu \mathrm{m}$ CML microspheres, $0.3-\mu \mathrm{m} C M L$ microspheres, and iodide in Experiment 13 (rotated Bullfrog tuff fracture). 


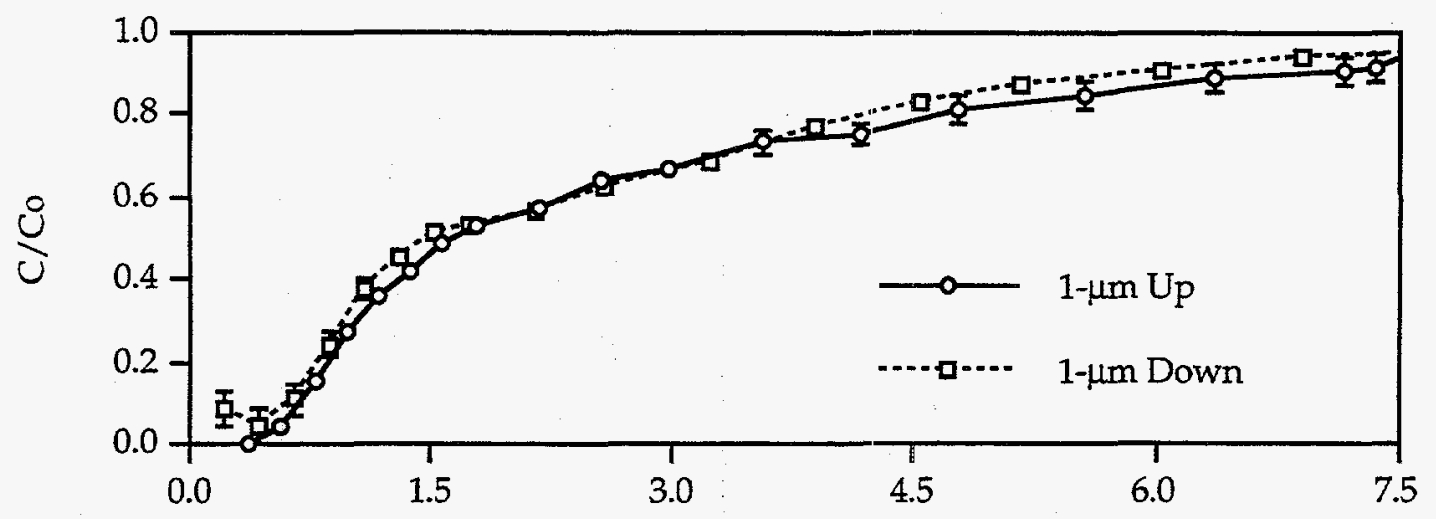

Volume Eluted, $\mathrm{ml}$

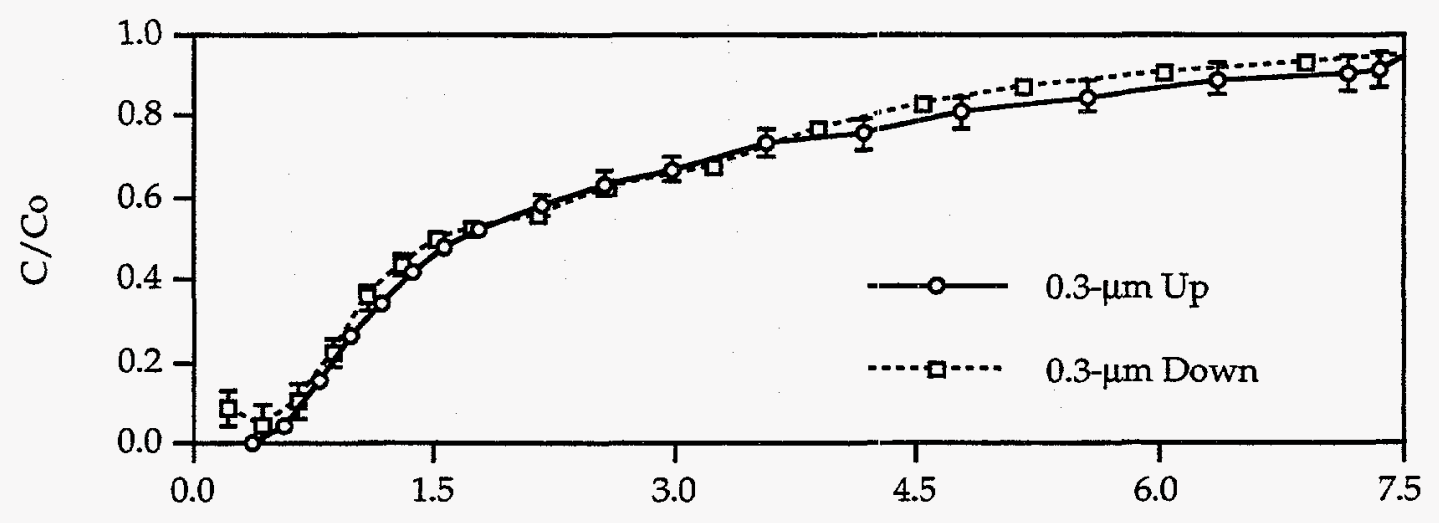

Volume Eluted, $\mathrm{ml}$

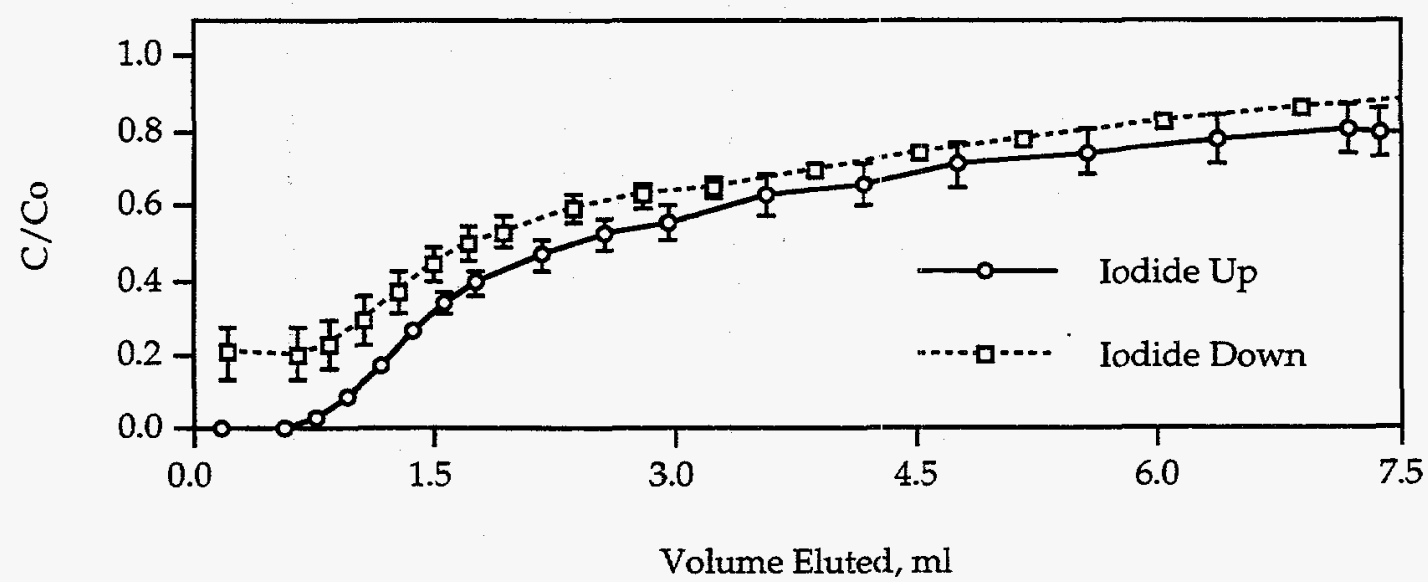

Figures B.58, B.59, B.60. Comparison of $\left(C / C_{0}\right)_{\text {step }}$ (up) and (1-C/ $\left.C_{0}\right)_{\text {flush }}$ (down)

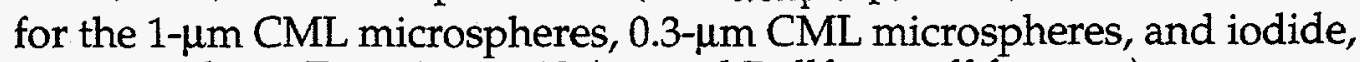
respectively, in Experiment 13 (rotated Bullfrog tuff fracture). 


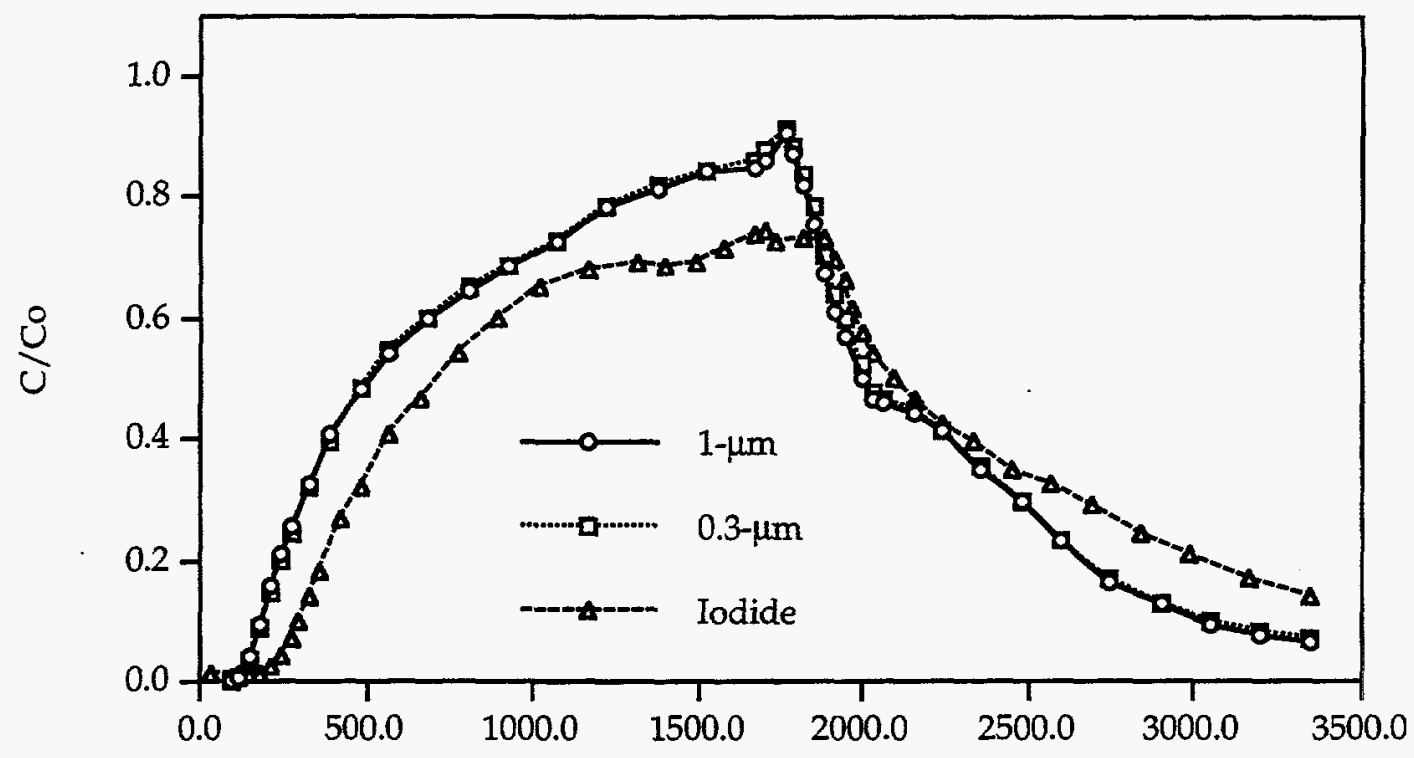

Time, sec

Figure B.61. Breakthrough curves of 1- $\mu \mathrm{m}$ CML microspheres, $0.3-\mu \mathrm{m}$ CML microspheres, and iodide in Experiment 14 (rotated Bullfrog tuff fracture).

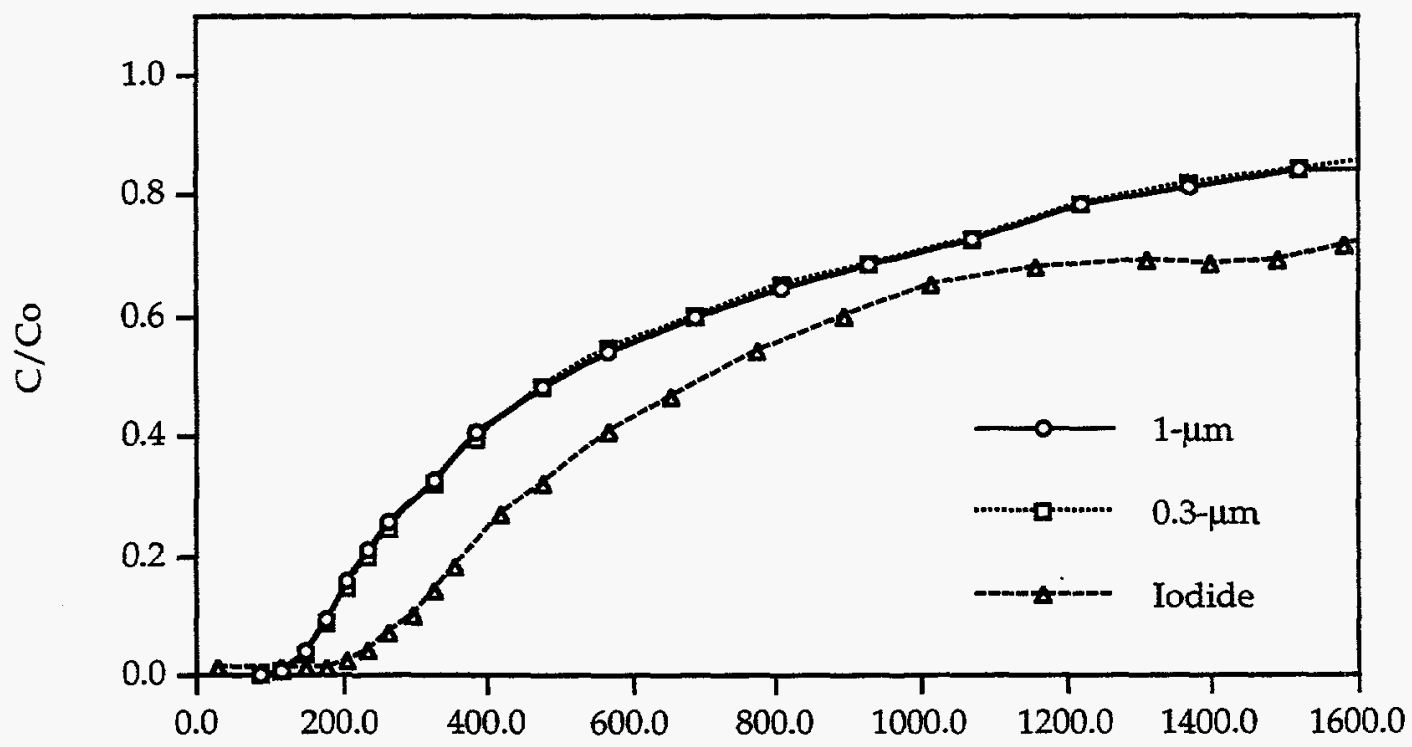

Time, sec

Figure B.62. Step portion of breakthrough curves for 1- $\mu \mathrm{m}$ CML microspheres, $0.3-\mu \mathrm{m}$ CML microspheres, and iodide in Experiment 14 (rotated Bullfrog tuff fracture). 


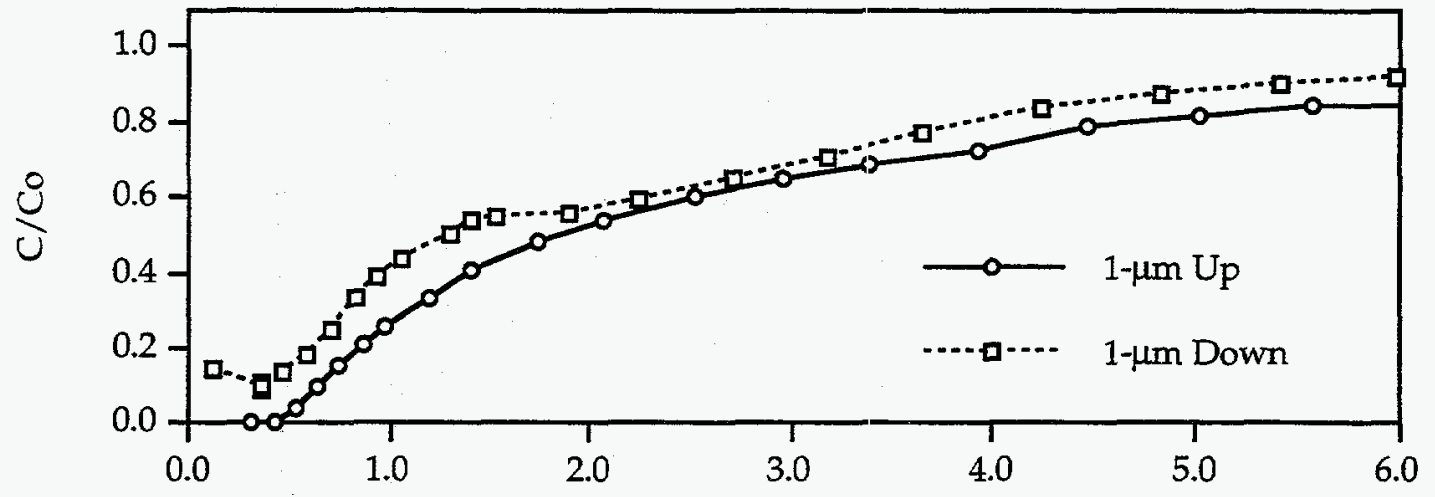

Volume Eluted, $\mathrm{ml}$

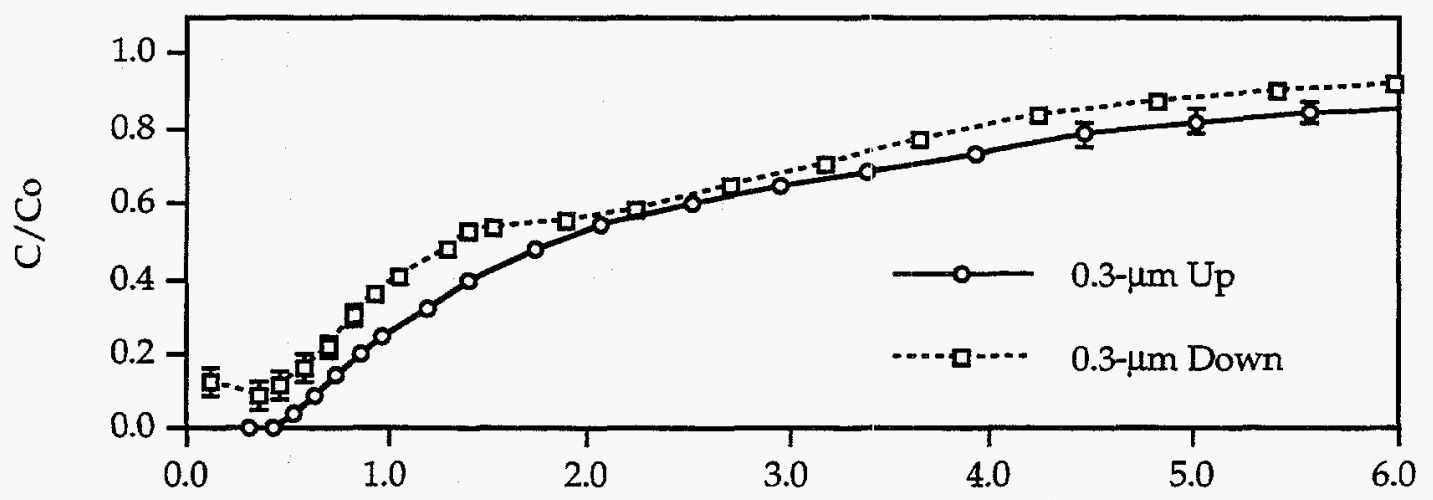

Volume Eluted, $\mathrm{ml}$

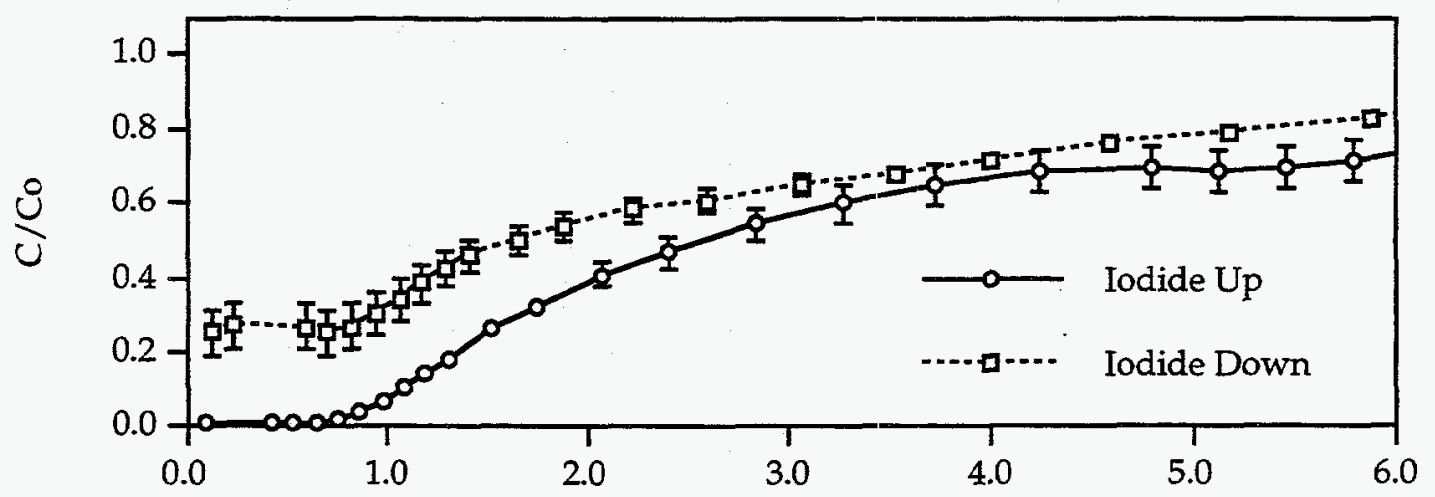

Volume Eluted, $\mathrm{ml}$

Figures B.63, B.64, B.65. Comparison of $\left(\mathrm{C} / \mathrm{C}_{0}\right)_{\text {step }}$ (up) and $\left(1-\mathrm{C} / \mathrm{C}_{0}\right)_{\text {flush }}$ (down) for the 1- $\mu \mathrm{m} C M L$ microspheres, $0.3-\mu \mathrm{m} C \mathrm{CML}$ microspheres, and iodide, respectively, in Experiment 14 (rotated Bullfrog tuff fracture). 


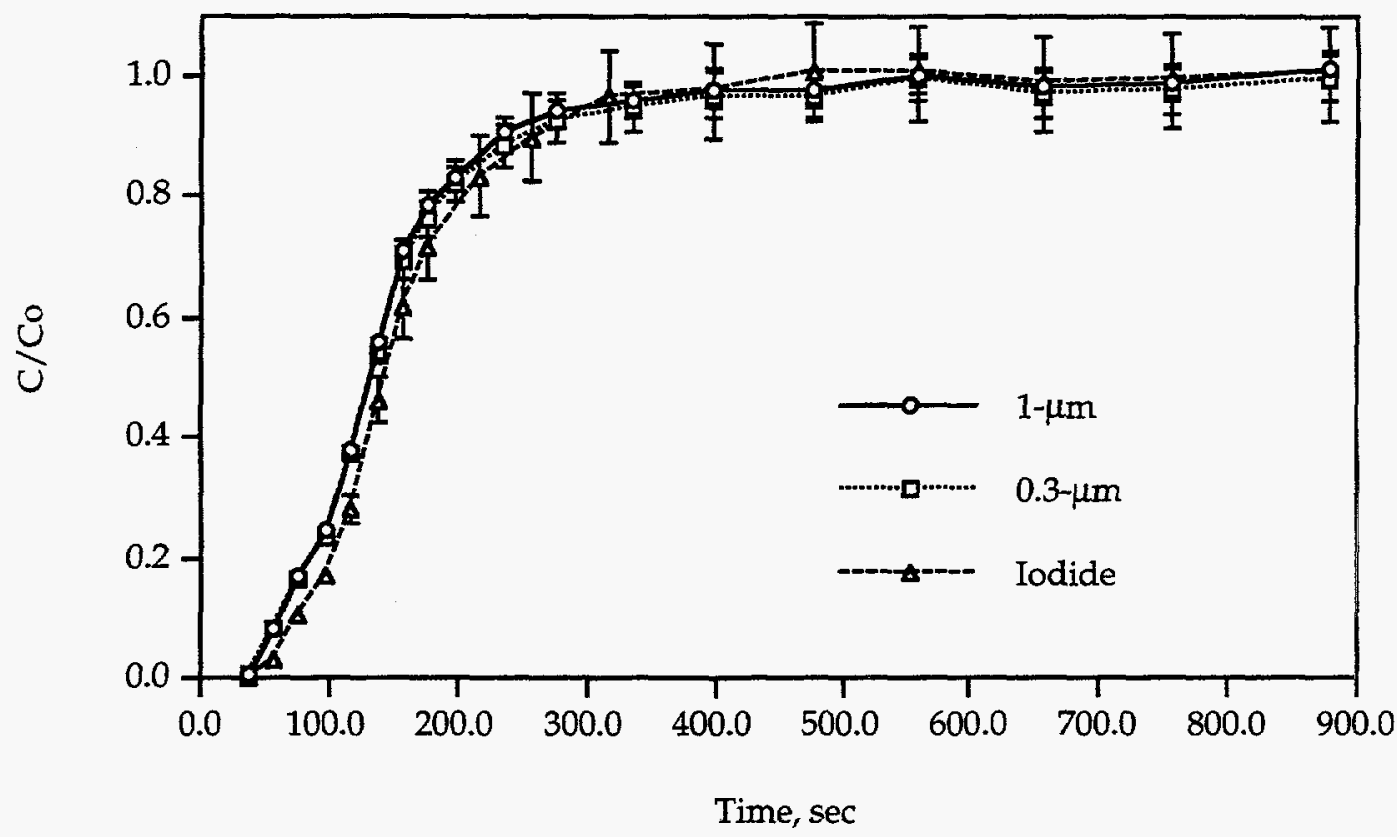

Figure B.66. Breakthrough curves of 1- $\mu \mathrm{m}$ CML microspheres, 0.3- $\mu \mathrm{m}$ CML microspheres, and iodide in Experiment 15 (Bullfrog tuff fracture).

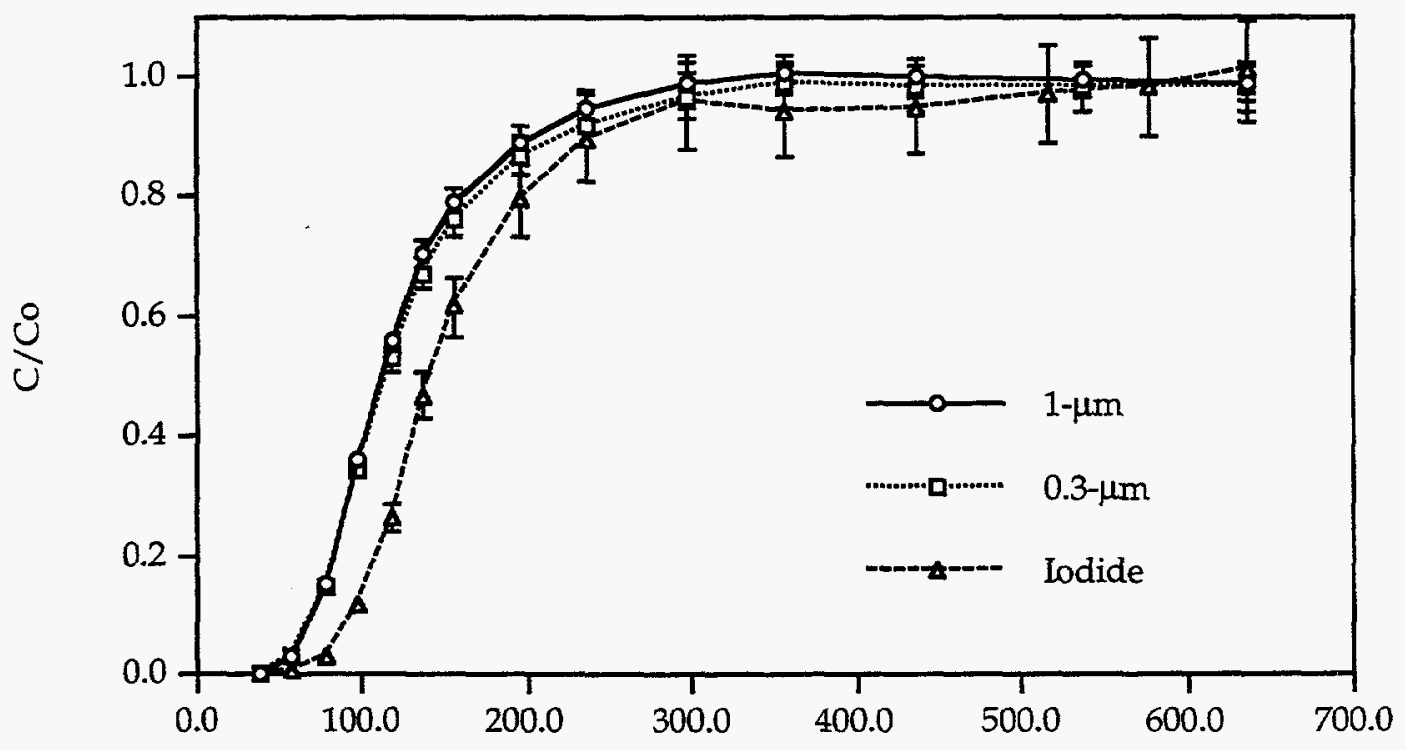

Time, sec

Figure B.67. Breakthrough curves of 1- $\mu \mathrm{m}$ CML microspheres, $0.3-\mu \mathrm{m} C \mathrm{CM}$ microspheres, and iodide in Experiment 16 (Tram tuff fracture). 


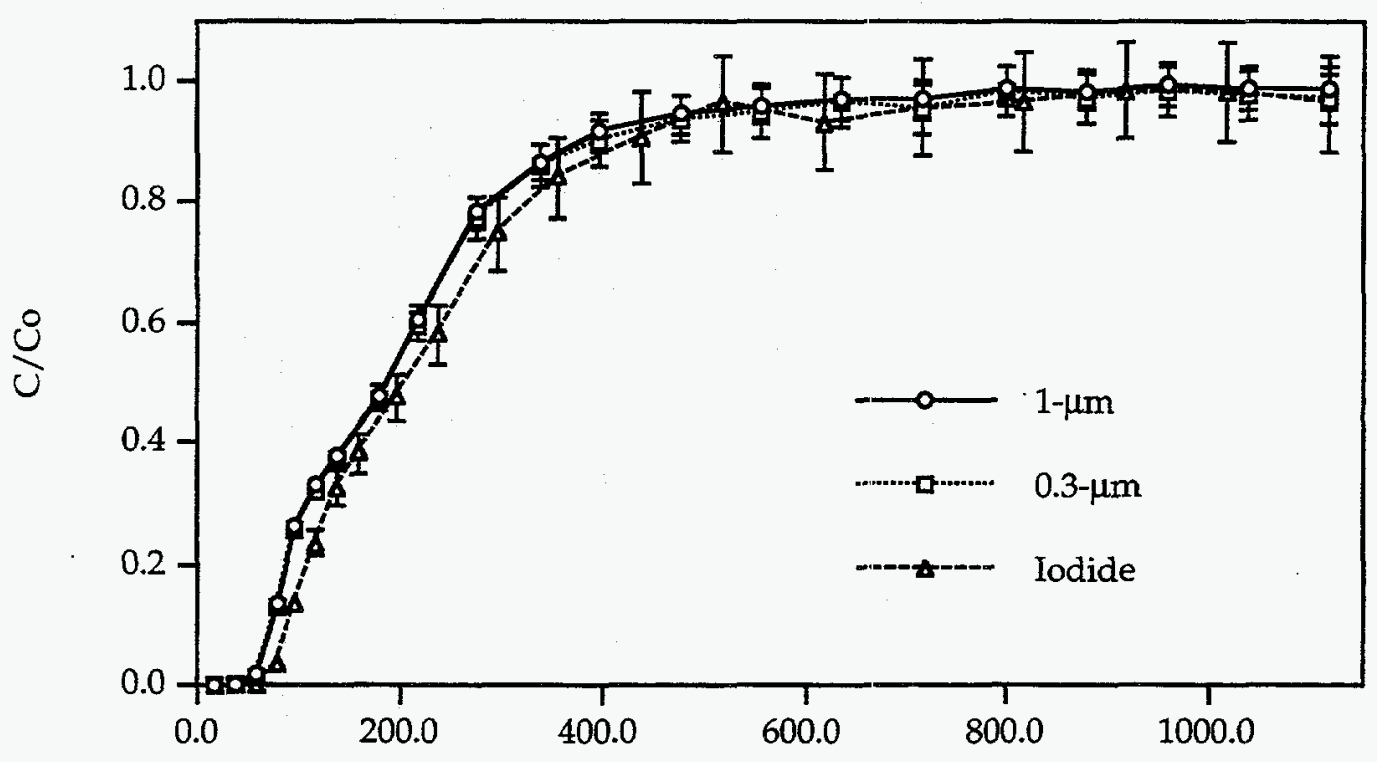

Time, sec

Figure B.68. Breakthrough curves of $1-\mu \mathrm{m}$ CML microspheres, $0.3-\mu \mathrm{m} \mathrm{CML}$ microspheres, and iodide in Experiment 17 (rotated Bullfrog tuff fracture).

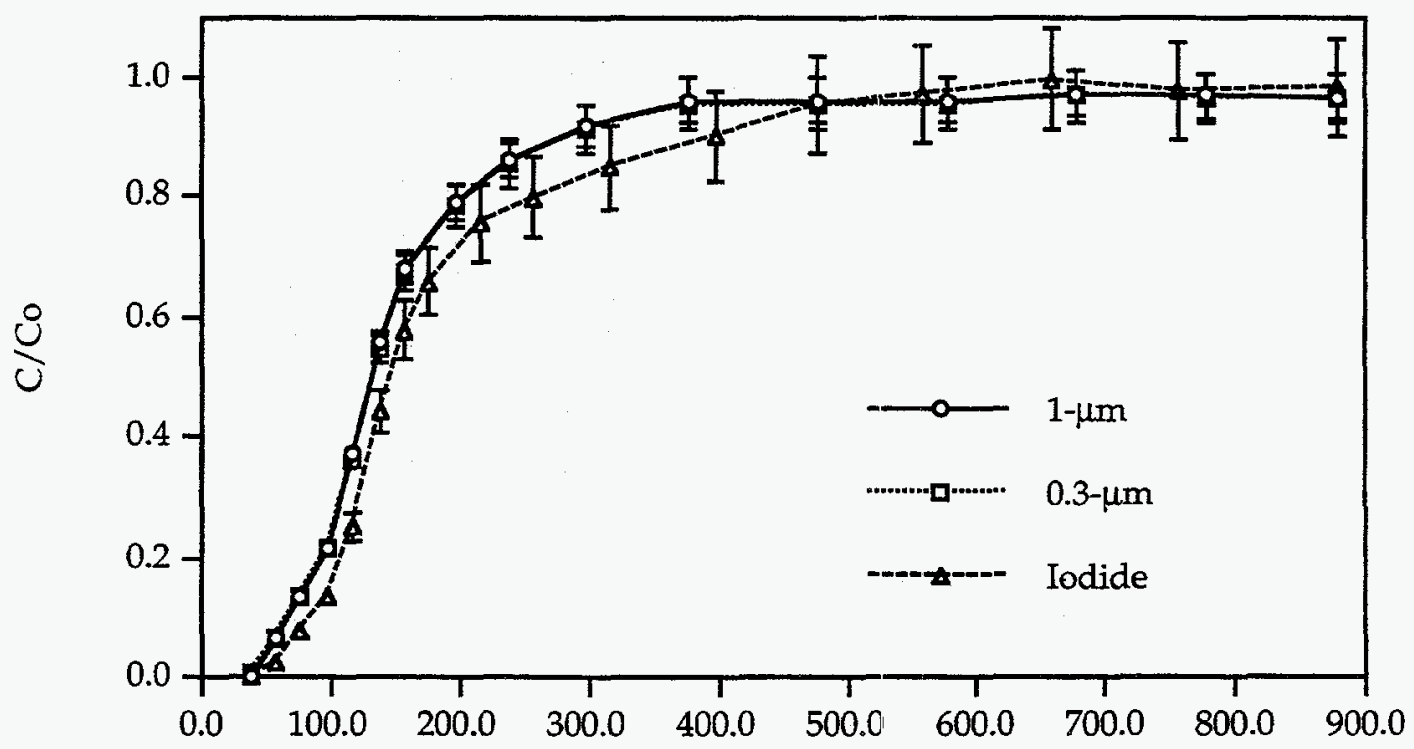

Time, sec

Figure B.69. Breakthrough curves of $1-\mu \mathrm{m}$ CML microspheres, $0.3-\mu \mathrm{m} C M L$ microspheres, and iodide in Experiment 18 (Bullfrog tuff fracture). 


\section{APPENDIX C: BREAKTHROUGH CURVES AT DIFFERENT FLOW RATES IN SAME FRACTURE}

This appendix contains plots of breakthrough curves in different experiments (at different flow rates) in the same fracture. The tracer concentrations are plotted as a function of volume eluted, and the curves for a given tracer (in a given fracture) are superimposed on each other to allow comparisons. The plots are intended to show the equivalence of the microsphere breakthrough curves at different flow rates (in the same fracture) and the dependence of the iodide breakthrough curves on flow rate. The plots for the Bandelier tuff fracture show the attenuation of the 1- $\mu \mathrm{m}$ non-CML microspheres and the $0.3-\mu \mathrm{m}$ silica microspheres in this fracture. The plots for the Bullfrog tuff fracture show how the dispersion of all tracers changed in this fracture after damage occurred to the surfaces. All concentrations in the breakthrough curves are normalized to the inlet concentration of the experiment, $\mathrm{C}_{\mathrm{o}}$. Error bars are not shown in any of the plots in this appendix. 


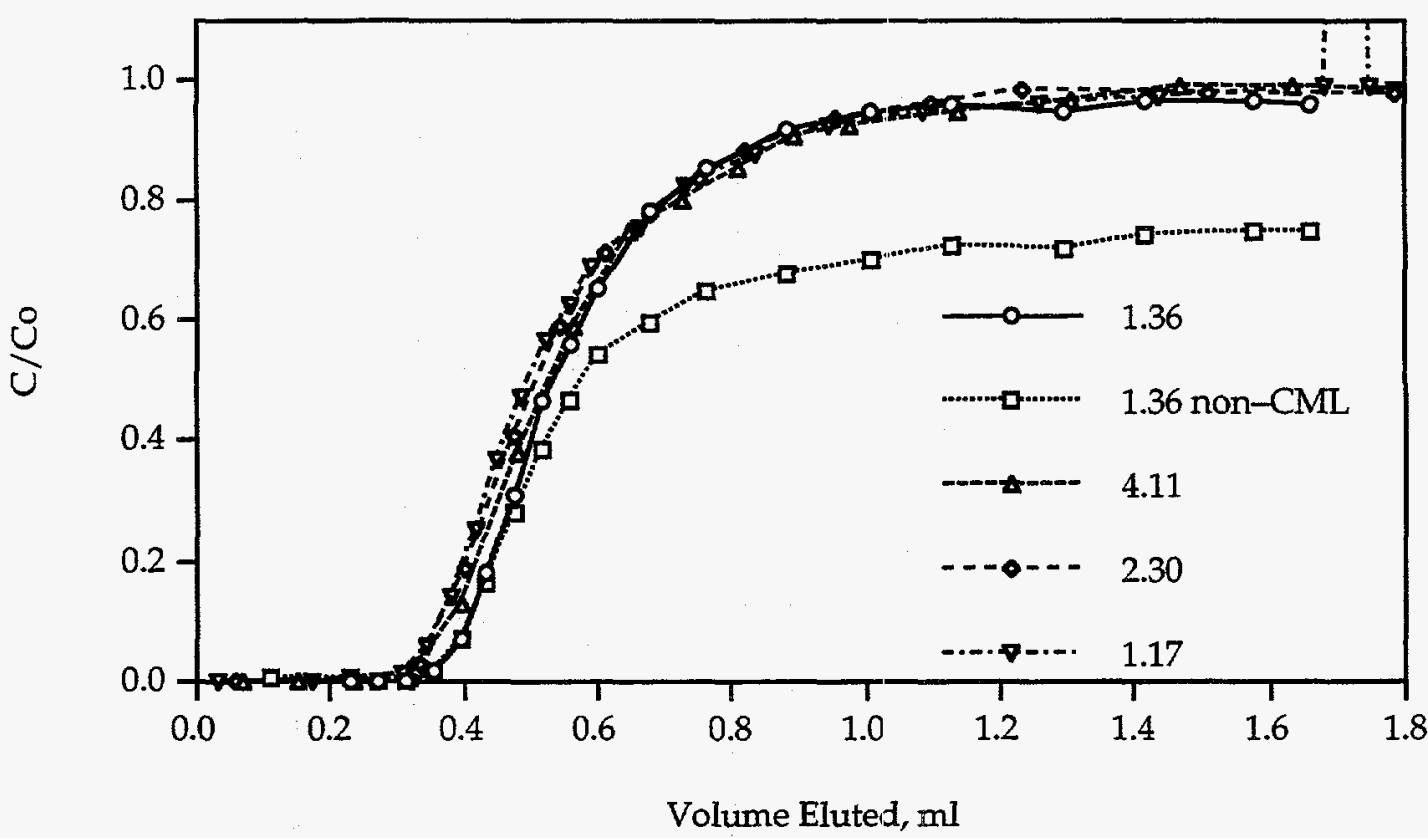

Figure C.1. Breakthrough curves of 1- $\mu \mathrm{m}$ microspheres in Bandelier tuff fracture at various flow rates $(\mu \mathrm{l} / \mathrm{sec})$. Except as noted, all microspheres were CML.

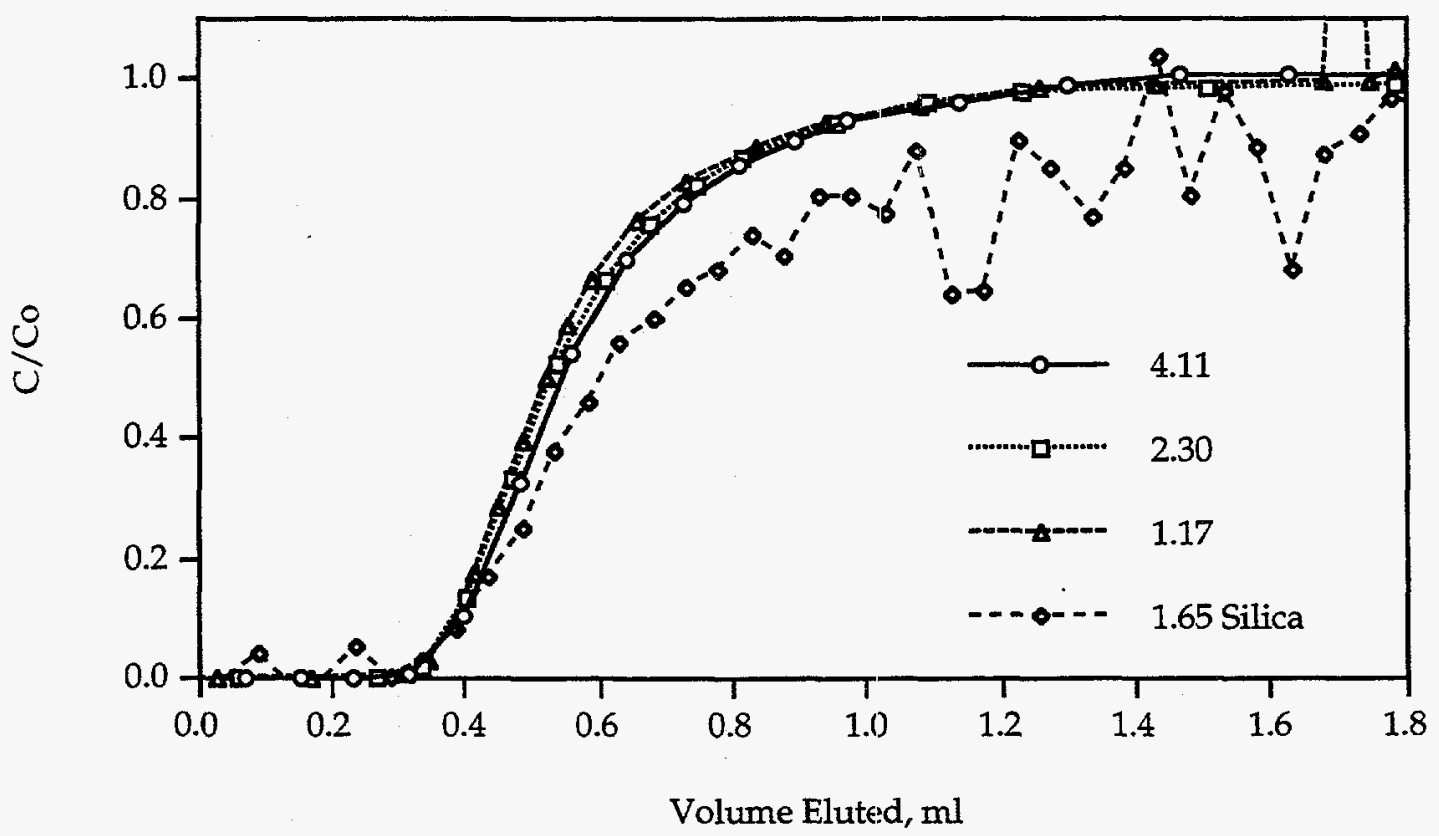

Figure C.2. Breakthrough curves of 0.3- $\mu \mathrm{m}$ microspheres in Bandelier tuff fracture at various flow rates $(\mu \mathrm{l} / \mathrm{sec})$. Except as noted, all microspheres were CML. 


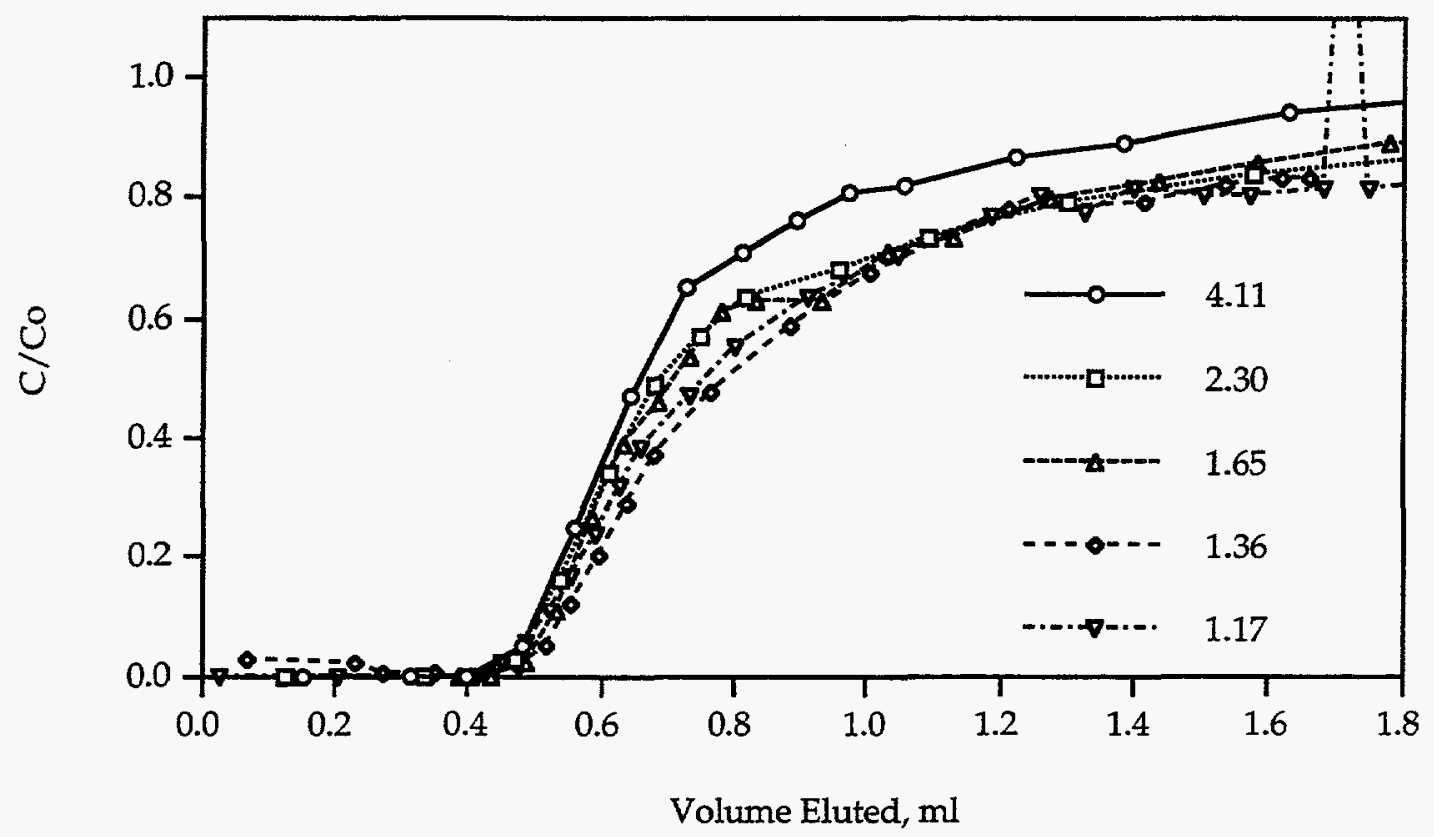

Figure C.3. Breakthrough curves of iodide in Bandelier tuff fracture at various flow rates $(\mu \mathrm{l} / \mathrm{sec})$. 


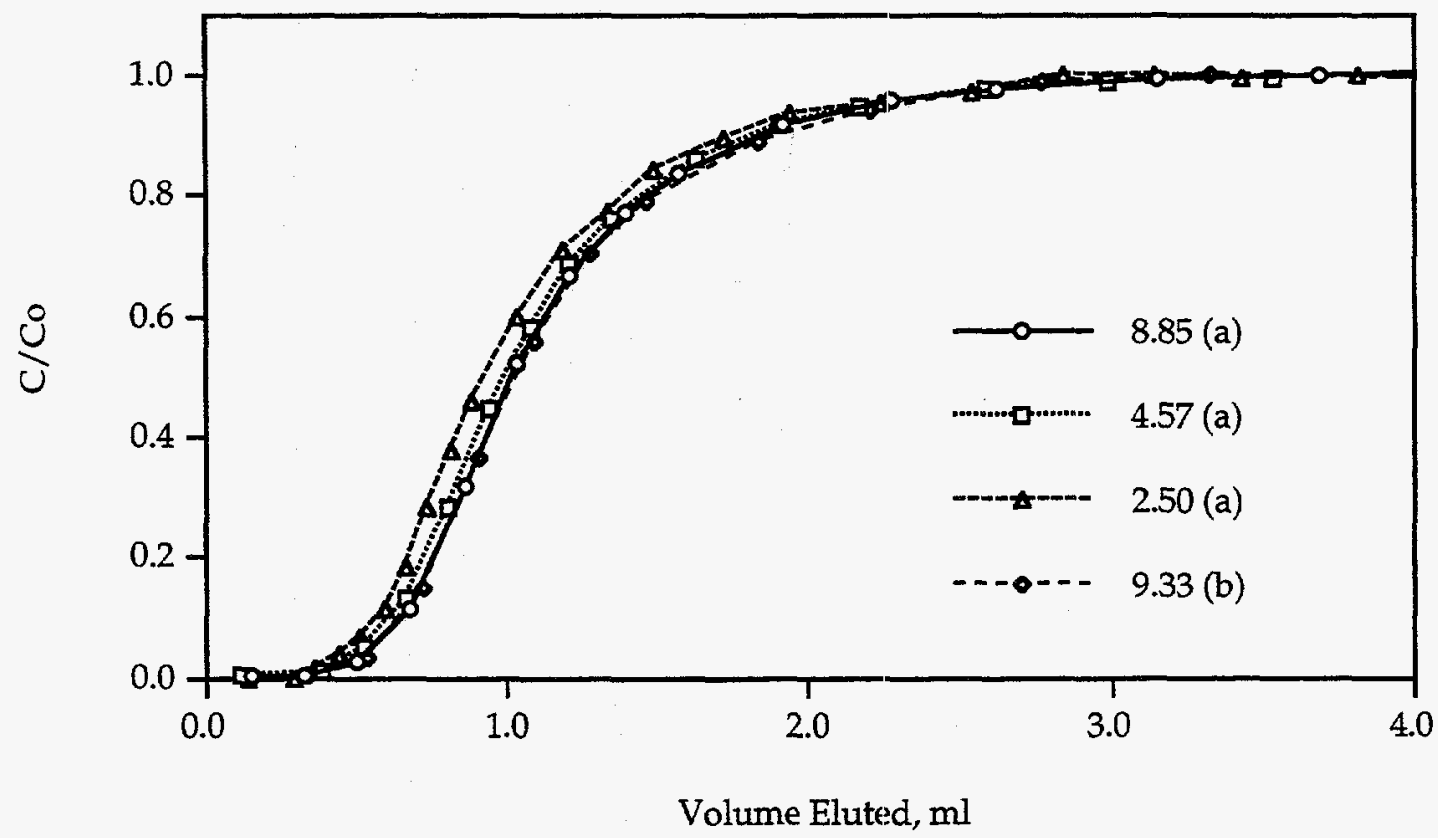

Figure C.4. Breakthrough curves of $1-\mu \mathrm{m}$ CML microspheres in Tram tuff fracture at various flow rates $(\mu \mathrm{l} / \mathrm{sec})$. (a) refers to Experiments 9-11, and (b) refers to Experiment 16 (a separate day).

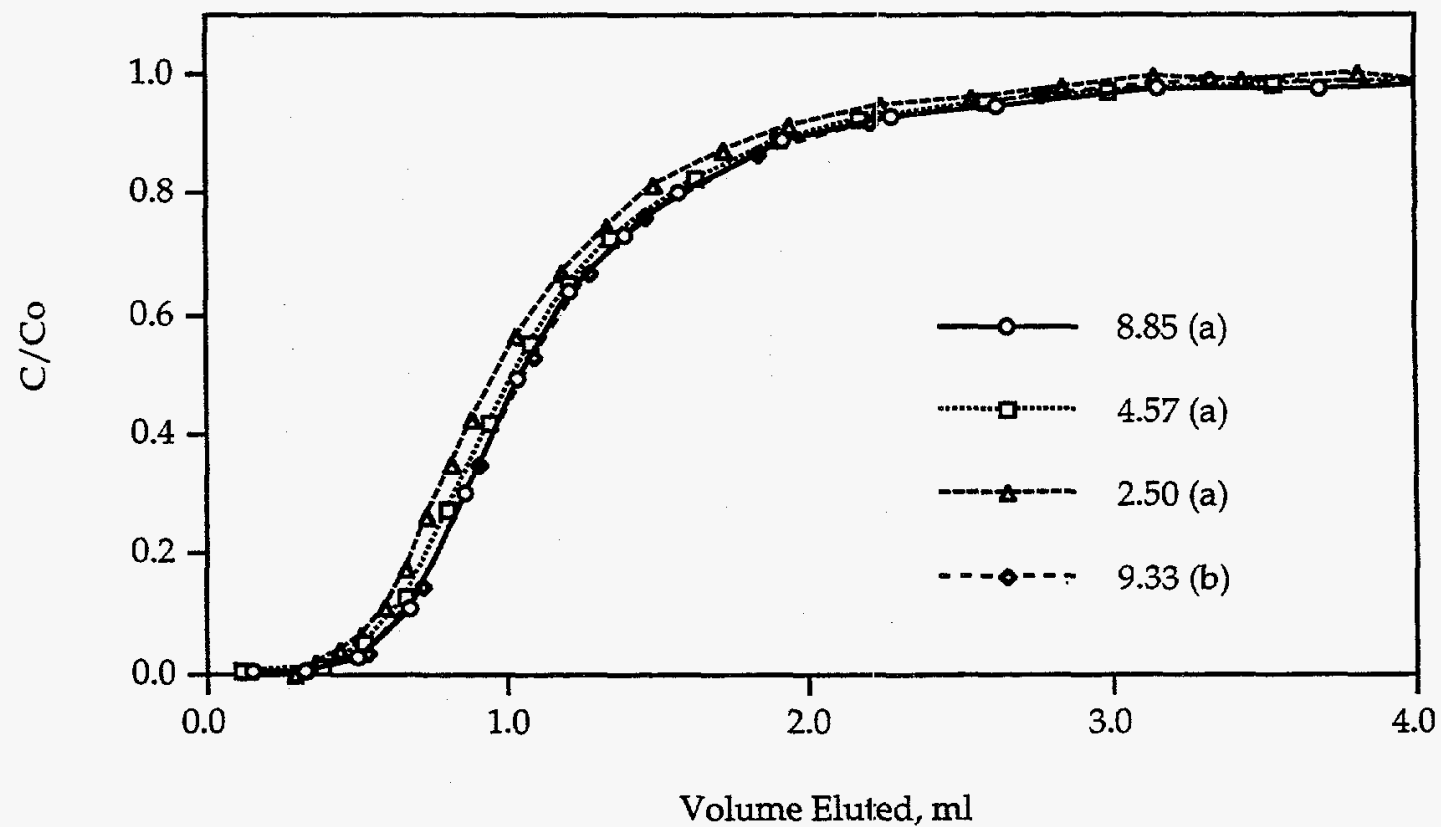

Figure C.5. Breakthrough curves of 0.3- $\mu \mathrm{m}$ CML microspheres in Tram tuff fracture at various flow rates $(\mu \mathrm{l} / \mathrm{sec})$. (a) refers to Experiments 9-11, and (b) refers to Experiment 16 (a separate day). 


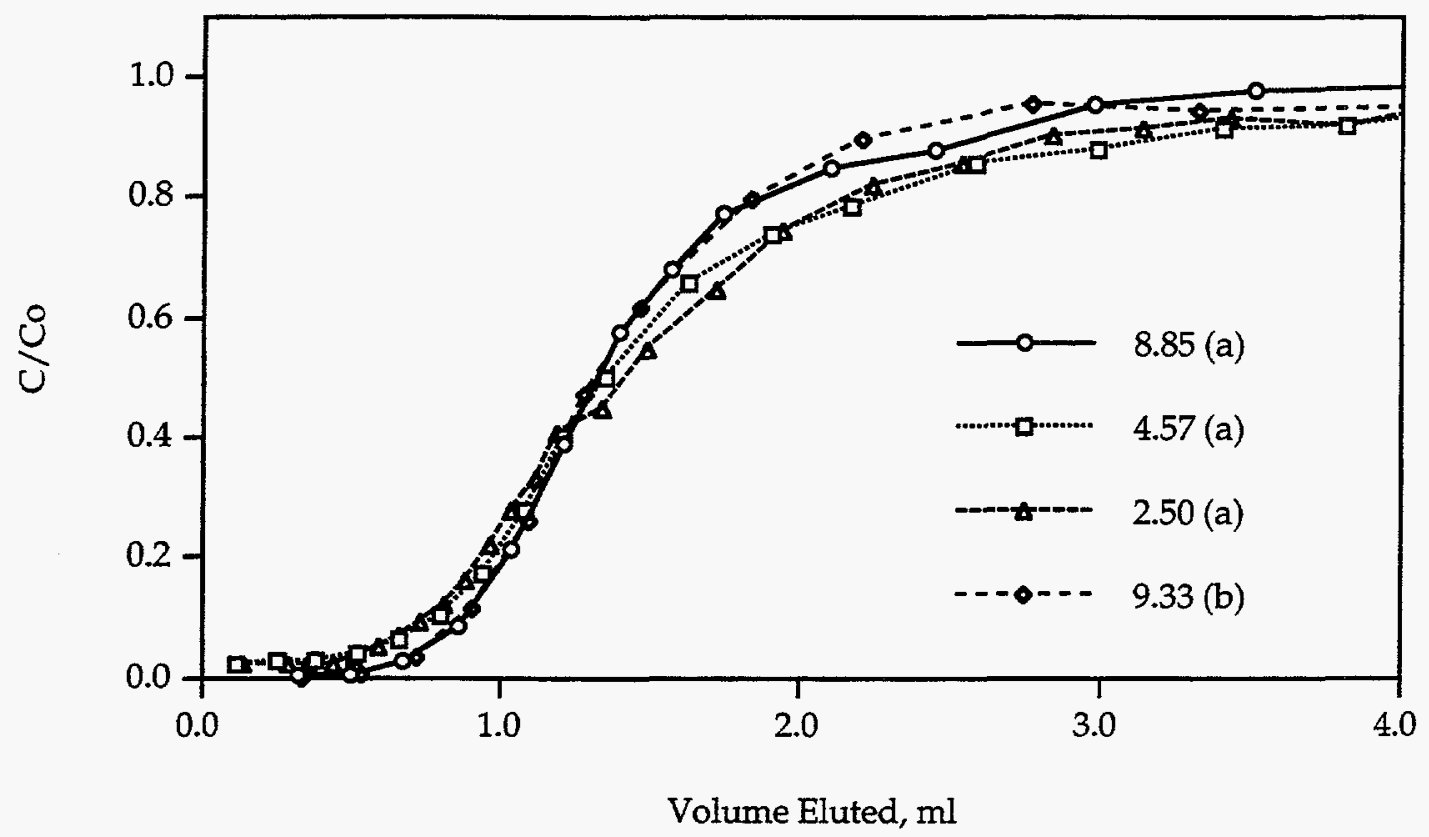

Figure C.6. Breakthrough curves of iodide in Tram tuff fracture at various flow rates $(\mu \mathrm{l} / \mathrm{sec})$. (a) refers to Experiments 9-11, and (b) refers to Experiment 16 (a separate day). 


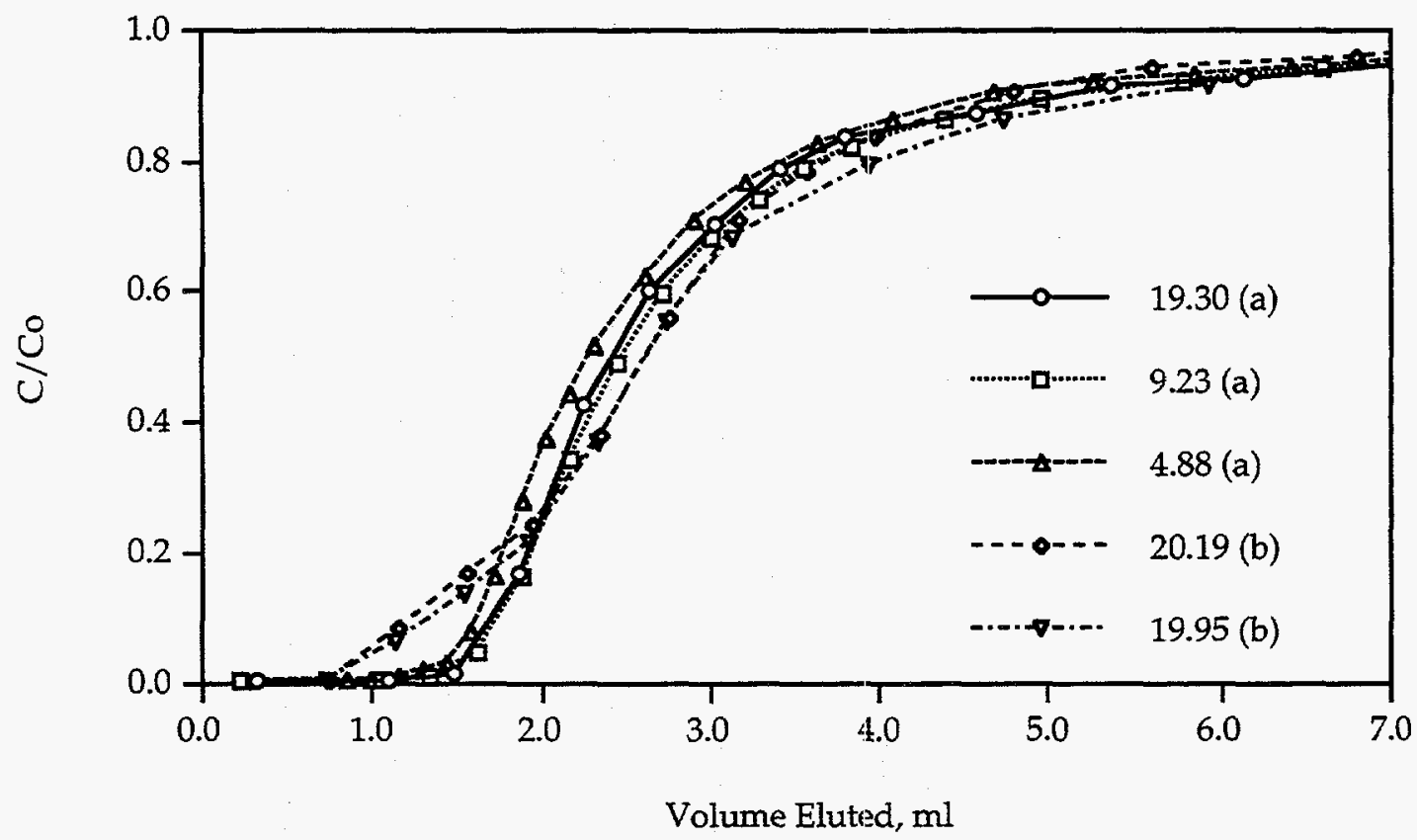

Figure C.7. Breakthrough curves of 1- $\mu \mathrm{m}$ CML microspheres in Bullfrog tuff fracture at various flow rates $(\mu \mathrm{l} / \mathrm{sec})$. (a) refers to Experiments 6-8, and (b) refers to Experiments 15 and 18 (separate days).

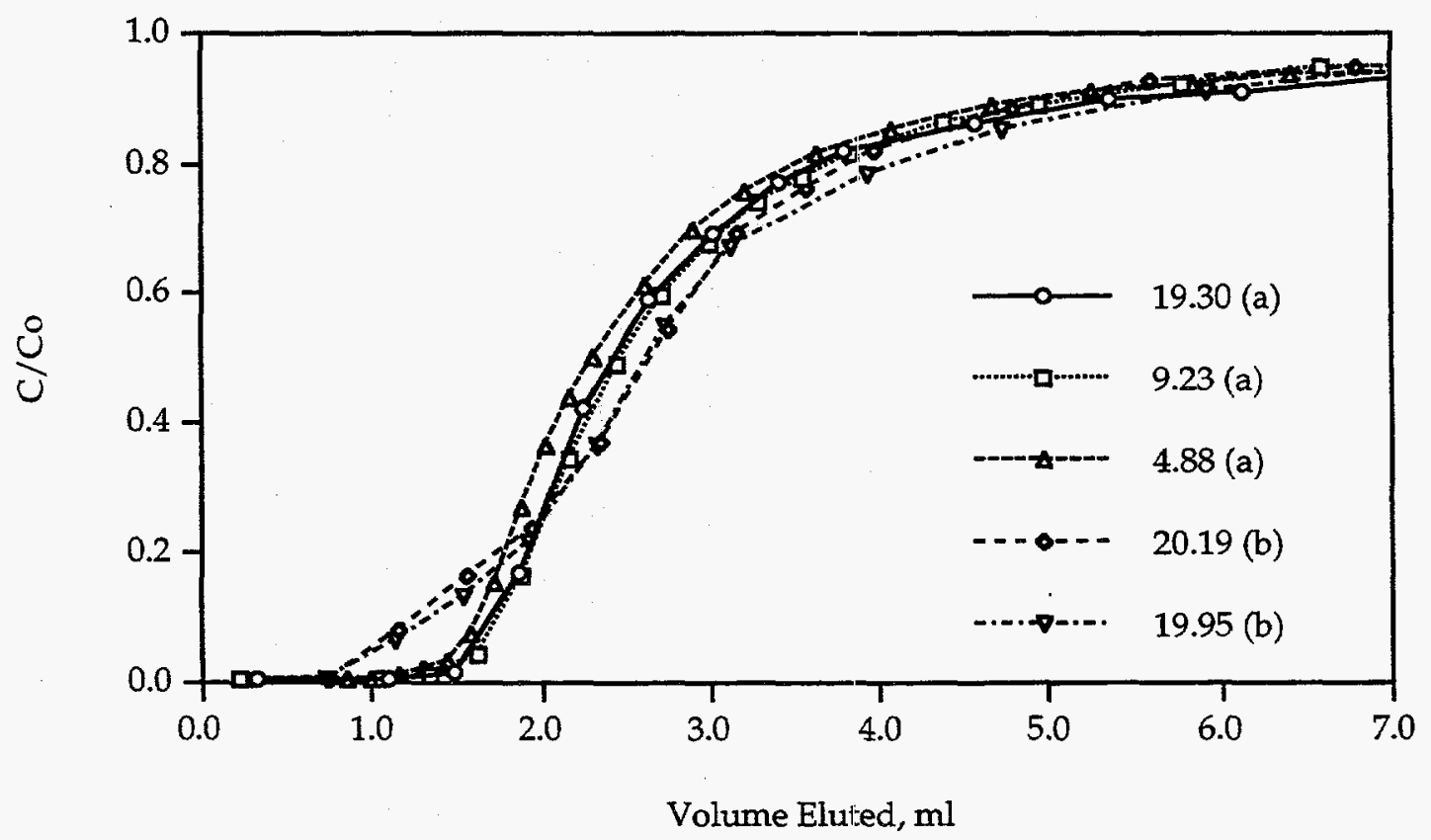

Figure C.8. Breakthrough curves of 0.3- $\mu \mathrm{m}$ CML microspheres in Bullfrog tuff fracture at various flow rates $(\mu \mathrm{l} / \mathrm{sec}$ ). (a) refers to Experiments 6-8, and (b) refers to Experiments 15 and 18 (separate days). 


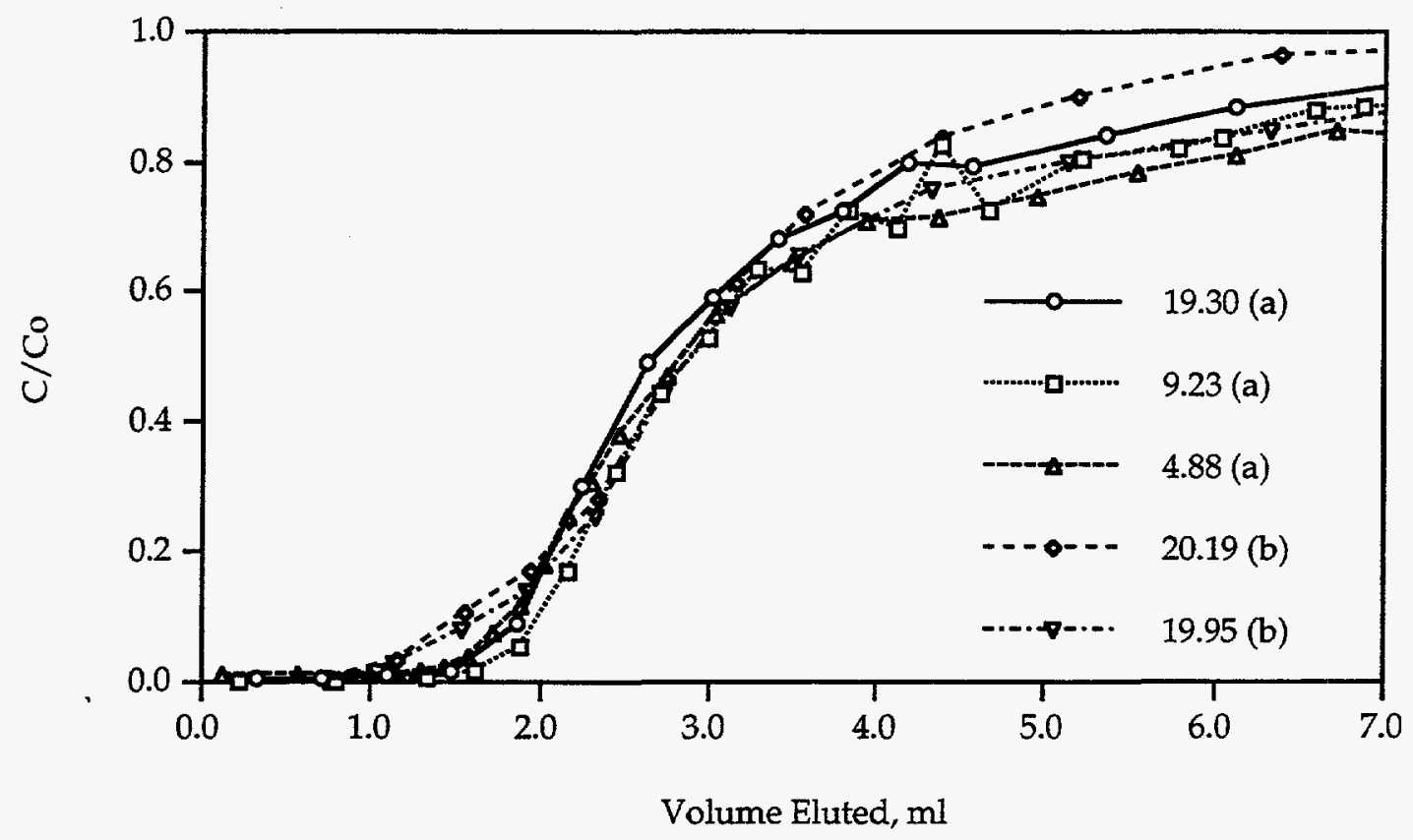

Figure C.9. Breakthrough curves of iodide in Bullfrog tuff fracture at various flow rates $(\mu \mathrm{l} / \mathrm{sec})$. (a) refers to Experiments 6-8, and (b) refers to Experiments 15 and 18 (separate days). 


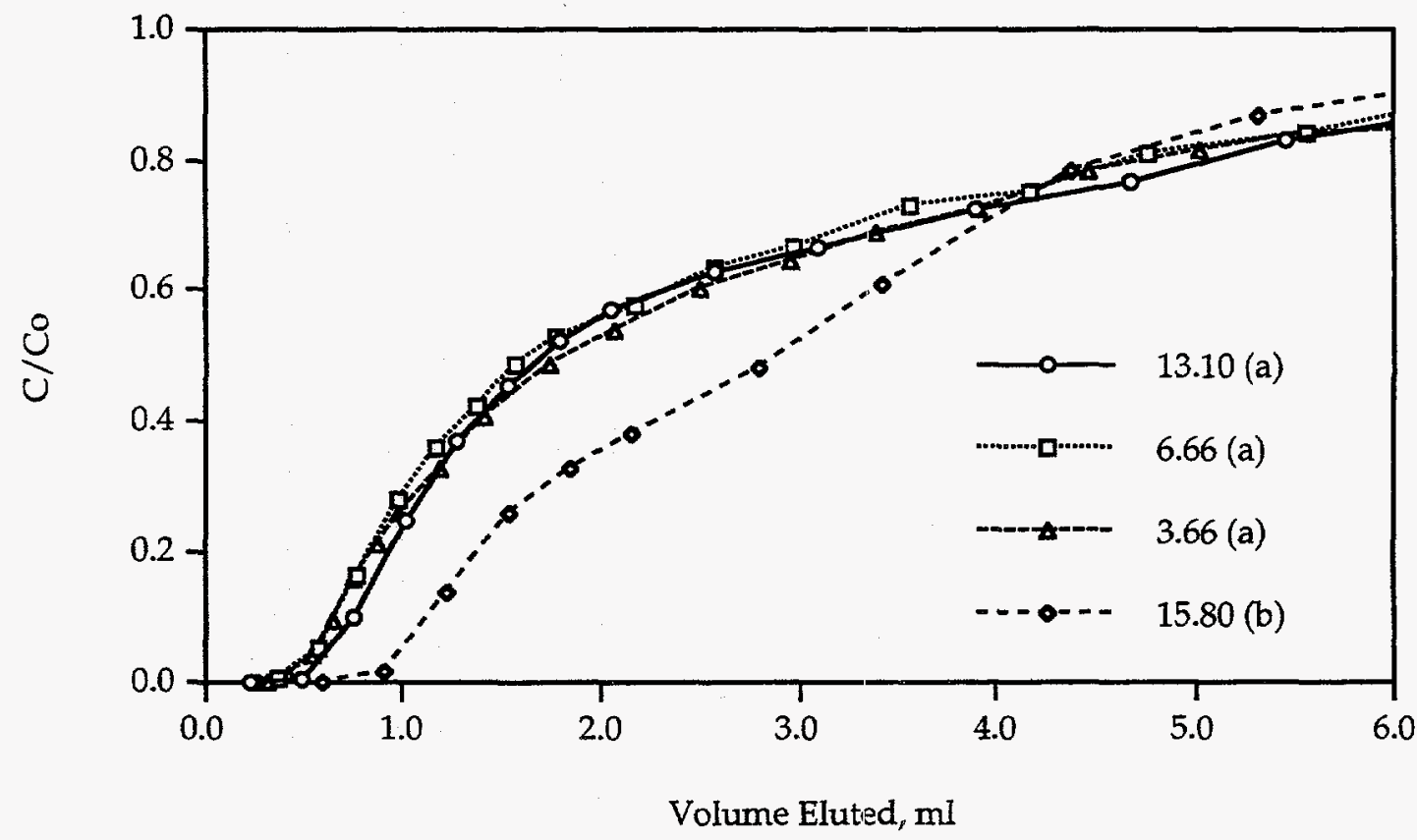

Figure C.10. Breakthrough curves of 1- $\mu \mathrm{m}$ CML microspheres in rotated Bullfrog tuff fracture at various flow rates $(\mu \mathrm{l} / \mathrm{sec})$. (a) refers to Experiments 12-14, and (b) refers to Experiment 17 (a separate day).

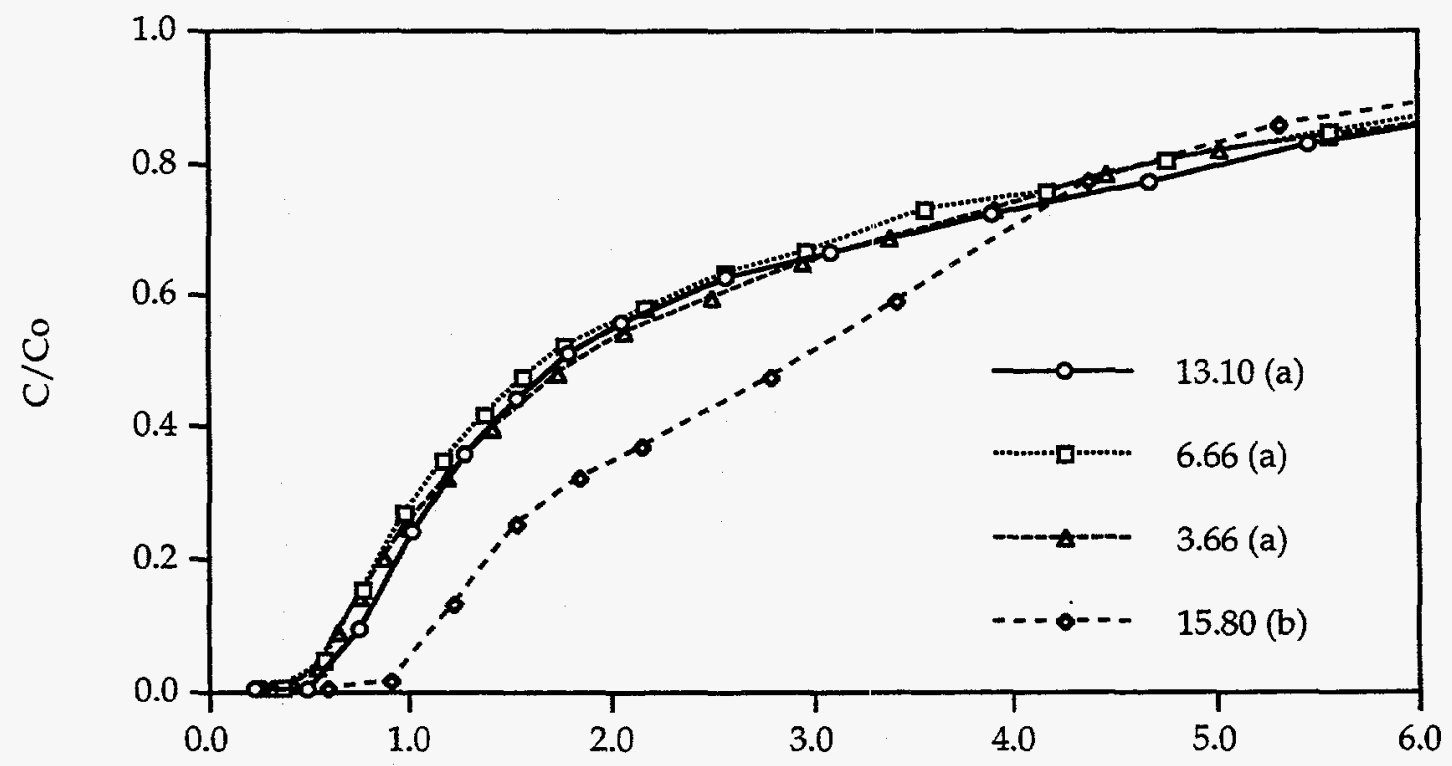

Volume Eluted, $\mathrm{ml}$

Figure C.11. Breakthrough curves of $0.3-\mu \mathrm{m}$ CML microspheres in rotated Bullfrog tuff fracture at various flow rates $(\mu \mathrm{l} / \mathrm{sec})$. (a) refers to Experiments 12-14, and (b) refers to Experiment 17 (a separate day). 


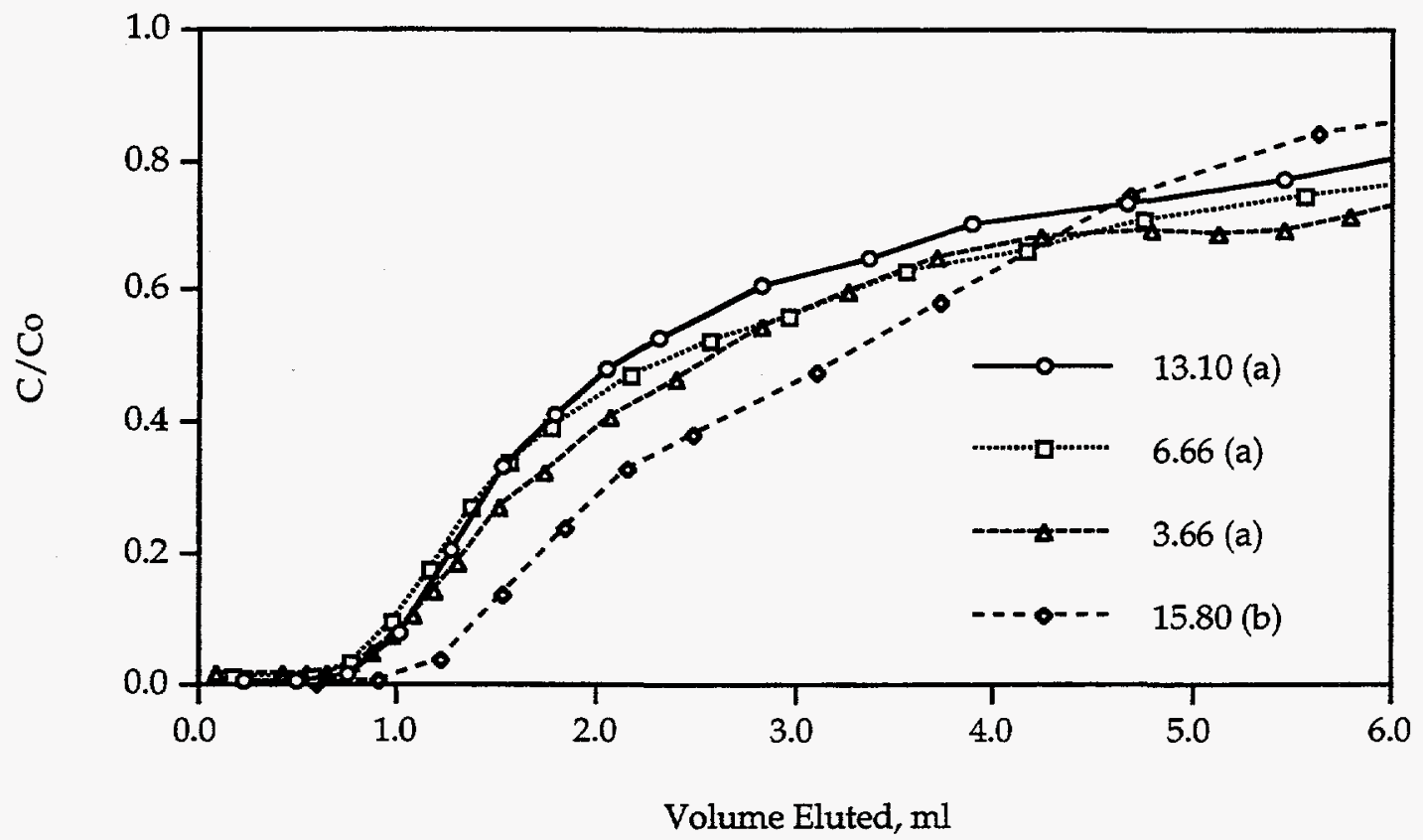

Figure C.12. Breakthrough curves of iodide in rotated Bullfrog tuff fracture at various flow rates $(\mu \mathrm{l} / \mathrm{sec}$ ). (a) refers to Experiments 12-14, and (b) refers to Experiment 17 (a separate day). 


\section{APPENDIX D: COMPARISON OF PARTICLE-TRACKING MODEL PREDICTIONS AND EXPERIMENTAL DATA}

This appendix contains plots of predicted breakthrough curves for all the tracers in each tracer experiment except Experiment 1 . The experimental breakthrough curves (with error bars) are shown in each plot for comparison. The particle-tracking models described in Section 4.2 were used to generate the predicted curves. The implications of the model predictions are discussed in Section 4.3.

A separate plot is shown for each tracer in each experiment (except the first experiment). The first experiment was not modeled because (1) it would have been necessary to account for colloid-wall interactions (i.e., attachment and detachment) to model the non-CML microspheres (the models were not capable of simulating such interactions), and (2) the 1- $\mu \mathrm{m}$ CML and iodide breakthrough curves in this experiment were nearly identical to the breakthrough curves of these tracers in Experiment 5, which was essentially a duplicate experiment. In all simulations, only the step phases of the tracer experiments were modeled. The flush phases were not modeled because they would have required more sophisticated logic to account for material that was already in the fractures when the flushes began. However, the fact that the step and flush breakthrough curves were essentially mirror images of each other suggests that the flush phase could have been modeled by subtracting one step phase curve from another, with a time offset between the two curves corresponding to the length of the step phase.

The following models were used to predict the microsphere breakthrough curves:

(1) the unaltered (or baseline) colloid model described in Section 4.2.5 -designated by (a) in the plot legends,

(2) the variation described in Section 4.2.6 in which colloids are excluded from a layer of fluid along the fracture walls -- designated by $(b)$ in the plot legends (the particles maintained a constant relative distance from the effective walls when crossing node boundaries in the Bandelier and Tram tuff fracture calculations and from the actual walls in the Bullfrog tuff fracture calculations), and

(3) a parallel-plate model in which the aperture is assumed to be equal to the average aperture estimated from the experimental breakthrough curves of the 1- $\mu \mathrm{m}$ CML microspheres (see Section 3.1.7) -- designated by (c) in the plot legends.

The following models were used to predict the iodide breakthrough curves:

(1) the solute particle-tracking method described in Section 4.2.3, which included the effects of matrix diffusion - designated by (a) in the plot legends,

(2) the solute particle-tracking method described in Section 4.2.4, which included a stagnant layer of fluid along the fracture walls as well as the effects of matrix diffusion -- designated by (b) in the plot legends,

(3) a parallel-plate particle-tracking model that included the effects of matrix diffusion -- designated by (c) in the plot legends, and

(4) the Starr method described in Section 4.2.3 (i.e., assumes that the Starr analytical expression is valid for every node in the flow domain) designated by "Starr" in the plot legends. 
The predicted fluid residence time distributions, calculated as described in Section 4.2.1, are also shown in each plot (designated by "Fluid" in the plot legends). These fluid residence time distributions provide perspective on when the tracers are predicted to arrive in the effluent relative to the fluid, and they also provide a basis for comparing model predictions associated with different tracers in the same fracture (which are shown on different plots). The microsphere and iodide breakthrough curves were constructed from the cumulative residence time distributions of 1000 particles in the particle-tracking simulations. The fluid and "Starr" breakthrough curves were constructed from the cumulative residence time distributions of 2000 particles. It was found that the breakthrough curves generated from simulations of 1000 particles and 10000 particles were essentially indistinguishable.

With the exception of the experiment involving the silica microspheres (Experiment 2), the experiments in the Bandelier tuff fracture were modeled using each aperture data set associated with this fracture. Thus, there are four sets of plots for each tracer in Experiments 3, 4, and 5, corresponding to the four aperture data sets. Only one aperture data set (the second PEAK set) was used for the silica experiment because it was obvious that the models would have overpredicted the silica breakthrough curve regardless of which data set was used. Also, the parallel-plate models were not exercised for the silica experiment. However, a plot of the predicted silica breakthrough curve without gravitational settling and without hydrodynamic corrections is shown for comparison with the curve predicted by the model that included these phenomena. The effect of gravitational settling should have been much greater for the silica microspheres than for the CML microspheres because of the much greater density of the former.

The particle diffusivities used in the simulations were $0.0015 \mathrm{~mm}^{2} / \mathrm{sec}$ for iodide, $4.36 \times 10^{-7} \mathrm{~mm}^{2} / \mathrm{sec}$ for the $1-\mu \mathrm{m}$ microspheres, and $1.45 \times 10^{-6} \mathrm{~mm}^{2} / \mathrm{sec}$ for the $0.3-\mu \mathrm{m}$ microspheres. Densities were assumed to be $1.055 \mathrm{~g} / \mathrm{cm}^{3}$ for the CML microspheres and $2.65 \mathrm{~g} / \mathrm{cm}^{3}$ for the silica. The volumetric flow rates in the simulations were matched to the experimental flow rates, which are given in Appendix A. The assumed values of the fracture-dependent parameters in the simulations are given in Table D.1.

The porosities in Table D.1 were measured experimentally (see Section 3.1.1). The values of the other parameters were arbitrarily adjusted to obtain reasonable fits to the experimental breakthrough curves. The predicted breakthrough curves were much more sensitive to the thickness of the colloid exclusion layer and/or the solute stagnant layer when the average aperture was smaller (because these layers then comprised a greater percentage of the fracture volume). Thus, the effect of a $5 \mu \mathrm{m}$ layer in the Bandelier tuff fracture (average aperture $=55 \mu \mathrm{m}$ ) was actually greater than the effect of a $20 \mu \mathrm{m}$ layer in the Bullfrog tuff fracture (average aperture $=555 \mu \mathrm{m}$ ). Whether or not these layer thicknesses were physically realistic could not be determined. Such a determination would require an examination/comparison of the fine-scale features of the fracture surfaces. However, a quick examination of the fracture surfaces under an optical microscope suggested that the assumed layer thicknesses were at least reasonable, if not realistic. 
Table D.1. Parameter Values Used in Simulations for Different Fractures.

\begin{tabular}{|c|c|c|c|}
\hline Parameter & Bandelier Tuff & Tram Tuff & Bullfrog Tuff \\
\hline Porosity & 0.089 & 0.192 & 0.208 \\
\hline $\begin{array}{c}\text { Transition Layer } \\
\text { Thickness }\end{array}$ & $10 \mu \mathrm{m}$ & $10 \mu \mathrm{m}$ & $10 \mu \mathrm{m}$ \\
\hline $\begin{array}{c}\text { Colloid Exclusion } \\
\text { Layer Thickness }\end{array}$ & $5 \mu \mathrm{m}$ & $10 \mu \mathrm{m}$ & $10 \mu \mathrm{m}$ \\
\hline $\begin{array}{c}\text { Solute Stagnant } \\
\text { Layer Thickness }\end{array}$ & $5 \mu \mathrm{m}$ & $10 \mu \mathrm{m}$ & $20 \mu \mathrm{m}$ \\
\hline
\end{tabular}




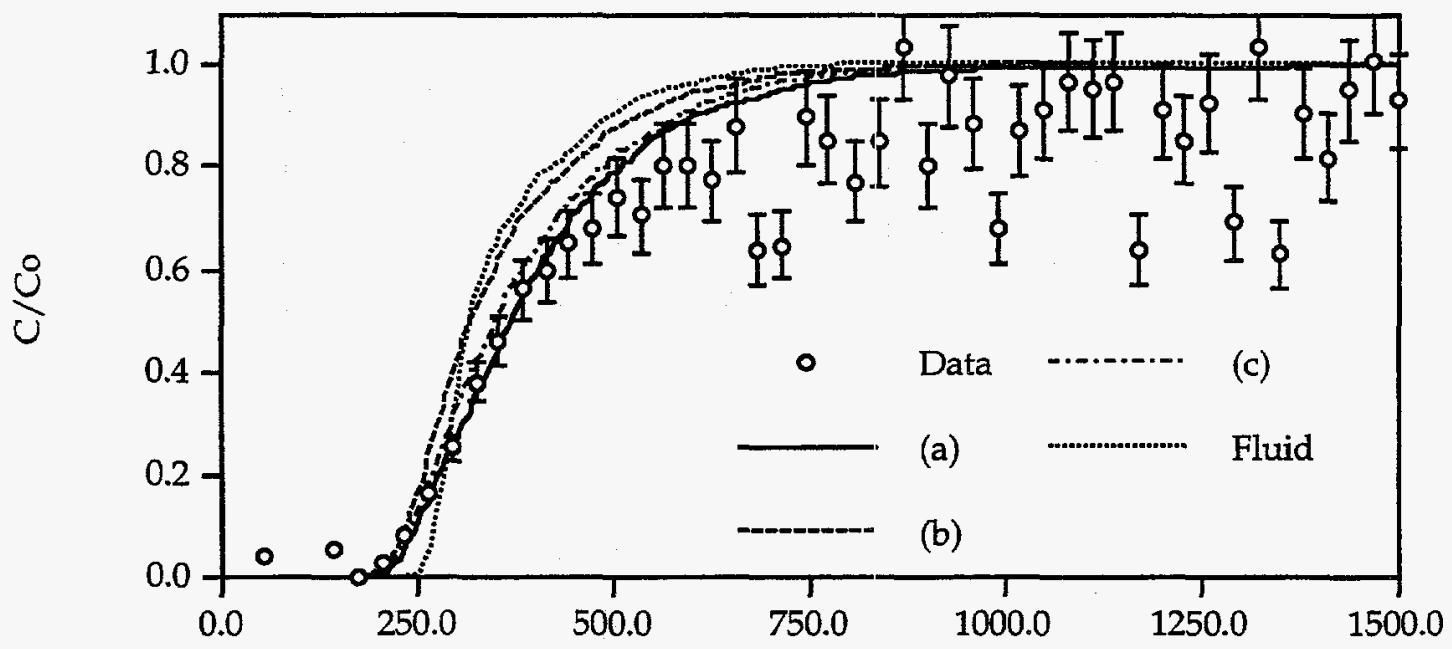

Time, sec

Figure D.1. Predicted and experimental breakthrough curves of $0.3-\mu \mathrm{m}$ silica microspheres in Experiment 2. See p. D-2 and Table D.1 for description of models and parameter values, respectively, corresponding to plot legend. Curve (c) is not a parallel-plate model; it is same as (a) but with gravitational settling and hydrodynamic corrections ignored in the calculations.

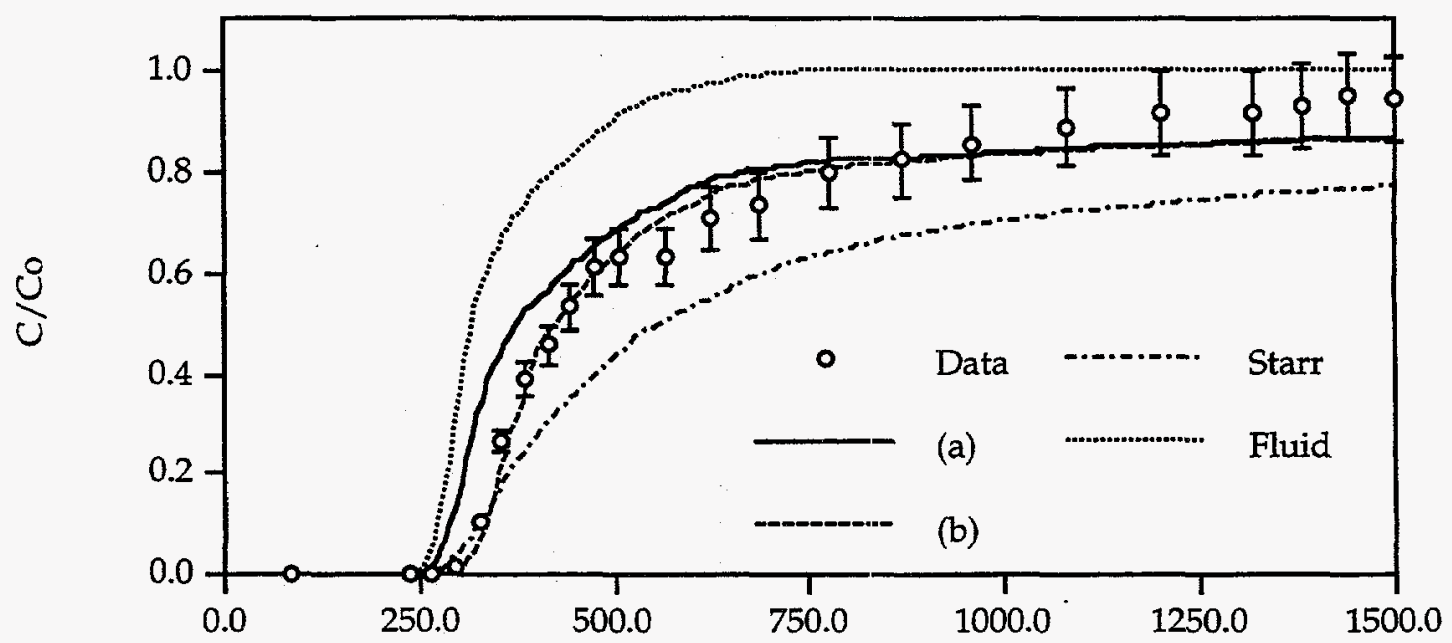

Time, sec

Figure D.2. Predicted and experimental breakthrough curves of iodide in Experiment 2. See p. D-2 and Table D.1 for description of models and parameter values, respectively, corresponding to plot legend (parallel-plate model was not exercised). 


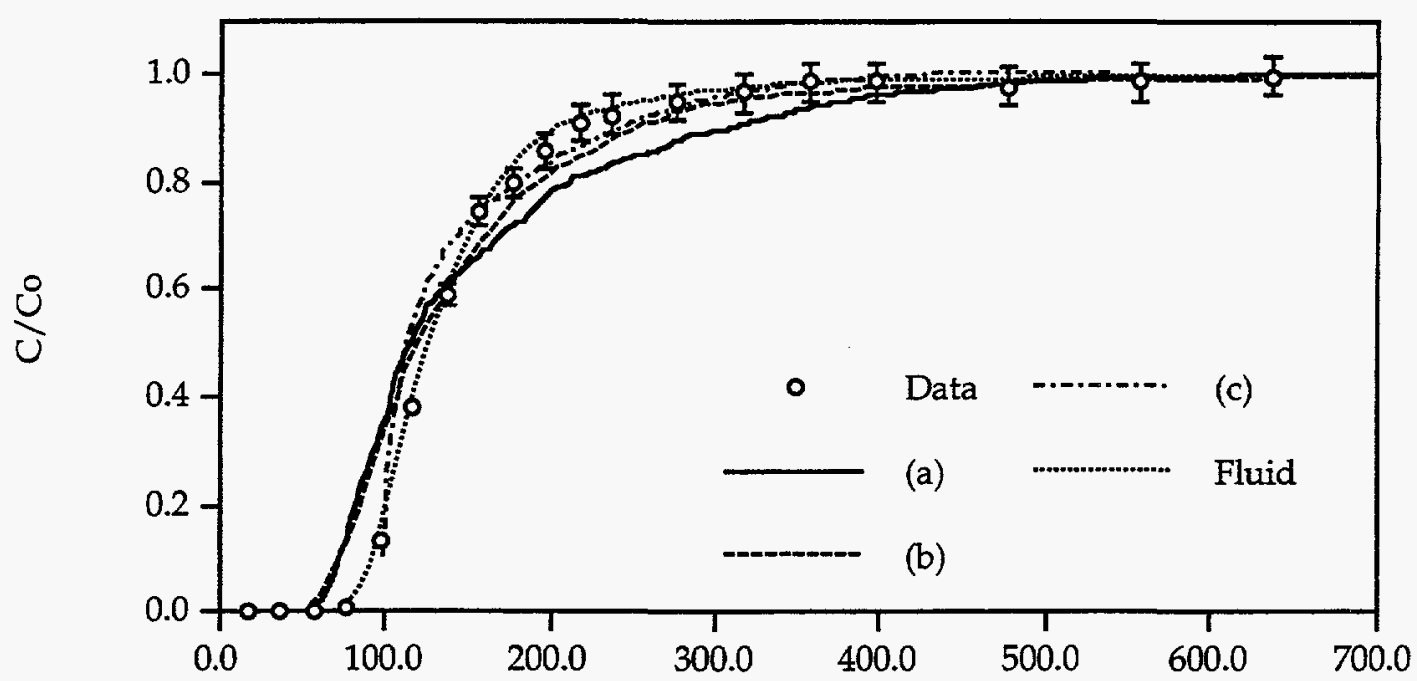

Time, sec

Figure D.3. Predicted and experimental breakthrough curves of 1- $\mu \mathrm{m} \mathrm{CML}$ microspheres in Experiment 3 (Bandelier tuff) using the laser profile aperture data set for the model predictions. See p. D-2 and Table D.1 for description of models and parameter values, respectively, corresponding to plot legend.

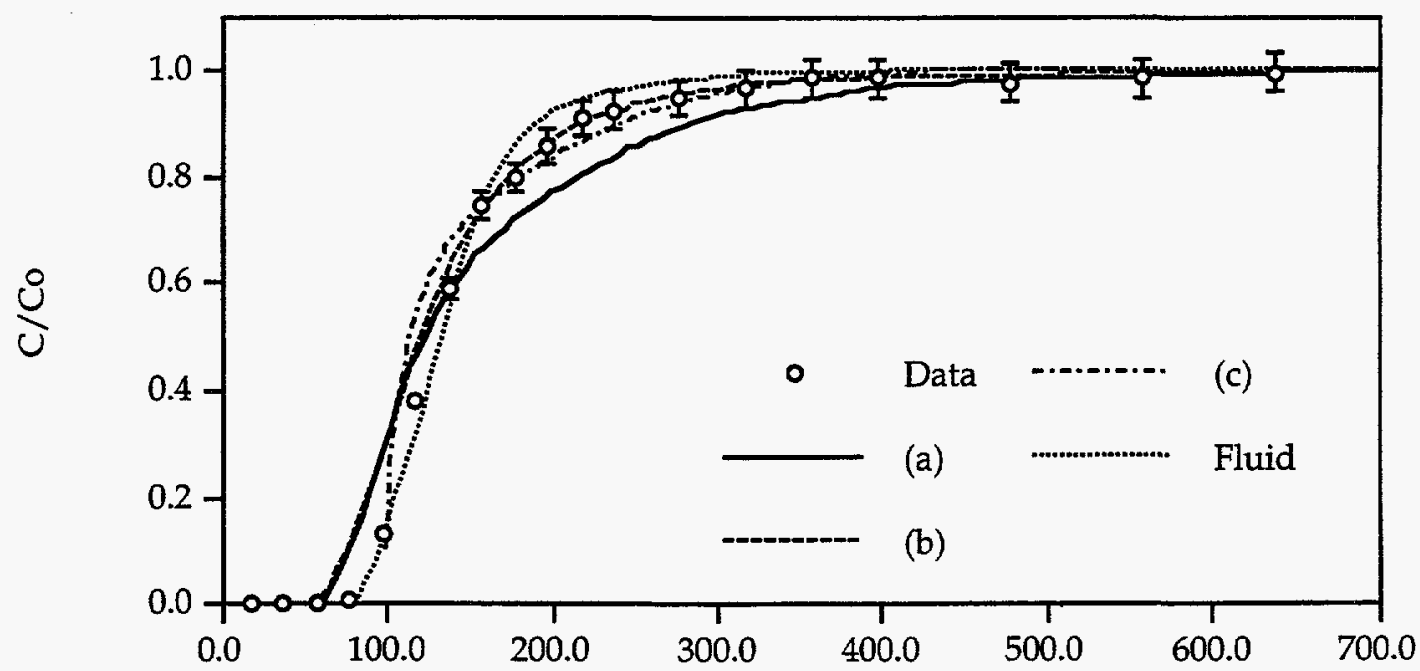

Time, sec

Figure D.4. Predicted and experimental breakthrough curves of 1- $\mu \mathrm{m}$ CML microspheres in Experiment 3 (Bandelier tuff) using the RTV aperture data set for the model predictions. See p. D-2 and Table D.1 for description of models and parameter values, respectively, corresponding to plot legend. 


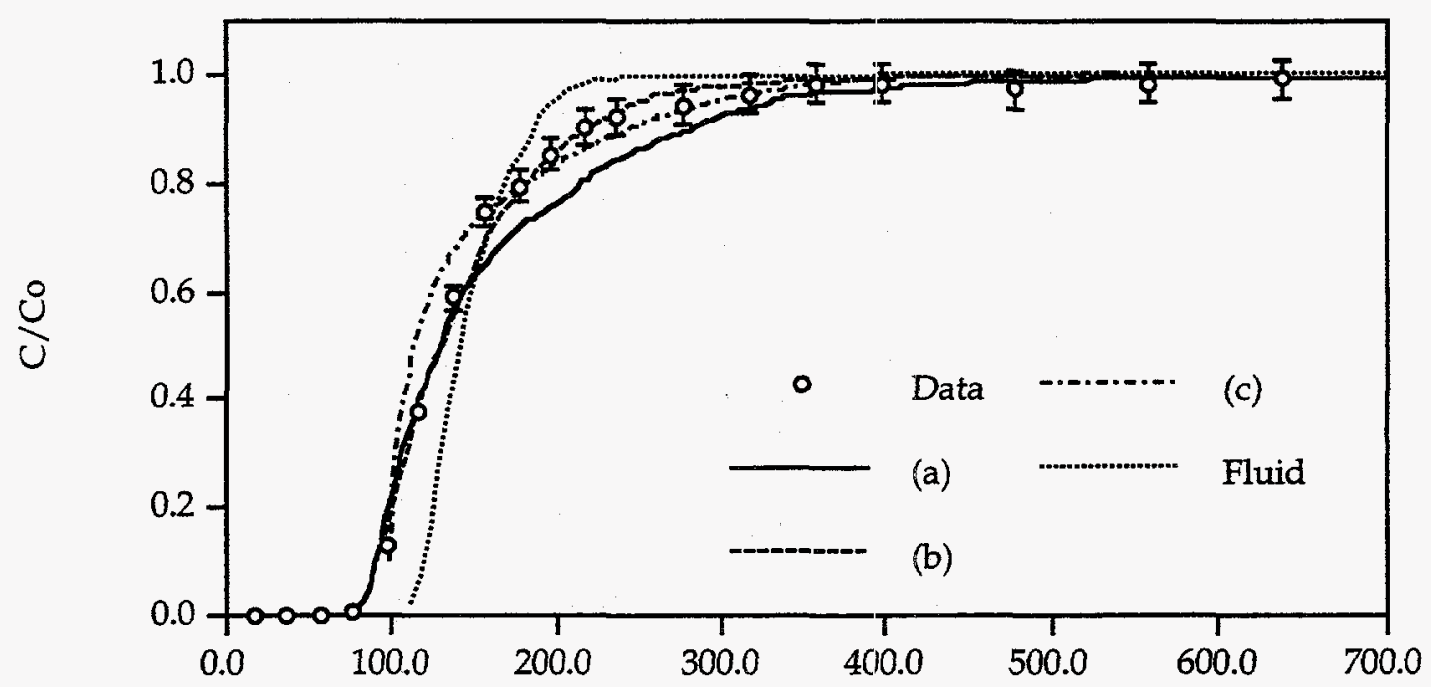

Time, sec

Figure D.5. Predicted and experimental breakthrough curves of $1-\mu \mathrm{m} C M L$ microspheres in Experiment 3 (Bandelier tuff) using the 1st PEAK aperture data set for the model predictions. See p. D-2 and Table D.1 for description of models and parameter values, respectively, corresponding to plot legend.

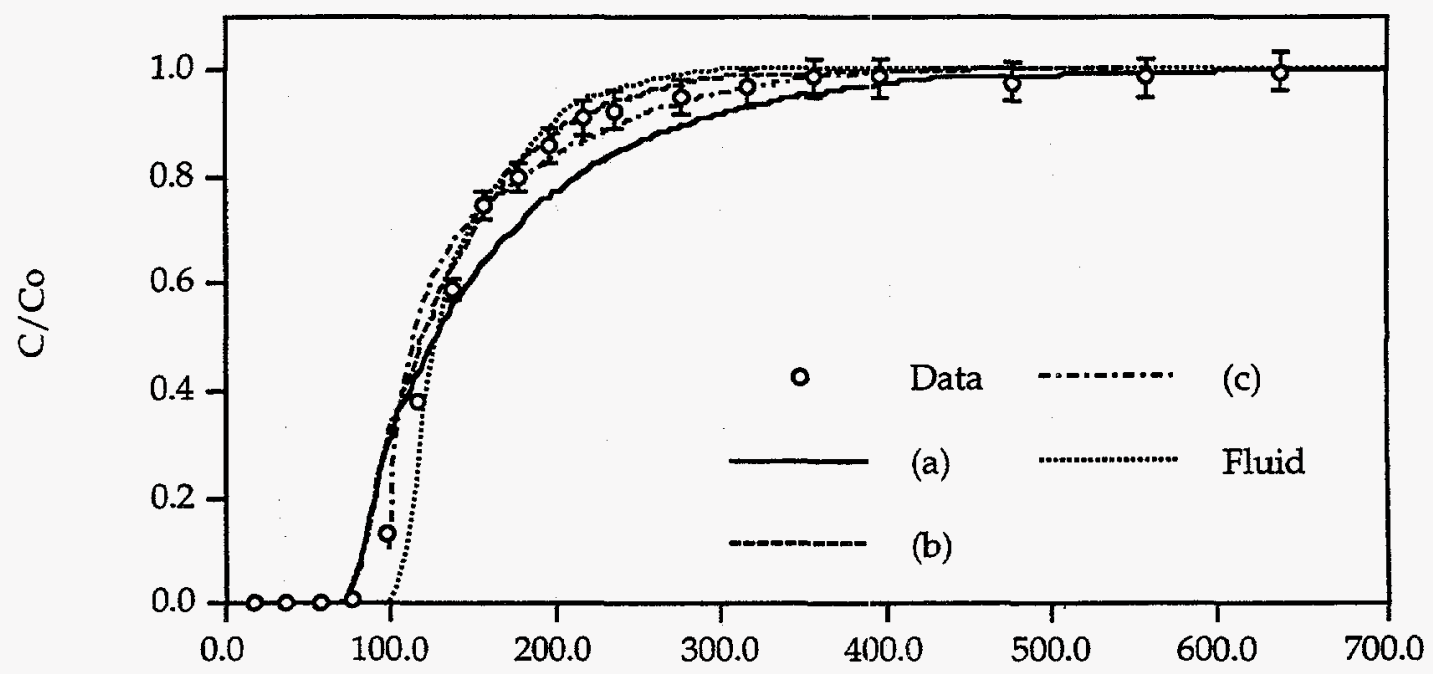

Time, sec

Figure D.6. Predicted and experimental breakthrough curves of 1- $\mu \mathrm{m} C \mathrm{CML}$ microspheres in Experiment 3 (Bandelier tuff) using the 2nd PEAK aperture data set for the model predictions. See p. D-2 and Table D.1 for description of models and parameter values, respectively, corresponding to plot legend. 


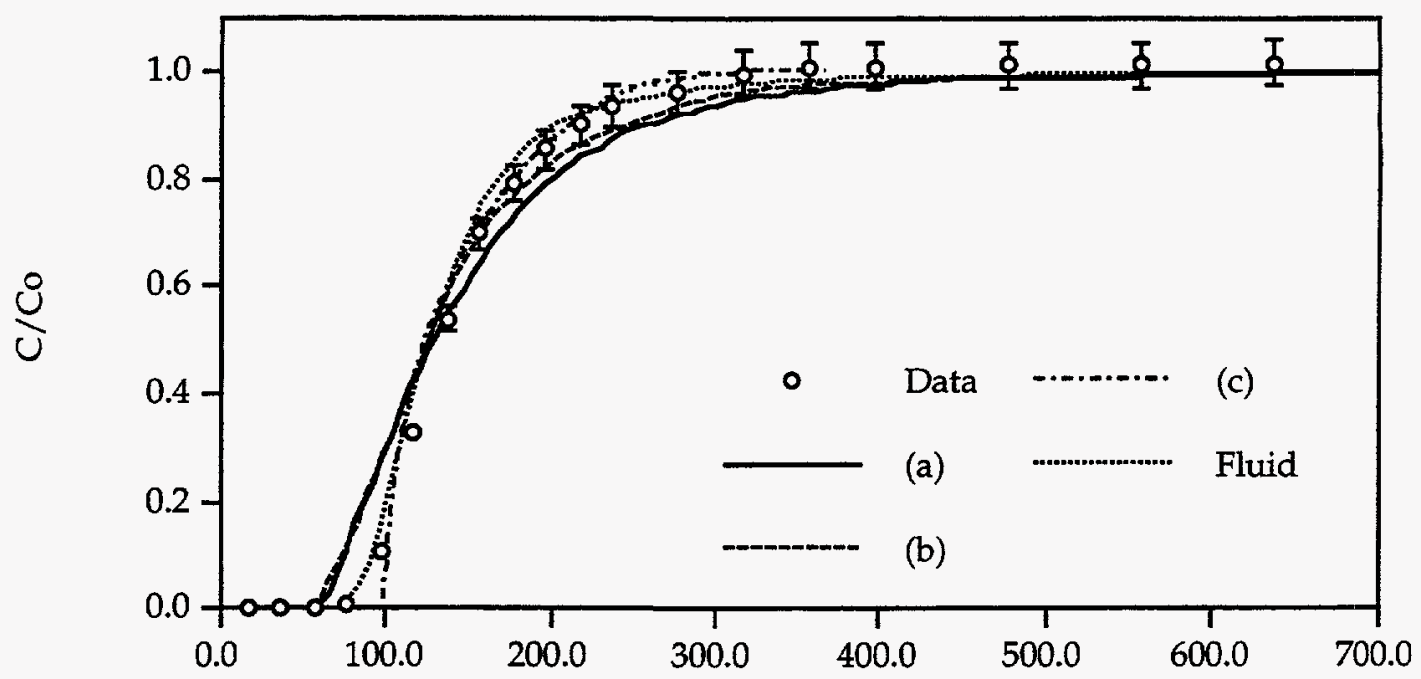

Time, sec

Figure D.7. Predicted and experimental breakthrough curves of $0.3-\mu \mathrm{m} \mathrm{CML}$ microspheres in Experiment 3 (Bandelier tuff) using the laser profile aperture data set for the model predictions. See p. D-2 and Table D.1 for description of models and parameter values, respectively, corresponding to plot legend.

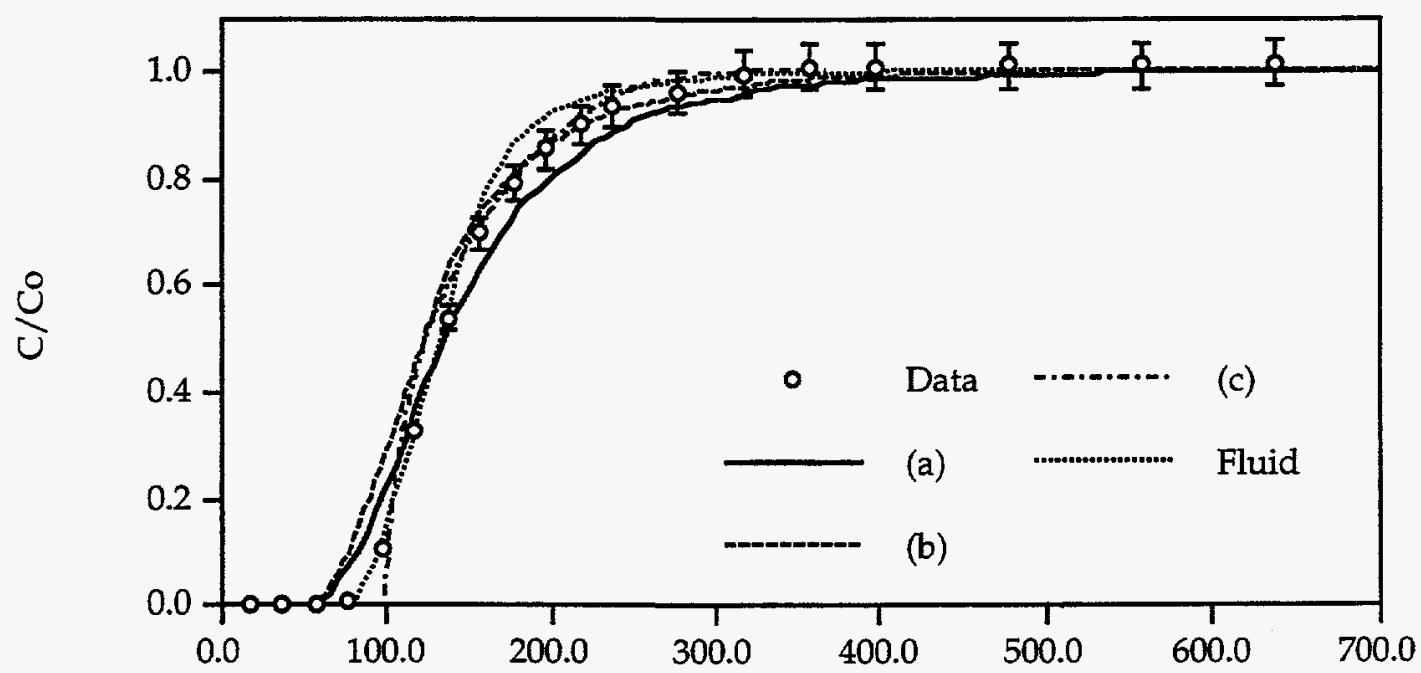

Time, sec

Figure D.8. Predicted and experimental breakthrough curves of $0.3-\mu \mathrm{m}$ CML microspheres in Experiment 3 (Bandelier tuff) using the RTV aperture data set for the model predictions. See p. D-2 and Table D.1 for description of models and parameter values, respectively, corresponding to plot legend. 


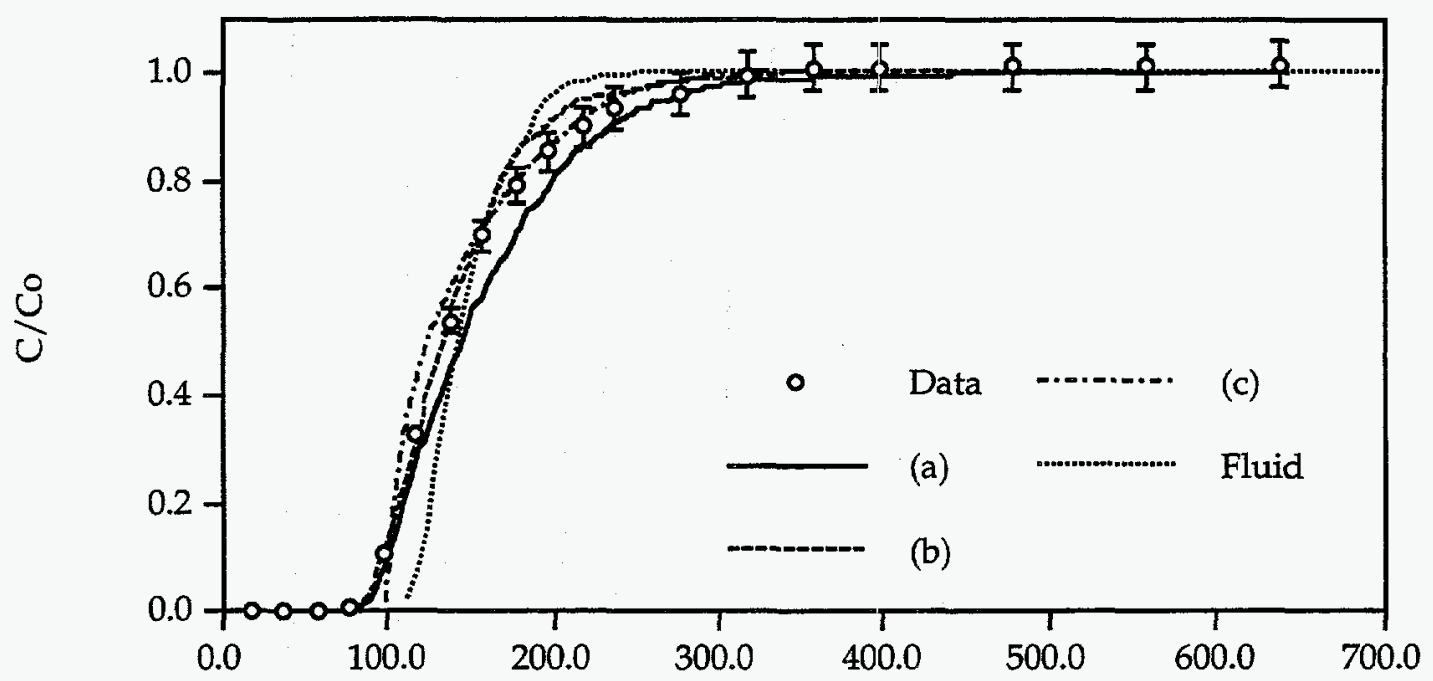

Time, sec

Figure D.9. Predicted and experimental breakthrough curves of $0.3-\mu \mathrm{m} \mathrm{CML}$ microspheres in Experiment 3 (Bandelier tuff) using the 1st PEAK aperture data set for the model predictions. See p. D-2 and Table D.1 for description of models and parameter values, respectively, corresponding to plot legend.

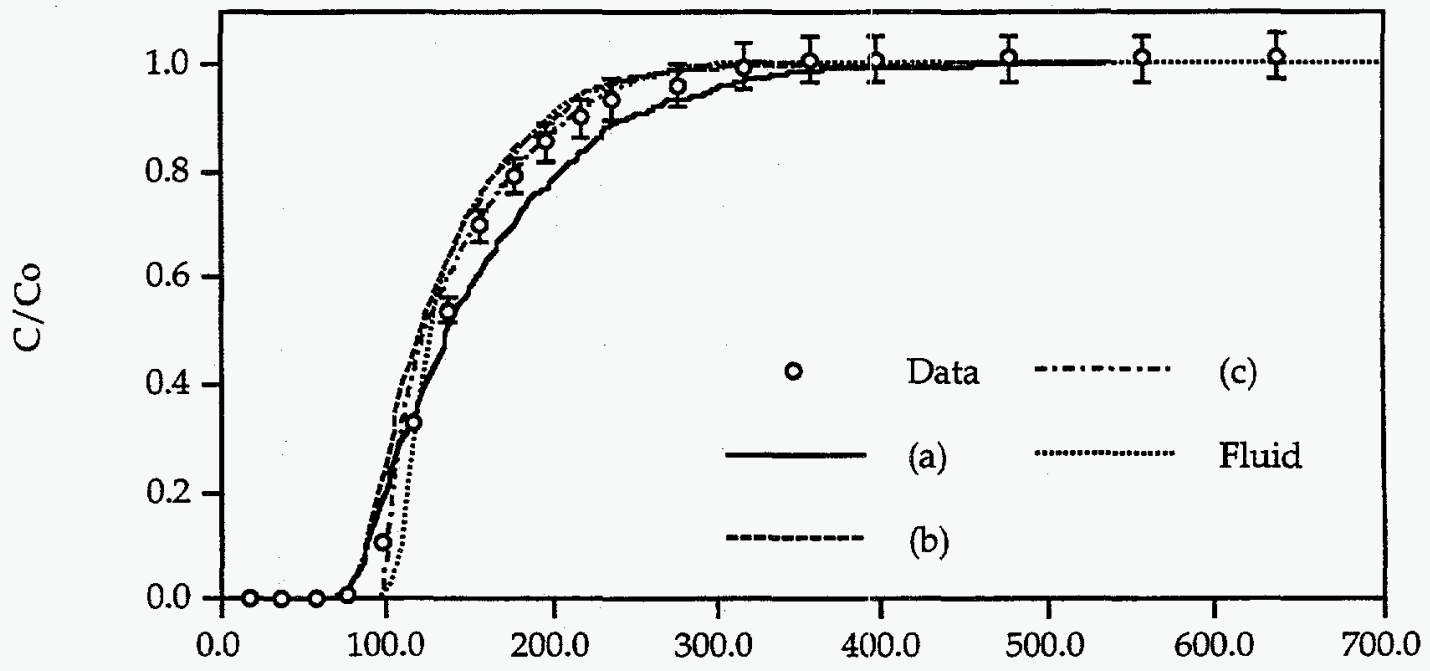

Time, sec

Figure D.10. Predicted and experimental breakthrough curves of $0.3-\mu \mathrm{m}$ CML microspheres in Experiment 3 (Bandelier tuff) using the 1st PEAK aperture data set for the model predictions. See p. D-2. and Table D.1 for description of models and parameter values, respectively, corresponding to plot legend. 


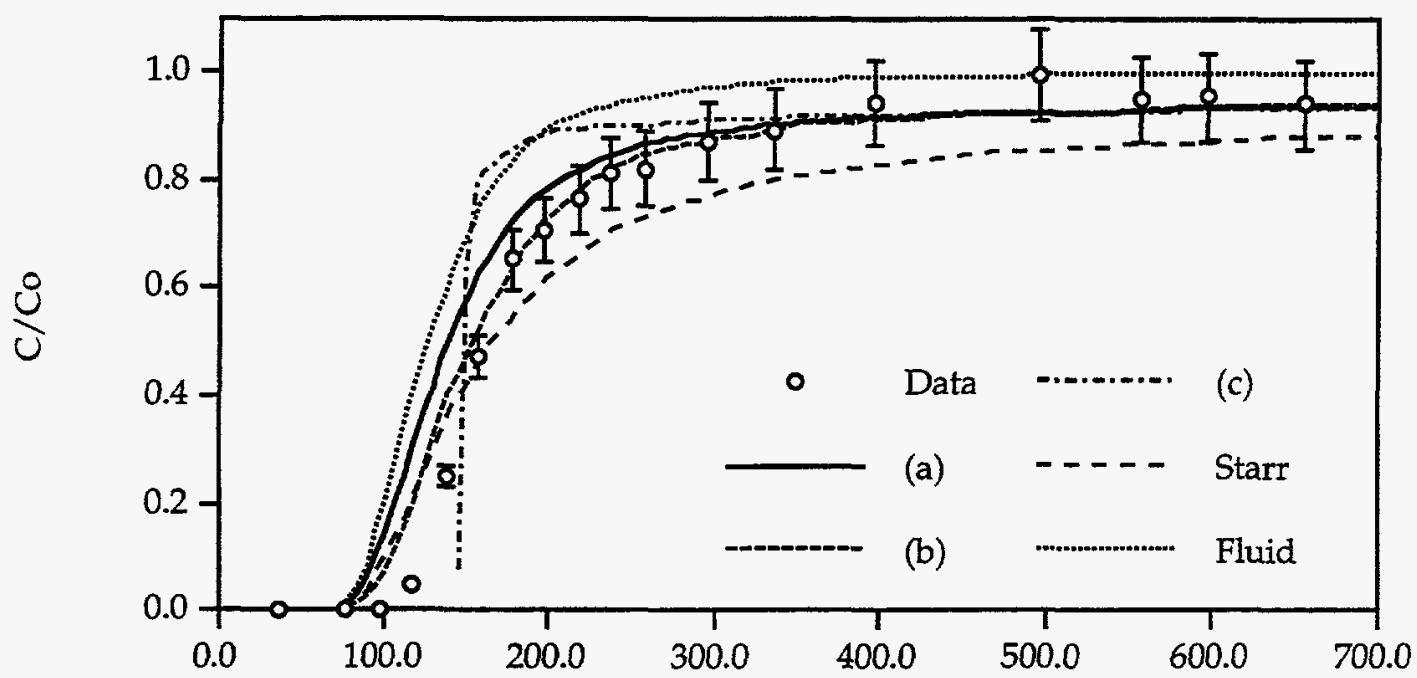

Time, sec

Figure D.11. Predicted and experimental breakthrough curves of iodide in Experiment 3 (Bandelier tuff) using the laser profile aperture data set for the model predictions. See p. D-2 and Table D.1 for description of models and parameter values, respectively, corresponding to plot legend.

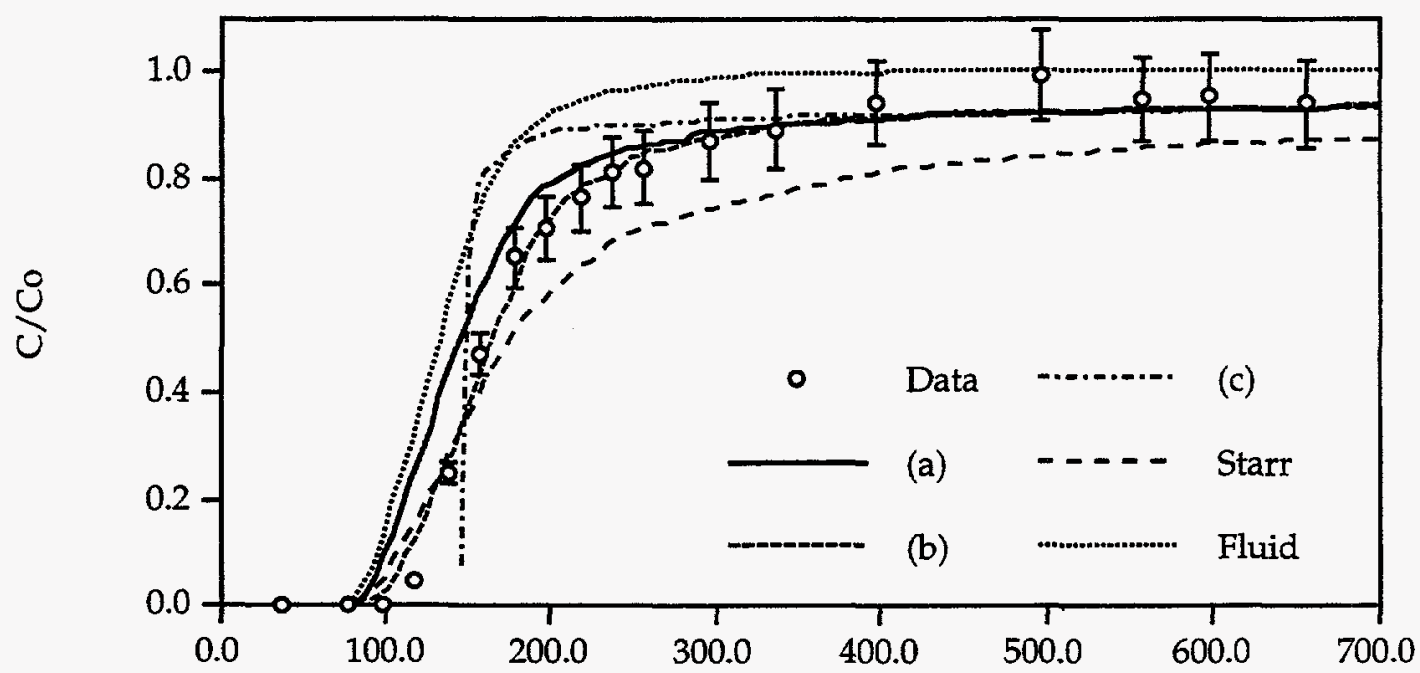

Time, sec

Figure D.12. Predicted and experimental breakthrough curves of iodide in Experiment 3 (Bandelier tuff) using the RTV aperture data set for the model predictions. See p. D-2 and Table D.1 for description of models and parameter values, respectively, corresponding to plot legend. 


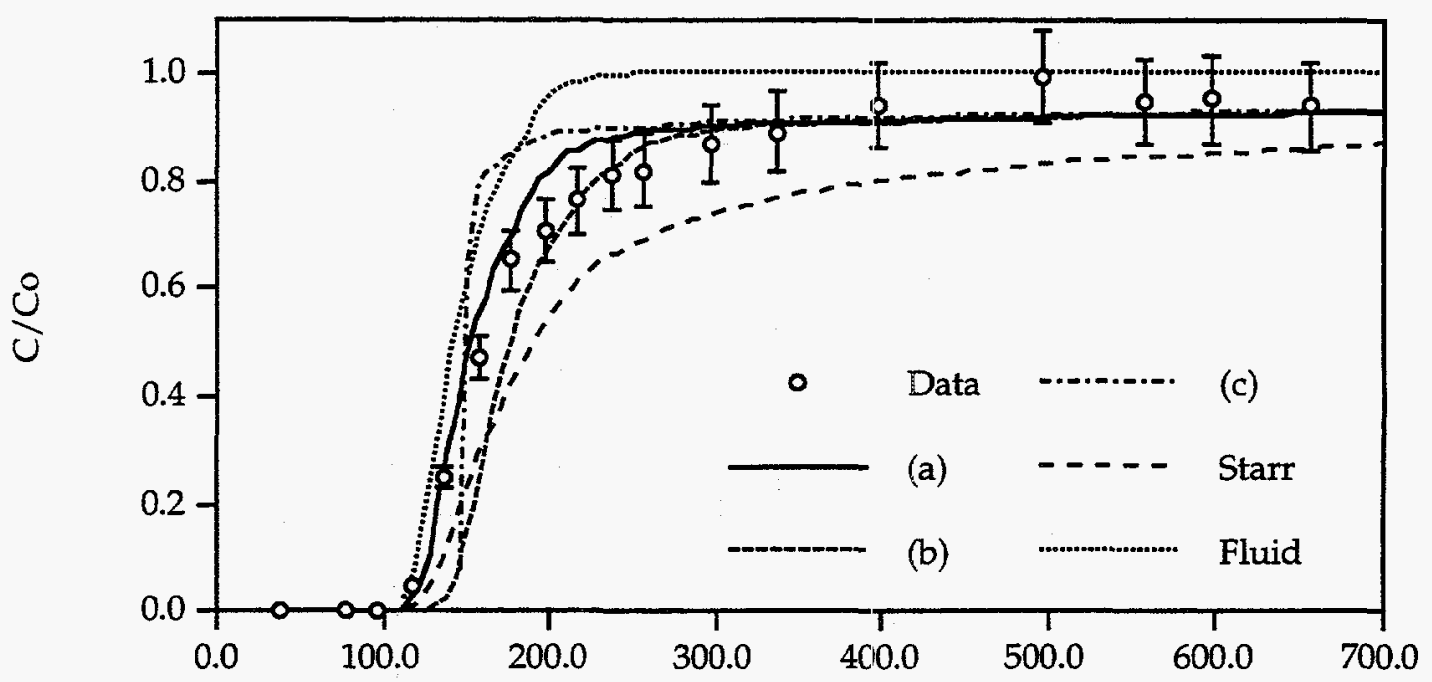

Time, sec

Figure D.13. Predicted and experimental breakthrough curves of iodide in Experiment 3 (Bandelier tuff) using the 1st PEAK aperture data set for the model predictions. See p. D-2 and Table D.1 for description of models and parameter values, respectively, corresponding to plot legend.

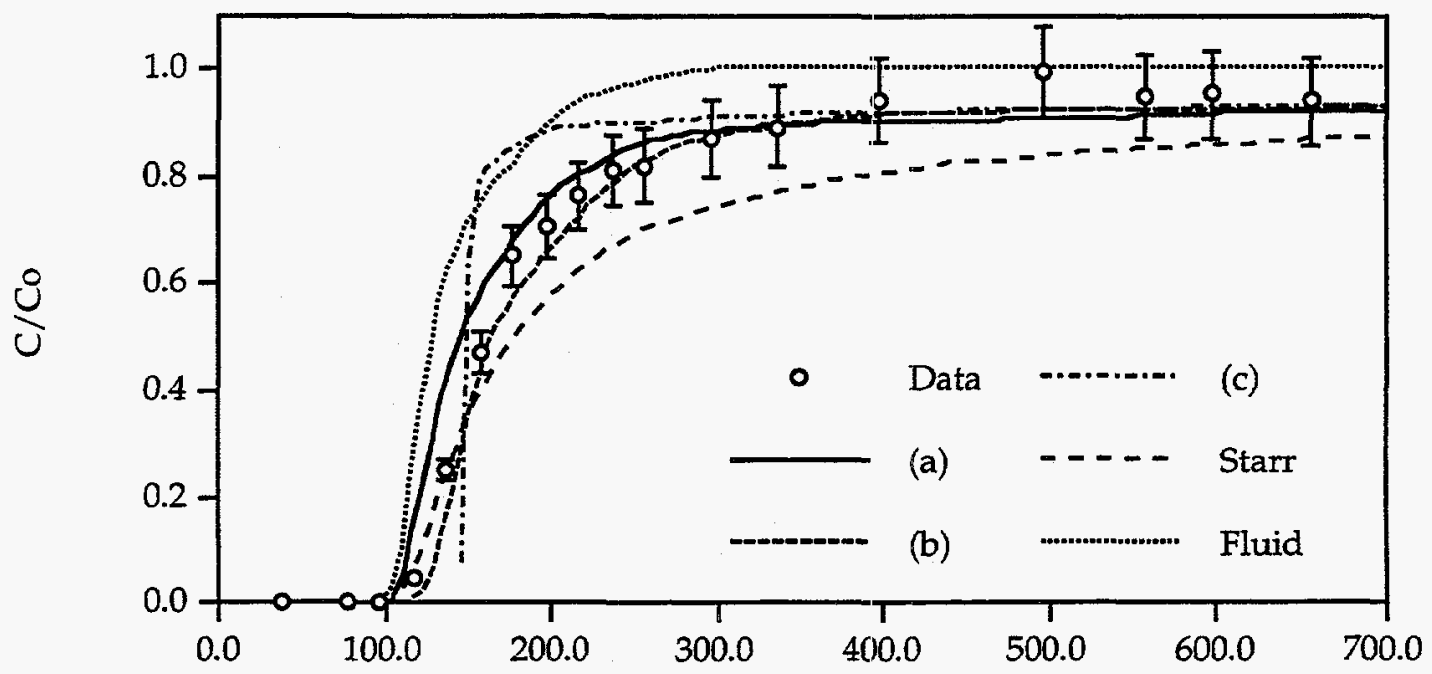

Time, sec

Figure D.14. Predicted and experimental breakthrough curves of iodide in

Experiment 3 (Bandelier tuff) using the 2nd PEAK aperture data set for the model predictions. See p. D-2 and Table D.1 for description of models and parameter values, respectively, corresponding to plot legend. 


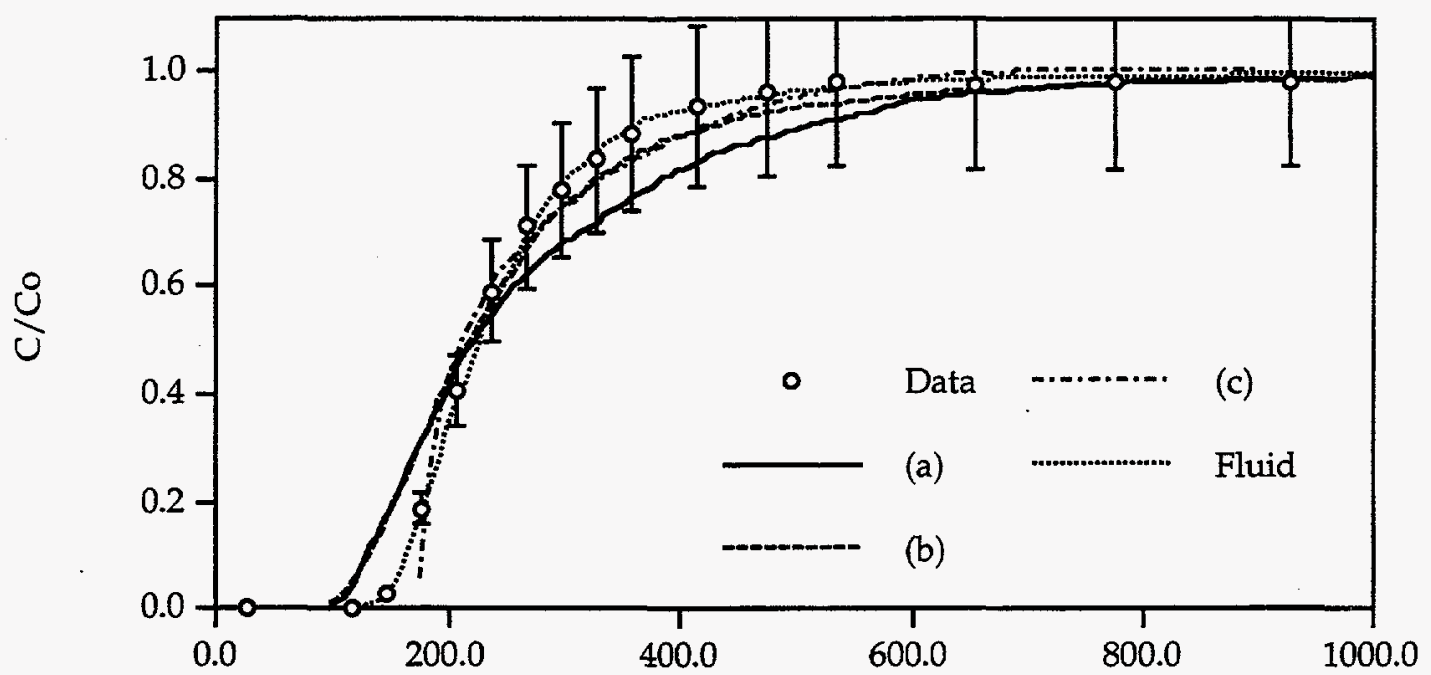

Time, sec

Figure D.15. Predicted and experimental breakthrough curves of 1- $\mu \mathrm{m}$ CML microspheres in Experiment 4 (Bandelier tuff) using the laser profile aperture data set for the model predictions. See p. D-2 and Table D.1 for description of models and parameter values, respectively, corresponding to plot legend.

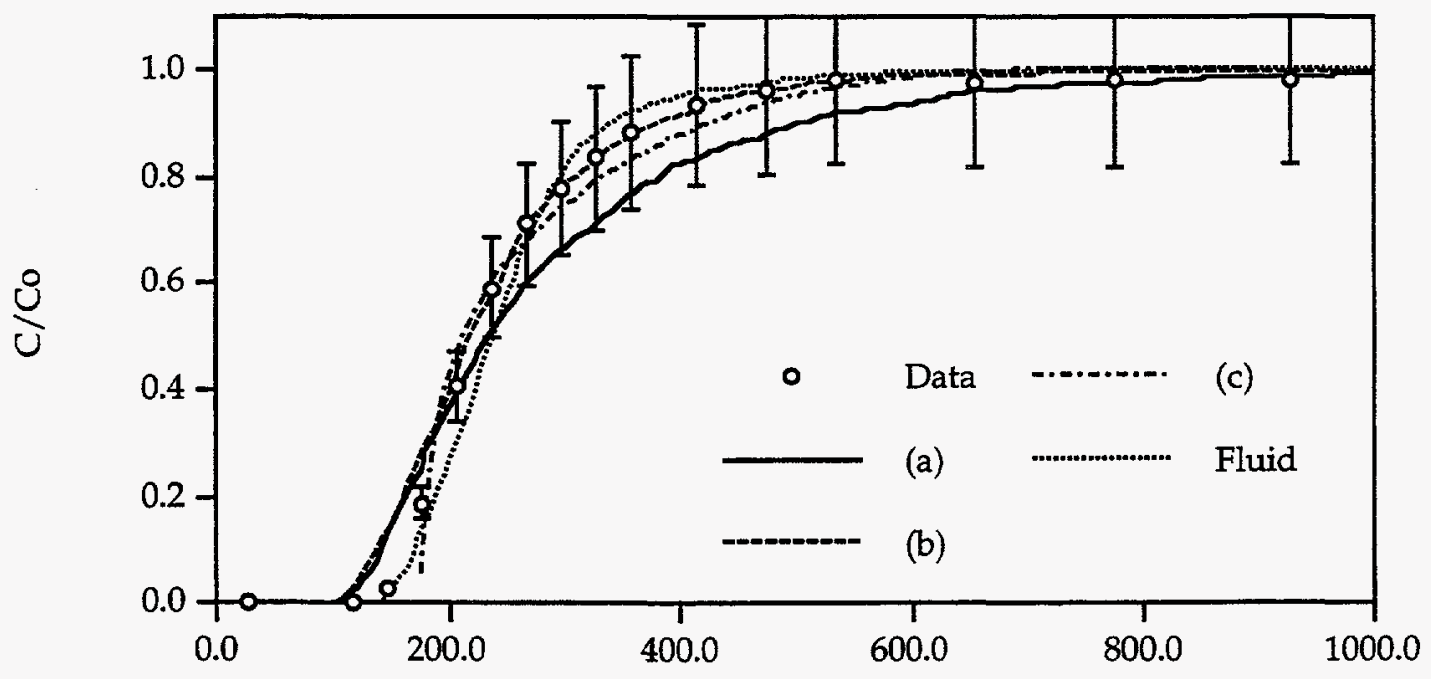

Time, sec

Figure D.16. Predicted and experimental breakthrough curves of $1-\mu \mathrm{m} \mathrm{CML}$ microspheres in Experiment 4 (Bandelier tuff) using the RTV aperture data set for the model predictions. See p. D-2 and Table D.1 for description of models and parameter values, respectively, corresponding to plot legend. 


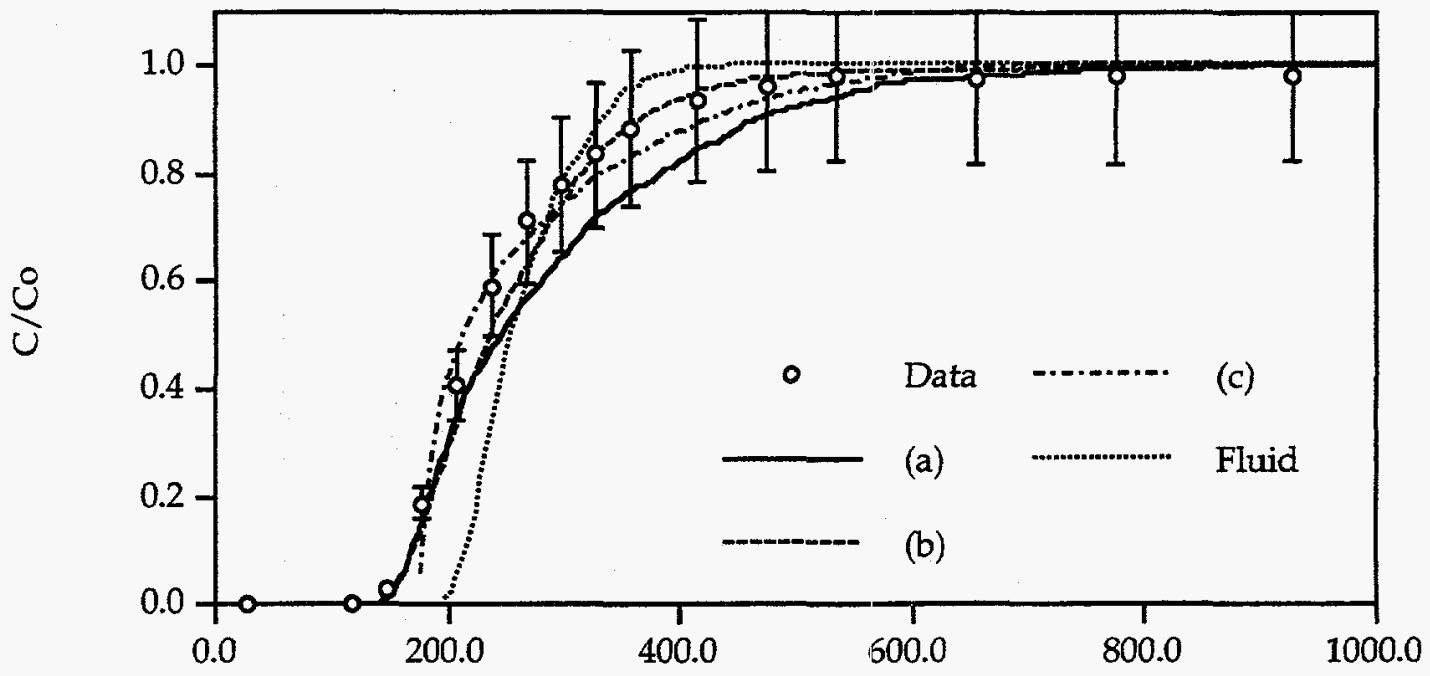

Time, sec

Figure D.17. Predicted and experimental breakthurough curves of $1-\mu \mathrm{m}$ CML microspheres in Experiment 4 (Bandelier tuff) using the 1st PEAK aperture data set for the model predictions. See p. D-2 and Table D.1 for description of models and parameter values, respectively, corresponding to plot legend.

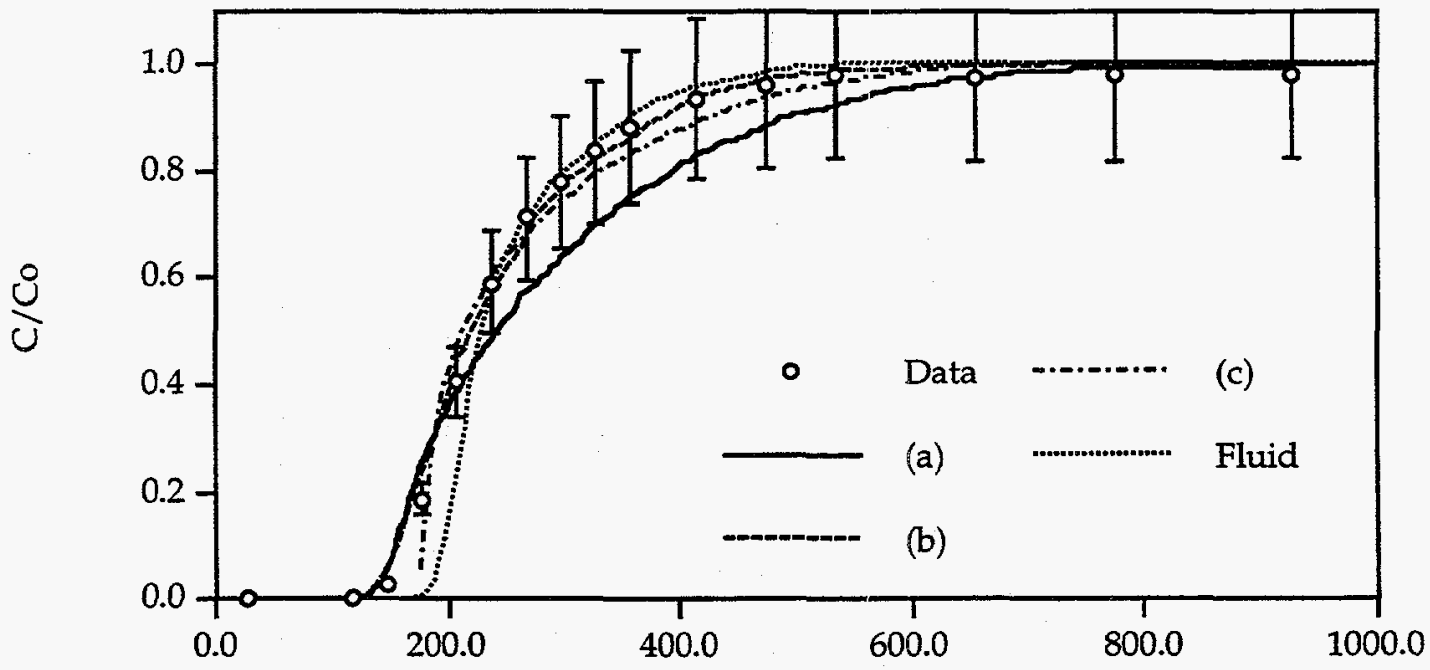

Time, sec

Figure D.18. Predicted and experimental breakthrough curves of 1- $\mu \mathrm{m}$ CML microspheres in Experiment 4 (Bandelier tuff) using the 2nd PEAK aperture data set for the model predictions. See p. D-2. and Table D.1 for description of models and parameter values, respectively, corresponding to plot legend. 


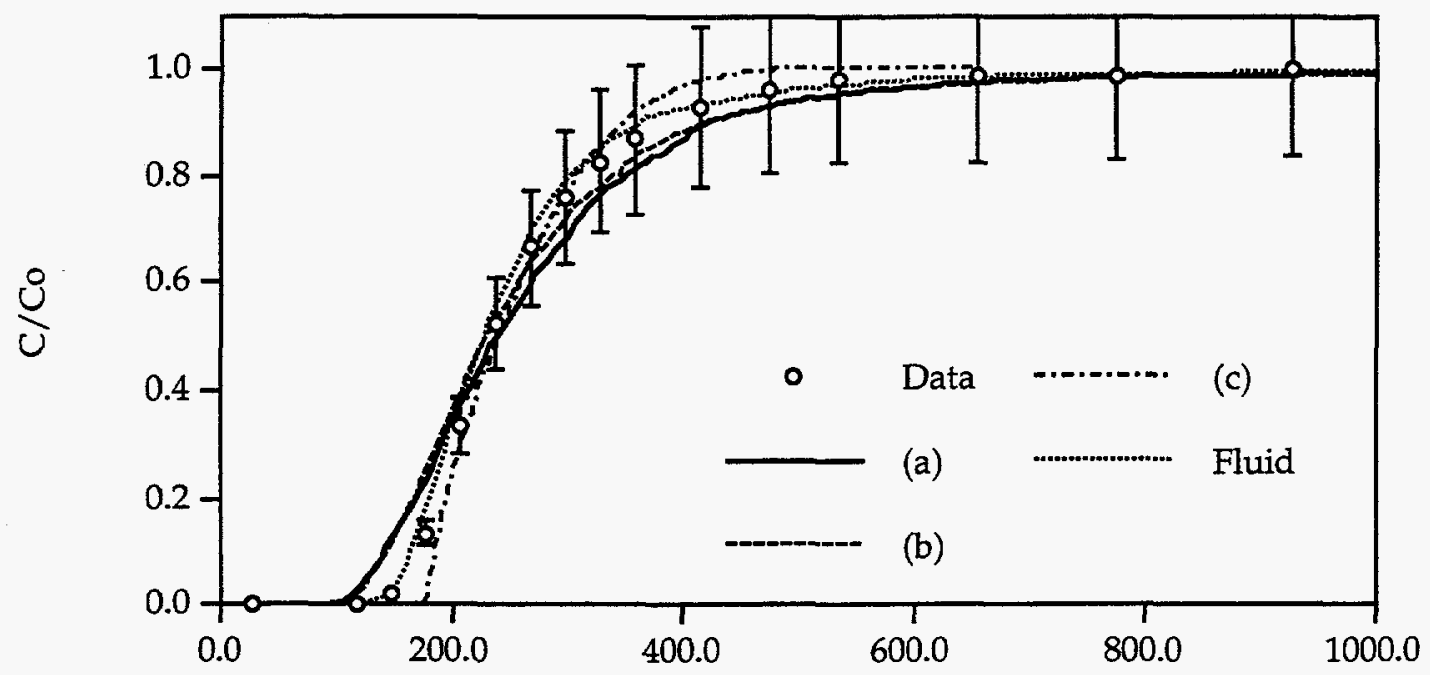

Time, sec

Figure D.19. Predicted and experimental breakthrough curves of $0.3-\mu \mathrm{m} C M L$ microspheres in Experiment 4 (Bandelier tuff) using the laser profile aperture data set for the model predictions. See p. D-2 and Table D.1 for description of models and parameter values, respectively, corresponding to plot legend.

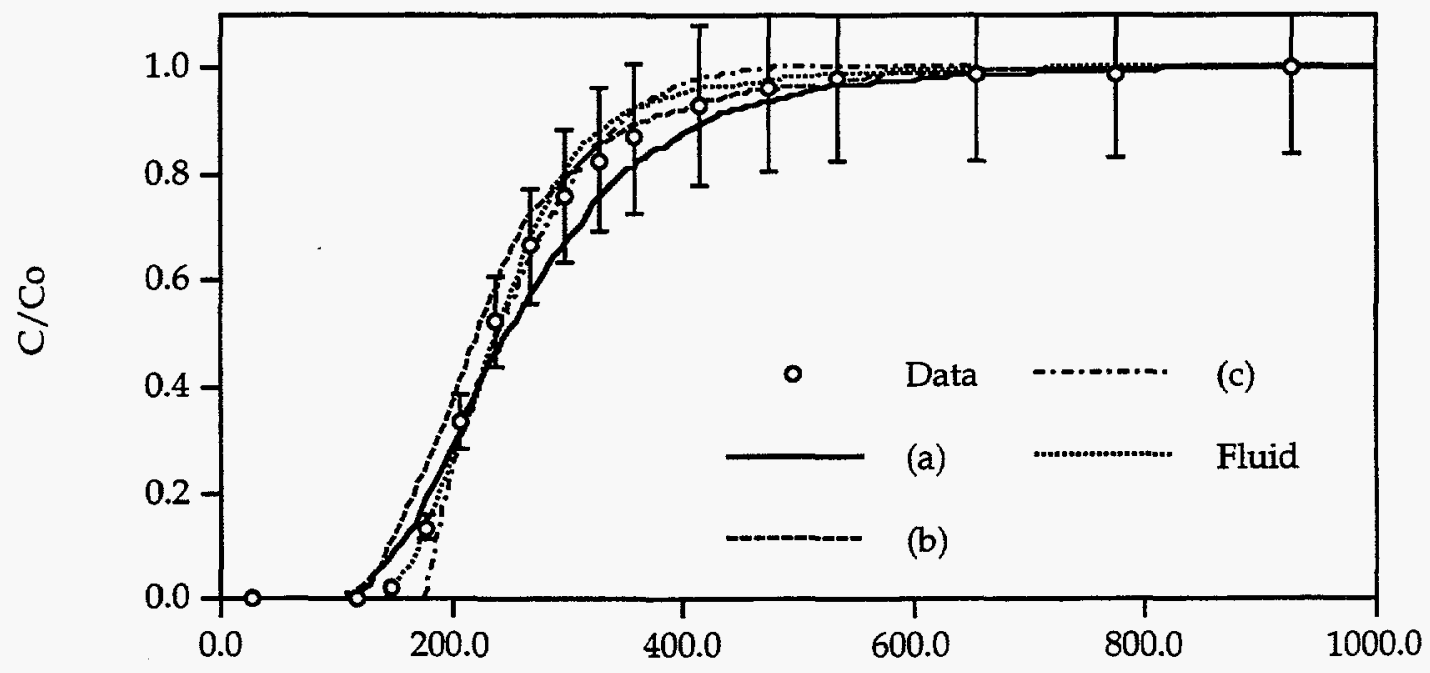

Time, sec

Figure D.20. Predicted and experimental breakthrough curves of $0.3-\mu \mathrm{m} C M L$ microspheres in Experiment 4 (Bandelier tuff) using the RTV aperture data set for the model predictions. See p. D-2 and Table D.1 for description of models and parameter values, respectively, corresponding to plot legend. 


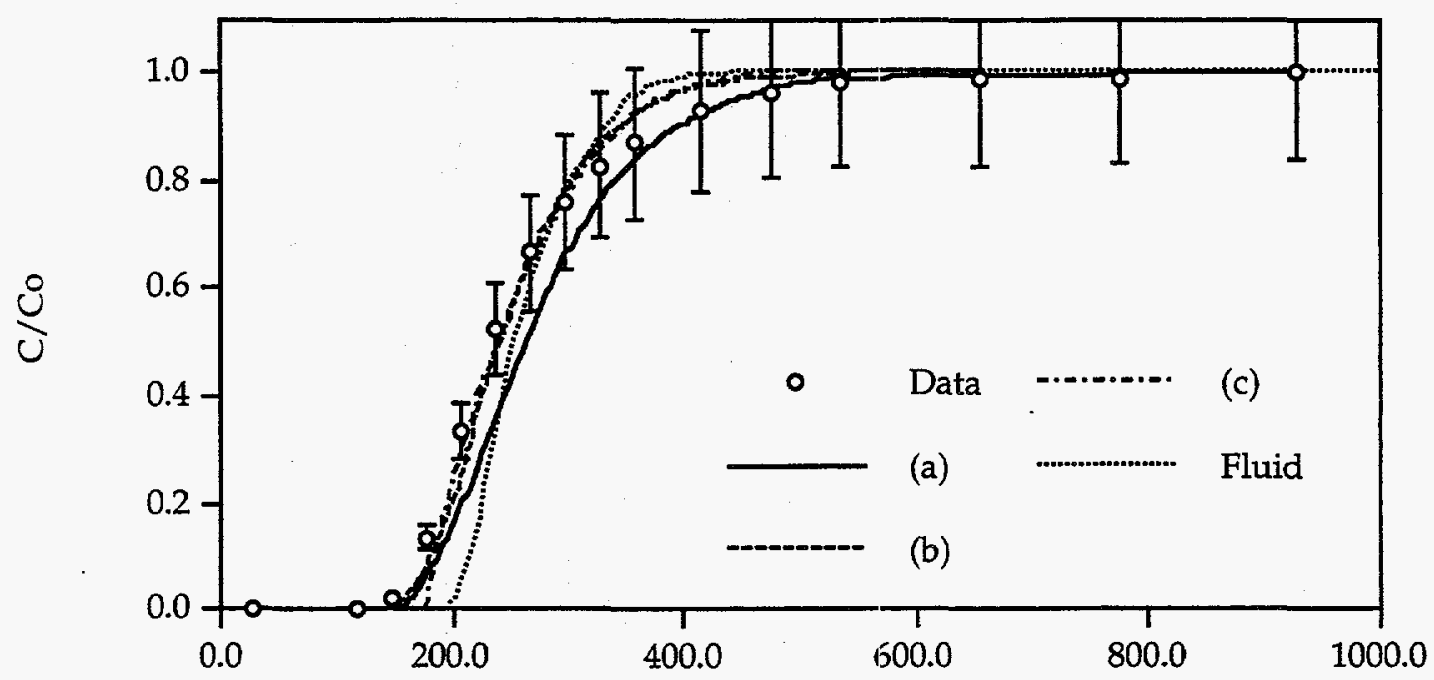

Time, sec

Figure D.21. Predicted and experimental breakthrough curves of $0.3-\mu \mathrm{m} \mathrm{CML}$ microspheres in Experiment 4 (Bandelier tuff) using the 1st PEAK aperture data set for the model predictions. See p. D-2 and Table D.1 for description of models and parameter values, respectively, corresponding to plot legend.

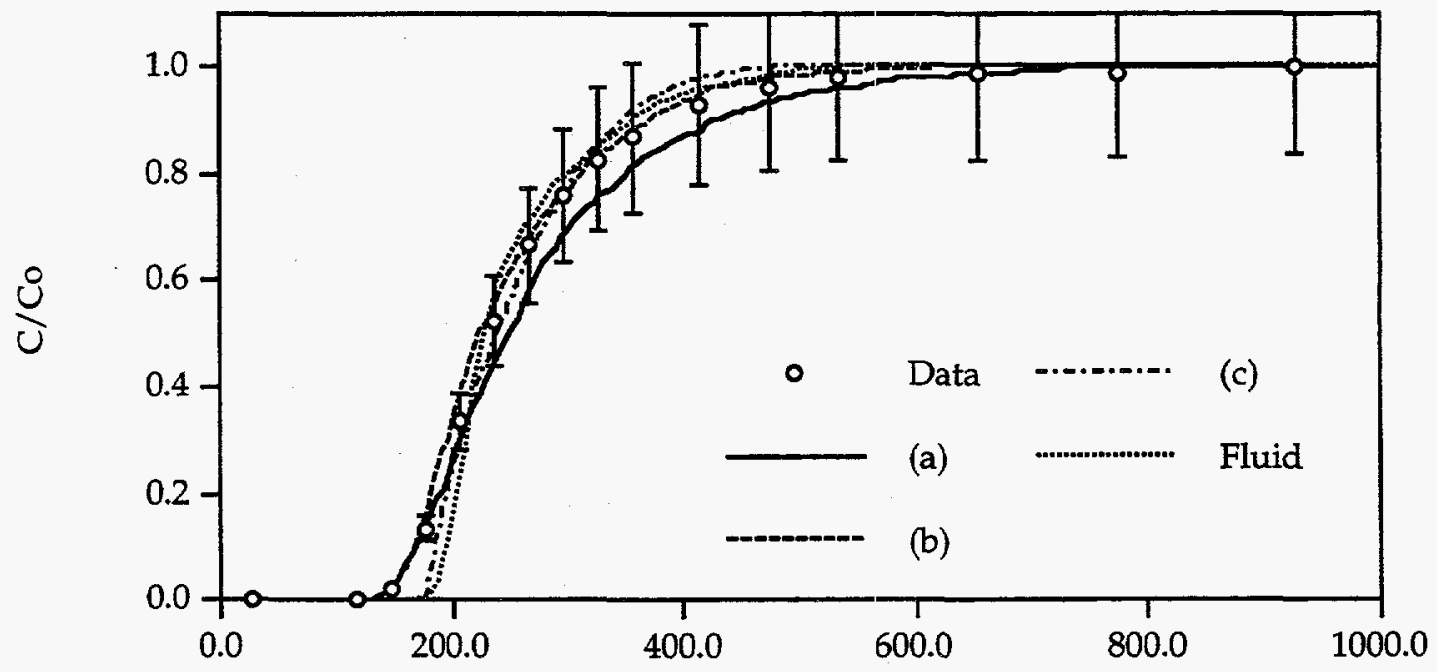

Time, sec

Figure D.22. Predicted and experimental breakthrough curves of $0.3-\mu \mathrm{m}$ CML microspheres in Experiment 4 (Bandelier tuff) using the 2nd PEAK aperture data set for the model predictions. See p. D-2 and Table D.1 for description of models and parameter values, respectively, corresponding to plot legend. 


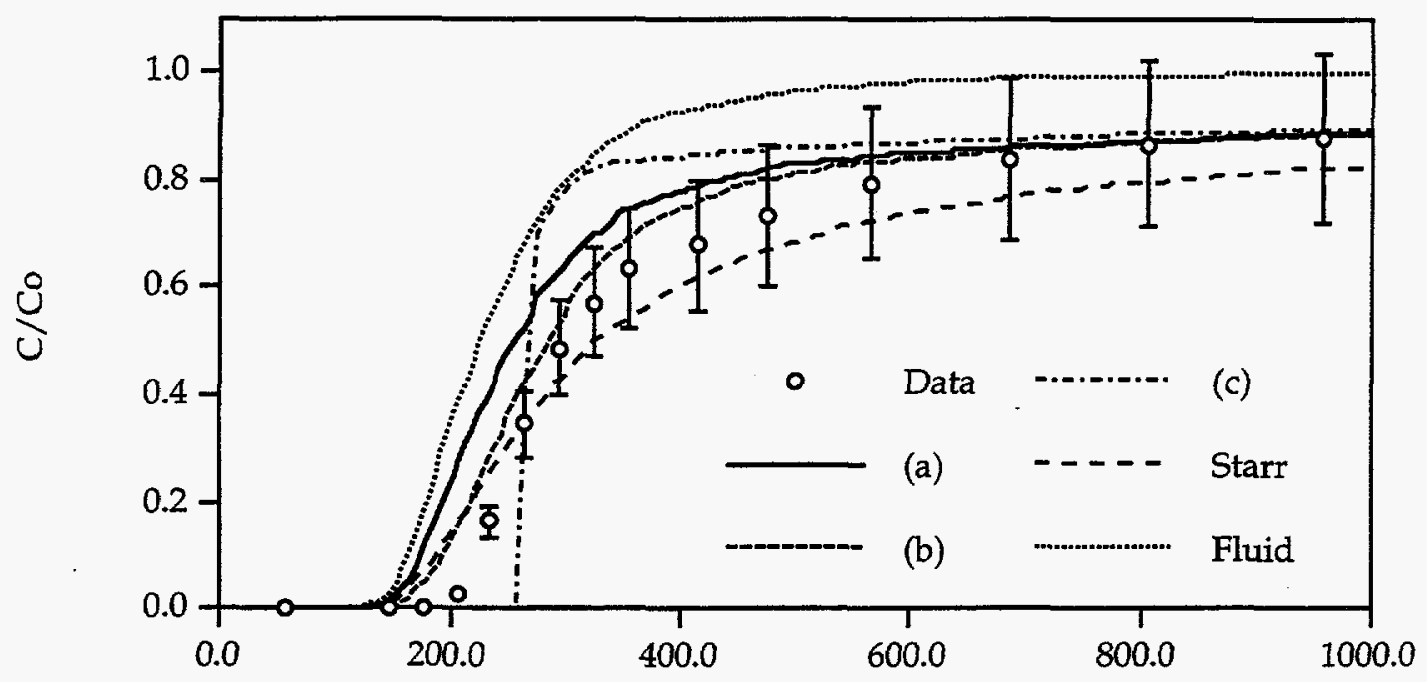

Time, sec

Figure D.23. Predicted and experimental breakthrough curves of iodide in Experiment 4 (Bandelier tuff) using the laser profile aperture data set for the model predictions. See p. D-2 and Table D.1 for description of models and parameter values, respectively, corresponding to plot legend.

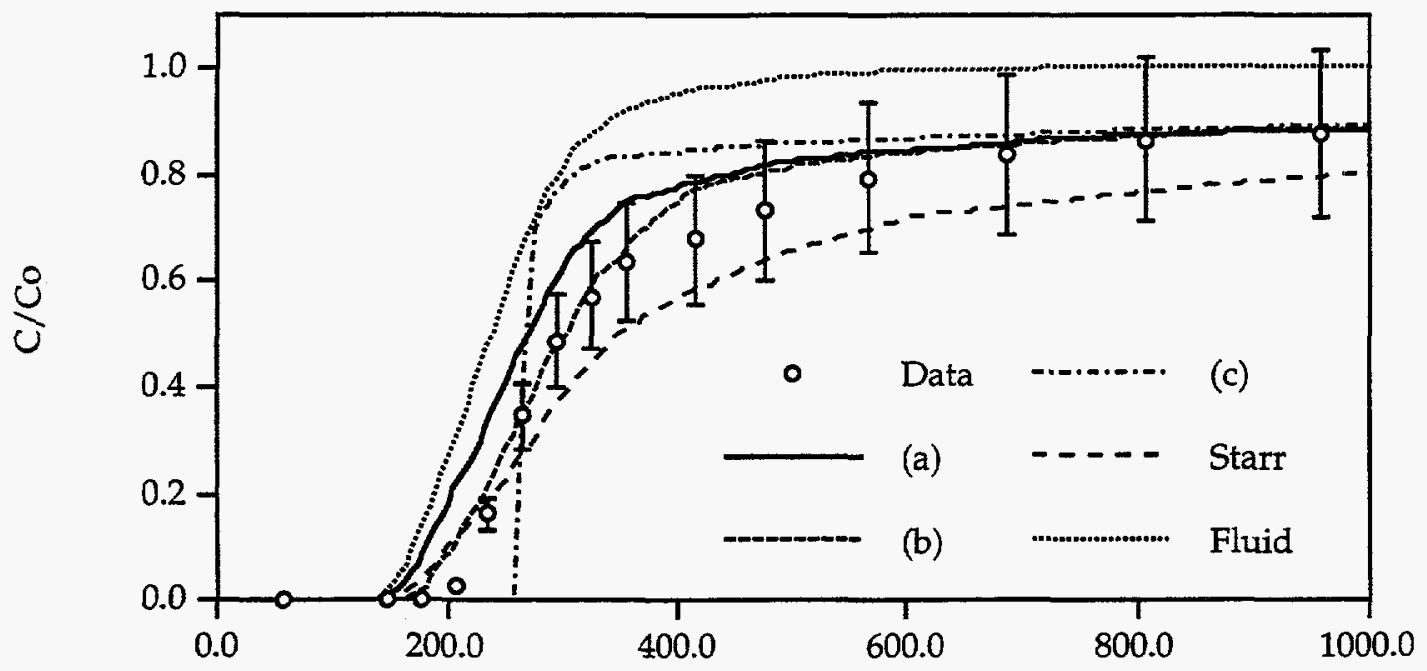

Time, sec

Figure D.24. Predicted and experimental breakthrough curves of iodide in Experiment 4 (Bandelier tuff) using the RTV aperture data set for the model predictions. See p. D-2 and Table D.1 for description of models and parameter values, respectively, corresponding to plot legend. 


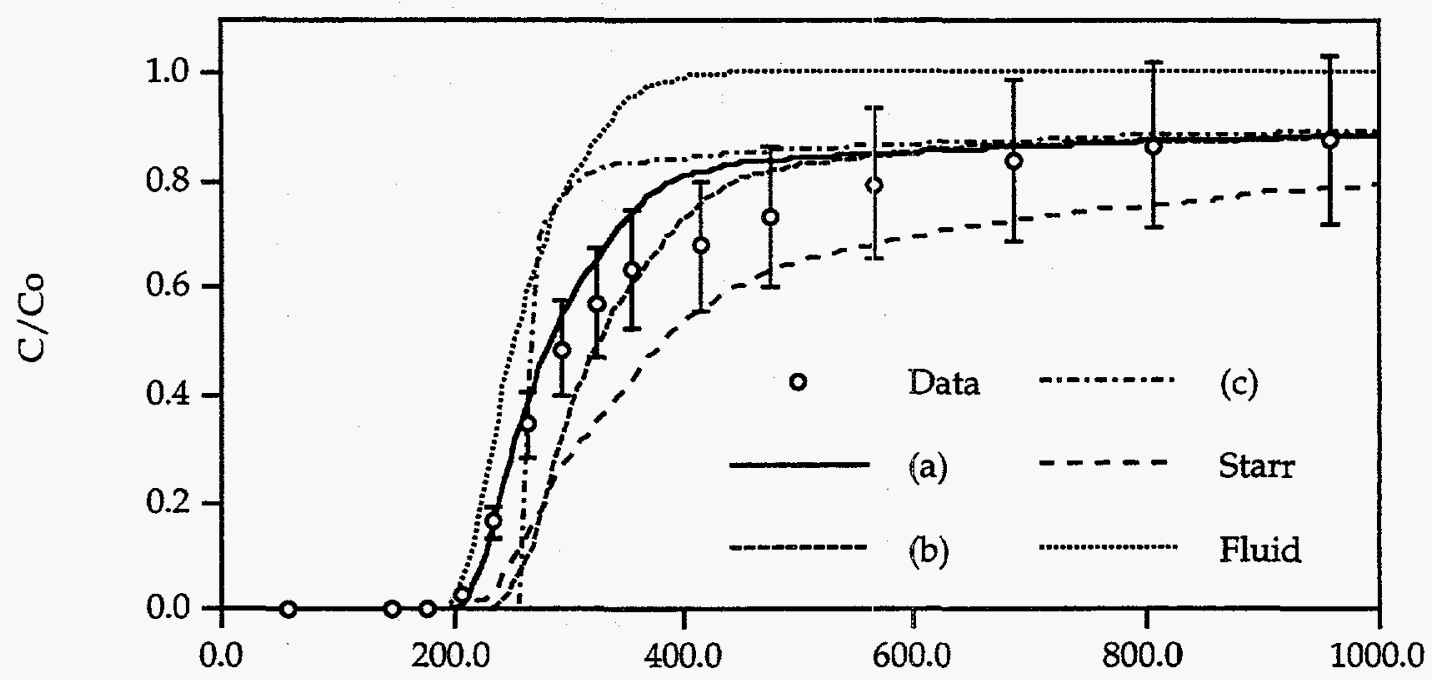

Time, sec

Figure D.25. Predicted and experimental breakthrough curves of iodide in Experiment 4 (Bandelier tuff) using the 1st PEAK aperture data set for the model predictions. See p. D-2 and Table D.1 for description of models and parameter values, respectively, corresponding to plot legend.

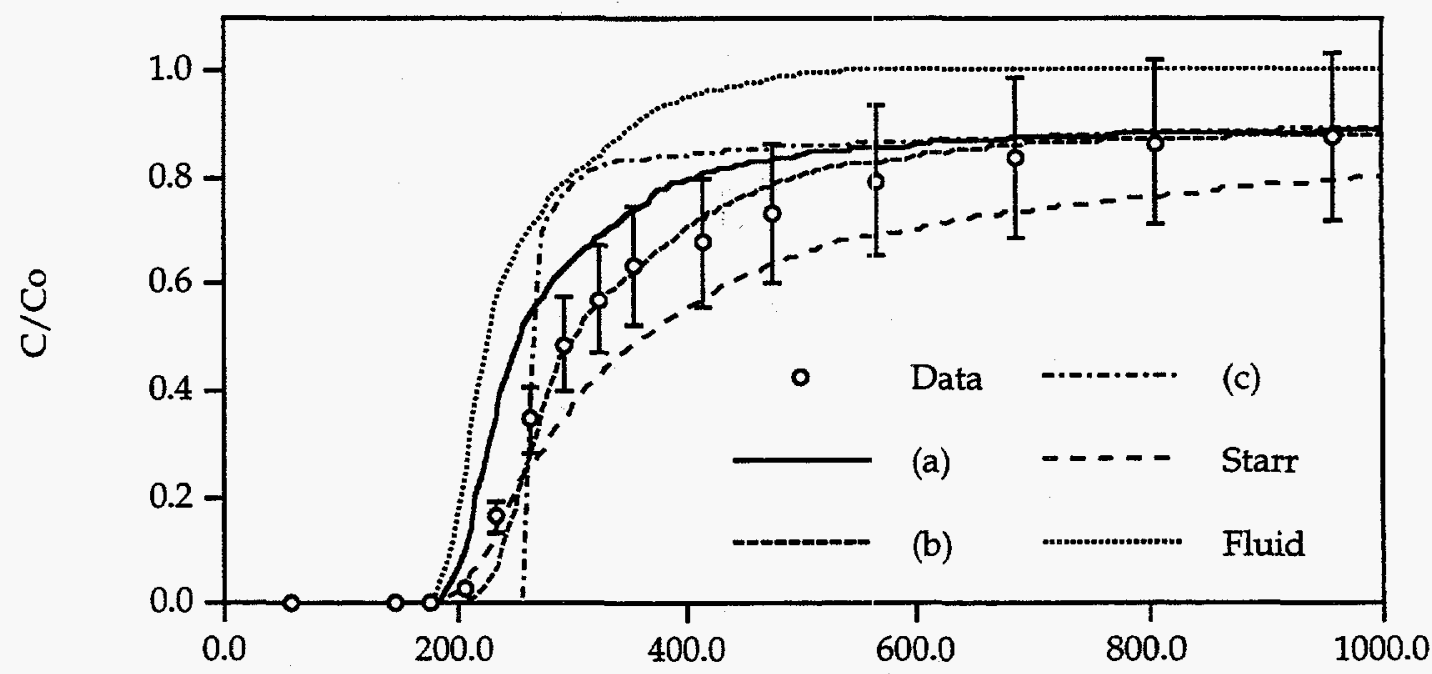

Time, sec

Figure D.26. Predicted and experimental breakthrough curves of iodide in Experiment 4 (Bandelier tuff) using the 2nd PEAK aperture data set for the model predictions. See p. D-2 and Table D.1 for description of models and parameter values, respectively, corresponding to plot legend. 


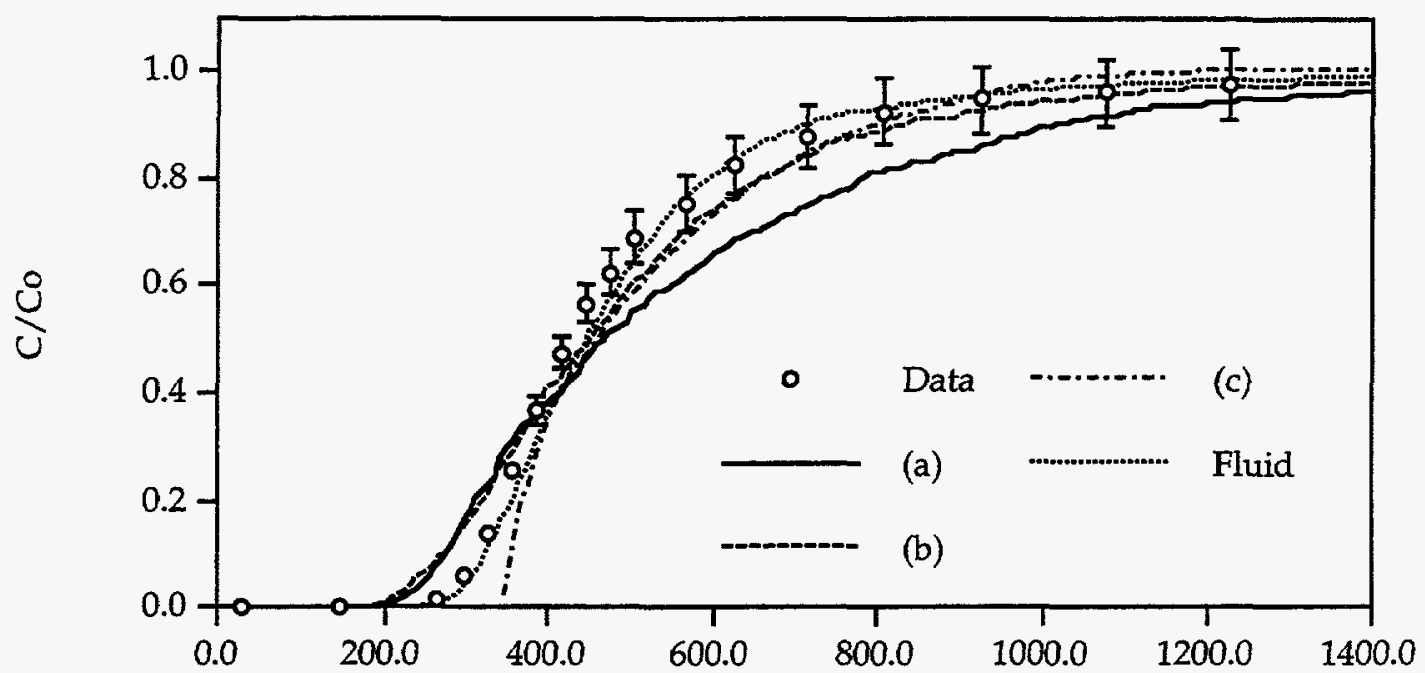

Time, sec

Figure D.27. Predicted and experimental breakthrough curves of 1- $\mu \mathrm{m}$ CML microspheres in Experiment 5 (Bandelier tuff) using the laser profile aperture data set for the model predictions. See p. D-2 and Table D.1 for description of models and parameter values, respectively, corresponding to plot legend.

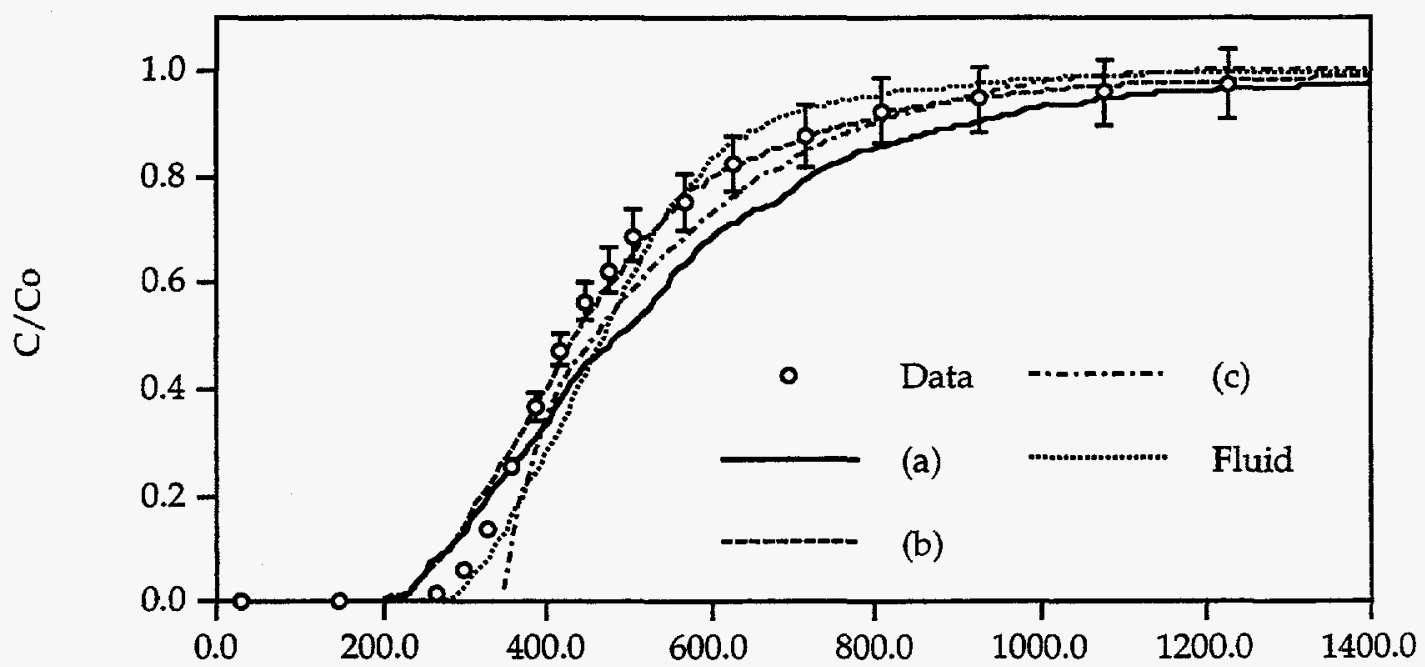

Time, sec

Figure D.28. Predicted and experimental breakthrough curves of 1- $\mu \mathrm{m} \mathrm{CML}$ microspheres in Experiment 5 (Bandelier tuff) using the RTV aperture data set for the model predictions. See p. D-2 and Table D.1 for description of models and parameter values, respectively, corresponding to plot legend. 


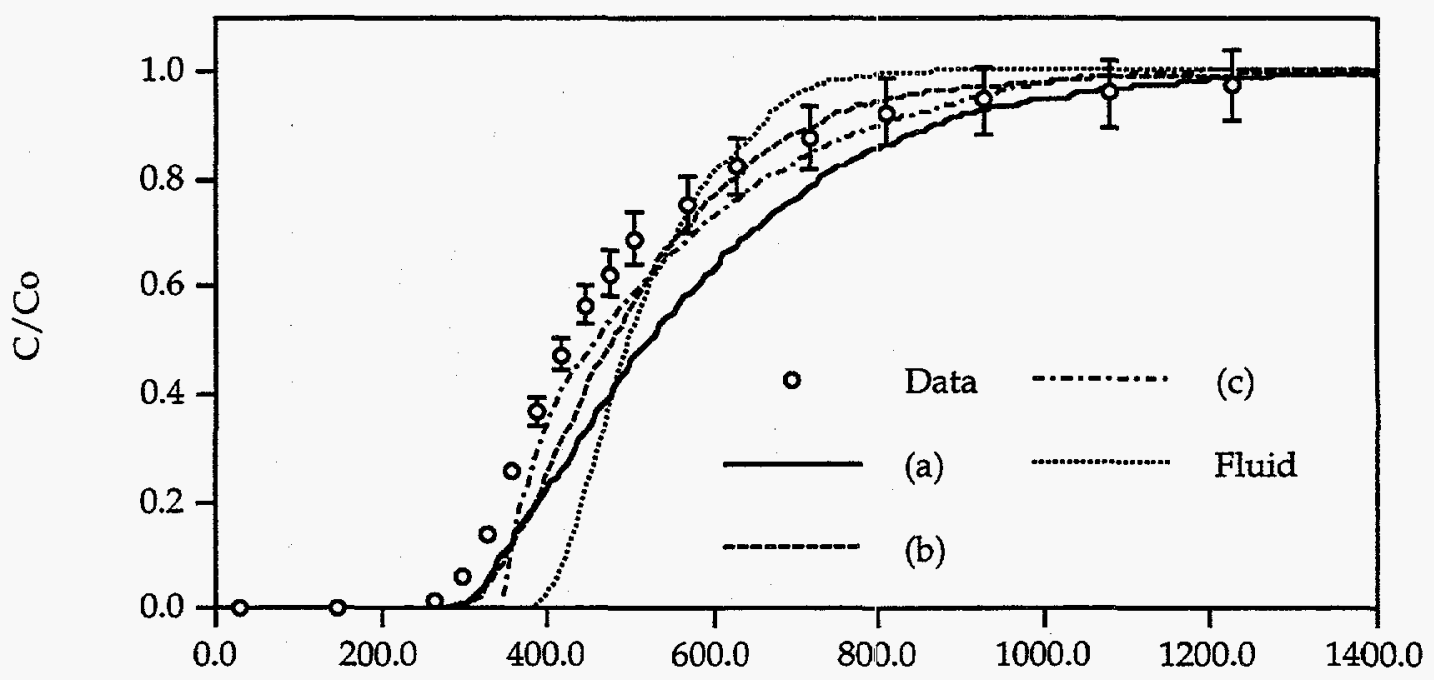

Time, sec

Figure D.29. Predicted and experimental breakthrough curves of 1- $\mu \mathrm{m} C \mathrm{CML}$ microspheres in Experiment 5 (Bandelier tuff) using the 1st PEAK aperture data set for the model predictions. See p. D-2 and Table D.1 for description of models and parameter values, respectively, corresponding to plot legend.

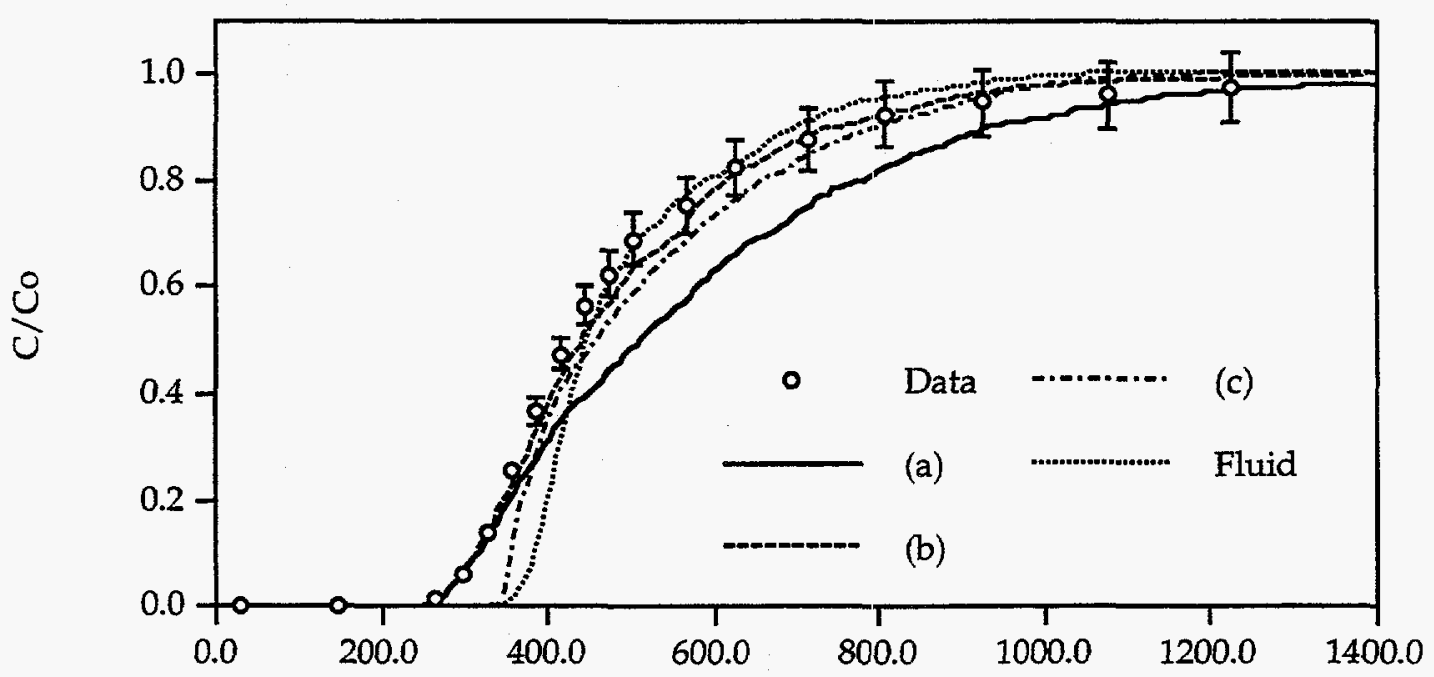

Time, sec

Figure D.30. Predicted and experimental breakthrough curves of 1- $\mu \mathrm{m} C \mathrm{CML}$ microspheres in Experiment 5 (Bandelier tuff) using the 2nd PEAK aperture data set for the model predictions. See p. D-2 and Table D.1 for description of models and parameter values, respectively, corresponding to plot legend. 


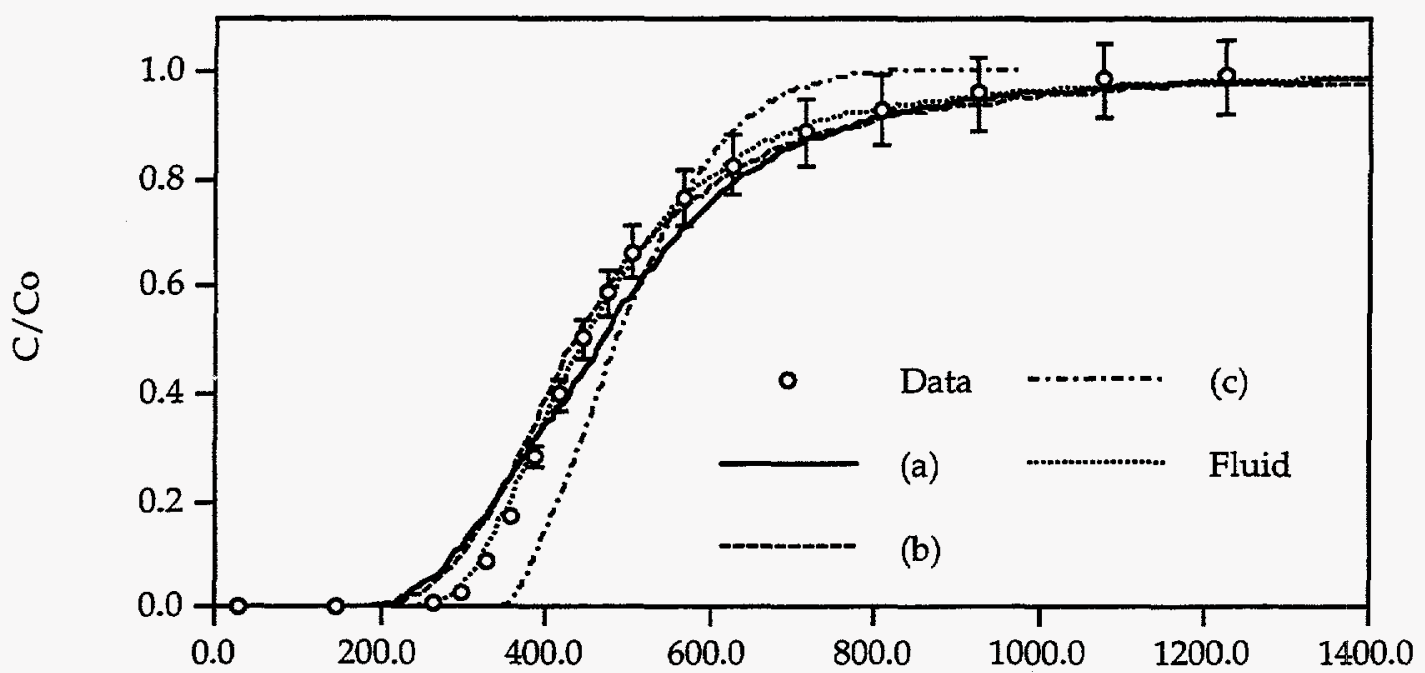

Time, sec

Figure D.31. Predicted and experimental breakthrough curves of $0.3-\mu \mathrm{m} C M L$ microspheres in Experiment 5 (Bandelier tuff) using the laser profile aperture data set for the model predictions. See p. D-2 and Table D.1 for description of models and parameter values, respectively, corresponding to plot legend.

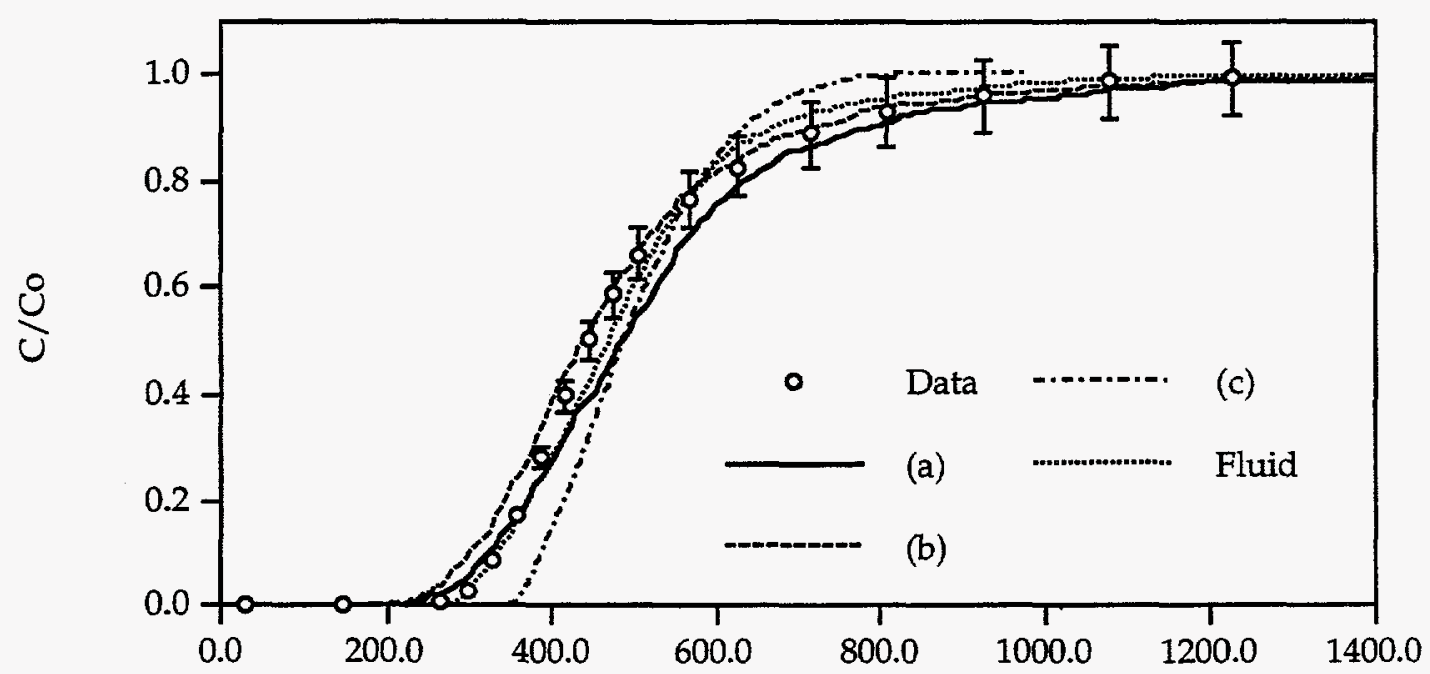

Time, sec

Figure D.32. Predicted and experimental breakthrough curves of $0.3-\mu \mathrm{m} \mathrm{CML}$ microspheres in Experiment 5 (Bandelier tuff) using the RTV aperture data set for the model predictions. See p. D-2 and Table D.1 for description of models and parameter values, respectively, corresponding to plot legend. 


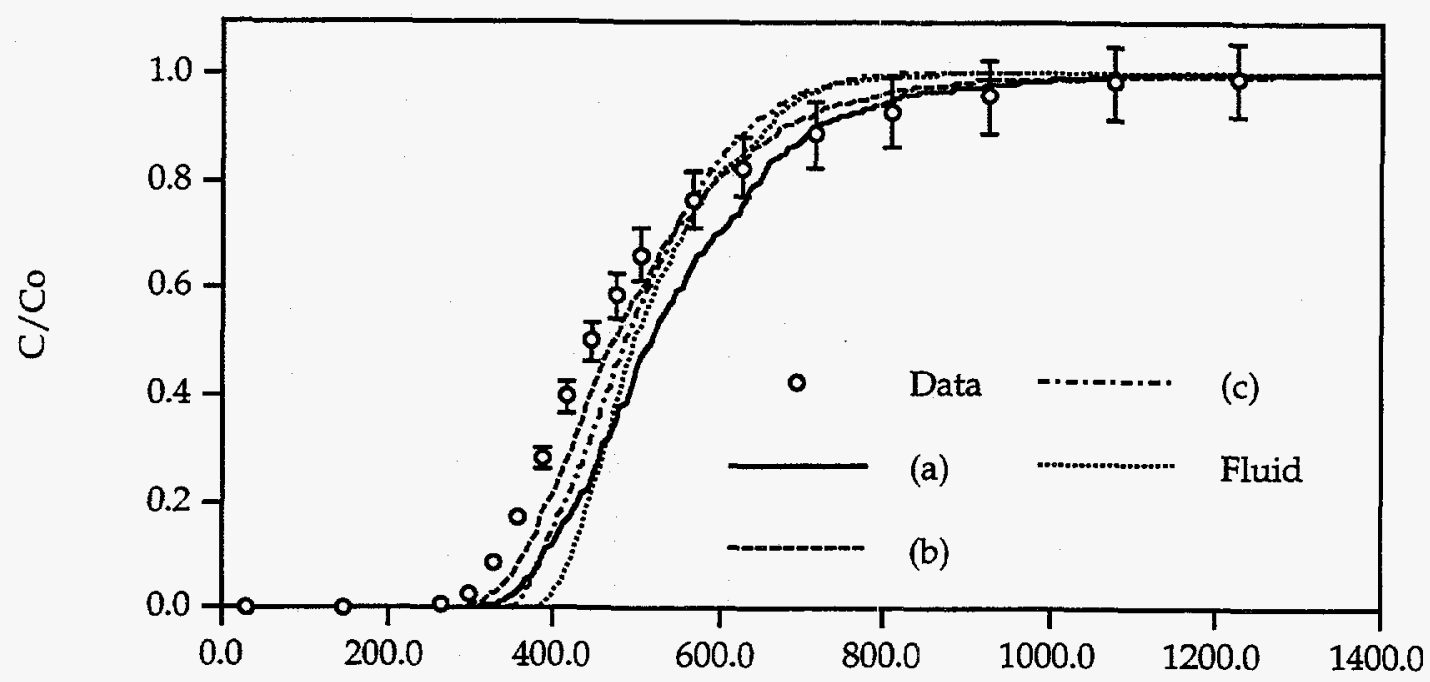

Time, sec

Figure D.33. Predicted and experimental breakthrough curves of $0.3-\mu \mathrm{m} C M L$ microspheres in Experiment 5 (Bandelier tuff) using the 1st PEAK aperture data set for the model predictions. See p. D-2 and Table D.1 for description of models and parameter values, respectively, corresponding to plot legend.

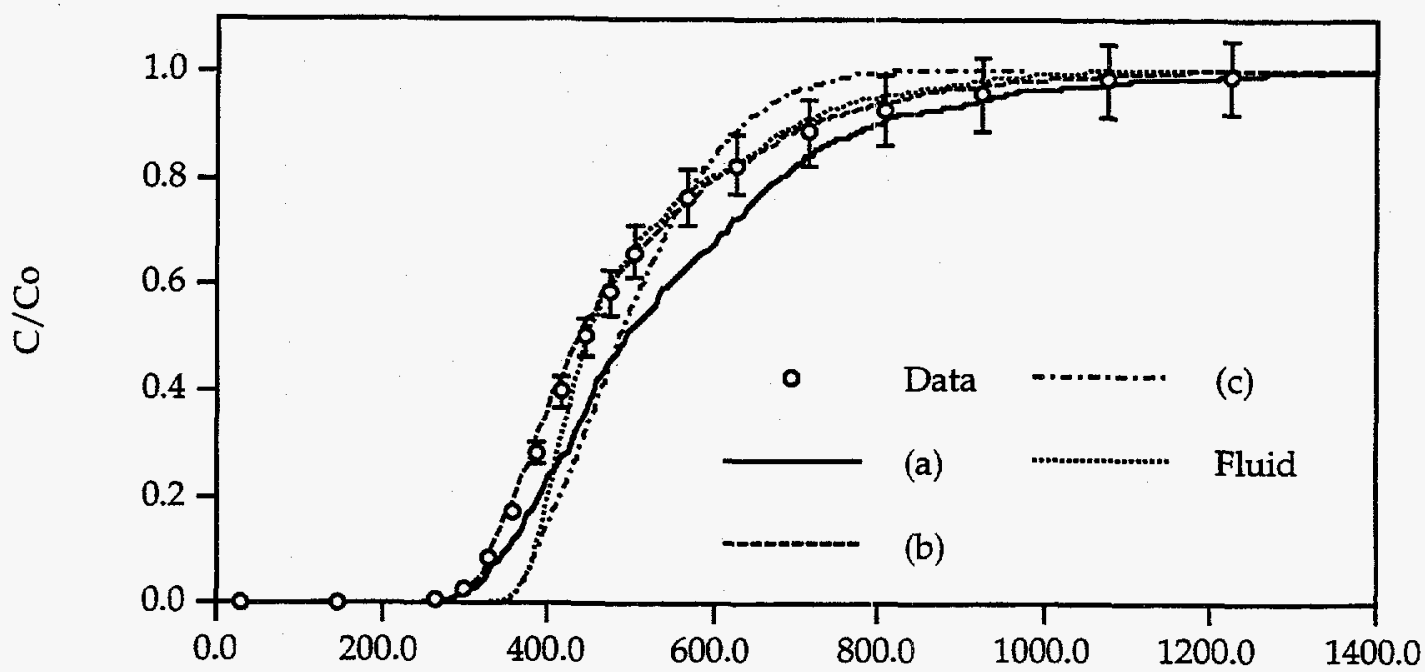

Time, sec

Figure D.34. Predicted and experimental breakthrough curves of $0.3-\mu \mathrm{m} C \mathrm{CM}$ microspheres in Experiment 5 (Bandelier tuff) using the 2nd PEAK aperture data set for the model predictions. See p. D-2 and Table D.1 for description of models and parameter values, respectively, corresponding to plot legend. 


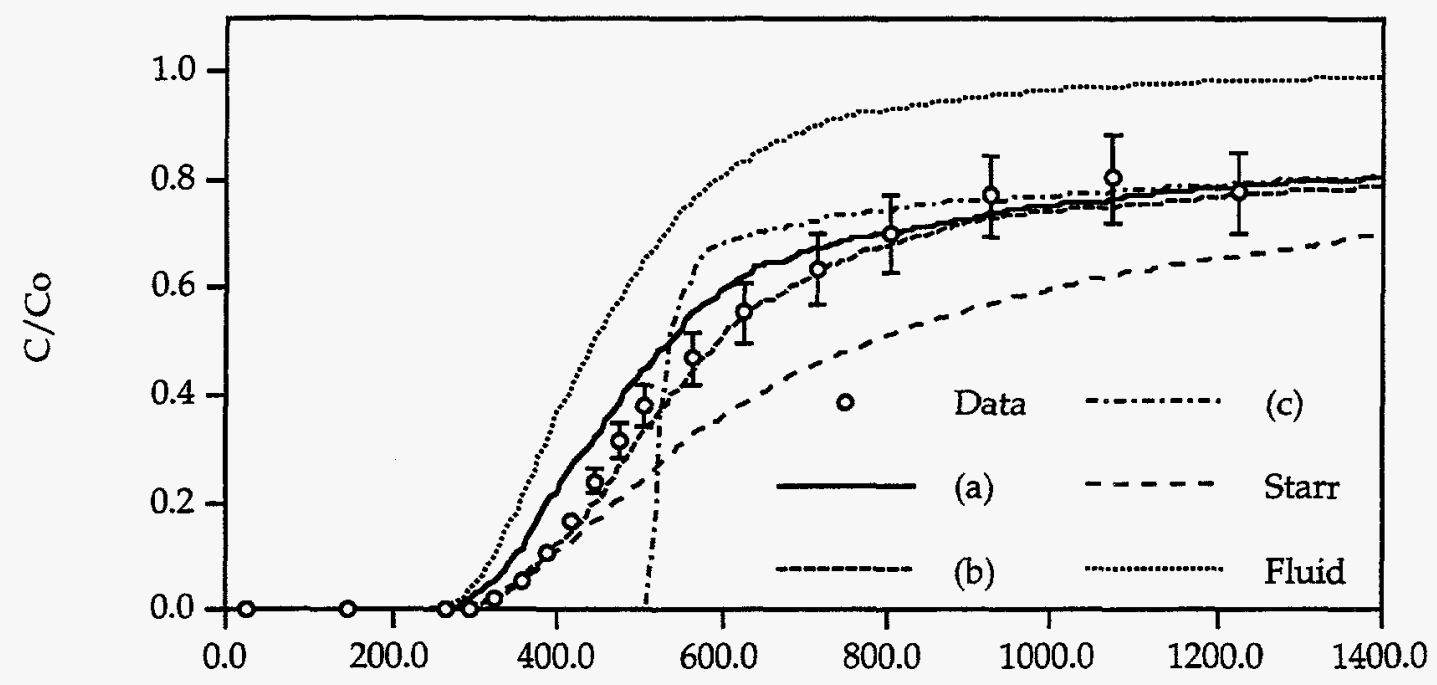

Time, sec

Figure D.35. Predicted and experimental breakthrough curves of iodide in

Experiment 5 (Bandelier tuff) using the laser profile aperture data set for the model predictions. See P. D-2 and Table D.1 for description of models and parameter values, respectively, corresponding to plot legend.

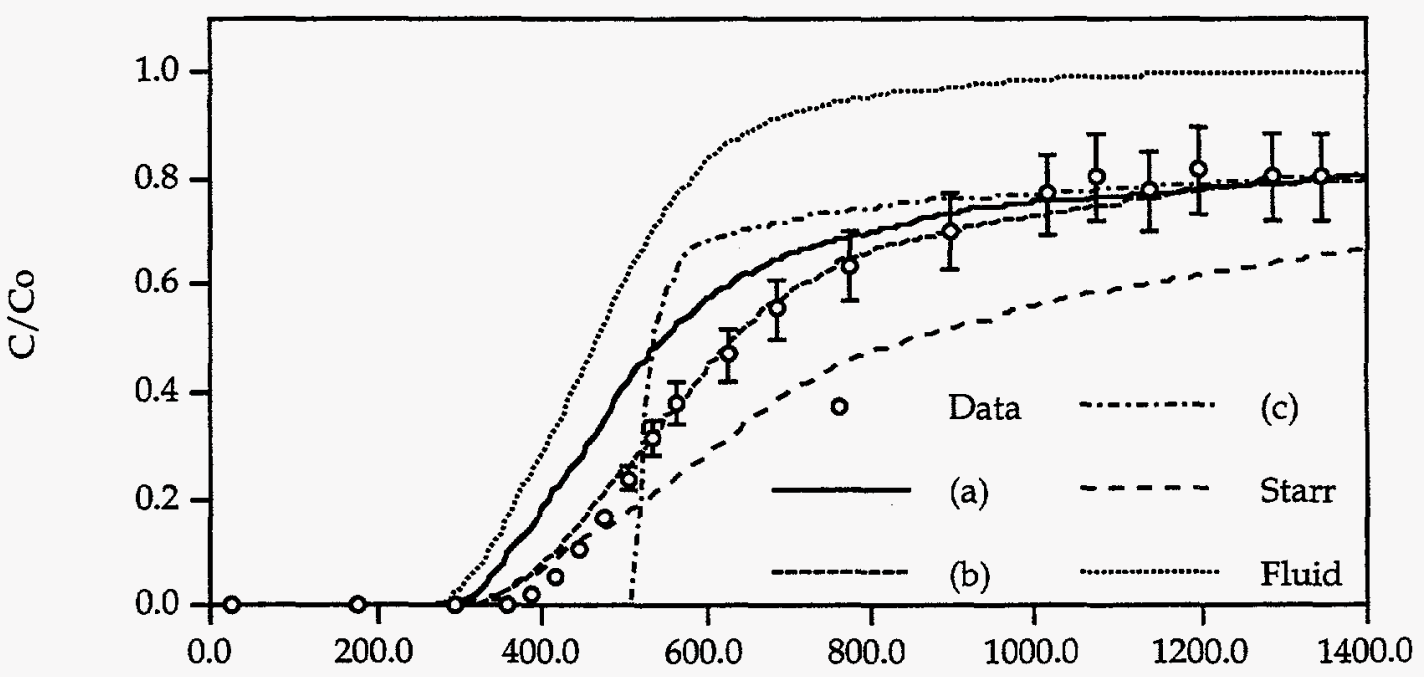

Time, sec

Figure D.36. Predicted and experimental breakthrough curves of iodide in Experiment 5 (Bandelier tuff) using the RTV aperture data set for the model predictions. See p. D-2 and Table D.1 for description of models and parameter values, respectively, corresponding to plot legend. 


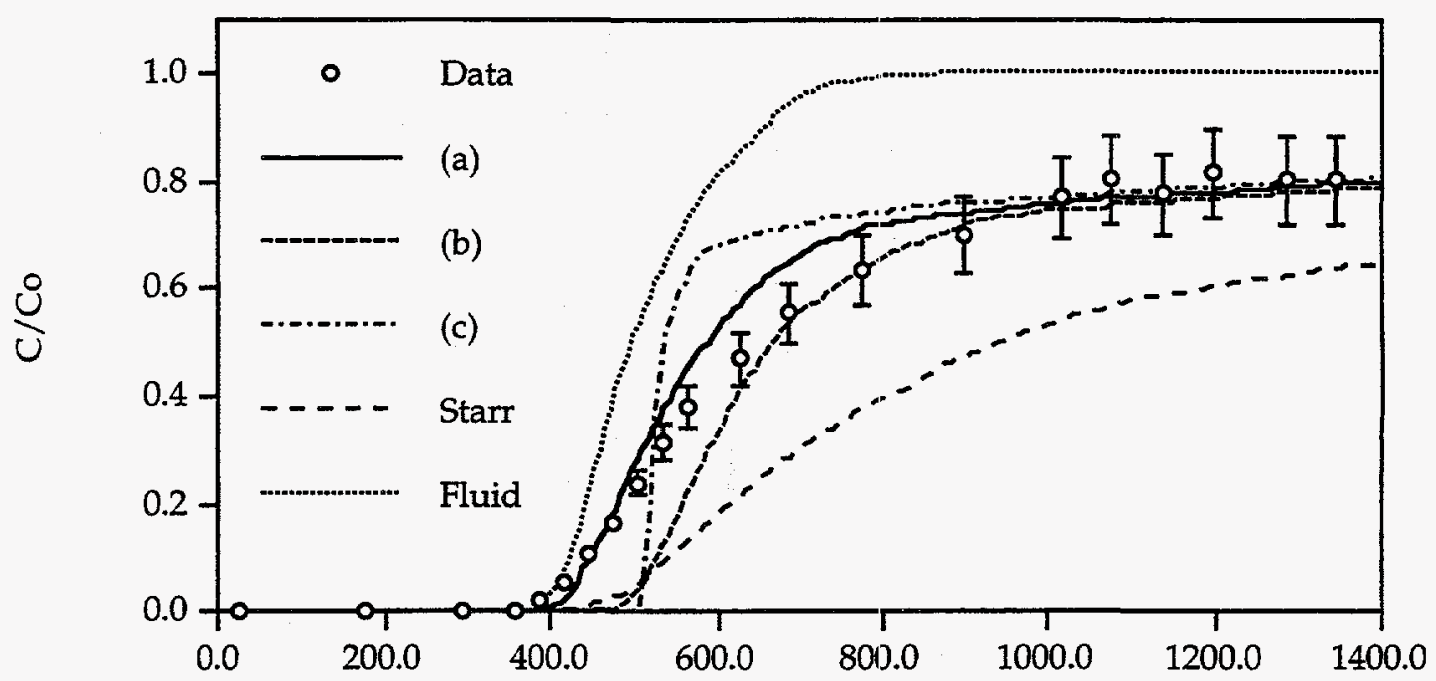

Time, sec

Figure D.37. Predicted and experimental breakthrough curves of iodide in Experiment 5 (Bandelier tuff) using the 1st PEAK aperture data set for the model predictions. See p. D-2 and Table D.1 for description of models and parameter values, respectively, corresponding to plot legend.

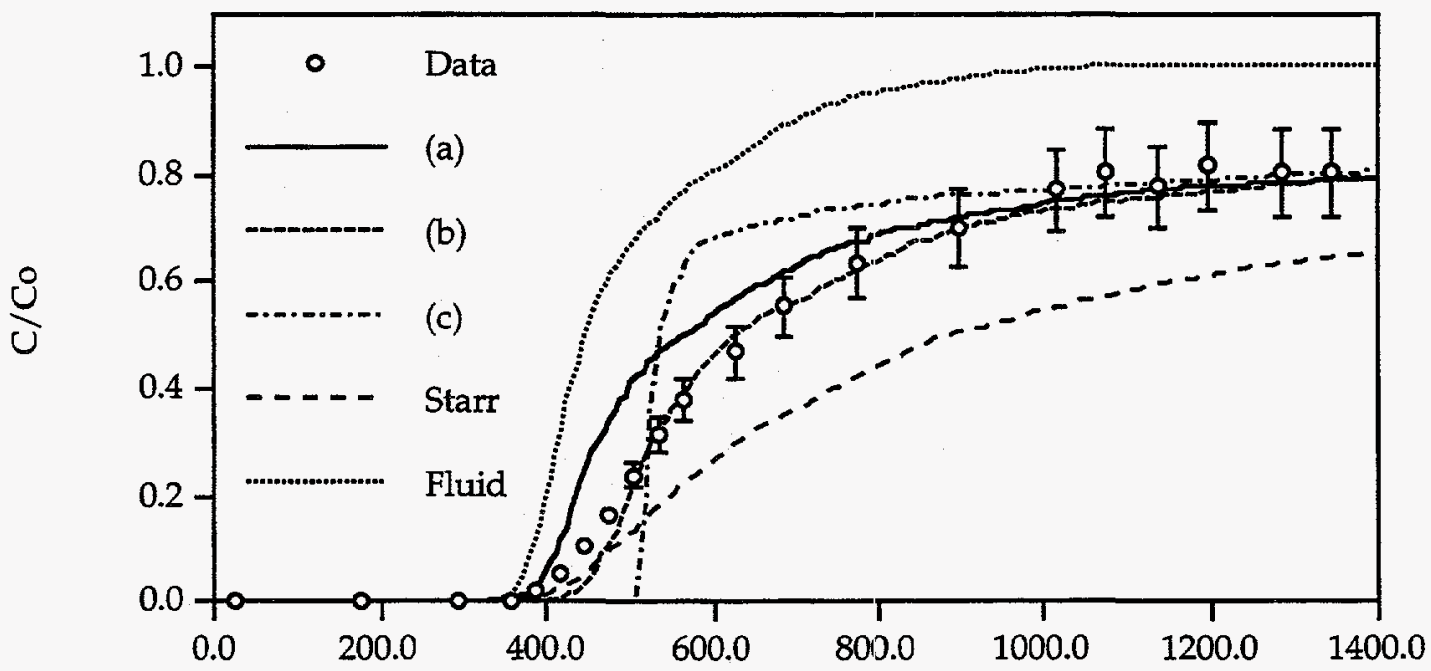

Time, sec

Figure D.38. Predicted and experimental breakthrough curves of iodide in

Experiment 5 (Bandelier tuff) using the 2nd PEAK aperture data set for the model predictions. See p. D-2 and Table D.1 for description of models and parameter values, respectively, corresponding to plot legend. 


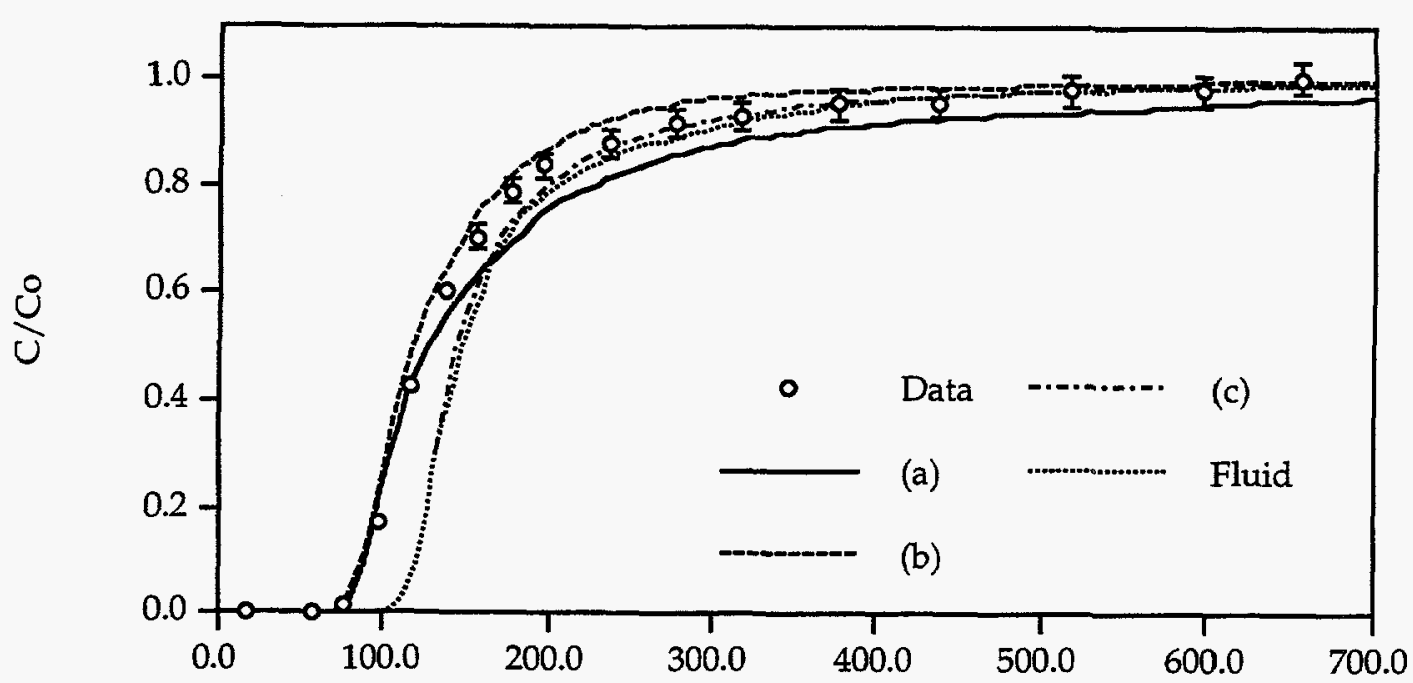

Time, sec

Figure D.39. Predicted and experimental breakthrough curves of 1- $\mu \mathrm{m}$ CML microspheres in Experiment 6 (Bullfrog tuff). See p. D-2 and Table D.1 for description of models and parameter values, respectively, corresponding to plot legend.

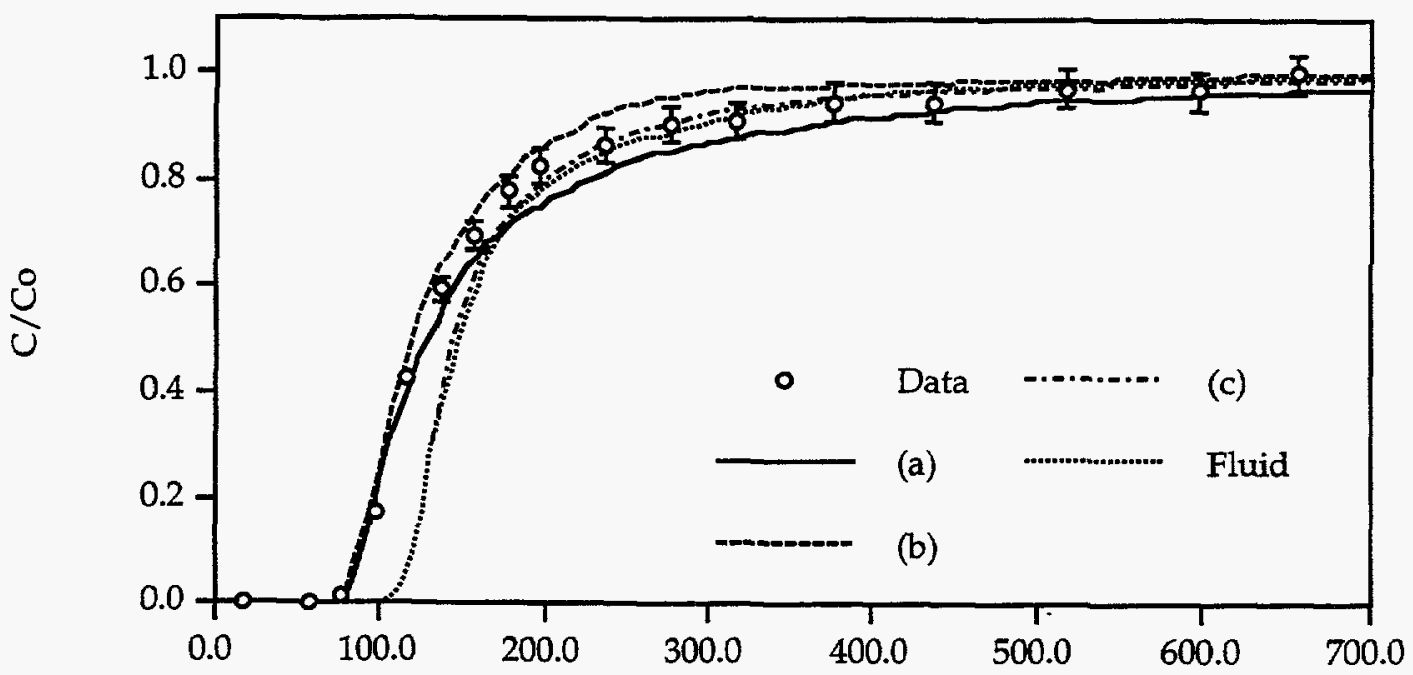

Time, sec

Figure D.40. Predicted and experimental breakthrough curves of $0.3-\mu \mathrm{m} \mathrm{CML}$ microspheres in Experiment 6 (Bullfrog tuff). See p. D-2 and Table D. 1 for description of models and parameter values, respectively, corresponding to plot legend. 


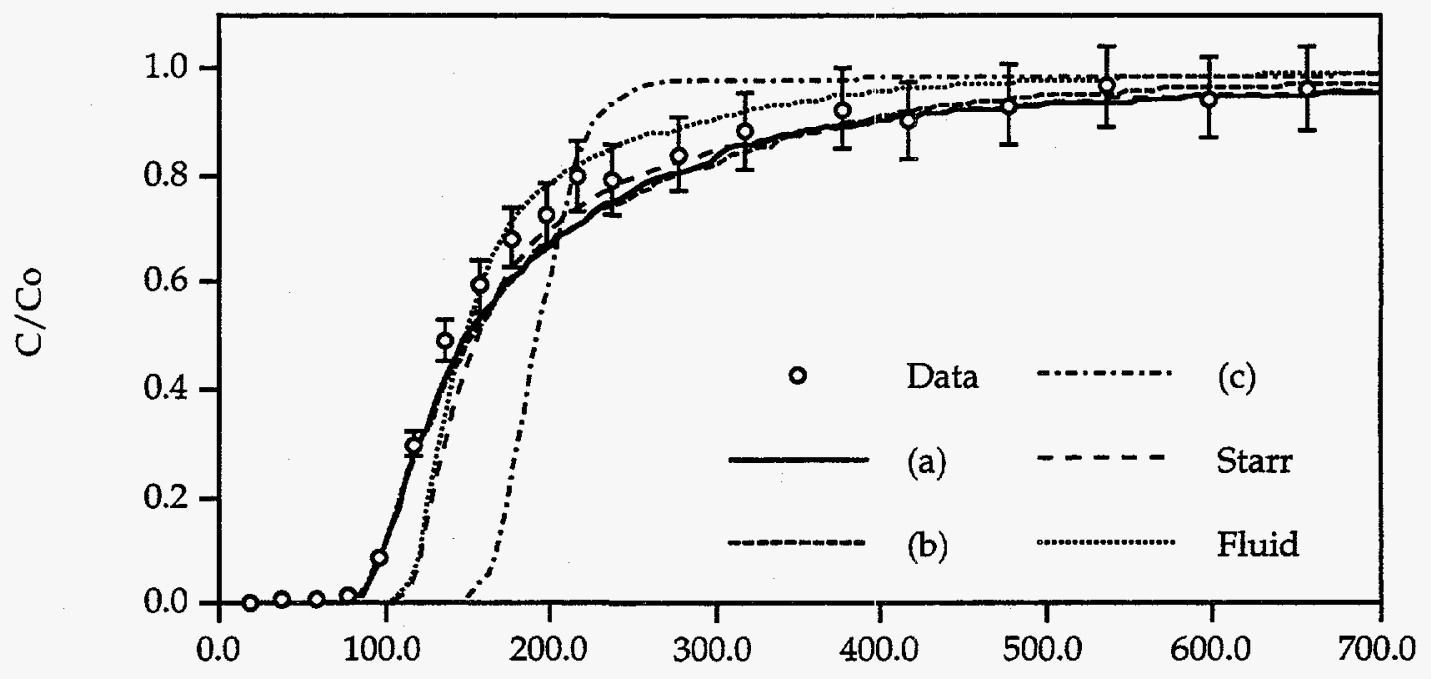

Time, sec

Figure D.41. Predicted and experimental breakthrough curves of iodide in Experiment 6 (Bullfrog tuff). See p. D-2 and Table D.1 for description of models and parameter values, respectively, corresponding to plot legend. 


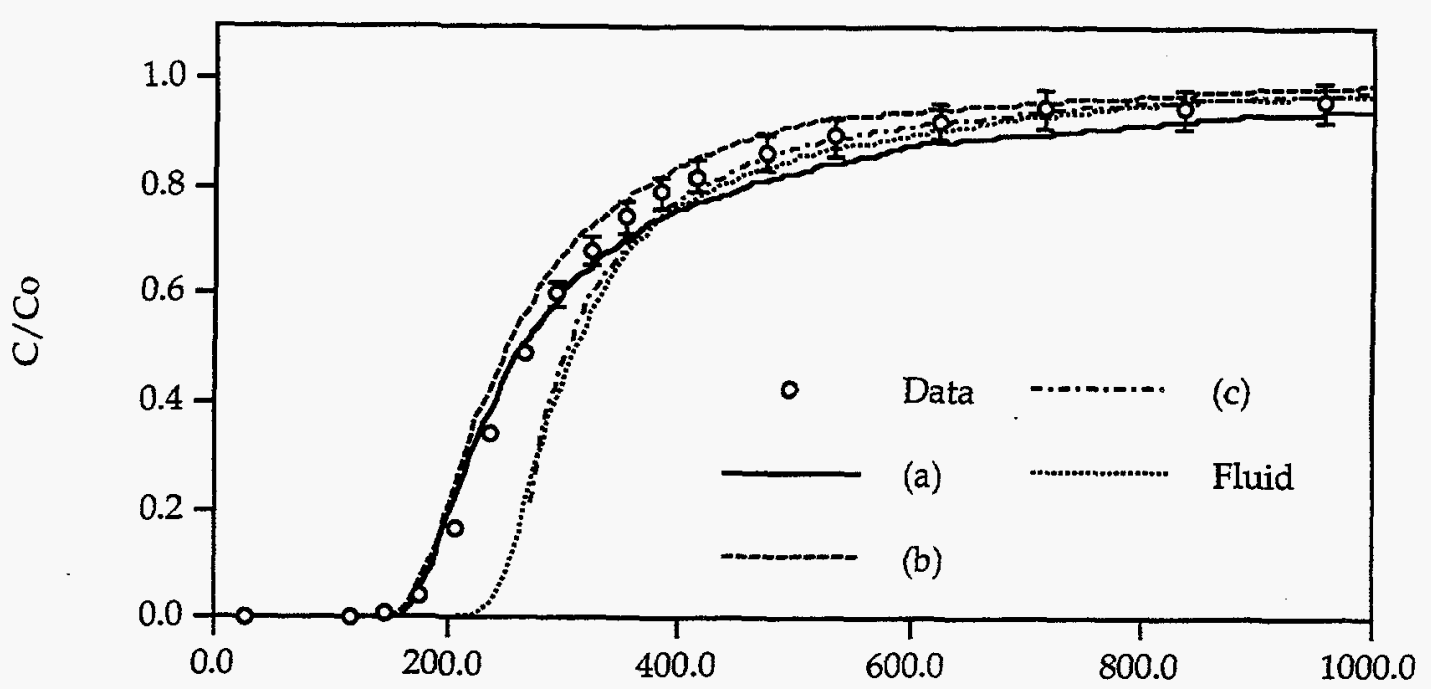

Time, sec

Figure D.42. Predicted and experimental breakthrough curves of 1- $\mu \mathrm{m} C \mathrm{CML}$ microspheres in Experiment 7 (Bullfrog tuff). See p. D-2 and Table D.1 for description of models and parameter values, respectively, corresponding to plot legend.

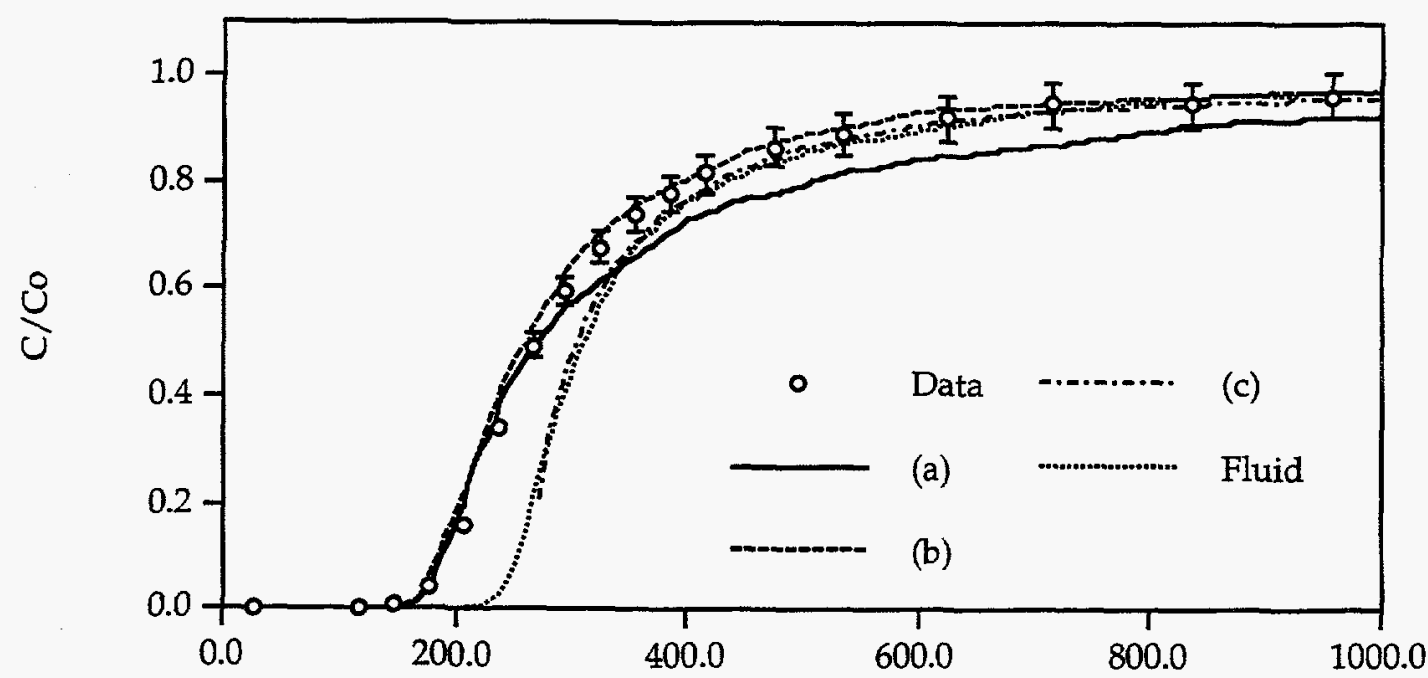

Time, sec

Figure D.43. Predicted and experimental breakthrough curves of $0.3-\mu \mathrm{m} \mathrm{CML}$ microspheres in Experiment 7 (Bullfrog tuff). See p. D-2 and Table D.1 for description of models and parameter values, respectively, corresponding to plot legend. 


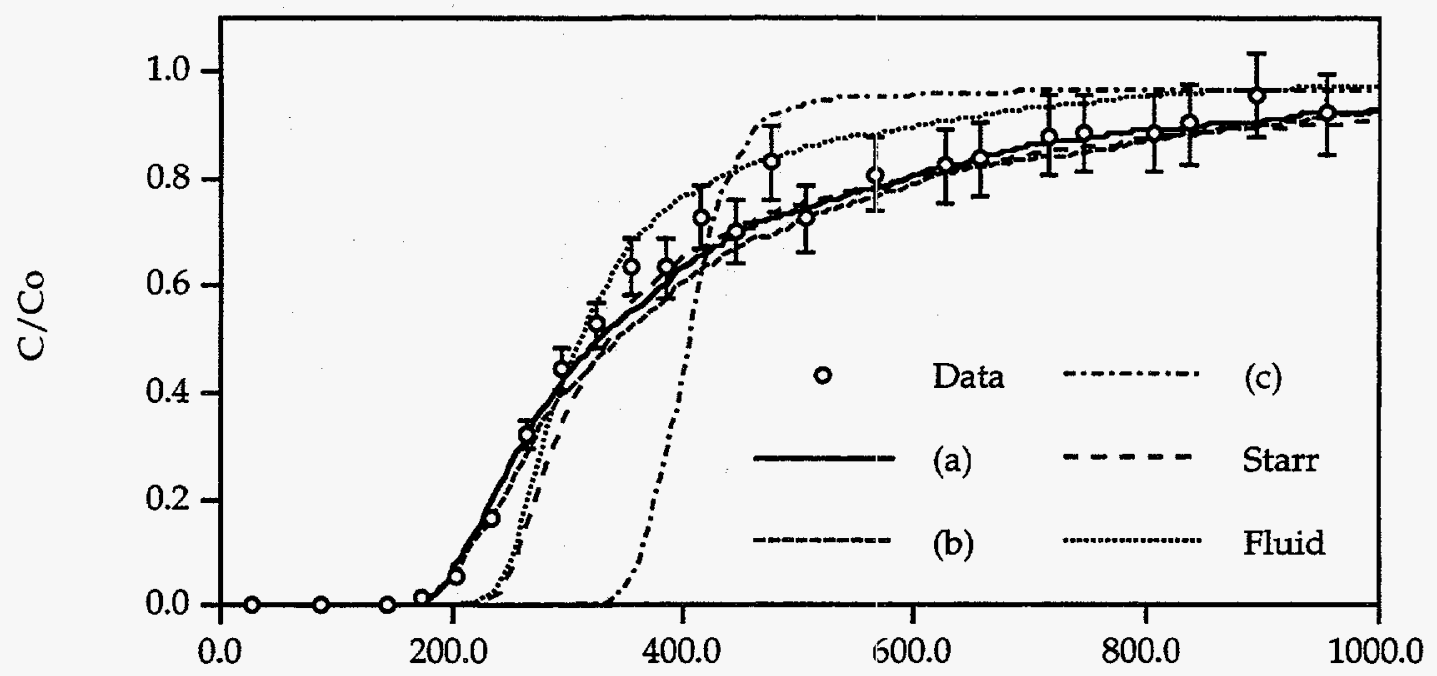

Time, sec

Figure D.44. Predicted and experimental breakthrough curves of iodide in Experiment 7 (Bullfrog tuff). See p. D-2 and Table D.1 for description of models and parameter values, respectively, corresponding to plot legend. 


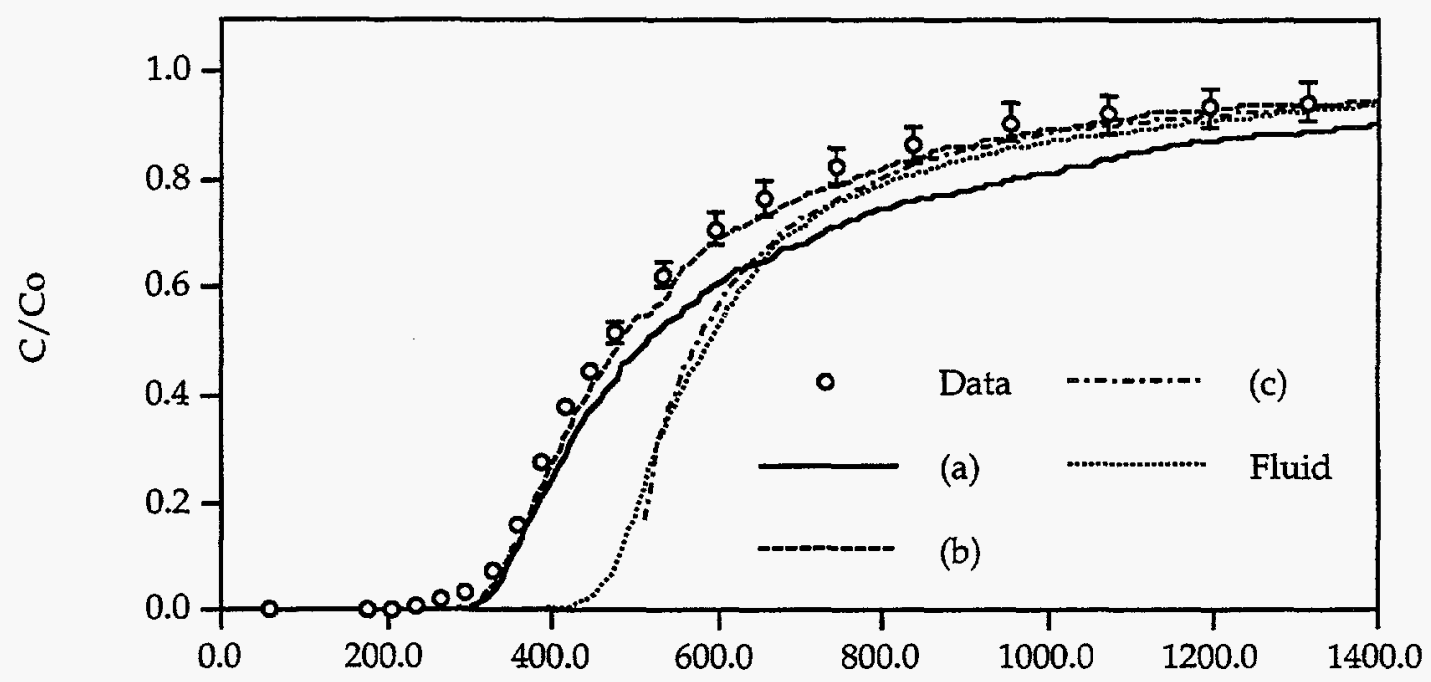

Time, sec

Figure D.45. Predicted and experimental breakthrough curves of 1- $\mu \mathrm{m}$ CML microspheres in Experiment 8 (Bullfrog tuff). See p. D-2 and Table D.1 for description of models and parameter values, respectively, corresponding to plot legend.

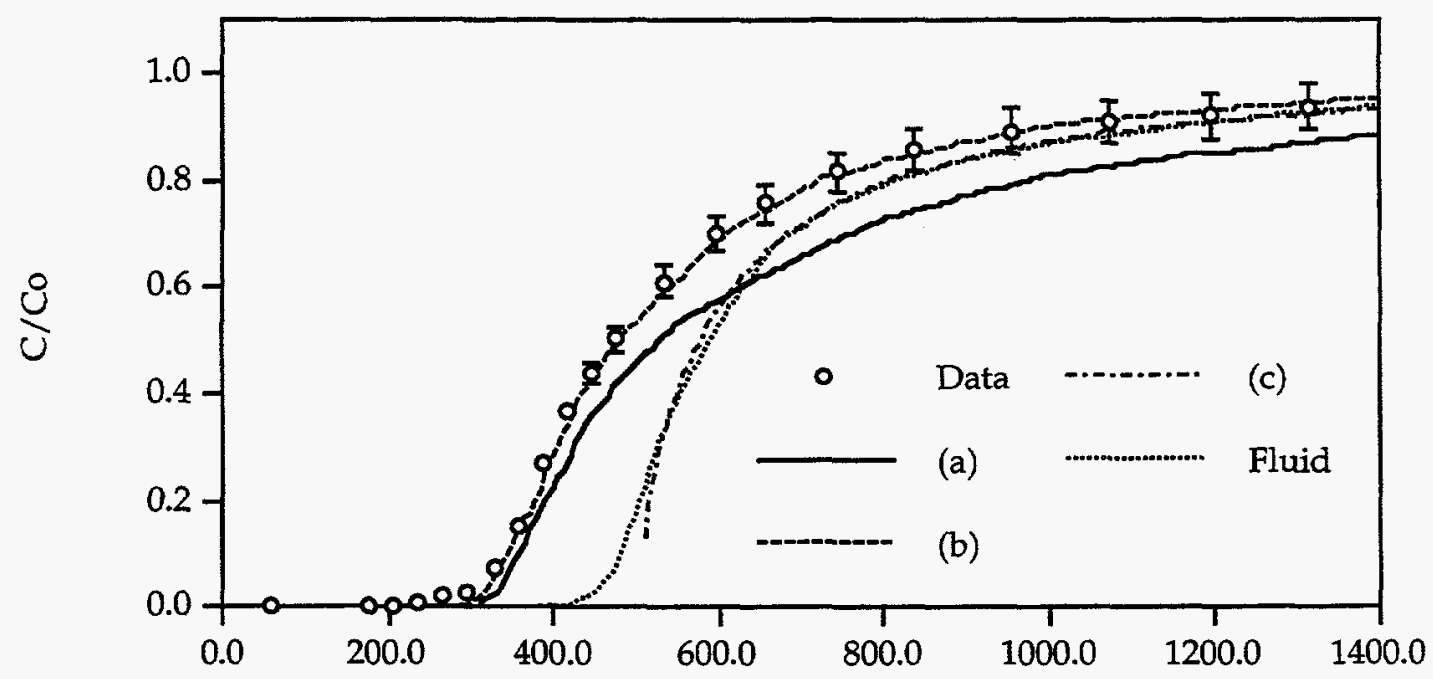

Time, sec

Figure D.46. Predicted and experimental breakthrough curves of $0.3-\mu \mathrm{m} C \mathrm{CML}$ microspheres in Experiment 8 (Bullfrog tuff). See p. D-2 and Table D.1 for description of models and parameter values, respectively, corresponding to plot legend. 


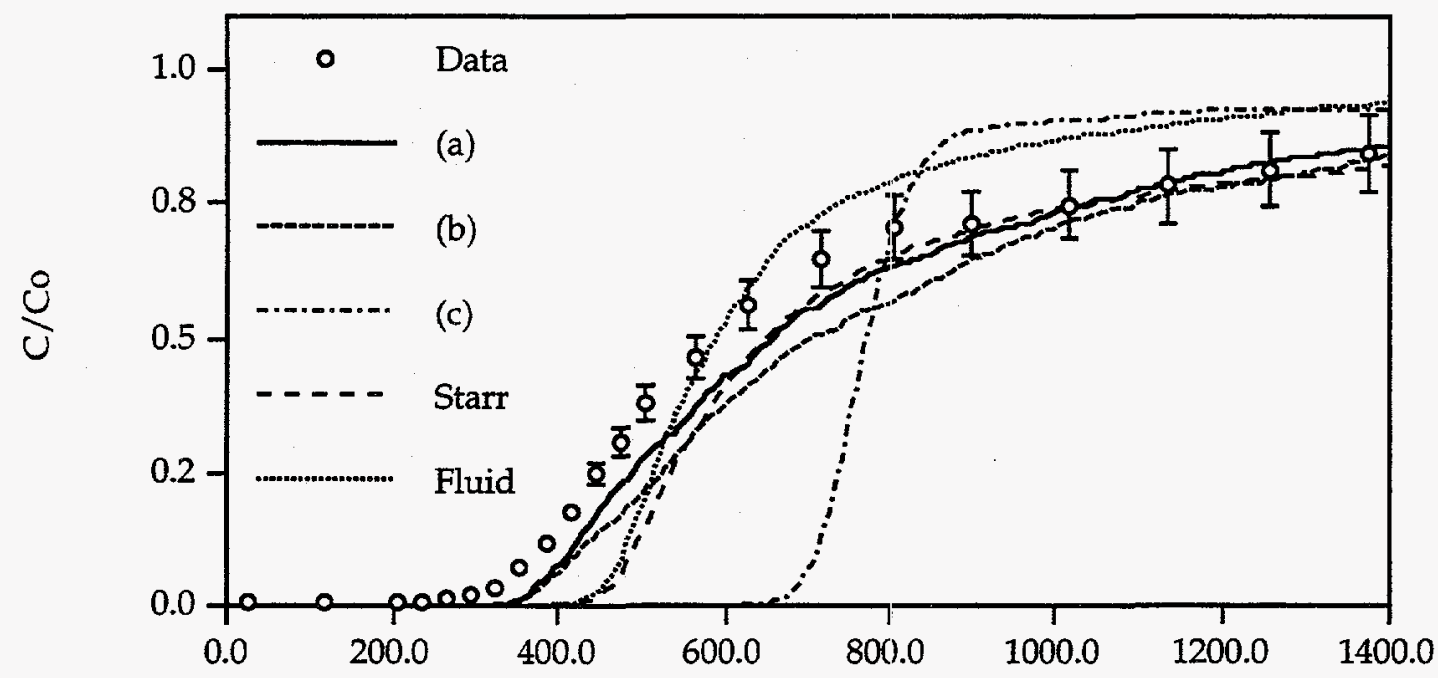

Time, sec

Figure D.47. Predicted and experimental breakthrough curves of iodide in Experiment 8 (Bullfrog tuff). See p. D-2 and Table D.1 for description of models and parameter values, respectively, corresponding to plot legend. 


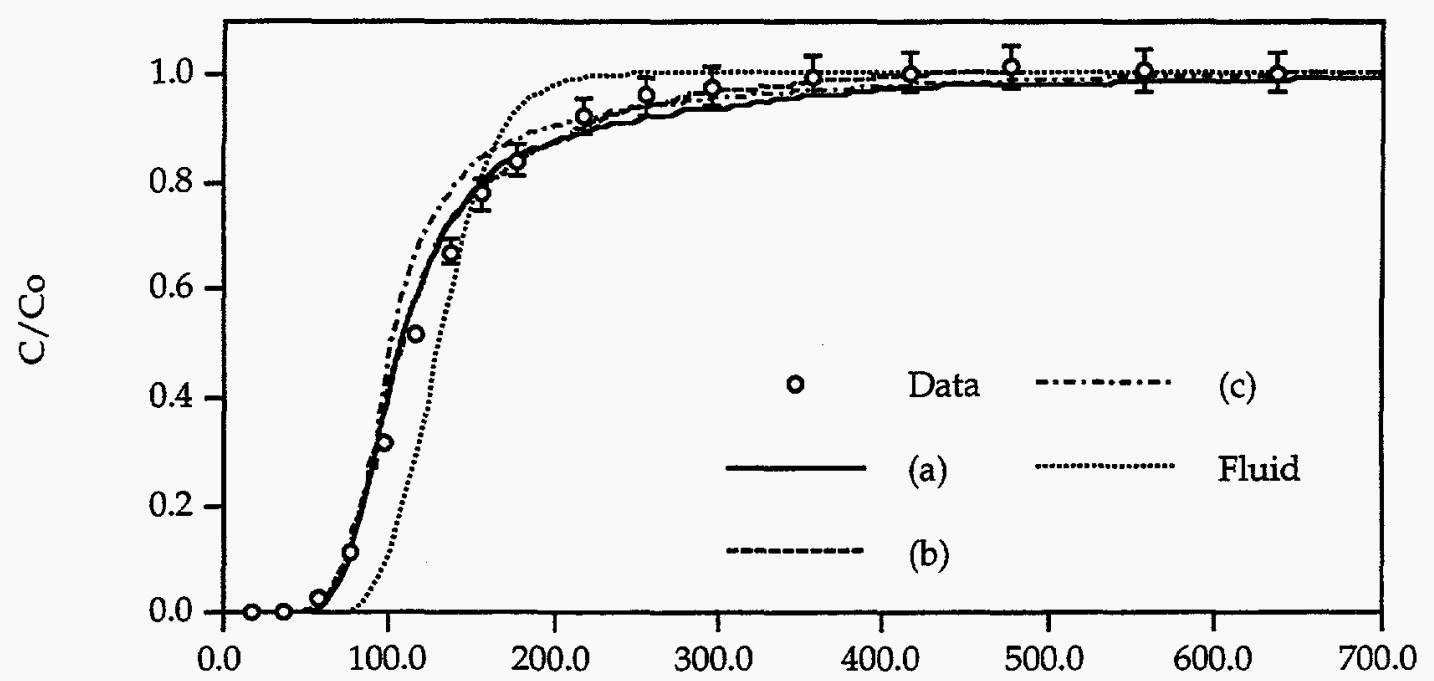

Time, sec

Figure D.48. Predicted and experimental breakthrough curves of $1-\mu \mathrm{m} C \mathrm{CML}$ microspheres in Experiment 9 (Tram tuff). See p. D-2 and Table D.1 for description of models and parameter values, respectively, corresponding to plot legend.

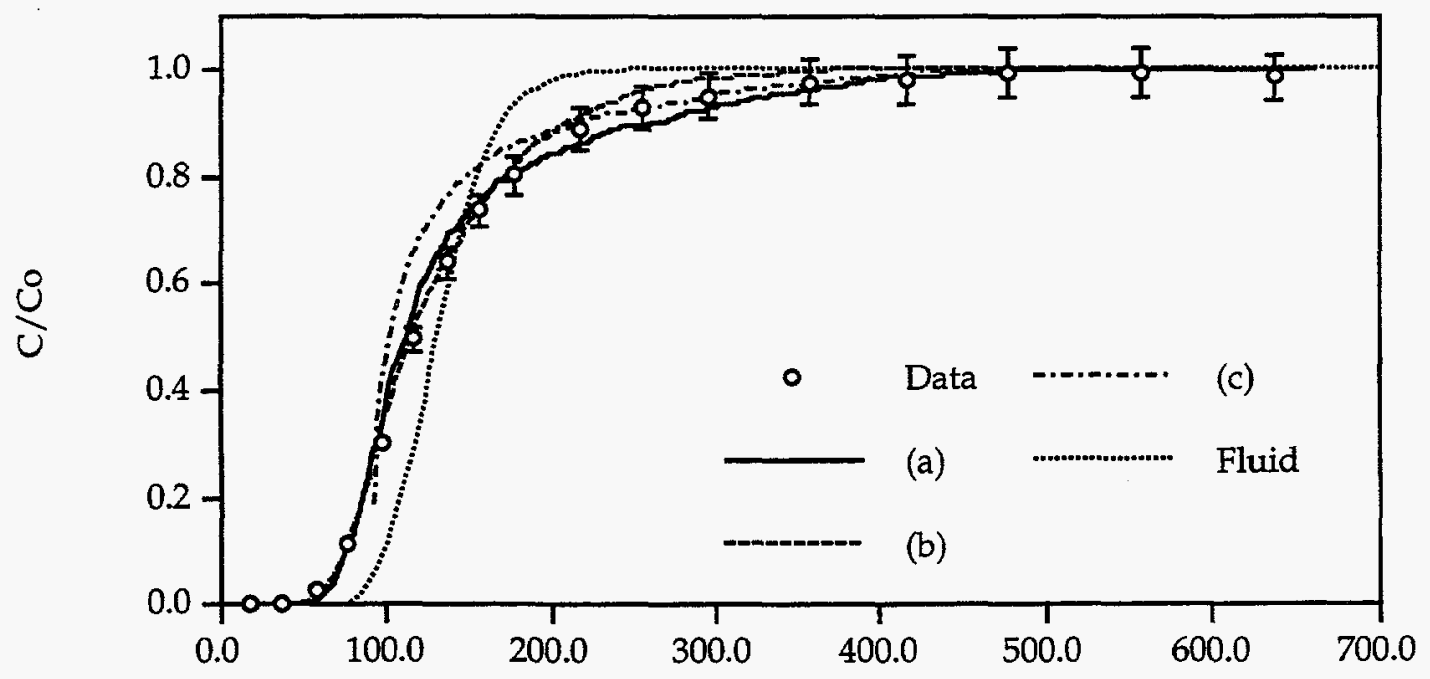

Time, sec

Figure D.49. Predicted and experimental breakthrough curves of $0.3-\mu \mathrm{m}$ CML microspheres in Experiment 9 (Tram tuff). See p. D-2 and Table D.1 for description of models and parameter values, respectively, corresponding to plot legend. 


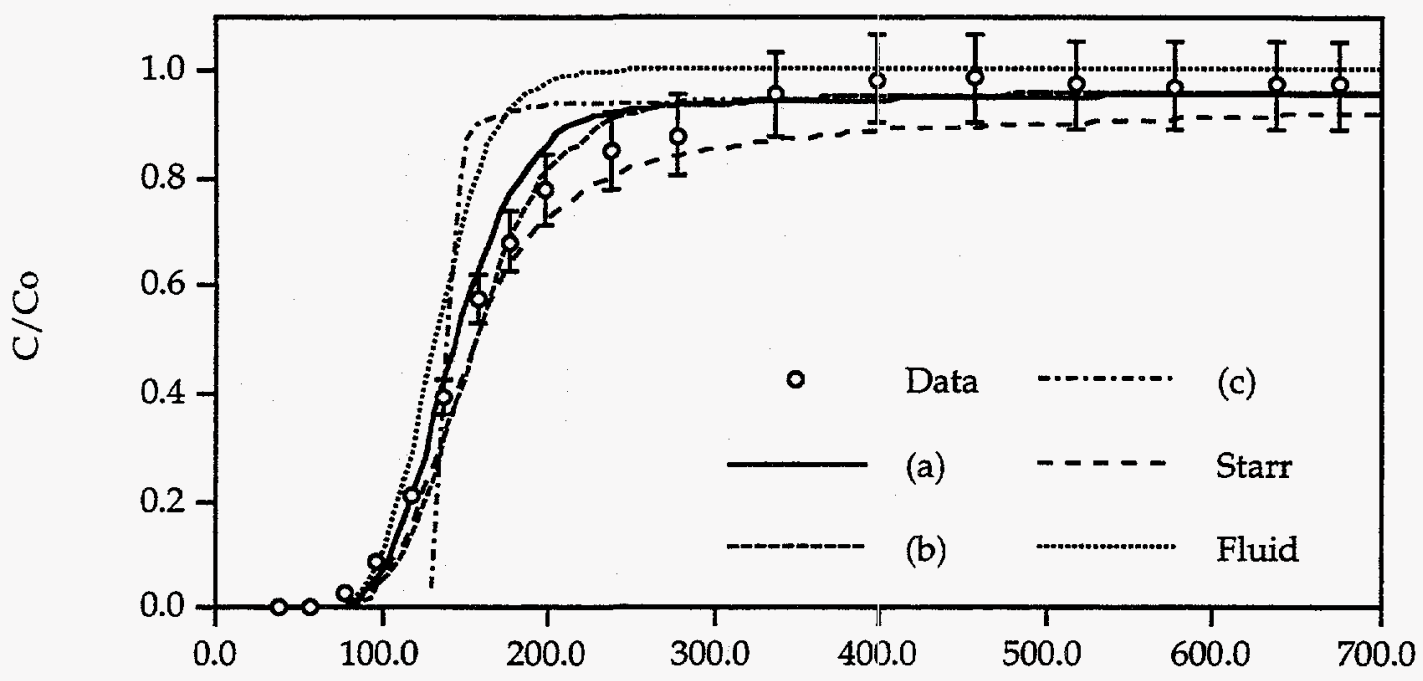

Time, se:

Figure D.50. Predicted and experimental breakthrough curves of iodide in Experiment 9 (Tram tuff). See p. D-2 and Table D. 1 for description of models and parameter values, respectively, corresponding to plot legend. 


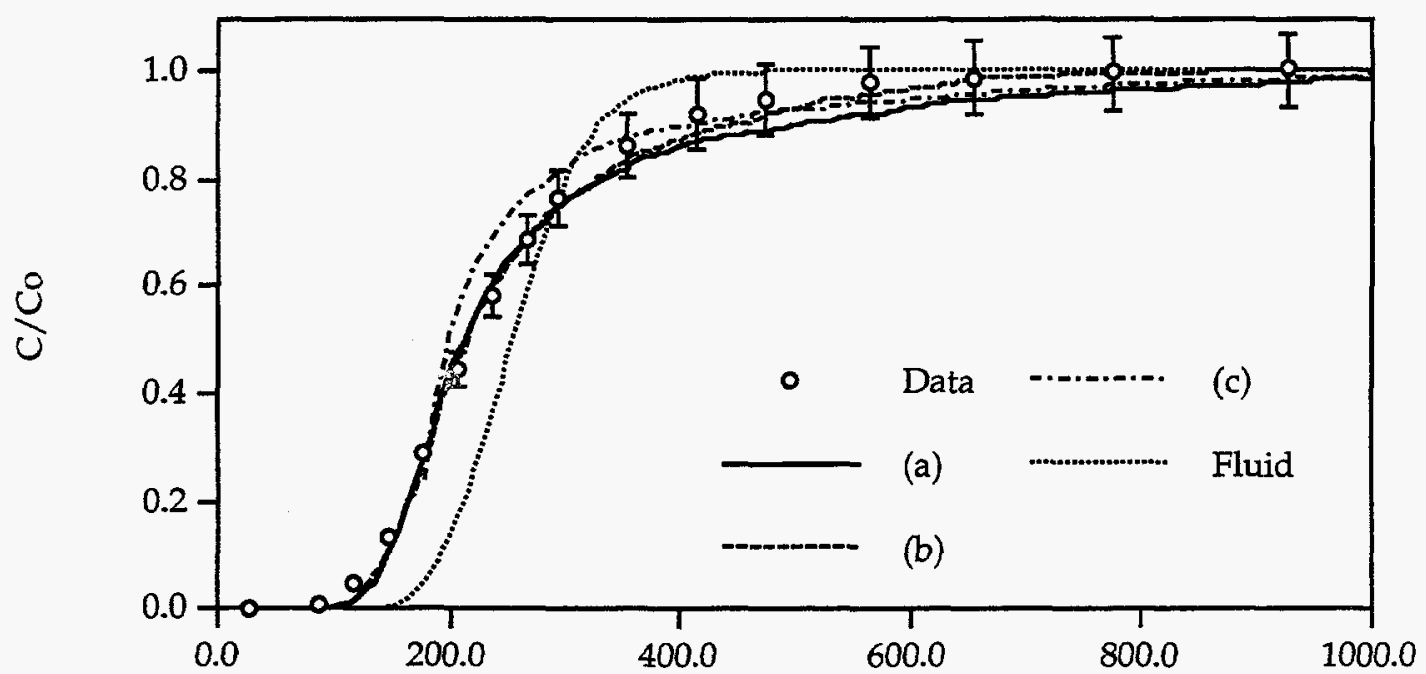

Time, sec

Figure D.51. Predicted and experimental breakthrough curves of $1-\mu \mathrm{m} C M L$ microspheres in Experiment 10 (Tram tuff). See p. D-2 and Table D.1 for description of models and parameter values, respectively, corresponding to plot legend.

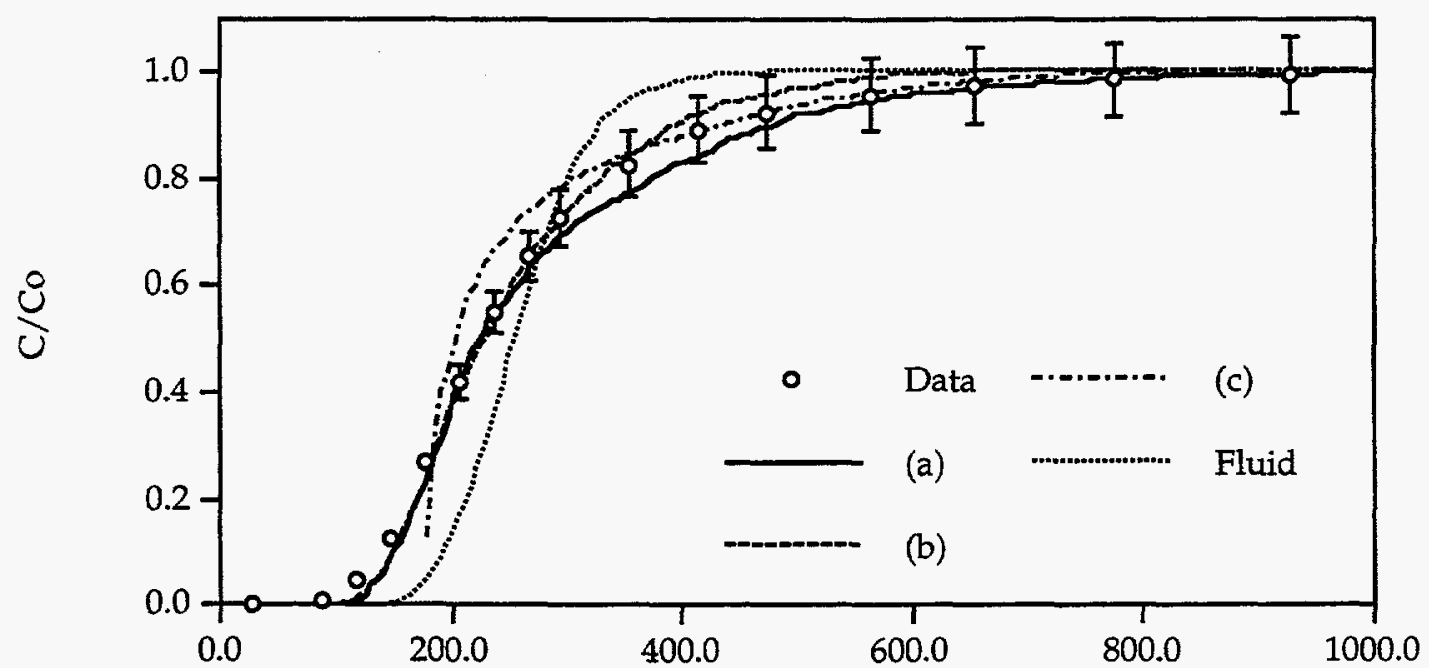

Time, sec

Figure D.52. Predicted and experimental breakthrough curves of $0.3-\mu \mathrm{m} C \mathrm{CML}$ microspheres in Experiment 10 (Tram tuff). See p. D-2 and Table D.1 for description of models and parameter values, respectively, corresponding to plot legend. 


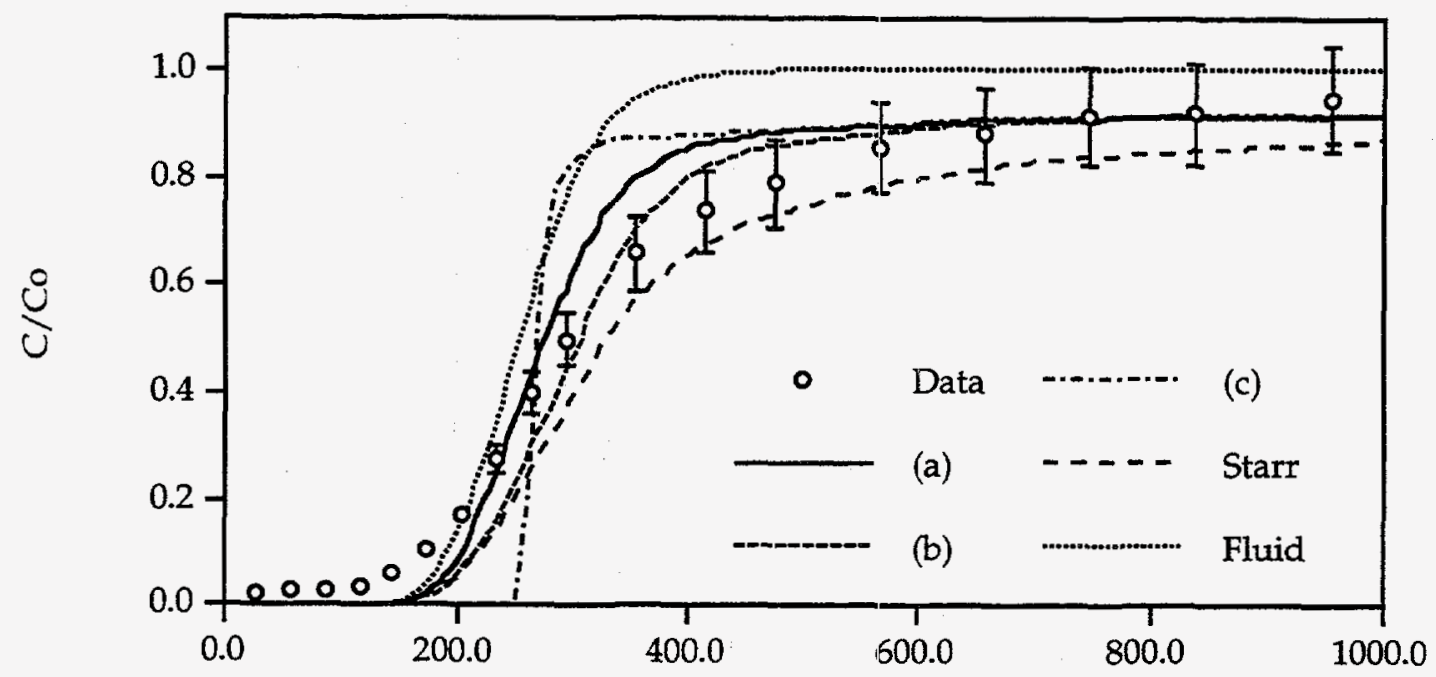

Time, sec

Figure D.53. Predicted and experimental breakthrough curves of iodide in Experiment 10 (Tram tuff). See p. D-2 and Table D.1 for description of models and parameter values, respectively, corresponding to plot legend. 


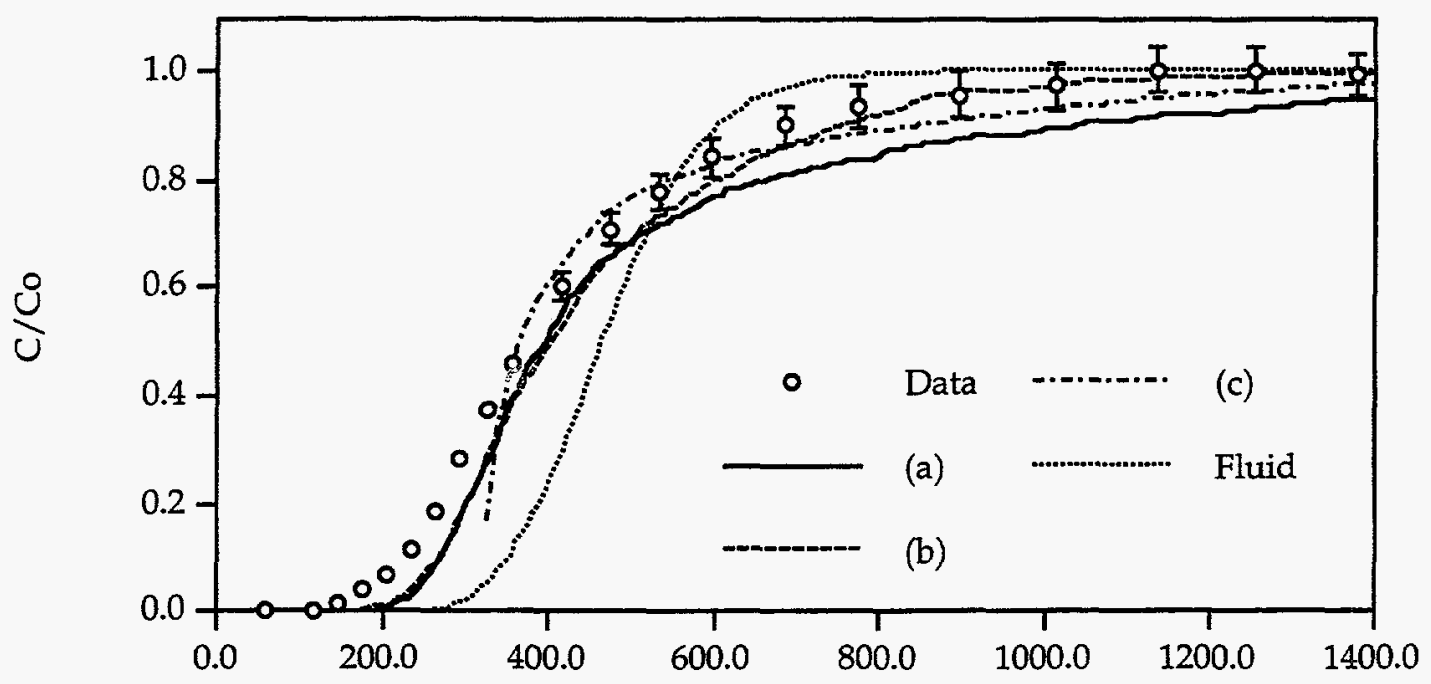

Time, sec

Figure D.54. Predicted and experimental breakthrough curves of 1- $\mu \mathrm{m} C \mathrm{CML}$ microspheres in Experiment 11 (Tram tuff). See p. D-2 and Table D.1 for description of models and parameter values, respectively, corresponding to plot legend.

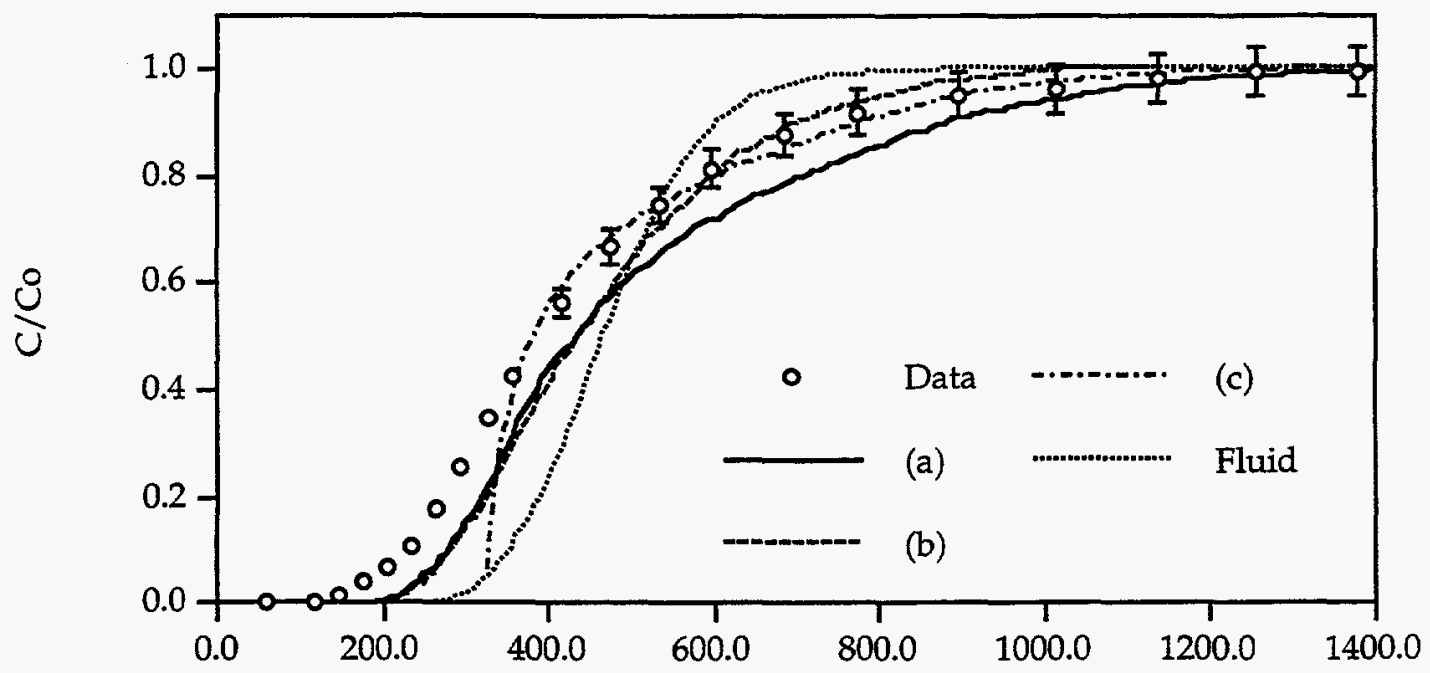

Time, sec

Figure D.55. Predicted and experimental breakthrough curves of $0.3-\mu \mathrm{m} C \mathrm{CML}$ microspheres in Experiment 11 (Tram tuff). See p. D-2 and Table D.1 for description of models and parameter values, respectively, corresponding to plot legend. 


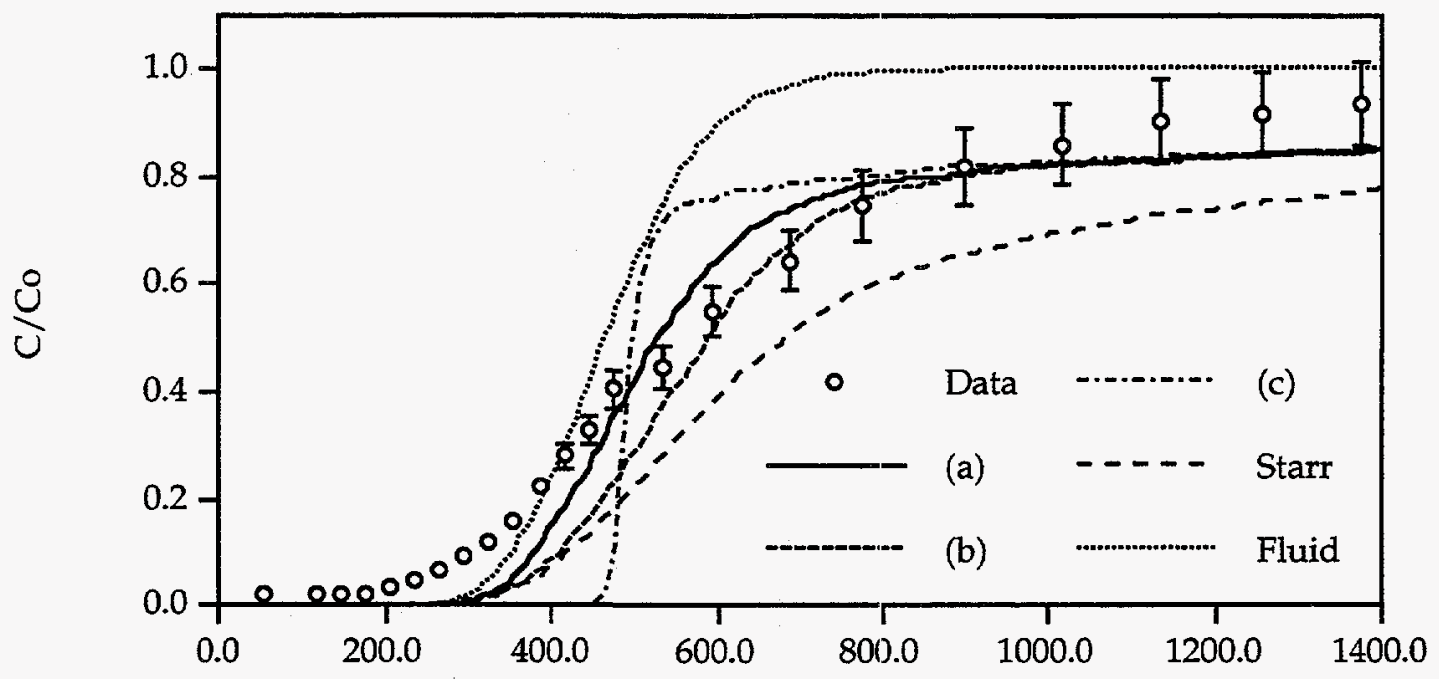

Time, sec

Figure D.56. Predicted and experimental breakthrough curves of iodide in Experiment 11 (Tram tuff). See p. D-2 and Table D.1 for description of models and parameter values, respectively, corresponding to plot legend. 


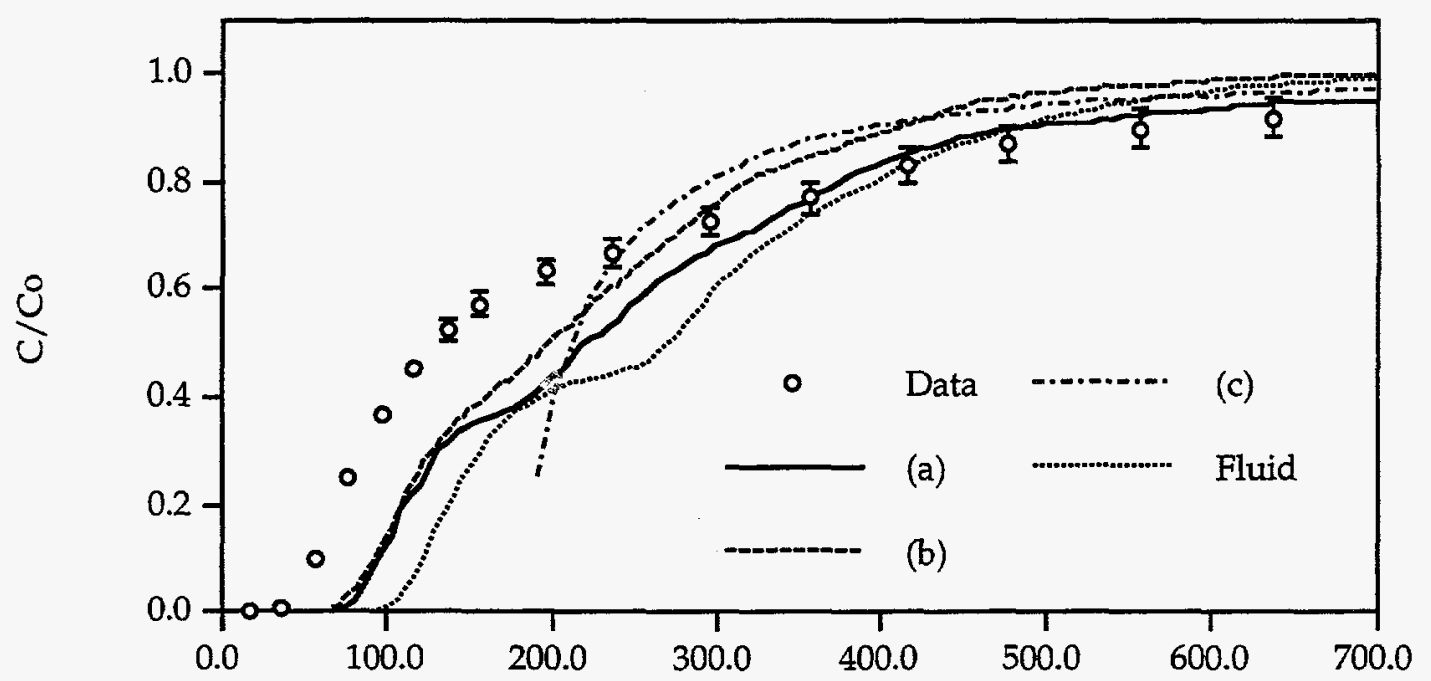

Time, sec

Figure D.57. Predicted and experimental breakthrough curves of 1- $\mu \mathrm{m} \mathrm{CML}$ microspheres in Experiment 12 (rotated Bullfrog tuff). See p. D-2 and Table D.1 for description of models and parameter values, respectively, corresponding to plot legend.

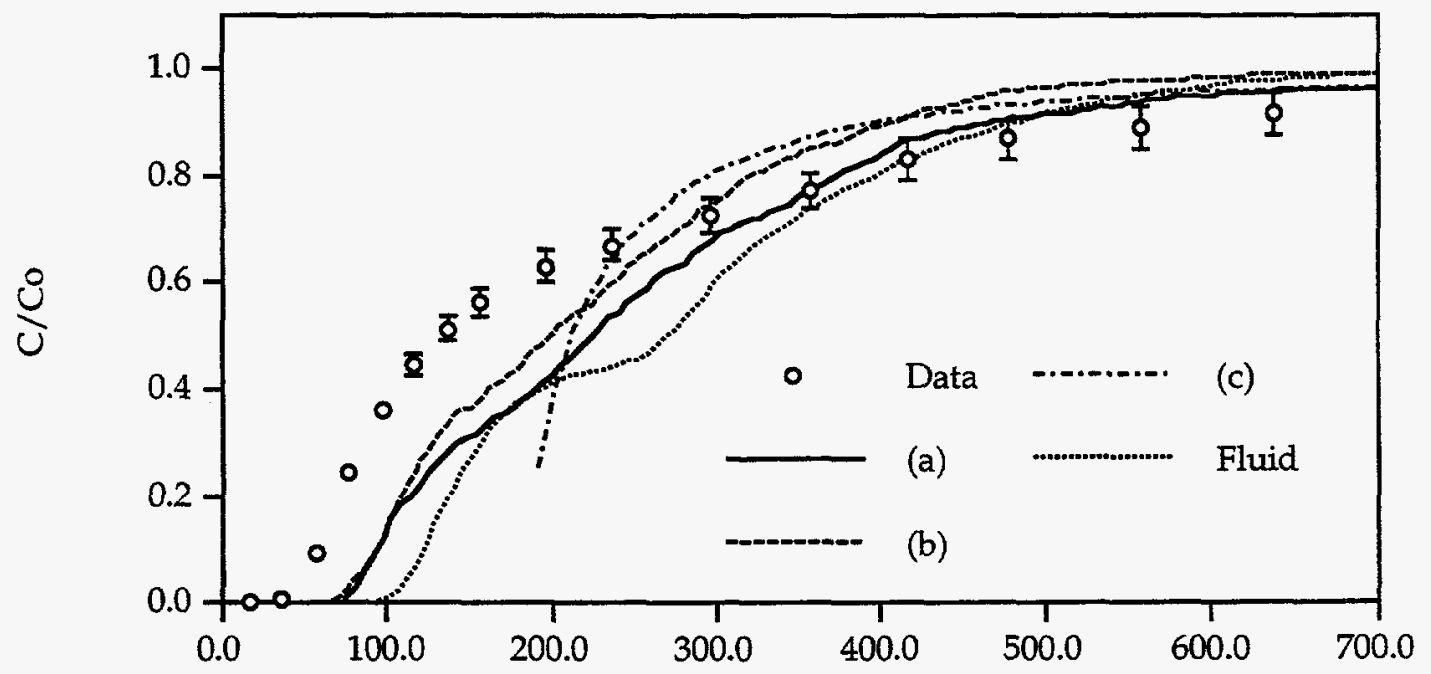

Time, sec

Figure D.58. Predicted and experimental breakthrough curves of $0.3-\mu \mathrm{m} C M L$ microspheres in Experiment 12 (rotated Bullfrog tuff). See p. D-2 and Table D.1 for description of models and parameter values, respectively, corresponding to plot legend. 


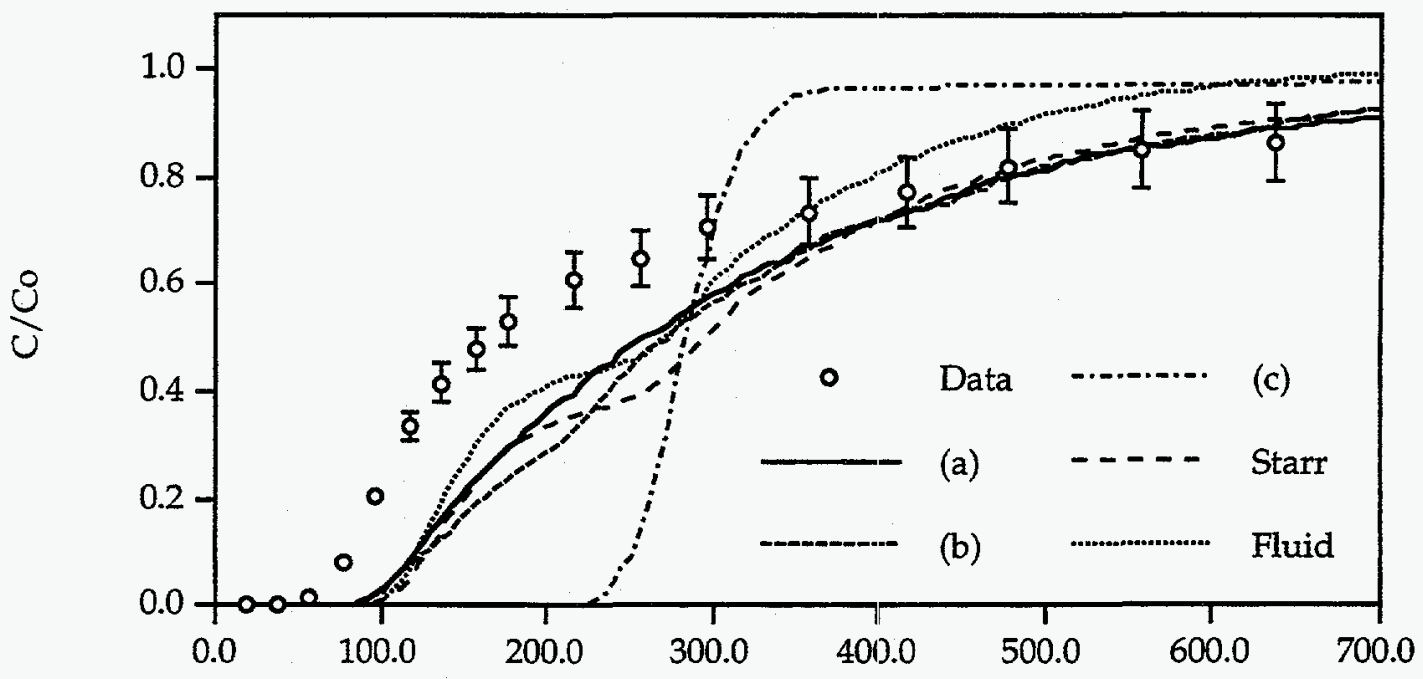

Time, sec

Figure D.59. Predicted and experimental breakthrough curves of iodide in Experiment 12 (rotated Bullfrog tuff). See p. D-2 and Table D.1 for description of models and parameter values, respectively, corresponding to plot legend. 


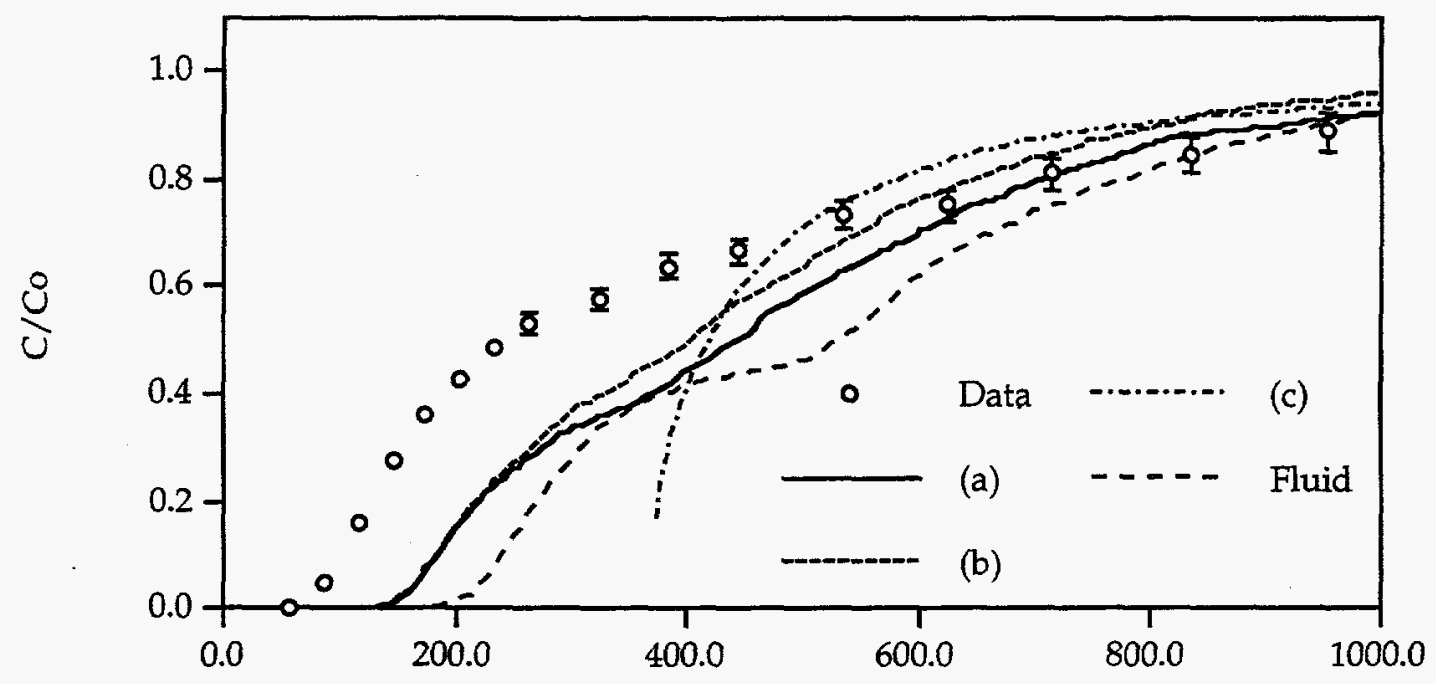

Time, sec

Figure D.60. Predicted and experimental breakthrough curves of 1- $\mu \mathrm{m} C \mathrm{CML}$ microspheres in Experiment 13 (rotated Bullfrog tuff). See p. D-2 and Table D.1 for description of models and parameter values, respectively, corresponding to plot legend.

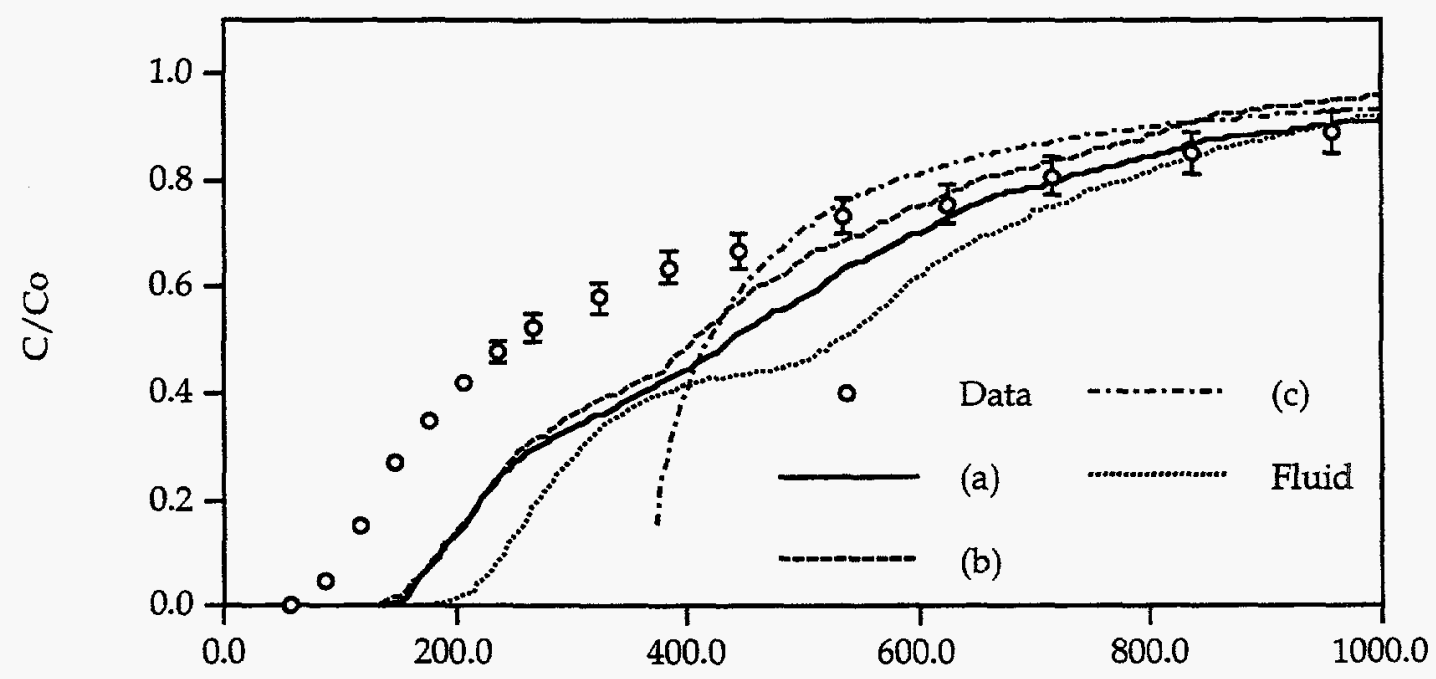

Time, sec

Figure D.61. Predicted and experimental breakthrough curves of $0.3-\mu \mathrm{m}$ CML microspheres in Experiment 13 (rotated Bullfrog tuff). See p. D-2 and Table D.1 for description of models and parameter values, respectively, corresponding to plot legend. 


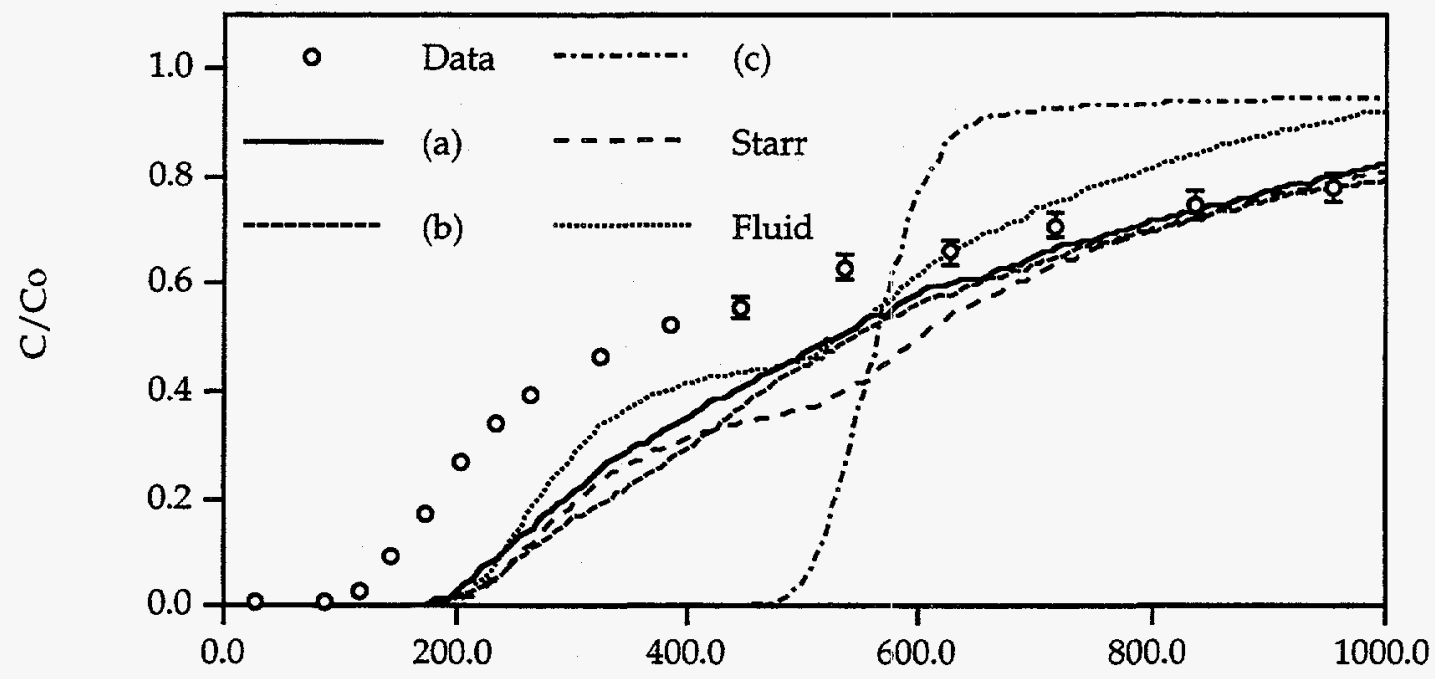

Time, sec

Figure D.62. Predicted and experimental breakthrough curves of iodide in Experiment 13 (rotated Bullfrog tuff). See p. D-2 and Table D.1 for description of models and parameter values, respectively, corresponding to plot legend. 


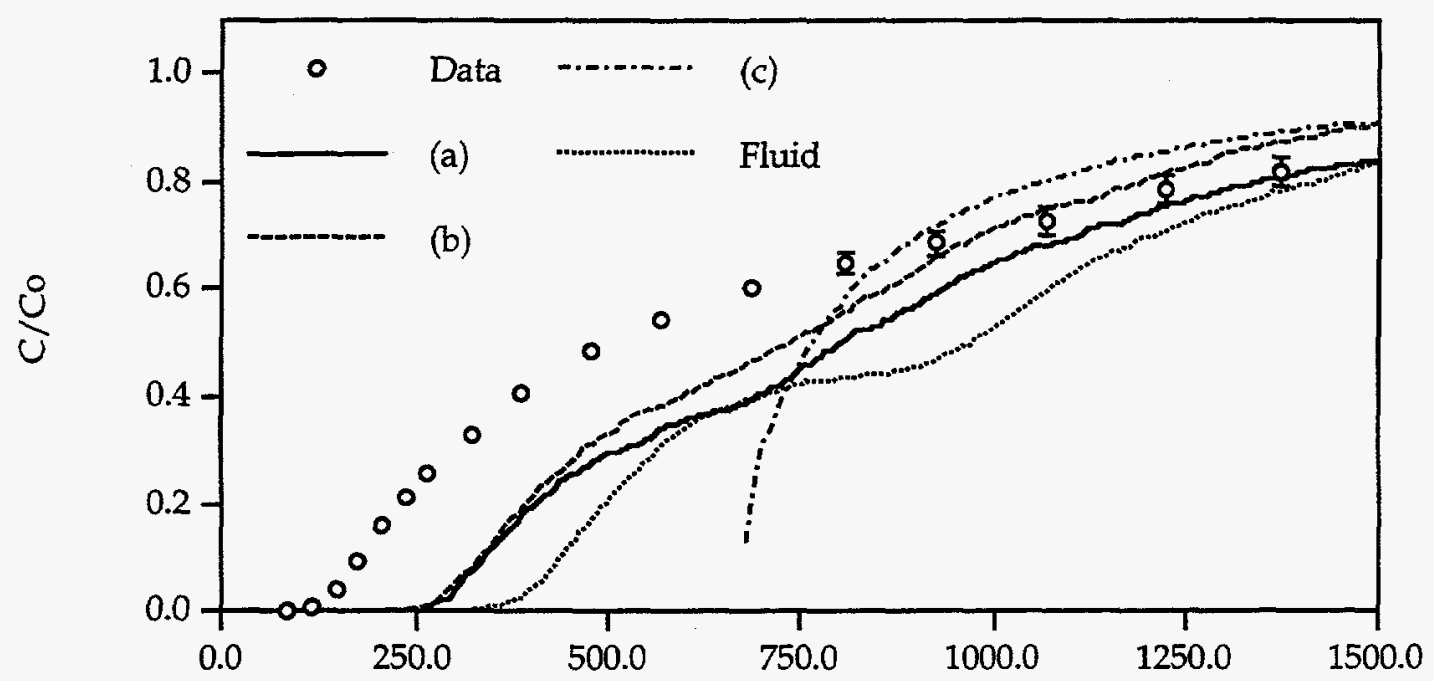

Time, sec

Figure D.63. Predicted and experimental breakthrough curves of 1- $\mu \mathrm{m} \mathrm{CML}$ microspheres in Experiment 14 (rotated Bullfrog tuff). See p. D-2 and Table D.1 for description of models and parameter values, respectively, corresponding to plot legend.

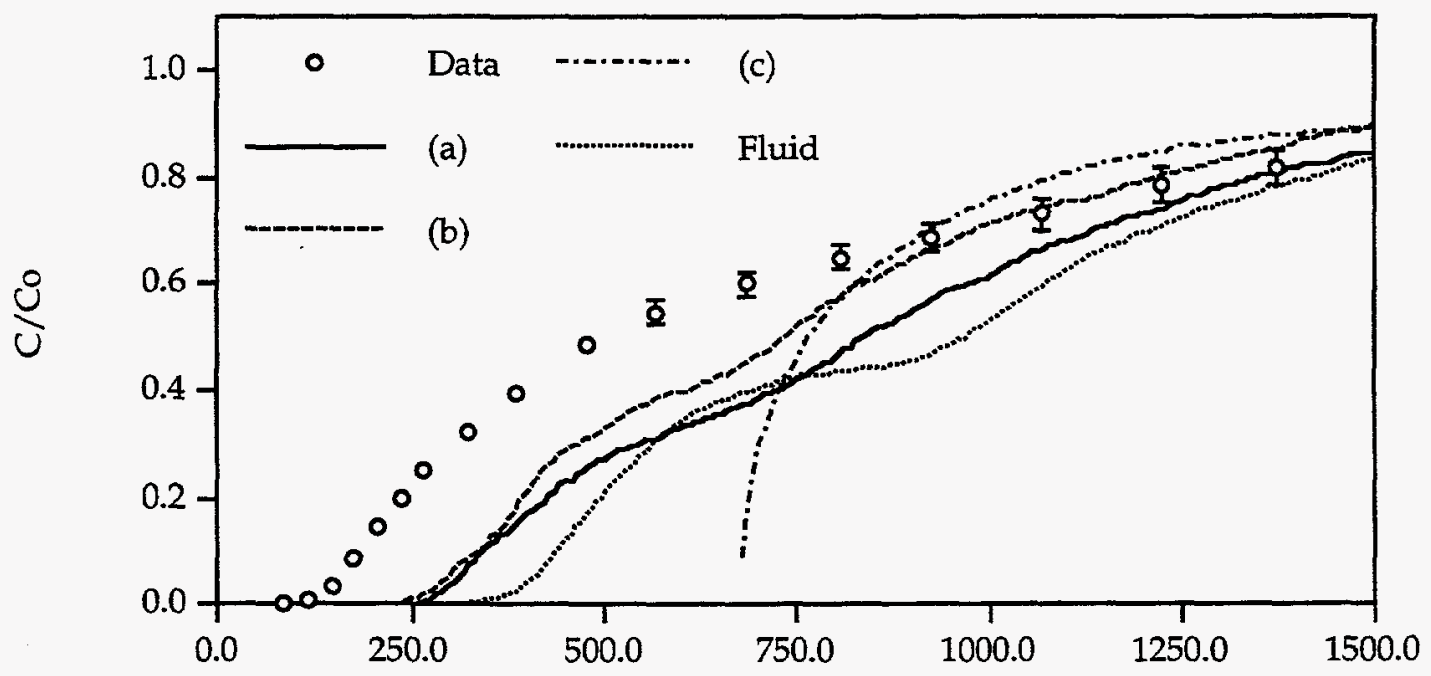

Time, sec

Figure D.64. Predicted and experimental breakthrough curves of $0.3-\mu \mathrm{m} \mathrm{CML}$ microspheres in Experiment 14 (rotated Bullfrog tuff). See p. D-2 and Table D.1 for description of models and parameter values, respectively, corresponding to plot legend. 


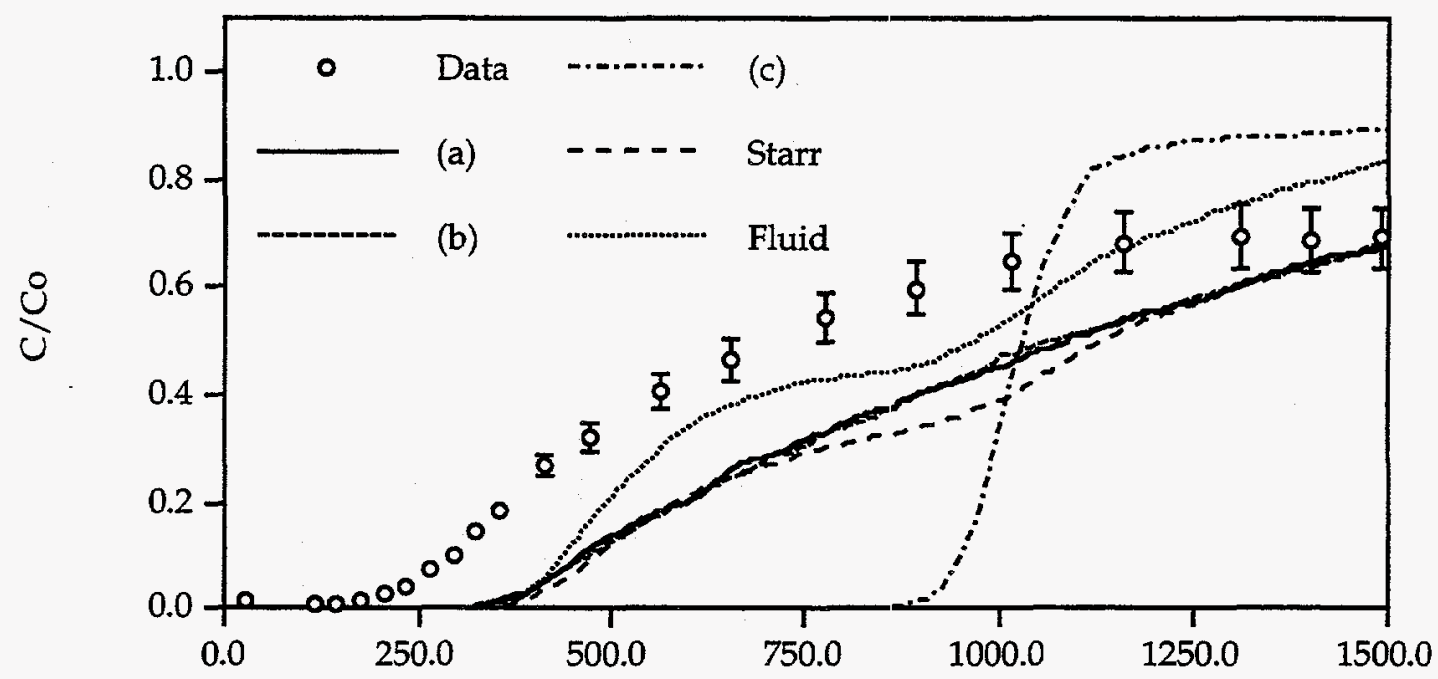

Time, sec:

Figure D.65. Predicted and experimental breakthrough curves of iodide in Experiment 14 (rotated Bullfrog tuff). See p. D-2 and Table D.1 for description of models and parameter values, respectively, corresponding to plot legend. 


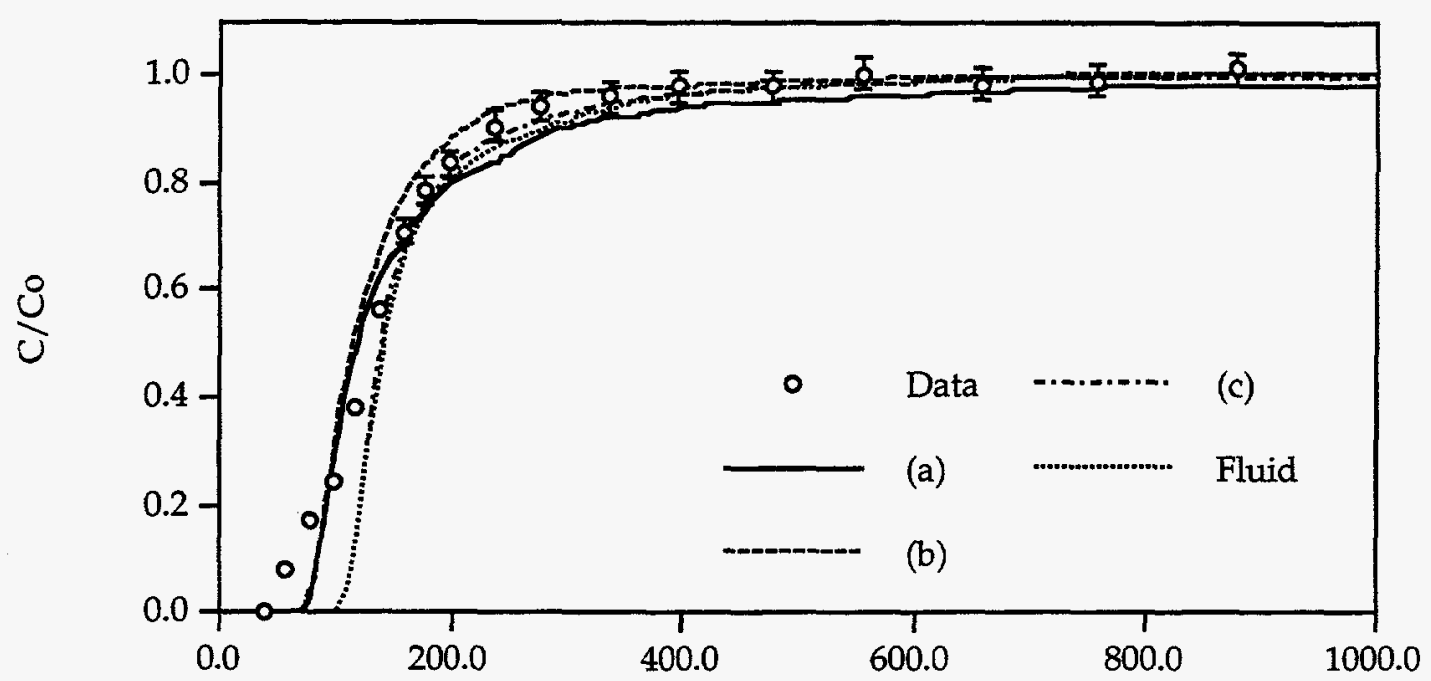

Time, sec

Figure D.66. Predicted and experimental breakthrough curves of $1-\mu \mathrm{m} \mathrm{CML}$ microspheres in Experiment 15 (Bullfrog tuff). See p. D-2 and Table D.1 for description of models and parameter values, respectively, corresponding to plot legend.

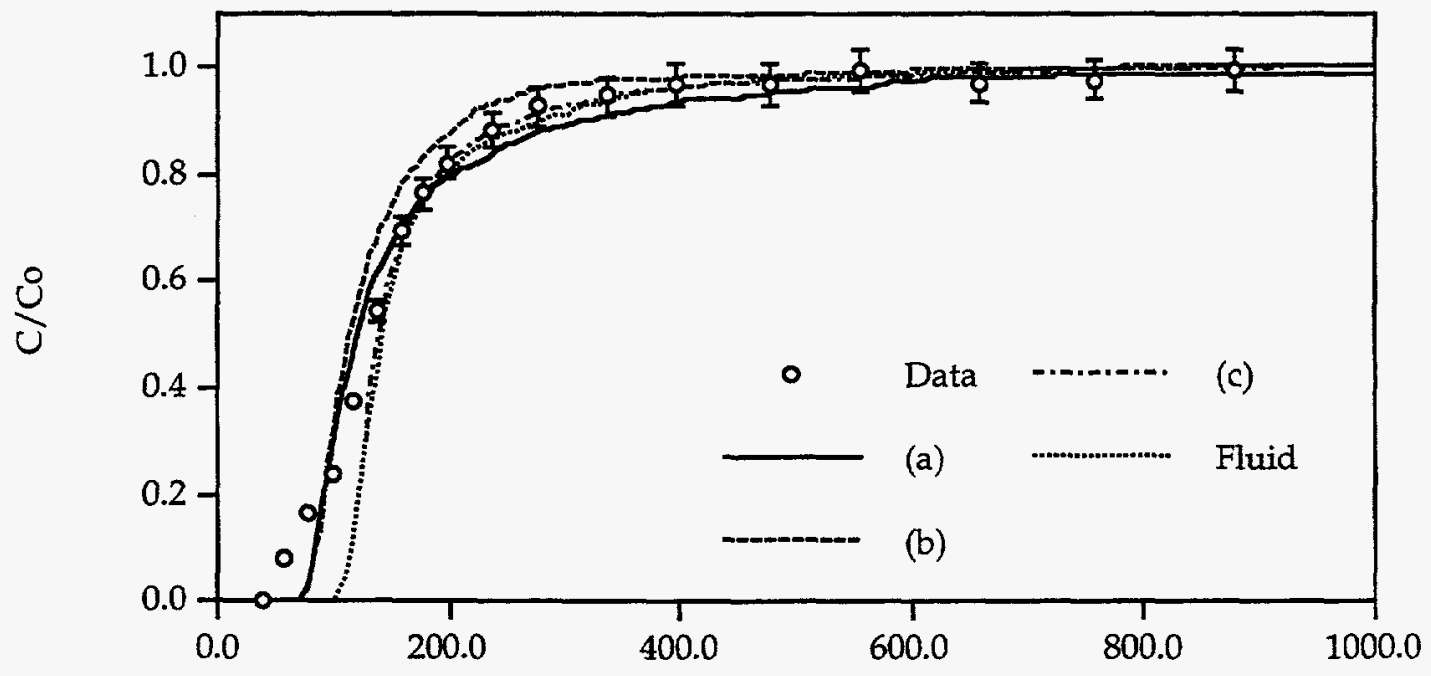

Time, sec

Figure D.67. Predicted and experimental breakthrough curves of $0.3-\mu \mathrm{m} \mathrm{CML}$ microspheres in Experiment 15 (Bullfrog tuff). See p. D-2 and Table D.1 for description of models and parameter values, respectively, corresponding to plot legend. 


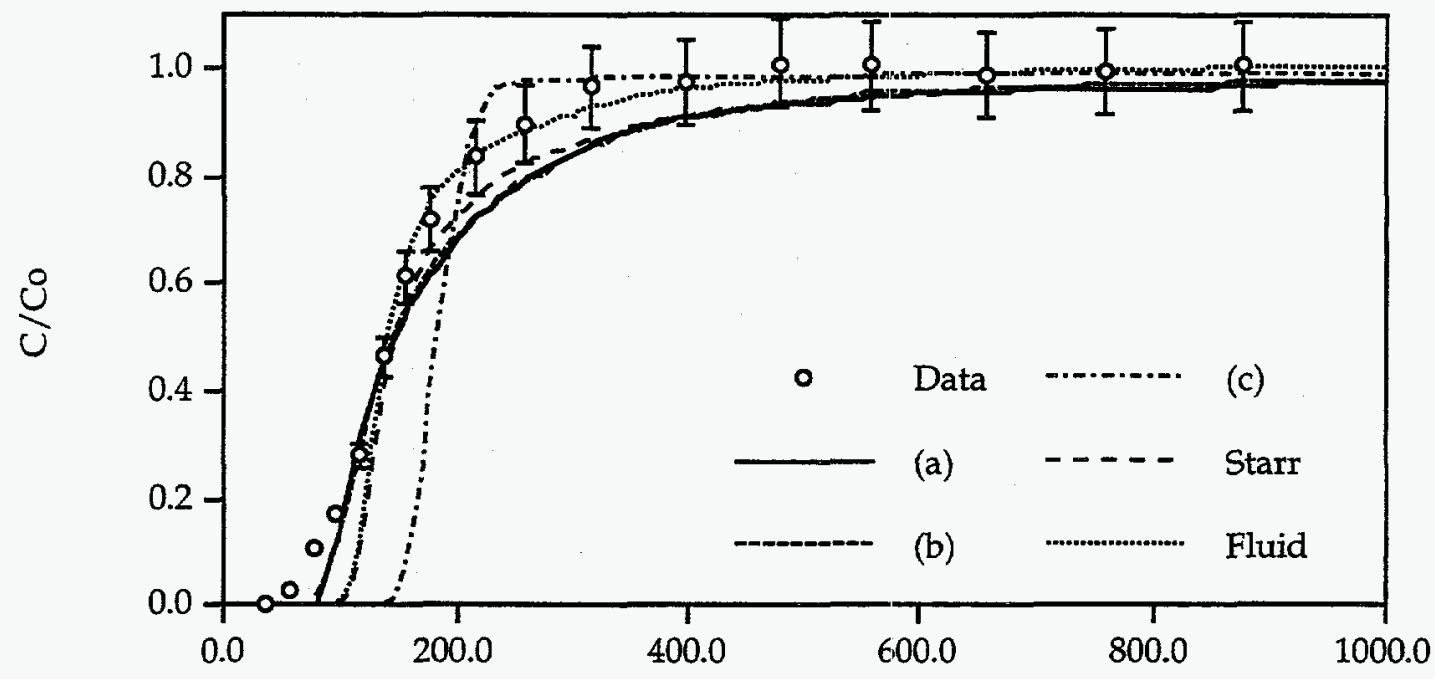

Time, sec

Figure D.68. Predicted and experimental breakthrough curves of iodide in Experiment 15 (Bullfrog tuff). See p. D-2 and Table D.1 for description of models and parameter values, respectively, corresponding to plot legend. 


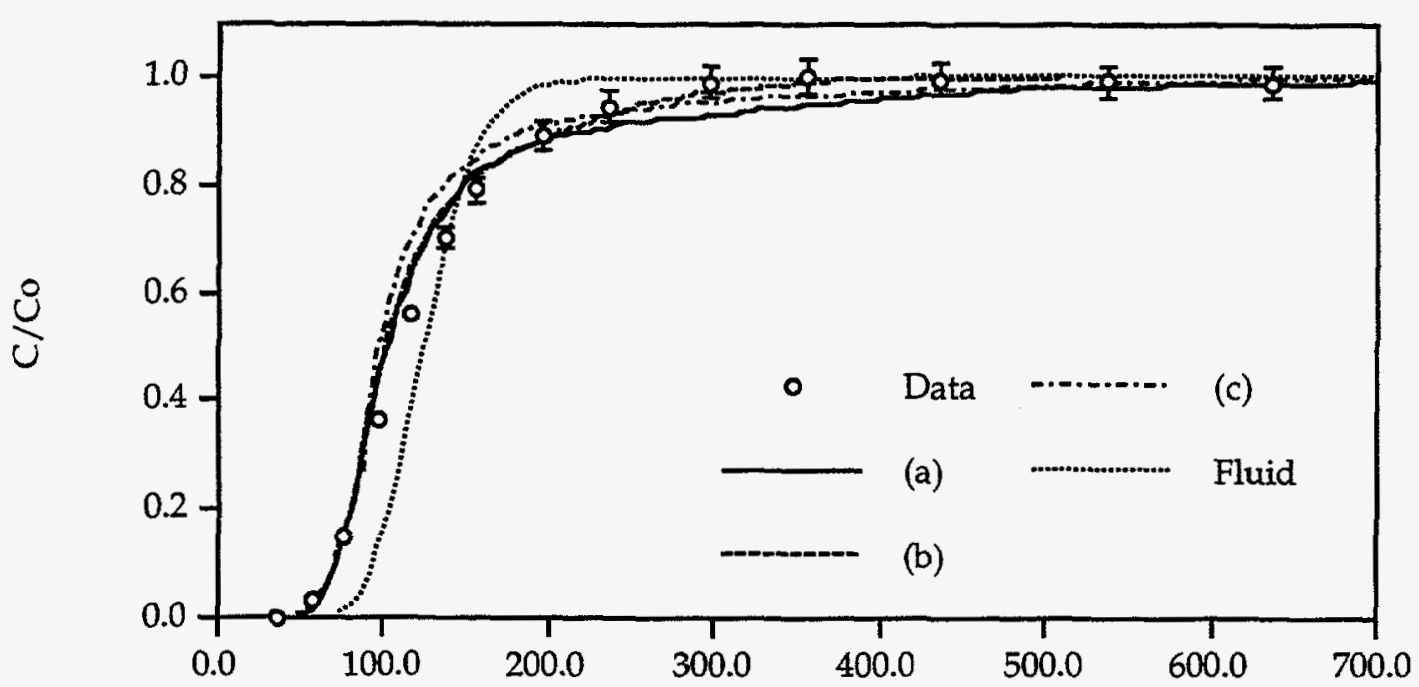

Time, sec

Figure D.69. Predicted and experimental breakthrough curves of $1-\mu \mathrm{m} C M L$ microspheres in Experiment 16 (Tram tuff). See p. D-2 and Table D.1 for description of models and parameter values, respectively, corresponding to plot legend.

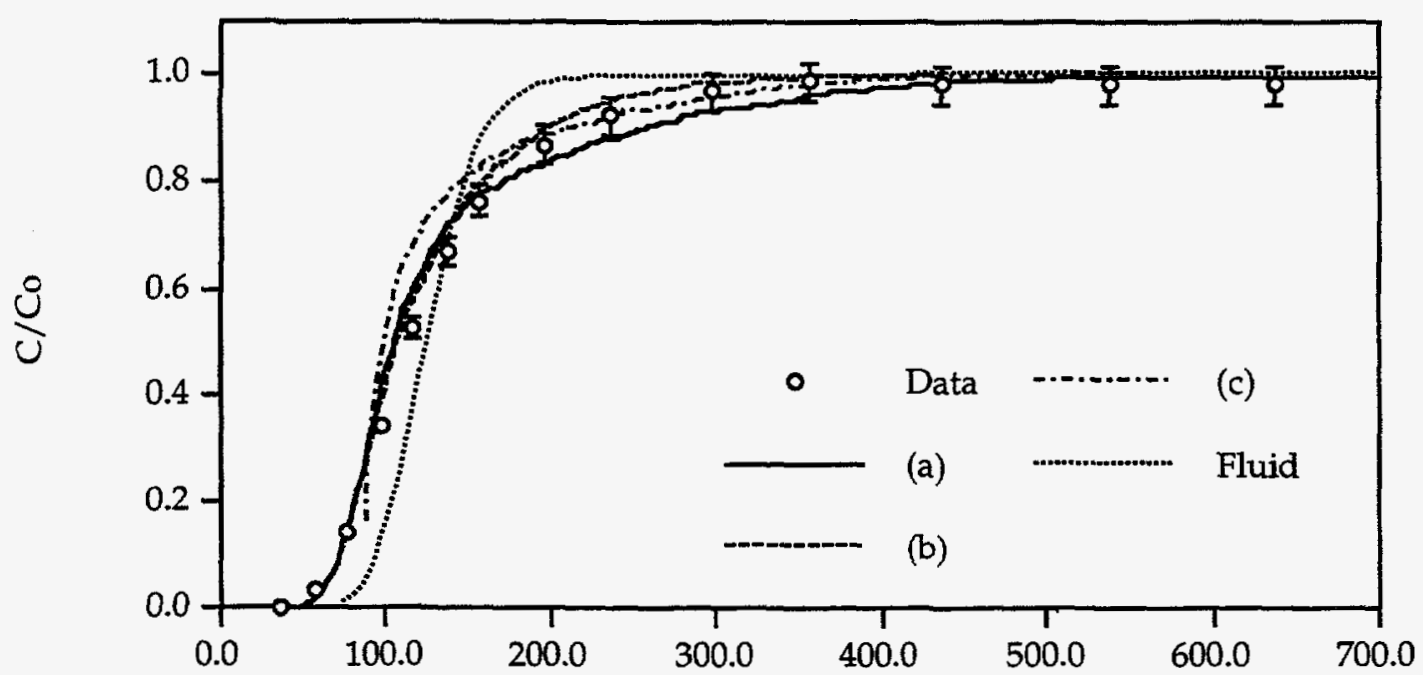

Time, sec

Figure D.70. Predicted and experimental breakthrough curves of $0.3-\mu \mathrm{m} C M L$ microspheres in Experiment 16 (Tram tuff). See p. D-2 and Table D. 1 for description of models and parameter values, respectively, corresponding to plot legend. 


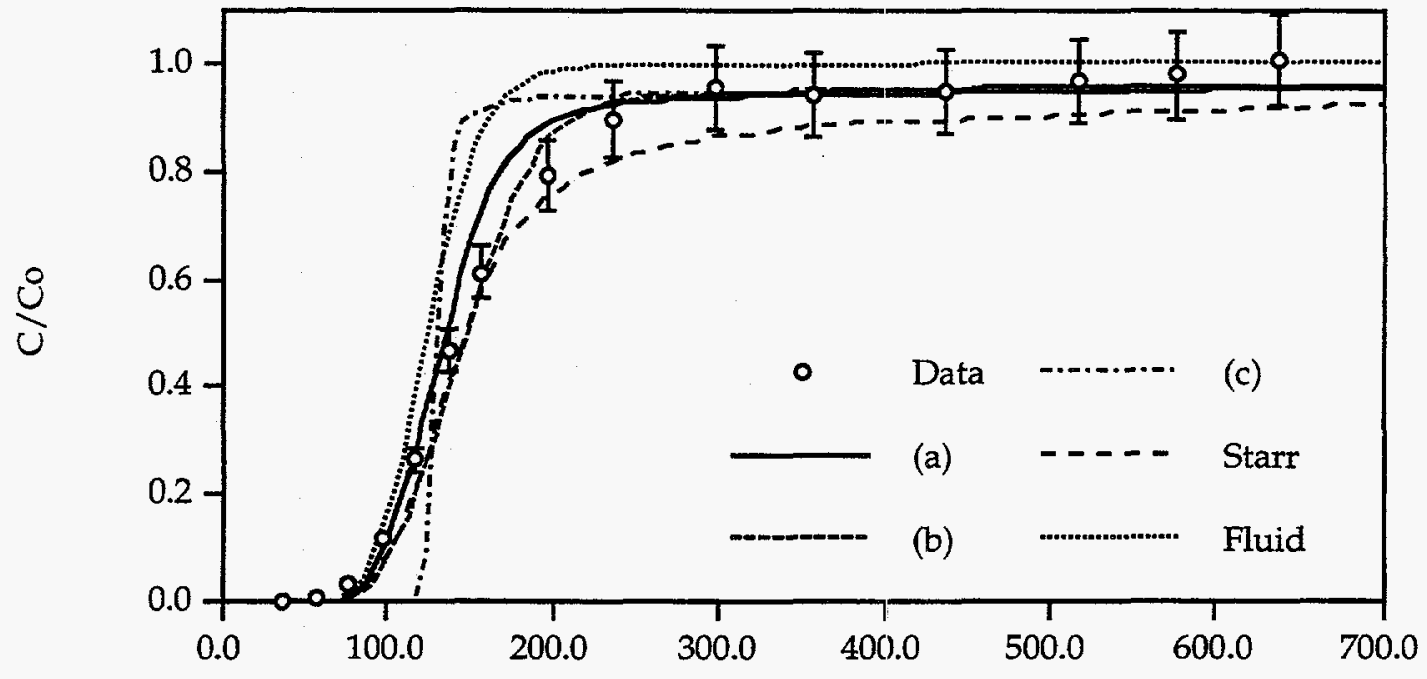

Time, sec

Figure D.71. Predicted and experimental breakthrough curves of iodide in Experiment 16 (Tram tuff). See p. D-2 and Table D. 1 for description of models and parameter values, respectively, corresponding to plot legend. 


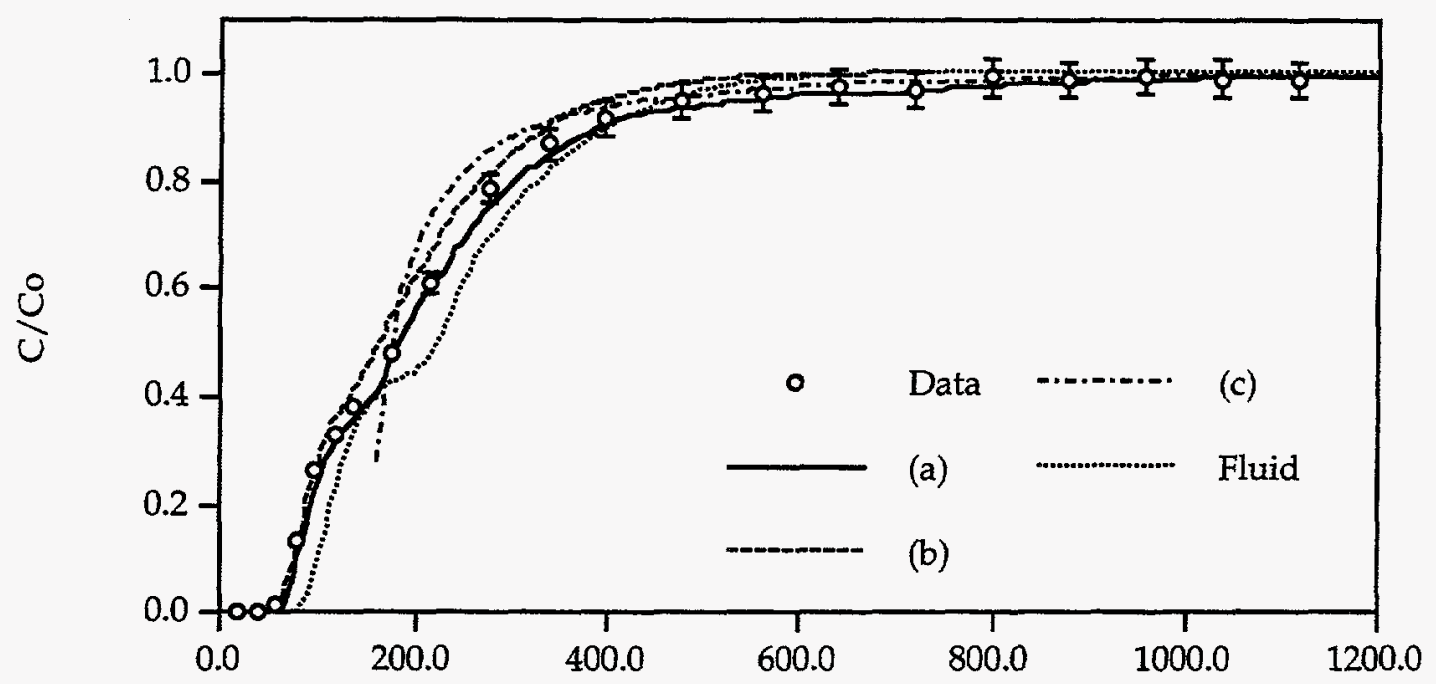

Time, sec

Figure D.72. Predicted and experimental breakthrough curves of 1- $\mu \mathrm{m} C \mathrm{CML}$ microspheres in Experiment 17 (rotated Bullfrog tuff). See p. D-2 and Table D.1 for description of models and parameter values, respectively, corresponding to plot legend.

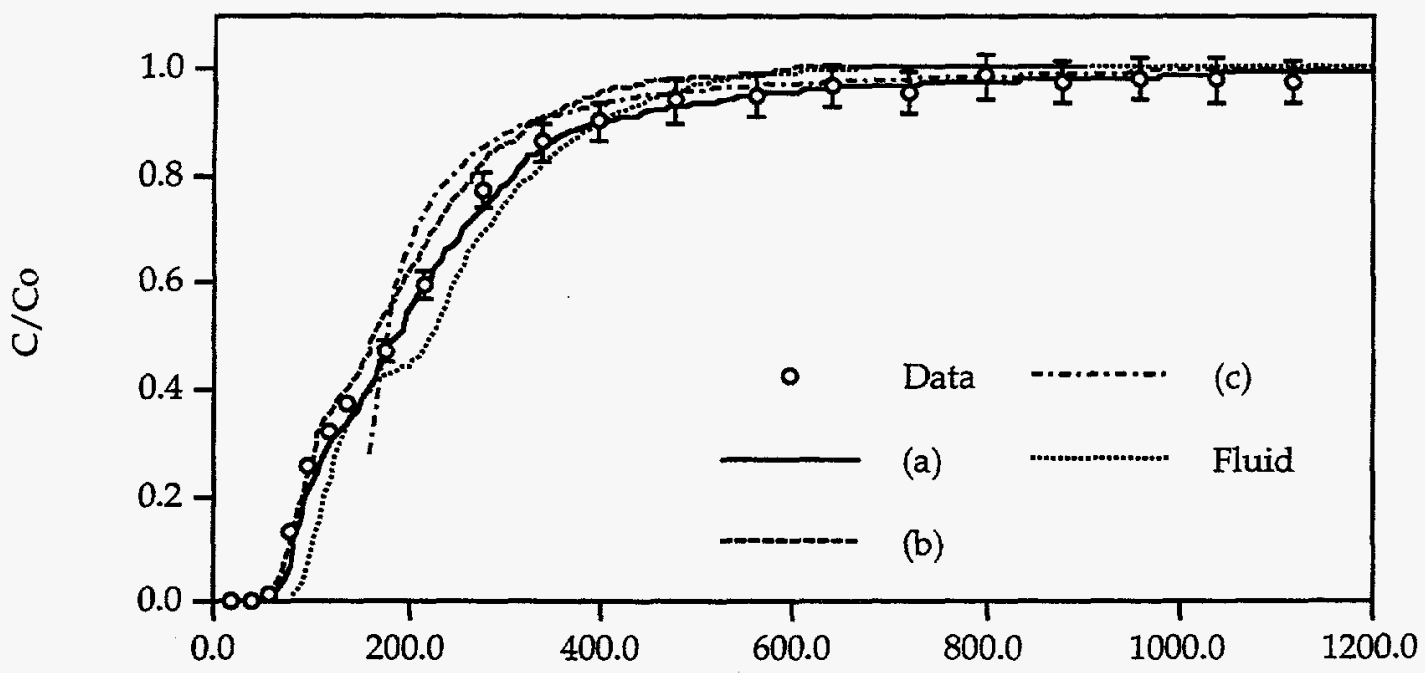

Time, sec

Figure D.73. Predicted and experimental breakthrough curves of $0.3-\mu \mathrm{m}$ CML microspheres in Experiment 17 (rotated Bullfrog tuff). See p. D-2 and Table D.1 for description of models and parameter values, respectively, corresponding to plot legend. 


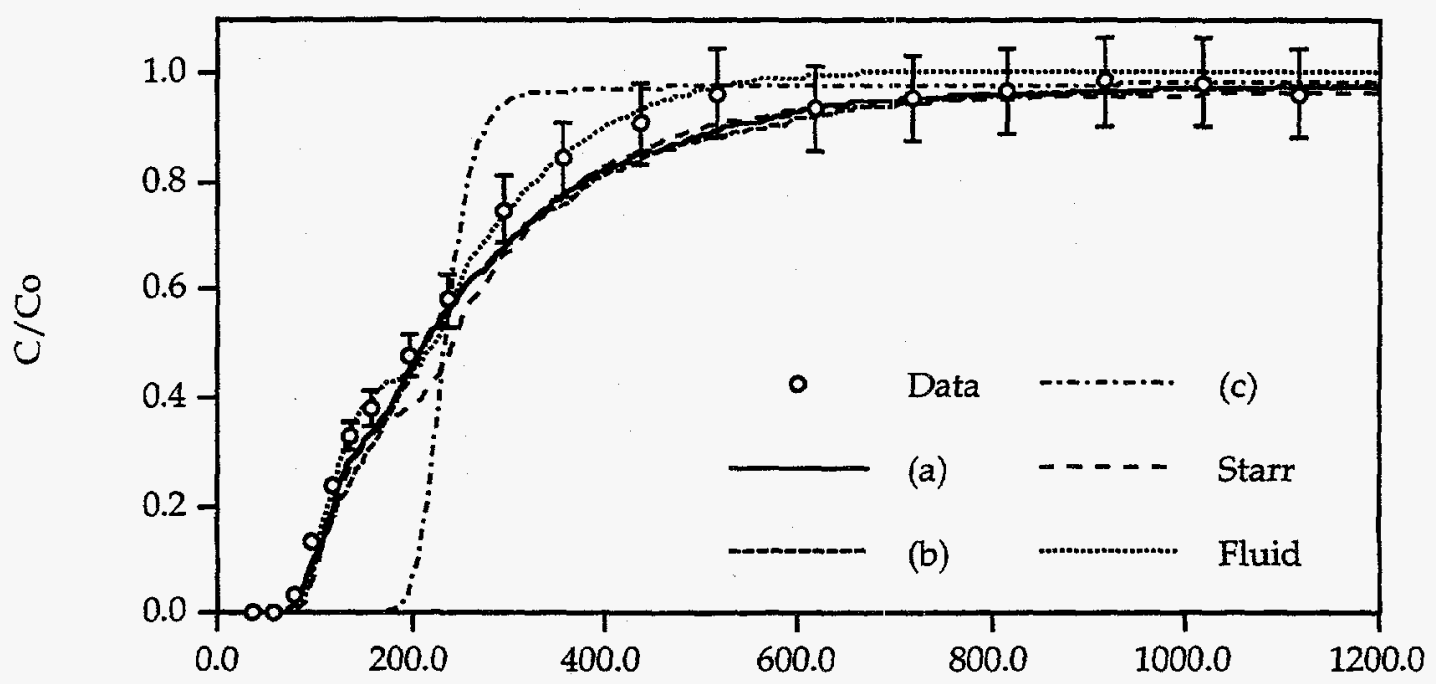

Time, sec

Figure D.74. Predicted and experimental breakthrough curves of iodide in Experiment 17 (rotated Bullfrog tuff). See p. D-2 and Table D.1 for description of models and parameter values, respectively, corresponding to plot legend. 


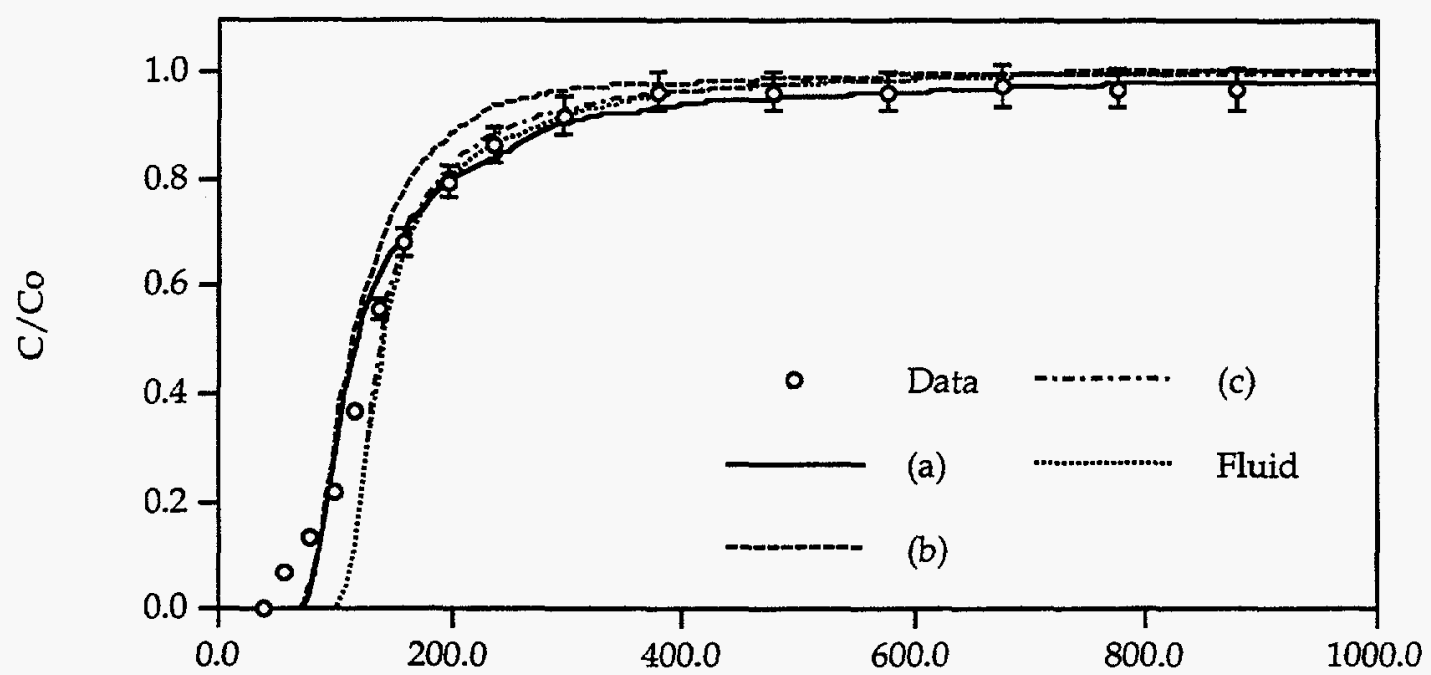

Time, sec

Figure D.75. Predicted and experimental breakthrough curves of 1- $\mu \mathrm{m}$ CML microspheres in Experiment 18 (Bullfrog tuff). See p. D-2 and Table D.1 for description of models and parameter values, respectively, corresponding to plot legend.

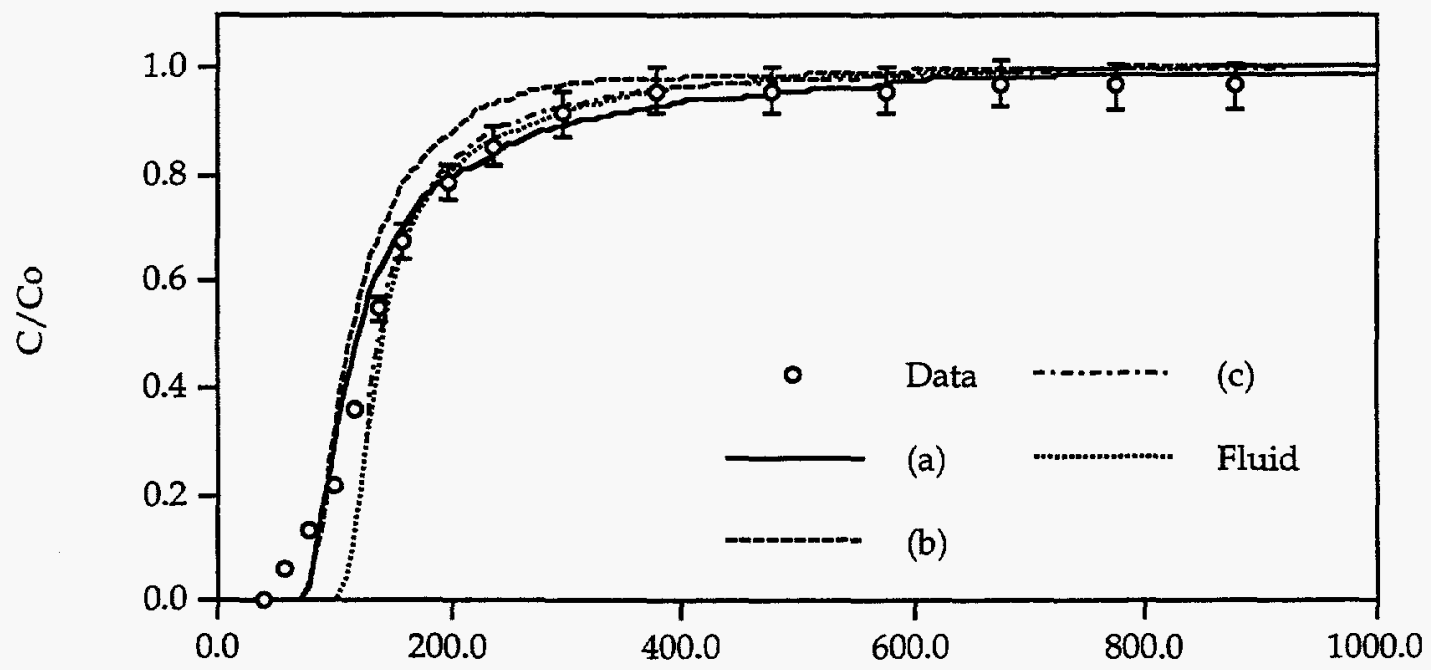

Time, sec

Figure D.76. Predicted and experimental breakthrough curves of $0.3-\mu \mathrm{m} C M L$ microspheres in Experiment 18 (Bullfrog tuff). See p. D-2 and Table D.1 for description of models and parameter values, respectively, corresponding to plot legend. 


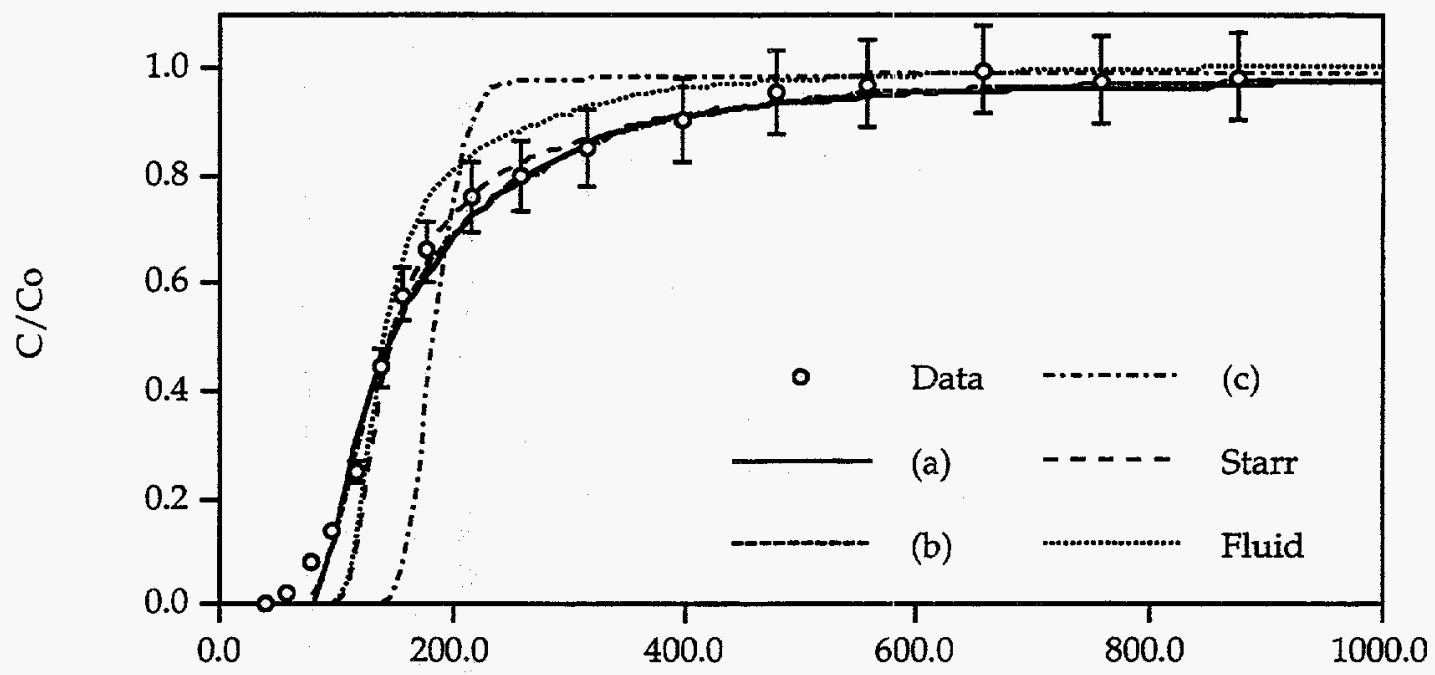

Time, sec

Figure D.77. Predicted and experimental breakthrough curves of iodide in Experiment 18 (Bullfrog tuff). See p. D-2 and Table D.1 for description of models and parameter values, respectively, corresponding to plot legend. 